\title{
Haas, Elisabeth
}

\section{Mentoringprozesse in der Lehrer:innenausbildung. Gelingensbedingungen für Schulpraktika}

Bad Heilbrunn : Verlag Julius Klinkhardt 2021, 265 S. - (klinkhardt forschung)

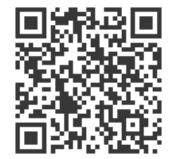

Quellenangabe/ Reference:

Haas, Elisabeth: Mentoringprozesse in der Lehrer:innenausbildung. Gelingensbedingungen für Schulpraktika. Bad Heilbrunn : Verlag Julius Klinkhardt 2021, 265 S. - (klinkhardt forschung) - URN: urn:nbn:de:0111-pedocs-227864 - DOI: 10.25656/01:22786

https://nbn-resolving.org/urn:nbn:de:0111-pedocs-227864

https://doi.org/10.25656/01:22786

in Kooperation mit / in cooperation with:

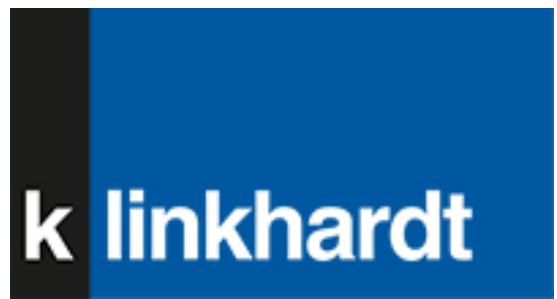

http://www.klinkhardt.de

\section{Nutzungsbedingungen}

Dieses Dokument steht unter folgender Creative Commons-Lizenz: http://creativecommons.org/licenses/by-nc-nd/4.0/deed.de - Sie dürfen das Werk bzw. den Inhalt unter folgenden Bedingungen vervielfältigen, verbreiten und öffentlich zugänglich machen: Sie müssen den Namen des Autors/Rechteinhabers in der von ihm festgelegten Weise nennen. Dieses Werk bzw. dieser Inhalt darf nicht für kommerzielle Zwecke verwendet werden und es darf nicht bearbeitet, abgewandelt oder in anderer Weise verändert werden.

Mit der Verwendung dieses Dokuments erkennen Sie die Nutzungsbedingungen an.

\section{Terms of use}

This document is published under following Creative Commons-License: http://creativecommons.org/licenses/by-nc-nd/4.0/deed.en - You may copy, distribute and transmit, adapt or exhibit the work in the public as long as you attribute the work in the manner specified by the author or licensor. You are not allowed to make commercial use of the work or its contents. You are not allowed to alter, transform, or change this work in any other way.

By using this particular document, you accept the above-stated conditions of use.

\section{Kontakt / Contact:}

\section{peDOCS}

DIPF | Leibniz-Institut für Bildungsforschung und Bildungsinformation Informationszentrum (IZ) Bildung

E-Mail: pedocs@dipf.de

Internet: www.pedocs.de

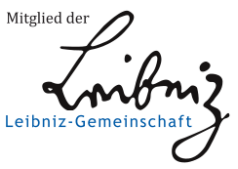


Haas

Mentoringprozesse in der Lehrer:innenausbildung 



\section{Elisabeth Haas}

\section{Mentoringprozesse in der Lehrer:innenausbildung}

Gelingensbedingungen für Schulpraktika 
Die vorliegende Arbeit wurde von der Fakultät für LehrerInnenbildung der Universität Innsbruck unter dem Titel „Gelingensbedingungen schulpraktischer Mentoringprozesse in der Lehrer/innenausbildung“ als Dissertation angenommen.

Gutachter: Univ.-Prof. Mag. Dr. Christian Kraler.

Tag der Disputation: 30.06.2020.

Dieser Titel wurde in das Programm des Verlages mittels eines Peer-Review-Verfahrens aufgenommen. Für weitere Informationen siehe www.klinkhardt.de.

Bibliografische Information der Deutschen Nationalbibliothek Die Deutsche Nationalbibliothek verzeichnet diese Publikation in der Deutschen Nationalbibliografie; detaillierte bibliografische Daten sind im Internet abrufbar über http://dnb.d-nb.de.

2021.kg. (C) by Julius Klinkhardt.

Bildnachweis Umschlagseite 1: Claudia M. Haas, Innsbruck.

Druck und Bindung: Bookstation GmbH, Anzing.

Printed in Germany 2021.

Gedruckt auf chlorfrei gebleichtem alterungsbeständigem Papier.

(c) (i) $\odot$ Die Publikation (mit Ausnahme aller Fotos, Grafiken und Abbildungen) ist veröffentlicht unter der Creative Commons-Lizenz: CC BY-NC-ND 4.0 International https://creativecommons.org/licenses/by-nc-nd/4.0/ 


\section{Zusammenfassung}

Schulpraktisches Mentoring findet in Österreich in den Curricula der neuen Lehrer/innenausbildung mit der Implementierung der Pädagogisch-Praktischen Studien eine strukturelle Verankerung. Partnerschulen der Pädagogischen Hochschulen und Universitäten bieten mit ihren Lern- und Erfahrungsräumen Studierenden die Möglichkeit, die normativ-curricularen schulischen Anteile im Sozialisationsfeld Schule zu absolvieren. Mentor/innen begleiten und unterstützen im Professionalisierungsprozess und treten vor dem Hintergrund normativ-curricularer Anforderungsstrukturen sowie subjektiver Deutungsmuster, Ermöglichungs- und Erwartungshaltungen im Regelfall in eine wechselseitige Lern- und Entwicklungsbeziehung ein. Transformationales Mentoring mit einer kategorialen Aufschlüsselung zur Anleitung von Selbstreflexion wird als eine mögliche Form des Mentorings vorgestellt und diskutiert.

Im Forschungsansatz wurden Interviews mit Mentor/innen und Studierenden geführt und mit Grounded Theory ausgewertet. Zentrales Ergebnis der Studie ist, dass die Beteiligten in der dyadischen Beziehung einen professionsspezifischen Lern- und Entwicklungsprozess mit dem Ziel der Selbstwirksamkeits- und Professionsentwicklung aufbauen bzw. eingehen wollen. Daraus ableitend wurden (Ausbildungs-)Modelle für Mentoring-Programme konstruiert. 


\begin{abstract}
School mentoring in Austria is structurally anchored in the curricula of the new teacher training with the establishment/implementation of pedagogical-practical studies. Partner schools of universities of teacher education and universities offer students space for learning experience through practice and opportunity to complete the curricular parts of school in social environment of schools. Mentors accompany and support the professionalization process and enter into a mutual learning and developmental relationship against the background of curricular requirement structures as well as subjective interpretative patterns. Transformational mentoring with a categorical breakdown to guide self-reflection is presented and discussed as a possible form of mentoring.

In the research approach, interviews with mentors and students were conducted and evaluated with Grounded Theory. The central result of the study is that those involved in the dyadic relationship want to build up or want to enter into a profession-specific learning and development process with the aim of furthering their own effectiveness and professionalism. Emanating from these studies, (training) models for mentoring programs were constructed.
\end{abstract}




\section{Christian Kraler}

\section{Zur Einleitung - Mentoring im Kontext formaler Bildung}

Mentor/innentätigkeit an und für sich ist im Bildungssystem nicht unbekannt. Seit der Antike begleiten Lehrpersonen Lernende und erschließen im Unterricht die Welt, insbesondere bezogen auf Inhalte, Normen und Werte. In jüngster Zeit bekam der mentoriale Aspekt des Lehrer/ innenberufs durch den "paradigmatic shift from teaching to learning " rund um die Jahrtausendwende eine noch stärkere Bedeutung. Dies vor allem mit dem Übergang von der kompetenzorientierten Individualisierung hin zu einer Personalisierung von Lernprozessen auch innerhalb des formalen Bildungssystems. Der sich seit langem langsam vollziehende Wandel von der Lehrhin zu einer Lernzentrierung (konkrete moderne Ansätze finden sich zumindest bereits bei Comenius) bedingt, dass spätestens seit der zweiten empirischen Wende rund um 2000 die Begleitungsfunktion bei Lernprozessen, damit auch mentor/innenbezogene Tätigkeiten des Lehrer/ innenberufs, bei der Arbeit im Klassenraum eine zunehmend gewichtigere Rolle spielen.

Innerhalb der bildungswissenschaftlichen Forschung lässt sich über den Diskurs der letzten etwa 20 Jahre gut nachzeichnen, wie sich im Feld der formalen Bildung der Fokus koevolutiv sukzessive erweitert hat: Beginnend mit Schülerinnen und Schülern, insbesondere über die großen internationalen Schulleistungsvergleichsstudien sowie umfangreiche Untersuchungen zur Unterrichtsqualität, übergehend auf Lehrpersonen sowohl im Ausbildungskontext als auch im Berufsfeld und schließlich hin zur Gruppe der Lehrerbildner/innen.

Hier setzt die vorliegende Arbeit von Frau Elisabeth Haas, $\mathrm{PhD}$ thematisch an und ist damit, wie die skizzierte Entwicklung zeigt, hochaktuell. Frühe systematische Berichte zum (schulpraktischen) Mentoring in der Lehramtsausbildung reichen im deutschen Sprachraum bis in die 1980iger Jahre zurück. „Fahrt aufgenommen“ hat die Thematik ab etwa 2010, unter anderem mit vermehrten Veröffentlichungen in Zeitschriften, facheinschlägigen Tagungen sowie Sammelbänden. Die umfangreichen Reformen der Lehrer/innenbildung im Gefolge von PISA und „Bologna“ ungefähr zur selben Zeit mündeten automatisch auch in einen Handlungsdruck auch im Bereich der Begleitung Studierender in ihrer schulpraktischen Ausbildung sowie in den ersten Berufsjahren.

Bis in die 1980/90iger Jahre bestand die Qualifizierung von mentoriell tätigen Lehrpersonen in der Regel in ihrer eigenen langjährigen Berufserfahrung. Warum sollte das gegenwärtig nicht mehr ausreichen? Schulpraktische Erfahrung dürfte heute eine grundlegende Expertise sein wie vor 50 Jahren ... Elisabeth Haas würde auf diese Frage wohl mit einem fundiert begründeten „Ja, aber ..." antworten. - Eine detaillierte Antwort ist sowohl theoretisch begründet wie empirisch untersucht, im vorliegenden Band zu finden. Schule und formale Bildung, damit auch die Lehrer/innenbildung, sind Frau Haas zufolge immer auch Resonanzräume gesellschaftlicher Entwicklungen. Rahmenbedingungen, berufliche Anforderungen, strukturelle wie gesellschaftliche Herausforderungen erfordern heute eine andere, auch umfassendere Form der Professionalisierung in der Begleitung auszubildender wie in den Beruf einsteigender Kolleg/innen.

Elisabeth Haas zeichnet mit ihrer Arbeit insbesondere für Österreich wohl erstmalig in einer Zusammenschau die historische Genese schulpraktischer Begleitung, deren inhaltlichen Dimensionen sowie die aktuellsten Entwicklungen und gesetzlichen Rahmenbedingungen aus einer ganzheitlichen Perspektive auf und führt so verschiedene Aspekte fokussierend zusammen. 
Selbst facheinschlägig kundige Interessierte finden hier vermutlich neue, bisher wenig bekannte Hinweise. Frau Haas bleibt jedoch - auch das dürfte die Arbeit für Leser/innen interessant machen - nicht bei grundlegenden Analysen stehen. Die Autorin legt mit den ersten 140 Seiten vielmehr ein theoretisches Fundament für ihren empirischen Zugang. Die Untersuchung bezieht sich zwar auf eine spezifische Ausbildungskohorte und Ausbildungsstätte, ist jedoch hinsichtlich ihrer Anlage und Fragestellung prototypisch und damit wohl für viele in der Lehrer/ innenbildung Tätige mit Gewinn zu lesen. Die bereits im Buchtitel anklingende Ausrichtung fragt im Kern nach dem, was es für nachhaltige Mentoringprozesse braucht bzw. was solche ausmacht. - Was kann zum Gelingen beitragen?

Frau Haas betont mehrfach, dass schulpraktische Mentoringprozesse in der Lehrer/innenausbildung vielschichtig und multifaktoriell und situativ bedingt sind, es wohl keine einfachen Antworten gibt. Gerade deshalb kann der konzeptionelle Zugang über Gelingensbedingungen ungemein anregend sein, sowohl hinsichtlich wissenschaftlicher Fragestellungen wie auch mit Blick auf die Praxis.

$\mathrm{Ob}$ man das Buch nun von vorne bis hinten durchliest, in einzelne Kapitel blättert oder sich auf die Interpretation der Untersuchungsergebnisse, die modellhafte Konzeptionierung der Integration oder das Resümee konzentiert, spannend und gewinnbringend zu lesen, ist es wohl in jedem Fall. Das Thema Mentoring wird in der Lehrer/innenbildung in den kommenden Jahren jedenfalls eine gewichtige Rolle spielen.

Elisabeth Haas liefert mit ihren Überlegungen, Modellierungen und Befunden einen für die scientific community wie Praktikerinnen und Praktikern gewichtigen und mit Gewinn zu lesenden Beitrag. 


\section{Inhalt}

Einführung und Kontextualisierung der Fragestellung $\ldots \ldots \ldots \ldots \ldots \ldots \ldots \ldots \ldots, 11$

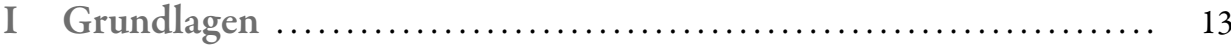

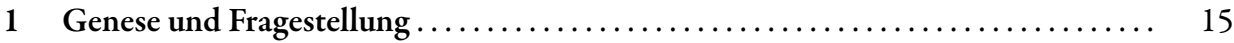

1.1 Zur Relevanz der Pädagogisch-Praktischen Studien im Lehramtsstudium ...... 16

1.1.1 Historiografische Betrachtung von Schulpraktika .............. 17

1.1.2 Gesetzliche Bestimmungen für Ausbildungscurricula in Österreich ..... 19

1.1.3 Zielsetzungen und Modelle der Pädagogisch-Praktischen Studien ...... 23

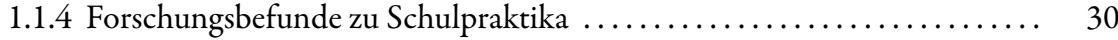

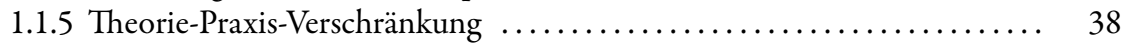

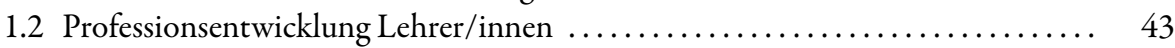

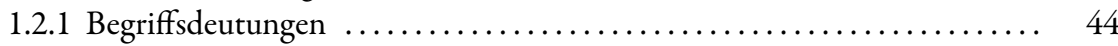

1.2.2 Forschungsansätze zur Professionalität im Lehrberuf ............. 46

1.2.3 Modelle zu Lehrer/innenkompetenzen .................. 48

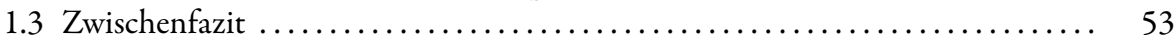

2 Mentoring als Begleitform für Schulpraktika $\ldots \ldots \ldots \ldots \ldots \ldots \ldots \ldots \ldots \ldots$

2.1 Mentoring unter begriffsentwickelnder Perspektive ................. 57

2.2 Mentoring in unterschiedlichen Kontexten $\ldots \ldots \ldots \ldots \ldots \ldots \ldots \ldots \ldots \ldots .60$

2.3 Forschungsbefunde zum schulpraktischen Mentoring $\ldots \ldots \ldots \ldots \ldots \ldots \ldots \ldots$

2.4 Mentoringprozess in der Lehrer/innenausbildung $\ldots \ldots \ldots \ldots \ldots \ldots \ldots \ldots . \ldots \ldots$

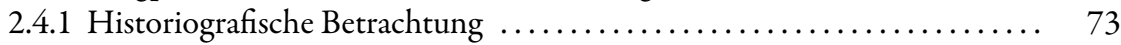

2.4.2 Kontextuelle Einbettung schulpraktischen Mentorings ............ 80

2.4.3 Gestaltungsparameter schulpraktischen Mentorings ............ 83

2.4.4 Phasen und Modelle im Mentoringprozess .................. 95

2.4.5 Beziehungsstruktur Mentor/in und Mentee ................. 102

2.4.6 Personale Lern- und Entwicklungsförderung ................ 112

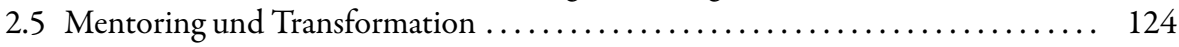

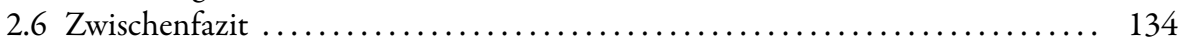


II Empirischer Zugang ................................... 139

3 Forschungsfrage $\ldots \ldots \ldots \ldots \ldots \ldots \ldots \ldots \ldots \ldots \ldots \ldots \ldots \ldots \ldots \ldots \ldots, 140$

4 Methodologischer Zugang und Datenerhebung $\ldots \ldots \ldots \ldots \ldots \ldots \ldots \ldots \ldots$

4.1 Expert/inneninterviews als Ausgangsbasis ..................... 142

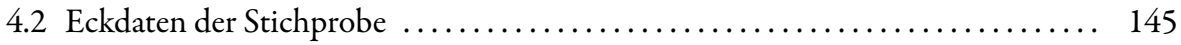

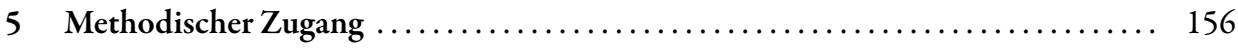

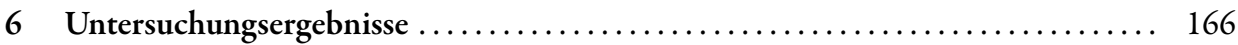

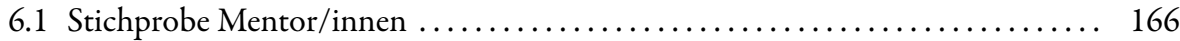

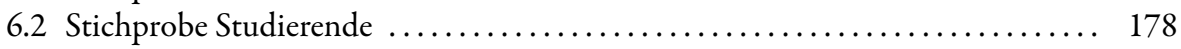

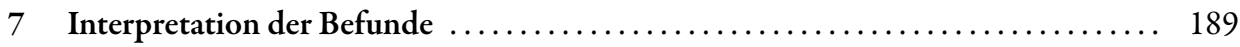

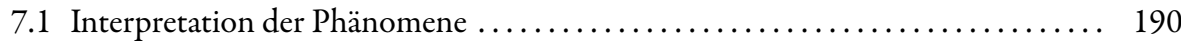

7.2 Interpretation der ursächlichen Bedingungen ................... 195

7.3 Interpretation der Handlungsstrategien $\ldots \ldots \ldots \ldots \ldots \ldots \ldots \ldots \ldots \ldots \ldots . \ldots \ldots$

7.4 Interpretation des Kontextes und der intervenierenden Bedingungen ....... 201

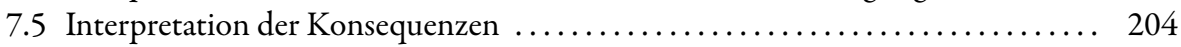

8 Integration und Kontextualisierung der Befunde $\ldots \ldots \ldots \ldots \ldots \ldots \ldots \ldots \ldots$

8.1 Befund 1: Gelingensbedingungen schulpraktischer Mentoringprozesse im Rahmen der Metakernkategorie $\ldots \ldots \ldots \ldots \ldots \ldots \ldots \ldots \ldots \ldots \ldots . \ldots \ldots$

8.2 Befund 2: Gelingensbedingungen schulpraktischer Mentoringprozesse

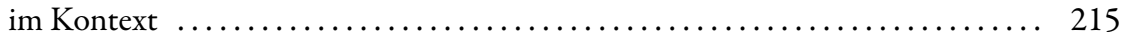

8.3 Befund 3: Gelingensbedingungen schulpraktischer Mentoringprozesse

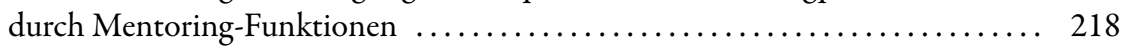

8.4 Befund 4: ATM-Modell - Analyse Transformationales Mentoring im berufsfeldbezogenen Wirkungskontext ...................... 224

8.5 Befund 5: MIW-Modell - Mentoringinduziertes Wirkungsmodell zum

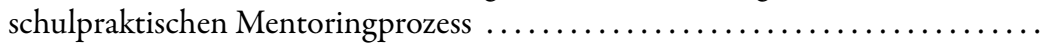

III Resüimee und Ausblick ............................. 231

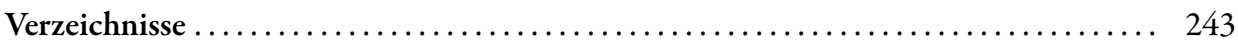

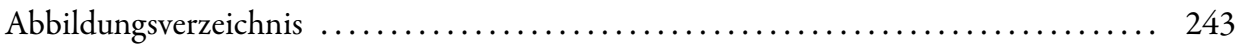

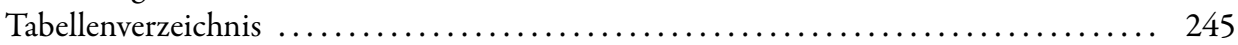

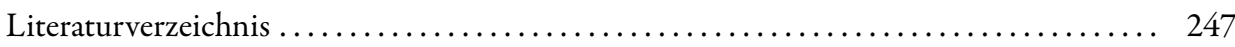

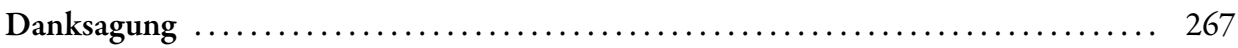




\section{Einführung und Kontextualisierung der Fragestellung}

In der Odyssee ist Mentor der Freund der Hauptfigur Odysseus und Lehrer seines Sohnes Telemachos. Auch die Göttin Athene verkörpert häufig die Gestalt des väterlichen Freundes Mentor. Hierbei berät sie Telemachos in Lebenssituationen und weist ihm den richtigen Weg. In Anlehnung an die griechische Mythologie bezeichnet man eine lehrende Person Mentor und eine lernende Person Mentee. Die/Der Mentor/in und die/der Mentee gehen in der Regel für eine bestimmte Zeit in einem bestimmten Tätigkeitsfeld eine Förderbeziehung ein.

Im schulischen Kontext kann das bedeuten, dass die/der Lehrende die/den Lernende/n in der Ausbildung begleitet und unterstützt. Umgekehrt kann auch die/der Lehrende neue Impulse von der/vom Lernenden erhalten und so selbst immer ein/e Lernende/r bleiben.

Im europäischen Hochschulraum, so auch in Österreich, wurden im Rahmen einer grundlegenden Lehrer/innenausbildungsreform die schulpraktische Ausbildung und das begleitende Mentoring grundlegend rekonzipiert. Im Rahmen Pädagogisch-Praktischer Studien erfolgt einer über die traditionell bereits gut verankerte Zusammenarbeit zwischen Hochschulen und Partnerschulen hinaus vertiefende Verschränkung von Schulpraktika und hochschulbasierter Ausbildung. Die Einbeziehung der Mentor/innen in den Ausbildungsprozess bedingt eine gegenseitige Abstimmung und Verknüpfung der einzelnen Ausbildungsbereiche (Schützenmeister 2008).

Ziel der vorliegenden Arbeit ist es, zentrale Gelingensbedingungen schulischer Mentoringprozesse in der Lehrer/innenausbildung empirisch zu rekonstruieren.

Ausgangspunkt hierfür ist die Beobachtung, dass aus Sicht von Lehramtsstudierenden Praktika häufig als zentrales Element der Ausbildung (Hascher 2006; Arnold et al. 2011; Schubarth et al. 2012; Ostermann 2015) wahrgenommen werden. Studierende legen besonders Wert darauf, Einblick in den beruflichen Alltag und die Möglichkeit zum Unterrichten zu bekommen (Hascher et al. 2007; Bach 2013, 106). Hierbei wird der/dem Mentor/in eine besondere Rolle zugeschrieben. Durch sie/ihn erhalten Studierende „Tipps und Hinweise“ (Hascher 2006; Bach 2013 , 107) für das pädagogische Handeln. Auf dieser Grundlage wird im Rahmen der vorliegenden Arbeit der zentralen Frage und den zwei binnendifferenzierten Fragen nachgegangen:

- Forschungsfrage: Welche Gelingensbedingungen lassen sich im Mentoringprozess der Lehramtsausbildung rekonstruieren bzw. identifizieren?

- Weiters: Welche Gelingensbedingungen lassen sich aus Sicht der Mentor/innen rekonstruieren bzw. identifizieren? Welche Gelingensbedingungen lassen sich aus Sicht der Studierenden rekonstruieren bzw. identifizieren?

Diese Fragestellungen bilden die Basis für die vorliegende Arbeit. Ziel ist die Identifikation von Gelingensbedingungen im Kontext der Lehramtsausbildung mittels Interviewdaten. Die Samplings wurden aus dem unmittelbaren Umfeld Schule und Hochschule bzw. Universität ausgewählt. Zunächst erfolgte eine qualitative Erhebung über Interviews bei Lehrpersonen bzw. Mentor/innen $(n=12)$ und Studierenden bzw. Mentee $(n=12)$. Die Interviews fanden im Juni 2017 statt. Die Inhalte der Interviews wurden mit Hilfe der Software MAXQDA ausgewertet. Als methodische Herangehensweise wurde Grounded Theory (Strauss et al. 1996) verwendet. Datenerhebung und Vorgehensweise der Auswertung basieren auf dem Prinzip der theoretischen Sättigung.

Die Arbeit ist in drei Hauptkapitel unterteilt. Wie ein Leitfaden zieht sich hierbei die historiografische, systemische und personalisierte Betrachtungsweise von Pädagogisch-Praktischen Studien und Mentoring durch das Betrachtungs- und Forschungsfeld. 
Im Kapitel I Grundlagen werden die Relevanz der Pädagogisch-Praktischen Studien und Mentoring als Begleitform der Schulpraktika dargestellt. Untersuchungsbefunde untermauern Konzepte, Modelle und Ansätze. Hierbei wird aufgezeigt, dass die Begleitung von zukünftigen Lehrpersonen im historischen und systemischen Kontext unterschiedliche Traditionen hatte und in Österreich mit Pädagog/innenbildung NEU ein neuerlicher Wandel eingeleitet worden ist. Mit Einführung des Bachelor-Master-Studiums in der Lehrer/innenausbildung erhalten Praktika als Teil der Ausbildung - einen besonderen Stellenwert. In diesem Zuge werden Zielsetzungen und zwei Modelle von Umsetzungskonzepten der Pädagogisch-Praktischen Studien innerhalb des westösterreichischen Entwicklungsverbundes bzw. der sogenannten Lehrer/innenbildung WEST beispielhaft angeführt und diskutiert. Sie zeigen auf, wie Studierende in professionelles pädagogisches Handeln eingeführt und theoriebasiert begleitet werden. Mentor/innen unterstützen hierbei Studierende im Sozialisationsfeld Schule, stellen ihr Handlungs- und Wirkungsfeld zur Verfügung und aktivieren Lern- und Entwicklungsmöglichkeiten für angehende Lehrpersonen. Die Qualität der schulischen Lerngelegenheiten hängt sehr stark von der Art und Weise der Begleitung ab, je nachdem wie der Mentoringprozess gestaltet wird. Die Betrachtung der Subjektivität und/oder personalisierte Begleitformen stehen in Ansätzen zur Diskussion. Werthaltung und Führungskompetenz der Mentor/innen nehmen nach Literaturrecherche und erfahrungsgemäß einen großen Stellenwert ein und tragen zur Lern- und Entwicklungsförderung bei. Abgerundet wird das Kapitel mit einem Diskurs zu Mentoring und Transformation. Hierbei stehen Überlegungen zur Selbstreflexion und der soziale Prozess ausgerichtet auf Zieldimensionen und Innovationen im schulpraktischen Kontext des Mentorings im Mittelpunkt. Aus unterschiedlichen Literaturangaben rekonstruierte Kategorien mit Fragestellungen für Selbstreflexionsprozesse für Mentor/innen und Mentee runden das Kapitel ab.

Im Kapitel II Empirischer Zugang werden die methodologischen und methodischen Zugänge erläutert (qualitative Methode) und Ergebnisse der Untersuchung beschrieben. Hintergrund des Rekonstruktions- und Identifikationsprozesses aus den Interviewdaten zu Gelingensbedingungen im schulpraktischen Mentoring bildet die Forschungsmethode Grounded Theory. Mit ihr werden Phänomene, ursächliche Bedingungen, Kontext und intervenierende Bedingungen, Handlungsstrategien und Konsequenzen ermittelt. Weitere Kapitel beschäftigen sich mit der Feinanalyse der Daten der Kodierparadigmata und Kernkategorien aus den Forschungsbefunden. Eine weitere Verdichtung und Ordnung der Daten zu den Bereichen Phänomene, ursächliche Bedingungen, Kontext und intervenierende Bedingungen, Handlungsstrategien und Konsequenzen ergibt sich im Kapitel Interpretation der Befunde. Im Kapitel Integration und Kontextualisierung der Befunde erfolgt eine Erörterung und Zusammenfassung der empirischen Befunde unter Berücksichtigung theoretischer Fundierung und vorhandener Forschungserkenntnisse. Perspektiven und Impulse für den schulischen und hochschulischen Kontext runden die Thematik Gelingensbedingungen im schulpraktischen Mentoringprozess in der Lehrer/innenausbildung jeweils ab. Resümierend zu den Untersuchungsergebnissen erfolgt die Darstellung und Beschreibung zweier Modellbildungen zu schulpraktischen Mentoringprozessen. Das erste Modell (ATM-Modell) fordert Mentor/innen in Hinblick auf die rekonstruierten Ergebnisfelder zu Selbstreflexionsprozessen auf. Im zweiten Modell (MIW-Modell) werden Ausgangslagen und Positionen im Hinblick auf eine Erhöhung eines mentoringinduzierten Wirkungsgrades diskutiert.

Im Kapitel III Resümee und Ausblick werden anhand der gewonnenen Erkenntnisse mögliche Konsequenzen erörtert, auf zukünftige Mentor/innen-(Ausbildungs-)Modelle hingewiesen, mögliche Forschungsvorhaben angeführt und Grenzen der vorliegenden Arbeit diskutiert. 


\section{Grundlagen}

Historische, bildungspolitisch orientierte und/oder kulturelle Deutungswelten prägten das national strukturierte Bildungssystem über viele Jahrhunderte hinweg. Gegenwärtig hat auch die Globalisierung in der Bildungspolitik Einzug gehalten. Der europäische Hochschulraum sucht eine Verständigung zwischen den Staaten und verabschiedet Reformen in der formalen Bildungslandschaft mit dem Ziel, „zur konkurrenzfähigsten und dynamischesten Wissensgesellschaft der Welt zu werden." (Prisching 2008, 223)

So wie in anderen europäischen Ländern kam es auch in Österreich um die Wende zum 21. Jahrhundert in der im tertiären Bereich des formalen Bildungskontextes integrierten Lehrer/innenausbildung durch die Änderung der Gesetzeslagen - wie des Universitätsgesetzes 2002, des Hochschulgesetzes 2005 und des Hochschul-Qualitätssicherungsgesetzes (BMB 2013) - zu markanten strukturellen und institutionellen Veränderungen. Im Hintergrund standen die bildungspolitischen Forderungen des Bologna-Systems aus dem Jahre 1999 und der Lissabon-Strategien aus dem Jahre 2000. Die Absicht der Implementierung der europäischen Richtlinien in das österreichische tertiäre Lehrer/innenausbildungssystem beschleunigten die Refomprozesse. Pädagog/innenbildung NEU wurde im Jahre 2015/16 flächendeckend in Österreich eingeführt. Die neuen Studienpläne enthalten die Bereiche Allgemeine Bildungswissenschaftliche Grundlagen, Fachwissenschaften, Fachdidaktiken und mit einem hohen Anteil Pädagogisch-Praktische Studien. Eine besondere Gewichtung werden dabei den schulischen und hochschulischen Anteilen schulpraktischer Prozesse eingeräumt. Das Erleben des Lern- und Erfahrungsortes Schule sowie theoriegeleitete Reflexionen während der Ausbildungszeit sollen Studierenden helfen, zu einer hohen Lehrer/innenselbstwirksamkeit zu gelangen. Hierbei unterstützen Mentor/innen (= der verwendete Begriff in der vorliegenden Arbeit für Synonyme wie Besuchsschullehrer/in, Praxislehrer/in, Praxislehrperson usw.) Studierende im pädagogischen Handlungsfeld und im Hinblick auf reflexiv-forschendes Lernen während der Zeit der Ausbildung. Forschungsbefunde geben Informationen und Hinweise zur Wirkungsweise von schulpraktischem Mentoring, zu prozessbegleitenden Formaten und zur Erfolgsgenerierung. Aus Sicht der Studierenden etabliert sich der Wunsch, Praktika im Sinne des humboldtschen Bildungsansatzes zu verstehen. Humboldt (1767-1835) verband mit Bildung die Möglichkeit der Entfaltung der persönlichen Fähigkeiten und Talente (von Humboldt 1810, 61f).

Während im formalen Bildungssystem gegenwärtig eher Wert auf die Überprüfbarkeit von Leistungen nach gewissen Standards gelegt wird, gibt es im Mentoring verschiedene Konzepte und Vorstellungen zur personalen Lern- und Entwicklungsförderung - wie das Konzept der Entwicklungsaufgaben (Hericks 2006; Keller-Schneider 2010; Kraler 2009, 2012; Ostermann 2015; Köffler 2015), Gedankenkonstrukte zur bedürfnisorientierten (Dreer 2018), typenorientierten (Košinár 2018) und/oder kontextsensiblen (Hofmann 2019) Begleitung, das Konzept der Selbstwirksamkeitserwartung (Bandura 1977) und das Prinzip des Empowerments (Masschelein et al. 2006; Arnold et al. 2011). (Genauere Erklärungen dazu werden im Kapitel I/2.4.6 beschrieben.) Norman et al. (2004) unterstreichen die Bedeutung eines humanistisch geprägten personenzentrierten Ansatzes, wenn sie schreiben: 
"The humanistic, person-centered perspectives of counseling can help maintain the soul of teacher mentoring as it evolves to meet needs of a changing society for well-trained teachers." (Norman et al. 2004, 129)

Die Lehrer/innenausbildung und das schulpraktische Mentoring sind gut beraten, diese Forderung und Annahme in das jeweilige Programm aufzunehmen. Für die Lehrer/innenausbildung rücken daher Fragestellungen in den Blick wie: Welchen Ansatz vertritt eine Lehrer/innenausbildung? Wie kann die Ausbildung aussehen? Ist jemand bildbar und wie ist jemand bildbar? Für den Mentoringprozess können folgende Fragen zum Nachdenkprozess anregen: Was ist das Bildbare im Mentoringprozess? Welche Bildungsansprüche haben Mentor/innen und Mentee im wechselseitigen Austausch? Welche Ziele lassen sich durch Begleitung erreichen? Ist ein Bildungserfolg durch Mentoring gegeben und kann er überprüft werden?

Die strukturelle Verankerung von Mentoring ist in der Lehrer/innenausbildung angesiedelt, die institutionelle Verankerung in der Schule. Mit schulischen Anteilen in Form von Schulpraktika geht auch eine Begleitform innerhalb des schulpraktischen Mentorings einher. Mentor/innen stehen den Studierenden in der Zeit der Ausbildung an Schulen zur Seite. Forschungsbefunde skizzieren den Bedeutungsgehalt, zeigen aber auch Desiderate auf. In der empirischen Untersuchung werden durch die Auswertung qualitativer Daten von Lehrenden- und Studierendeninterviews zum Mentoringprozess Impulse für (Ausbildungs-)Modelle im Rahmen des Mentoringprozesses abgeleitet.

Im Folgenden wird zunächst auf die Relevanz der Pädagogisch-Praktischen Studien eingegangen, bevor im weiteren theoretischen Teil das Thema schulpraktisches Mentoring näher in den Fokus rückt. 


\section{Genese und Fragestellung}

In diesem Kapitel erfolgt eine inhaltliche Darstellung der Bedeutung der Pädagogisch-Praktischen Studien hinsichtlich unterschiedlicher inhaltlicher Schwerpunkte wie Historiografie, gesetzliche Vorgaben, Zielsetzungen und Modelle. Im Weiteren werden Forschungsbefunde zu Schulpraktika thematisiert, deren Forschungsergebnisse für Überlegungen zum Mentoring herangezogen werden. Das Unterkapitel Professionsentwicklung von Lehrpersonen zeigt mit den Modellen die Komplexität von Kompetenzentwicklungen auf und lässt erahnen, dass Mentoring eine Form ist, die unterstützend dazu wirken kann.

Seit Bestehen der Europäischen Union wird eine Annäherung des europäischen Hochschulraumes in den Fragen der Ausbildung zum Lehrberuf forciert. Diese Diskussion und die unzureichenden Ergebnisse von Leistungsvergleichsstudien (PISA, TIMSS, PIRLS) von Schüler/ innen zeigten Defizite in der österreichischen Bildungslandschaft auf. Es deckte sich vielfach mit jenen Punkten, die „die OECD bereits 1965 im Bericht ,Bildungsplanung und Wirtschaftswachstum in Österreich““ (Seel 2010, 81) anführte und als Folgewirkung eine geringe Wettbewerbsfähigkeit bescheinigte. Nach Übereinstimmung der politischen Parteien sollte nun das postsekundäre Schulsystem im Pflichtschulbereich (Pädagogische Akademie) in eine tertiäre Ausbildungsform (Pädagogische Hochschule) überstellt werden.

Einen wesentlichen Schritt für den Beginn der Umstrukturierung der Lehrer/innenausbildung in eine tertiäre Bildungsform bildete die Unterzeichnung des Bologna-Prozesses im Mai 1999 von damals 29 Bildungsminister/innen in der Stadt Bologna. Die mittlerweile auf 48 Mitgliedstaaten gewachsene Organisation (Stand 2020) unterzeichnete im Abkommen die Schaffung von Leitlinien im europäischen und nationalen Hochschulraum, die im Laufe der Jahre erweitert und ausgebaut wurden. Wesentliche Eckpunkte sind die Einführung des dreistufigen Studienabschlusssystems (Bachelor-Master-PhD), die Einführung von Lehr-, Lern- und Bewertungsprozessen zur Planung, Durchführung und Evaluierung von Studienprogrammen und zur Student/innenmobilität in Form von ECTS-AP (European Credit Transfer System Anrechnungspunkte), die Förderung der Zusammenarbeit und Beschäftigungsfähigkeit (Employability), die Vermittlung von Kompetenzen auf Basis eines selbstbestimmten lebenslangen Lernens und die Förderung des Forschungsraumes (BMWFW 2016).

In Österreich wurde mit dem Studienjahr 2015/16 durch das Konzept Pädagog/innenbildung NEU eine Neustrukturierung und Neugestaltung in der Lehrer/innenausbildung eingeläutet: das Bachelor-Master-Ausbildungskonzept zur Primarstufenlehrperson an den Pädagogischen Hochschulen und das Bachelor-Master-PhD-Ausbildungskonzept zur Sekundarstufenlehrperson (Sekundarstufe Allgemeinbildung) an den österreichischen Universitäten in Kooperation mit den Pädagogischen Hochschulen (BMB 2013). Grundsätzlich wäre die Ausbildung zur Primarstufenlehrperson auch an den österreichischen Universitäten in Kooperation mit den Pädagogischen Hochschulen möglich, was de facto aber nicht der Fall ist.

Ziele von Pädagog/innenbildung NEU sind eine Qualitätssteigerung durch wissenschaftlich fundierte Theorie-Praxis-Transformation, eine Qualitätssicherung durch Begutachtung der Curricula durch eine unabhängige Personengruppe in Form des Qualitätssicherungsrates (QSR), die Umsetzung von kompetenzorientierten Standards (allgemeine und spezielle pädagogische Kompetenzen, fachliche und didaktische Kompetenz, inklusive und interkulturelle, soziale Kompetenz, Beratungskompetenz und Professionsverständnis) während der Ausbildungsphase, die gegebene Mobilität und Internationalisierung aufgrund des Aufbaus der Stu- 
dien nach Bologna-Struktur (Bachelor-Master-PhD) und die zukünftige Gleichwertigkeit von Lehrpersonen aufgrund gleicher Ausbildungsstätten. Fester Bestandteil in den Curricula sind auch die zu integrierenden Pädagogisch-Praktischen Studien.

In der Bildungsforschung wird gegenwärtig die Annahme getätigt, dass Schüler/innenleistungen nur gesteigert werden können, wenn die Unterrichtsqualität verbessert wird (Werler 2014, 110). Diese Denkfigur wird durch die Wirkungskettenannahme erweitert, dass eine hochwertig qualifizierte und professionsorientierte (Aus-)Bildung von Lehrpersonen Schüler/innenleistungen positiv beeinflussen kann (Herzmann et al. 2016, 133). Befunde zur Wirkungskette „mit den Stichwörtern Lehrerausbildung - Lehrerhandeln - Schülerleistung (Blömeke 2005; Lipowsky 2006; Terhart 2012)“ (Herzmann et al. 2016, 133) fehlen. Was bleibt, ist die Frage nach der Wirksamkeit von Lehrer/innenausbildung bzw. Schule und den institutionell-ausbildungsspezifischen Bedingungen und Möglichkeiten zur Hervorbringung „guter“ Lehrer/innen und „guter“ Schüler/innen. Verantwortliche der Institutionen beschäftigten sich bei der Implementierung der Curricula intensiv mit diesen Thematiken.

Welche Kompetenzen sollen Lehramtsstudierende im Rahmen des Studiums unbedingt erwerben? Welchen Beitrag können Praktika dazu leisten? Welche Bedeutung haben sie in der Lehramtsausbildung? Wann und wie sollen sie angelegt sein, damit Lehramtsstudierende das Handlungsfeld Schule in der Perspektive und Rolle der Lehrperson erfahren? Wie kann ein optimaler Begleitungsprozess gestaltet sein? Zur Beantwortung dieser Fragen werden und wurden oftmals bildungshistorische Argumente (Zymek 2008, 203) herangezogen und ein interdisziplinäres Forschungsfeld mit unterschiedlichen Forschungsschwerpunkten aufgebaut (Zymek 2008, 203). Das folgende Kapitel beschäftigt sich nun mit der Relevanz der Pädagogisch-Praktischen Studien innerhalb der Lehrer/innenausbildung.

\subsection{Zur Relevanz der Pädagogisch-Praktischen Studien im Lehramtsstudium}

Pädagogisch-Praktische Studienanteile innerhalb des Bachelor-Master-Studiums der Lehrer/innenausbildung bilden sich in einem schulischen und hochschulischen Anteil ab. Die schulischen Anteile werden oftmals als Praktikum, Praktika, Schulpraktika, Praxis oder Schulpraxis bezeichnet. Häufig werden bzw. wurden sie als Antipoden zur Theorie gesehen. Im neuen Ausbildungsmodus (Pädagog/innenbildung NEU) wird das simultane Studienmodell (Eurydice 2002) mit der Berücksichtigung der Vernetzung der schulpraktischen Ausbildungsanteile mit dem wissenschaftlichtheoretischen Studium herangezogen. So erklärt sich, dass die Pädagogisch-Praktischen Studien in den Studienarchitekturen in allen Bereichen ihren Platz finden und dass sie Praktika sowie ausgewählte praxisrelevante theoretische Seminare einschließen. Im schulischen Setting erfahren Studierende die Schulstruktur bzw. Schulkultur und bekommen Einblick in Handlungsabläufe des Unterrichtens. Sätze wie „Da lernt man, wie es geht.“ sind zum Leidwesen der Ausbildner/innen aus dem Mund von Studierenden, nicht selten auch von Mentor/innen zu hören. Was ist damit gemeint? Praktika bieten die Gelegenheit, Erfahrungen sammeln zu können, in den Austausch mit Lehrpersonen und Schülerinnen und Schülern zu gehen, fachwissenschaftliche und fachdidaktische Konzepte auszuprobieren, pädagogisch-psychologische Theorien umzusetzen, organisatorische Abläufe auszuführen, Schulstrukturen kennenzulernen, die eigene Wirksamkeit zu überprüfen und die Ergebnisse pädagogischen Handelns kritisch zu hinterfragen. Eine entsprechend fundierte Ausbildung bildet wohl die Basis für eine zielführende professionelle Umsetzung.

Die Bildungsforschung setzt sich mit Konzepten und Fragestellungen zu Schulpraktika bzw. schulischen Lerngelegenheiten auseinander. Hierbei stehen vor allem Fragen im Mittelpunkt 
wie: Was lernen Studierende in den Praktika? Welche Lernprozesse vollziehen sich in den Praktika? Welchen Beitrag können Schulpraktika zum Kompetenzerwerb leisten? Wie sollen Schulpraktika optimal angelegt werden? Wie sollte der Begleitungsprozess gestaltet sein? Oftmals bleibt in der Forschungslandschaft die Frage offen, ob Ansprüche, die an Schulpraktika gestellt werden, erfüllt werden bzw. erfüllt werden können (Bach 2013, 110f).

In den folgenden Unterkapiteln werden nach einem historischen Zugang zu Schulpraktika die gesetzlichen Vorgaben zur Implementierung Pädagogisch-Praktischer Studien in Curricula und darauf folgend Zielsetzungen im Rahmen der Praktika und zwei Konzeptionsmodelle der Lehrer/innenbildung WEST (= ein Verbund von vier in Österreich) vorgestellt. Danach werden Forschungsbefunde zu Schulpraktika aufgezeigt und Ergebnisse der Untersuchungen diskutiert. In einem abschließenden Abschnitt folgen Überlegungen zur Theorie-Praxis-Verschränkung.

\subsubsection{Historiografische Betrachtung von Schulpraktika}

Bildungshistorisch gesehen erfolgte zunächst die Ausbildung zur/zum Lehrer/in über einen Nachahmungs- und Anlernprozess bei Ordensleuten. Die Zeiten der „Meisterlehre“ (Topsch 2004b, 477) können somit als die ersten Praktikumsformen angesehen werden. Die/Der Novizin/Novize, die/der Lesen, Schreiben und Rechnen beherrschte (Seel 2010, 178), beobachtete Unterricht und lernte aus seinen eigenen Unterrichtserfahrungen (Bach 2013, 81). Ernst Christian Trapp (1780), erster Professor für Pädagogik an der Universität Halle, schrieb dazu:

„Aber was gehört dazu, um richtige Beobachtungen, zuverläßige (sic!) Erfahrungen anzustellen? Sehr viel. Ein Geist von Vorurtheilen (sic!) und Parteilichkeit frei; ein geübtes Auge; ein durchdringender Blick; eine weiche Seele, die alle Eindrücke annimmt, welche die beobachteten Gegenstände auf sie machen; eine lebhafte Vorstellung von der Möglichkeit sich zu irren, die zur Behutsamkeit und Wiederholung der angestellten Versuche leitet.“ (Trapp 1977, 63f; De Boer et al. 2012, 282)

Dieses Zitat unterstreicht die Bedeutung der situationsbezogenen Wahrnehmung und wertfreien Beschreibung pädagogischer Handlungen und im Weiteren die Wichtigkeit für den Aufbau einer reflexiven Grundeinstellung. Es zeigt auch die Möglichkeit des Versuch-Irrtumslernens im Lernprozess pädagogischer Aktivitäten auf (De Boer et al. 2012, 282). Trapp (1780) lieferte - wie wir heute sagen würden - ein Konzept für Praxisreflexion und Praxisbegleitung. Ob es angewandt wurde? „Praxis hatte in dieser frühen Phase der Lehrer/innenbildung eine unmittelbare berufsqualifizierende Funktion." (Bach 2013, 81; Bach et al. 2014, 46) Im Sinne von Abschauen - Umsetzen - Aneignen (Homfeld 1978; Bach 2013; Bach et al. 2014, 46) wurde Unterrichtswissen aufgebaut.

In den Hauptstädten der Habsburgermonarchie waren um 1774 die „Normalschulen“ die Träger der Lehrerausbildung (sic!). Nach einer dreimonatigen, später halbjährlichen Unterweisung in Rechte und Pflichten, Lehrgegenständen und Lehrmethoden in den Präparandenkursen waren die Absolvent/innen als Schulgehilfen in den Trivialschulen eingesetzt. Sie unterzogen sich somit wieder einer mindestens einjährigen Meisterlehre (Seel 2010, 178). In Tirol erfolgte die Einrichtung von 24 Musterschulen, an denen vor allem die neuen Unterrichtsmethoden und Lehrarten (Zusammenunterrichten, Katechisieren, Buchstabenmethode, Tabellenmethode) nach der Vorstellung von Philipp Jakob Tangl umgesetzt wurden bzw. werden sollten. Die Musterschullehrpersonen erhielten eine finanzielle Entschädigung (Juen 1986, 20).

Im Zuge der Festlegung der allgemeinen achtjährigen Schulpflicht im Jahre 1869 und der Neuetablierung von Lehrerbildungsanstalten im Jahre 1874 nach dem Reichsvolksschulgesetz konnten Lehramtsstudierende erstmals die vorgeschriebenen Praktika in den angeschlossenen 
Übungsschulen der Lehrerbildungsanstalt absolvieren. Zwölf Jahre nach Einführung der Anstalten wurde in einem Reformprozess den praktischen Inhalten (musikalische Ausbildung, Instrumentalunterricht, Landwirtschaftslehre) mehr Gewichtung als den wissenschaftlichen Fächern gegeben (Seel 2010, 181). Hospitationen, pädagogisches Handeln im Umgang mit Kindern und Übungseinheiten praktisch pädagogischer Lehrtätigkeit wurden von den zukünftigen Lehrpersonen verlangt (Juen 1986, 30).

Gymnasiallehrpersonen hatten nach Absolvierung der Lehrbefähigungsprüfung ab 1856 eine Probelektion zu gestalten und zu bestehen. Das eigentliche Schulpraktikum wurde im ersten Dienstjahr in Form des sogenannten Probejahres vollzogen. Die Begleitung und Beaufsichtigung unterstanden der jeweiligen Direktion (Seel 2010, 192). Im Jahre 1971 wurde das Lehramtsstudium für mittlere und höhere Schulen an den Universitäten neu konzipiert und „durch eine schulpraktische Ausbildung im Ausmaß von 8 Semesterwochenstunden (12 Wochen)“ (Seel 2010, 194) und ab 1988 durch „ein von der Schulverwaltung betreutes einjähriges ,Unterrichtspraktikum' als Einführungsdienst“" (Seel 2010, 194) ergänzt.

Mit der Einführung der Pädagogischen Akademien im Jahre 1968 war die „Schulpraktische Ausbildung" (damalige Bezeichnung) im Lehrplan der angehenden Pflichtschullehrpersonen ein Eckpfeiler neben Humanwissenschaften, Didaktik und Methodik und den ergänzenden Lehrveranstaltungen. Die Absolvierung der Praktika erfolgte an den institutionell angegliederten Übungsschulen der Akademien oder an Besuchsschulen in der Umgebung der Akademie. Die Praktika waren erstmals semesterweise vorgesehen. Im Anschluss an die absolvierten Praktika erfolgte eine Bewertung der Praxisleistung mit einer Ziffernnote.

Auch in den Rahmenlehrplänen der Pädagogischen Akademien Anfang der 80er Jahre des 20. Jahrhunderts waren die schulpraktischen Anteile Bestandteil der Ausbildung. Das Konzept der Schulpraktischen Ausbildung war im Lehrplan verankert. Es verwies auf die „Leitidee des reflektierten beruflichen Handelns.“ (Buchberger et al. 1987, 22,230)

„Die Praktika gewinnen auch theoretisch an Bedeutung (...) Praktika dienen der Erfahrungsbildung und der Reflexion von Erfahrung. (...) Nicht das Praktizieren als solches oder die Einübung praktischer Gewohnheiten kann das Ziel von Praktika im Rahmen der Lehrerinnen- und Lehrerbildung sein, sondern die Entwicklung der Fähigkeit, die eigene Praxis konstruktiv zu reflektieren." (Herzog 1995, 266, 271)

Die Betreuung der Studierenden in der Schulpraktischen Ausbildung bildete - so wie auch in den Pädagogischen Hochschulen - eine Triade zwischen Studierende/r, Hochschuldozent/in und Mentor/in bzw. Besuchsschullehrperson. Die Funktion, Aufgaben und Schulung von Mentor/innen in dieser Zeit werden im Kapitel I/2 näher beschrieben.

Auch mit der Errichtung der Pädagogischen Hochschulen im Jahre 2007 waren die gegenwärtigen „Schulpraktischen Studien“ (Akademien-Studiengesetz 1999; Akademien-Studienordnung 2000) weiterhin Ausbildungsteile und somit ein Teil des Studiums. Praktika waren vor Ort in den Schulen vorgesehen. Im Wesentlichen wurden Hospitationsphasen, mehr oder weniger angeleitete Unterrichtsversuche und längerfristige eigenverantwortliche Unterrichtstätigkeit gefordert, die in Form von Tagespraktika während des gesamten Semesters oder in zwei-, drei- oder vierwöchigen geblockten Praktika durchgeführt wurden. Die angegliederten Übungsschulen blieben als „Versuchsstätten“ für die Lehramtsstudierenden des Pflichtschulbereiches bestehen. Weitere Praxisschulen mit Praxislehrpersonen standen in Kooperation mit der Hochschule zur Verfügung. „Diese enge Verbindung zwischen Schule und Hochschule unterstreicht den hohen Stellenwert, der der praxisorientierten Ausbildung zukommt, und kann als eine Stärke der Pädagogischen Hochschulen gewertet werden." (Bach et al. 2014, 58) 
Auch mit Pädagog/innenbildung NEU nehmen Schulpraktika - integriert in den PädagogischPraktischen Studien - einen hohen Stellenwert ein. Laut Curricula der Lehrer/innenbildung WEST für die Sekundarstufe Allgemeinbildung sind für Studierende verpflichtende Schulpraktika in allen genannten Schultypen zu absolvieren. Qualifizierte Mentor/innen stehen in allen Stufen und in allen Schultypen zur Verfügung. Auch in den Curricula der Primarstufe sind für Studierende während der Ausbildung Praktika in unterschiedlichem Ausmaß an den Partnerschulen vorgesehen.

Bei der Konzeptentwicklung stehen meist zwei Denkfiguren im Mittelpunkt: der forschende und distanzierte Blick auf das zukünftige Handlungsfeld und die Erprobung des pädagogischen Handelns im sozialen Gefüge Schule (Leonhard 2018, 13f). Wie die Umsetzung der gesetzlichen Vorgaben in den Curricula von Pädagog/innenbildung NEU erfolgen kann, zeigen nun folgend nach den einleitenden Worten und Erklärungen zwei Beispiele im Entwicklungsverbund Lehrer/innenbildung WEST.

\subsubsection{Gesetzliche Bestimmungen für Ausbildungscurricula in Österreich}

Im österreichischen Bundesgesetz 2013 sind für das aufbauende Bachelor-Master-Studium Rahmenvorgaben für die Studienarchitektur des Lehramtes im Bereich der Primarstufe und im Bereich der Sekundarstufe Allgemeinbildung definiert (124. Bundesgesetz: Bundesrahmengesetz zur Einführung einer neuen Ausbildung für Pädagog/innen - Bundesgesetzblatt, Bundeskanzleramt 2013), die folgendermaßen lauten:

„Für Bachelor- und Masterstudien zur Erlangung eines Lehramtes im Bereich der Primarstufe Bachelorstudium im Umfang von 240 ECTS-Credits; davon:

- 40 bis 50 ECTS-Credits für allgemeine bildungswissenschaftliche Grundlagen;

- 120 bis 130 ECTS-Credits für Elementar- und Primarstufenpädagogik und -didaktik mit Schwerpunk im jeweiligen Altersbereich (Elementar- oder Primarstufe);

- 60 bis 80 ECTS-Credits Schwerpunktsetzung (z.B. in einem fachlichen Bildungsbereich, in Inklusiver Pädagogik, in Sonder- und Heilpädagogik, in Sozialpädagogik, in Mehrsprachigkeit usw.);

- Pädagogisch-Praktische Studien sind zu integrieren. [...]

Masterstudium im Umfang von mindestens 60 ECTS-Credits:

- Bezug zur pädagogischen Tätigkeit und zur Wissenschaft;

- der Anteil für allgemeine bildungswissenschaftliche Grundlagen muss so groß sein, dass zusammen mit dem Anteil im Bachelorstudium mindestens 60 ECTS-AP-Credits im Gesamtstudium enthalten sind;

- falls nach Absolvierung eines Bachelorstudiums für die Primarstufe, die Elementar- und die Primarstufe abgedeckt werden sollen, erhöht sich der Aufwand des Masterstudiums auf mindestens 90 ECTS-Credits. [...]

Für Bachelor- und Masterstudien zur Erlangung eines Lehramtes im Bereich der Sekundarstufe (Allgemeinbildung) - Ein „Studienfach“ bezieht sich im Folgenden immer auf einen Unterrichtsgegenstand im Bereich der Sekundarstufe (Allgemeinbildung).

Bachelorstudium im Umfang von 240 ECTS-Credits; davon:

- 40 bis 50 ECTS-Credits für allgemeine bildungswissenschaftliche Grundlagen;

- pro Studienfach 95 bis 100 ECTS-Credits für studienfachbezogene Fachdidaktik und Fachwissenschaften bzw. 190 bis 200 ECTS-Credits für mehr als zwei sich gegenseitig inhaltlich überschneidende Fächer (kohärentes Fächerbündel); 
- oder statt 2. Studienfach Spezialisierungen im Umfang von 95 bis 100 ECTS-Credits (z.B. Inklusive Pädagogik, Sonder- und Heilpädagogik, Sozialpädagogik; Berufsorientierung, Mehrsprachigkeit, Medienpädagogik usw.);

- Pädagogisch-Praktische Studien sind zu integrieren.

Masterstudium im Umfang von mindestens 90 ECTS-Credits:

- Bezug zur pädagogischen Tätigkeit und zur Wissenschaft;

- der Anteil für allgemeine bildungswissenschaftliche Grundlagen muss so groß sein, dass zusammen mit dem Anteil im Bachelorstudium mindestens 60 ECTS-Credits im Gesamtstudium enthalten sind;

- im Gesamtstudium müssen mindestens 115 ECTS-Credits studienfachbezogene Teile pro Studienfach enthalten sein.“ (124. Bundesgesetzblatt, Bundeskanzleramt 2013)

Die Hierarchisierung des Lehrberufs (Zweiteilung in niedrige und höhere Lehrämter) scheint mit der Umsetzung von Pädagog/innenbildung NEU im Jahre 2015/16 und somit durch die Gleichsetzung der Lehrämter durch Bachelor-Master-Abschlüsse zumindest formal, institutionell und gehaltsmäßig aufgehoben zu sein. Die gesetzlichen und studienrechtlichen Rahmenbedingungen waren die Grundlage für die Konzipierung der neuen Lehramtscurricula in den tertiären Bildungseinrichtungen (Pädagogische Hochschule und Universität). Für die Pädagogischen Hochschulen trat insbesondere auch die Verordnung des Bundesministeriums für Unterricht, Kunst und Kultur über die Curricula der Pädagogischen Hochschulen (HochschulCurriculaverordnung 2013, HCV 2013) in Kraft (BGBl. II Nr. 335/2013 vom 7.11.2013). Bei der Ausgestaltung der Curricula kam es in den vier österreichischen Entwicklungsverbünden zu einer Generierung unterschiedlicher Theoriebildungen, standortspezifischen Ressourcennutzungen und zum Festhalten von generativen Prinzipien (Bruner 1960) sowie zur Berücksichtigung von Diversifizierung im Professionalisierungsprozess. In den Verbünden wurden Kooperationsvereinbarungen unterzeichnet.

„Gemeinsames Ziel ist eine zukunftsweisende, qualitätsvolle, professionsorientierte und forschungsgeleitete Aus- und Weiterbildung künftiger Lehrkräfte (...), welche den fachwissenschaftlichen, fachdidaktischen, bildungswissenschaftlichen, schulpraktischen und dienstrechtlichen Anforderungen gerecht wird." (Kraler et al. 2017, 82)

Im Gesetz wurde auch die Forderung der Integration der Pädagogisch-Praktischen Studien verankert. Die Auslegung dieser Forderung wurde in den Entwicklungsverbünden (insgesamt vier Verbünde WEST, MITTE, NORD-OST, SÜD-OST) unterschiedlich umgesetzt.

Die zwei anschließenden Beispiele zeigen modellhaft die strukturelle Umsetzung der gesetzlichen Vorgaben im Entwicklungsverbund Lehrer/innenbildung WEST auf - Bachelor-MasterStudium Primarstufe und Bachelor-Master-Studium Sekundarstufe Allgemeinbildung.

\section{Beispiel Bachelor-Master-Studium Primarstufe}

Im Entwicklungsverbund Lehrer/innenbildung WEST können Interessierte des Lehramtsstudiums Primarstufe das deckungsgleiche Studienangebot der Pädagogischen Hochschule Tirol und der Pädagogischen Hochschule Vorarlberg oder das alternative Studienangebot an der Privaten Pädagogischen Hochschule Edith Stein wählen. Tabelle 1 gibt eine Übersicht über die Studienarchitektur des Bachelor-Master-Studiums Primarstufe an der Privaten Pädagogischen Hochschule Edith Stein (KPH Edith Stein). 
Tab. 1: Studienarchitektur Bachelor-Master-Studium Primarstufe ab dem Studienjahr 2015/16 (Curriculum Primarstufe KPH Edith Stein)

\begin{tabular}{|c|c|c|}
\hline \multicolumn{3}{|c|}{$\begin{array}{l}\text { Bachelorstudium Primarstufe ( } 240 \text { ECTS-AP) } \\
\text { an der Privaten Pädagogischen Hochschule Edith Stein }\end{array}$} \\
\hline $\begin{array}{l}\text { Allgemeine } \\
\text { Bildungswissenschaftliche } \\
\text { Grundlagen } \\
\text { (BWG) }\end{array}$ & $\begin{array}{l}\text { Primarstufenpädagogik und } \\
\text {-didaktik } \\
\text { (PPD) }\end{array}$ & $\begin{array}{l}\text { Schwerpunktsetzungen } \\
\text { (Beginn: 3. Semester) } \\
\text { (SP) }\end{array}$ \\
\hline $\begin{array}{l}50 \text { ECTS-AP } \\
\text { davon } 5 \text { ECTS-AP (PPS) }\end{array}$ & $\begin{array}{l}130 \text { ECTS-AP } \\
\text { davon } 25 \text { ECTS-AP (PPS) }\end{array}$ & $\begin{array}{l}60 \text { ECTS-AP } \\
\text { davon } 10 \text { ECTS-AP (PPS) }\end{array}$ \\
\hline $\begin{array}{l}\text { darin inkludiert } \\
\text { 1. Bachelorarbeit } \\
5 \text { ECTS-AP }-7 \text {. Semester }\end{array}$ & & $\begin{array}{l}\text { darin inkludiert } \\
\text { 2. Bachelorarbeit } \\
\text { 5ECTS-AP }-8 \text {. Semester }\end{array}$ \\
\hline \multicolumn{3}{|c|}{$\begin{array}{l}\text { Masterstudium Primarstufe (60 ECTS-AP) } \\
\text { an der Privaten Pädagogischen Hochschule Edith Stein }\end{array}$} \\
\hline $\begin{array}{l}\text { Allgemeine } \\
\text { Bildungswissenschaftliche } \\
\text { Grundlagen } \\
\text { (BWG) }\end{array}$ & Masterthesis und Defensio & $\begin{array}{l}\text { Bezug zur pädagogischen Tätigkeit } \\
\text { und zur Wissenschaft }\end{array}$ \\
\hline 10 ECTS-AP & 30 ECTS-AP & 20 ECTS-AP \\
\hline
\end{tabular}

Das vierjährige Bachelorstudium Primarstufe umfasst 240 ECTS-AP - davon entfallen 50 ECTS-AP auf die Allgemeinen Bildungswissenschaftlichen Grundlagen (BWG), 130 ECTS-AP auf Primarstufenpädagogik und -didaktik (PPD) und 60 ECTS-AP auf einen ab dem 3. Semester wählbaren Schwerpunkt (SP) des Studiums. Der Bereich Primarstufenpädagogik und -didaktik (130 ECTS-AP) enthält Seminare und Lehrveranstaltungen zu allen Unterrichtsfächern, die als Klassenlehrperson einmal in der Primarstufe unterrichtet werden. Die Pädagogisch-Praktischen Studien (PPS) sind im Bachelorstudium mit 40 ECTS-AP ausgewiesen. Sie sind in den bildungswissenschaftlichen (5 ECTS-AP), fachwissenschaftlichen und fachdidaktischen (25 ECTS-AP) Bereichen sowie in den Schwerpunktsetzungen (10 ECTS-AP) fester Bestandteil. Die Pädagogisch-Praktischen Studien (PPS) haben somit einen hohen Anteil hochschulischer und schulischer Ausbildungsanteile und stellen eine wichtige Verknüpfung zwischen Theorie, Praxis und Forschung dar. Das Bachelorstudium ist als vierjähriges und das Masterstudium als einjähriges Studium in Ausbildung konzipiert.

Im Masterstudium absolvieren die Studierenden insgesamt 60 ECTS-AP - davon sind 10 ECTS-AP den Allgemeinen Bildungswissenschaftlichen Grundlagen, 30 ECTS-AP für die Masterarbeit mit Masterthesis und 20 ECTS-AP den Seminaren mit Bezug zur pädagogischen Tätigkeit und zur Wissenschaft zugeschrieben. Im Masterstudium wurden keine PädagogischPraktischen Studienanteile vorgesehen und somit auch keine schulischen Anteile wie Praktika.

\section{Beispiel Bachelor-Master-Studium Sekundarstufe Allgemeinbildung}

Pädagogische Hochschulen, Hochschulen und Universitäten schlossen sich im Zuge der bildungspolitischen Neugestaltung des Lehramtes Sekundarstufe Allgemeinbildung in den Regionen zu Entwicklungsverbünden zusammen, und die Expert/innen der einzelnen Bildungsinstitutionen erarbeiteten in einem gemeinsamen Prozess Curricula und Anforderungsprofile für Schulpraktika. Tabelle 2 zeigt die Studienarchitekur des Bachelor-Master-Studiums des Ent- 
wicklungsverbundes Lehrer/innenbildung WEST. Beteilitgt sind die fünf Partnerinstitutionen Private Pädagogische Hochschule Edith Stein, Pädagogische Hochschule Tirol, Pädagogische Hochschule Vorarlberg, Universität Innsbruck und Universität Mozarteum.

Tab. 2: Studienarchitektur Bachelor-Master-Studium Sekundarstufe Allgemeinbildung Lehrer/innenbildung WEST ab dem Studienjahr 2015/16 (Curriculum 2016)

\begin{tabular}{|c|c|c|c|c|}
\hline \multicolumn{5}{|c|}{$\begin{array}{l}\text { Bachelorstudium Sekundarstufe Allgemeinbildung (240 ECTS-AP) } \\
\text { Lehrer/innenbildung WEST }\end{array}$} \\
\hline $\begin{array}{l}\text { Allgemeine } \\
\text { Bildungswissenschaftliche Grund- } \\
\text { lagen } \\
\text { (BWG) }\end{array}$ & \multicolumn{2}{|c|}{$\begin{array}{l}\text { Fachliche Ausbildung (FA) } \\
\text { Zwei Fächer zur Wahl } \\
\text { oder ein Fach + eine Speziali- } \\
\text { sierung }\end{array}$} & \multicolumn{2}{|c|}{$\begin{array}{l}\text { Fachdidaktische Ausbildung (FD) in } \\
\text { jedem Fach }\end{array}$} \\
\hline $\begin{array}{l}40 \text { ECTS-AP } \\
\text { davon 23,5 ECTS-AP (PPS) }\end{array}$ & \multicolumn{2}{|c|}{$\begin{array}{l}\text { je } 80 \text { ECTS-AP } \\
\text { davon je Fach } 5 \text { ECTS-AP } \\
\text { (PPS) }\end{array}$} & \multicolumn{2}{|c|}{ je 20 ECTS-AP } \\
\hline \multicolumn{5}{|c|}{$\begin{array}{l}\text { darin inkludiert } \\
1 \text { Bachelorarbeit pro Unterrichtsfach bzw. Spezialisierung }\end{array}$} \\
\hline \multicolumn{5}{|c|}{$\begin{array}{l}\text { Masterstudium Sekundarstufe Allgemeinbildung (120 ECTS-AP) } \\
\text { Lehrer/innenbildung WEST }\end{array}$} \\
\hline $\begin{array}{l}\text { Allgemeine } \\
\text { Bildungswissen- } \\
\text { schaftliche Grundlagen } \\
\text { (BWG) }\end{array}$ & $\begin{array}{l}\text { Masterthesis } \\
\text { und Defensio }\end{array}$ & \multicolumn{3}{|c|}{$\begin{array}{ll}\text { Fachliche Ausbildung und Freies Wahlmodul und } \\
\text { fachdidaktische Ausbil- } & \text { individuelle Schwer- } \\
\text { dung in jedem Fach } & \text { punktsetzung }\end{array}$} \\
\hline $\begin{array}{l}20 \text { ECTS-AP } \\
\text { davon } 5 \text { ECTS-AP (PPS) }\end{array}$ & 30 ECTS-AP & 25 ECTS-Al & & 20 ECTS-AP \\
\hline
\end{tabular}

Im vierjährigen Bachelorstudium mit 240 ECTS-AP absolvieren Lehramtsstudierende neben den zwei Fachbereichen zu je 100 ECTS-AP (80 ECTS-AP Fachwissenschaft in jedem Unterrichtsfach, 20 ECTS-AP Fachdidaktik in jedem Unterrichtsfach) auch Bildungswissenschaftlichen Grundlagen (BWG) mit 40 ECTS-AP. Im zweijährigen Masterstudium sind weitere 120 ECTS-AP zu erfüllen - davon entfallen 20 ECTS-AP auf die Bildungswissenschaftlichen Grundlagen, 20 ECTS-AP auf das Freie Wahlmodul, 25 ECTS-AP auf den Fachbereich (Fachliche Ausbildung 15 bis 20 ECTS-AP und fachdidaktische Ausbildung 5 bis 10 ECTS-AP) und 30 ECTS-AP auf die Masterarbeit mit Defensio. Die Pädagogisch-Praktischen Studien (PPS) beinhalten - so wie im Primarstufencurriculum - hochschulische und schulische Anteile und sind im Bachelorstudium in den Bildungswissenschaftlichen Grundlagen (23,5 ECTS-AP) und der Fachdidaktik (mit 10 ECTS-AP) integriert. Im Masterstudium sind weitere 5 ECTS-AP den Pädagogisch-Praktischen Studien zugeordnet.

Den Pädagogisch-Praktischen Studien kommt in der Pädagog/innenbildung NEU eine besondere Bedeutung zu. In beiden vorgestellten Curricula ist der relative schulische Anteil hoch. In diesen Anteilen werden die Studierenden von Mentor/innen an den Partnerschulen begleitet. Die Anforderungen in den jeweiligen Praktika ergeben sich aus den zugeordneten Bereichen wie Bildungswissenschaftliche Grundlagen, Fachdidaktiken bzw. Schwerpunktsetzung oder Spezialisierung. Anbei sind zwei Modelle von Praktika mit ihren Anforderungskatalogen der Lehrer/innenbildung WEST angeführt. 


\subsubsection{Zielsetzungen und Modelle der Pädagogisch-Praktischen Studien}

Schulpraktika, als ein Teil der Pädagogisch-Praktischen Studien, nehmen in der derzeitigen Reformdiskussion der Lehrer/innenausbildung eine besondere Stellung ein (Herzmann et al. 2016, 155). Wie bereits erwähnt, sind in Österreich laut Hochschul-Qualitätssicherungsgesetz (2013) Pädagogisch-Praktische Studien (PPS) in den Curricula des Bachelor-Master-Studiums $\mathrm{zu}$ integrieren.

„Pädagogisch-Praktische Studien definieren sich (...) als jene Disziplin an der Schnittstelle der akademischen und beruflichen Welt, die dafür sorgt, dass multiple Perspektiven und Wissensressourcen zu einem zukunftsfähigen Professionswissen verschmelzen." (Fraefel et al. 2017, 7)

Ein bedeutendes Kernprinzip liegt nach dieser Aussage in der Relationierung von Theorie und Praxis im Sinne einer Professionsentwicklung. In der konkreten Umsetzungsform gliedern sich Pädagogisch-Praktische Studien nun in einen schulischen Anteil mit Praktika direkt vor Ort an den Schulen und einen hochschulinternen Anteil mit begleitenden Lehrveranstaltungen. Dabei wird darauf geachtet, dass Praxiserfahrungen „durch Reflexion und Verbindung mit professionellen Wissensbeständen für eine gelingende professionelle Kompetenzentwicklung genutzt werden." (Schnebel 2019, 14) Angesprochen wird in dieser Aussage auch die Wichtigkeit der Begleitung und Unterstützung der Studierenden im Rahmen der Pädagogisch-Praktischen Studien (siehe Kapitel I/2).

Aus wissenschaftlicher Sicht besteht Einigkeit, dass Praktika während der Ausbildungszeit die Aufgabe und Zielsetzung der Berufswahlentscheidung, des Kompetenzerwerbes, der Kompetenzerweiterung und der Verknüpfung von Theorie und Praxis (Bach 2013, 90; Bach 2020, 623) sowie den Aufbau einer offenen, reflexiven und forschenden Grundhaltung haben. Umfang, Organisationsformen und Begrifflichkeiten von Praktika sind den institutionellen Ausbildungsprofilen angepasst. In den einzelnen Entwicklungsverbünden Österreichs gestalten sich auch Anforderungen und Zielsetzungen im Orientierungspraktikum, Tagespraktikum, geblockten Tagespraktikum, Schulpraktikum, Initialpraktikum, Fachpraktikum, Blockpraktikum, Schwerpunktpraktikum, Professionalisierungspraktikum, Abschlusspraktikum u.ä. und den einhergehenden begleitenden Lehrveranstaltung sehr unterschiedlich. Mit der Unterscheidung gehen immer auch studienrechtliche und prüfungsrelevante Bestimmungen einher.

Studierende sammeln in den Praktika unterschiedliche Erfahrungen und entwickeln Kompetenzen für ein professionelles Handeln. Sind die Praktika zunächst ein „Hineinschnuppern“ in schulpraktische Lernsettings und Möglichkeit der Berufsorientierung durch Beobachtung, um sich mit der Frage der Berufswahlentscheidung intensiv auseinandersetzen zu können, werden spätere Praktika geprägt durch Erproben und Einsetzen von theoriebezogenen, fachwissenschaftlichen und fachdidaktischen Konzepten und deren forschungsgeleiteter Reflexionseinheiten. Die zentralen Elemente für die Entwicklung professionellen Handelns in den Lehramtsstudien sind laut Topsch (2004b) und Herzmann et al. (2016):

1. Schaffung von Möglichkeiten zur berufsfeldbezogenen Orientierung z.B. durch Hospitation und die Auseinandersetzung mit der eigenen Berufswahlentscheidung

2. Erweiterung der persönlichen und professionsspezifischen Kompetenzen durch Beobachtungsmöglichkeiten von Unterrichtsgeschehen und eigenem pädagogischen Handeln

3. Gestaltung von Lern- und Lehrprozessen und Erwerb der Methoden- und Sozialformenvielfalt

4. Auseinandersetzung mit dem eigenen pädagogischen Handeln und Gewinnen einer Reflexionsfähigkeit (Topsch 2004b, 484f; Herzmann et al. 2016, 155) 
Die Umsetzung dieser Elemente sieht je nach Institution und gewachsener Struktur sehr unterschiedlich aus. Dies zeigt sich am folgenden Beispiel bezogen auf Punkt 1: Das Ansetzen des ersten Praktikums mit begleitenden Lehrveranstaltungen wird in der österreichischen Ausbildungslandschaft bipolar diskutiert. Eine Gruppe von Expert/innen unterstreicht mit einem frühzeitigen Beginn von Praktika im Lehramtsstudium die Bedeutung der Praxis, die Möglichkeit der Kontaktaufnahme mit Schülerinnen und Schülern und betont die Wichtigkeit des Kennenlernens des Schul- und Berufsalltages. Die andere Gruppe geht davon aus, dass für ein kompetentes pädagogisch-praktisches Handeln ein theoretisch-inhaltlicher Kompetenzaufbau die Voraussetzung ist und plädiert für einen späteren Beginn im Bachelorstudium.

In Anlehnung an Bach (2020), der die vier Dimensionen: temporale, inhaltlich-konzeptionelle, begleitungsbezogene und aufgabenbezogene als Kategorien für Realisierungsformen zu Praktika diskutiert (Bach 2020, 621), wäre es wertvoll, diese Überlegungen aufzugreifen und bezogen auf die Pädagogisch-Praktischen Studien zu diskutieren. Im Resümee zeigt die Autorin Überlegungen auf.

Anbei werden zwei konkrete Beispiele des Entwicklungsverbundes Lehrer/innenbildung WEST angeführt, die die Umsetzung und Zielsetzung erläutern und die Wesenszüge der PädagogischPraktischen Studien mit schulischen und hochschulischen Anteilen unterstreichen.

\section{Modell Primarstufe an der Privaten Pädagogischen Hochschule Edith Stein}

Zunächst wird ein Modell vorgestellt, das an der Privaten Pädagogischen Hochschule Edith Stein im Bachelorstudium Lehramt Primarstufe Anwendung findet. Es zeigt die Kombination von schulischen und hochschulischen Anteilen als ein wesentliches Kriterium in den Pädagogisch-Praktischen Studien und verdeutlicht einen personenspezifischen Ansatz während der Zeit der Begleitung.

Ausgehend von den Inhalten des Curriculums, den Zielvorstellungen der Verantwortlichen und den Überlegungen zur Gestaltung der Praktika an den Schulen entstand an der Privaten Pädagogischen Hochschule Edith Stein unter Beteiligung von Expert/innen ein Konzept zu den Pädagogisch-Praktischen Studien als eine Orientierungshilfe für alle in diesem Kontext handelnden Personen. Dieses sieht die Ausbildung im Wesentlichen als einen Teil des berufsbiografischen Entwicklungsprozesses (Huberman 1989; Terhart 2001; Herzog 2011) an, in dem unterschiedliche Bedingungen ineinandergreifen und sich gegenseitig beeinflussen.

Die Pädagogisch-Praktischen Studien (PPS) an der Privaten Pädagogischen Hochschule Edith Stein (KPH Edith Stein) des Bachelorstudiums Primarstufe konzentrieren sich in den einzelnen Ausbildungsphasen im Wesentlichen auf die Beschreibungen der Ziele und der Kernelemente von Praktika nach Topsch (2004b, 484f) und Herzmann et al. (2016, 155). Im Curriculum wird die Basis für die Umsetzung dargestellt. Zunächst werden drei Phasen beschrieben:

- Orientierungsphase im 1. Semester

- Professionalisierungsphasen I, II und III im 2., 3., 5., 6. und 8. Semester

- Schwerpunktphasen I und II im 4. und 7. Semester

Tabelle 3 gibt einen Einblick über die Einteilung und den Inhalt der Phasen der Schulpraktika in den Pädagogisch-Praktischen Studien (PPS) des Bachelorstudiums Primarstufe: 
Tab. 3: Phasenmodell der Pädagogisch-Praktischen Studien an der KPH Edith Stein (Curriculum Primarstufe 2016)

\begin{tabular}{|c|c|c|}
\hline Semester & Phasen & Inhalte \\
\hline 1. & Orientierungsphase & $\begin{array}{l}\text { Theorie- und forschungsgeleitete Beobachtung und Reflexion von Lern- } \\
\text { und Lehrprozessen - Reflexion der eigenen Lernbiografie und Rollenbil- } \\
\text { der - Potentialanalyse sowie Klärung der Berufswahl }\end{array}$ \\
\hline 3. & $\begin{array}{l}\text { Professionalisierungs- } \\
\text { phase I - Lehr- und } \\
\text { Lernprozesse }\end{array}$ & $\begin{array}{l}\text { Planung und Durchführung von Lern- und Lehrprozessen - theorie- und } \\
\text { forschungsgeleitete Reflexion - Entwickeln von fachwissenschaftlichen } \\
\text { und fachdidaktischen Perspektiven sowie kompetenzorientierten persönli- } \\
\text { chen Schwerpunkten (Leitung und Begleitung, Kommunikation, Sprache } \\
\text { und Ausdrucksfähigkeit, Beobachtung und Reflexion...) }\end{array}$ \\
\hline 4. & tphase I & $\begin{array}{l}\text { Theoriegeleitete und metakognitive Reflexion sowie Planung und } \\
\text { Durchführung von Lern- und Lehrprozessen - Rolle und Funktion als } \\
\text { pädagogisch Denkende/r und Handelnde/r in Bezug auf den Schwer- } \\
\text { punkt und Reflexion }\end{array}$ \\
\hline 5. & $\begin{array}{l}\text { Professionalisierungs- } \\
\text { phase II - Lehr- und } \\
\text { Lernprozesse, Schul- } \\
\text { organisation }\end{array}$ & $\begin{array}{l}\text { Planung und Durchführung von Lern- und Lehrprozessen im Diversitäts- } \\
\text { kontext Schule - forschungsmethodologisch-wissenschaftstheoretische } \\
\text { Vergewisserung - Vertiefen und Weiterentwickeln von fachwissenschaft- } \\
\text { lich und fachdidaktisch relevanten sowie anderer professionsspezifischer } \\
\text { Kompetenzen (Leitung und Begleitung, Kommunikation, Sprache und } \\
\text { Ausdrucksfähigkeit, Beobachtung und Reflexion...) - Kommunikation } \\
\text { und Kooperation in der Schule - Schulorganisation und -entwicklung }\end{array}$ \\
\hline 7. & Schwerpunktphase II & $\begin{array}{l}\text { Planung, Durchführung und Reflexion von Lern- und Lehrprozessen - } \\
\text { Entwickeln von Professionsverständnis und Kompetenzen in Bezug auf } \\
\text { den Schwerpunkt }\end{array}$ \\
\hline 8. & $\begin{array}{l}\text { Professionalisierungs- } \\
\text { phase III }\end{array}$ & $\begin{array}{l}\text { Eigenständige Planung, Durchführung und theoriegeleitete Reflexion von } \\
\text { Lern- und Lehrprozessen - Reflexion von Rolle, Funktion, professions- } \\
\text { spezifischen und persönlichen Kompetenzen im schulischen Kontext - } \\
\text { Resümee und Ausblick }\end{array}$ \\
\hline
\end{tabular}

In der Orientierungsphase erfolgen zunächst Hospitationseinheiten. Die Studierenden hospitieren insgesamt fünf Mal je zwei Unterrichtseinheiten bei einer/einem Mentor/in in Begleitung einer Hochschulprofessorin oder eines -professors. Sie erhalten hierbei Beobachtungsaufgaben mit der Aufforderung einer gezielten Wahrnehmungsbeobachtung zu einem gewissen Setting, welche in den begleiteten hochschulischen Lehrveranstaltungen analysiert werden. Grundlage bildet das Modell nach Lunkenbein (2012). Nach dem Autor sollte der Blick der Beobachtung auf Lehrperson, sich Selbst, Unterricht und Schüler/innen gerichtet sein. Reines Beobachten ist im Sinne des Aufbaus einer Beobachtungskompetenz nicht ausreichend, sondern es braucht nach Topsch (2004) ein geführtes und direkt geleitetes Beobachten mittels verschiedener Aufgabenstellungen (Rahm et al. 2014, 243) und die Zielvorgabe einer Dokumentation. Der Idee des Beobachtens am Beginn des Studiums liegt auch die Vorstellung zugrunde, dass für das pädagogische Handeln bedeutsame Erkenntnisse gewonnen werden können (Köck et al. 1994, 74). Für Abel et al. (2007, 40) und Flagmeyer et al. $(2002,83)$ ist die „Beobachtungskompetenz [...] die Grundlage qualifizierten Lehrer/innenhandelns." (Rahm et al. 2014, 243) Im Weiteren werden in den begleitenden hochschulischen Lehrverstaltungen Fragestellungen zur eigenen Lernbiografie, zu Potentialen, zu Rollenbildern und zum Perspektivenwechsel aufgegriffen. Die- 
se erste Phase bietet somit Gelegenheit, sich im Berufsfeld zu orientieren und ein Professionsverständnis zu entwickeln.

In den Professionalisierungsphasen I, II und III werden Studierende an die Ausübung der Unterrichtstätigkeit und andere schulische Aufgabenbereiche herangeführt und sollen lernen, unter bildungswissenschaftlichen, fachwissenschaftlichen, fachdidaktischen und pädagogisch-didaktischen Gesichtspunkten Unterricht zu beobachten, durchzuführen und eine theoriebasierte Forschungshaltung aufzubauen. Hierbei erhalten sie aus wissensbasiert-theoretischen Lehrveranstaltungen Arbeitsaufträge und setzen sich in den Praktika professions- und personenspezifische Ziele (Entwicklungsaufgaben), um so einen Lern- und Entwicklungsprozess erfolgreich zu gestalten. Ostermann (2015) konnte in ihrer Studie aufzeigen, dass die Entwicklungsaufgaben Reflexion, Perspektivenwechsel, Schüler/innenzentrierung, Vermittlung, Verhaltenskontrolle, Sicherheit und Selbstverantwortlichkeit mit ihren professionsspezifischen Anforderungen für Studierende eine wichtige Rolle auf dem Weg zum Lehrer/in-Werden spielen (Ostermann 2015, 162). Ausgearbeitete Fragestellungen zu den einzelnen Aufgaben bieten Hilfestellung für Studierende (KPH Edith Stein 2020) und eine Grundlage im Mentoring innerhalb des Beziehungsgefüges (siehe Kapitel I/2.4.5).

Die Schwerpunktphasen I und II finden im jeweiligen Schulkontext des gewählten Schwerpunktes statt und haben Planung und Gestaltung von Lernsettings sowie die kritische Auseinandersetzung in Form von theoriegeleiteter Reflexion zum pädagogischen Handeln und zur eigenen Person als Hauptaufgaben. Studierende werden durch Arbeitsaufträge angehalten, sich im Sinne des Professionalisierungsprozesses mit Fragen zu unterschiedlichen gesellschaftsrelevanten Perspektiven und mit Fragen zum eigenen Entwicklungsprozess (Entwicklungsaufgaben mit professionsspezifischen Anforderungen; Ostermann 2015) zu befassen und sowohl mit der/dem Mentor/in als auch in den begleitenden Lehrveranstaltung mit der/dem Hochschullehrenden in Austausch zu gehen.

Im Fokus der unterschiedlichen Phasen steht die Entwicklung der pädagogischen Handlungsfähigkeit im Sinne einer Transformation und eines reflexiv-forschenden Lernansatzes im Berufsalltag. Im Professionalisierungsprozess werden Studierende angeleitet, sich mit pädagogischem Handeln und selbstregulativen Lern- und Entwicklungsprozessen auseinanderzusetzen. Die Tätigkeiten in den Praxiszeiten werden von ausgebildeten Mentor/innen und Lehrenden der Hochschule (Beziehungsgefüge) in intensiven Vor- und Nachbesprechungen dialogorientiert begleitet - ganz im Sinne des Empowerment-Konzepts (Arnold et al. 2011, 240). Neben Planung und Gestaltung von Lehr-Lernprozessen zielen die reflexiven Praxis- und Beratungsgespräche mit Studierenden auf: Ausbau subjektiven Berufswissens, Auseinandersetzung mit bildungswissenschaftlichem und fachbezogenem Wissen und mit Didaktiken sowie mit Kompetenzbereichen, auf Setzen und Reflektieren von individuellen Entwicklungsaufgaben, Darstellung und Klärung der Wertvorstellungen und Haltungen zu Schule und Lehren-Lernen im Blick auf Profession. Die Beziehungsebene Praxislehrer/in und Praxisberater/in (der Hochschule) schafft für Studierende (Beziehungsgefüge) im Optimalfall eine Lernumgebung, die den Lernprozess und die Entwicklung zur/zum Pädagogin/ Pädagogen explizit unterstützt und personenspezifisch begleitet. Letztendlich wird für das Wirksamkeitserleben entscheidend sein, wie die interpersonale und zeitliche Ausformung der Begleitung gestaltet und erlebt wird (Arnold et al. 2011, 240) bzw. ob bei Studierenden eine Kongruenz zwischen Persönlichkeitseigenschaften und Umweltbedingungen im Sinne des Person-UmweltModells nach Holland (1997) stattfindet. 
„Gegeben ist eine solche Kongruenz, wenn personale Interessen, Fähigkeiten und Eigenschaften den Befriedigungsmöglichkeiten bzw. Anforderungen der jeweiligen Umwelt entsprechen' (Bergmann 1998, 29). Die Herstellung von Kongruenz erfolgt über die Auswahl von solchen Umwelten, in denen Personen ihre Persönlichkeitseigenschaften am besten nutzbar machen können.“ (Wisniewski 2016, 18)

Professionelles pädagogisches Handeln vollzieht sich im unmittelbaren Tun in den Erprobungs- und Studienfeldern und bedarf nach der unterrichtlichen Tätigkeit einer selbstkritischen Reflexion (Altrichter et al. 2004a). Die unterschiedlichen Phasen bieten Gelegenheit zur Kompetenzentwicklung und -erweiterung zur Profession Lehrer/in-Sein. Bei genauer Konzeptbetrachtung auf Organisations- und Inhaltsebene sind auch die Handlungsbereiche Unterrichten, Erziehen, Beurteilen und Innovieren in Anlehnung an die deutsche Kultusministerkonferenz (KMK Modell 2004) für das Konzept der Pädagogisch-Praktischen Studien (PPS) an der Privaten Pädagogischen Hochschule Edith Stein (KPH Edith Stein) übernommen und um den Handlungsbereich Beobachten erweitert worden. Pädagogisches Beobachten bedarf einer Schulung, um qualitative Unterschiede zu erkennen (Bromme et al. 2008) und in einen zirkulären Prozess des Beschreibens, Deutens und Handelns einsteigen zu können (De Boer et al. 2012, 282). Für alle fünf Handlungsbereiche liegen Definitionen für Standards für Studierende vor, die einerseits als Möglichkeit des selbstreflexiven theoriegeleiteten Erkundens und andererseits als Bewertungsgrundlage im Ausbildungsprozess Verwendung finden.

Zusammenfassend kann gesagt werden: Das Konzept der Pädagogisch-Praktischen Studien (PPS) an der Privaten Pädagogischen Hochschule Edith Stein (KPH Edith Stein) verknüpft in der Gestaltung und Ausführung der hochschulischen und schulischen Ausbildungsanteile mehrere Theoriekonzepte. Im Hintergrund stehen die Bildungsgangtheorie (Schenk 2005, 2012) mit prozess- und personenorientierten Konzepten wie der Berufsbiografische Entwicklungsprozess, die Person-Umwelt-Theorie nach Holland (1997), das Konzept der Entwicklungsaufgaben (Havighurst 1948, 1953) im Sinne der Kompetenz- und Identitätserweiterung (Hericks 2004, 2006) und die/der Studierende (die/der Lehrer/in nach Kordes 1996) als Bildungsgangforscher/in (Kordes 1996) durch Aufbau einer forschenden-reflektierten Haltung.

In der Sekundarstufe wurden von einem Expert/innenteam der Universitäten und Pädagogischen Hochschulen Anforderungsprofile und Konzepte zu den Pädagogisch-Praktischen Studien ausgearbeitet, die im Folgenden erläutert werden.

\section{Modell Sekundarstufe Allgemeinbildung in der Lehrer/innenbildung WEST}

Das zweite Modell weist auf die Verwobenheit der unterschiedlichen Studienanteile mit den Pädagogisch-Praktischen Studien hin, das auch im Mentoringprozess zum Tragen kommen soll. Im Zuge der Neugestaltung der Curricula im Entwicklungsverbund Lehrer/innenbildung WEST kam das integrative Studienmodell (Eurydice 2002) zum Tragen. Expert/innen der Pädagogischen Hochschulen und Universitäten konzipierten ein Curriculum, das schulpädagogisch-praktische Ausbildungsanteile mit dem theoretisch-wissenschaftlichen Studium bzw. den hochschulischen Ausbildungsanteilen verschränkt. Die Pädagogisch-Praktischen Studienanteile, die im Bachelor-Master-Studium integriert sind, dienen zunächst der Orientierung im Berufsfeld Schule, zielen im Weiteren auf den Erwerb und die Entwicklung von Kompetenzen und betonen den Ansatz der Theorie-Praxis-Verknüpfung. Die vorgeschriebenen ECTS-Anrechnungspunkte der Pädagogisch-Praktischen Studien finden sich in den Bildungswissenschaftlichen Grundlagen (BWG) und den jeweiligen Fachbereichen und Spezialisierungen (Inklusive Pädagogik, Medienpädagogik). Schulpraktika sind in den einzelnen Modulen der Curricula thematisch und normativ-curricular nach einem Spiralprinzip und dem Vertiefungs- und Ver- 
netzungsaspekt verortet. Das Curriculumhandbuch (2016), das für die Bildungswissenschaftlichen Grundlagen erstellt wurde, dient als weiteres Entwicklungsinstrument. Darin werden die Anforderungen und Zielsetzungen der schulpraktischen Anteile zum Schulpraktikum I (1,5 ECTS-AP), Schulpraktikum II (1,5 ECTS-AP), Schulpraktikum IIIa und IIIb (mit je 2,5 ECTSAP) und Schulpraktikum IV (5 ECTS-AP) beschrieben. All diese genannten Praktika werden den Bildungswissenschaftlichen Grundlagen zugeordnet, während Fachpraktikum I und II (mit je 5 ECTS-AP) in den Curricula der Fachbereiche bzw. der Spezialierung implementiert sind. Bereits in den Modulbeschreibungen der Bildungswissenschaftlichen Grundlagen wird darauf geachtet, dass Studierende an allen unterschiedlichen Schultypen schuladäquate Erfahrungen sammeln können. Hervozuheben ist auch Schulpraktikum IV im Masterstudium (5 ECTS-AP). Dieses wird als Möglichkeit der Vertiefung von Erfahrungsprozessen wissensorientiertem und pädagogischem Handeln gesehen. Die Aufgabe ist die Konzeption eines evidenzbasierten Entwicklungsprojektes mit dem Ziel der Professionalisierung unter schulartspezifischen und altersadäquaten Bedingungen.

Praktika in den Bildungswissenschaftlichen Grundlagen verfolgen in den Anforderungen ein Fünf-Themenbereichs-Konzept: Unterrichtsbeobachtung, Schule als Organisation, Reflexion und Dokumentation, Schule als Forschungsfeld, Unterrichten und Classroom-Management. Als Gestaltungsart wurden fünf Zahnräder gewählt (Abbildung 1):

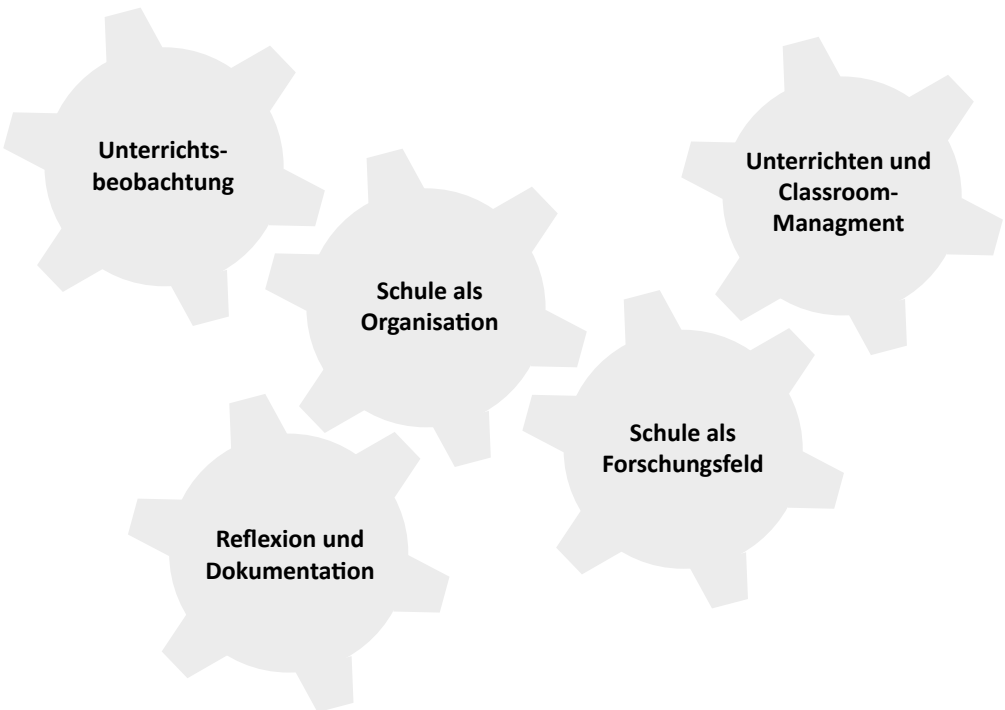

Abb. 1: Fünf-Themenbereichs-Konzept Lehrer/innenbildung WEST (Curriculumhandbuch 2016)

Die fünf Themenbereiche sind jene Bereiche, in denen Studierende im Unterricht und im Sozialisationsfeld Schule verstärkt eingebunden sind, Erfahrungen durch direktes pädagogisches Handeln sammeln können und die subjektiv-objektive Entwicklung voranschreiten soll. In den begleitenden hochschulischen Lehrveranstaltungen wird darauf geachtet, dass eine Vernetzung von Theorie, Praxis und Forschung gelingen kann. Im Curriculumhandbuch (2016) sind zu allen Praktika und zu allen Bereichen Zielsetzungen angeführt. Exemplarisch werden die Zielsetzungen des Schulpraktikums I bezogen auf die fünf Themenbereiche vorgestellt (Tabelle 4): 
Tab. 4: Zielsetzungen Schulpraktikum I Lehrer/innenbildung WEST (Curriculumhandbuch 2016,38)

\begin{tabular}{ll}
\hline Bereich & Zielsetzungen \\
\hline Unterrichts- & - Unterrichtliches Handeln als Zusammenspiel von Aktion und Reaktion zielgerichtet \\
beobachtungen & wahrnehmen und teilnehmend lehrseitig und lernseitig beobachten \\
& - Erkennen von unterschiedlichen Unterrichtsphasen \\
& - Wahrnehmung der Aspekte der Diversität und des sozialen Lernens \\
\hline Unterrichten & - Sammlung von ersten Erfahrungen in Unterrichtssequenzen individuell und im Team \\
und Classroom- & - Schriftliche Planung von Unterrichtssequenzen auf der Basis unterschiedlicher Bausteine \\
Management & - Umsetzung von Konzepten für das Planen von Unterricht \\
& - Schaffung eines Einblicks in Methodenvielfalt und Sozialformen \\
\hline Reflexion und & - Aufgaben und Rolle der Lehrperson \\
Dokumentation & - Schüler/innenverhalten \\
& - Gestaltete Lernumgebungen \\
& - Lehrerinnen- und Lehrerbild zwischen Fremd- und Selbstbedeutung \\
& - Bildungsbiografie \\
\hline Schule als & - Schule als bildende Institution \\
Organisation & - Einblick in den zukünftigen Arbeitsplatz \\
\hline Schule als & - Relevanz von Forschungskompetenz für Lehrpersonen \\
Forschungsfeld & - Beobachtungsmodelle und Beobachtungsmethoden \\
& - Empirischer Zugang zu forschendem Lernen \\
\hline
\end{tabular}

Ziele des Schulpraktikums I sind das Kennenlernen einer Schulkultur, das Sich-Auseinandersetzen mit den Beobachtungseinheiten in den Vor- und Nachbesprechungen im Sinne der Weiterentwicklung, das Gestalten und Durchführen von Unterrichtseinheiten, das kritische Reflektieren von eigener Unterrichtstätigkeit sowie das Entwickeln eines forschenden Habitus. In den begleitenden Lehrveranstaltungen werden neben einer theoretischen Fundierung Grundkenntnisse zu Unterrichtsplanung, Durchführung von Unterricht, Methoden von Unterrichts- und Schüler/innenbeobachtungen und Methoden zur Förderung einer theoriebasierten Reflexionsfähigkeit (z.B. reflexives Schreiben usw.) vermittelt. Die Auseinandersetzung mit der eigenen Lernbiografie und mit subjektiven Theorien zu Lehren und Lernen skizzieren ebenso die Inhalte der ersten Lehrveranstaltungen im Lehramtsstudium Sekundarstufe Allgemeinbildung. Auf Organisationsebene wurden auf Basis der Curricula sowie der Zielsetzungen innerhalb des Konzepts und der Lehrveranstaltungen Anforderungsprofile für jedes Praktikum erstellt. Damit diese in den Praktika erreicht werden können, werden Studierende von erfahrenen Lehrpersonen im Rahmen ihrer Mentoring-Funktion in den Lernarrangements und an unterschiedlichen Schulen begleitet und unterstützt.

Erste Forschungsbefunde zum Schulpraktikum I Sekundarstufe Allgemeinbildung der Lehrer/ innenbildung WEST (angesetzt zwischen dem ersten und zweiten Semester), die mittels qualitativer Inhaltsanalyse von Haas et al. (2018) ausgewertet wurden, zeigen drei wesentliche Komponenten. Die Ergebnisse verdeutlichen die Wichtigkeit des Austausches aller Beteiligten im Rahmen einer qualitätsoffensiven Lehrer/innenbildung.

(1) Ergebnis der Studie: Studierende $(n=30)$ fühlen sich nach Absolvierung des Schulpraktikums I in der Berufswahlentscheidung bestätigt. Fragestellungen und Diskussionen zur Berufswahlentscheidung sollten somit Gegenstand der ersten begleitenden Seminare sein und auch Mentor/innen müssen mit dieser Thematik vertraut sein. 
(2) Ergebnis der Studie: Abläufe und Aufgabenstellungen zum Schulpraktikum I lassen Studierende $(n=30)$ in einen Lernprozess eintauchen, bei denen sie von hochschulischer Seite gut vorbereitet (vor allem im Bereich Fachdidaktik) und von Seiten der Mentor/innen intensiv begleitet werden wollen. Die Ergebnisse zeigen, dass es eine Klärung zu Zielsetzungen und Anforderungen zum Schulpraktikum I mit allen Beteiligten benötigt.

(3) Ergebnis der Studie: Spektren der Emotionen wie Freude und Angst kommen zum Tragen. Studierende $(\mathrm{n}=30)$ freuten sich auf die Herausforderung und darauf, den Perspektivenwechsel vollziehen zu können. Ängste plagten Studierende vor allem in den Bereichen: die eigene Person betreffend (Leitung einer Gruppe), Unterrichtsfach (zu wenig Fachwissen, wenig didaktisches Verständnis), im Umgang mit den Schülerinnen und Schülern (fehlender Respekt, Umgang mit der Altersgruppe) und in Zusammenhang mit schulinternen Abläufen (Teamteaching, Kollegium) (Haas et al. 2018, 54f). Das Ansprechen der emotionalen Komponenten verlangt neben Behutsamkeit und Achtsamkeit auch ein Instrument für Mentor/innen und Hochschullehrenden.

Diese Erkenntnisse weisen darauf hin, dass schulpraktisches Mentoring vorbereitet, strukturiert und im gemeinsamen Austausch mit den Ausbildungsinstitutionen erfolgen muss. Weiters ist im schulpraktischen Mentoring - und vielleicht besonders in den ersten Praktika - auf affektivmotivationale Aspekte seitens der Mentee Rücksickt zu nehmen. Dazu wird im Kapitel I/2 näher eingegangen.

Im Folgenden werden Forschungsbefunde zu Schulpraktika diskutiert.

\subsubsection{Forschungsbefunde zu Schulpraktika}

In der Forschungslandschaft zu Schulpraktika liegen im deutschsprachigen Raum vor allem Ergebnisse zum Stellenwert der Praktika und zu Erfahrungen aus Sicht der Studierenden (1) und zur Lerneffektivität von schulischen Lernmöglichkeiten (2) vor (Bach 2013, 106).

Aus der Perspektive von Studierenden (1) wird betont bzw. zeigen Studien und Befragungen von Studierenden (Ostermann 2015; Cramer 2012) immer wieder auf, dass Pädagogisch-Praktische Studienanteile auf große Akzeptanz (Ostermann 2015, 148) stoßen und sie meist von Studierenden als das „Kernstück der Lehrerbildung in der Hochschule“ (Arnold et al. 2011, 9) betitelt werden.

„Über diese Attraktivität hinaus ist die Möglichkeit der Mitgestaltung beruflicher Praxis in den Augen der Studierenden aber ein hochgradig effektives Setting des Erwerbs beruflicher Handlungsfähigkeit und der damit verbundenen Handlungssicherheit." (Leonhard et al. 2018, 12)

Cramer (2012) ging in Interviews der Frage nach der hohen Bedeutung bei Studierenden nach. Als großen Nutzen führen Studierende mehrere Gründe an: Überprüfung der Berufswahlentscheidung, Neudefinition der eigenen Lehrer/innenrolle und Vollziehen des Perspektivenwechsels, Bewusstwerden der Komplexität von Unterrichtsanforderungen, Vertrautwerden mit der Schulorganisation, Erkennen der Notwendigkeit einer Selbstorganisation, Verknüpfung von Theorie und Praxis (Cramer 2012, 460f). Kreis (2012) dokumentierte, dass Studierende darauf verweisen, dass es aus ihrer Sicht in den Praktika zu einer Erweiterung des allgemein-didaktische Wissens sowie der Planungs- und Reflexionsstrategien kommt (Kreis 2012, 302). Demzufolge können Mentor/innen annehmen, dass Studierende sich auf Praktika freuen und sich mit Planungs- und Reflexionsstrategien auseinandersetzen werden. Leonhard et al. (2018) spricht auch den Erwerb der Handlungssicherheit (Leonhard et al. 2018, 12) an, der in der Literatur 
als Selbstwirksamkeit diskutiert und in der vorliegenden Arbeit noch thematisiert wird (Kapitel I/2.4.6).

Studierende hegen oftmals den Wunsch, mehr und längere Praktika während der Zeit der Ausbildung zu absolvieren. Im internationalen Kontext fehlen dazu Untersuchungen, wonach die Lehrer/innenausbildung durch Verlängerung von Praxisanteilen bessere Ergebnisse erzielt (Rothland et al. 2018, 1). Es sind ebenso Befunde ausständig, die eine Verbindung zwischen Berufswahlmotivation und Absolvierung einiger Praktika haben (Mertens 2018, 69).

Neben den Befunden zu motivationalen Variablen wie Berufswahlmotivation oder Selbstwirksamkeit werden auch emotionale Spektren in Studien nach Praktikumsabläufen untersucht (Haas et al. 2018, 54f).

Zum Nutzen und zur Wirkung von Schulpraktika (2) konnte Bach (2013) bestätigen, dass es eine Korrelation zwischen der Beziehung zur/zum Mentor/in und der allgemeinen didaktischen Planungskompetenz gibt (Bach 2013, 106f). Untersuchungen von Hascher (2012) und Arnold et al. (2014) verdeutlichen die Notwendigkeit von Reflexionsteilen zur Erhöhung der Lerneffektivität. König et al. (2012) bestätigten nach ihren Untersuchungen eine Steigerung des pädagogischen Unterrichtswissens und des bildungswissenschaftlichen Wissens aufgrund von Praktika (König et al. 2012). Diese genannten Befunde weisen für das schulpraktische Mentoring darauf hin, dass Mentor/innen eine Kompetenz zur Planungsberatung sowie Kenntnisse und Zeit zu Abläufen von Reflexionsprozessen haben müssen, um im Sinne der Lerneffektivität zu handeln und Studierende entsprechend zu begleiten. Demzufolge sind Ausbildungsinstitutionen gefordert, Angebote der Weiterbildung und Instrumente der Beratung für Mentor/innen zu etablieren.

Im Folgenden werden acht Forschungsergebnisse dargelegt, verstärkt aus den Forschungssupports des in einigen deutschen Bundesländern neu eingeführtem Praxissemesters, die vor allem in Rothland et al. (2018) „Forschung zum Praxissemester in der Lehrerbildung“ und in König et al. (2018) „Learning to Practice, Learning to Reflect?“ diskutiert werden. Das Praxissemester (meist pro Woche ein Praxistag und ein zweiwöchiges Blockpraktikum) bzw. Langzeitpraktika zählen als „Königsweg neuer Lehrerbildungsmodelle“ (Schubarth et al. 2012, 202) oder als „Herzstück der Lehrerbildungsreform“ (Floß et al. 2010). Die Beschreibung erfolgt nach dem Prinzip Forschungsfrage, Aufbau des Untersuchungsdesigns, zentrale Ergebnisse und endet immer mit der Kernidee als Versuch der Interpretation der Bedeutung für das schulpraktische Mentoring.

Die ersten drei angeführten Untersuchungen konzentieren sich auf personenbezogene Empfindsamkeiten der Studierenden bzw. Mentee während der Zeit der Praktika. Es wird die Bedeutung der Mentor/innen ersichtlich, wenn es darum geht, Studierende in den Praktika zu stärken.

(1) Forschungsfrage: An Studierende $(n=4)$ wurde die offene Fragestellung „Wie läuft es im Partnerschulprojekt?" bzw. „Wie war es?" gestellt.

Aufbau des Untersuchungsdesigns: Nach Absolvierung des Vertiefungspraktikums (ein Tag pro Woche und zweiwöchiges Blockpraktikum) erfolgte an der Pädagogischen Hochschule FHNW eine Befragung von Studierenden $(n=4)$. Die Gesprächsaufzeichnungen wurden transkribiert und mittels Objektiver Hermeneutik ausgewertet.

Zentrale Ergebnisse: Studierende erwähnen als Besonderheit die „Zugewinne an Sicherheit und Routine der eigenen Performanz" (Leonhard et al. 2018, 13) aufgrund einer län- 
geren Anwesenheit in der Klasse im Rahmen der Vertiefungspraktika oder Langzeitpraktika bzw. des Praxissemesters (Bezeichnung je nach Literatur). Sie bezeichnen die Klasse als „Cool“, ein Verantwortungsgefühl gegenüber der Gruppe der Lernenden in dieser Zeit wird nicht thematisiert. Das Nachdenken über den eigenen Handlungsprozess und die damit einhergehende Verschriftlichung in Form von Reflexionsarbeiten wird von Studierenden als belastend betrachtet und abgelehnt (Leonhard et al. 2018, 5f).

Kernidee: Mentor/innen sollen in den Praktika auf die Bedeutung von Reflexionsphasen und Bildungszielen, wie sie im Lehrplan dokumentiert sind, eingehen. Außerdem soll im Mentoring die Lehrer/innen-Schüler/innen-Beziehung thematisiert werden.

(2) Forschungsfrage: „Stellen eine intrinsische Berufswahlmotivation, eine hohe Selbstregulation sowie hohe Kompetenzselbsteinschätzungen Prädiktoren für das konzeptionelle bildungswissenschaftliche Professionswissen und die professionelle Unterrichtswahrnehmung zu Beginn und am Ende des Praxissemesters dar?" (Mertens et al. 2018, 72)

Aufbau des Untersuchungsdesigns: Für die jeweils einstündige Untersuchung mittels Fragebogen und Videografieinterpretationen mit anschließenden Itembewertungen wurden zwei Zeitpunkte gewählt. Für die Auswertung konnten 142 Fragebögen herangezogen werden. Die anschließende Tabelle (Tabelle 5) stellt die verwendeten Diagnoseinstrumente dieser Studie vor:

Tab. 5: Diagnoseinstrumente mit Kompetenzfacetten zur Studie (Basierend auf Mertens et al. $2018,72 \mathrm{f})$

\begin{tabular}{|c|c|c|}
\hline Kompetenzfacette & Diagnoseinstrument & Besonderheit \\
\hline Berufswahlmotivation & $\begin{array}{l}\text { Fragebogen zur Erfassung der } \\
\text { Motivation (FEMOLA) (Pohl- } \\
\text { mann \& Möller 2010) }\end{array}$ & $\begin{array}{l}6 \text { Skalen mit } 41 \text { Items zu: } \\
7 \text { zu Pädagogisches Wissen, } 6 \text { zu Fachliches Wis- } \\
\text { sen, } 6 \text { zu Fähigkeitsüberzeugung, } 9 \text { zu Nützlich- } \\
\text { keit, } 5 \text { Schwierigkeit des Studiums, } 6 \text { zu sozialen } \\
\text { Einflüssen }\end{array}$ \\
\hline Selbstregulation & $\begin{array}{l}\text { Selbsteinschätzung mit Skalen } \\
\text { und vierstufiger Bewertung } \\
\text { (Grob \& Maag-Merki 2001) }\end{array}$ & $\begin{array}{l}\text { 4 Skalen mit } 21 \text { Items: } \\
5 \text { zu Planung, } 5 \text { zu Monitoring, } 5 \text { zu Evaluation, } 6 \\
\text { zu Selbstreflexion }\end{array}$ \\
\hline $\begin{array}{l}\text { Praxisbezogene Kom- } \\
\text { petenzselbst- } \\
\text { einschätzungen }\end{array}$ & $\begin{array}{l}\text { Fragebogen in Anlehnung an } \\
\text { KMK-Modell (Gröschner 2009) }\end{array}$ & $\begin{array}{l}4 \text { Skalen mit } 33 \text { Items: } \\
7 \text { zu Unterrichten, } 11 \text { zu Erziehen, } 9 \text { zu Beurteilen, } \\
6 \text { zu Innovieren }\end{array}$ \\
\hline $\begin{array}{l}\text { Konzeptuelles } \\
\text { bildungswissenschaftli- } \\
\text { ches Professionswissen }\end{array}$ & $\begin{array}{l}\text { Fragebogen: Wissenstest von } \\
\text { Seifert \& Schaper }(2010,2012)\end{array}$ & $\begin{array}{l}3 \text { Anforderungsbereiche mit Items: } \\
7 \text { zu Reproduktion, } 11 \mathrm{zu} \text { Anwendung, } 5 \mathrm{zu} \\
\text { Bewertung }\end{array}$ \\
\hline $\begin{array}{l}\text { Professionelle Unter- } \\
\text { richtswahrnehmung }\end{array}$ & $\begin{array}{l}\text { Diagnoseinstrument Obser- } \\
\text { ver (Seidel et al., 2010) mit } \\
\text { Videotools }\end{array}$ & $\begin{array}{l}3 \text { Skalen mit } 36 \text { Items mit Likert-Skala: } \\
\text { Situationsanalyse, Ergebniserklärung, Formulieren } \\
\text { von Lernerfolgsvorhersagen }\end{array}$ \\
\hline
\end{tabular}

Zentrale Ergebnisse: Mittels eines Fragebogens zu zwei Zeitpunkten (vor und nach dem Praxissemester) mit Kurzversionen der in Tabelle 5 genannten Fragebögen und Diagnoseinstrumente werden folgende Ergebnisse erzielt: 
Tab. 6: Mittelwerte (M) und Standardabweichungen (SD) der untersuchten Kompetenzaspekte zu Beginn (t1) und am Endes (t2) des Praxissemesters (Mertens et al. 2018, 76)

\begin{tabular}{lrrrr}
\hline \multirow{2}{*}{ Prädikatoren } & \multicolumn{3}{c}{$\mathbf{t}_{\mathbf{1}}$} & \multicolumn{3}{c}{$\mathbf{t}_{2}$} \\
\cline { 2 - 5 } & $\mathbf{M}$ & $\mathbf{S D}$ & $\mathbf{M}$ & $\mathbf{S D}$ \\
\hline Motivation & 3,37 & .33 & & \\
Selbstregulation & 2.89 & .38 & 2.92 & .38 \\
Selbsteinschätzung & 4.30 & .73 & 5.07 & .65 \\
Kriteriumsvariablen & & & & \\
(4.1) Konzeptuelles Wissen & 64.75 & 10.48 & 66.19 & 10.75 \\
(4.2) Unterrichtswahrnehmung & .36 & .15 & .41 & .18 \\
\hline
\end{tabular}

Steigerungen in den Mittelwertsunterschieden ergeben sich bei Unterrichtswahrnehmungen und Kompetenzselbsteinschätzungen. Korrelationen mittlerer Stärke bestehen zwischen Motivation, Selbstregulation und Selbsteinschätzungen. In Zusammenhang mit Selbstregulation konnte keine Signifikanz zum Professionswissen und zur Unterrichtswahrnehmung festgestellt werden (Mertens et al. 2018, 79). Zum ersten Zeitpunkt der Messung konnte „keine Veränderung des konzeptuellen bildungswissenschaftlichen Professionswissens (...) und der Unterrichtswahrnehmung (...) im Praxissemester" (Mertens et al. 2018, 77f) vorhergesagt werden. „Die Analysen zeigen, dass erst nach Absolvieren des Praxissemesters die subjektiven Kompetenzeinschätzungen in einem systematischen Zusammenhang zu der professionellen Unterrichtswahrnehmung standen." (Mertens et al. 2018, 66) Forschendes Lernen und das Anwenden von Lernstrategien zielen daher auf ein effektives und zielgerichtetes Handeln (Schiefele et al. 2003). Somit ergeben sich nach dieser Studie vor allem Fragestellungen nach Art und Optimierung von Lernangelegenheiten hinsichtlich einer Kompetenzentwicklung (Mertens et al. 2018, 81).

Kernidee: Mentor/innen leiten zum forschenden Lernen an, setzen Schwerpunkte und suchen Verbindungen zwischen subjektiver Kompetenzeinschätzung und Unterrichtswahrnehmung.

(3) Forschungsfrage: „Wie entwickelt sich die Lehrerselbstwirksamkeitsüberzeugung (sic!) (LSWE) in den Subfacetten Classroom Management, Instructional Strategies und Student Engagement bei Studierenden im Praktikum und darüber hinaus über den Zeitraum von insgesamt 12 Monaten (...) und ergeben sich hierbei Unterschiede in Abhängigkeit von der Länge der Praxisphasen?“ (Böhnert et al. 2018, 85)

Aufbau des Untersuchungsdesigns: Die Befragung der LSWE wurde mittels der Scale for Teacher Self-Efficacy (Pfitzner-Eden et al. 2016) vor, während und nach den Praktikumsphasen mit zwei Gruppen, jene die ein Blockpraktikum $(n=56)$ und jene, die das Praxissemester $(\mathrm{n}=203)$ absolvieren, durchgeführt.

Zentrale Ergebnisse: Je länger anhaltend Praxisphasen gestaltet sind, desto eher ist eine Erhöhung der Lehrer/innenselbstwirksamkeitsüberzeugung (LSWE). Böhnert et al. (2018) weisen darauf hin, dass es während des Praxissemesters zu Veränderungen von Überzeugungen und Erwartungen kommt, wenn auch nicht von Dauerhaftigkeit (Böhnert et al. 2018, 103). Beim Engagement der Studierenden zeigt sich keine Veränderung in den Gruppen und über die Zeit hinweg. Eine Uneinheitlichkeit ergibt sich bei Ergebnissen zu Studien zum Aufbau von Selbstwirksamkeitsüberzeugungen in Verbindung mit Praxisphasen. Böhnert et al. (2018) schlagen deshalb Konzepte mit Längsschnittuntersuchungen und der 
Beachtung der Dauer der Praktika vor (Böhnert et al. 2018, 86f). Im Forschungssupport wurde auch nicht der Frage nachgegangen, welchen Einfluss begleitende Lehrveranstaltungen oder Mentor/innen haben (Böhnert et al. 2018, 104f).

Kernidee: Mentor/innen brauchen Informationen zu Lehrer/innenselbstwirksamkeitsüberzeugung. Die Thematik sollte in Gesprächen mit Mentee zur Selbstverständlickeit innerhalb des Mentoringprozesses werden.

Die folgenden zwei Untersuchungen beschäftigen sich mit dem Aufbau des Professionswissens während der Zeit der Praktika. Mentor/innen können dabei einen wichtigen Aufgabenbereich übernehmen.

(4) Forschungsfrage: Wie gestalten sich „komplexe Lerngelegenheiten im Masterstudium an den Universitäten Hamburg und Köln“ und wie werden „lernprozessbezogene Tätigkeiten der Studierenden in den Praktika gemäß der Angebots-Nutzungs-Konzeption als Nutzung der schulpraktischen Lerngelegenheiten" (Doll et al. 2018, 24) beschrieben?

Aufbau des Untersuchungsdesigns: Drei Wochen vor Semesterende im Jahr 2016 wurde mittels Online-Befragung der Fragebogen zu den lernprozessbezogenen Tätigkeiten (König et al. 2014) (Forschungsmethodischer Zugang, Handlungsplanung, Handlungsdurchführung, Theoriebezug, Situationsreflexion) an den Universitäten Hamburg und Köln durchgeführt. Weiters wurden Daten zur investierten Lernzeit (Hospitation, begleitendes Unterrichten, selbständiges Unterrichten) und zu den Angebotsmerkmalen Kohärenz zwischen Hochschullehre und Schulpraxis und Betreuungssituation durch Mentor/innen erhoben. Für die Auswertung lagen Rückmeldungen von 482 Studierenden vor.

Zentrale Ergebnisse: Die Ergebnisse zu Mittelwert (M) und Standardabweichung (SD) zeigen hohe zeitliche interindividuelle Unterschiede bei den schulischen Lerngelegenheiten und die hohen Standardabweichungen bei den lernbezogenen Tätigkeiten. Doll et al. (2018) „weisen auf die Bedeutung individueller Lernstrategien, Lernorientierungen und Lernmotivation bei der Nutzung der vielfältigen Lerngelegenheiten in Schulpraktika hin.“ (Doll et al. 2018, 42) Die Kohärenz zwischen schulischen und hochschulischen Anteilen beurteilen die Studierenden niedrig.

Kernidee: Mentor/innen können in der Begleitung einen Fokus legen auf Erkunden forschungsmethodischer Zugänge, Planen pädagogischer Handlungssituationen, Zusammenfügen von Theorien auf Situationen und auf einen Umgang mit reflexiven Situationen.

(5) Forschungsfrage: „Wie intensiv wird das Thema Klassenführung in den erziehungswissenschaftlichen und fachdidaktischen Veranstaltungen zu Beginn des Bachelorstudiums, im regulären Masterstudium (vor dem Praxissemester) und in den Begleitveranstaltungen des Praxissemesters (am Ende des Masterstudiums) thematisiert? Wie ist der Wissenstand zum Thema Klassenführung in den drei Studienkohorten ausgeprägt und wie entwickelt er sich über den Verlauf von einem Semester?" (Westphal et al. 2018, 136)

Aufbau des Untersuchungsdesigns: Lehramtsstudierende $(n=554)$ beurteilten den Umfang des Themenbezuges Klassenführung in erziehungswissenschaftlichen und fachdidaktischen Seminaren und führten den Test zum Klassenführungswissen (Lenske et al. 2015) durch.

Zentrale Ergebnisse: Das Thema Klassenführung wurde aus Sicht der Studierenden vermehrt in den erziehungswissenschaftlichen Lehrveranstaltungen $(\mathrm{M}=2.73, \mathrm{SD}=.82 \mathrm{vgl}$. fachdidaktische $\mathrm{LV}$ mit $\mathrm{M}=1.78, \mathrm{SD}=.73$ ) besprochen. Intensiv war vor allem die Aus- 
einandersetzung mit der Thematik in Lehrveranstaltungen des Bachelorstudiums und des Praktikumssemesters, weniger in Lehrveranstaltungen des Masterstudiums. Auch konnte im Vergleich der Bachelorkohorte mit der Masterkohorte nachgewiesen werden, dass das Klassenführungswissen zunimmt. Ein weiteres Ergebnis zeigt auf, dass weibliche Studierende im Vergleich zu ihren männlichen Mitstudierenden zunächst mehr Wissen nachweisen können, aber die männliche Kohorte einen stärkeren Wissensanstieg im Laufe der Ausbildung erzielt. Auch korrelieren gute Abiturnoten mit höherem Wissenserwerb (Westphal et al. 2018, 146).

Kernidee: Mentor/innen besprechen in den ersten Praktika mit der/dem Mentee Strategien zur Klassenführung, entwickeln Forschungsfragen und/oder setzen Schwerpunkte zu professionsspezifischem Handeln.

Die folgenden drei angeführten Untersuchungsbeispiele zeigen die inhaltliche Verknüpfung von hochschulischen und schulischen Anteilen. Auch hierbei agieren Mentor/innen als Brückenbauer/innen, wenn es darum geht eine forschende Haltung einzunehmen und in Austausch mit den Ausbildungsinstitutionen zu gehen.

(6) Forschungsfragen: Welche unterschiedlichen Varianten zum Forschenden Lernen zeigen sich in den Studienprojekten? Welche berufspraktische Bedeutsamkeit lässt sich für Studierende über die Bearbeitung von Studienprojekten rekonstruieren?

Aufbau des Untersuchungsdesigns: Im Forschungsprojekt StiPS (Studienprojekte im Praxissemester) wurden Texte $(\mathrm{n}=35)$ und Poster $(\mathrm{n}=11)$ von Studierenden $(\mathrm{n}=46)$, die eine Lehrveranstaltung im Masterstudium als Vorbereitung für das Praxissemester besuchten, nach der qualitativen Inhaltsanalyse ausgewertet.

Zentrale Ergebnisse: Die inhaltsanalytische Auswertung der Studienprojekte der Fachdidaktik und Sonderpädagogik ergab in Zusammenhang mit Wirkungsforschung und Forschendem Lernen, dass Studierende ihren Blick auf Schülerin und Schüler als Lernende werfen und in den Prozess des Forschenden Lernens einsteigen. 74 Prozent der Studienprojekte $(\mathrm{n}=34)$ verbinden Praktika mit Evidenz und der Möglichkeit zur wirksamkeitsorientierten Forschung. Als Varianten der Wirksamkeitsforschung wurden die Klassifikationsdimensionen affektive bzw. kognitive Lernleistungen von Schülerinnen und Schülern, affektive und kognitive Dispositionen von Schülerinnen und Schülern erhoben. Studierende setzten unterschiedliche grafische Elemente zur Klärung der Memorierfähigkeit ein, ein Förderprogramm zur Verhaltensregulierung oder zu veränderten Schriften beim Lesen und überprüften dazu die Einstellungen und Interessen der Schüler/innen. Mittels Testverfahren und Evaluierungen wurden Rückschlüsse gezogen und im Forschungsprojekt unter dem Begriff Wirkungsforschung zusammengefasst. 26 Prozent der Studienprojekte $(\mathrm{n}=12)$ nahmen keine ablehnende Haltung gegenüber dem eigenen Forschungssupport an, wenngleich dieser ein Teil der Prüfungsleistung war und somit die Ergebnisse kritisch betrachtet werden müssen. Studierende beschrieben in den Studienprojekten, dass das Reflektieren von Handlungen für weitere Handlungspraktiken von Nutzen sein kann, eine Gruppe von Studierenden stand dem jedoch skeptisch gegenüber (Herzmann et al. 2018, 61).

Kernidee: Mentor/innen setzen gemeinsam mit der/dem Mentee einen Schwerpunkt für professionelles Handeln, reflektieren gemeinsam den Prozess und wiederholen Handlungsabläufe. Somit wird ein reflektiertes Handlungsrepertoire erstellt. 
(7) Forschungsfrage: Welche Bedeutung haben motivationale Prozesse der Studierenden hinsichtlich der Begleitveranstaltungen zu den Praktika, die „das Erlernen als auch das Anwenden der Reflexion von konkreten Unterrichtssituationen ermöglichen“ (Dehne et al. 2018, 113) auf Basis des Erwartungs-Wert-Ansatzes?

Aufbau des Untersuchungsdesigns: Die Interventionsgruppe $(n=52)$ reflektierte ihre videografierten Unterrichtssequenzen und gab bzw. erhielt Feedback; die Kontrollgruppe $(n=73)$ traf sich zu Feedbackrundengesprächen, aber ohne Videomaterial. Anschließende Fragebögen und quantitative Auswertungsmethoden zu subjektiven Aufgabenwerten (Skalen von Gaspard et al. 2015), Kompetenzeinschätzungen und Erfolgserwartungen (nach Gorges et al. 2012) mit Adaption der Skalen zu Unterrichtsreflexion folgten. Die zwei Messzeitpunkte lagen vor und nach dem Praktikum.

Zentrale Ergebnisse: Es zeigt sich eine Verbindung zwischen Nutzen und Kosten hinsichtlich weiterer beruflicher Anforderungen. Der motivationale Aspekt kann damit nicht isoliert betrachtet werden, sondern in der Komplexität der Ausbildung (Dehne et al. 2018, 124). Signifikante Ergebnisse zeigen sich in beiden Gruppen in Kompetenzeinschätzung und Erfolgserwartung. Die Interventionsgruppe beurteilt den Nutzen der videobasierten Reflexion für den Alltag und für die Zukunft höher als die Kontrollgruppe. Erfolge und Nutzen von Praktika sind somit sehr stark mit den Fragen verbunden, wie der theoriegeleitete Reflexionsteil aufbereitet wird bzw. was im Reflexionsteil von Studierenden in den begleitenden Lehrveranstaltungen verlangt wird (Hascher 2012; Arnold et al. 2014). Somit kommt der Begleitung der Praktika durch den hochschulischen Anteil eine besondere Bedeutung zu (Dehne et al. 2018, 109). Der Nutzen von Begleitreflexionen bei Praktika $(n=125)$ wird in Bezug auf Nutzen für die Zukunft als relativ hoch eingeschätzt, die Kosten (emotionale Kosten, Opportunitätskosten) weisen eine relative Stabilität auf (Dehne et al. 2018, 126). Im Fokus dieser Betrachtungsweise dürfen Motivation und Emotionen der/des Studierenden nicht vernachlässigt werden (Hascher et al. 2016; Dehne et al. 2018, 109). Nach Kunter et al. (2011) sind sie mitbestimmend für den Lernertrag auf dem Weg zur Professionalisierung (Dehne et al. 2018, 124). Weitere Untersuchungen vor allem in Bezug auf den Entwicklungsaspekt zu diesen Bereichen sind wünschenswert (Dehne et al. 2018, 127).

Kernidee: Mentor/innen videografieren, besprechen Handlungssequenzen mit der/dem Mentee auf theoriebegründeten Konzepten. Mentor/innen berücksichtigen affektiv-motivationale Faktoren der Mentee.

(8) Forschungsfrage: „(1) Welche psychometrischen Eigenschaften (faktorielle Struktur, Reliabilität) weist eine neu entwickelte Likert-Skala zur Erfassung der Einstellungen von Lehramtsstudierenden zur Theorie- und Praxis-Orientierung des Lehramtsstudiums auf? (2) Über welche Einstellung zur Theorie- und Praxisorientierung des Lehramtsstudiums verfügen Lehramtsstudierende am Anfang des Praxissemesters und wie verändern sich diese Einstellungen im Verlauf der Praxisphase?" (Fischer et al. 2018, 156)

Aufbau des Untersuchungsdesigns: Bei beiden Studien über die Einstellungen zur TheoriePraxis-Orientierung nahmen Studierende des 3. Semesters Masterstudium ((1) n = 294; (2) $\mathrm{n}=260$ ) der Universität Flensburg teil. Nach der Ermittlung der Faktorenstruktur erfolgte eine Absicherung der Einstellungsdimensionen durch eine Faktorenanalyse. Zur weiteren Vorgangsweise wurde das Zwei-Faktoren-Modell herangezogen. 
Zentrale Ergebnisse:

Tab. 7: Subdimensionen der Skala und Kennwerte (Fischer et al. 2018, 162); Anmerkungen: 4-stufige Skala ( 1 = stimme nicht zu, $4=$ stimme genau $\mathrm{zu}$ )

\begin{tabular}{llccccc}
\hline $\begin{array}{l}\text { Messzeit- } \\
\text { punkt }\end{array}$ & Subdimension & $\begin{array}{c}\text { Item- } \\
\text { anzahl }\end{array}$ & $\mathbf{n}$ & $\mathbf{M}$ & SD & $\begin{array}{c}\text { McDo- } \\
\text { nalds }\end{array}$ \\
\hline M.A. Prä & $\begin{array}{l}\text { Theoretisch-reflexive } \\
\text { Professionalisierungsfunktion }\end{array}$ & 3 & 294 & 3.01 & .54 & .70 \\
\cline { 2 - 7 } & $\begin{array}{l}\text { Berufseinführende } \\
\text { Professionalisierungsfunktion }\end{array}$ & 4 & 294 & 2.84 & .79 & .86 \\
\hline M.A. Post & $\begin{array}{l}\text { Theoretisch-reflexive } \\
\text { Professionalisierungsfunktion }\end{array}$ & 3 & 259 & 2.87 & .65 & .75 \\
\cline { 2 - 7 } & $\begin{array}{l}\text { Berufseinführende } \\
\text { Professionalisierungsfunktion }\end{array}$ & 4 & 260 & 2.71 & .90 & .91 \\
\hline
\end{tabular}

Die Mittelwerte liegen vor und nach dem Praxissemester über 2,7.

\begin{tabular}{lccccc}
\hline & $\begin{array}{c}\text { M(SD) } \\
\text { Prä }\end{array}$ & $\begin{array}{l}\text { M (SD) } \\
\text { Post }\end{array}$ & $\mathbf{t}(\mathbf{d f})$ & p & $\begin{array}{l}\text { Cliff's } \\
\text { delta }\end{array}$ \\
\hline Theoretisch-reflexive & 3.02 & 2.87 & 2.75 & .006 & .13 \\
Professionalisierungsfunktion & $(.55)$ & $(.65)$ & $(256)$ & & \\
\hline Berufseinführende & 2.85 & 2.72 & 1.77 & .078 & \\
Professionalisierungsfunktion & $(.77)$ & $(.90)$ & $(258)$ & & \\
\hline
\end{tabular}

Im Rahmen des Projektes ISabEL (Intensität und Stabilität berufsbezogener Einstellungen im Lehramtsstudium) an der Universität Flensburg zeigten die Studierenden nach dem Praktikum eine ablehnende Haltung gegenüber der Bearbeitung einer Forschungsfrage angesichts der Belastungen in der Zeit der Praktikumsabwicklung, und sie zeigten auch Umsetzungsschwierigkeiten auf. Der Vorbereitung und Begleitung im Sinne der Verständniserklärung kommt demnach eine besondere Bedeutung zu (Fischer et al. 2018, 163).

Kernidee: Mentor/innen sollen über Konzipieren von Forschungsfragen zu bildungswissenschaftlichen, fachwissenschaftlichen und fachdidaktischen Fragestellungen Bescheid wissen und beim unterrichtspraktischen Handeln Mentee auf die eine oder andere Fragestellung aufmerksam machen.

Die angeführten Untersuchungen berücksichtigen in ihren Forschungsdesigns drei wesentliche Komponenten in den schulischen Lerngelegenheiten: (1) personenbezogene Empfindsamkeiten zu Praktika und während der Zeit der Absolvierung der Praktika, (2) Aufbau von Professionswissen und Kompetenzerweiterung und (3) Relationierung zwischen Theorie und Praxis. Die dargestellten Forschungsbefunde verdeutlichen auch: Schulische Lerngelegenheiten sind in den Curricula der Lehrer/innenausbildung etabliert. Die angeführten Untersuchungsreihen sind ein weiterer Versuch, schulische Lerngelegenheiten während der Zeit der Ausbildung vor allem auch aus dem Blickwinkel der Forschung zu betrachten und Aussagen über die Lernwirksamkeit zu erhalten.

„Das Konzept der Lerngelegenheiten stellt seit Jahren einen wichtigen Ansatz der Bildungsforschung dar, um das Curriculum zu beschreiben und zu analysieren, das das Lernen sowie die Kompetenzentwicklung angehender Lehrkräfte bestimmt (McDonnell, 1995; Floden, 2015; Scheerens, 2017).“ (König et al. 2018, 23) 
Um die Effektivität der Lerngelegenheiten zu erhöhen, nehmen Mentor/innen eine bedeutende Funktion ein. Die angeführten Befunde weisen durch die Interpretation der Ergebnisse in den Kernideen auch auf mögliche Aufgabenbereiche für Mentor/innen und Mentee in den Praktika hin. In einer Zusammenschau der angeführten Forschungsbefunde ergibt sich eine Vierteilung von Bereichen, in denen Mentor/innen unterstützend agieren und somit Lern- und Entwicklungserfahrung ermöglichen können:

1. Erweiterung der Planungskompetenz und des Professionswissens

2. Aufbau und Ausbau der Reflexions- und Analysekompetenz

3. Vertiefen des Ansatzes forschenden Lernens

4. Umgang mit Lehrer/innenselbstwirksamkeit und affektiv-motivationalen Faktoren

Die Aufgabe der/des Mentee/Mentees in den Praktika besteht letztlich darin, Selbstverantwortung zu übernehmen. Mentor/innen bieten einen Lern- und Erfahrungsraum, schaffen situativ angemessene Lerngelegenheiten für Mentee und gehen in Kooperation. Die zentrale Frage für eine Konzeption von Praktika, für weitere Forschungsdesigns und schulpraktisches Mentoring mit all seinen Facetten ist weiterhin: Wie können Praktika angelegt sein, damit Studierende professionsspezifisches Handeln erproben und erwerben und Lernleistungen der Schüler/innen berücksichtigt werden?

Nach diesen resümierenden Aussagen zu Forschungsbefunden bzw. zur Anführung der Fragestellung wird der Fokus auf eine weitere Thematik im Professionalisierungsprozess der Lehrer/ innenausbildung gelegt: Theorie-Praxis-Verschränkung. Das folgende Kapitel eruiert, wie Konzepte und Ansätze der Theorie-Praxis-Verschränkung zum Professionalisierungsprozess beitragen und welche Aufgabe Mentor/innen dabei übernehmen können.

\subsubsection{Theorie-Praxis-Verschränkung}

Pädagogisch-Praktische Studien mit ihren schulischen Anteilen werden oftmals als Bindeglied zwischen dem Schulalltag (sogenannte Praxis) und den hochschulischen Anteilen in den Lehrveranstaltungen (mit verstärktem Bezug zur Theorie) gesehen. Mentor/innen nehmen in der Vermittlerfunktion eine nicht unbedeutende Rolle ein. Dieses Kapitel skizziert kurz den bildungshistorischen Verlauf und konzentriert sich auf gegenwärtige Sichtweisen zur TheoriePraxis-Verschränkung. Modelle von Meyer (2004), Wildt (2005), Patry (2014) und Fichten (2017) zeigen gangbare Wege für Mentor/innen in der Begleitung auf. Die Autor/innen setzen den Schwerpunkt auf reflexiv-forschendes Lernen als Möglichkeit zur Vertiefung der Verschränkung.

In die immer wiederkehrenden Diskussionen zur optimalen Entwicklung des professionsspezifischen Denkens und Handelns mischt sich das Thema der Theorie-Praxis-Verbindung während der Zeit des Studiums. Diese Thematik keimte vor allem durch die Institutionalisierung der Ausbildung zur Lehrperson auf, denn aus bildungshistorischer Sichtweise ist eine Veränderung und Weiterentwicklung der Theorie-Praxis-Relation zu erkennen.

„Von einem Theorie-Praxis-Verhältnis kann in der Ausbildungsform der Meisterlehre nicht gesprochen werden, denn Praxis bildete das konstitutive und meist alleinige Lernfeld (Bennack, 1989). Das Wissen zum Unterrichten beschränkte sich im Wesentlichen auf berufspraktische Fertigkeiten, erworben durch Belehrung bzw. Anschauung von Musterlektionen des Schulmeisters und deren reproduzierende Umsetzung in eigenes Lehrerhandeln (Homfeld, 1978). Allenfalls wurde das Lernen in der Praxis durch zunfteigene Methoden im Sinne von ,rezepthaften' Regeln ergänzt.“ (Bach 2013, 81) 
Praxis wurde damals als Berufsqualifikation verstanden. Durch Schaffung von Universitätsprofessuren und Einrichtung von Lehrstühlen für Pädagogik bzw. Erziehungswissenschaft erfolgte die Einbeziehung von theoretischen Inhalten, und es bildeten sich eigene Formen von Praxisanteilen heraus (Bach, 2013, 81). Die Folge war das Aufkeimen unterschiedlicher

„Diskurse im praktisch-professionellen und wissenschaftlich-disziplinären Kontext, wobei eine Unterscheidung solcher Diskurse nicht nur auf den deutschsprachigen Raum beschränkt zu sein scheint (Cochran-Smith/Lytle 1993; Fenstermacher 1994). Kennzeichnend dabei ist, dass die Genese, die Inhalte und die Funktion von Wissen stark vom jeweiligen Kontext bzw. Handlungsfeld abhängig sind und daher unterschiedlichen Gesetzmäßigkeiten unterliegen." (Herzmann et al. 2016, 149)

Heutige Denkfiguren beschäftigen sich mit Verbindungsmöglichkeiten und mit der Verringerung der Kluft von Theorie und Praxis (Cramer 2012, 449; Bach 2013, 92). Gibt es überhaupt eine Kluft? Können Theorie und Praxis eine Beziehung eingehen? Erklärungen und Diskussionsbeiträge zu diesen Fragen liefern die Bildungswissenschaft mit den Ansätzen zur Professionsforschung, die Kognitionspsychologie mit der Wissenspsychologie und die Wissensverwendungsforschung.

Wenn man in der Bildungswissenschaft von Theorie spricht, dann wird darunter ein Aussagesystem verstanden. Hierbei wird zwischen wissenschaftlichen Theorien, die allgemeinen Kriterien unterzogen sind, und subjektiven Theorien, die das eigene Handeln erklären oder beschreiben, unterschieden. Die Praxis zeichnet sich als ein Tun aus, das auf ein oder mehrere Ziele gerichtet ist (Patry 2014, 31f). Nach Ryle (1970) ergibt sich für die Theorie-Praxis-Beziehung bzw. Theorie-Praxis-Kluft ein Kategorienfehler, weil auf eine Theorie nicht nur eine Antwort fallen kann. Theorie (Aussagesysteme) und Praxis (Tun) sind divergente Kategorien. Das Tun in der Praxis ist vielleicht auf Grund der Theorie entstanden, kann somit als theoriegeleitet betrachtet werden. Weiters leitet und erklärt auch der Einbezug von subjektiven Theorien des Tuns im Sinne von „Kognitionen der Handelnden, welche die Selbst- und Weltsicht zum Gegenstand haben“ (Patry 2014, 32), das Tun. Patry (2014) spricht in diesem Zusammenhang von der „Theorie der Praxis“ (Patry 2014, 31). Jedoch nicht alle wissenschaftlichen Theorien können zunächst in der Ausbildung vorgestellt und übernommen bzw. in die Praxis übertragen werden. Phänomene wie Unvollständigkeit, fehlender Bedeutungsgehalt, Selektion und Legierung bestimmen die Aufnahme und Anwendung von wissenschaftlichen Theorien (Arnold et al. 2011, 86).

Leonhard (2018a) bringt gegenwärtig in seinem Diskurs zu Theorie und Praxis die Alternativen von Wissenschaftspraxis und Berufspraxis mit ins Spiel. Dabei „soll die Gleichrangigkeit beider Praxen“ (Leonhard 2018a, 19f) betont werden. Wie das Konstrukt der Zwei-Praxen-Rahmung weiter diskutiert wird, bleibt abzuwarten.

Im Kern der Lehrer/innenausbildung stellt sich die Frage, wie viel Theorie eine Lehrperson braucht. Für Terhart (1993) und Neuweg (2004) sind beide Formen des Lehrer/innenwissens das theoretisch-formale Wissen und das praktische Wissen und Können (Terhart 1993; Fenstermacher 1994; Baumert et al. 2006) - unverzichtbar. In der Bildungsforschung sind Konzepte und „Figuren der Relationierung von Lehrerwissen (sic!) und Lehrerkönnen (sic!)“ (Neuweg, 2004, 2) entstanden, die Fragen zur Theorie-Praxis-Problematik, bezogen auf die Relation, erörtern. Hierbei sind vor allem zwei konzeptuelle Gegenentwürfe vorherrschend: das Integrationsund das Differenzkonzept.

„Integrationskonzepte unterstellen deskriptiv wie präskriptiv einen relativ engen Zusammenhang zwischen Ausbildungswissen und berufspraktischem Können und zielen deshalb in der einen oder anderen Form darauf ab, Wissen und Können zu integrieren. Dagegen setzen Differenzkonzepte an einer Beob- 
achtung an, die auch Vertretern (sic!) des Integrationsgedankens durchaus zugänglich ist (...). Abseits des Anspruches, der Professionelle müsse Theorie und Praxis gleichsam innerpsychisch legieren, werden die Welten des Wissens und des praktischen Könnens different betrachtet." (Neuweg 2004, 2)

Die Überlegungen zu den Konzepten beruhen auf unterschiedlichen Annahmen zum Lehrer/ innenhandeln und nehmen ihren Stellenwert in der Wissenspsychologie (Integrationsthese) oder Wissensverwendungsforschung (Differenzthese) ein.

Dewe et al. (1990) legen ein weiteres Modell mit drei Vorstellungsideen der Vermittlung von Theorie und Praxis vor: das Transfermodell, das Transformationsmodell und das Relationierungsmodell (Vermittlung dritter Bereiche) (Abbildung 2).

(1) Technischer Transfer
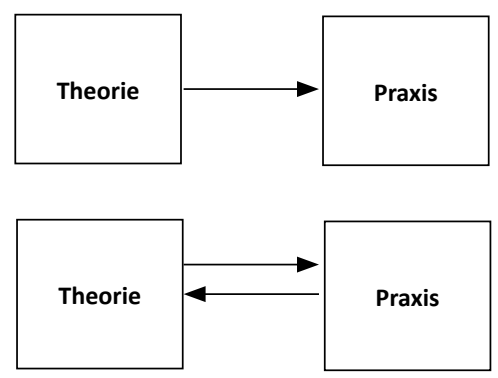

(2) Transformation

(3) Vermittlung dritter Bereiche

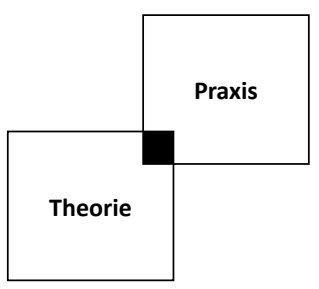

Abb. 2: Bestimmungsversuche des Verhältnisses von wissenschaftlichem und handlungsbezogenem Wissen (Dewe et al. 1990; Herzmann et al. 2016, 152)

Die Grundannahme im Transfermodell ist die Vorstellung, dass wissenschaftliches Wissen auf Praxis übertragbar und anwendbar ist. Ziel des Transfers ist die theoretische Absicherung der Praxis und die Anhebung der Handlungsrationalität auf Basis wissenschaftlicher Erkenntnisse. Theorie wird somit zur primären Grundlage für pädagogisches Handeln in der Praxis.

Im Transformationsmodell wird davon ausgegangen, dass Theorie und Praxis nicht übertragbar sind. Praxis wird als unvorhersehbar mit fehlender Antizipation für die Wissenschaft gesehen. Ziele sind Vermittlung von Theorie und Praxis und Reflexion von Alltagstheorien mit Hilfe des wissenschaftlichen Wissens.

Im Relationierungsmodell (Vermittlung dritter Bereiche) steht die Schnittmenge zwischen Theorie und Praxis als dritter Wissensbereich im Mittelpunkt.

„Relationierung ist der meta-reflexive Akt, denselben Gegenstand aus unterschiedlichen Perspektiven zu betrachten, (In-)Kongruenzen zu erkennen und sich der eingegnommenen Perspektive bewusst zu sein." (Cramer 2020, 271) 
Die verschiedenen Vorstellungen der Vermittlungsprozesse von Theorie und Praxis sind bei Konzeption von Curricula und Konzepten der Pädagogisch-Praktischen Studien mitbestimmend. Wie wird die Theorie-Praxis-Verknüpfung vollzogen?

Die „Theorie-Praxis-Verknüpfung hat sich unter dem Label des forschenden Lernens bereits in den 1970erJahren entwickelt (vgl. dazu Boelhauve, 2005; Fichten, 2010) und als hochschuldidaktisches Konzept seitdem etabliert (vgl. z.B. Wissenschaftsrat, 2001; zuletzt Schüssler et al., 2017).“ (Herzmann et al. 2018, 46)

Während Autor/innen wie Van Ophuysen et al. (2017) forschendes Lernen als einen Prozess sehen, „in dem bestimmte Elemente empirischen wissenschaftlichen Handelns in Kombination mit theoretischem Wissen und individuellen Erfahrungen zur Beantwortung beruflich relevanter Fragen genutzt werden“ (Van Ophuysen et al. 2017, 276), verstehen Schüssler et al. (2017) forschendes Lernen nur als „Mittel zum Zweck“ (Schüssler et al. 2017, 41), wobei nicht das Ergebnis, sondern vielmehr der „Prozess der Erkenntnisgewinnung“ (Schüssler et al. 2017, 41) und der „Lernprozess der Studierenden“ (Schüssler et al. 2017, 41) im Mittelpunkt stehen.

Nach Van Ophuysen et al. (2017) kann forschendes Lernen nicht nur ein Teil der hochschulischen Anteile in der Zeit des Studiums sein: Inputs müssen auch im schulischen Teil erfolgen. Hier nehmen Mentor/innen eine bedeutende Funktion ein. Stadelmann (2006) konnte nachweisen, dass jene Gruppe von Mentor/innen, die sich auf eine Theorie-Praxis-Verschränkung einlässt, eine intensivere Arbeitsbeziehung in der Begleitung eingehen und mit Ausbildungsinstitutionen stärker kooperieren (Stadelmann 2006, 176; Kreis 2012, 50). Demzufolge kommt es beim schulpraktischen Mentoring nun darauf an, Mentor/innen zu finden, die eine positive Haltung gegenüber einer Theorie-Praxis-Verschränkung einnehmen und mit Ausbildungsinstitutionen zusammenarbeiten. Im Folgenden werden nun Modelle zur Theorie-Praxis-Verschränkung in Schulpraktika vorgestellt und diskutiert.

\section{Modelle zur Theorie-Praxis-Verschränkung in Praktika}

Nach Wildt (2005) wird das Prozessmodell forschenden Lernens als wiederkehrender Lernzirkel (Abbildung 3) dargestellt. Die beschriebene Abfolge im Modell gibt Anregungen für Konzepte der schulischen Anteile bzw. Begleitungsprozesse seitens der Mentor/innen.

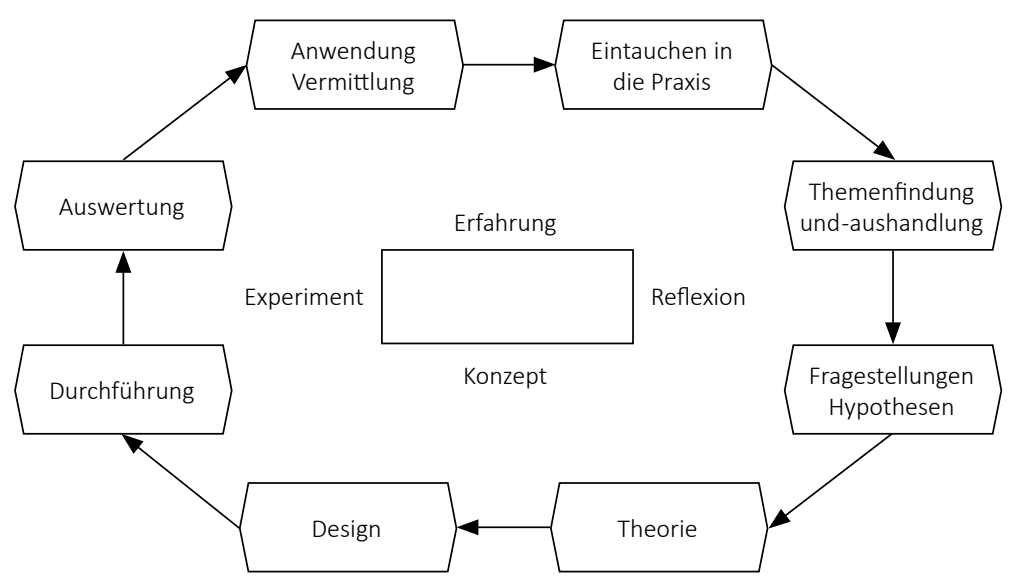

Abb. 3: Prozessmodell forschenden Lernens (Wildt 2005, 187; Herzmann et al. 2016, 162) 
Im Rahmen des Praktikums erfolgt zunächst das „Eintauchen“ in die Praxis. Nach der Themenfindung z.B. durch Mentor/innen folgt das Gestalten eines sozialwissenschaftlichen Untersuchungsprozesses mit Entwickeln von Fragestellungen bzw. Hypothesen, Theoriebildung, Designentwicklung, Durchführung und Auswertung. Die Ergebnisse sollen demnach so aufbereitet werden, dass Inhalte angewendet und/oder vermittelt werden können (Herzmann et al. 2016, 162). In den Praktika erfahren Studierende den Schulalltag, reflektieren mit der/dem Mentor/ in, entwickeln ein gemeinsames Konzept und experimentieren in der Anwendung und Durchführung. In diesem Fall unterstützen Mentor/innen den zirkulären Prozess und treten vermutlich selbst in einen Lernkreis ein.

Nach Fichten (2017) soll in den Schulpraktika eine Haltung herausgebildet werden, indem kritische Fragen gestellt werden (Fichten 2017, 30) und Studierende in weiterer Folge einen prozessorientierten Weg beschreiten. Hierbei übernehmen Mentor/innen einen wichtigen Aufgabenbereich. Patry (2014) beschreibt vier Funktionen von deskriptiven wissenschaftlichen Theorien und gibt Beispiele für Fragestellungen innerhalb der Praktika (Patry 2014, 32) - vielleicht auch ein Anreizmodell für Mentor/innen (Tabelle 8):

Tab. 8: Funktionen und Fragestellungen (Adapiert nach Patry 2014, 32)

\begin{tabular}{ll}
\hline Funktion & Fragestellung \\
\hline Beschreibung von Phänomen & Was ist der Fall? \\
Erklärung & Warum ist etwas der Fall? \\
Prognose & Was wird der Fall sein? \\
Handlungsprinzipien & Was kann ich tun, um ein bestimmtes Ziel zu erreichen? \\
\hline
\end{tabular}

Zieldimensionen des Umgangs mit diesen Fragestellungen durch Mentor/innen können das Generieren von Wissen, das Anwenden von wissenschaftlichen Arbeits- und Denkweisen (Fichten 2017) und die theoriegeleitete-reflexive und systematische Umsetzung in kontextspezifischen Handlungsausführungen des Schulalltags sein. Diese Auflistung an Fähigkeiten und Fertigkeiten deckt sich mit dem Verständnis des kompetenzorientierten Ansatzes der Professionsentwicklung.

„Kompetent ist im Lehrberuf demnach, wer die Fähigkeiten und Fertigkeiten besitzt, Aufgaben oder Probleme zielorientiert und verantwortungsbewusst zu lösen, sein Vorgehen zu reflektieren sowie zu bewerten vermag und dabei das eigene Verhaltensrepertoire weiterentwickelt.“' (Cramer 2012, 35)

Aus ausbildungsinstitutioneller Sicht benötigen der Ansatz des forschenden Lernens und die Einübung einer wissenschaftlich-reflexiven Grundhaltung die frühzeitige Kenntnis von Forschungsmethoden und Forschungsdesigns im Ausbildungsmodus der Lehramtsstudierenden (Herzmann et al. 2016, 163). Damit Mentor/innen Studierende adäquat unterstützen können, werden diese Kenntnisse auch von Mentor/innen erwartet.

In diesem Sinn fördern Schulpraktika eine Theorie-Praxis-Verknüpfung. Anbei folgt ein weiteres Modell zur Verdeutlichung (Abbildung 4): 


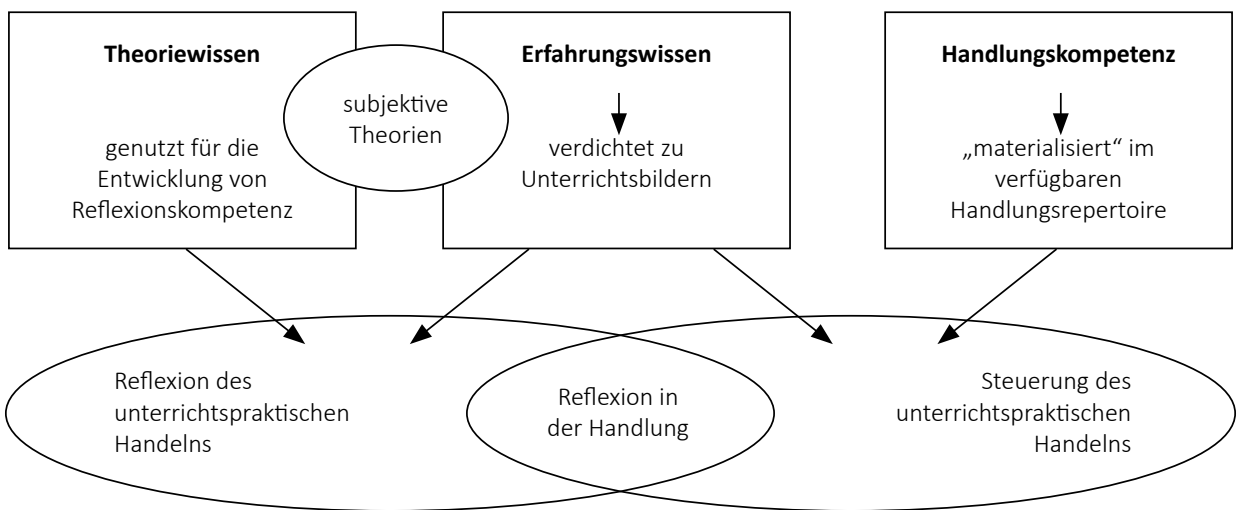

Abb. 4: Zusammenspiel von Theoriewissen, Erfahrungswissen, Handlungskompetenz (Meyer 2004, 137; Standop et al. 2015, 252)

Meyer (2004) liefert mit seinem Modell (Abbildung 4) ein Zusammenspiel von Theoriewissen, Erfahrungswissen und Handlungskompetenz und unterstreicht die Bedeutung im Zuge von Unterrichtsnachbereitung und -reflexion. Hierbei kommt der Reflexionsfähigkeit für das unterrichtspraktische Handeln eine besondere Bedeutung zu, die letztendlich das unterrichtspraktische Handeln in einem weiteren Prozess erneut steuert (Meyer 2004, 137; Standop et al. 2015, 252). Mentor/innen greifen wesentlich in diesen Prozess ein. Sie reflektieren auf Basis theoriebegründeter Konzepte und Erfahrungen handlungsgeleiteter Prozesse und erweitern bzw. steuern zukünftiges unterrichtspraktisches Handeln. Ein Sichtbar-Werden dieses Prozesses ist in einer reflektierten Handlung möglich. Die gelungene Umsetzung lässt dann auf einen erfolgreichen Lernprozess schließen.

Die eingangs gestellte Frage: „Können Theorie und Praxis eine Beziehung eingehen?“ muss, wie Modelle aufzeigen, bejaht werden. Es kommt auf die Haltung der Beteiligten und auf die Etablierung von Möglichkeiten zur Reflexionsfähigkeit an. Mentor/innen können wesentlich zur Förderung beitragen, indem sie „Inhalte und Standards der Ausbildung“ (Kreis 2012, 50) in der Begleitung aufnehmen und sich damit auseinandersetzen. Mentor/innen brauchen allerdings auch ein Rüstzeug zum Anleiten von reflexiv-forschenden Prozessen. Letztlich kommt beim schulpraktischen Mentoring die Frage der konstruktiven Kooperation und Kollaboration zwischen Ausbildungsinstitutionen, Schule und Mentor/in ins Spiel.

Das folgende Kapitel der vorliegenden Arbeit beschäftigt sich mit Profession und Professionsentwicklung von Lehrperson und resümiert dabei, dass Mentor/innen Kenntnisse von Konzepten und Modellen haben sollten, um in einen qualitätsvollen Prozess einsteigen zu können.

\subsection{Professionsentwicklung Lehrer/innen}

Das Kapitel Professionsentwicklung Lehrer/innen diskutiert die Begriffe Profession, Professionalisierung und Professionalität, zeigt Forschungsansätze zur Professionalität im Lehrberuf und Modelle zu Lehrer/innenkompetenzen.

Die folgenden Leitfragen werden in der Bildungsgangforschung und im Speziellen in der Lehrer/innenprofessionsforschung als zentrale Forschungsfragen thematisiert: Wann und wie entwickelt sich die Profession der Lehrperson? Ist Lehrer/in-Sein überhaupt als Profes- 
sion anzusehen? Welches Wissen und welche Kompetenzen sind für die Ausübung des Lehrberufs wichtig? Welchen Beitrag leistet die Lehrer/innenausbildung zur Professionsentwicklung? Eingebunden in diesen Diskurs sind Fragen zu den Päagogisch-Praktischen Studien und zum Mentoring: Welchen Beitrag leisten Schulpraktika? Wie können Reflexionsfähigkeit und Handlungskompetenz erweitert werden? Was tragen Mentor/innen zur Professionsentwicklung bei? Basierend auf diesen Fragestellungen werden in diesem Kapitel Professionsentwicklung in Zusammenhang mit Pädagogisch-Praktischen Studien und schulpraktischem Mentoring diskutiert.

Das Lehrer/innenkönnen „als Funktion von Fachkompetenz und Persönlichkeit“ (Neuweg 2011, 451) galt im bildungshistorischen Kontext bis in die 70iger Jahre des letzten Jahrhunderts als oberstes Leitmotiv der Profession. Ab den 1980iger Jahren wurde unter Lehrer/innenwissen (Neuweg 2011) oder Professionswissen (Baumert et al. 2006) auch das Anwenden von situationsangepasstem und flexiblem Verhalten in den unterschiedlichen Kontexten des Lehrens und Lernens verstanden. Seit der Jahrtausendwende werden die Ergebnisse der Schüler/innenleistungstests als Indikatoren für die Lehrkompetenz bzw. didaktische Kompetenz von Lehrpersonen herangezogen. In der Aus-, Fort- und Weiterbildung werden „Entwicklungen in Gang gesetzt (...), um die für ein optimales Lernen der Schüler/innen notwendigen Kompetenzen der Lehrperson theoretisch zu beschreiben, empirisch zu erfassen und in die Richtung eines gewünschten Zustandes zu lenken." (Maag Merki et al. 2011, 573) Ziele sind eine optimale Gestaltung von Lernsettings und Lernangeboten für das einzelne Schulkind in den heterogenen Schulklassen und „systematische Reflexion und Diskussion der geleisteten Arbeit“ (Maag Merki et al. 2011, 573) im Sinne „schulischer Qualitätsentwicklung“ (Maag Merki et al. 2011, 573).

Der folgende Abschnitt gibt einen Überblick, wie Profession und Professionalität definiert werden, zeigt Forschungsansätze zu Professionalität auch in Verbindung mit Schulpraktika für Studierende auf und geht der Fragen nach, welche Kompetenzen im Laufe der Lehrer/innenaus-, -fort- und -weiterbildung erworben werden sollen.

\subsubsection{Begriffsdeutungen}

Der Begriff „Profession“ (lateinisch professio: „öffentliches Bekenntnis“) ist ein theoretisches Konstrukt aus der Soziologie und dokumentiert „ein eigenständiges gesellschaftliches Funktionsprinzip neben Markt und Hierarchie bzw. Bürokratie (Freidson 2001, 2003; Mieg \& Pfadenhauer 2003).“ (Dick 2016, 19) Auch die Pädagogik verwendet den Begriff und beschäftigt sich mit der Anwendbarkeit des erworbenen Wissens je nach Situation und Interaktionen (Combe et al. 1996; Nittel 2000; Helsper et al. 2011). Beide Disziplinen entwickelten und entwickeln Theorien und Konzepte zum Begriff Profession. Die Disziplin Psychologie wiederum geht im Rahmen der Expertiseforschung vor allem auf Verständnisfragen von professionellem Wissen und Lernen ein (Ericsson et al. 2006). Alle drei Disziplinen vereint die Frage nach einer konstruktiven Wechselbeziehung zwischen der/dem Professionellen und dem Gegenüber (Dick 2016, 19).

In der Historie lässt sich erkennen, dass in der Theorie zwei zentrale Fragen zur Profession immer wieder diskutiert wurden bzw. werden, und zwar ob der Lehrberuf als eigene Profession gedeutet werden kann bzw. welche Professionalität dem Lehrberuf zugrunde liegt (Zlatkin-Troitschanskaia et al. 2009; Helsper et al. 2011; Terhart 2013). Die Professionsforschung hat lange Zeit nur Professionen wie Arzt, Richter oder Geistlichkeit aufgrund der beruflichen Anforderungen und Qualitätsmerkmale als privilegierte Berufsgruppen an- 
gesehen, die sich vor allem durch Macht, Status und Selbstbestimmung auszeichneten (Mieg 2016, 27). Der Lehrberuf hingegen war nur ein Zuverdienst zu den kirchlichen Diensten, zum Handwerksberuf oder für Geistliche. Da die Entlohnung auch nur sehr mäßig war, war der Beruf lange Zeit nicht sehr attraktiv. Erst mit der Einführung der Schulpflicht und der Institutionalisierung des Schulwesens kann von einem Beginn der eigentlichen Professionalisierung des Lehrberufs gesprochen werden (Engelbrecht 1986; Enzelberger 2001; Seel 2010; Terhart 2013).

Mieg (2016) geht davon aus, dass die Begriffe Profession, Professionalisierung und Professionalität/professionalism auf der institutionellen und individuellen Ebene betrachtet werden müssen (Mieg 2016, 29) und gibt Erklärungen (Tabelle 9).

Tab. 9: Begriffsdefinitionen Profession, Professionalisierung, Professionalität (Mieg 2016, 30)

\begin{tabular}{llll}
\hline & Profession & Professionalisierung & $\begin{array}{l}\text { Professionalität/ } \\
\text { professionalism }\end{array}$ \\
\hline $\begin{array}{l}\text { Institutioneller } \\
\text { Aspekt }\end{array}$ & privilegierte Berufsgruppe & $\begin{array}{l}\text { Übergang einer Berufs- } \\
\text { gruppe in eine Profession: } \\
\text { Zuständigkeitsmonopol }\end{array}$ & $\begin{array}{l}\text { Organisationsform von } \\
\text { Wissensarbeit: Bearbeitung } \\
\text { durch Profession }\end{array}$ \\
\hline $\begin{array}{l}\text { Individueller } \\
\text { Aspekt }\end{array}$ & $\begin{array}{l}\text { selbständig, } \\
\text { Experte/Expertin, } \\
\text { gemeinwohlorientiert, } \\
\text { zuständig }\end{array}$ & $\begin{array}{l}\text { an bereichsspezifischen } \\
\text { Leistungsstandards } \\
\text { orientierte Berufsarbeit }\end{array}$ & $\begin{array}{l}\text { professionelles } \\
\text { Handeln: } \\
\text { Kompetenzanwendung }\end{array}$ \\
\hline
\end{tabular}

Würde nur der institutionelle Aspekt bzw. der in der Literatur als klassisch professionstheoretisch beschriebene Ansatz (Parsons 1939, 1968) betrachtet werden, dann sind Professionen privilegierte Berufsgruppen mit einem hohen Ansehen aufgrund der Qualifikationen und aufgrund der Tatsache, dass sie über ein Wissen in spezifischen Bereichen und damit auch über Risikoeinschätzungen verfügen. Auf individueller Ebene zählen zur Profession die Merkmale Autonomie im Sinne von Selbstorganisation (Freidson 2001; Abbott 2005), Abstraktion im Sinne von Expertin/Experte über gewisse Wissensgebiete (Abbott 1988; Evetts 2003a), Altruismus im Sinne von Gemeinwohlorientierung (Parsons 1968) und Autorität im Sinne von Zuständigkeit für bestimmte Bereiche und Problembereiche der Gesellschaft (Freidson 1994).

Noch um die Jahrtausendwende wurde im klassisch professionstheoretischen Ansatz die Diskussion zur „Semi-Profession“ des Lehrberufes geführt (Terhart 2001; Blömeke 2002; Kemnitz 2011) - die Lehrperson, die im Unterricht Autonomie ausleben kann, aber ansonsten Pflichterfüller/in der staatlichen Behörden ist.

Ein definitorischer Ansatz der Profession Lehrberuf aus der Soziologie ist zwar vorhanden (Mieg 2016, 28), offen bleibt aber „inwieweit Lehrerhandeln professionellen ethischen Standards folgt und vorliegende wissenschaftliche Orientierungspotentiale auch genutzt werden“ (Lehmann-Grube et al. 2009, 59). Für Ilien (2008) sollte der Lehrberuf unbedingt einen Professionsstatus erhalten, da dadurch „die Komplexität, die öffentliche Bedeutung und die durch gesellschaftliche Prozesse selbst verursachten Handlungsprobleme des Lehrerberufs besser“ (Ilien 2008, 236) wahrgenommen werden.

Im aktuellen Diskurs der Professionssoziologie meint der Begriff Professionalität bzw. professionalism: 
„Aus wissenssoziologischer Interpretation geht es hierbei um einerseits die Ausdifferenzierung des wissenschaftsgestützten Wissenssystems (institutionelle Ebene) und andererseits um professionelles Handeln im Sinne einer Suche für Darstellung und Anwendung der professionellen Kompetenz (Pfadenhauer 2003).“ (Mieg 2016, 30)

Soziologisch orientierte Autor/innen diskutieren weiters die spezifischen Problematiken innerhalb der Berufsrolle der Lehrperson (Reinisch 2009, 40).

Der Begriff Professionalisierung beschreibt auf der institutionalen Ebene den Übergang in den eigentlichen Zuständigkeitsbereich einer Berufsgruppe und auf individueller Ebene eine bereichsspezifische Tätigkeit mit meist vorgegebenen Leistungsstandards für diese Berufsgruppe (Mieg 2016, 30). Die Professionalisierung im Lehrberuf meint im historischen Kontext die zunehmende Akademisierung des Lehrberufs für alle Lehrämter (Lemmermöhle et al. 2003; Sandfuchs 2004; Cramer 2012) und im individuellen Kontext den Weg der Entwicklung zur Lehrperson durch die Ausbildungsinstitutionen (Cramer 2012, 23). In der Literatur wird der Begriff Professionalisierung von Lehrpersonen vor allem im Kontext der Akademisierung gesehen. Hierbei gilt es, verschiedene Wissensbereiche wie Fachwissen, fachdidaktisches Wissen und allgemein pädagogisches Wissen (Shulman 1987) zu verknüpfen bzw. professionelles Wissen (Baumert et al. 2006; Blömeke et al. 2008) situationsgerecht anwenden zu können.

„Dabei wird Lehrer-Professionalisierung als Entwicklungsprozess verstanden, der sowohl die System- bzw. Makroebene als auch die Individual- bzw. Mikroebene im Blick hat und somit gleichermaßen die Entwicklung eines Berufsstandes und die Entwicklung von Personen thematisiert." (Ortenburger 2016, 559)

Nach Terhart (2009) ist unter Professionalisierung von Lehrpersonen auf der individuellen Ebene das Ankommen in der Rolle der Lehrperson, das Erkennen der Statusmerkmale und das Erarbeiten der Kompetenzen gemeint (becoming professional) (Terhart 2009, 64). Ausbildungsinstitutionen achten vor allem auch in den Pädagogisch-Praktischen Studien durch unterschiedliche Maßnahmen darauf, dass Studierende in einen Entwicklungsprozess eintreten. Das schulpraktische Mentoring im Rahmen der Praktika unterstützt Studierende beim Perspektivenwechsel, bei der Rollenübernahme von Studierender/m als Lehrperson und beim Erarbeiten und Reflektieren von Handlungsstrategien. Welche Forschungsansätze liegen dazu vor? Das ist Teil der Ausführungen im folgenden Unterkapitel.

\subsubsection{Forschungsansätze zur Professionalität im Lehrberuf}

In der Bildungsforschung gibt es vor allem seit der Jahrtausendwende und dem „PISA-Schock“ unzählige Publikationen mit Forschungsbeiträgen zum Thema Lehrprofessionalität (Baumert et al. 2006; Lipowsky 2006; Reinisch 2009, 33). Die Forschungsdokumentationen zeigen auf, dass dem Lehrer/innenhandeln eine bedeutende und für Lernende oftmals eine entscheidende Rolle zugewiesen wird (Zlatkin-Troitschanskaia et al. 2009, 13). Professionalität von Lehrpersonen wird im Sinne der Verantwortlichkeit für den Einzelnen und die Gesellschaft somit zwingend eingefordert und vielfach unter psychologischen Gesichtspunkten diskutiert:

„Professionalität wird so quasi als Endprodukt eines individuellen Entwicklungsprozesses gedeutet, in dem spezifisches Wissen, spezielle Fertigkeiten und Einstellungen erworben werden, die es nunmehr zu erforschen gilt.“ (Reinisch 2009, 37) „Der Prozess des Kompetenzerwerbs von Lehrkräften wird hingegen konsequent als, Entwicklung von Professionalität' oder synonym als ,professionelle Entwicklung bezeichnet." (Cramer 2012, 23) 
Lehrer/innenbildungssysteme und im Besonderen die Lehrer/innenausbildung leisten einen spezifischen Beitrag zur Entwicklung und Ausprägung von Lehrer/innenprofessionalität. Kompetenzorientierte und professionsorientierte Gestaltung der Studienangebote, ausformulierte Standards, forschungs- und evidenzbasierte Lehre, strukturelle Verankerung von Mentoring sowie Modelle der Subjektiven Theorien werden als Grundlage für die Curricula in den tertiären Ausbildungsstätten herangezogen. Hintergrund bilden oftmals mehr oder weniger forcierte Forschungsansätze von Lehrprofessionalität, wie z.B. der praxistheoretische Ansatz, der Persönlichkeitsansatz, der berufsbiografische Ansatz, der Expertiseansatz, das Prozess-Produkt-Paradigma, der kompetenzorientierte Ansatz und/oder der strukturtheoretische Ansatz. Die beiden letztgenannten sind richtungsweisend im Diskurs zur Professionalität (Cramer 2012, 24).

In der vorliegenden Arbeit werden Mentoringprozesse beleuchtet, die in Verbindung mit Schulpraktika in der Erstausbildung stehen. Daher wird nun der Frage nachgegangen, welche Forschungsbefunde vorliegen, die den Anteil der Pädagogisch-Praktischen Studien in Zusammenhang mit der Profession und Professionsentwicklung beleuchten. Wenngleich empirische Befunde für eine evidenzbasierte Professionalisierung der Lehrer/innenausbildung durch die Eingliederung umfangreicherer Praxisanteile in das Studium ein Desiderat darstellen (Rothland et al. 2018, 1), wird im Folgenden ein erster Überblick zu Untersuchungsergebnissen gegeben. Eine ausführliche Darstellung der Befundlage zur Relevanz der Pädagogisch-Praktischen Studien im Allgemeinen wird im Kapitel I/1.1.4 referiert.

Forschungsbefunde zur Professionsentwicklung in Zusammenhang mit Schulpraktika ergeben sich auf der Mikroebene Person (1) z.B. bei Untersuchungen zu individuellen Eingangsbedingungen oder von Einzelmerkmalen. So zeigt die Berufswahlmotivationsforschung einen konstanten Verlauf der Motivation (vor allem intrinsisch-pädagogische Motive - wie Umgang mit Kindern und Jugendlichen) während der Zeit der Ausbildung und ein konkretes Interesse mit steigender Qualität an einzelnen Lerninhalten auf (Rothland et al. 2010, 793f; Brühwiler 1999, 123). Ein weiteres Einzelmerkmal betrifft die Selbstwirksamkeitserwartung (2), die vor allem auch durch Coaching bzw. Mentoring unterstützt werden kann (Warner et al. 2009, 629). Eine hohe Selbstwirksamkeit steht für Regulationsfähigkeit von Berufsbelastung (Baumert et al. 2006, 503), zeichnet sich durch hohes Engagement und Berufszufriedenheit bei Lehrpersonen aus (Schmitz et al. 2002) und wirkt sich positiv auf Schüler/innenbeziehung (Podell et al. 1993) und auf ein innovatives Unterrichtsgeschehen (Allinder 1994) aus (Cramer 2012, 96f). Untersuchungen zu berufsbiografischen Entwicklungen bei Studierenden wären eine lohnende Aufgabe. Fortbildungskonzepte für Mentor/innen sollen die Bedeutung von Selbstwirksamkeitserwartung herauskristallisieren.

Auf der Meso - und Makroebene Ausbildung (3) werden in Zusammenhang mit der Professionsentwicklung vor allem Untersuchungen zur Wirksamkeit der Lehrer/innenausbildung getätigt. Hierbei fällt auf:

„Problematisch ist, dass die eigentliche Leistung der Lehrkräfte für den Erfolg ihrer Schüler (sic) nicht isoliert werden kann und damit auch nicht eindeutig auf ein bestimmtes Unterrichtshandeln zurückzuführen ist, das in der Lehrerbildung (sic!) seinen Anfang nehmen könnte (Kyriakides 2007)“. (Cramer 2012, 100)

Die Evaluation von Lehrer/innenausbildungssystemen zeigt, dass sich aus Sicht Studierender eine erfolgreiche Ausbildung dadurch auszeichnet, dass Wissen vermittelt wurde und Studierende von kompetenten Personen (vor allem Mentor/innen) unterstützt wurden (Fried 2004, 
240). Schulpraktika haben - wie oftmals von Studierenden erwähnt - den bedeutendsten Studienanteil während der Ausbildung (Reintjes 2007). Geklärt werden muss in diesem Zusammenhang, inwiefern die Bedeutsamkeitseinschätzungen - gepaart mit hoher Qualitätsund Nutzenzuschreibung - mit der späteren Lehrtätigkeit in Verbindung stehen (Cramer 2012, 108).

Es ergeben sich somit folgende Fragestellungen für Ausbildungsinstitutionen: Wie können in der Erstausbildung berufsrelevante Kompetenzen entwickelt werden? Wie kann es gelingen, angehende Lehrpersonen auf Paradoxien und Antinomien (Combe et al. 1996a) des Lehrberufs vorzubereiten? Wie kann eine „Steigerung der Reflexivität“ (Reh 2004, 369) bei Lehramtsstudierenden forciert werden? Ob trotz Berücksichtigung dieser Fragestellungen im Implementierungsprozess der Ausbildung, wie z.B. durch vermehrte Anteilen an Pädagogisch-Praktischen Studien, die Lernwirksamkeit gegeben ist, bleibt weiterhin Forschungsgegenstand. Bach (2013) weist in diesem Zusammenhang auf einen Mangel in der bildungsbezogenen Forschungsaktivität trotz vieler Forschungsvorhaben im Bereich der Lehrprofessionalität hin, der auch dadurch gekennzeichnet ist, dass letztendlich Verknüpfungen der Projekte in theoretischer wie methodischer Hinsicht fehlen (Bach 2013, 47). Dieses Defizit zeigt sich auch, wenn Forschungen im transnationalen Kontext gesehen werden sollten (Cramer 2012, 95). Weiters beschäftigen sich unterschiedliche Wissenschaften mit Lehrer/innenausbildung und dem Lehrberuf. Dies ist befruchtend, kann aber auch aufgrund der unterschiedlichen Schwerpunkte, Ansätze und methodischen Herangehensweisen Fragen unbeantwortet lassen.

Im Folgenden werden Modelle zu Lehrer/innenkompetenzen im Rahmen eines Kompetenzorientierten Ansatzes vorgestellt und mit schulpraktischem Mentoring in Verbindung gebracht. Als Auswahlkriterium wurden jene Modelle gewählt, die Professionsentwicklung durch die Auseinandersetzung mit Theoriewissen und Erfahrungswissen (Meyer 2004, 137) und durch die Verschränkung von institutionellen und individuellen Aspekten (Mieg 2016, 30) als einen Lernprozess deuten.

\subsubsection{Modelle zu Lehrer/innenkompetenzen}

Der Kompetenzorientierte Ansatz in der Bildungswissenschaft geht von der Annahme aus, dass erfolgreiches pädagogisches Handeln auf den Prinzipien des Wissens, Könnens und eines werteorientierten Handelns beruht. Die Lehrperson besitzt demnach

„die Kompetenz, so tätig zu werden, dass sie eine Absicht, ein Ziel oder einen Zweck unter Beachtung von Handlungsprinzipien, Werten, Normen und Regeln mit Bezug auf konkrete, die jeweiligen Handlungssituationen bestimmende Bedingungen, zu erreichen vermag." (Frey 2006, 31)

Die Grundlegung des Kompetenzerwerbs erfolgt für Lernende in erster Linie in der Zeit der Ausbildung und entwickelt sich im Laufe der Berufsjahre weiter (Bromme 2008, 159; Baumert et al. 2006, 481). Demgegenüber steht die Forderung von Dozierenden der Ausbildungsinstitutionen „ein Repertoire von Instrumenten an die Hand“ (Eilerts et al. 2010, 111) zu bekommen, „mit denen sie ihre Studenten (sic!) kompetenzorientiert auf ihre berufliche Zukunft vorbereiten und prüfen können." (Eilerts et al. 2010, 111) Gegenwärtig stellen sich für den Ausbildungsprozess die Fragen: Wie werden Handlungswissen und berufliche Kompetenzen definiert? Welches Handlungswissen und welche konkreten professionsspezifischen Kompetenzen sollen forciert werden? (Baumert et al. 2006; Cramer 2012, 37) 
In Darstellungen von unterschiedlichen Modellen - Strukturmodell, Niveaumodell, Entwicklungsmodell - werden Bedeutung und Erwerb von Kompetenzen bei Lehrpersonen beschrieben. Beispielhaft werden einige Modelle angeführt und in Zusammenhang mit PädagogischPraktischen Studien beleuchtet.

Ausgangspunkt ist das Modell von Shulman $(1986,1987)$ mit der Klassifizierung von Lehrer/ innenwissen. Terhart (1991) und Bromme $(1992,1997)$ entwickelten die Taxonomie Shulmans für den deutschsprachigen Raum weiter. Das Professionswissen gilt als ein wesentlicher Bestandteil der Lehrer/innenexpertise und soll in der Zeit der Lehrer/innenausbildung angeeignet werden (Baumert et al. 2006; Lipowsky 2006; Blömeke et al. 2008).

Das folgende Modell (Abbildung 5) veranschaulicht in einem Beziehungsgefüge die Annahme, dass Erwerb und Aneignung von objektivem Wissen (vor allem fachwissenschaftliches, fachdidaktisches und pädagogisch-psychologisches Wissen) (Wissen 1) sich auf das Wissen im subjektiven Sinne (Wissen 2 als Konstrukt gesehen) auswirken. Eine Verknüpfung beider Wissensarten zeigt sich in den Handlungsepisoden (Wissen 3) und ist „Erzeugungsgrundlage für kompetentes Handeln, das die Bedeutsamkeit der Ziele und Inhalte, die Effizienz des Unterrichts und das Lernklima (Kramis 1990) und damit die Lernprozesse von Schülern (sic!) beeinflusst." (Neuweg 2011, 453)

\section{LERNEN}

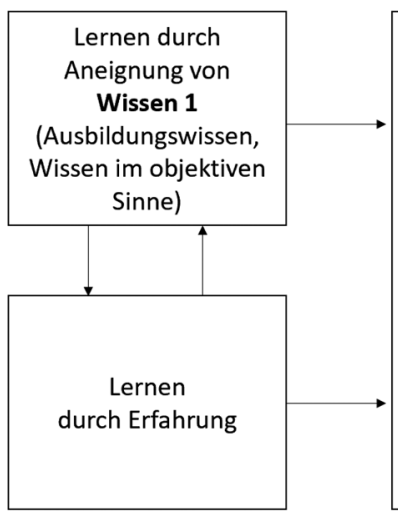

WISSEN

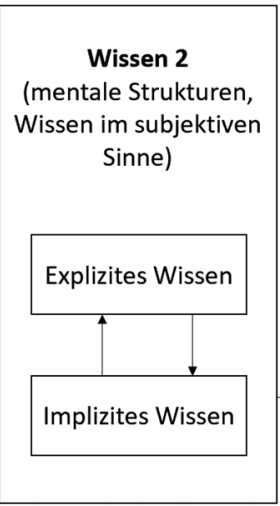

HANDELN

Wissen 3

(Können, von außen rekonstruierte Logik des Handelns)

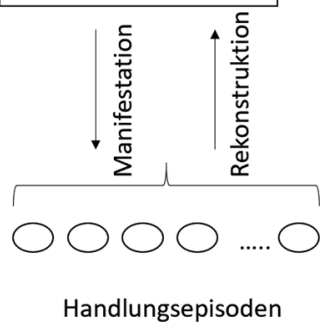

Abb. 5: Konzepte des „Lehrer/innenwissens“ (Neuweg 2011, 453)

Demzufolge kann im Rahmen des schulpraktischen Mentorings davon ausgegangen werden, dass nach Handlungsepisoden Reflexionsprozesse verankert werden sollen, die die Handlung in der perspektivischen Betrachtung von Wissen 2 und Wissen 1 deuten.

Baumert et al. (2006) verwenden in ihrem heuristischen Modell eine Typologie von professionellen Wissensdomänen. Diese Wissensdomänen sind Teil der professionellen Handlungskompetenz im Lehrberuf. Voss et al. (2015) betonen, dass die Entwicklung durch institutionelle und individuelle Faktoren beeinflusst wird (Kunter et al. 2011). Im Folgenden wird das Modell grafisch vorgestellt (Abbildung 6): 


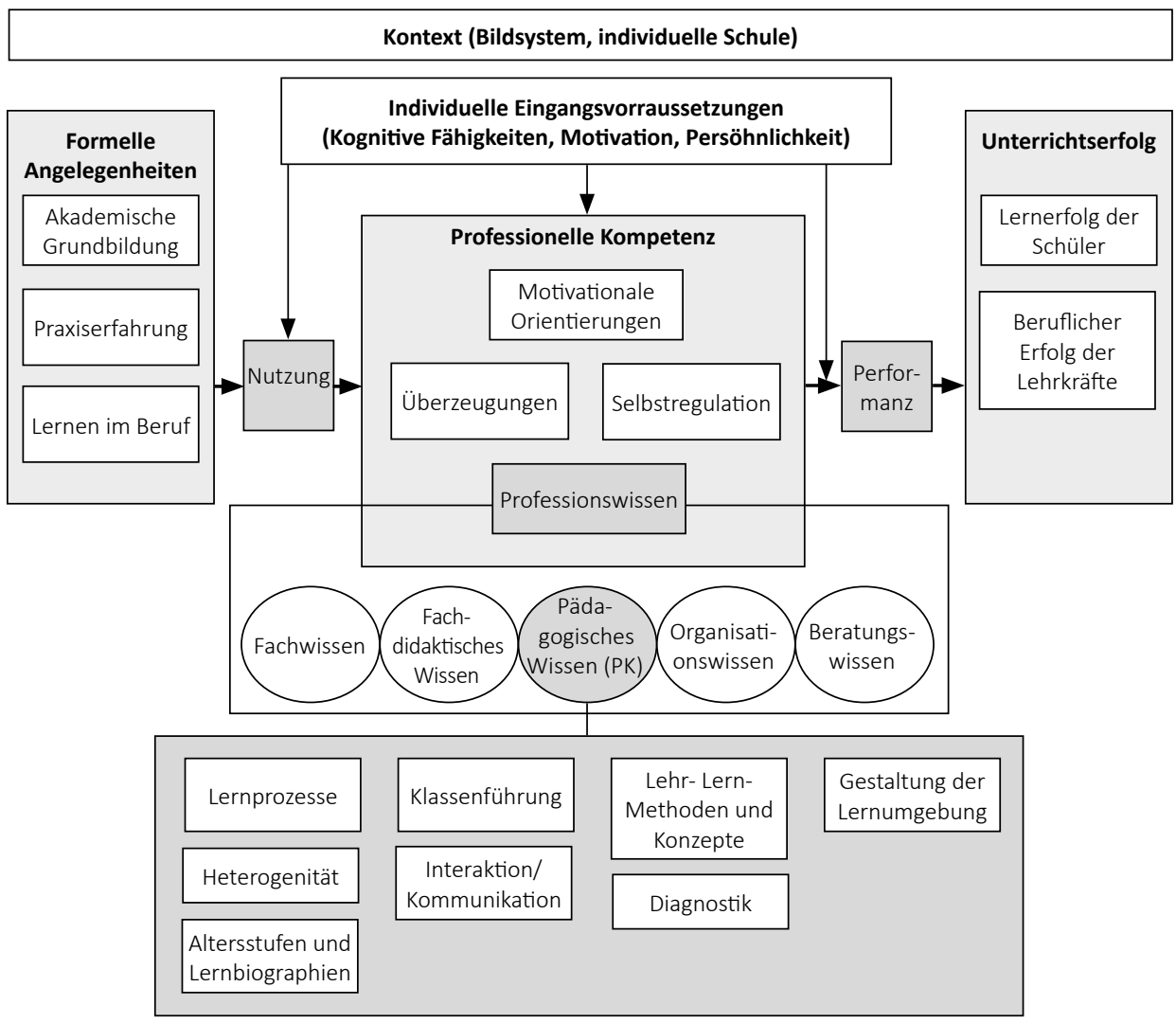

Abb. 6: Pädagogisches Wissen von Lehrkräften (Voss et al. 2015)

Interindividuelle Unterschiede in den professionellen Kompetenzen äußern sich divergierend in der Nutzung der Lerngelegenheiten (Mertens et al. 2018, 66). Für Baumert et al. (2011) und Kunter et al. (2017) zählen zu den professionellen Kompetenzen das Professionswissen, Überzeugungen, motivationale Orientierungen und die Selbstregulation. Sie bilden die Grundlage für den Unterrichtserfolg seitens der Schüler/innen und Lehrkräfte. Für die Autorin und den Autor beinhaltet das Professionswissen wesentliche Wissensinhalte und Wissensformen: das Fachwissen, das fachdidaktische Wissen, das pädagogische Wissen, das Organisationswissen und das Beratungswissen (Baumert et al. 2011; Kunter et al. 2017), die nach der Kompetenzdefinition nach Weinert (2001) erlernbar und vermittelbar sind (Weinert 2001).

In der Literatur wird Professionswissen auch in konzeptuelles (domänenspezifisches Wissen und deren Zusammenhänge) und prozedurales Wissen (handlungs- und situationsbezogenes Wissen) unterteilt. Im Zuge der Professionalisierung ergibt sich eine deutliche Verschränkung dieser beiden Arten von Wissen und eine differenziertere Analysefähigkeit von Unterrichtssituationen (Van Es et al. 2008; Mertens 2018, 68). Welchen Beitrag die motivationale Orientierung wie die Berufswahlmotivation zur Professionsentwicklung und zum Prozess des Aneignens von Professionswissen leistet, ist Gegenstand von Untersuchungen. Laut König et al. (2013) gibt es nur „geringe signifikante Zusammenhänge zwischen einer hohen intrinsischen Berufswahlmotivation und dem 
pädagogischen Professionswissen bei angehenden Lehrkräften zu Beginn ihres Studiums.“ (Mertens 2018, 69) Auch die Dimension Selbstregulation, die selbständiges Lernen forciert, ist bedeutend, wenn es um die Entwicklung des Professionswissens oder um Wahrnehmung von Unterrichtsprozessen geht. Hierbei soll die/der Lernende sich Ziele definieren und bestrebt sein, diese zu erreichen, indem die Handlungen darauf ausgerichtet sind (Mertens 2018, 70). Überzeugungen können mit Selbstwirksamkeitserwartungen gleichgesetzt werden. Sie regulieren das kontextspezifische Handeln, steuern Handlungen und verändern handlungsorientierte Prozesse aufgrund vorheriger Erfahrungsrückmeldungen (Bach 2013, 62f). Befunde zu „Kompetenzselbsteinschätzungen und Professionswissen verweisen (...) darauf, dass bei zunehmender Praxiserfahrung subjektive Kompetenzeinschätzung und Unterrichtswahrnehmung stärker in Zusammenhang stehen." (Mertens et al. 2018, 71) Auch für das schulpraktische Mentoring kann dieses Modell von Bedeutung sein und es lassen sich Schlüsse ziehen. Das Aufgabenfeld der Mentor/innen könnte sein, in Planungs- und Reflexionssettings mit Mentee auf Bereiche des Pädagogischen Wissens, des Organisationswissens und Beratungswissens einzugehen. Dabei sollte der Blick immer auf den Lernerfolg der Schüler/innen gerichtet sein. Die/Der erfahrene Mentor/in wird in der Begleitung diesen Aspekt berücksichtigen und an Mentee rückmelden. In der dyadischen Begleitung werden auch Bereiche der professionellen Kompetenz, wie motivationale Orientierungen, Überzeugungen bzw. Werthaltungen und Formen der Selbstregulation thematisiert werden.

Cramer (2012) ging in seiner Studie vom Berliner Anfangsmodell Baumerts und Kunters (2006) aus und erweiterte sein Modell um die subjektiven Aspekte: Eingangsbedingungen und Ausbildungserfahrungen bei Studierenden. Nach Cramer (2012) sind sie entscheidende Einflussfaktoren in der professionellen Entwicklung. Alle sechs Aspekte bezeichnet er in seinem Modell als „Dimensionen der Professionalität im Lehrerberuf (sic!)“ (Cramer 2012, 41).

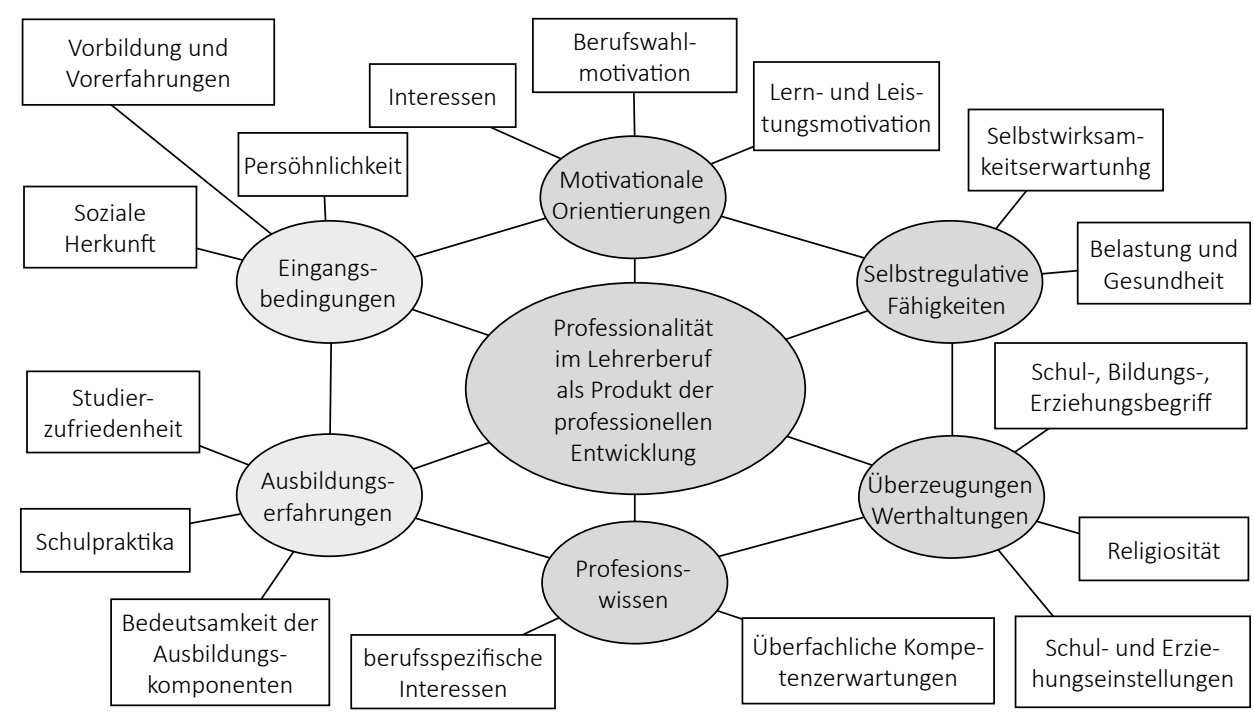

Abb. 7: Modell der Professionalität im Lehrer/innenberuf (Cramer 2012, 42)

Wenn dieses Modell im Kontext des schulpraktischen Mentorings Eingang findet, dann sind neben den professionellen Kompetenzen - wie bei Baumert et al. (2006) beschrieben - auch die 
Ausbildungserfahrungen und Eingangsbedingungen mitzuberücksichtigen. Mentor/innen setzen sich mit den Anforderungen des Studiums und der Praktika auseinander. Bei den Eingangsbedingungen wird im schulpraktischen Mentoring auf die Vorerfahrungen und Persönlichkeit Bezug genommen.

Nach Cramer (2012) bleibt die Frage nach der Art und Weise der Einbindung der subjektiven Dimensionen in das Ausbildungskonzept in der näheren Beschreibung seines Modells unbeantwortet. Dazu liefert Ostermann (2015) nach einer qualitativen Analyse von dreißig Studierendeninterviews mit den rekonstruierten Entwicklungsaufgaben im Ausbildungsprozess Antworten. Sie ging der Frage nach, „wie die individuellen Entwicklungs- und Kompetenzerwerbsprozesse zusammen mit den normativ-curricularen professionsspezifischen Vorgaben im Endeffekt zu Professionalisierungsschritten führen können." (Ostermann 2015, 181) Auf der ausbildungsinstitutionellen Seite werden studierendenbezogene und studierendenseitige professionsspezifische Entwicklungsaufgaben (Reflexion, Perspektivenwechsel, Schüler/innenzentrierung, Vermittlung, Verhaltenskontrolle, Sicherheit, Selbstverantwortlichkeit) in die curricularen hochschulischen und schulischen Ausbildungsbereiche (Allgemeine Bildungswissenschaftliche Grundlagen, Fachdidaktiken, Fachwissenschaft/Kunstfächer, Pädagogisch-praktische Studien) miteinbezogen. Abbildung 8 veranschaulicht das Gesamtkonstrukt Entwicklungsaufgaben und Studienbereiche im Lehramtsstudium.

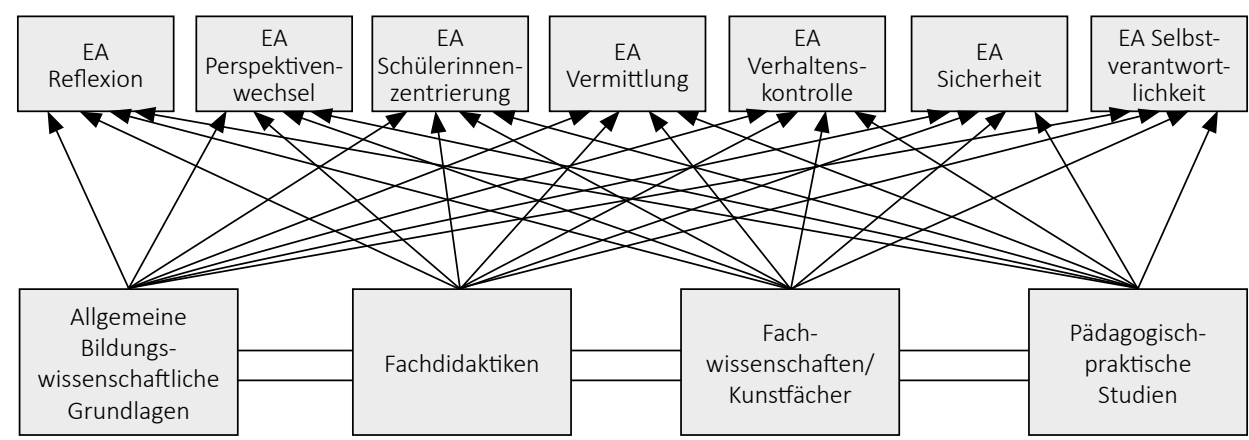

Abb. 8: Ausbildungsvorgaben und professionsspezifische Entwicklungsaufgaben (Ostermann 2015, 184)

Es ist darauf ausgelegt, eine reflexiv-forschende Haltung zu entwickeln, aufzubauen, zu fördern und letztendlich im Sinne der Professionalität diese in der zukünftigen Lehrtätigkeit zu etablieren. Wie dieses Modell in den Mentoringprozess integriert werden kann, ist im Kapitel I/2.4.6 näher erläutert.

Professionalisierungsmodelle (Baumert et al. 2006; Kunter 2015; Cramer 2012; Ostermann 2015; Köffler 2015) stellen nicht nur Professionswissen als Dimension dar, um erfolgreich zu handeln, sondern betrachten auch selbstbezogene und selbstregulative Prozesse als einen wesentlichen Teil der Professionalisierung in der Lehrer/innenausbildung. Vor allem beeinflussen diese Faktoren die Berufszufriedenheit und Gesundheit (Rothland et al. 2018) und tragen zum Aufbau eines positiven Selbstkonzepts bei.

„Das, professionelle Selbst' ist (...) fortwährend nach Kompetenz strebend, gleichzeitig aber auch steuernder Navigator, der den Professionalisierungsprozess organisiert und koordiniert sowie mit Ressourcen im Zuge dieser (Re-)Organisation haushaltet." (Köffler 2015, 190; Bauer 1998, 356f) 
Es kann davon ausgegangen werden, dass die Aktivierung von selbstregulativen Prozessen sicherlich durch die Unterstützung seitens der Ausbildungsinstitutionen oder im Rahmen der Schulpraktika durch Mentor/innen gefördert wird. Dazu benötigt es aber eine Atmosphäre, die Wert auf Zusammenarbeit, auf Offenheit und auf lösungsorientierte Ansätze legt.

Die in diesem Abschnitt dargestellten Modelle verbindet eine bedeutende Annahme, und zwar, dass Kompetenzen „grundsätzlich (...) erlern- und vermittelbar“ (Kunter et al. 2009, 154) sind. Dazu ergeben sich wohl die Fragestellungen, „für bzw. an wen welche Kompetenzen in welchem Maße erlern- und vermittelbar sind?" (Neuweg 2011, 471) und welchen Beitrag Schulprakti$\mathrm{ka}$ in der Lehrer/innenausbildung zur Professionsentwicklung leisten? Im Mentoring werden jedenfalls Themen zum Professionswissen, zur Professionalisierung und zur professionellen Kompetenz behandelt, da es nicht auszuschließen ist, dass Spannungsfelder zwischen Ausbildungsstätte und dem Lernort Schule auftreten werden. Die/Der Mentor/in fungiert durch die Integration schulischer und hochschulischer Anteile in das Ausbildungsmodell als mögliches Bindeglied.

\subsection{Zwischenfazit}

Um 2000 gab es in der Bildungs- und Hochschulpolitik entscheidende Weichenstellungen zur Lehrer/innenausbildungsreform. Die Ergebnisse in internationalen Leistungsvergleichsstudien und die Diskussionen im Bologna-Prozess forcierten die Neustrukturierung der Lehrer/innenausbildung in Österreich. Durch die gesetzliche Verankerung im Bundesrahmengesetz von 2013 (Bundesgesetzblatt 2013; vgl. Parlament Republik Österreich 2017) erfolgte im Studienjahr 2015/16 die Etablierung von Pädagog/innenbildung NEU. Mit Einführung des verpflichteten Bachelor-Master-Systems kam bzw. kommt es zu einer Angleichung und Statusannäherung der Lehrämter, um eine zureichende Qualifizierung aller Lehrprofessionen zu sichern. Die Umstellung auf verpflichtende Bachelor-Master-Abschlüsse bedeutet eine tiefgreifende Veränderung in der historischen Entwicklung der Lehrer/innenausbildung in Österreich und brachte eine Veränderung der Verantwortlichkeit der Ausbildungsstätten. Als Besonderheit gilt auch, dass in Österreich vier Entwicklungsverbünde der Lehrer/innenbildung geschaffen wurden. Tabelle 11 stellt eine Übersicht über die gegenwärtige Lehrer/innenausbildung in Österreich dar:

Tab. 10: Übersicht zur österreichischen Lehrer/innenausbildung

\begin{tabular}{ll}
\hline Primarstufe & Sekundarstufe \\
\hline Pädagogische Hochschule & $\begin{array}{l}\text { Gemeinsam eingerichtetes Studium von Universitäten } \\
\text { und Pädagogischen Hochschulen }\end{array}$ \\
$\begin{array}{l}\text { Bachelor of Education (BEd) und Master of Educa- } \\
\text { Bachelor of Education (BEd) und Master of Educa- } \\
\text { tion (MEd) }\end{array}$ & $\begin{array}{l}\text { tion (MEd) und Doctor of Philosophy (PhD) } \\
\text { 8 Semester Bachelorstudium mit 240 ECTS-AP }\end{array}$ \\
$\begin{array}{l}\text { 2 Semester Bachelorstudium mit 240 ECTS-AP } \\
\text { ECTS-AP für den erweiterten Altersbereich }\end{array}$ & 6 Semester Masterstudium mit 120 ECTS-AP \\
\hline
\end{tabular}

Seit dem Studienjahr 2015/16 werden an den Pädagogischen Hochschulen Österreichs Primarstufenlehrer/innen ausgebildet, während die Ausbildung für zukünftige Lehrpersonen der Sekundarstufe, welche nun zwei beliebige Fächer oder ein Fach und eine Spezialisierung (z.B. Inklusive Pädagogik, Medienpädagogik in der Lehrer/innenbildung WEST) für das Lehramt 
wählen, in den Zuständigkeitsbereich der unterschiedlichen Fakultäten der Universitäten in Zusammenarbeit mit den Pädagogischen Hochschulen fällt. Die Pädagogisch-Praktischen Studienanteile werden in das Bachelor-Master-Studium integriert.

Mit der Umstrukturierung der Ausbildungsschiene entfacht auch wiederum die Diskussion um die Wirksamkeit der Lehrer/innenausbildung. Hierbei entwickelten sich unterschiedliche Ansätze, bekannt aus der Bildungsforschung und Bildungspraxis des deutschsprachigen Raumes, mit dem Fokus auf berufliche Rahmenbedingungen oder auf lehrer/innenseitige Merkmale. Strukturtheoretische oder berufsbiografische Ansätze betrachten das soziale Gefüge und die Rollenerwartungen (Helsper 2014) bzw. den berufsbezogenen Sozialisationsprozess (Terhart 2001) als wesentliche Komponente für das berufliche Handeln. Beim Persönlichkeitsansatz werden die eigenen stabilen und situationsübergreifenden Merkmale als Garant für pädagogisches Handeln angesehen. Beim Expertiseansatz gelingt es Lehrpersonen, kognitive Prozesse und berufsrelevante Informationen gekonnt in Verbindung zu setzen und entsprechend zu handeln. Der Kompetenzansatz mit dem Konzept der erlernbaren und vermittelbaren Kompetenz bei Lehrpersonen rückt in den letzten Jahren in den Blickpunkt der Forschung und Professionalitätsentwicklung. Terhart (2013) meint dazu:

„Schwierige, komplexe, riskante Aufgaben und Probleme können nur auf der Basis einer in anspruchsvoller Ausbildung und sorgfältiger beruflichen Sozialisation erworbenen Wissensgrundlage sowie entsprechender Haltungen, Fähigkeiten und Fertigkeiten bewältigt werden. Man ist umso professioneller, je kompetenter man diese beruflichen Aufgaben erfüllt. Da diese sich immer schneller wandeln, ist die Bereitschaft zur Weiterentwicklung seiner Kompetenzen ein entscheidendes Kriterium für dieses Verständnis von Professionalität." (Terhart 2013, 78)

In der Literatur zu Kompetenzentwicklung geht man der Annahme nach, dass angehende Lehrpersonen ihre professionelle Kompetenz in unterschiedlichen Lerngelegenheiten entwickeln und aufbauen können. Als zentraler Aspekt der professionellen Kompetenz wird das professionelle Wissen als Voraussetzung für erfolgreiches Handeln gesehen, das Wissen zu Fakten, Handlungsabläufen und Schemata beinhaltet. Das ursprüngliche Konzept mit Fachwissen, fachdidaktischem Wissen und pädagogischem Wissen geht auf Shulman $(1986,1987)$ zurück. In der Literatur werden je nach Konzept und Begriffsverständnis unterschiedliche Begrifflichkeiten zum professionellen Wissen verwendet: Pädagogisches (Professions-)Wissen (König et al. 2009), Pädagogisches Unterrichtswissen (König et al. 2012), Pädagogische Kompetenz (Seifert et al. 2009), Erziehungswissenschaftliches Wissen (Blömeke et al. 2008), Adaptive Lehrerkompetenz (Beck et al. 2008), Bildungswissenschaftliches Wissen (Terhart et al. 2012), Pädagogisch-Psychologisches Wissen (Voss et al. 2011).

Zusammenfassend kann gesagt werden:

„Pädagogisches Wissen von Lehrkräften umfasst Kenntnisse über das Lernen und Lehren, die sich auf die Gestaltung von Unterrichtssituationen beziehen und die fachunabhängig, das heißt auf verschiedene Fächer und Bildungsbereiche anzuwenden sind. Pädagogisches Wissen ist ein Bereich professioneller Kompetenz, spezifisch für den Lehrerberuf (sic!), erlern- und vermittelbar und von pädagogischen Überzeugungen und beruflichen Verhaltensweisen (Performanz) abzugrenzen. Es umfasst Wissen über Fakten/Inhalte (deklaratives Wissen), Handlungsabläufe (prozedurales Wissen) und Verknüpfungen/ Schemata (konzeptuelles Wissen).“ (Voss et al. 2015)

Welchen Beitrag können Pädagogisch-Praktische Studien und insbesondere auch Schulpraktika zum Wissenserwerb während der Zeit der Ausbildung leisten? Pädagogisch-Praktische Stu- 
dien unterteilen sich in einen schulischen und hochschulischen Anteil mit unterschiedlichen Lernangeboten. Empirische Befunde für eine evidenzbasierte Professionalisierung der Lehrer/ innenausbildung durch die Eingliederung umfangreicherer Praxisanteile in das Studium stellen ein Desiderat dar (Rothland et al. 2018, 1). In der Forschung zu Schulpraktika liegen vor allem Ergebnisse zum Stellenwert der Praktika aus Sicht der Studierenden und über die Lernwirksamkeit schulischer Lerngelegenheiten vor (Bach 2013, 106). Praktika haben bei Studierenden einen hohen Stellenwert mit Anfangsheroisierung der Mentor/innen. Nach einer gewissen Anzahl an Praktika beurteilen Studierende „die Zufriedenheit mit dem Praktikum als auch in Bezug auf die eigenen Lernerfolge und die Betreuungsqualität“ (Bach 2013, 107) die schulischen Anteile kritischer (Hascher 2006). Welche Lernwirksamkeit Praktika erfüllen, ist nicht ausreichend erforscht (Bach 2013, 110). Fest steht: Die Qualität der Lernprozesse (Gröschner et al. 2015) und die Lernerfolge im Praktikum (Dieck et al. 2009; Müller 2010) sind entscheidend für Erfolg und Nutzen von Praktika. Hierbei kommt nun die qualitätsvolle Arbeit der Mentor/ innen ins Spiel. Bach (2013) meint dazu,

„die Lernwirksamkeit von Schulpraktika ist wiederum davon abhängig, inwiefern es Mentor/innen gelingt, Studierende über das Imitationslernen unterrichtspraktischer Fertigkeiten hinaus zu einer theoriebezogenen und selbstreflexiven Haltung gegenüber der Unterrichtspraxis anzuregen.“ (Bach 2013, 121)

Der Aufbau einer Reflexionskompetenz bzw. einer analytisch-reflektierten Haltung (Herzmann et al. 2016) gelten als Schwerpunkte forschenden Lernens in Zusammenhang mit Lehrer/innenausbildung (Bromme 1992) und schulischen Lerngelegenheiten (Zeichner 2010). Damit Lehrpersonen ihre Tätigkeiten professionell ausführen können, müssen sie „über wissenschaftlich abgesicherte Wissensbestände und Reflexionsmöglichkeiten verfügen.“ (Helsper 2001, 11) Handlungsfähigkeiten und praktisches Können sollen in den schulischen Lerngelegenheiten erprobt und erforscht und Erfahrungen im Dialog mit Mentor/innen reflexiv besprochen werden. Es ist anzunehmen, dass Schulpraktika wesentlich zum Erwerb professioneller Kompetenzen beitragen, wenngleich exakte Befunde zur empirischen Wirkung fehlen. Nach Gröschner et al. (2015), Hesse et al. (2020), Bach (2020) kommt es auf qualitätsvolle Lernprozesse im Schulpraktikum und den begleitenden Lehrveranstaltungen an. Ersteres bedingt wiederum eine qualitätsvolle Begleitung bzw. Ausbildung der Mentor/innen. Die Auseinandersetzung mit Modellen zu Lehrer/innenkompetenzen können Anhaltspunkte in den Ausbildungskonzepten für Mentor/innen sein.

Die Gestaltung von Praktika in den Ausbildungsinstitutionen vor dem Hintergrund einer theoriegeleiteten analytischen Berufspraxis ist sehr unterschiedlich strukturiert und organisiert. Beim Vergleich der Schulpraktika im Bachelor-Master-Studium der Lehrer/innenbildung WEST (Primarstufe, Sekundarstufe) lassen sich folgende gemeinsame Zielvorstellungen identifizieren:

- Orientierungspraktika als Möglichkeit, den Berufswunsch kritisch zu hinterfragen

- Berufserprobung aufgrund vielfältig angelegter Praktikumsformen (Tagespraktikum, geblocktes Tagespraktikum, Blockpraktikum)

- Kompetenzerwerb in den zentralen Handlungsbereichen (Beobachten, Unterrichten, Erziehen, Beurteilen, Innovieren)

- Erwerb von Reflexionsfähigkeit durch forschungsgeleitete Auseinandersetzung mit Theorien

- Theorie-Praxis-Verschränkung durch Überprüfung von Theorien und Konzepten im schulischen Setting und durch Ergänzung von Fragestellungen im weiteren Studienverlauf mit der Zieldimension der Verknüpfung von Wissen und schulpraktischer Erfahrung 
Die akademische Lehrer/innenausbildung nach Lehrer/innenbildung NEU versucht, in Fachwissenschaft und Fachdidaktik, Bildungswissenschaftlichen Grundlagen und PädagogischPraktischen Studien

„forschungs- und nicht subjektiv-erfahrungsfundiert (wie im Falle der Meisterlehre) die Grundlagen für das Verstehen und Erklären, für die Analyse und Reflexion von Schule, Unterricht, Lehren und Lernen samt den bedingenden Faktoren und der intendierten wie nicht intendierten Folgen" (König et al. 2018,7)

zu vermitteln und selbstreflexive und entwicklungsbezogene Aspekte (Weyland 2010) im Sinne der Professionsentwicklung zu forcieren. Pädagogisch-Praktische Studienanteile nehmen in der gegenwärtigen Lehrer/innenausbildung einen hohen Stellenwert ein, wenngleich Praktika nicht die primäre Aufgabe übernehmen, berufsqualifizierende Funktionen einzuüben und zu trainieren, „sondern die Aspekte Berufserkundung und Berufserprobung bilden zentrale Zielbestimmungen (vgl. Topsch, 2004b).“ (Bach 2013, 90) Die koexistierenden Zielperspektiven „Distanz und Einlassung" (Neuweg 2011, 33) innerhalb der Schulpraktika sind für Hochschulen und Universitäten bei der derzeitigen Konzeptentwicklung maßgebend. Die Einbeziehung der Mentor/innen in diese Ausbildungskonzepte ist unabdingbar.

Zusammenfassend kann gesagt werden: Qualitätskriterien für Praktika sind der Implementierungsprozess der Konzepte der Pädagogisch-Praktischen Studien, die systematische Umsetzung begleitet mit Evaluierungen und anschließenden Qualitätsverbesserungsmaßnahmen, die Einbeziehung der Perspektive der Hochschullehrenden sowie die Einbeziehung von innovativen Schulen und ausgebildeten Mentor/innen (historiografischer und systemischer Zugang), eine dialogorientierte kritisch-reflexive Feedbackkultur durch Mentor/innen und der Aufbau einer forschenden Haltung bei Studierenden (Ronfeldt et al. 2015; Hascher et al. 2017) (personalisierter Zugang). Somit kommt der Begleitungs- und Betreuungsqualität (Wilson et al. 2005; Schubarth et al. 2012) eine besondere Bedeutung zu.

Nach einem Überblick zu Einbindung von Pädagogisch-Praktischen Studien in die Lehrer/innenausbildung wird nun auf das Kernthema Bezug genommen: Mentoring als Begleitform in den Schulpraktika. Als Mentor/in wird jene Lehrpersonen definiert, die zu ihrer/seiner Lehrtätigkeit die Aufgabe der Mentor/in übernimmt. Die Grundlage der folgenden Ausführungen bilden historiografische, systemische und personalisierte Betrachtungsweisen untermauert mit Forschungsbefunden, bevor die Ergebnisse zur vorliegenden Studie vorgestellt werden. 


\section{Mentoring als Begleitform für Schulpraktika}

Schulpraktisches Mentoring spielt in der Diskussion um die Wirksamkeit der PädagogischPraktischen Studien im Ausbildungskonzept eine konstitutive Rolle. Mentor/innen unterstützen den Lernprozess. Sie sind Expert/innenlehrpersonen am Lernort Schule (Arnold et al. 2011, 13), nehmen in der Ausübung ihrer Tätigkeit unterschiedliche Funktionen ein und bilden eine Schnittstelle zwischen den hochschulischen und schulischen Anteilen sowie zwischen Theorie, Praxis und Forschung während der Zeit der Ausbildung. Im Kontext von Lehrer/ innenausbildung und von Anteilen Pädagogisch-Praktischer Studien stehen Paradigmen wie: forschende-reflexive Haltung, entwicklungsfördernde und entwicklungsforcierende Aspekte, problemlösende Strategien, theoriegeleitete Analyseprozesse, ausbauende Reflexionsfähigkeit, erklärungsorientierte Handlungskompetenz und dialogorientierte kritisch-reflexive Feedbackkultur.

Diese Paradigmen bedingen sowohl die Schaffung einer förderlichen Lerngelegenheit für Studierende als auch das Bewusstsein der Mentor/innen, Begleitung als Lern- und Entwicklungsprozess in der beruflichen Sozialisation anzusehen. Doch wie kann Mentoring den Prozess des Lernens bei Studierenden unterstützen? Was kann als Aufgabe der/des Mentorin/Mentors gesehen werden? Welche Bereiche sollen bei Studierenden gefördert werden? Wie kann Förderung während der Zeit der Praktika umgesetzt werden? Welchen Beitrag hat die/der Mentee zu leisten? Welche Rolle nimmt die/der Mentor/in und welche die/der Mentee ein? Was zeichnet Mentoring aus? Wann kann Mentoring im schulischen Kontext als erfolgreich angesehen werden? Zur Beantwortung dieser Fragen werden im Folgenden Mentoring und der Mentoringprozess in der Lehrer/innenausbildung näher beleuchtet.

Mentoring ist ein Begleitungsmodell, das in den letzten Jahrzehnten in den USA und Europa eine hohe Verbreitung und eine besondere Beliebtheit erfahren hat. Im Laufe der Zeit kam es zur Ausdifferenzierung unterschiedlicher Zielgruppen und Formate. Das Angebot einer professionellen Begleitung in den Pädagogisch-Praktischen Studien wird als essentiell erachtet. Aus bildungspolitischer Sicht werden enorme Ressourcen für praxisbezogene Ausbildungsteile zur Verfügung gestellt (Arnold et al. 2011, 21).

Im Zuge der vorliegenden Arbeit ist unter Mentor/in jene Person gemeint, die unmittelbar im Umfeld Schule agiert und von den Pädagogischen Hochschulen und/oder Universitäten für die Begleitungsfunktion von Studierenden in der Zeit der Praktika beauftragt wird.

In den folgenden Kapiteln werden die definitorische Sicht, die historiografische, theoretischkonzeptionelle, strukturelle und personelle Betrachtungsweise zum Mentoring und zum schulpraktischen Mentoring ausführlicher dargestellt, da sie essentiell für die Durchführung der Untersuchung, Auswertung und Interpretation der Ergebnisse sind.

\subsection{Mentoring unter begriffsentwickelnder Perspektive}

Der Begriff Mentoring geht bereits auf die Antike zurück. Im zwölften vorchristlichen Jahrhundert taucht der Begriff in der griechischen Mythologie als Gestalt von „Mentor“, dem Freund von Odysseus, auf (siehe Einführung). „Im traditionellen Verständnis hat der Mentor (sic!) ge- 
genüber dem Mentee (sic!) einen gewissen Alters-, Erfahrungs-, Wissens- und Machtvorsprung, was ihn auch als Rollenmodell prädestiniert." (Fuge 2016,33)

Tab. 11: Theoretische Hintergründe des Mentoring (in Anlehnung an Fuge 2016, 76)

\begin{tabular}{llllll}
\hline Dimensionen & $\begin{array}{l}\text { Begriffs- } \\
\text { verständnis }\end{array}$ & $\begin{array}{l}\text { Theoretische } \\
\text { Zugänge }\end{array}$ & Funktionen & Einflussfaktoren & $\begin{array}{l}\text { Didaktisches } \\
\text { Potential }\end{array}$ \\
\hline $\begin{array}{l}\text { Theoretische } \\
\text { Hintergründe }\end{array}$ & $\begin{array}{l}\text { Dyadische } \\
\text { Beziehung }\end{array}$ & $\begin{array}{l}\text { Griechische } \\
\text { Mythologie }\end{array}$ & $\begin{array}{l}\text { Erziehungsfunk- } \\
\text { tion }\end{array}$ & $\begin{array}{l}\text { Erfahrung der/ } \\
\text { des Mentorin/ } \\
\text { Mentors }\end{array}$ & $\begin{array}{l}\text { Förderung } \\
\text { des Lern- und } \\
\text { Entwicklungs- } \\
\text { prozesses }\end{array}$ \\
\hline
\end{tabular}

Tabelle 11 zeigt die theoretischen Hintergründe des ursprünglichen Mentoring-Begriffs auf. Es ist eine dyadische Beziehung, in der die/der erfahrene Mentor/in eine Erziehungsfunktion innehat. Als Ziele gelten die Förderung des Lernens und Sich-Entwickelns. Der Ursprung des Gedankengutes stammt aus der griechischen Mythologie.

Die Geschichtsschreibung zeigt auf, dass „der Begriff des Mentors (sic!) als Synonym für einen geachteten und gebildeten Menschen“ (Oettler 2009, 12) verwendet wird. Die/Der Mentor/ in agiert in der Rolle der/des Beraterin/Beraters, Begleiterin/Begleiters und Beschützerin/Beschützers. Die/Der Mentor/in agiert im Idealfall im Sinne eines Vorbildes und verfolgt einen prozess- und förderungsorientierten Ansatz (Faix et al. 2017, 36).

Ab den 1970er-Jahren erlebte Mentoring vor allem in amerikanischen Großkonzernen eine Wiederbelebung. Als beispielhaft ist der Unternehmer Max von Dach zu erwähnen, der seine Aufgabe darin sah, Nachwuchskräften eine optimale Laufbahnentwicklung zu ermöglichen und beratend und unterstützend zur Seite zu stehen. Das erfolgreiche Konzept des Mentorings fand somit als Methode zur Personalentwicklung in Wirtschaftsunternehmen Einzug, ,aber auch als Förderinstrument im Bereich der Bildung und der sozialen Arbeit für Angehörige unterrepräsentierter oder unterprivilegierter Gruppen." (Magg-Schwarzbäcker 2014, 15)

Die ersten Forschungsbefunde stammen auch aus jenem Zeitabschnitt. Levinson u.a. analysierten 1978 in der Studie „The Seasons of a Man's Life“vierzig Biografien und kamen zum Ergebnis, dass die Relevanz von Mentor/innen mit Eltern, die Kinder begleiten, gleichgesetzt werden kann (Eby et al. 2007, 8; Ragins et al. 2007a, 4; Magg-Schwarzbäcker 2014, 17).

„Ein Mentor (sic!) wird dort beschrieben als Führer (sic!), Lehrer (sic!), Berater (sic!) und Entwickler (sic!) von Fähigkeiten, der die Realisierung des Traums, der Vision, die jemand von seinem Erwachsenenleben hat, ermöglicht (Eby et al. 2007, 8).“ (Magg-Schwarzbäcker 2014, 17)

Als Grundlage in der Mentoring-Forschung (Fuge 2016, 34) gilt das Werk „Mentoring to Work“ von Kram (1985). In der qualitativen Interviewstudie mit 18 Mentoring-Partner/innen arbeitete Kram (1985) Mentoring-Funktionen, Phasen in der Mentoring-Beziehung und mögliche Auswirkungen von Mentoring heraus. Die Mentoring-Funktionen kategorisierte sie in Psychosoziale Funktionen und Karrierefunktionen (Tabelle 12). 
Tab. 12: Mentoring-Funktionen nach Kram (Kram 1985, 23f; Übersetzung Magg-Schwarzbäcker 2014, 18)

\begin{tabular}{ll}
\hline Mentoring-Funktionen & \\
\hline Psychosoziale Funktionen & Karrierefunktionen \\
- Role Modelling (Vorbild) & - Sponsorship (Förderung, Unterstützung) \\
- Acceptance-and-Confirmation & - Exposure-and-Visibility (Konfrontation und Sichtbarkeit) \\
(Akzeptanz und Bestätigung) & - Coaching (Coaching) \\
- Counseling (Beratung) & - Protection (Schutz) \\
- Friendship (Freundschaft) & - Challenging Assignments (Herausfordernde Aufgaben) \\
\hline
\end{tabular}

Den Karrierefunktionen wurden jene Kategorien zugeordnet, in denen die/der Mentor/in ihre/seine Schützlinge innerhalb der Organisation fördert, beim Vorankommen unterstützt und durch besondere Aufgabenerfüllung deren/dessen Kompetenzen und Leistungen sichtbar machen kann. Durch Coaching ist es der/dem Mentee möglich, Strukturen rascher zu erkennen und zu durchschauen. Die Schutzfunktion durch die/den Mentor/in und die Bewältigung von herausfordernden Aufgaben wirken sich je nach Bedarfslage positiv oder negativ auf die/den Mentee aus.

Die meistgenannte Kategorie bei den Psychosozialen Funktionen ist die Vorbild- oder Rollenmodellfunktion, die als erfolgreich gilt, wenn sie mit einer emotionalen Bindung einhergeht. Weitere Kategorien bilden die Akzeptanz und Bestätigung, die Beratung und Freundschaft als erfolgversprechende Besonderheiten innerhalb der Mentoring-Beziehung. Organisatorische und psychologische Elemente beeinflussen jede Mentoring-Beziehung und im Kontext werden Funktionen unterschiedlich verstanden. Als weiteres Ergebnis ihrer Untersuchung bildet Kram (1985) eine Prozesshaftigkeit im Mentoring ab, indem die Phasen Initiation, Cultivation, Separation, Redefinition (Kram 1985, 48f) konstruiert wurden (Magg-Schwarzbäcker 2014, 19f). Aus den USA gelang in den 1980er-Jahren das Konzept des Mentorings über die skandinavischen Länder und Großbritannien auf den Kontinent Europa. In Betrieben wurden Mentoring-Konzepte als Personalentwicklungsmodelle, vor allem aber auch Frauenförderprogramme installiert und weiterentwickelt. Nicht unerwähnt kann die oftmals divergierende Betrachtungsweise bleiben.

"In the US, the macho, protective aspects of Athena dominated the perception of the mentoring relationship. Not surprising, then that the recipient of mentoring become known as a protégé (someone, who is sponsored and/or protected). In Europe, by contrast, the emphasis of the role was given to the development of wisdom - hence the term, mentee (someone who thinks)." (Clutterbuck 2017,310)

Im internationalen Kontext gibt es nach Clutterbuck (2017) Kombinationen beider Modelle. Mentoring ist mittlerweile in Europa Bestandteil in Wirtschaftsbetrieben, Verwaltungen und Organisationen, Hochschulstrukturen und im Bildungsbereich (Magg-Schwarzbäcker 2014, 16). Bedeutung erfährt die besondere Form der Begleitung durch den Struktur- und Gesellschaftswandel in der Arbeits- und Lebenswelt, in der „Flexibilität, Kreativität, Individualismus, Bildung und Kommunikation“" (Faix et al. 2017, 23) im Vordergrund stehen und seit das „Zeitalter der Wissensökonomie“ (Faix et al. 2017, 23) eingeläutet wurde. „Wissen wird zum entscheidenden Produktionsfaktor und zur zentralen Schlüsselressource. Gemeint ist allerdings nicht formelles, abrufbares Wissen, sondern Erfahrung, Urteilsvermögen und Selbstorganisation." (Demographie Netzwerk 2019) Mentoring zeigt sich in diesem Zusammenhang als idealtypische Lernform. Auch das schul- 
praktische Mentoring gewinnt in den letzten beiden Dekaden des 21. Jahrhunderts an Bedeutung. "In teacher training, there is now more emphasis placed on the school as a learning environment (Brouwer, 2007; Mantle-Bromley, 2003; Smith, 2003).” (Hennissen et al. 2008, 169)

Graf et al. (2017) beschreiben zwei wesentliche Veränderungen im heutigen Mentoring: die Installierung von strukturierten Programmen statt einer losen unsystematischen Beziehung und die Erwartungshaltung eines proaktiven und interessierten Verhaltens seitens der/des Mentee/ Mentees statt eines „demütigen, lernenden Rollenverständnisses“(Graf et al. 2017, 5).

Eine einheitliche Definition aus (Teil)Wissenschaften ist bisher nicht gegeben. Die heutige Verwendung des Begriffs Mentoring wird von vielen Autor/innen in Anlehnung an Homers Sage weiterhin eingesetzt. Dazu beschreibt Ziegler (2009) mit seiner Definition den „Begriffskern eines typischen Mentorings" (Ziegler 2009, 11):

„Mentoring ist eine zeitlich relativ stabile dyadische Beziehung zwischen einem/einer erfahrenen MentorIn (sic!) und seinem/r/ihrem/r weniger erfahrenen Mentee. Sie ist durch gegenseitiges Vertrauen und Wohlwollen geprägt, ihr Ziel ist die Förderung des Lernens und der Entwicklung sowie das Vorankommen des/der Mentees." (Ziegler 2009, 11)

Die Definition nach Ziegler (2009) wird in den folgenden Ausführungen - vor allem im Kapitel Mentoringprozess in der Lehrer/innenausbildung (II/2.4) - als Grundlage verwendet. Durch die Betonung auf "gegenseitiges Vertrauen und Wohlwollen“ (Ziegler 2009, 11) wird der Beziehungsaspekt in den Vordergrund gestellt und diskutiert. Die Zieldimension „Förderung des Lernens und der Entwicklung“ (Ziegler 2009, 11) erweist sich in der Umsetzung als multiperspektivischer Ansatz, der dem humanistischen Bildungsbegriff zugrunde liegt.

Diesbezüglich stellt sich die Frage, welche Inhalte gerade auch im schulpraktischen Mentoring dargeboten werden sollen, damit ein Lern- und Entwicklungsprozess stattfinden kann. Theorien, Konzepte und Forschungsbefunde werden dazu im weiteren Verlauf der Arbeit diskutiert. Die Definition nach Ziegler (2009) weist letztendlich auf die Bedeutung des Beziehungs- und Inhaltsaspekts in der Begleitung hin. Wie werden nun diese Intentionen in unterschiedlichen Kontexten und speziell im schulpraktischen Mentoringprozess verstanden und umgesetzt?

\subsection{Mentoring in unterschiedlichen Kontexten}

Mentoring setzt da an, wo Menschen Unterstützung, Entscheidungshilfen, Förderung, Strukturierung, Lösungsoptionen u.ä. benötigen. Daher begegnet man Mentoring im Kontext verschiedener Felder (wirtschaftlicher, hochschulischer und universitärer, psychologischer usw.). Seit etwa dreißig Jahren weisen Publikationen zunehmend auf die Bedeutung und Aktualität von Mentoring-Programmen für Unternehmen sowie Hochschulen und Universitäten hin und beschreiben unterschiedliche Formen und Ausprägungen (Graf et al. 2017; Pflaum et al. 2019). Ausgangspunkt für erfolgreiches Mentoring im Managementbereich waren zwei Artikel in Harvard Business Review im Jahre 1977. Darin waren Studien von 1000 Führungspersönlichkeiten aufgezeigt, welche „underscored the association between having a sponsor, or mentor, and achieving success in business." (Jacobi 1991, 506) Mentoring ist seither für Unternehmen eine Methode der beruflichen und persönlichen Personalentwicklung und -förderung - vor allem auch seit ca. 1990 zur Förderung von Frauen in den unterschiedlichsten Berufen. Auch im hochschulischen und universitären Bereich werden Mentoring-Programme als Instrument für Geschlechtergleichstellung und in jüngster Zeit auch für die Nachwuchsförderung und bei 
Transitionsprozessen etabliert (Magg-Schwarzbäcker 2014, 16). Ausgangspunkt war der Einsatz von Interventionsstrategien in den MINT-Fächern der Universitäten. Ziel war bzw. ist es, Schülerinnen (sic!) in den postsekundären Schulen und Frauen die Naturwissenschaften näher zu bringen, das Selbstvertrauen zu stärken, die weiblichen Begabungspotentiale auszuschöpfen, einem Fachkräftemangel entgegenzuwirken und die fachbezogene männerdominierte Rollenzuschreibung zu verändern (Sander 2009, 55f). Hierbei entstanden bzw. entstehen unterschiedliche Formen von Mentoring: Netzwerk-Mentoring, Cross-Mentoring, Peer-Mentoring, Gruppen-Mentoring, Team-Mentoring, virtuelles Mentoring, Career Mentoring usw. Universitäten und Hochschulen kooperieren mit Firmen und Wirtschaftsunternehmen. Mentee erleben „einen breiten fachlichen Zugang zu Kontakten, Erfahrung und Wissen“ (Pflaum 2017, 46) durch ihre Mentor/innen.

Bedeutung erlangen in wirtschaftlichen Feldern gegenwärtig auch Reverse Mentoring-Programme im Bereich der Digitalisierung und Social-Media-Kompetenz. „In diesen Programmen sind die Mentoren (sic!) meist zwischen 20 und 30 Jahre und die Mentees zwischen 40 und 65 Jahre alt." (Pflaum et al. 2019, 2) Unternehmen reagieren mit dem Instrument Mentoring auf rasche Veränderungen innerhalb der Organisationsstrukturen und kulturell-gesellschaftlicher Rahmenbedingungen. Durch den Strukturwandel kommt nach Higgins und Kram (2001) vor allem Netzwerk-Beziehungen eine besondere Bedeutung zu, wie sie in ihren Studien resümierten. Die Besonderheit beim Mentoring als Entwicklungsnetzwerk sind mehrfache dyadische Beziehungen oder Netzwerk-Beziehungen, die den theoretischen Ursprung aus der griechischen Mythologie angepasst an heutige strukturelle Veränderungen haben. Die Bildung erfolgt zu jeder Zeit im Berufsleben mit dem Ziel der Gemeinsamkeit und Reziprozität. „Die beiden Dimensionen Vielfalt des Entwicklungsnetzwerks und Stärke (Umfang (...) der Kommunikation, emotionale Nähe und Grad der Reziprozität in der Entwicklungsbeziehung) bilden die Basis der Typologie von Entwicklungsnetzwerken." (Magg-Schwarzbäcker 2014, 36; Higgins et al. 2001, 268)

Auch im schulpraktischen Mentoring kann davon ausgegangen werden, dass Netzwerk-Beziehungen von Bedeutung sind. Gegenwärtig sind sie meist im schulpraktischen Mentoring noch unterrepräsentiert. Die Lehrer/innenausbildung mit dem Institutionalisierungsprogramm Mentoring kann sich als Brückenfunktion zwischen der Makroebene Gesellschaft und der Mikroebene Praktika an Schulen verstehen. Es kann als Aufgabe der Lehrer/innenausbildung betrachtet werden, gesellschaftliche Veränderungen, Diversitäten und Herausforderungen zu berücksichtigen und personenspezifisch darauf einzugehen. Abbildung 9 verdeutlicht diesen Zusammenhang (Abbildung 9):
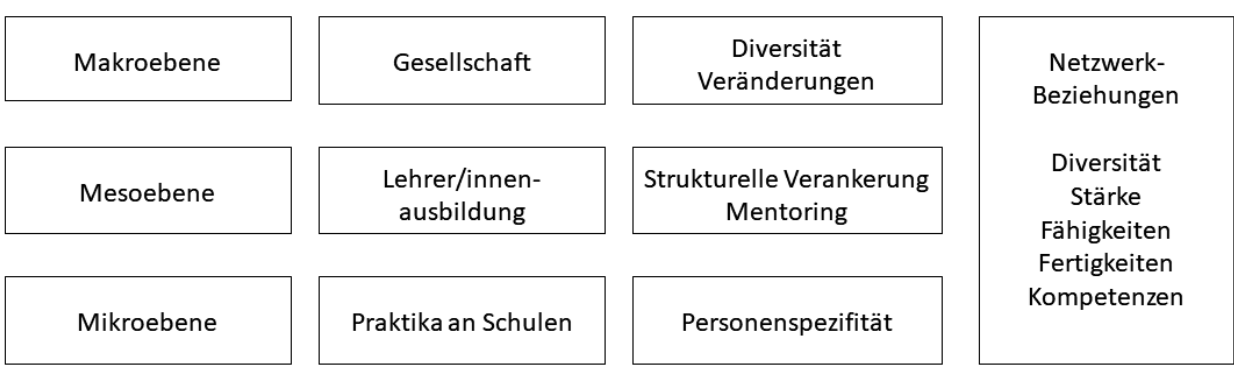

Abb. 9: Mentoring in der Lehrer/innenausbildung aus dem Kontext wirtschaftlicher Felder 
Die Etablierung von Netzwerk-Beziehungen als Mentoring-Instrument und institutionalisierte Einrichtung innerhalb der Lehrer/innenausbildung, wie sie vor allem auch in wirtschaftlichen Feldern zur Personalentwicklung und Personalförderung vorkommen, die auf Diversitäten der Gesellschaft reagieren und Potentiale sowie Stärken, Fähigkeiten, Fertigkeiten und Kompetenzen jeder/jedes einzelnen hervorheben, kann als besondere Form des Mentorings herangezogen werden. Es liegt an den Ausbildungsinstitutionen, dass Studierende verschiedene Begleitformen und Unterstützungsmaßnahmen erleben. Lehrer/innenausbildung kann somit auch verdeutlichen, dass Institutionen und Schulen Spezifitäten zulassen und auf reziproke fördernde Beziehungen - verglichen mit der Beschreibung des Reverse Mentoring Programmes - bauen.

Auch im Kontext psychologischer Felder und hier vor allem die Teildisziplinen Entwicklungspsychologie, Allgemeine Psychologie (Lernpsychologie) und Sozialpsychologie werden Anhaltspunkte zum Mentoring und zur Konzeption von Mentoring-Programmen sichtbar. Fuge (2016) resümiert in diesem Zusammenhang Dimensionen und Potentiale aus psychologischer Perspektive folgendermaßen (Tabelle 13):

Tab. 13: Hintergründe des Mentorings aus psychologischer Perspektive (Fuge 2016, 76)

\begin{tabular}{llll}
\hline Mentoring als ... & & & \\
\hline & $\begin{array}{l}\text { Übergangshilfe } \\
\text { (Entwicklungspsychologie) }\end{array}$ & $\begin{array}{l}\text { Lernhilfe } \\
\text { (Lernpsychologie) }\end{array}$ & $\begin{array}{l}\text { Unterstützung } \\
\text { (Sozialpsychologie) }\end{array}$ \\
\hline Begriffsverständnis & $\begin{array}{l}\text { Entwicklungs- } \\
\text { Beziehung }\end{array}$ & $\begin{array}{l}\text { Lern- } \\
\text { Beziehung }\end{array}$ & $\begin{array}{l}\text { Zwischenmenschliche } \\
\text { Beziehung }\end{array}$ \\
& Konzept der & Scaffolding & Soziale Unterstützung \\
Theoretische & Entwicklungsaufgaben & Lernen am Modell & Soziale Netzwerke \\
& Einfluss von Peer-Beziehungen & Kognitionsmodell & \\
\hline Funktionen & Bewältigungs- & Vorbild- & Unterstützungs- \\
& Funktion & Funktion & Funktion \\
\hline Einfluss- & Einfluss des & Positive Persönlichkeit & Vertrauens- \\
Faktoren & Mentors (sic!) & des Mentors (sic!) & Verhältnis \\
\hline Didaktisches & Orientierung & Anleitung und & Stressbewältigung, \\
Potential & und Führung & Training & soziale Integration \\
\hline & & &
\end{tabular}

Entwicklungspsychologischer Blickwinkel: Die/Der Mentor/in hat aus entwicklungspsychologischer Sicht und im traditionellen Verständnis einen großen Einfluss auf die/den Mentee im Hinblick auf Gestaltung von Veränderungsprozessen. Sie/Er baut eine vorübergehende Beziehung mit dem Fokus der Bewältigungsfunktion der Aufgaben durch den Mentee auf. Im Hintergrund steht das Konzept der Entwicklungsaufgaben nach Havighurst (1948).

„Entwicklungsaufgaben sind gesellschaftliche Anforderungen an Menschen in je spezifischen Lebenssituationen, die individuell als Aufgaben eigener Entwicklung gedeutet werden (können). Entwicklungsaufgaben müssen wahrgenommen und bearbeitet werden, wenn es zu einer Progression von Kompetenz und Stabilisierung von Identität kommen soll.““ (Hericks 2004, 117)

Mentor/innen können hierbei Vorschläge zur Bearbeitung von Entwicklungsaufgaben machen. Einen bedeutenden Einfluss gerade im Hochschulbereich und im Rahmen der Pädagogisch- 
Praktischen Studien haben auch Peer-Beziehungen. Peers sind wichtige Partner/innen, wenn es darum geht, in der beruflichen Sozialisation Verankerung zu finden, und sie tragen wesentlich zur Entwicklung des Selbstkonzepts und der Identitätsfindung bei (Fuge 2016, 48). Peers sind in den Pädagogisch-Praktischen Studien meist Mitstudierende. Sie zeigen so wie die/der Mentor/in gewisse Anhaltspunkte auf und/oder kennzeichnen sich durch ein bestimmtes Führungsverhalten aus.

Lernpsychologischer Blickwinkel: Aus lernpsychologischer Sicht vollzieht sich im Mentoring ein Lernprozess und je nach Ausdifferenzierung der Gestaltung werden Reflexionskompetenz und die Fähigkeit eines Perspektivenwechsels gefördert. Im Hintergrund der lernpsychologischen Betrachtungsweise von Mentoring stehen Konzepte des Konstruktivismus (Kognitionsmodell), der sozial-kognitiven Lerntheorie (Lernen am Modell) und der Interaktion und Kommunikation (z.B. Scaffolding). Nach dem Kognitionsmodell beobachten und analysieren Mentees das Verhalten der/des Mentorin/Mentors, gehen in einen Interaktions- und Kommunikationsprozess und entscheiden auf Basis bereits gemachter Erfahrungen über die Effektivität und folglich also darüber, „ob sie die neuen Erfahrungen mit ihren bisherigen Erfahrungen zu Wissen verknüpfen möchten." (Fuge 2016, 51) Beim Modelllernen nach Bandura (1976) steht die/der Mentor/in im Mittelpunkt des Geschehens. Gelingt es der/dem Mentor/in, als Vorbild anerkannt zu werden, wird die/der Mentee versuchen, Erlebtes in ihren/seinen eigenen Handlungen umzusetzen (Richert 2006, 47; Fuge 2016, 53). Hinter dem Verständnis Scaffolding (engl. Gerüst) steht die Theorie des russischen Psychologen Vygotskij, der behauptet, dass es Niveauunterschiede zwischen selbstständigem Problemlösen und Lernen aufgrund der Anleitung einer kompetenten Person zugunsten der Begleitung gibt. Die/Der Mentor/in könnte nach dieser Ansicht zur/zum Förderin/Förderer der Erweiterung der kognitiven Fähigkeiten werden. Nach Mc Kenzie (1999) sind Merkmale für ein gelungenes Scaffolding: Bereitstellung einer eindeutigen Anleitung, Offenlegung des Zwecks von Aufgaben, Verhinderung einer Abweichung von der Aufgabenstellung, Verdeutlichung der Erwartungen, Nennung von Informationsquellen zum Thema sowie Vermeidung von Unsicherheiten, Überraschungen und Enttäuschungen. Im Mentoring könnten die Umsetzung dieser Anhaltspunkte zunächst Stütze sein und Anwendung finden. Allmählich sollten sie aber wieder zurückgenommen (fading)werden, damit die/der Mentee sich persönlich entfalten kann (McKenzie 1999).

Sozialpsychologischer Blickwinkel: Im Mentoring unter dem Aspekt der Sozialpsychologie bauen Mentor/innen ein Vertrauensverhältnis zum Mentee auf und agieren in kommunikativer, unterstützender und wohlwollender Interaktion. Ziele sind das Wohlbefinden der/des Mentee/ Mentees und die Betonung einer zwischenmenschlichen Beziehung. Dazu braucht es vor allem ein Erkennen der möglichen Problembereiche (Aymanns 1995, 25).

„Nach der Puffereffekt-These mildert soziale Unterstützung die gesundheitsgefährdenden Wirkungen vieler Arten von Umweltstress, indem sie den Einfluss des Stress auslösenden (sic!) Ereignisses und der Stressreaktion verändert. Dies wirkt sich in der Folge positiv auf die Befindlichkeit aus. Demgegenüber besagt die Direkteffekt-These, dass soziale Unterstützung generell, unabhängig von einer spezifisch stressreichen Situation, das seelische und körperliche Wohlbefinden positiv beeinflusst (u.a. House, 1981; Röhrle, 1994)“. (Fuge 2016, 53f)

Für Schweer (2004) geht soziale Unterstützung mit der Komponente Vertrauen zwischen den Aktionspartner/innen einher (Schweer 2004, 280f). Je höher das Vertrauensverhältnis ist, desto eher wird soziale Unterstützung seitens der Mentee in Anspruch genommen (Fuge 2016, 54). 
Die angeführten Sichtweisen aus den Teildisziplinen der psychologischen Perspektiven haben auch für das schulpraktische Mentoring und innerhalb der Lehrer/innenausbildung eine große Bedeutung. Die Ansätze verkörpern unterschiedliche Funktionen: Bewältigungs-Funktion, Vorbild-Funktion, Unterstützungs-Funktion. Abbildung 10 veranschaulicht wiederum einen Zusammenhang der Lehrer/innenausbildung mit den gesellschaftlichen Herausforderungen, Veränderungen und Diversitäten und die Möglichkeit, Studierende in den Praktika im Sinne der unterschiedlichen Funktionen zu fördern.
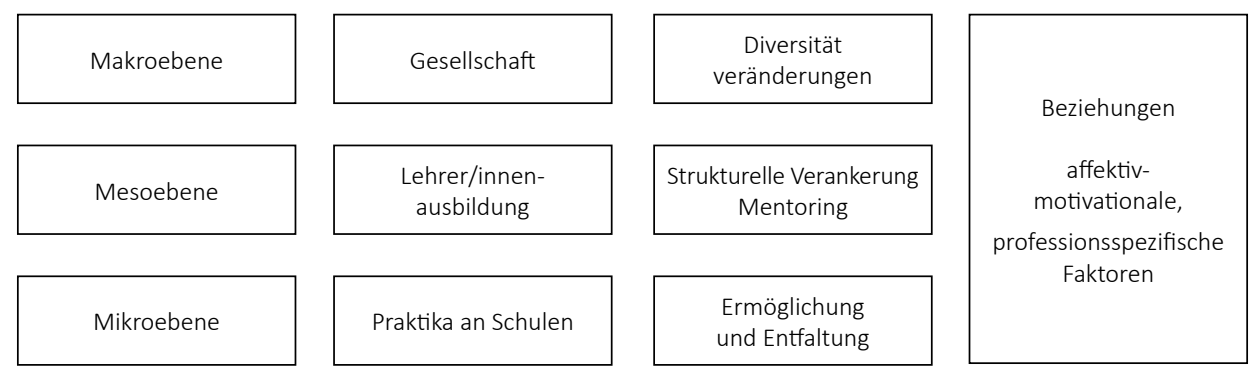

Abb. 10: Mentoring in der Lehrer/innenausbildung zur Stärkung affektiv-motivationaler und professionsspezfischer Faktoren

Bewältigungs- und Unterstützungs-Funktionen beziehen sich im Kontext von Mentoring auf das Eingehen von affektiv-motivationalen Aspekten zur Entfaltung der Persönlichkeit. VorbildFunktionen können professionsspezifische Faktoren stärken, indem pädagogisches Wirken und Handeln im Rahmen der Intersubjektivität betrachtet und analysiert wird. Es obliegt nun den Institutionen der Lehrer/innenausbildung bzw. -fortbildung, welche Strukturen und Konzepte oder welche Ausbildungsmodule in Fortbildungsformaten zu Mentoring konzipiert werden. Wird in den Programmen der Kontext des psychologischen Zuganges in den Fokus genommen, so zielt es auf die Berücksichtigung affektiv-motivationaler sowie professionsspezifischer Faktoren im Beziehungs- und Begleitungskontext.

Im Folgenden werden Forschungsbefunde zum schulpraktischen Mentoring und anschließend der Mentoringprozess in der Lehrer/innenausbildung näher beleuchtet.

\subsection{Forschungsbefunde zum schulpraktischen Mentoring}

Erfolgsgenerierende Befunde zu Schulpraktika sind stark an die Frage der Begleitung und an den Anteil der theoriegeleiteten Reflexionsteile gekoppelt (Hascher 2012; Arnold et al. 2014). Mentor/innen schaffen Rahmenbedingungen im schulischen Setting und unterstützen im Begleitungsprozess die berufsbiografische Entwicklung (Terhart 2001).

Ausgangspunkt der ersten Untersuchungen zu Mentoring waren Fallstudien ab 1970, die der Begleitform eine Effektivität zuerkannten. Ein häufig genanntes Beispiel für Effektivität wird mit dem Atomphysiker Ernest Rutherford (1871-1937) verbunden, der als herausragende Persönlichkeit geehrt wurde, zumal er neben seinen beruflichen Erfolgen elf seiner Schüler zu Trägern des Nobelpreises verhalf (Ziegler 2009, 11). Im anglo-sächsischen Raum mehren sich seither Studien zu Mentoring im Allgemeinen, zu Mentoring in der Induktionsphase und zum schulpraktischen Mentoring. Die Fülle an Beschreibungen zum Thema Mentoring ist wohl 
kaum mehr überschaubar (Ziegler 2009, 12). Wie sieht nun die Forschungslandschaft im schulpraktischen Mentoring aus?

Bei einer Zusammenschau der Studien kann festgestellt werden, dass sich Autor/innen auf unterschiedliche Forschungsschwerpunkte konzentrieren. Eine inhaltliche Grobeinteilung lässt sich mit den Überschriften (1) Wirkungsweise von schulpraktischem Mentoring oder lernwirksame Schulpraxis durch Mentoring, (2) prozessbegleitende Formate und (3) Erfolgsgenerierung versehen. In den Designs werden jeweils unterschiedliche Aspekte berücksichtigt und Untersuchungen können den erwähnten Hauptfaktoren zugeordnet werden.

Wird die Wirkungsweise (1) betrachtet, so sprechen Autor/innen oftmals von einer wechselseitigen positiven Wirkung. Studierende und Mentor/innen gehen in den Praxisphasen eine Symbiose ein. Wenn Mentor/innen die optimale Sozialisationseingliederung der Studierenden in den Schulalltag gelingt (Crisp 2010), können Studierende ihr Selbstvertrauen steigern, das professionelle Handeln und die Selbstreflexion verbessern (Hobson et al. 2009, 209). Laut Untersuchungen von König et al. (2018) haben Mentor/innen einen sehr großen Einfluss auf die „Stärkung des beruflichen Selbstkonzeptes und dem Erleben eigener Unterrichtskompetenzen, aber auch in Form der Minderung von Beanspruchungserleben." (König et al. 2018, 44) Mentor/innen haben sich ein implizites Wissen aufgrund ihrer Erfahrungen in der Interaktion mit anderen und mit der Umwelt - in diesem Fall mit der Schule - aufgebaut, das ,in einem hohen Maße personen- und situationsgebunden ist und das wissenschaftliche Wissen in der Alltagspraxis in sinnvoller Weise ergänzt.“ (Magg-Schwarzbäcker 2014, 82) Hobson et al. (2009) erwähnen, „that 70 \% of mentors claimed to have benefited professionally from mentoring." (Hobson et al. 2009, 209) Einige Mentor/innen "have talked about a revitalisation of their enthusiasm for teaching “ (Moor et al. 2005), becoming „re-energised“ or „re-engaged“ with the profession (Hobson et al. 2007) and „being more committed to teaching." (Hobson et al. 2009, 210) In den Untersuchungen wird meist von Einzelfallbeschreibungen ausgegangen und auf eine Verallgemeinerung geschlossen.

Untersuchungen zu prozessbegleitenden Formaten des Mentorings (2) beschäftigen sich mit Mentoring-Beziehungen und Gesprächsverläufen. Forschungsbefunde zu Gesprächen von Mentor/innen zeigen die Rollenfunktion auf, stellen Inhalte dar und beschreiben den individuellen Prozessverlauf (Jünger et al. 2017, 114). Hierbei ist auffallend, dass Mentor/innen, bei denen Studierende eine positiv erlebte mentorielle Unterstützung erfahren bzw. erfahren haben und Freiräume gegeben sind bzw. waren, als besonders erfolgreich gelten (Duffield 2006; Cherian 2007; Schubarth et al. 2014). Mentoring-Beziehungen mit dem Fokus sozio-konstruktivistisch orientierter Perspektive konzentrieren sich auf einen beidseitig gewinnbringenden Lern- und Entwicklungsprozess (Schüssler et al. 2017). Studien sind nun um diese Thematiken ausgelegt, beispielhafte Anleitungen von erfolgreichen Mentoring-Beziehungen und Gesprächsverläufen fehlen.

Hobson et al. (2009) zählen zur Erfolgsgenerierung im Mentoring (3) vier wesentliche Faktoren: Kontextuelle Unterstützung im Mentoring, Mentor/innenauswahl und Matchingprozess, Mentoring-Strategien und Vorbereitung und Ausbildung zum Mentoring (Hobson et al. 2009, 211f). Die Relevanz der einzelnen Faktoren wird in den nachstehenden Kapiteln noch ausführlicher diskutiert. Im Folgenden werden acht exemplarische Forschungsdesigns vorgestellt und Ergebnisse in Bezug auf das schulpraktische Mentoring dokumentiert. Die Beschreibung erfolgt wie bei den Forschungsbefunden zu Schulpraktika (siehe Kapitel I/ 1.1.4) nach dem Prinzip Forschungsfrage(n), Aufbau des Untersuchungsdesigns, zentrale Ergebnisse und endet immer mit der Kernidee als Versuch der Interpretation der Bedeutung für das schulpraktische Mentoring. Die angeführten Untersuchungen wurden nach den Kategorien menteebezogene, mentor/innenbezogene sowie kombinatorische mentee- und mentor/innenbezogene Untersuchungen gewählt. 
Das erste Untersuchungsdesign weist auf ein menteebezogenes Format hin. Mentor/innen sind demnach aufgefordert, im Begleitungsprozess auf Persönlichkeitsstrukturen der Mentee zu achten.

(1) Forschungsfragen: „Welche Bedeutung haben die Praktika für Studierende? Wie konstituieren sich Anforderungen in den Praktika im Erleben der Studierenden und wie bearbeiten sie diese? Welche Rolle haben die Praxislehrpersonen im Rahmen der Konstituierung von Anforderungen in den Praktika?" (Košinár 2018,72)

Aufbau des Untersuchungsdesigns: 14 Interviews wurden auf unterschiedliche Dimensionen (Bedeutung der Praktika, Konstituierung und Bearbeitung von Anforderungen, Rolle der Praxislehrperson) einer komparativen Analyse unterzogen.

Zentrale Ergebnisse: Es wurden die vier Typen Selbstverwirklichung, Vermeidung, Entwicklung und Bewährung von Studierenden identifiziert. Die Studierenden sehen in den Praktika unterschiedliche Bedeutsamkeiten und bearbeiten die Anforderungen aus unterschiedlichen Motiven (Košinár 2018, 72). Der Typologieansatz hat demzufolge Auswirkungen auf das Verhalten der Mentor/innen. Aus diesem Grund folgt eine Detailbeschreibung im Kapitel I/2.4.6.

Kernidee: Im schulpraktischen Mentoring muss auf die in den Eingangsbedingungen (Cramer 2012) beschriebene Persönlichkeit Rücksicht genommen werden.

Die folgenden vier Untersuchungen zeichnen mentor/innenbezogene Forschungsdesigns. Dabei werden Motive und Einstellung von Mentor/innen erhoben und Aufgabenfelder evaluiert.

(2) Forschungsfragen: Die Fragen sind an Mentor/innen gerichtet: „Aus welchen Motiven heraus möchten Sie Praktikanten (sic!) betreuen? Was erhoffen Sie von der Übernahme dieser Aufgabe? Welche Befürchtungen haben Sie bzgl. der Rolle/Aufgabe?" (Weyland et al. 2011)

Aufbau des Untersuchungsdesigns: Die Auswertung der Fragen erfolgte im Erstfall qualitativ-inhaltsanalytisch, bei beiden letztgenannten Fragen mittels Häufigkeit der kategorisierten Nennungen.

Zentrale Ergebnisse: Die Ergebnisse der 96 Mentor/innen zeigen auf, dass $52 \%$ selbstbezogene Motive (Anregungen erhalten, Interesse an der Lehrer/innenausbildung, Interesse an der Aufgabe, Kontakt zu jüngeren Kolleg/innen, Reflexion der eigenen Tätigkeit), $22 \%$ praktikant/innenbezogene (Hilfestellung für Berufsanfänger/innen, Hilfestellung bei Berufswahl, Wissen/Erfahrung weitergeben) und $18 \%$ systembezogene Motive (Mitwirken an der Ausbildung, Ausbildung von Lehrer/innen wichtig) haben. Ähnliche Prozentzahlen weisen mit $60 \%$ selbstbezogene, $22 \%$ praktikant/innenbezogene und $14 \%$ systembezogene Hoffnungen auf sowie $66 \%$ Befürchtungen im Blick auf die eigene Person, $10 \%$ auf Praktikant/innen und 19\% auf das System (Weyland et al. 2011).

Kernidee: Motiverforschung zur Betreuungstätigkeit der Mentor/innen kann in reflexivbiografischen Ansätzen erkundet werden und Unterstützungsmaßnahmen für Mentor/ innen können in den Ausbildungskonzepten verankert werden.

(3) Forschungsfrage: „Wie entwickeln Mentor/innen positive Mentor/in-Mentee-Beziehungen?" (Hudson 2013, 1)

Aufbau des Untersuchungsdesigns: Auf der Grundlage von schriftlichen Kommentaren $(n>200)$, offenen Gesprächsrunden $(n=19)$ und Interviews von zwei Mentor/innenMentee-Paaren $(n=4)$ wurden im Rahmen einer qualitativ angelegten Untersuchung Fragen zur Mentor/in-Mentee-Beziehung gestellt. Die Daten wurden mittels Grounded Theory ausgewertet. Alle befragten Lehrpersonen sind im MET (Mentoring for Effecti- 
ve Teaching) Programm involviert. In Schulungen wurden Mentor/innen mit Themen zu Persönlichkeitsaspekten, systemischen Anforderungen, Pädagogischem Wissen, zur Rollenfunktion und Feedbackqualität auf die Aufgabe vorbereitet (Hudson 2013, 6f).

Zentrale Ergebnisse: Hudson (2013) stellt in einer grafischen Darstellung (Abbildung 11) die Phänomene und Kategorien vor. Die Phänomene Respekt (Respect) und Vertrauen (Trust) bilden „das Zentrum der Zwei-Weg-Interaktion“ (Hudson 2013, 16).

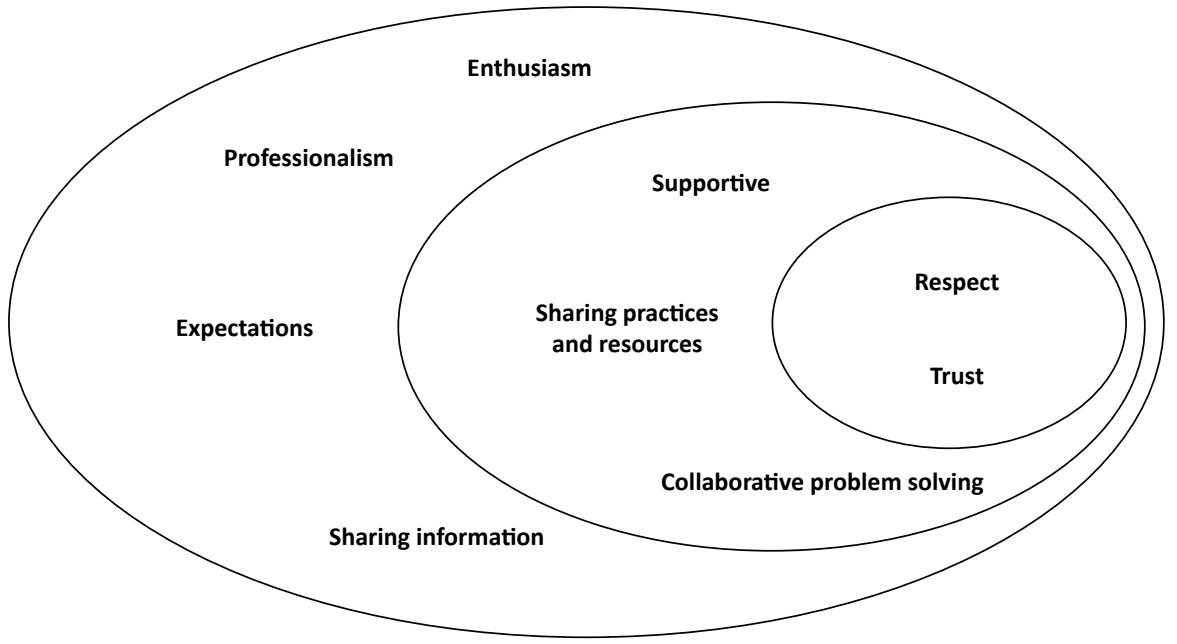

Abb. 11: Modell für Mentor/innen-Mentee-Beziehung (Hudson 2013, 17)

Sie sind die Basis für Kommunikation und den Umgang miteinander. Mentor/innen brauchen Vertrauen in Mentee, dass sie/er die Klasse führt. Mentee setzen Vertrauen in die Begleitperson, dass sie die Möglichkeit erhalten, sich weiterzuentwickeln. Ein wechselseitiger respektvoller Umgang ermöglicht eine konstruktive Zusammenarbeit. Die Kategorien Unterstützung, gemeinsame Herangehensweise zur Lösung von Problemen und der Austausch bilden die erste Untergruppe. Die weiteren Indikatoren einer erfolgreichen Mentor/in-Mentee-Beziehung sind Begeisterung und Professionalität von beiden, Klärung der Erwartungen in der Zusammenarbeit und das Teilen von Informationen.

Kernidee: Mentor/innen führen am Beginn des schulpraktischen Mentoringprozesses Gespräche über Rahmenbedingungen, klären Vorstellungen, Erwartungen und Vorlieben und betonen den Einsatz der persönlichen Komponenten zur Gestaltung einer guten Beziehung.

(4) Forschungsfragen: „Wie gestalten die schulischen Mentorinnen und Mentoren (sic!) die (unterrichtsbezogenen) Lerngelegenheiten in den Praxisphasen der Waldorf-Lehrpersonenausbildung? Inwiefern gibt es Zusammenhänge zwischen den personalen Merkmalen der Mentorinnen und Mentoren (sic!) und der Gestaltung unterrichtsbezogener Lerngelegenheiten in den Praxisphasen?" (Reintjes et al. 2018, 171)

Aufbau des Untersuchungsdesigns: Von den befragten 69 Mentor/innen wurden mittels Online-Fragebogen Aussagen zu den Personenmerkmalen, „zu Rahmenbedingungen des 
Mentorings und zur Gestaltung der Lerngelegenheiten" (Reintjes et al. 2018, 171) erhoben. Verschiedene statistische Verfahren (Deskription, t-Test, Korrelationen, Clusteranalyse) führten zu Befunden in Bezug auf Mentoring.

Zentrale Ergebnisse: Über drei Fünftel der Mentor/innen wurden für die Tätigkeit vorgeschlagen, der Rest hat sich beworben oder wurde von Studierenden angefragt. Die Skalen Beanspruchung, Stabilität, Selbstkontrolle, Kontaktbereitschaft, Selbstreflexion, Expert/ innentum und Selbstwirksamkeit sind stark ausgeprägt.
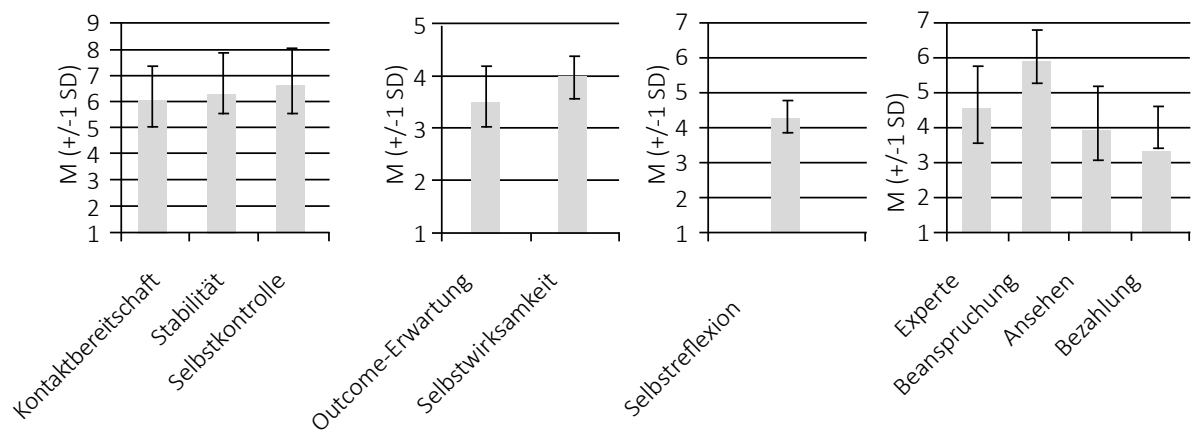

Abb. 12: Lehrpersönlichkeit der Mentor/innen, Selbstwirksamkeit als Mentor/in, Selbstreflexion, Vorstellungen der Mentor/innen über den Lehrberuf (Reintjes et al. 2018, 174)

Hingegen schätzen Mentor/innen die Outcome-Erwartung, das Ansehen und die Bezahlung als eher gering ein. Für drei Fünftel der Mentor/innen dauert die Vorbesprechung zwischen 10 bis 30 Minuten, während drei Viertel die Nachbesprechungszeit mit einer Dauer von 15 bis 45 Minuten angeben. Mit längerer Dauer der Vorbesprechung ergibt sich ein Zusammenhang zur Thematisierung von „Anregungen zur Materialsuche, methodischen Fragen und Zielsetzungen der Stunde." (Reintjes et al. 2018, 182) Unterschiede ergeben sich auch bei Mentor/innen mit unterschiedlich langen Erfahrungswerten. So weisen die Befunde der erfahrenen Mentor/innen einen Zusammenhang mit Kontaktbereitschaft, dem Besprechen von „fachwissenschaftlichen Aspekten, Zielsetzungen der Stunde und Lernvoraussetzungen der Lerngruppe“ (Reintjes et al. 2018, 183) auf.

Kernidee: Mentor/innen sollen selbst längere Zeit Berufserfahrung aufweisen. Inhalte von Vorbesprechungen könnten systematisiert werden, z.B. durch konkrete Planungsvorhaben. Zur Frage der Belastung durch Betreuungstätigkeit soll die Schulleitung miteinbezogen werden. Sie kann Koordinationsaufgaben übernehmen, z.B. durch Stundenplanregelungen, Klassenzusammensetzungen usw.

(5) Forschungsfragen: „Über welche Einstellungen zur Theorie- und Praxisorientierung des Lehramtsstudiums verfügen Mentorinnen und Mentoren (sic!) im Praxissemester? Wie schätzen Mentorinnen und Mentoren (sic!) die Intensität ihrer Betreuung von Studierenden im Praxissemester ein? Welchen Effekt haben die Einstellungen von Mentorinnen und Mentoren (sic!) auf die Betreuung von Studierenden im Praxissemester?" (Bach et al. 2018, 193f)

Aufbau des Untersuchungsdesigns: 223 Mentor/innen nahmen am Ende des zehnwöchigen Praktikums der Studierenden an der Studie teil. Das Instrument von Fischer et al. 
(2018) wurde zur Erhebung von Einstellungen herangezogen und das zweifaktorielle Modell mit der konfirmatorischen Faktorenanalyse ausgewertet (Bach et al. 2018, 196).

Zentrale Ergebnisse: Mentor/innen wenden zwei bis drei Stunden pro Woche für Studierende auf. Durchschnittlich beträgt die Nachbesprechungsdauer 35.49 Minuten, wenn Studierende unterrichten. Nach dem Unterricht von Mentor/innen beträgt die durchschnittliche Nachbesprechungszeit 14.10 Minuten. Mentor/innen stimmen „der Praxisorientierung des Lehramtsstudiums („Berufseinführende Professionalisierungsfunktion“; $\mathrm{M}=3.58, \mathrm{SD}=.52)$ signifikant stärker zu (...) als der Theorieorientierung des Lehramtsstudiums („Theoretisch-reflexive Professionalisierungsfunktion“, $\mathrm{M}=2.96, \mathrm{SD}=.60$; $\mathrm{t}(221)=11.34, \mathrm{p}<.001, \mathrm{~d}=1.11$ )“ (Bach et al. 2018, 197). Sie unterstützen auch häufiger in schulischen $(\mathrm{M}=3.09, \mathrm{SD}=.51)$ als in universitären $(\mathrm{M}=2.06, \mathrm{SD}=.61)$ Bereichen. Effekte zur Erklärung der Betreuungsqualität durch die Einstellungen zur Theorieorientierung liegen in Bezug auf schulische Unterstützung $(\beta=.22$; $S E=0.8)$ und Unterstützung bei universitären Leistungsanforderungen $(\beta=.33$; $S E=0.9)$ vor (Bach et al. 2018, 200).

Tab. 14: Unterstützung in schulischen und universitären Bereichen (Bach et al. 2018, 198) Anmerkung: Vierstufige Skala ( $1=$ nie bis $4=$ sehr oft)

\begin{tabular}{lccccc}
\hline Dimension „Schulische Unterstützung“ $(\alpha=.70)$ & n & M & SD & Min. & Max. \\
\hline Gemeinsame Reflexion des Unterrichts & 223 & 3.31 & .61 & 2 & 4 \\
Beratung in unterrichtlichen Belangen & 218 & 3.30 & .62 & 1 & 4 \\
Beratung im Hinblick auf die Berufseignung & 219 & 2.59 & .95 & 1 & 4 \\
Einbezug in schulische Angelegenheiten & 221 & 3.35 & .75 & 1 & 4 \\
Beratung zu der Beziehung zu den Schülerinnen und Schülern & 217 & 2.86 & .80 & 1 & 4 \\
Gesamt „Schulische Unterstützung“ & 223 & 3.09 & .51 & 1.60 & 4 \\
\hline
\end{tabular}

Dimension „Unterstützung bei universitären

Leistungsanforderungen" $(\alpha=.63)$

\begin{tabular}{llllll}
\hline Hilfestellungen bei der Anfertigung von Unterrichtsentwürfen & 215 & 2.62 & .79 & 1 & 4 \\
Beratung bei der Forschungsaufgabe & 217 & 1.99 & .82 & 1 & 4 \\
Beratung beim Portfolio & 217 & 1.57 & .67 & 1 & 4 \\
Gesamt „Unterstützung universitärer Leistungsanforderungen“ & 223 & 2.06 & .61 & 1 & 4
\end{tabular}

Kernidee: Ausbildungsinstitutionen können Mentor/innen in Fragen der Beratung bei Forschungsaufgaben und beim Verfassen des Portfolios unterstützen. Mentor/innen setzen sich mit der Bedeutung dieser Aufgabenbereiche auseinander.

In den folgenden drei Forschungsdesigns wurden Mentee und Mentor/innen befragt und beide Perspektiven erhoben. Die Kernaussagen zeigen, dass Mentoring eine wechselseitige Bereicherung ist.

(6) Forschungsfrage: „Welchen Nutzen haben Mentoren (sic!) und Mentees von der Teilnahme?" (Pflaum 2017, 267)

Aufbau des Untersuchungsdesigns: Die Evaluationsstudie wurde an der LMU München durchgeführt. Dort gibt es seit 1996 ein Mentoringprogramm. Die Mentee wurden nach Abschluss des Studiums am Beginn des Berufseintritts von Mentor/innen begleitet. Zur 
Erhebung dienten Fragebögen, Dokumentationen im Forschungstagebuch des Autors und Interviews (Pflaum 2017, 136). Folgend ein Auszug der Studie:

Zentrale Ergebnisse: Der Lernprozess war in Bezug auf Inhalt, Methode und Ergebnisorientierung im Sinne des konstruktivistischen Lernens ausgelegt. Laut dieser Untersuchung (Abbildung 13) zeigt sich, dass Mentor/innen für sich einen allgemeinen Nutzen (91\%) erkennen und sich neue Karriereperspektiven eröffnen (78\%). Übertragen auf den schulischen Sektor ist wohl die neue Rolle der/des Mentorin/Mentors neben der Rolle der Lehrperson zu verstehen. Die Mentee profitieren vor allem im psychosozialen Bereich wie Persönlichkeitsentwicklung (79\%), mehr Selbstbewusstsein (79\%), Bestärkung (88\%) und erfolgreiche Hilfe (62\%). Auch sehen Mentee einen karrierebezogenen Nutzen durch allgemeine Profitierung (89\%), besseres Selbstmanagement (62\%) und neue Karriereperspektiven (80\%) (Pflaum 2017, 270f).

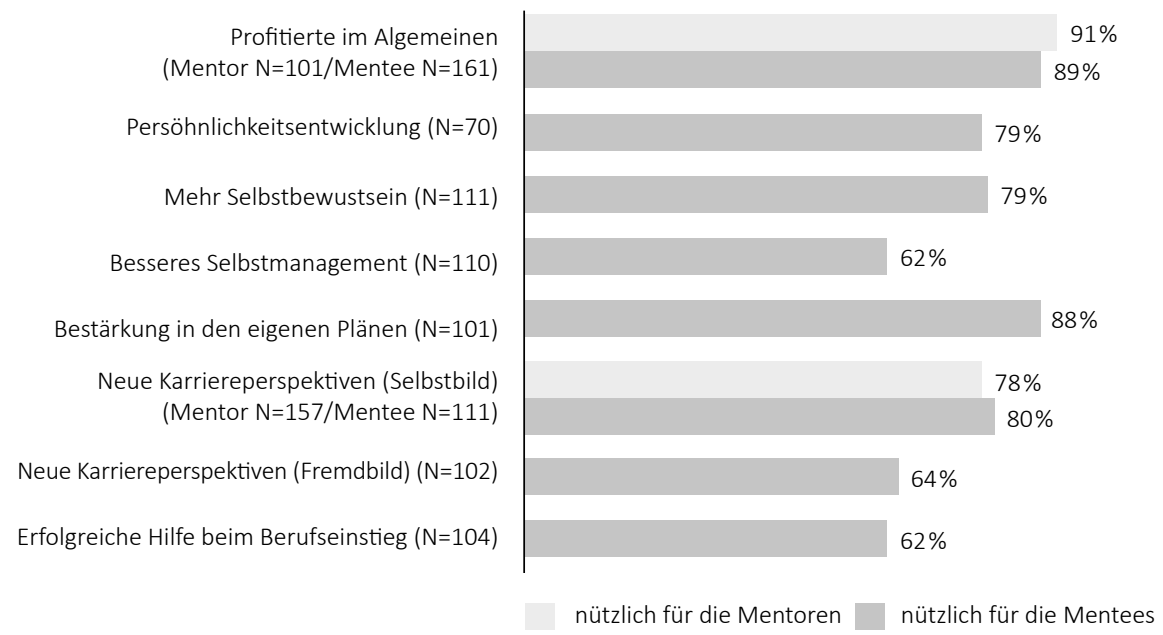

Abb. 13: Übersicht über Nutzen von Mentor/innen und Mentee (Pflaum 2017, 271)

Kernidee: Im Rahmen der Schulentwicklung werden professionelle Lerngemeinschaften bzw. schulpraktisches Mentoring als Bereicherung wahrgenommen und anerkannt sowie in Schulen durch den positiven Mehrwert institutionell etabliert.

(7) Forschungsfrage: Findet sich innerhalb einer Stichprobe von Mentor/innen „für die Selbsteinschätzung eine mit den Fremdeinschätzungsdaten der Studie von Brandau et al. (2017) vergleichbare Skalenstruktur“ (Brandau et al. 2018, 212)? Der Frage liegt das Ziel, „die Überprüfung der Konstruktvalidität der Selbsteinschätzungsversion des Mentoring-Inventars in einer Stichprobe von Mentorinnen und Mentoren (sic!)“ (Brandau et al. 2018, 212) zugrunde. Aufbau des Untersuchungsdesigns: Studie eins verfolgte eine mögliche Replikation der fünf Stildimensionen (Professionelle Unterstützung, Partnerschaftliche Kollegialität, Arbeits- und Lernebenen, Vertrauen, Direktivität) aufgrund von Fremdeinschätzungswerten durch Mentee auf die Selbsteinschätzungswerte der Mentor/innen. 206 Mentor/innen füllten einen Selbsteinschätzungsfragebogen (31 Items, sechsstufige Likert-Skala mit 1-6 ( 1 trifft nie zu/6 trifft immer zu)) aus. Studie zwei versuchte mittels ,explorativer Fakto- 
renanalyse zweiter Ordnung über die Items des Mentoring-Inventars“" (Brandau et al. 2018, 218) Aussagen zu treffen. 397 Studierende beantworten 35 Items (sechsstufige Likert-Skala mit 1-6 (1 trifft nie zu/6 trifft immer zu)) im Fremdeinschätzungsfragebogen.

Zentrale Ergebnisse:

Tab. 15: Zusammenhang der Ergebnisse der Multi-Item Multi-Trait-Analyse für das Mentoring-Inventar in der Stichprobe der Mentor/innen ( $\mathrm{N}=206)$ (Brandau et al. 2018, 217) Anmerkung: Der mögliche Wertebereich der Skalen beträgt $1=$ „trifft nie zu“ bis $6=$ „trifft immer zu“

\begin{tabular}{lccccc}
\hline Skala & $\begin{array}{c}\text { Anzahl } \\
\text { der Items }\end{array}$ & M & SD & $\begin{array}{c}\text { Cronbachs } \\
\text { Alpha }\end{array}$ & $\begin{array}{c}\text { Anzahl der } \\
\text { Fehler }\end{array}$ \\
\hline Professionelle Unterstützung & 10 & 5.31 & 0.53 & 0.83 & $3(7.5 \%)$ \\
Partnerschaftliche Kollegialität & 9 & 5.39 & 0.49 & 0.73 & $1(2.8 \%)$ \\
Arbeits- und Lernebenen & 6 & 5.20 & 0.63 & 0.74 & $3(12.5 \%)$ \\
Vertrauen & 3 & 4.34 & 0.83 & 0.62 & $0(0.0 \%)$ \\
Direktivität & 3 & 3.05 & 1.01 & 0.59 & $1(8.3 \%)$ \\
\hline
\end{tabular}

Nach der Überprüfung mittels Faktorenanalyse wurde die Konstruktvalidität/Konstruktstabilität des Mentorings-Inventars bestätigt. Brandau et al. (2018) sehen nur eine Ausweitung des Faktors „Vertrauen“ als notwendige Maßnahme bei einer Inventarrevision.

Tab. 16: Varimax-rotierte Faktorladungsmatrix der Faktorenanalyse zweiter Ordnung über die Dimension des Mentoring-Inventars (Brandau et al. 2018, 221)

\begin{tabular}{lccc}
\hline & Faktor 1 & Faktor 2 & Kommunalität \\
\hline Faktor 1: „Professionelle Unterstützung“ & 0.91 & 0.01 & 0.83 \\
Faktor 2: „Arbeits- und Lernebenen“ & 0.86 & 0.15 & 0.77 \\
Faktor 3: „Partnerschaftliche Kollegialität“ & 0.67 & 0.54 & 0.74 \\
Faktor 4: „Vertrauen“ & 0.31 & 0.72 & 0.61 \\
Faktor 5: „Direktivität“ & 0.01 & -0.88 & 0.77 \\
\hline
\end{tabular}

Vor allem die Dimensionen „Professionelle Unterstützung“, „Arbeits- und Lernebenen“ und „Partnerschaftliche Kollegialität“ bestimmen den Faktor 1 mit Blickrichtung auf einen „professionellen Arbeitsfaktor“ (Brandau et al. 2018, 221), „Vertrauen“ und „Direktivität“ den Faktor 2 mit Blickrichtung auf „sozial-emotionale Aspekte“ (Brandau et al. 2018, 221).

Kernidee: Im Mentoring sollen professions- und personenspezifische Unterstützungsmaßnahmen erfolgen und Inhalte dazu transparent kommuniziert werden.

(8) Forschungsfragen: „Do the SBTEs (school-based teacher educators) experience any personal ontological and conceptual shift in relation to both their own teaching and their new and wider role within teacher education as a consequence of working in a Third Space? Is there any evidence to suggest that Third-Space activity brings benefits and, if so, to whom? Does there appear to be a need for the involvement of a university perspective to guide the SBTEs and act as boundary broker? Is this involvement necessary initially and/or over time?" (Jackson et al. 2018, 6) 
Aufbau des Untersuchungsdesigns: Forscher/innen beschreiben Fallstudien von fünf Lehrpersonen und Mentee, in denen Unterricht nach dem Motto, was gelingt und was nicht gelingt, reflektiert wurde. Anschließend wurden Interviews geführt. Ziel war die Generierung einer Theorie ohne Allgemeingültigkeit aufgrund der beschränkten Samples (Jackson et al. 2018, 18f). Zentrale Ergebnisse: In erster Linie teilten Mentor/innen praktisches Wissen mit den Studierenden und gaben Antworten auf Fragestellungen der Mentee. Die Mentor/innen vermieden im Gespräch die Auseinandersetzung mit Theorien. Sie fühlten sich in der Identität sehr sicher. Die in den letzten fünf Monaten kennengelernten Theorien wurden aber von den/dem Mentor/innen in der Praxis verknüpft. Das weist auf eine neue Identität in der Begleitung hin. Der Boundary Broker motivierte die Lehrpersonen, sich Gedanken um die Weiterentwicklung der eigenen Praxis zu machen.

Kernidee: Die Ergebnisse schließen darauf, dass man in Ausbildungskonzepten zu Mentoring auch die Weiterentwicklung der Mentor/innen fördern soll. Weiters braucht es einen regelmäßigen Austausch zwischen Mentor/innen und Ausbildungsinstitutionen, damit Theorien verankert werden.

Die dargestellten Forschungsbefunde zeigen auf: Motivationsaspekte, Bedeutungsgehalt der Praktika, eine positive Beziehungsentwicklung sowie Gestaltung einer optimalen Lerngelegenheit und Lernumgebung sind wesentliche Faktoren im schulpraktischen Mentoring. Die eingangs zum Kapitel erwähnten Forschungsschwerpunkte zum schulpraktischen Mentoring mit (1) Wirkungsweise von schulpraktischem Mentoring oder lernwirksame Schulpraxis durch Mentoring, (2) prozessbegleitende Formate und (3) Erfolgsgenerierung können nach Literaturrecherche und Auflistung der exemplarisch angeführten Forschungsergebnisse mit (4) Motivlage und (5) Einstellungen der Mentor/innen erweitert werden. Die Einteilung von Forschungen im schulpraktischen Mentoring könnte auch nach der Dreiteilung menteebezogene, mentor/innenbezogene sowie die kombinierte Form der mentee- und mentor/innenbezogene Untersuchungen haben.

Jackson (2018) bringt durch seine Untersuchung den Gedanken in die Diskussion, dass Mentor/innen sich in einem Third-Space befinden und eine Zusammenarbeit mit der Ausbildungsinstitution bzw. eine vertiefende Ausbildung für Mentor/innen zur Identitätsbildung „Mentor/ in und Lehrer/innenausbildner/in" führen kann bzw. soll.

„Bei der Third-Space-Aktivität geht es im Wesentlichen darum, dass zwischen Universität und Schule eine intensive Zusammenarbeit erfolgen muss, da es durch die Ausbildungskooperation zu einer persönlichen und konzeptionellen Veränderung kommt. Studierende sollten mehr Einheit zwischen Theorie und Praxis erleben können.“ (Jackson et al. 2018, 26f).

Mentor/innen haben in ihrer Vermittlungsfunktion zwischen der Ausbildungsinstitution und der Schule somit einen weiteren Aufgabenbereich. Sie sind Teil der Lehrer/innenausbildner/innen und unterstützen Studierende im prozesshaften Voranschreiten innerhalb des Lehrberufs. An sie wird die Forderung nach einer Theorie-Praxis-Verschränkung herangetragen, was wiederum ihrerseits die Haltung einer/eines forschenden Lernerin/Lerners bedingt, und ausgelegt ist auf einen Mentoringprozess, der auf Erkenntnisgewinnung und wechselseitigem Lernen aufgebaut ist.

Nach den Forschungsschwerpunkten und -befunden sollen bei der Konzeption von MentoringProgrammen die fünf angeführten Forschungsschwerpunkte (1-5) inhaltlich miteinbezogen werden. Sie bilden die Basis und Grundlage.

Die angeführten Forschungsbefunde liefern auch Hinweise für mögliche weitere Fragehorizonte im schulpraktischen Mentoringprozess: zu Beziehungs- und Inhaltsaspekten. Beziehungsaspekte spielen im Mentoringprozess eine große Rolle. Die Identifizierung von unterschiedlichen 
Typen von Mentee, die Konstrukte Vertrauen und Respekt und die Motivlage der Mentor/ innen bedingen sich in ihrer Wechselwirkung. Beziehungsverhalten und Beziehungskultur bedürfen somit eines intensiven Reflexionsprozesses und im Forschungssupport der Klärung der Anwendung von Forschungsmethoden zur Erzielung von Ergebnissen. Der Inhaltsaspekt unterstreicht die Bedeutung der Ausbildung für Mentor/innen und betont das eigene konstruktivistische Lernen jeder/jedes Mentorin/Mentors. Auch diese Aspekte sollten bei der Konzeption von Mentoring-Programmen miteinbezogen werden. Es bleibt abzuwarten, wie sich die Themenforschungslandschaft rund um das schulpraktische Mentoring in den nächsten Jahren vor allem auch in Österreich - weiterentwickelt und nach welchen theoriebegründeten Ausbildungskonzepten schulpraktisches Mentoring angelegt wird.

Es lohnt sich im Folgenden, inhaltlich verstärkt in den schulpraktischen Mentoringprozess der Lehrer/innenausbildung einzutreten und historiografische, systemische und personalisierte Betrachtungsweisen zu erörtern.

\subsection{Mentoringprozess in der Lehrer/innenausbildung}

Praktika für Studierende, die durch Lehrer/innenbildner/innen und Mentor/innen begleitet werden, ,gelten sowohl aus lerntheoretischer als auch aus empirischer Sicht als für die Kompetenzentwicklung bedeutsame Lerngelegenheiten" (Reintjes et al. 2018, 8) in der Lehrer/innenausbildung. Studierende werden auf dem Weg zur Professionalisierung durch theoriegeleitete hochschulische Lehrveranstaltungen und schulische Anteile unterstützt, um das unterrichtspraktische Handeln und die Reflexionsfähigkeit zu fördern (Dehne et al. 2018, 110). Im schulischen Setting sind Lehrpersonen beauftragt, als Mentor/in zu fungieren. Diese/r gestaltet im Klassenzimmer ,weitgehend autonom die Lernumgebung, schafft Spielräume oder engt sie ein, leitet das Handeln an, steuert die Gespräche, gibt Feedbacks und bewertet." (Fraefel 2018, 42) Wie der Prozess (lateinisch „procedere“ vorwärts gehen) im Einzelnen abläuft bzw. gestaltet wird, hängt von verschiedenen Faktoren ab, die in weiteren Kapiteln diskutiert werden. Möglicherweise treffen subjektive Sichtweisen der/des Mentorin/Mentors auf subjektive Erwartungen und Vorstellungen der/des Mentee/Mentees und geraten in ein Spannungsfeld. Dazu kommen curriculare, formale und normative Vorgaben der Institutionen, die Bedingungen für die Prozessgestaltung schaffen. Es bedarf nun eines Zusammenspiels aller Beteiligten, um im Sinne von Hegel (17701831) zu einer „Bewegung“, in diesem Fall ausgerichtet auf die Professionalisierung zu kommen. Um ein Gesamtbild zur Thematik Mentoringprozess in der Lehrer/innenausbildung zu gewinnen, werden auch in den folgenden Kapiteln - wie im gesamten Verlauf der Arbeit - historiografische, systemische und personalisierte Sichtweisen herangezogen, dargestellt und interpretiert. Den Anfang in der Beschreibung bildet die historiografische Betrachtungsweise.

\subsubsection{Historiografische Betrachtung}

Die Ausgangslage der Lehrer/innenbildung kann in Österreich so wie auch in Deutschland (Bach 2013, 125) von der Praxis her angesehen werden. Wer Lehrer/in werden wollte, hatte eine Lehrzeit bei Ordensleuten, Handwerkern oder Studierenden und wurde vom Rat bestellt. Das pädagogische Rüstzeug erfolgte in einem Aneignungsprozess. Die „Meisterlehre“ mit dem Konzept des Vor- und Nachmachens von Fertigkeiten, des Übernehmens von Verhaltensmustern, des Einübens praktischer Handlungen, des Meisterns jeglicher Unterrichtssituationen, des spontanen Unterrichtens, des Erhaltens von Tipps und Ratschlägen prägte lange Zeit Teile der Ausbildung (Enzelberger 2001, 19f). Im weitesten Sinn kann man annehmen, dass dies das erste Mentoring im schulischen Kontext ist. 
Mit der Diskussion um die Qualitätssteigerung der Lehrer/innenausbildung ging und geht auch die Diskussion um die Schwerpunktsetzung der Studierenden für schulpraktische Lerngelegenheiten und damit die Frage der Begleitung durch Lehrpersonen einher. Sollten in der Ausbildung mehr wissenschaftliche oder mehr praktische Kompetenzen vermittelt werden? (Bäuerle $1989,5)$ Wie sollten Praxisanteile verlaufen? Welche Schwerpunkte könnten in der Schulpraxis gesetzt werden? Welchen Beitrag können Mentor/innen leisten? Welche Lehrpersonen übernehmen die Aufgabe der Begleitung und Betreuung? Das sind nur einige Fragen, die Ausbildner/innen beschäftigten.

In den fünführigen Lehrerbildungsanstalten (LBA) und an den Pädagogischen Akademien (Pädak) waren sogenannte Übungsschulen den Institutionen angeschlossen oder Praxisschulen standen im urbanen und ländlichen Raum zur Verfügung. Übungsschullehrpersonen wurden mit der Aufgabe der Studierendenbegleitung beauftragt. Aufgabenbereiche wurden von den jeweiligen Institutionen vorgegeben. Lechthaler (1966) macht in seinem Schreiben aus dem Jahr 1955 auf die Belastungssituation der Übungsschullehrpersonen (damalige Bezeichnung) aufmerksam: Neben den vielen zu unterrichtenden Schülerinnen und Schülern kommen viele zeitgleiche Hospitant/innen und viele Unterrichtspraktikant/innen, für die/den Unterrichtsthemen erstellt, Planungsentwürfe korrigiert und Zeit für die Absolvierung ihrer/seiner Probeauftritte zur Verfügung gestellt werden müssen. Vor- und Nachbesprechungen zu den Unterrichtsstunden, Führen von Protokollen und die Teilnahme an Konferenzen der Ausbildungsanstalt nehmen sehr viel Zeit in Anspruch (Lechthaler 1966, 337f). Seitens der Übungsschullehrperson ist die Betreuungstätigkeit mit viel Zeit und vielen Ressourcen verbunden. 1968 forderte die UNESCO (United Nations Educational, Scientific and Cultural Organization) in den Lehrer/innenausbildungsprogrammen einen engen Theorie-Praxis-Transfer und eine professionelle Begleitung der Studierenden. „Fundamentally, a teacher-preparation programme should include [...] practice in teaching and in conducting extra curricular activities under the guidance of fully qualified teachers." (UNESCO 1968, 13) Die Forderung nach qualifizierten Lehrpersonen als Mentor/innen blieb in Österreich lange Zeit ungehört.

In Österreich gab es in einigen Studienrichtungen - z.B. in der Religionspädagogik, Musikerziehung - schon in den 1980er-Jahren Ausbildungsprogramme für Mentor/innen (mit teils wechselnden Bezeichnungen). Insbesondere seit der Einführung des sogenannten Unterrichtspraktikums (UP) an den Allgemeinbildenden Höheren Schulen wurden an den damaligen Pädagogischen Instituten (PI) und Religionspädagogischen Instituten (RPI) spezifische Kurse und Fortbildungsveranstaltungen für sogenannte Betreuungslehrer/innen angeboten.

Im Pflichtschulbereich wurden als Besuchsschullehrpersonen (die gegenwärtige Bezeichnung für Übungsschullehrpersonen) weiterhin jene Lehrpersonen ausgewählt, die von der Schulinspektion als "gute" Lehrperson oder Lehrperson mit Fachkompetenz angesehen wurden (Teml 2002, 9). Eine spezielle Ausbildung war weiterhin noch nicht vorgesehen. Als Meilenstein der schulpraktischen Ausbildungstätigkeit gilt der „Erlass zu Aufgaben des Besuchsschullehrers (sic!) einer Pädagogischen Akademie im Rahmen der Schulpraktischen Ausbildung (Z. 17.154/36-31/1980)“ (Brenn et al. 1996, 186f) aus dem Jahre 1980 mit folgenden Forderungen:

1. „Vorführen/Demonstrieren von Unterricht bzw. von m.o.W. stark idealisierten Teilaspekten von Unterricht

2. Einführen in die Schul- und Klassensituation zu Beginn des jeweiligen Praxisabschnittes

3. Beraten bei der kurz- und mittelfristigen Unterrichtsplanung sowie Kontrollieren der schriftlichen Unterrichtsvorbereitung

4. Unterstützung der Studierenden bei der Beschaffung von Lehr- und Lernmitteln 
5. Beobachten und verantwortliches Überwachen der Lehrübungen

6. Rückmeldung geben bezüglich vereinbarter Kategorien und Kriterien

7. Beurteilen der Gesamtleistung der Studierenden während des ganzen Praxisabschnittes

8. Einweisen in die Führung von Amtsschriften sowie Vertrautmachen mit weiteren Aktivitäten (Aufgaben) des Lehrers (sic!)

9. Berücksichtigen und Unterstützen der besonderen, von der Pädagogischen Akademie gewählten Schwerpunkte bezüglich der Schulpraktischen Ausbildung

10. Teilnahme an Kontakt- und Fortbildungsveranstaltungen der Pädagogischen Akademie (ein- bis zweimal jährlich) sowie selbständiges und eigenverantwortliches Weiterbilden

11. Teilnehmen und Mitarbeiten an akademieinternen oder akademieübergreifenden Forschungsprojekten zur Weiterentwicklung von Didaktik und Unterrichtspraxis“" (Brenn et al. 1996, 186f)

„Dabei wird in den elf Überschriften zwar noch die vorgebende, kontrollierende und überwachende Funktion der AusbildnerInnen (sic!) deutlich sichtbar, es zeichnet sich aber bereits die Beratungsfunktion sowie die Orientierung an gemeinsam vereinbarten Kriterien der Unterrichtsgestaltung ab." (Teml 2002, 11) Ausbildungsinstitution und Schule entwickelten integrative Konzepte für Schulpraktische Studien, die ein Teil der Curricula der Pädagogischen Akademien waren. Von Praxisausbildnerinnen und Praxisausbildnern (gegenwärtige Bezeichnung) wurde erwartet, dass sie Prozesse leiten, die zum reflektierten Handeln und Bilden von subjektiven Theorien führen (Klement et al. 1996, 16; Brenn et al. 1996, 24f). Das Aufgabenfeld vergrößert sich für Praxisausbildner/innen und aufgrund der Verzahnung mit den Schulen auch für Praxisberater/innen, die an die Praxisschulen gingen und Unterricht der Studierenden beobachteten und reflektierten. Somit trat anstelle der Praxisbegleitung die Praxisberatung. Sie soll Studierende zur Eigenverantwortung und zur Selbständigkeit im Handeln anhalten. Als Merkmale werden verankert:

„Lern- und Entwicklungsprozesse von Lehrerstudenten (sic!) stehen im Mittelpunkt der Bemühungen. Praxisberater (sic!) und Ausbildungslehrer (sic!) stellen förderliche Lernsituationen für diese Lern- und Entwicklungsprozesse her. Beratung bedeutet eine Hilfestellung für Lern- und Entwicklungsprozesse." (Brenn et al. 1996, 2)

Ein weiterer Erlass aus dem Jahre 1998 zur „Qualifikation von AusbildungslehrerInnen“ (sic!) betonte die Notwendigkeit einer reflexiven, forschenden Haltung und einen Verweis der Bereitschaft zur persönlichen Weiterentwicklung im Sinne der Profession. An den Pädagogischen Hochschulen wurden nun auch Ausbildungs- und Fortbildungsformate für Praxisausbildungslehrer/innen geschaffen. Das Curriculum der Lehrgänge sah Praxisreflexion, beratungsorientierte Inhalte, Praxisforschung und erstmalig auch kollegiale Hospitationen im zeitlichen Ausmaß von zwei bis vier Semestern vor (Klement et al. 2002, 14). Es zeichnete sich ein Bild der Veränderung ab. Gewünscht waren Ausbildungslehrpersonen, die Studierenden einen handlungsorientierten Spielraum ermöglichen, Fragen zum Unterrichtsgeschehen und -handeln kritisch kommentieren und gemeinsam beantworten sowie lernförderliche Lernsituationen bereitstellen. Bis in die Mitte der zweiten Dekade des 21. Jahrhunderts wurden für Mentor/innen Qualifizierungsprogramme in Form von Praxislehrer/innen- und Betreuungslehrer/innenlehrgängen an den Pädagogischen Hochschulen im Ausmaß von 6 bis 10 ECTS-AP angeboten. Neben Reflexionseinheiten und kollegialen Hospitationen wurden strukturelle Rahmenbedingungen und Fragen zur Kommunikation und Konfliktbewältigung erörtert. Eine theoretische Fundierung und der Blick auf einen Forschungssupport waren allerdings nicht gegeben. 
$\mathrm{Zu}$ jener Zeit bildeten im internationalen Kontext Untersuchungsbefunde zur effektiven Qualifizierung von Mentor/innen die Grundlage für Ausbildungsformate. Die drei Modelle (1) "the knowledge transmission model“, (2) „the theory-and-practice connection model“ und (3) "the collaborative inquiry model“ (Wang et al. 2002, 525f) nach Wang et al. (2002) verfolgen hierbei unterschiedliche Annahmen und Theoriekonzepte. Vor allem im anglo-amerikanischen Raum fanden diese Modelle Beachtung und Umsetzung.

Um ca. 1990 wurde im Sinne des ausbildungsdidaktischen Ansatzes das „4-K-Modell“ entwickelt und vom Unterrichtsministerium den Pädagogischen Akademien empfohlen (Brenn 1991, Brenn et al. 1996; Klement et al. 1996; Klement et al. 2002; Teml 2011).

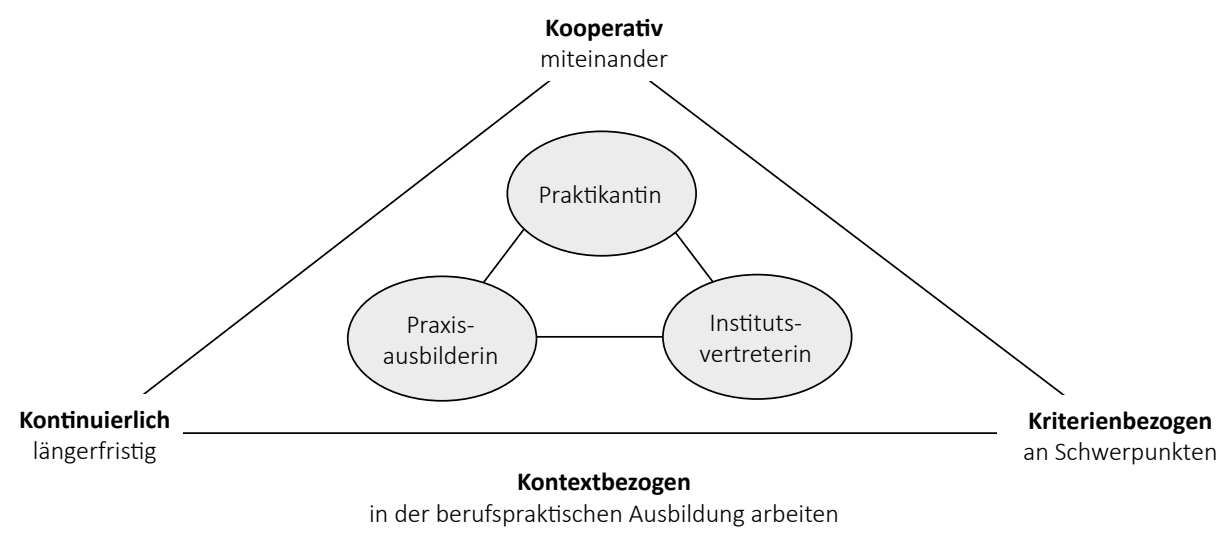

Abb. 14: 4-K-Modell der Praxisberatung (Teml et al. 2011, 51)

Die Elemente des Modells sind Kooperation, Kontinuität, Kriterien- und Kontextbezogenheit (Abbildung 14). Das Schulpraxisteam (Studierende/r, Institutionsvertreter/in, Praxisausbildner/in) gestaltet die Beratungseinheiten im Sinne der Elemente des Modells zu den Handlungsbereichen Vorbesprechen, Beobachten, Nachbesprechen, Beurteilen (Brenn et al. 1996, 89). In einer Ergänzung zum bestehenden Modell wurde in einer Revision noch das Element Kreativität (lateinisch „creare“ für schaffen, erzeugen, gestalten; verwandt auch das lateinische Wort „crescere“ für wachsen, gedeihen, entstehen (Hölzle 2011, 81)) hinzugefügt. Im Folgenden werden die Inhalte der Elemente kurz skizziert (Tabelle 17). Hierbei werden auch Empfehlungen für mögliche Inhalte zur Ausbildner/innenschulung erwähnt.

Tab. 17: Beschreibung der Elemente des 4 bzw. 5-K-Modells (Adaptiert nach Teml 2011, 51f; Brenn et al. 1996, 44f; Klement et al. 1996, 193f)

\begin{tabular}{lll}
\hline Elemente & Bedeutung für Schulpraxis & $\begin{array}{l}\text { Mögliche Inhalte für Ausbildner/ } \\
\text { innenschulung(en) }\end{array}$ \\
\hline Kooperation & Partnerschaftliche Beziehung & Rollenklärung \\
& Teamgedanke & Kooperation als Lernprozess \\
& Ko-Konstruktion & Aufgaben im Prozess \\
& Offenheit und Angstfreiheit & Gemeinsame Arbeitsstruktur \\
& & Partnerzentrierte (sic!) Gespräche \\
\hline
\end{tabular}




\begin{tabular}{|c|c|c|}
\hline Elemente & Bedeutung für Schulpraxis & $\begin{array}{l}\text { Mögliche Inhalte für Ausbildner/ } \\
\text { innenschulung(en) }\end{array}$ \\
\hline $\begin{array}{l}\text { Kriterien- } \\
\text { Bezogenheit }\end{array}$ & $\begin{array}{l}\text { Festlegung von Schwerpunkten } \\
\text { Auseinandersetzung mit Inhalten der } \\
\text { Schwerpunkte } \\
\text { Reflexion zur Schwerpunktsetzung }\end{array}$ & $\begin{array}{l}\text { Kriterienfindung } \\
\text { Entwicklung von Bausteinen } \\
\text { Kriterienbeschreibung } \\
\text { Ko-Konstruktiver Prozess }\end{array}$ \\
\hline Kontinuität & $\begin{array}{l}\text { Personelle, didaktische und organisatori- } \\
\text { sche längerfristige Auseinandersetzung mit } \\
\text { Schwerpunkten }\end{array}$ & $\begin{array}{l}\text { Weiterentwicklung der Schwerpunktsetzung } \\
\text { und Schulpraxis } \\
\text { Team(weiter)entwicklung } \\
\text { Bedachtnahme auch des schulischen Curri- } \\
\text { culums }\end{array}$ \\
\hline $\begin{array}{l}\text { Kontext- } \\
\text { Bezogenheit }\end{array}$ & $\begin{array}{l}\text { Beachtung der Schulpraxisanforderungen } \\
\text { und des individuellen Ausbildungsstandes } \\
\text { Bereitstellung angemessener Lernsituationen } \\
\text { in Klassen und während des Studienverlaufes }\end{array}$ & $\begin{array}{l}\text { Förderliche Beratungsgespräche } \\
\text { Klasse als Ort professionellen Lernens }\end{array}$ \\
\hline Kreativität & $\begin{array}{l}\text { Ganzheitlicher Prozess der Neugestaltung } \\
\text { Sich-Einlassen auf neue Lernsituationen } \\
\text { Überprüfen von Hypothesen }\end{array}$ & $\begin{array}{l}\text { Forschende Haltung } \\
\text { Forschende Gemeinschaft } \\
\text { Kreativitätszirkel (Denken, Fühlen, Intuition, } \\
\text { Empfinden) }\end{array}$ \\
\hline
\end{tabular}

$\mathrm{Zu}$ den vier Elementen wurde von den Autor/innen ein Fragenkatalog zu den Handlungsbereichen (Vorbesprechen, Beobachten, Nachbesprechen, Beurteilen) für das Schulpraxisteam entwickelt. Exemplarisch wird der Handlungsbereich Beobachten (Tabelle 18) angeführt. Beobachten ist nicht nur ein wesentlicher Aufgabenbereiche der Mentor/innen im Begleitungsprozess. Auch Studierende sollten durch Beobachten lernen, Sachverhalte im Unterrichtsgeschehen wahrzunehmen, zu erkennen und Unterrichtsgeschehen adäquat optimal auf Bedürfnisse abzustimmen.

Tab. 18: Fragestellungen zur Analysemöglichkeit zum Handlungsbereich Beobachten (Brenn et al. 1996, 125)

\begin{tabular}{|c|c|c|c|}
\hline $\begin{array}{l}\text { Beobach- } \\
\text { ten }\end{array}$ & $\begin{array}{l}\text { Was? } \\
\text { Wozu? } \\
\text { Didaktische Analyse } \\
\text { und Lernziele }\end{array}$ & $\begin{array}{l}\text { Wie? } \\
\text { Womit? } \\
\text { Methoden und Medien }\end{array}$ & $\begin{array}{l}\text { Wo? } \\
\text { Wohin? } \\
\text { Lernkontrollen und } \\
\text { Konsequenzen }\end{array}$ \\
\hline $\begin{array}{l}\text { Koopera- } \\
\text { tion }\end{array}$ & $\begin{array}{l}\text { Z.B.: Wurden die Beobachtungs- } \\
\text { schwerpunkte gemeinsam mit } \\
\text { den Studierenden ausgehandelt? }\end{array}$ & $\begin{array}{l}\text { Z.B.: Wurde gemeinsam } \\
\text { ein Beobachtungsverfahren } \\
\text { erstellt? }\end{array}$ & $\begin{array}{l}\text { Z.B.: Werden die Ergebnisse } \\
\text { der Beobachtung gemeinsam } \\
\text { ausgewertet? }\end{array}$ \\
\hline Kontinuität & $\begin{array}{l}\text { Z.B.: Werden gewählte } \\
\text { Schwerpunkte für einen länge- } \\
\text { ren Zeitraum ins Auge gefaßt } \\
\text { (sic!)? }\end{array}$ & $\begin{array}{l}\text { Z.B.: Ermöglichen die verwen- } \\
\text { deten Beobachtungsbehelfe } \\
\text { eine längerfristige kontinuierli- } \\
\text { che Beobachtung? }\end{array}$ & $\begin{array}{l}\text { Z.B.: Lassen sich im Verlaufe } \\
\text { eines bestimmten Zeitrahmens } \\
\text { Veränderungen, Lernfortschrit- } \\
\text { te feststellen? }\end{array}$ \\
\hline $\begin{array}{l}\text { Kriterien- } \\
\text { Bezogen- } \\
\text { heit }\end{array}$ & $\begin{array}{l}\text { Z.B.: Werden für Beobach- } \\
\text { tungsschwerpunkte konkrete } \\
\text { Kriterien erarbeitet? }\end{array}$ & $\begin{array}{l}\text { Z.B.: Ermöglicht das verwende- } \\
\text { te Beobachtungsverfahren ein } \\
\text { kriterienbezogenes Beobachten? }\end{array}$ & $\begin{array}{l}\text { Z.B.: Erlauben die vereinbarten } \\
\text { Kriterien das Festlegen von } \\
\text { begründeten Konsequenzen? }\end{array}$ \\
\hline $\begin{array}{l}\text { Kontext- } \\
\text { Bezogen- } \\
\text { heit }\end{array}$ & $\begin{array}{l}\text { Z.B.: Stehen die gewählten } \\
\text { Beobachtungskriterien und } \\
\text {-formen im Kontext des Aus- } \\
\text { bildungsgangs? }\end{array}$ & $\begin{array}{l}\text { Z.B.: Läßt (sic!) sich das einge- } \\
\text { setzte Beobachtungsverfahren } \\
\text { im Kontext begründen? }\end{array}$ & $\begin{array}{l}\text { Z.B.: Welche Folgerungen oder } \\
\text { Auswirkungen ergeben sich } \\
\text { für die konkrete Unterrichtssi- } \\
\text { tuation? }\end{array}$ \\
\hline
\end{tabular}


Die angeführten Fragestellungen zum Handlungsbereich Beobachten im Rahmen des 4-KModells lenken auf einen auf Austausch basierenden gemeinsamen intensiven Reflexionsprozess hin. Die Dreiteilung mit didaktischer Analyse, Umsetzung und letzlich Lernkontrolle lässt vermuten, dass hierbei auf den unmittelbaren Entwicklungsprozess im engeren Sinn Bezug genommen wird.

Wie die Autor/innen selbst kritisch anmerken, wurden die Empfehlungen der Inhalte zur Ausbildung und Schulpraxis „nur“ in Buchform dokumentiert und festgehalten. Der Weg zur konkreten Umsetzung des 4 bzw. 5-K-Modells in die Ausbildungsschiene ist am Ende des 20. Jahrhunderts dann im Sande verlaufen (Klement et al. 1996, 194).

In Österreich gestaltet sich gerade seit Pädagog/innenbildung NEU (2015/16) ein Paradigmenwechsel sowohl in den Ausbildungsinstitutionen Universität und Pädagogische Hochschule und im Weiteren auch im System Schule. Die Forderung nach einer gezielten Ausbildung zur Ausübung der Tätigkeit als Mentor/in wird immer wieder in der Literatur (Stöger et al. 2009, 325) diskutiert. Seitens des Bildungsministeriums gibt es für die Konzeption der Mentor/innenhochschullehrgänge (15, 30 bzw. 90 ECTS-AP) an den Pädagogischen Hochschulen - gewünscht in Kooperation mit Universitäten - strukturelle Vorgaben (Abbildung 15) und seit Jänner 2019 auch inhaltliche Empfehlungen (Tabelle 19).

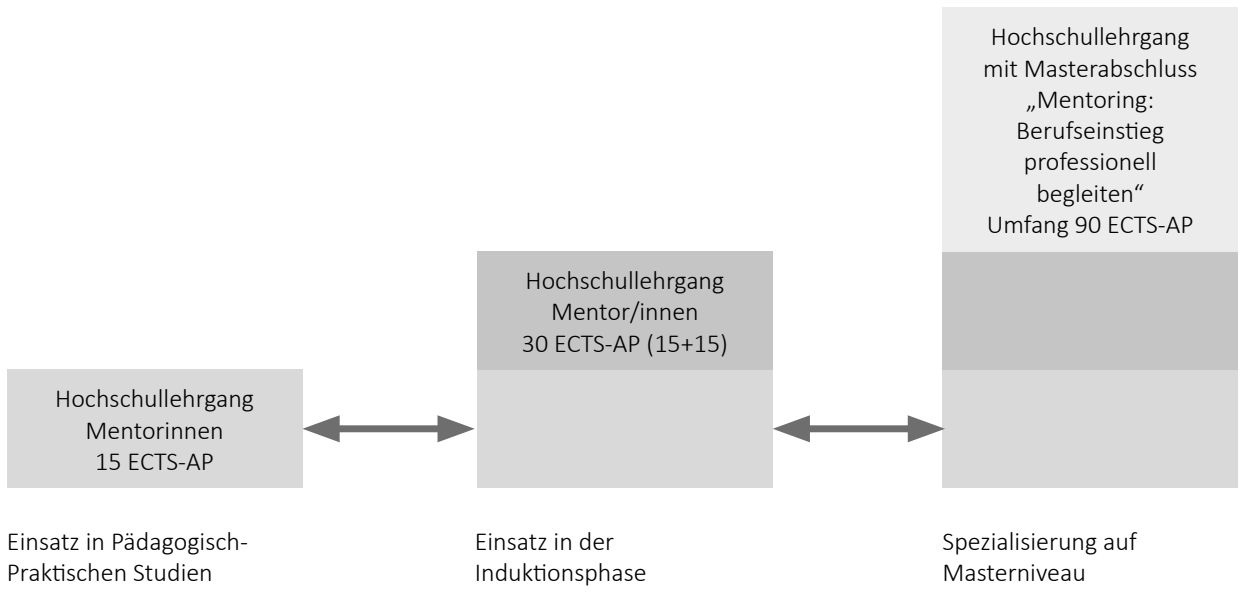

Abb. 15: Studienarchitektur zum Hochschullehrgang für Mentor/innen (BMBWF 2019)

Der Hochschullehrgang qualifiziert Lehrpersonen für die Begleitung von Studierenden in den Pädagogisch-Praktischen Studien im Kontext der Ausbildung (15 ECTS-AP) und er qualifiziert Lehrpersonen für die Begleitung von Vertragslehrpersonen in der Induktionsphase $(15+15$ ECTS-AP). Eine weitere Möglichkeit zur inhaltlichen Vertiefung ist durch die Absolvierung des Hochschullehrganges mit Masterabschluss „Mentoring: Berufseinstieg professionell begleiten“ $(15+15+60$ ECTS-AP) gegeben.

„Das Studienangebot basiert auf einer ausbalancierten Mischung aus forschungs- und theoriebasierter Wissensvermittlung, gemeinsamer Analyse- und Theoriebildung, praktischen Übungen und Arbeiten an Fallbeispielen aus der eigenen Praxis. Darüber hinaus soll es Phasen der Erprobung und der reflektierten Weiterentwicklung eigener Handlungsmöglichkeiten in Praxissituationen sowie Phasen teamorien- 
tierten Lernens geben. Der Praxis- und Forschungsbezug ist in allen Kompetenzbereichen herzustellen. (Empfehlungsschreiben BMBWF 2019)

In den Entwicklungsverbünden sind bzw. werden unterschiedliche Programme ausgearbeitet und den Lehrpersonen mit mindestens 5-jähriger Berufserfahrung angeboten. Die inhaltliche Empfehlung seitens des Bildungsministeriums beruht auf einer Tagung zum Thema „Mentoring in Österreich" am 18. Jänner 2019 in Salzburg. In einer Expert/innengruppe wurden Kriterien diskutiert. Anbei die empfohlenen Kompetenz- und Inhaltsbereiche (Tabelle 19):

Tab. 19: Empfehlung für Kompetenz- und Inhaltsbereiche zum Hochschullehrgang Mentor/ innen (BMBWF 2019)

\begin{tabular}{lll}
\hline & 15 & 15 \\
& ECTS-AP & ECTS-AP \\
\hline Professionsverständnis für Lehrer/innen/Rolle von Mentor/innen & $2-5$ & $2-5$ \\
\hline Kommunikation und Interaktion & $2-5$ & $2-5$ \\
\hline Begleiten und Beraten & $2-5$ & $2-5$ \\
\hline $\begin{array}{l}\text { Lehren und Lernen in einem Schwerpunktbereich } \\
\begin{array}{l}\text { Wahlpflichtbereiche mit Fokus auf die Schulformen bzw. Primar- oder Sekun- } \\
\text { darbereich sowie Fachbereiche und in Fachgruppen in der Berufspädagogik }\end{array}\end{array}$ & $2-5$ \\
\hline
\end{tabular}

Die viersemestrige Ausbildung sollte berufsbegleitend absolviert werden können. Neben den Präsenzanteilen sollten auch berufsbegleitende Studienanteile und Selbststudienanteile enthalten sein (BMBWF 2019). Wie die konkrete Umsetzung aussieht, obliegt den Verantwortlichen in den Verbünden bzw. den Pädagogischen Hochschulen mit Partneruniversitäten.

Der Blick in die Geschichte zeigt einerseits auf, dass schulpraktisches Mentoring mit der Diskussion um die Ausbildung der Mentor/innen einhergeht. Mentoring wird als Möglichkeit gesehen, die Lern- und Entwicklungsprozesse (Brenn et al. 1996, 2) der Studierenden zu fördern. Dabei wird die Notwendigkeit einer professionellen Beratung und Begleitung als essenziell erachtet. In diesem Bewusstsein stellt sich die Frage nach der Auswahl geeigneter Mentor/innen. Gewünscht wurden Lehrpersonen, die Erprobungsräume zur Verfügung stellen und in einen Reflexionsprozess treten. Die Forderung nach einer fundierten Ausbildung wurde lange Zeit in Österreich vernachlässigt. Nach ersten Ausbildungsprogrammen für Mentor/innen ab den 1980er-Jahren gab es ab 2010 spezielle Qualifizierungsprogramme im Rahmen von Lehrgängen im Ausmaß von 6 bis 10 ECTS-Anrechungspunkten. Der Ausbildung der Mentor/innen wurde zusehends eine größere Bedeutung beigemessen.

Eine vertiefende inhaltliche Auseinandersetzung mit unterschiedlichen Schwerpunkten und Reflexionsanteilen sowie einer forschungsgeleiteten Lehre erhofft man sich auch in der gegenwärtigen Mentor/innenausbildung, für die eine Konzeptionsempfehlung für Curricula seitens des Bildungsministeriums seit Jänner 2019 vorliegt. Die Absolvierung wird als ein Mehrwert in der Begleitung und Unterstützung von Studierenden und nun auch von Junglehrer/innen in der Induktionsphase auf dem Weg der Professionalisierung gesehen.

Der Blick in die Geschichte veranschaulicht andererseits auch, dass allgemeine theorie- und forschungsgeleitete Konzepte zur Praxisgestaltung Entwicklungspotential haben. Zu sehr prägen noch gewachsene Strukturen, institutionelle Vorgaben und persönliche Vorlieben das Handeln der beteiligten Personen im schulpraktischen Mentoring. 
Inhaltliche Standardisierungen im schulpraktischen Ausbildungsformat des Lehramtsstudiums fehlen in Österreich. Dadurch gibt es in den Verbünden unterschiedliche Umsetzungen der Pädagogisch-Praktischen Studien und unterschiedliche Anforderungsprofile für Studierende und im Weiteren für die Begleitung der Mentor/innen im Mentoringprozess. Formale Standardisierungen fehlen auch für Qualifizierungsprogramme zum Mentoring. An den unterschiedlichen Institutionen werden zwar die Empfehlungen (BMBWF 2019) eingearbeitet, die Programme in den einzelnen Verbünden sind dennoch sehr divergierend.

Das folgende Kapitel und das angeführte Mentoring-Modell verdeutlichen eine allgemeine Einbettung des schulpraktischen Mentoringprozesses im Rahmen personaler und systemischer Bedeutungsträger.

\subsubsection{Kontextuelle Einbettung schulpraktischen Mentorings}

Die Einbettung des schulpraktischen Mentorings bedingt eine Analyse des Kontextes des Mentoringprozesses. Der schulpraktische Mentoringprozess im Rahmen der Ausbildung findet unter besonderen personellen, institutionellen und formalen Bedingungen statt, wie in der Abbildung (Abbildung 16) grafisch zum Ausdruck gebracht wird. Die Abbildung verdeutlicht die Rahmung, in dem der schulpraktische Mentoringprozess eingebettet ist. Im Zentrum sind die Akteurinnen/ Akteure: die/der Mentor/in und die/der Mentee. Sie bringen unterschiedliche Lebenserfahrungen und berufsbiografische Entwicklungen mit und treten in eine Wechselbeziehung. Die Institutionalisierung des Mentorings hat sich im System Lehrer/innenausbildung und Schule etabliert. Hierbei spielen Ansätze der formalen Bildung sowie historiografische Zugänge oftmals eine nicht zu unterschätzende Rolle. Die einzelnen Bereiche werden nun detailliert analysiert (Abbildung 16):

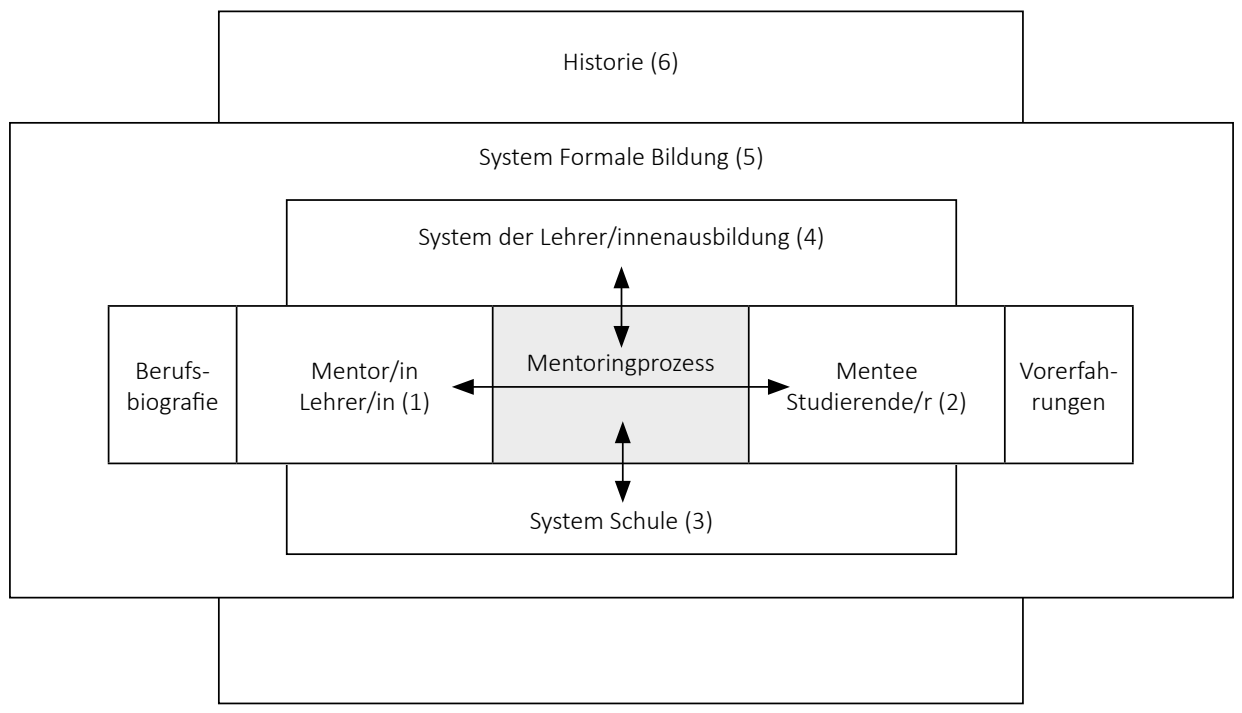

Abb. 16: Mentoring-Modell unter Einbindung der Personen und Systeme (Adaptiert nach Oettler 2009, 36)

Die Beschreibung der Bereiche wird in der folgenden Reihenfolge vorgenommen:

(1) Mentor/in bzw. Lehrperson mit Berufsbiografie

(2) Mentee bzw. Studierende/r mit Vorerfahrungen 
(3) System Schule

(4) System Lehrer/innenausbildung

(5) System Formale Bildung

(6) Historie

(1) Mentor/in bzw. Lehrperson mit Berufsbiografie: Die/Der Mentor/in ist bei der Ausübung der Begleitungstätigkeit mit Systemen und intra- und interpersonellen Perspektiven konfrontiert. Oettler (2009) spricht von „einem dreifachen Abhängigkeitsverhältnis“ (Oettler 2009, 35) (Schule, Ausbildungsuniversität, Mentoring-Beziehung). Die Autorin der vorliegenden Arbeit erweitert dies und spricht in diesem Zusammenhang von einer sechsfachen Perspektivenbetrachtung: bezogen auf eigene Wertevorstellung mit der eigenen Berufsbiografie, Perspektive auf die/den andere/n, Einbindung in die Systeme Schule sowie Lehrer/innenausbildung bzw. das System Formale Bildung und die historische Sichtweise von Lehrer/innenausbildung, Schule und Mentoring. Im Mentoringprozess richtet die/der Mentor/in den Blick auf sich selbst und auf das zu begleitende/das anvertraute Gegenüber vor dem Hintergrund institutioneller Systeme mit Berücksichtigung des historischen Kontextes.

„Die Besonderheit des systemischen Blickes besteht darin, dass die Persönlichkeit eines Menschen sehr stark aus den Wechselwirkungen mit ihrer Umgebung hergeleitet wird. Verhalten ist sowohl eine Reaktion auf ein Verhalten einer anderen Person als auch Ursache von neuen Verhaltensweisen. Und genau darin liegt auch die Chance auf Veränderung: mit einer neuen Verhaltensweise einer Person beginnt automatisch das System sich zu verändern." (Faix et al. 2017, 16)

Der/Dem Mentor/in wird im Mentoringprozess eine tragende Rolle zugeschrieben. Sie/Er verfügt über mehrere Jahre Berufserfahrung und kann auf ein Repertoire unterschiedlicher pädagogischer Handlungsmuster zurückgreifen, das sie/er sich in der Grundausbildung, im schulischen Alltag, durch verschiedene formale, informale (z.B. Fortbildungsveranstaltungen, Schulveranstaltungen) und nonformale (z.B. Internetrecherche) Lerngelegenheiten in einem Entwicklungsprozess aufgebaut hat (Richter 2011; Cramer 2012, 34).

Die Berufsbiografie der Lehrperson ist individuell verlaufen und im Idealfall wurden/werden normative Elemente (Strukturtheoretischer Ansatz) und empirische Elemente (Kompetenzorientierter Ansatz) miteinander verbunden. „Professionalität entsteht dann in der wissens- aber auch erfahrungsgestützten produktiven Verarbeitung beruflichen Erlebens.“ (Cramer 2012, 34) Durch die berufsbiografische Prägung im Rahmen der Professionsentwicklung wird vermutet, dass bestimmte Dimensionen (Classroom-Management, Umgang mit Konflikten, Haltung zu lernpsychologischen Ansätzen, Sozialisation der Lehrperson in der Schule, Fortbildungswille der Lehrperson usw.) auch im Mentoringprozess eine Rolle spielen. Untersuchungen dazu bilden aber ein Desideratum.

(2) Mentee bzw. Studierende/r mit Vorerfahrungen: Die/Der Mentee steht in Ausbildung zur/zum Lehrer/in. Je nach Studienarchitektur sind unterschiedliche lange und intensive Praxisphasen im schulischen Setting vorgesehen. Studierende erhalten Anforderungskataloge seitens des Systems Lehrer/innenausbildung, in denen die Anzahl der auszuübenden Tätigkeiten und Aufgabenstellungen jedes Praktikums aufgelistet sind. Zu den Aufgaben zählen Hospitationen, Vor- und Nachbesprechungen, Unterrichtstätigkeiten, Forschungsaktivitäten, Begleitung schulbezogener Veranstaltungen, Reflexionsphasen, Projektleitungen usw. in unterschiedlichem Stunden- und ECTS-AP-Ausmaß. Die/Der Mentee erlebt Schulalltag und gestaltet Lerngelegenheiten in Begleitung. Der Mentoringprozess gestaltet sich nun für die/den Mentee in 
Abhängigkeit zu Institutionen und zur/zum Lernbegleiter/in, und im Optimalfall erhält sie/er die Möglichkeit, „Kompetenzen selbstorganisiert, in einem kommunikativen Prozess mit Lernpartnern (sic!) (Netzwerk), aufzubauen." (Erpenbeck et al. 2015, 20) Wie bereits erwähnt, hat $\mathrm{die} /$ der Mentee im Laufe der eigenen Schulzeit pädagogische Vorerfahrungen gesammelt, die zu gewissen Vorstellungen, Erwartungen und Haltungen führen (Kraler 2009). Im Mentoringprozess wird ein Aufbrechen der bereits „,vorkonstruierten“ Bilder zu Schule und Unterrichtsettings nötig sein, um Entwicklung zu ermöglichen.

(3) System Schule: Im schulischen Umfeld (System Schule) erfolgt die institutionalisierte Verankerung des Mentoringprozesses. Hierbei nehmen die unterschiedlichen Ebenen des Systems Schule (Mikro-, Meso-, Exo-, Makro-, Chronosystem nach Bronfenbrenner) Einfluss auf die Gestaltung des Mentoringprozesses und der Mentoring-Beziehung. Jede Schule hat ihr eigenes Charakteristikum, geprägt durch Größe, Gesamtschüler/innenzahl, Einzugsgebiet, Lage, Gestalt, Architektur, Organisationsform, Bildungsauftrag, Bildungsverständnis usw. (Beyer et al. 2006, 21). Seitens der Schulleitung und Verwaltungsebene sollen Anstrengungen unternommen werden, Mentor/innen optimale Rahmenbedingungen an den Schulen zur Verfügung zu stellen. Die/Der Mentee nimmt im System Schule auch die Besonderheiten gelebter Schulkultur wahr. Hierbei kann Mentoring „helfen, Räume für gegenseitiges Vertrauen, Verständnis und Förderung zu schaffen bzw. zu kultivieren. Somit bietet es eine Form an, durch die vorhandene Bedürfnisse zum Ausdruck gebracht werden können.“ (Schmid et al. 2011, 30) Mentoring kann z.B. durch verstärkte Aktivitäten bzw. durch Zusammenarbeit mit der Hochschule als Systempartner einen Beitrag zur Kulturentwicklung (Schmid et al. 2011,29) im System Schule liefern. „Aus diesem Grund ist von einer „win-win-win-“ Situation zu sprechen (...), da zusätzlich neben dem Mentoringpaar die Organisation erhebliche Vorteile (...) zieht." (Oettler 2009, 31)

(4) System Lehrer/innenausbildung: Mit Pädagog/innenbildung NEU und damit der Neukonzeption der Lehrer/innenausbildung in Österreich rückt das simultane Studienmodell (Eurydice 2002), das Integration von Theorie und Praxis und damit einhergehend Vernetzung von hochschulischen und schulischen Studienanteilen, Theorie-Praxis-Transfer und Aufwertung der Pädagogisch-Praktischen Studien im Ausbildungskonzept zum Ziel hat, in den Vordergrund. Erkenntnisse der Fachwissenschaften, Fachdidaktiken und Bildungswissenschaften sowie Fragen zum Theorieverständnis, zur Möglichkeit der Verortung von Praktika in Schulen, zum Gestaltungs- und Reflexionsprozess prägen und gestalten den Mentoringprozess auf inhaltlicher, organisatorischer, methodisch-didaktischer und schließlich personeller Ebene. Lehrer/innenausbildung erfolgt auf Hochschulebene an den Universitäten oder Pädagogischen Hochschulen. Art und Dauer der Praktika sind in Österreich regional und strukturell sehr unterschiedlich. Im Entwicklungsverbund Lehrer/innenbildung WEST werden Schulpraktika und Fachpraktika der Sekundarstufe Allgemeinbildung vom Praktikumsbüro aus organisiert, das eigens mit einer Leitungsfunktion eingerichtet wurde. Für die Primarstufe sind an den Pädagogischen Hochschulen Institute oder Departments eingerichtet worden, die jegliche Organisations- und Kooperationsaufgaben mit Mentor/innen und Studierenden übernehmen. Die strukturelle Verankerung von Mentoring liegt der Lehrer/innenausbildung zugrunde.

(5) System Formale Bildung: Das Schulsystem ist in Primarstufe, Sekundarstufe, postsekundäre und tertiäre Ausbildung untergliedert. Das österreichische Bildungssystem wird auf Bundesebene durch das Bundesministerium für Bildung, Wissenschaft und Forschung gesteuert. $\mathrm{Zu}$ den wesentlichen Aufgaben im schulischen Bereich zählen Schulgesetzgebung, Verwaltung des Bildungsapparates und Gehälter der Bundeslehrpersonen (AHS/BMHS). Auf Landesebene regeln die neuerrichteten Bildungsdirektionen (1. Jänner 2019) schulstrukturelle Maßnahmen 
und Kosten der Lehrpersonengehälter der Pflichtschulen. Innerhalb der Landesebene gibt es Bildungsregionen, denen Schulqualitätsmanager/innen zugeordnet sind. Sie beraten Lehrpersonen in Fragen der Schul- und Unterrichtsentwicklung und helfen bei der Erstellung eines Schulentwicklungsplans. Auf Gemeindeebene liegt die Zuständigkeit der räumlichen, technischen und gegenständlichen Ausstattung. Zur institutionellen Ebene Schule mit einer/einem Schulleiter/in an der Spitze gehören Lehrpersonen, Eltern und Schüler/innen und gegebenenfalls Verwaltungspersonal. Das österreichische Ausbildungs- und Bildungswesen sieht Mentoring sowohl in der Ausbildung als auch in der Induktionsphase verankert. Der Bildungsrahmen wird vom europäischen Hochschulraum geprägt (Terhart 2007, 62).

(6) Historie: Viele Phänomene der Systeme Schule, Lehrer/innenausbildung, Formale Bildung und Mentoring können aus der Historie begründet werden. Es „,ist das Resultat politischer, gesellschaftlicher, kultureller und theologisch-philosophischer sowie wissenschaftlicher Entwicklungen." (Beyer et al. 2006, 22)

Zusammenfassend kann gesagt werden: Schulpraktisches Mentoring versteht sich als ein Prozess, der in Systeme zwischen Personen, Kontexten und Institutionen mit geschichtlichem Hintergrund eingebunden ist und sich in einem Zusammenspiel bzw. Wechselspiel innerhalb eines Tätigkeitsrahmens vollzieht. Ittel et al. (2009) sind der Meinung, dass

„eine Ausweitung und Umwandlung des Mentoring-Prozesses (sic!) in eine dauerhafte Einrichtung (...) deshalb erstrebenswert (ist), um althergebrachte Denkmuster aller Beteiligten dauerhaft zu modifizieren und damit eine nachhaltige Befähigung zur kooperativen und verantwortungsbewussten Gestaltung von Schulkultur zu erreichen.“ (Ittel et al. 2009, 203)

Die Beteiligten öffnen sich „gegenüber dem jeweils anderen und lernen neue Perspektiven, Wege und Lösungen kennen, die ihnen sonst verschlossen bleiben." (Ittel et al. 2009, 199) Normen et al. (2005) erkennen im Mentoringprozess ebenfalls die Chance für Veränderungen und Weiterentwicklungen. "If mentoring is to function as a form of individualizes professional development, it must be guided by a vision of the kind of teaching to be developed." (Norman et al. 2005, 695) Mentoring unterstützt demnach personelle und institutionelle Entwicklungen und trägt zur Qualitätssicherung bei. In diesen Aussagen wird verdeutlicht, dass Mentoring auch zur Schulentwicklung beiträgt. Ein weiteres Forschungsfeld das sich gegenwärtig öffnet.

Im Folgenden werden nun Gestaltungsparameter des schulpraktischen Mentorings, wie Auswahl und Aufgaben der Mentor/innen, Beobachtet-Werden und Beobachten als Aufgabenfeld, Rolle und Funktion der/des Mentorin/Mentors sowie Rollenverhalten der/des Mentorin/ Mentors in Dialogen näher beleuchtet. Die Wahl der mentor/innenseitigen Parameter fiel auf systematische und systemische Bereiche im Mentoring, die wesentlich zur Wirksamkeit beitragen können.

\subsubsection{Gestaltungsparameter schulpraktischen Mentorings}

Mentor/innen nehmen in der Gestaltung des schulpraktischen Mentorings eine bedeutende Position ein. Nach dem Modell von Rhodes (2002) beeinflussen sie Mentee in drei wesentlichen Bereichen: „(1) improving social skills and emotional well-being; (2) (...) developing cognitive skills through dialogue and listening; (3) identity development." (Schnautz 2014, 19) Welche Voraussetzungen, Persönlichkeitsmerkmale, Kompetenzen und Qualifikationen müssen oder sollen nun Mentor/innen mitbringen bzw. entwickeln, damit sie die Verantwortung übernehmen können und dem Anspruch und den Erwartungen, denen sie im Mentoringprozess begegnen, gerecht werden? Es werden ausgewählte Gestaltungsparameter wie 
Auswahl, Aufgaben, Rolle und Funktion sowie Rollenverhalten der/des Mentorin/Mentors beschrieben (Tabelle 20).

Tab. 20: Auflistung der folgenden Gestaltungsparameter und Kurzbeschreibungen zu schulpraktischem Mentoring

\begin{tabular}{ll}
\hline Gestaltungsparameter & Kurzbeschreibung \\
\hline Mentor/innenauswahl & Diskurs zu möglichen Ansätzen zur Auswahl \\
Aufgaben für Mentor/innen & Unterschiedliche Bereiche je nach \\
& Anforderungsprofil(e) \\
Beobachtet-Werden und Beobachten & Bereiche für Analyse und Reflexion im Rahmen der \\
als Aufgabenfeld & Begleitungstätigkeit \\
Rolle und Funktion der/des Mentorin/ & Analyse zu Rolle und Funktion im schulpraktischen \\
Mentors im schulpraktischen Mentoring & Mentoring \\
Rollenverhalten der/des & Befunde zur Gesprächsführung \\
Mentorin/Mentors in Dialogen & \\
\hline
\end{tabular}

Es liegt in der Verantwortung der Ausbildungsinstitutionen Mentor/innen mit der Aufgabe zu betrauen und zu beauftragen. Die Reihenfolge der Beschreibungen richtet sich nach der in Tabelle 20 ausgerichteten Anordnung. Der Gestaltungsparameter Auswahl wird als erstes thematisiert.

\section{Mentor/innenauswahl}

Welche Kriterien sollen für Auswahl einer/eines Mentorin/Mentors herangezogen werden? Welche Funktion im Mentoring sollen Mentor/innen erfüllen? Diese Fragen wird jede Organisation bzw. Institution im Bildungsbereich für sich entscheiden. Hierbei werden unterschiedliche Ansätze, Rollen und Denkweisen verfolgt. Die gesetzliche Basis bildet $\$ 39$ des Bundesgesetzblattes der Republik Österreich (Bundesgesetzblatt 2013). Darin wird als „Voraussetzung für die Bestellung zur Mentorin oder zum Mentor [ist] eine mindestens fünfährige Berufserfahrung als Lehrperson an einer Schule“ (Bundesgesetzblatt 2013) gefordert. Für die Bestellung als Mentor/in in der Induktionsphase gilt außerdem:

„\$39 a (4) Bis zum Schuljahr 2029/2030 dürfen auch Lehrpersonen als Mentorinnen oder als Mentoren eingesetzt werden, die

1. zu Betreuungslehrkräften im Unterrichtspraktikum oder im Rahmen der schulpraktischen Ausbildung bestellt sind oder

2. einen einschlägigen Lehrgang im Umfang von mindestens 30 ECTS absolviert haben." (Bundesgesetzblatt 2013)

Ausschlaggebend für eine Bestellung ist aber immer die Bereitschaft der/des Mentorin/Mentors, diese Aufgabe zu übernehmen. „Effective mentors must (...) be willing and able to 'make their work public' and make explicit the factors underlying their classroom practices (Simpson et al. 2007)." (Hobson et al. 2009, 212) Oettler (2009) betont die Freiwilligkeit und zeigt eine Korrelation in diesem Zusammenhang mit dem Item „Mentor (sic!) profitiert vom Ausbildungswissen des Studenten (sic!)“ auf (Oettler 2009, 241).

Zur Ausübung der Tätigkeit benötigt die/der Mentor/in Professionalität, Persönlichkeit, Idealismus, zeitliche und individuelle Ressourcen sowie ein solides Umfeld. „Mentoring ist 
durch Diskussionen, Reflexion, kritisches Feedback und konkrete Aufgaben eine mitunter (...) anstrengende und fordernde persönliche Arbeitsbeziehung." (Pflaum 2017, 65) Herausforderungen sind vielfältig und Anforderungen unterschiedlich. Nicht jede/r Mentor/in, die/der sich zur Verfügung stellt, ist auch geeignet (Graf et al. 2017, 147).

Theoretische Erklärungsansätze können bei der Auswahl der/des Mentorin/Mentors zum schulpraktischen Mentoring von Institutionen zum Tragen kommen. Anbei eine kurze Darstellung ohne Anspruch auf Vollständigkeit (Tabelle 21):

Tab. 21: Verschiedene Ansätze für die Auswahl von Mentor/innen

\begin{tabular}{|c|c|}
\hline Ansatz & $\mathrm{d}$ und Besonderheiten in der Profession Mentoring \\
\hline $\begin{array}{l}\text { xpertise- } \\
\text { leoretischer }\end{array}$ & $\begin{array}{l}\text { Das Modell nach Shulman }(1986,1987) \text { um berufsspezifisches Wissen bildet die } \\
\text { Grundlage für diesen Ansatz. Er unterscheidet die Wissensbestände: general pedagogical } \\
\text { knowledge, content knowledge, curriculum knowledge, pedagogical content know- } \\
\text { ledge, knowledge of education context, pedagogical reasoning (Shulman 1986, 1987). } \\
\text { Nach Hudson (2013b) brauchen Mentor/innen für die Ausübung ihrer Tätigkeit einen } \\
\text { pädagogischen Wissensrahmen und ein Repertoire an pädagogischen Wissensstrategien } \\
\text { (Hudson 2013b, 2). Diese Kriterien könnten für die Auswahl herangezogen werden. }\end{array}$ \\
\hline $\begin{array}{l}\mathrm{z}- \\
\mathrm{r}\end{array}$ & $\begin{array}{l}\text { aumert et al. (2006) beschreiben das Modell professioneller Handlungskompetenz mit } \\
\text { ofessionswissen, Überzeugungen und Werthaltungen, motivationaler Orientierung und } \\
\text { lbstregulativen Fähigkeiten (siehe Kapitel I/1.2.3). Die Elemente können durch refle- } \\
\text { ve Auseinandersetzung erweitert werden. Darauf muss sich die/der Mentor/in einlassen. }\end{array}$ \\
\hline$r$ & cctellt sich die Eroge ob fochfremde oder nur fochaucohildete Dercone \\
\hline $\begin{array}{l}\text { zio- } \\
\text { ologischer }\end{array}$ & $\begin{array}{l}\text { „... verwendet als Grundlage der Argumentation Prinzipien der Evolutionstheorie und } \\
\text { Evolutionsbiologie, bei denen insbesondere Reproduktionsstrategien und Selektionskrite- } \\
\text { rien von Bedeutung sind.“ (Schneider et al. 2009, 147) Demzufolge würde ein/e Mentor/ } \\
\text { in Handlungen beim Mentee beobachten, reflektieren, sich damit auseinandersetzen - vor } \\
\text { allem auch unter dem Blickwinkel der Selektion. Wären solche Eigenschaften von der Insti- } \\
\text { tution gewünscht, würden Mentor/innen mit Blick zu diesem Ansatz zum Einsatz kommen. }\end{array}$ \\
\hline Ansatz & $\begin{array}{l}\text { in, sich mit Entwicklungsaufgaben zu beschäftigen } \\
\text { d zu unterstützen. Ziel ist, im gemeinsamen Prozess die } \\
\text { gen. }\end{array}$ \\
\hline $\begin{array}{l}\text { Sozial- } \\
\text { psychologischer } \\
\text { Ansatz }\end{array}$ & $\begin{array}{l}\text { ie/Der Mentor/in zeigt Interesse an den Anliegen des Gegenübers und ist gewillt, ein } \\
\text { Tertrauensverhältnis aufzubauen. Sie/Er bemüht sich um eine gute zwischenmenschliche } \\
\text { eziehung. }\end{array}$ \\
\hline $\begin{array}{l}\text { Systemischkon } \\
\text { struktivistische } \\
\text { Ansatz }\end{array}$ & $\begin{array}{l}\text { Die/Der Mentor/in leitet und berät Mentee bei Handlungen, die ein Teil der Aufga- } \\
\text { benstellung sind. Die/Der Mentee kann sich frei bewegen. Aus Sicht der/des Mentorin/ } \\
\text { Mentors wird angenommen, dass die/der Lernende auf Grundlage von Erfahrungen lernt. } \\
\text { In der Begleitung wird meist eine enge Beziehung aufgebaut. }\end{array}$ \\
\hline
\end{tabular}

Nach Hobson et al. (2009) ist erfolgreiches Mentoring vor allem davon abhängig, welche Mentor/innen ausgewählt werden (Hobson et al. 2009, 211). Sie zählen dazu folgende ideale Charakterzüge auf:

“They must be supportive, approachable, nonjudgemental and trustworthy, have a positive demeanour, and possess good listening skills and the ability to empathize, as well as the willingness and ability to take an interest in beginning teachers." (Hobson et al. 2009, 212) 
Gesetzliche Bestimmungen, Beobachtungen von Charakter- und Persönlichkeitszügen bei Lehrpersonen sowie Berücksichtigung von Erklärungen theoretischer Ansätze, wie in Tabelle 21 angeführt, zeigen Bereiche auf, welche Überlegungen für die Wahl angestellt werden müssen bzw. könnten. Wahrscheinlich ist es ein Konglomerat aus mehreren Aspekten und Ansätzen, die schließlich zur Beauftragung führen. Wesentlich sind in der Interaktion und Kommunikation „positive Persönlichkeitseigenschaften wie Empathie, Herzlichkeit, Ehrlichkeit, Zuverlässigkeit und Vertrauenswürdigkeit“" (Fuge 2016, 42) seitens der Mentor/innen.

In der Mentoring-Funktion sind die Begleiter/innen in einer anderen Rolle als in jener der Lehrperson. Gelten sie in der Funktion als Lehrer/in als Expert/in/Experte, so wird im Rahmen sozio-konstruktivistischer Forschung die Annahme getätigt, dass sie in der Begleitungstätigkeit zunächst Noviz/innen-Mentor/innen sind und sich erst durch Ausbildung, Wissenserfahrungen und Interaktion im Laufe des Mentoringprozesses zu Expert/innen-Mentor/innen weiterentwickeln (Garvey 2000, 7).

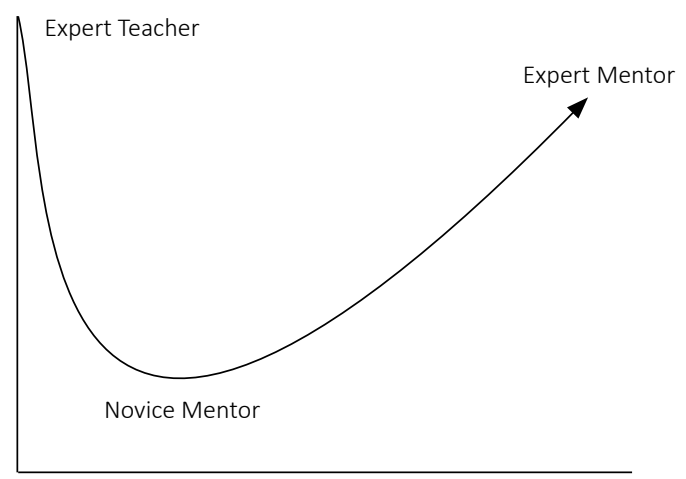

Abb. 17: Entwicklung zur/zum Expert/innen-Mentor/in (Garvey 2000,7)

Die Ansicht der Professionsentwicklung und die Vorstellung des Expert/innen-Paradigmas wohl analog zum Noviz/innen-Expert/innen-Modell bei Lehrpersonen nach Kraler (Kraler 2012b, 562) - legen den Schluss nahe, dass professionsspezifische Kompetenzen durch die Tätigkeit entwickelt werden. Gerade auch bei der Auswahl der Mentor/innen sollte auf die Möglichkeit des Entwicklungsprozesses Bedacht genommen werden.

Bei Bestellung einer/eines Mentorin/Mentors haben Mentee oft eine anspruchsvolle Erwartungshaltung. So soll sie/er über Kompetenzen verfügen und ideale Persönlichkeitsmerkmale für die Zusammenarbeit aufweisen (Ittel 2009, 194). „Menschen mit hoher Kompetenz sind stets auch qualifiziert, Hochqualifizierte sind jedoch nicht zwangsläufig auch kompetent." (Erpenbeck et al. 2018, 10) Idealerweise ist es günstig, wenn Kompetenzen (zu den Basiskompetenzen zählen personale Kompetenz, Aktivitäts- und Handlungskompetenz, Fach- und Methodenkompetenz, sozial-kommunikative Kompetenz) auch mit Werten und Wertvorstellungen verknüpft sind (Erpenbeck et al. 2018, 11).

\section{Aufgaben im schulpraktischen Mentoring für Mentor/innen}

Die Aufgabenstellung für eine/n Mentor/in ist im Wesentlichen an Zielsetzungen der unterschiedlichen Anforderungskataloge der Praktika der Pädagogischen Hochschulen und Univer- 
sitäten gekoppelt. Diese sind in die Pädagogisch-Praktischen Studien oder/und Fachdidaktiken eingebunden und verfolgen unterschiedliche Intentionen.

„Voraussetzung für eine gelingende Verknüpfung zwischen Praxiserfahrungen und an der Ausbildungsinstitution gelehrtem Theoriewissen ist eine hohe Kongruenz zwischen den Zielen und Standards der Ausbildungsinstitution und den unterrichtsrelevanten Überzeugungen und dem Handeln der" (Kreis 2012, 87) Begleitperson.

Es erfordert im Idealfall einen intensiven Austausch. Mentor/innen stellen ihr Berufsfeld zur Verfügung, um Studierenden Gelegenheit zur Schulerkundung, zum Hospitieren, zum Unterrichten und zum Reflektieren zu bieten (Arnold et al. 2011, 62f) und zum Aufbau von professionellen Kompetenzen beizutragen. Formelle und informelle Gespräche unterstützen den Austausch bzw. ko-konstruktive Aktionen (Kreis 2012, 43) und zielen auf „Optimierung von Unterrichtshandeln.“ (Kreis 2012, 87) Schnebel (2018) stellt eine „Verbindung zwischen den intendierten Lernprozessen der Studierenden in Praxisphasen, der Lernbegleitung sowie erforderlichen Kompetenzen“" (Schnebel 2018, 24) der Mentor/innen her (Tabelle 22):

Tab. 22: Verbindung von intendierten Lernprozessen der Studierenden in Praxisphasen und Lernbegleitung sowie erforderlichen Kompetenzen (Schnebel 2018, 24)

\begin{tabular}{|c|c|c|}
\hline $\begin{array}{l}\text { Ziele, Lernfelder im } \\
\text { Praktikum }\end{array}$ & Aufgabe & $\begin{array}{l}\text { Erforderliche Kompetenz bzw. } \\
\text { Qualifikation }\end{array}$ \\
\hline $\begin{array}{l}\text { Einblicke in und } \\
\text { Erfahrungen mit Aufga- } \\
\text { ben von Lehrpersonen }\end{array}$ & $\begin{array}{l}\text { Bereitstellen von Situationen; } \\
\text { Einführung ins Praxisfeld; } \\
\text { Informationen geben über Schule, } \\
\text { Lernende, Umfeld; } \\
\text { Unterstützung der Reflexion eigener } \\
\text { Erfahrungen; }\end{array}$ & $\begin{array}{l}\text { Kenntnis über Schulfeld und Bedingun- } \\
\text { gen der LehrerInnenausbildung (sic!); } \\
\text { Reflexion eigener beruflicher Praxis und } \\
\text { Berufsauffassung; }\end{array}$ \\
\hline $\begin{array}{l}\text { Erweiterung allgemein- } \\
\text { und fachdidaktischer } \\
\text { Planungs- } \\
\text { kompetenz }\end{array}$ & $\begin{array}{l}\text { Planungsgespräche ko-konstruktiv } \\
\text { durchführen; } \\
\text { Unterstützung der Reflexion eigener } \\
\text { Erfahrungen; }\end{array}$ & $\begin{array}{l}\text { Fach- und allgemeindidaktische Pla- } \\
\text { nungskompetenz; } \\
\text { Gesprächsführungskompetenz; }\end{array}$ \\
\hline $\begin{array}{l}\text { Erweiterung didakti- } \\
\text { scher und pädagogischer } \\
\text { Handlungskompetenz }\end{array}$ & $\begin{array}{l}\text { Modellieren; } \\
\text { Unterstützung der Eigenverantwortung } \\
\text { bei gleichzeitiger Reduktion der Hand- } \\
\text { lungskomplexität; } \\
\text { Feedback geben; }\end{array}$ & $\begin{array}{l}\text { Verfügen über Strategien des } \\
\text { Feedbacks; } \\
\text { Kenntnisse über Kriterien und Indikato- } \\
\text { ren professioneller } \\
\text { Handlungskompetenz; }\end{array}$ \\
\hline $\begin{array}{l}\text { Überprüfung } \\
\text { Berufswunsch }\end{array}$ & $\begin{array}{l}\text { Unterstützung der Reflexion eigener } \\
\text { Erfahrungen; } \\
\text { Beurteilung; } \\
\text { Unterstützung in Problem- und } \\
\text { Konfliktfällen; }\end{array}$ & $\begin{array}{l}\text { Gesprächsführungskompetenz; } \\
\text { Kenntnisse über Anforderungen und } \\
\text { Kompetenzbeschreibungen der Univer- } \\
\text { sität/Hochschule; }\end{array}$ \\
\hline $\begin{array}{l}\text { Erarbeiten von Perspek- } \\
\text { tiven hinsichtlich der } \\
\text { weiteren professionellen } \\
\text { Entwicklung }\end{array}$ & $\begin{array}{l}\text { Unterstützung in Problem- und } \\
\text { Konfliktfällen; } \\
\text { Unterstützung in der Entwicklung von } \\
\text { Perspektiven; }\end{array}$ & $\begin{array}{l}\text { Gesprächsführungskompetenz; Kenntnis- } \\
\text { se über Kriterien und Indikatoren professi- } \\
\text { oneller Handlungskompetenz; Kenntnisse } \\
\text { über Anforderungen und Kompetenzbe- } \\
\text { schreibungen der Universität/Hochschule }\end{array}$ \\
\hline
\end{tabular}


Die angeführten Aufgabenfelder in den Bereichen Planungs-, Reflexions-, professionsspezifische Handlungs- und Beratungskompetenz zeigen, dass der Aufgabenbereich sich intensiver auf schulische Angelegenheiten konzentriert. Auch Bach et al. (2018) erhoben in ihrer Untersuchung, dass Mentor/innen Studierende mehr in schulischen Bereichen unterstützen, wie gemeinsame Reflexion des Unterrichts, Beratung in unterrichtlichen Belangen, Einbezug in schulische Angelegenheiten, Beratung im Hinblick auf die Berufseignung, Beratung zur Beziehung zu Schülerinnen und Schülern als in universitären Bereichen wie Beratung bei der Forschungsaufgabe, Beratung beim Portfolio, Hilfestellung bei der Anfertigung von Unterrichtsentwürfen (Bach et al. 2018, 198). Die Vermutung liegt nahe, dass Mentor/innen „spezifisches Wissen, spezielle Fertigkeiten und Einstellungen erworben“ (Reinisch 2009, 37) haben. Beispielhaft kann ein ausreichendes Klassenführungswissen angeführt werden: Klassenführungswissen ist nach Baumert et al. (2006) oder Voss et al. (2015) eine Facette des Pädagogischen Wissens. Die inhaltliche Ausdifferenzierung erinnert an das Modell von Kounin (1970), der vorbeugende und aktive Maßnahmensetzungen wie Allgegenwärtigkeit, Überlappung, Momentum, Reibungslosigkeit und Gruppenaktivierung für einen störungsfreien Unterricht nennt. Seidel (2015) spricht von einer Dreidimensionalisierung der Klassenführung: Umgang mit Unterrichtsstörungen (Regeln, Transparenz, Rückmeldekultur), bewusste Lernzeitphasen (klare Struktur und Handlungen) und unterstützende Lernprozessbegleitung (Westphal et al. 2018, 132). Gold et al. (2013) zeigen in ihrer Studie auf, dass durch Trainingseinheiten mittels Videosequenzen Klassenführungswissen verbessert werden kann. König et al. (2012) konnte im Rahmen der LEK-Studie nachweisen, dass das Klassenführungswissen je nach Ausbildungsinstitution mit einer Effektstärke von 0.4 bis 0.8 in den ersten vier Semestern zunimmt. Es ist anzunehmen, dass Mentor/innen Klassenführungsstrategien kennen, mit dem prozeduralen Wissen somit vertraut sind und in Reflexionsprozessen die Besonderheiten zur Klassenführung mit den Studierenden thematisieren und es als wesentliche Aufgabe erachten, Expertise einzubringen.

Studierende erproben sich also im Handlungsfeld Schule. Bezüglich des Begleitungsprozesses werden seitens der Mentor/innen Kompetenzen und Qualifikationen für die Betreuungsaufgabe erwartet. Schnebel (2018) führt einige an (Tabelle 22). Für die genannten Aufgabenfelder „Unterstützung in Problem- und Konfliktfällen, Unterstützung in der Entwicklung von Perspektiven“ (Schnebel 2018, 24) ergeben sich besondere Herausforderungen. Sowohl zu einer typen- und kontextsensiblen Begleitung (Hofmann 2019) als auch zur Erarbeitung „,on Perspektiven hinsichtlich der weiteren professionellen Entwicklung" (Schnebel 2018, 24) bedarf es eines Handlungswissens und Fachwissens. Es ist Aufgabe der Ausbildungsinstitutionen, Mentor/innen in den Fort- und Weiterbildungsprogrammen zu schulen, um Sicherheit und Kompetenz in der Begleitung zu vermitteln. Im Rahmen des Austausches mit Ausbildungsinstitutionen besteht auch die Möglichkeit, „Kriterien und Indikatoren professioneller Handlungskompetenz" (Schnebel 2018,24) zu diskutieren und festzulegen.

Im Folgenden wird auf die spezifische Situation Beobachtet-Werden und auf den Aufgabenbereich Beobachten näher eingegangen, da dieser Bereich von Mentor/innen in der Rolle als Mentor/in und im Rahmen der Lehrtätigkeit wichtig ist.

\section{Beobachtet-Werden und Beobachten als Aufgabenfeld}

Hospitationen und Gestaltung von Unterrichtssettings zählen vielfach zum Anforderungsprofil der Praktika der Studierenden. Mentor/innen kommen somit im Begleitungsprozess in die Rolle der/des Beobachteten und in die Rolle der/des Beobachterin/Beobachters. Im Anforderungsprofil der Pädagogisch-Praktischen Studien der Lehrer/innenbildung WEST sind Hos- 
pitationen bzw. Unterrichten und Classroom-Management zwei wichtige Bereiche der schulischen Anteile der Praktika.

Ersteres setzt im Allgemeinen die Bereitschaft voraus, dass sich Mentor/innen beim Lehrer/ innenhandeln beobachten lassen. Studierende erhalten meist die Aufgabe, ihre Beobachtungen in Schriftsprache überzuführen, in immer häufigeren Fällen auch videografisches Datenmaterial herzustellen. „Üblicherweise stehen die meisten Betroffenen der Aussicht, beobachtet zu werden, mindestens ambivalent, wenn nicht skeptisch gegenüber." (Bennewitz 2012, 205) Die Anwesenheit einer „schulfremden Person“ im Unterricht, die Tatsache, dass Beobachtungsaugen auf die eigene Person und das Handeln gerichtet sind und dass Notizen vom Geschehen verschriftlicht werden, verlangen Offenheit seitens der Beteiligten und Transparenz und Klarheit in der Aufgabenstellung. In der Rolle der/des Beobachterin/Beobachters muss man sich über Bedeutung von Deutungsmustern und -erklärungen bzw. Wahrnehmungsfehlern im Klaren sein und schulisch normative Erwartungshaltungen kritisch hinterfragen. De Boer (2012) schlägt in der persönlichen Auseinandersetzung mit dem Beobachtungsprozess folgende Fragestellungen vor:

„Was sind meine Beobachtungsmotive? Warum sehe ich, was ich sehe? Welches Verhalten erwarte ich? (...) Wann werde ich auf etwas aufmerksam? Wie deute ich das Geschehene? Was kommt mir bekannt vor und was ist mir fremd? Welche Annahmen liegen meinen Deutungen zugrunde?" (De Boer 2012, 220)

Dies sind Fragestellungen, die auch für Mentor/innen ein Anreiz für die Weiterentwicklung sein können. Breidenstein (2012) nennt für die teilnehmende Beobachtung drei wesentliche Aspekte vor dem Hintergrund der ethnografischen Beobachtung, und zwar „die Etablierung und Nutzung der Beobachterrolle (sic!), die extensive Verschriftlichung und die reflexiv-analytische Durchdringung der Beobachtungen." (Breidenstein 2012, 31) Ethnografisches Beobachten zeichnet sich dadurch aus, „das eigentlich Vertraute zu betrachten ,als sei es fremd“ (Amann und Hirschauer 1997, 12)، (Breidenstein 2012, 30) Wie gelingt es nun, eine Distanz zum Vertrauten im Beobachtungsprozess herzustellen? „Reines“ Beobachten verlangt in der Beobachtungsrolle Zeit, Raum und Beziehungsqualität. Durch intensivere und längerfristige Phasen der Teilnahme stellt sich ein Gewöhnungseffekt ein und Strukturen, Abläufe und Entwicklungen erschließen sich für die/den Betrachter/in. Ziel ist, zu „einer möglichst präzisen, übersichtlichen und verallgemeinerungsfähigen Beschreibung“" (Breidenstein 2012, 31) zu gelangen, die auch für nicht teilnehmende Personen verständlich und nachvollziehbar ist, und die somit einen Zugang zum Feld bekommen.

„Das Qualitätskriterium für die Verschriftlichung von Beobachtungen liegt einerseits in der Ausführlichkeit und Detaillierung des Schreibens und andererseits in der Reflexion und zunehmenden Fokussierung und analytischen Verdichtung des Schreibens." (Breidenstein 2012, 33)

In der anschließenden Phase geht es nun darum, Verschriftlichungen einer Analyse zu unterziehen. Im Analyseprozess genügen keine plakativen Erklärungen und Bewertungsversuche, sondern die/der Beobachter/in taucht in eine reflexive, sich Fragen stellende und forschende Haltung ein. Was als linearer Ablauf dargestellt wird, verläuft in der Praxis vielfach in einem zirkulären und somit immer wiederkehrenden Prozess (Fabel-Lamla et al. 2012, 282). Verschriftlichungen (Protokolle, Hospitationsberichte, Studienprojekte, Portfolios, Lernjournale, Lerntagebücher usw.) bedeuten, „sich intensiv mit einer Situation und ihren Protagonisten (sic!) auseinanderzusetzen, sensibel für die eigene Wahrnehmung und Versprachlichung zu werden 
und sie in diesem Prozess zu reflektieren und auszudifferenzieren." (De Boer 2012, $76 \mathrm{f}$ ) Bromme et al. (2008) verglichen Studien von Anfänger/innen und Expert/innen und zeigten hierbei „die Veränderung der kategorialen Wahrnehmung von Unterrichtssituationen“ (Bromme et al. 2008, 807) auf. Somit ist anzunehmen, dass Studierende sich eine Beobachtungs- und Wahrnehmungskompetenz im Studium aufbauen.

Idealerweise sollten Mentor/innen und Mentee den Prozess der „reflexiven Distanzierung“ (Breidenstein 2012, 33) oder „sequentiellen Interpretation protokollierter Praxis“ (Bennewitz 2012, 212) gemeinsam gestalten und sich im Anschluss Schwerpunkte für die Vertiefung einzelner Sequenzen oder Thematiken setzen. Für Mentor/innen und Studierende können somit ethnografische Beobachtungen „neue Perspektiven auf das allzu vertraute Geschehen eröffnen.“ (Breidenstein 2012, 43) Wichtig ist für die am Begleitungsprozess beteiligten Personen das Erkennen von Intention und Wirkung in der Handlungsausführung und das Erkennen von „Möglichkeiten und Grenzen bei der Umsetzung pädagogischer Konzepte“ (Bennewitz 2012, 213). Denn Lehrer/innenhandeln ist sehr komplex, „nicht standardisierbar und nicht technologisch steuerbar“ (Fabel-Lamla et al. 2012, 283) und „unter Berücksichtigung der jeweiligen Umstände des Einzelfalls und der jeweiligen Situation ausdeutungs- und konkretisierungsbedürftig." (Baumert et al. 2006, 476) Bereits 1780 beschrieb Trapp (1780, 1977) Aspekte des Beobachtens (siehe Kapitel I/2.4.3), die heute in der Lehrer/innenausbildung und insbesondere in den Praktika Bedeutung haben: „Beobachten wird als grundlegende Komponente einer forschenden, reflexiven Haltung gegenüber der eigenen Handlungspraxis konzipiert." (Fabel-Lamla et al. 2012, 286) Somit sind Hospitationseinheiten ein wichtiger Bestandteil im Anforderungskatalog der Schulpraktika, und Mentor/innen leisten einen wesentlichen Beitrag zur Kompetenzerweiterung.

\section{Rolle und Funktion der/des Mentorin/Mentors im schulpraktischen Mentoring}

Die/Der Mentor/in nimmt im Mentoringprozess eine entscheidende Position ein, bekleidet eine Mentoring-Funktion und besetzt eine Rolle. Der Begriff Rolle ist multiperspektivisch, da zur Rollentheorie aus soziologischer Sicht unterschiedliche Ansätze vorliegen, die die Interaktionsprozesse oder das Individuum als Teil der Gesellschaft betrachten. Diese Ansätze erklären Rollenfestlegungen, eröffnen aber auch Handlungsspielräume für jeden einzelnen (Verden 1997, 24f).

So vertritt Mead, der neben Linton als Urvater der modernen sozialpsychologischen Rollentheorie gilt, die Ansicht, dass das Selbst aus übernommenen Rollen besteht, die sich abgestimmt auf Handlungen mit dem Gegenüber entwickeln (Preyer 2012, 63f).

„Die Knotenpunkte der gesellschaftlichen Kommunikation und Kooperation nennt Mead ,Rollen'. Das Mitglied eines sozialen Systems versteht sich als eine besondere Person, die sich bestimmte Eigenschaften zuschreibt, indem es bestimmte Rollen übernimmt und spielt. Sie werden ihm auch von Anderen als seine Charaktereigenschaften zugeschrieben." (Preyer 2012, 63f)

Mead ist Vertreter des symbolischen Interaktionismus und verdeutlicht, dass Rollenstrukturen durch Interaktionen in der Gesellschaft entstehen (Jahnke 2006, 45). Für das schulpraktische Mentoring könnte die Annahme bedeuten, dass sich Mentor/in und Mentee in der Interaktion wechselseitig beeinflussen und Identität entwickeln.

Der strukturell-funktionalistische Ansatz vertritt die Annahme, dass „es in erster Linie nicht um die lebensweltliche Realisierung von Rolle geht, sondern um die Verdeutlichung grundlegender sozialer Strukturen, die das Handeln mittels Rollen prägen und steuern." (Jahnke 2006, 48) Die 
sozialpsychologische Perspektive des Rollenansatzes „,betont die heuristische Funktion von Rollen im individuellen Sozialverhalten" (Ziegler 2008, 4), indem bestimmte Verhaltensweisen in bestimmten Situationen wie eingeübte Muster angewendet werden (Gollwitzer et al. 2006, 80; Ziegler 2008, 4). Demnach werden Rollen aufgezwungen, möglichst erfüllt. Für das schulpraktische Mentoring würde dieser Ansatz bedeuten, dass Mentee bei unterschiedlichen Mentor/ innen Praktika absolvieren sollen, damit Mentee unterschiedliche Rollenbilder kennenlernen. Nach der Beschreibung der beiden Ansätze entspricht das Handeln einer Rollenkonformität oder die Rolle entsteht aus der Zusammenarbeit mit anderen (Ziegler 2008, 3). Empirische Befunde in Verbindung von Rollentheorien und schulpraktischem Mentoring stellen ein Desiderat dar. Es finden sich lediglich Befunde zu Rollenverhalten und Erwartungshaltungen. Graf et al. (2017) beschreiben Anforderungen für Funktion, Rolle und Mensch-Sein im Mentoringprozess. In Abbildung 18 werden Kriterien dargestellt.

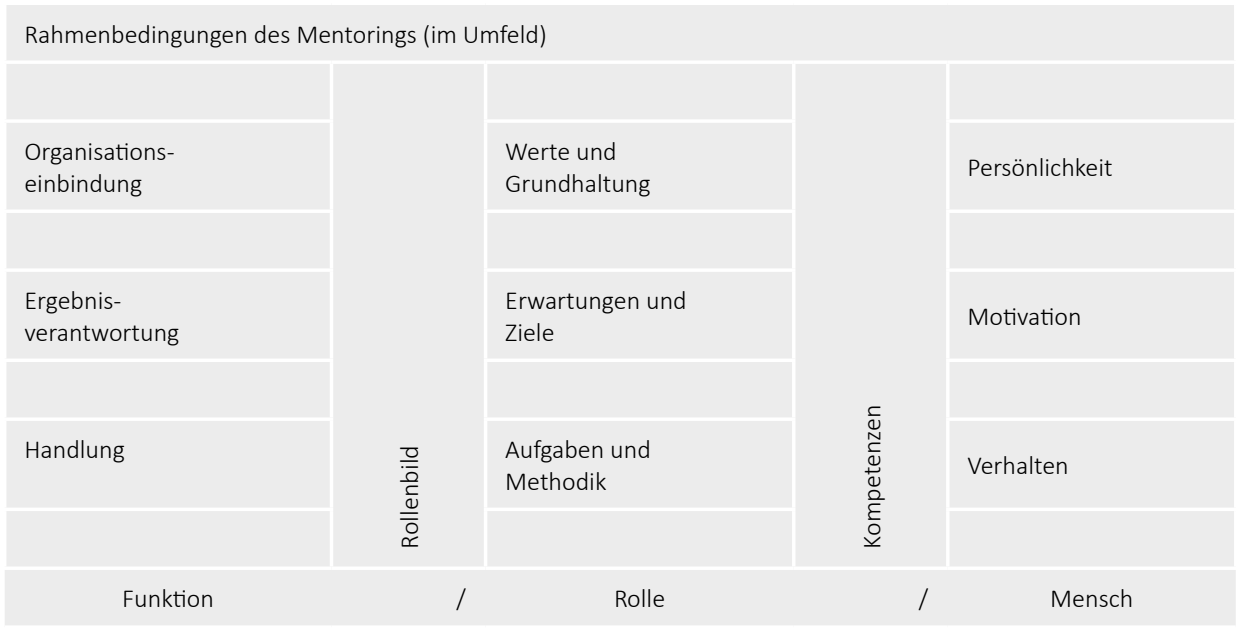

Abb. 18: Anforderungen an Funktion, Rolle und Mensch (Graf et al. 2017, 48)

In der Rolle der/des Mentorin/Mentors kommen immer Persönlichkeit, Motivgründe und ein bestimmtes situationsspezifisches und individuell gefärbtes Verhalten zum Ausdruck. Sie/Er muss ihre/seine Kompetenzen kennen und sich der Qualifikationen bewusst sein, um erwägen zu können, ob sie/er die Aufgabe der Begleitung und somit die Rolle erfüllen kann. Die Tätigkeit ist an Erwartungen und Anforderungen gebunden. Denn „unter der Rolle versteht man (...) den ,Satz von Erwartungen (von irgend jemandem) bezüglich des Inhabers einer Position (Sader 1969, 209)““ (Wellhöfer 2018, 31).

Im schulpraktischen Mentoring sind es eine Reihe von unterschiedlichen Bezugsgruppen, die eine Erwartungshaltung an die/den Inhaber/in der Mentoring-Rolle stellen. Zu ihnen zählen: Mentee, Praktikumsleitung, Ausbildungsinstitution, Schulleitung, Klasse, Schüler/innen, Eltern, Schulbehörde, Dienstgeber, Bildungspolitik (Perkhofer-Czapek 2016, 49). Rothland (2013) spricht bei Lehrpersonen aufgrund der vielfältigen Aufgabenbereiche und im Zuge der „Abhängigkeit von der Erwartung der Bezugsgruppe von Rollensektoren“ (Perkhofer-Czapek 2016, 49; Rothland 2013, 30). Die Bezeichnung Rollensektoren könnte auch auf die Mentor/innen-Rolle übertragen werden. Dazu passend soll die Studie von Clarke et al. (2014) genannt werden. Sie 
erfassen nach ihrem Forschungssupport von 183 Reviewstudien elf unterschiedliche Kategorien von Rollen im schulpraktischen Mentoring und beschreiben jeweils die inhaltliche Bedeutung. Die Autor/innen unterscheiden: "Providers of Feedback, Gatekeepers of the Profession, Modelers of Practice, Supporters of Reflection, Gleaners of Knowledge, Purveyors of Context, Conveners of Relation, Agents of Socialization, Advocates of the Practical, Abiders of Change, and Teachers of Children" (Clarke et al. 2014, 174f). Die Autor/innen kommen zum Entschluss, dass Besprechungsphasen aufgrund des Anleitens von Unterricht meist noch wie Lehrmeister/innenmodelle wirken und Mentor/innen diese Rolle gehäuft einnehmen (Clarke et al. 2014, 190f). Ist es das, was sich Mentee wünschen? Hennissen et al. (2011) betonen, dass sie sich von der/dem Träger/in der Rolle der/des Mentorin/Mentors Unterstützung im emotionalen Bereich (emotional support) und Hilfestellung bei der Bewältigung von anstehenden Aufgabenstellungen (task assistance) erwarten (Hennissen et al. 2011, 1050). Daraus lässt sich ableiten, dass sich die „Beteiligten (...) über ihre Werte und Grundhaltungen bewusst“ (Graf et al. 2017, 49) sein müssen und Erwartungen, Ziele, Aufgabenbereiche und das methodische Vorgehen immer gemeinsam mit der/dem Tandempartner/in absprechen sollen. Somit würde wohl ein idealtypisches Mentor/inRollenbild - geprägt durch eine „Kombination aus Erwartungen und Wahrnehmungen“ (Graf et al. 2017, 48) - entstehen. In der Funktion agiert die Person mit Handlungen, zielt in der Verantwortung auf ein Ergebnis und bindet das Organisationsfeld Schule intensiver im Prozess ein. Die strukturellen und persönlichen Rahmenbedingungen sind oftmals weitere wesentliche Faktoren für gelingendes Mentoring. Die gilt es in der Funktion zu erfassen und sich darin zu bewegen. Aus all den angeführten Erklärungen lässt sich schließen, dass Anforderungen an die Rolle und Funktion der/des Mentorin/Mentors komplex sind. Schmid et al. (2011) betonen die Notwendigkeit der Klärung eines gemeinsamen Rollenverständnisses und die Bereitschaft beider Partner/innen, Verantwortung zu übernehmen. Weiters schreiben die Autor/innen den Mentor/ innen noch Selbstreflexions-, Gesprächsführungs- und Problemlösekompetenz sowie Empathiefähigkeit zu; den Mentee einen Willen zur Aktivität und Initiativensetzung sowie die Auseinandersetzung mit Problemlösestrategien innerhalb ihrer Rolle (Schmid et al. 2011, 39). Preyer (2012) empfiehlt daher eine ständige Rollenreflexivität und gegebenenfalls eine Rollendistanz im Sinne der Salutogenese für sich zu überlegen (Preyer 2012, 61f).

Forschungsschwerpunkte zum schulpraktischen Mentoring könnten zukünftig in Zusammenhang mit dem Begriff der sozialen Rolle die Phänomene Rollenkompetenz, Rollenbilanz, Rollenkonflikte, Rollenstress, Rollendruck oder Rollendistanz werden. Forschungsbefunde zu diesen Bereichen im schulpraktischen Mentoring bilden ein Desideratum. Im nachfolgenden Abschnitt wird Bezug auf das Rollenverhalten der/des Mentorin/Mentors in Dialogen genommen. Kommunikationsstile und Dialoge prägen den Begleitungsprozess und sind am Gelingen oder Misslingen der Zusammenarbeit oftmals verantwortlich. Daher ist es notwendig, dass sich Mentor/innen damit auseinandersetzen.

\section{Rollenverhalten der/des Mentorin/Mentors in Dialogen}

Aus der Mentor/innenforschung geht hervor, dass es für die Gestaltung von Dialogen keinen einheitlichen konzeptionellen Rahmen gibt. Inhalte und Phasengestaltungen sind sehr unterschiedlich und weisen auf kein allgemeingültiges Verhalten der/des Mentorin/Mentors hin. In den meisten Mentoringdialogen wird nach Harrison et al. (2005) zwischen einer Phase des Zurückverweisens („Dekonstruktion“) und einer Phase des Vorausschauens („Konstruktion“) unterschieden. Die Art der Kommunikation spielt eine große Rolle. Die Forderung nach Gleichbehandlung, der Wunsch nach Akzeptanz oder einer wechselseitig profitierenden Unterhaltung liegen dem Mentoring zugrunde. 
„Der Mentor (sic!) ist zwar an Erfahrung und Wissen reicher, dennoch spielt beim Mentoring mehr als bei anderen Formen der Beratung und Unterstützung die reziproke, auf Augenhöhe stattfindende Kommunikation eine wichtige Rolle (u.a. Johnson 2003:130). Was den Mentor (sic!) von Coach (sic!) und Führungskraft unterscheidet ist die persönliche Nähe und der damit verbundene Austausch auf Augenhöhe." (Pflaum 2017, 50)

Für Hennissen (2008) ergeben sich im Mentoringdialog drei Schlüsselaspekte: „Stil/Supervision“, „Input“ und „Zeit“ und diese wurden im MERID-Modell (MEntor (teacher) Roles In Dialogues) (Abbildung 19) integriert.

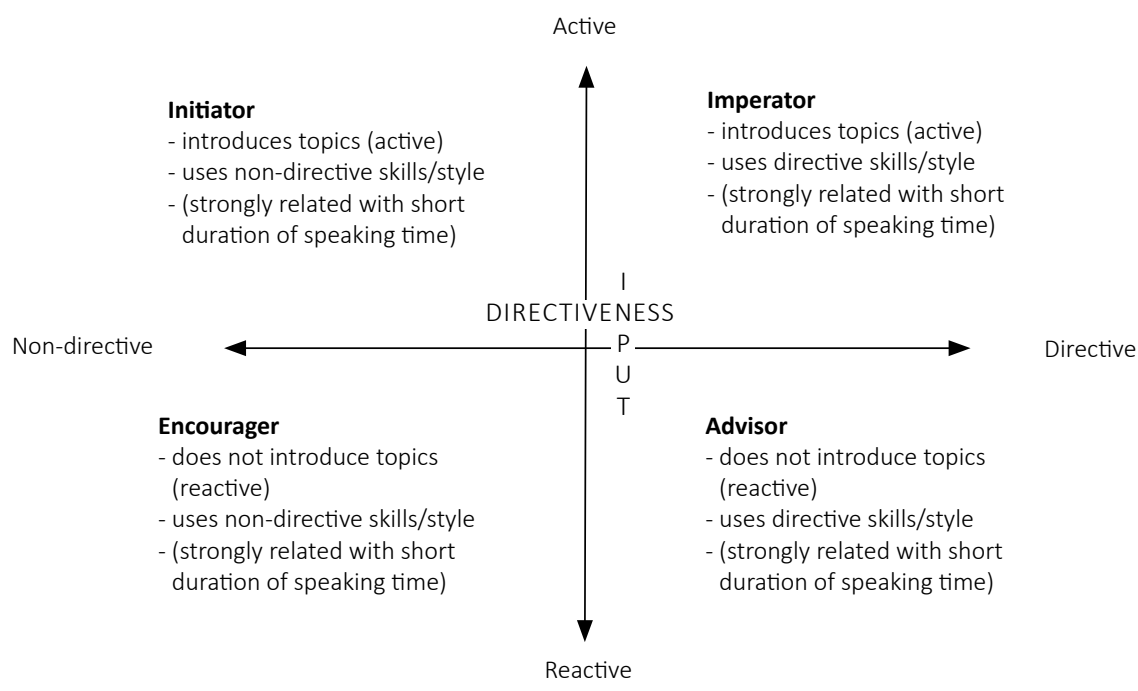

Abb. 19: The MERID-Modell (Hennissen et al. 2008, 177)

Das MERID-Modell als Dimensionsmodell umfasst den Grad der Direktivität (Directiveness) auf der horizontalen Achse und den Grad des Inputs auf der vertikalen Achse. Aus Sicht der/ des Mentorin/Mentors ergeben sich daraus vier verschiedene Rollen in Dialogen: initiator (Initiator/in), imperator (Imperator/in), advisor (Berater/in) and encouragor (Ermutigerin/Ermutiger). Die Dimension Direktivität mit den Polen Direktive/Nicht-Direktive zeigt das Steuerungsverhalten einer/eines Mentorin/Mentors in Gesprächssituationen auf.

"Most mentor teachers can be placed on the right side of the horizontal axis, the mentor teacher instructing the prospective teacher and talking from the standpoint of his or her own experience and evaluating the behaviour of the prospective teacher (Ben-Peretz \& Rumney, 1991; Edwards \& Protheroe, 2004; Evertson \& Smithey, 2001; Franke \& Dahlgren, 1996; Harrison et al., 2005; Hawkey, 1998b; Williams et al., 1998)." (Hennissen et al. 2008, 177)

Laut Studie von Hawkey (1998a) benötigt eine direktiv-orientierte/r Mentor/in mehr Redezeit als Mentor/innen, die Anregungen äußern. Bestätigt wurde die Annahme durch Studien von Crasborn et al. (2005), die aufzeigten, "that supervisory skills such as "giving advice" and "giving information", require more time than non-directive supervisory skills such as "asking questions" and "summarising content" (Hennissen et al. 2008, 177). Für Hennissen et al. (2008) ergibt sich 
daraus ein Zusammenhang zwischen der Dauer der Dialoge und dem Grad der Direktivität. Direktiv-orientierte Mentor/innen benötigen mehr Redezeit (im Modell als "strongly correlated with long duration of speaking time") als nicht-direktiv-orientierte Mentor/innen (im Modell als "strongly correlated with short duration of speaking time").

Die Dimension Input mit den Polen Active/Reactive verdeutlicht den Ausprägungsgrad wie Themenführung und aktive Beitragsetzung in Dialogen. Initiator/innen und Imperator/innen bestimmen die Themenwahl, leiten und koordinieren Themen verstärkter als advisors (Berater/innen) und encouragors (Ermutiger/innen) (Feiman-Nemser et al., 1992; Geldens et al., 2005; Haggarty, 1995; Wang et al., 2004). "According to Feiman-Nemser (2001), a mentor teacher can encourage prospective teachers to reflect on their own experience if he or she is able to understand his or her concerns." (Hennissen et al. 2008, 178) Die angeführten Befunde weisen darauf hin, dass die Themeneinführung in Dialoge ein wichtiger Indikator für die Rolle der/des Mentorin/Mentors ist.

Die vier Quadranten im MERID-Modell beschreiben die Rollen der/des Mentorin/Mentors mit den entsprechenden Aufgabenzuschreibungen (Tabelle 23):

Tab. 23: Rollen im MERID-Modell (Hennissen et al. 2008, 178f)

\begin{tabular}{ll}
\hline Rolle/n & Aufgabenbeschreibung zum MERID-Modell \\
\hline initiator & Einführen in das Thema \\
& Verwenden von direktiven Interventionen (Du solltest ...) \\
imperator & Kurzes Einführen in die Thematik \\
& Anleiten zur Beschäftigung mit Themen (Was würdest du ...) \\
advisor & Eingehen auf Fragen des/der Mentee/s \\
& Austeilen von Ratschlägen und Tipps im Sinne von Wirkungsmaßnahmen (Du kannst ...) \\
encouragor & Eingehen auf die Thematik der/des Mentee/Mentees \\
& Anleiten des Reflexionsprozesses \\
& Verwenden von nichtdirektiven Interventionen (Wie hast du ...) \\
\hline
\end{tabular}

Das Rollenverständnis unterscheidet sich in der Begegnung mit der/dem anderen im Hinblick auf das Hinführen zur Thematik, auf die Herangehensweise an unterschiedliche Thematiken und auf die sprachliche Anleitung von Prozessen. Forschungsbefunde zu den einzelnen Rollen erklären bestimmte Verhaltensweisen und geben Einsicht in Strukturabläufe.

Tab. 24: Forschungsbefunde zu Rollen im MERID-Modell (Hennissen et al. 2008, 178f)

\begin{tabular}{ll}
\hline Rolle/n & $\begin{array}{l}\text { Forschungsbefunde zu den Rollen im MERID-Modell } \\
\text { (Hennissen et al. 2008, 178f) }\end{array}$ \\
\hline initiator & $\begin{array}{l}\text { Nach dem Befund von Feiman-Nemser et al. (1992) geben Initiator/innen-Typ-Mentor/ } \\
\text { innen zunächst Komplimente ab und kommentieren anschließend alle Teile. Hughes (1998) } \\
\text { stellt fest, dass Mentor/innen zunächst Gesehenes besprechen, anschließend aus ihrer Sicht } \\
\\
\text { Ungeeignetes kommentieren und schließlich ihre (mögliche) Verhaltensweise darlegen. }\end{array}$ \\
\hline imperator & $\begin{array}{l}\text { Timperley (2001) weist darauf hin, dass Imperator/innen-Typ-Mentor/innen nach einer } \\
\text { Schulung/einem Training/einer Ausbildung in der Lage sind, adressatengerecht Themen der }\end{array}$ \\
& Mentee auszudrücken und zu formulieren. \\
\hline advisor & $\begin{array}{l}\text { Dunn et al. (1993) behaupten, dass Berater/innen-Typ-Mentor/innen den Prozess des Nach- } \\
\text { denkens beim Mentee nicht besonders fördern. Sie geraten schnell in das Beantworten von }\end{array}$ \\
& Fragen und Kommentaren.
\end{tabular}




\begin{tabular}{ll}
\hline Rolle/n & $\begin{array}{l}\text { Forschungsbefunde zu den Rollen im MERID-Modell } \\
\text { (Hennissen et al. 2008, 178f) }\end{array}$ \\
\hline encouragor & $\begin{array}{l}\text { Edwards et al. (1996) und Harrison et al. (2005) kehren die Bedeutung der Rolle der/des } \\
\text { Ermutigerin/Ermutigers hervor und betonen, dass jene Ermutiger/innen-Typ-Mentor/innen } \\
\text { einen Dialog auf Grundlage der Anliegen der Mentee gestalten. Hierbei fördern sie Reflexions- } \\
\text { fähigkeit im Sinne der Professionalisierung zur Lehrperson. }\end{array}$ \\
\hline
\end{tabular}

Hennisson et al. (2008) geben kein Urteil über eine positive oder negative Wirkungsweise - im Sinne von Schwarz-Weiß-Denken - der unterschiedlichen Rollen ab. Der Autor meint dazu: "How a person learns a particular set of knowledge and skills is strongly intertwined with the situation in which this person learns." (Hennisson et al. 2008, 180) Aus diesem Grund schlagen Hennisson et al. (2008) vor:

1. Mentee sollen die Möglichkeit haben, mit unterschiedlichen Typen von Mentor/innen zusammenzukommen.

2. Mentor/innen sollen in der Ausbildung die unterschiedlichen Rollen kennenlernen und Mentor/innen "should be able to take several roles in dialogues as distinguished in the MERID model.” (Hennisson et al. 2008, 180)

Nach Befunden von Crasborn et al. (2011) waren Mentor/innen ( $\mathrm{n}=20)$ im MERID-Modell vor allem im Quadranten der/des Imperatorin/Imperators positioniert (Crasborn et al. 2011, 327). Crasborn et al. (2011) bestätigten somit Befunde, wonach Mentor/innen ein direktives Gesprächsverhalten aufweisen und gerne Ratschläge und Tipps äußern (Dunne et al. 1997; Franke et al. 1996; Timperley 2001; Wang et al. 2004; Williams et al. 1998). Bezugnehmend zum MERID-Modell wurde das SMART-Trainingsprogramm (Supervisory Skills for Mentor Teachers to Activate Reflection in Teaching) konzipiert, das darauf ausgelegt ist, Mentor/innen ein sprachliches Werkzeug in die Hand zu geben und durch Fragestellungen den Reflexionsprozess bei Studierenden anzuleiten und zu fördern (Crasborn et al. 2014, 382).

Wie Dialoge aufgebaut werden und wie sich das Rollenverhalten von Mentor/in und Mentee äußert, hängt schließlich von beiden Partner/innen ab, die im Mentoring aufeinander treffen. Für einen gelingenden Prozess muss es Ziel sein, „ein Kooperationssystem aufzubauen, in dem sich beide mit ihren Erwartungen und Vorstellungen wiederfinden“" (Schmid et al. 2011, 41) und gegenseitige Entwicklungen zulassen.

Zusammenfassend kann gesagt werden: Rhodes (2002) und Schnautz (2014) weisen in Publikationen darauf hin, dass Mentor/innen vor allem zur „(1) Verbesserung der sozialen Fähigkeiten und des emotionalen Wohlbefindens, zur (2) Entwicklung kognitiver Fähigkeiten durch Dialog und Zuhören und zur (3) Identitätsentwicklung" (Schnautz 2014, 19; Rhodes 2002) bei Mentee beitragen. Damit diese Komponenten erfüllt werden, brauchen Mentor/innen die Bereitschaft zur Tätigkeit, Kenntnisse zu den Anforderungen in den Praktika und für sich eine klare Rollen- und Funktionsdefinition. Seitens der Ausbildungsinstitutionen benötigt es einen Weitblick bei Auswahl der Mentor/innen, Aufgabenstellung bzw. Fortbildungskonzepten für Mentor/innen.

Im Folgenden werden nun Phasen und Modelle im Mentoringprozess vorgestellt und diskutiert.

\subsubsection{Phasen und Modelle im Mentoringprozess}

In diesem Unterkapitel werden exemplarisch Konzepte zu Phasen und Modellen von Mentoring zunächst vor allem aus den Bereichen der Personalentwicklung für Unternehmen und der Bildungswissenschaften diskutiert. Die angeführten Beispiele wurden nach den Prinzipien Verständlichkeit und Umsetzbarkeit in den pädagogischen Alltag ausgewählt. Sie können somit Anhaltspunkte für 
schulische Mentoringprozesse liefern. Auffallend ist bei diesen Modellen, dass manche eine zeitliche Sequenzierung, manche die inhaltlichen Elemente eines Prozesses, andere die Beziehungsebene und einige alle Dimensionen berücksichtigen. Zunächst werden Modelle im Kontext der wirtschaftlichen Zugänge beschrieben. Im Anschluss werden aus dem Bereich der Bildungsforschung das DreiEbenen-Modell von Niggli (2005) und das Reflexionsmodell ALACT nach Korthagen (1985) vorgestellt. Nach jeder inhaltlichen Beschreibung erfolgt eine Deutung der Phasen bzw. Modelle in Form der Kernidee bezugnehmend auf schulpraktisches Mentoring. Folgende Konzepte zu Phasen bzw. Modellen im Mentoringprozess werden beschrieben (Tabelle 25):

Tab. 25: Auflistung der im folgenden ausgewählten Phasen bzw. Modelle

\begin{tabular}{|c|c|}
\hline Phasen bzw. Modelle & Kurzbeschreibung \\
\hline Phasen nach Kram & $\begin{array}{l}\text { Vier beobachtbare aufeinanderfolgende Phasen mit dem Ziel der Selbst- } \\
\text { ständigkeit und anschließender Auflösung }\end{array}$ \\
\hline Management-Modell nach Mentus & $\begin{array}{l}\text { Ständige Ist-Soll-Analyse und intensive Reflexion mit Ausrichtung auf } \\
\text { umsetzbare Zieldefinitionen }\end{array}$ \\
\hline GROW-Modell nach Whitmore & Systematische Prozessgestaltung ausgehend von Zieldefinition(en) \\
\hline 4R-Modell nach Logan & $\begin{array}{l}\text { Mentoringbeziehung mit Stärke-Schwäche-Analyse und Entwickeln von } \\
\text { Zielperspektive(n) }\end{array}$ \\
\hline Drei-Ebenen-Modell nach Niggli & Führung von individuellen Beratungsgesprächen auf unterschiedlichen Ebenen \\
\hline ALACT-Modell nach Korthagen & $\begin{array}{l}\text { Schritte zur Entwicklung der Selbstreflexion und handlungsorientierten } \\
\text { Selbstregulation }\end{array}$ \\
\hline
\end{tabular}

Die Kurzbeschreibungen weisen darauf hin, dass hinter allen angeführten Phasen und Modellen unterschiedliche Herangehensweisen in der Prozessgestaltung zu Grunde liegen. Für das schulpraktische Mentoring erlangen sie insofern Bedeutung, als dass sie in der Beschreibung auf einen ständigen Reflexionsprozess und auf das Wissen um personenspezifische Ressourcen hinweisen. Einige Modelle beschreiben auch die Bedeutung der Übertragung der Verantwortung von der/ vom Mentor/in auf die/den Mentee im Laufe des Prozesses. Die Reihenfolge der Beschreibungen richtet sich nach der in Tabelle 25 ausgerichteten Anordnung.

\section{Phasen nach Kram}

Kram (1983), die mittels einer qualitativen Studie psychosoziale und karrierebezogene Funktionen im Mentoring rekonstruierte, analysiert im Mentoringprozess vier aufeinanderfolgende Phasen (Fuge 2016, 34).

Tab. 26: Phasenbezeichnungen nach Kram (Kram 1983)

\begin{tabular}{llll}
\hline Phase der & Phase der & Phase der & Phase der \\
Initiation & Kultivation & Separation & Redefinition \\
\hline
\end{tabular}

Die vier Phasen: Initiation, Kultivation, Separation und Redefinition folgen nach einem linearen Prinzip für einen bestimmten Zeitabschnitt.

- Die Phase der Initiation ist geprägt vom Kennenlernen, vom Darlegen der Wünsche, Hoffnungen und Erwartungen, vom kommunikativen Austausch zu Fragen des Berufsalltags, von der Interaktion und dem Engagement von Mentor/in und Mentee. Die/Der Mentor/in übt 
die Rolle der/des Unterstützerin/Unterstützers aus. Sie/Er wird zur Identifikationsfigur aufgrund der beruflichen Kompetenzen (Oettler 2009, 20f).

- Die eigentliche Persönlichkeitsentwicklung im Mentoringprozess zeigt sich in der Phase der Kultivation. Die/Der Mentor/in ist zunächst in der Expert/innenrolle. Allmählich geben sie/er Verantwortung an Mentee ab. Das Gefühl des Wichtigseins, der persönlichen Zufriedenheit und des Gebrauchtwerdens ist kennzeichnend für die/den Mentor/in. Die/Der Mentee agiert im geschützten Raum, gewinnt an Selbstvertrauen und Eigenständigkeit und handelt aus der Rollenvorstellung der/des Mentorin/Mentors. Vertrauen, Respekt und eine gewisse Intimität prägen die Phase im Optimalfall (Oettler 2009, 21f).

- Die Phase der Separation ist gekennzeichnet durch ein Abnabeln zwischen den Beteiligten. Die/Der Mentor/in erkennt für sich, dass sie/er die Aufgabe des Beistandes bei der Kompetenzentwicklung zufriedenstellend erfüllt hat. Die/Der Mentee übernimmt Führungsrollen und ist bereit für psychologische Loslösung (Oettler 2009, 22).

- Die Phase der Redefinition ist nach dem bereits stattgefundenen Entwicklungsprozess beider beteiligten Mitglieder mit der Auflösung der offiziellen Mentor/in-Mentee-Beziehung verbunden. Auf informellem Weg könnte aufgrund von hohen Empathiewerten weiterhin eine Interaktion stattfinden (Oettler 2009, 22f).

Kernidee dieses rekonstruierten Phasenmodells liegt darin, dass es sich um ein Modell des Annäherns und Ablösens handelt. Im Laufe des gemeinsamen Prozesses - geprägt von einem werteorientierten Umgang - überträgt die/der Mentor/in die Verantwortung an die/den Mentee und zieht sich in der direkten Rolle als Vermittler/in und Unterstützer/in zurück. Die/Der Mentee erfüllt die Aufgabe in Eigenverantwortung.

\section{Management-Modell nach Mentus}

Das Modell „verknüpft ein vierstufiges organisationales Vorgehen für das Mentoring-Programm mit einem sechsstufigen Mentoring-Prozess." (Graf et al. 2017, 84f)

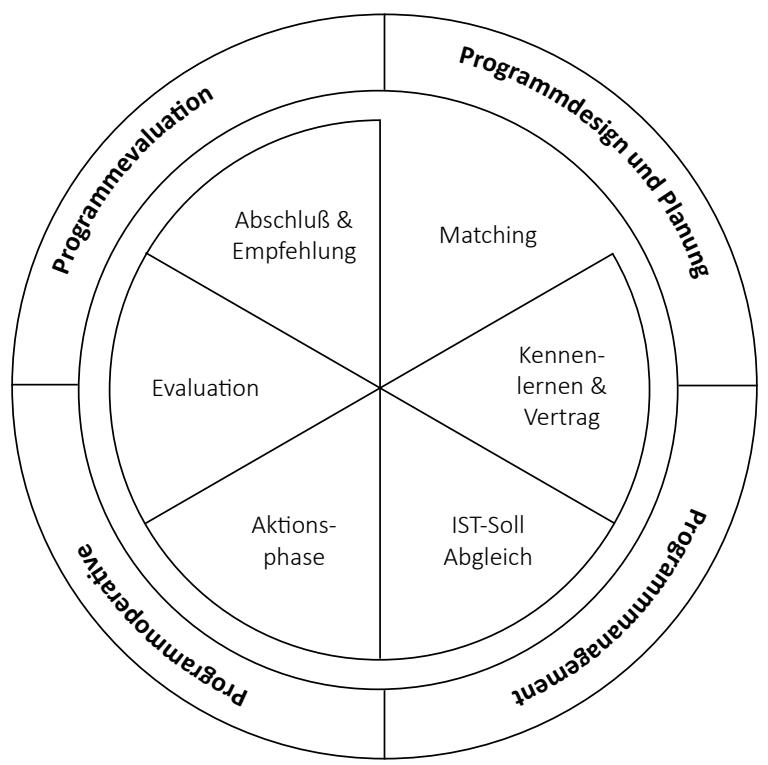

Abb. 20: Vorgehensmodell im Mentoring nach Mentus-Modell (Graf et al. 2017, 85) 
Hinter dem in Projekt- und Qualitätsmanagement zum Tragen kommenden Modell verbirgt sich die Logik der folgenden vier Schritte: Konzeption - Planen - Durchführung - Auswertung. Hierbei ist das Planen im Mentoringprozess ständig integriert und kann nicht als vorbereitetes Planen betrachtet werden.

Tab. 27: Management-Modell (Graf et al. 2017, 85)

\begin{tabular}{llll}
\hline Konzeption & Planen & Durchführung & Auswertung \\
\hline „Denken“ & „Planen“ & „Handeln“ & „Lernen“ \\
\hline
\end{tabular}

Im Mentus-Modell werden in der ersten Phase Ziele festgelegt und in ein Konzept zusammengefügt. Graf et al. (2017) betonen: „Das Matching, d.h. die Zusammenstellung der Tandems aus Mentor und Mentee, gehört zu den erfolgskritischen Schritten im Mentoring." (Graf et al. 2017, 137) Die Autorin und der Autor zählen in ihrer Publikation (2017) Beispiele von Unternehmen auf, wie Tandems im Matching entstehen: klassisch (eine Mentor/in stellt sich zur Verfügung), informell (Unternehmen schlägt Mentee erfahrene/n Mitarbeiter/in vor), cross (Programmleitung beachtet Ansätze wie Cross-Company, Cross-Sektor, Cross-Gender, CrossCulture), Peer-to-Peer (Mentor/in wählt aus zwei Vorschlägen; Auswahlkriterien sind Expertise, Werdegang, Persönlichkeit) (Graf et al. 2017, 138). Im Weiteren wird ein MentoringVertrag empfohlen, indem gemeinsame Vereinbarungen - Organisation des Prozesses, Inhalte und Entwicklungsziele, Erwartungshaltung und Regeln der Zusammenarbeit, Vereinbarungen bei Problemen - schriftlich festgehalten werden (Graf et al. 2017, 140; 185). Die zweite Phase ist, nachdem der Kooperationsvertrag vereinbart wurde, geprägt durch den Ist-Soll-Abgleich. Eine Ist-Analyse erhebt die derzeitigen Kompetenzen und Ressourcen und mündet in eine Soll-Definition mit dem Ziel eines Aktionsplanes (Graf et al. 2017, 190). Die Umsetzung der Entwicklungsziele und Erwartungen erfolgt mit der Aktionsphase in der Phase Programmoperative. Die temporäre und inhaltliche Ausgestaltung ist hierbei von Tandem zu Tandem unterschiedlich. Die Teamplayer vollziehen einen Lernprozess, der im Idealfall Reflexions- und Selbstreflexionseinheiten beinhaltet und zu einer Erweiterung des Handlungsspektrums und der Kompetenzentwicklung führt. Die Mentoring-Beziehung spielt in der Differenzierung eine wesentliche Rolle. Im Interesse des Teams und des Unternehmens raten Graf et al. (2017) zur Evaluierung des eigenen Prozesses (Graf et al. 2017, 209) und zur Erfassung eines Commitments mit der Ausrichtung auf weitere Maßnahmensetzungen (Graf et al. 2017, 213). Die Phase Programmevaluation bildet somit den Abschluss des Programmes.

Kernideen dieses Phasenmodells sind die ständigen Abgleichungen von Vereinbarung und Durchführung, von kritischer Betrachtung der Umsetzungsfähigkeit und fehlenden Voraussetzungen, von Begutachtung der Konzeption und Analyse. Die Überlegungen führen in einen intensiven Reflexionsprozess, der stets in den Aufbau gestaltbarer Zieldefinitionen mündet.

\section{GROW-Modell nach John Whitmore}

Das vom Sportpsychologen und Coach John Whitmore entwickelte Modell zielt darauf ab, Entscheidungen durch vorheriges Abklären von Realitätsstrukturen, Auflisten von unterschiedlichen Handlungsmöglichkeiten und Bereitschaft der Zielumsetzung zu treffen (Beraterkreis 2019). 
Tab. 28: GROW-Modell nach Whitmore (Faix et al. 2017)

\begin{tabular}{llll}
\hline Goals & Reality & Options & Will \\
\hline Ziele & Realität & Möglichkeiten, & Wille, \\
& & Optionen & Bereitschaft \\
\hline
\end{tabular}

Die Phasen Goals, Reality, Options und Will sind kennzeichnend für den Ablauf des Mentoringprozesses im GROW-Modell und unterstützen letztendlich Zielfindung und Problemlösung in der Eigenverantwortlichkeit. Ein Problem oder Sachverhalt wird schrittweise und systematisch in Teilaspekte aufgeteilt und durch Zusammenschau, Hinterfragen, Erstellen von unterschiedlichen Lösungsmöglichkeiten wird eine Zielfindung angestrebt (Schuy 2019). Für den schulpraktischen Mentoringprozess wurden folgende Überlegungen seitens der Autorin dieser Arbeit angestellt. Die angeführten Fragestellungen könnten für Mentor/innen im schulpraktischen Mentoring hilfreich sein:

Tab. 29: Fragestellungen und Absichten zum GROW-Modell im schulpraktischen Mentoringprozess

\begin{tabular}{|c|c|c|}
\hline Phasen & Fragestellungen & Absicht \\
\hline Goals & $\begin{array}{l}\text { Was möchten Sie in dieser Unterrichtseinheit/an diesem Schultag/in } \\
\text { dieser Schulwoche/in diesem Praktikum erreichen? Welche Zielsetzun- } \\
\text { gen erachten Sie als realistisch umsetzbar? }\end{array}$ & Zieldefinition \\
\hline Reality & $\begin{array}{l}\text { Was haben Sie bereits erreicht? Was steht Ihnen zur Verfügung, damit } \\
\text { Sie das Ziel erreichen? Was brauchen Sie, damit Sie das Ziel erreichen? } \\
\text { Wie wichtig ist Ihnen persönlich die Zielerreichung? Was tun Sie, } \\
\text { wenn Sie das Ziel nicht erreichen? }\end{array}$ & $\begin{array}{l}\text { Ist-Standanalyse } \\
\text { und Erkennung der } \\
\text { Ressourcennutzung }\end{array}$ \\
\hline Options & $\begin{array}{l}\text { Welche konkreten kurz-, mittel- und langfristigen Handlungen setzen } \\
\text { Sie, um das Ziel zu erreichen? Wie strukturieren Sie den Ablauf der } \\
\text { Umsetzung? Auf welche Ressourcen können Sie zurückgreifen? Wel- } \\
\text { che positiven Erfahrungen haben Sie bereits gemacht? Wie motiviert } \\
\text { und bereit sind Sie Handlungen in Eigenverantwortung zu initiieren? }\end{array}$ & $\begin{array}{l}\text { Selbstreflexion und } \\
\text { Finden von Handlungs- } \\
\text { optionen in Abgleichung } \\
\text { mit der Zieldefinition }\end{array}$ \\
\hline Will & $\begin{array}{l}\text { Was ist wann zu tun? Wer kann Sie unterstützen? Wie läuft der kon- } \\
\text { krete Handlungsprozess ab? Welche Handlungen setzen Sie, wenn sich } \\
\text { Schwierigkeiten ergeben? }\end{array}$ & $\begin{array}{l}\text { Umsetzen der Hand- } \\
\text { lungsschritte }\end{array}$ \\
\hline
\end{tabular}

Dieses Modell zeigt auf, wie durch schrittweises Heranführen mit unterschiedlichen Fragestellungen Mentor/innen Mentee führen und begleiten können, und sie sich über Handlungsstrukturen bewusst werden und in weiterer Folge in Eigenverantwortung Handlungen planen, reflektieren, organisieren und ausführen (Faix et al. 2017, 45).

Als Kernidee dieses Phasenmodells könnten für das schulpraktische Mentoring Überlegungen zur Erstellung von kurz-, mittel- und langfristigen Zielvorstellungen und Zieldefinitionen sein, die in konkrete und operierende Handlungsschritte umgewandelt werden. Die/Der Mentor/in zieht sich im Laufe des Prozesses aus der Verantwortung zurück und überlässt der/dem Mentee die Eigenverantwortung. Dieses Modell ist geprägt von wechselseitigem Vertrauen und positiver Haltung der/dem anderen gegenüber. 


\section{R-Modell nach Robert Logan}

Dieses Modell findet vor allem im persönlichkeitsbildenden und spirituellen Bereich Anwendung. In der Mentoring-Beziehung wird zu Beginn der Reflexionsprozess zu Fragen des alltäglichen und persönlichen Lebens in den Mittelpunkt gestellt und Ziele (Neuausrichtung) neu definiert. Das Aufzeigen und Anführen möglicher Ressourcen vertieft die Mentoring-Beziehung zwischen der/dem Mentor/in und der/dem Mentee.

Tab. 30: 4R-Modell nach Logan (Faix et al. 2017, 46)

\begin{tabular}{llll}
\hline Reflect & Refocus & Resources & Relate \\
\hline Reflexion & Neuausrichtung & Ressourcen & Mentoring-Beziehung \\
\hline
\end{tabular}

In der Kommunikation verwendet die/der Mentor/in die Strategien: „1. Stärke-SchwächenQuadrat, 2. EBV-Analyse, 3. Analyse der idealen Zukunft“ (Faix et al. 2017, 46). Im Gesprächsverlauf des Stärke-Schwächen-Quadrats werden zunächst Stärken hervorgehoben, anschließend werden etwaige Probleme angesprochen und wird nach Lösungen gesucht. Durch die EBVAnalyse (Erreichen - Bewahren - Vermeiden) kann die/der Mentee Vorstellungen äußern, Ziele festlegen, Ängste besprechen und Lösungswege konkretisieren. Liebsames wird von der/ dem Mentee aufgenommen und Unliebsames verworfen. Gerade diese Art der Analyse benötigt eine gute Mentoring-Beziehung, da viele persönliche Vorlieben angesprochen werden. Die/Der Mentor/in benötigt Feinfühligkeit und Aufmerksamkeit (Faix et al. 2017, 46). Mentor/in und Mentee besprechen gemeinsam weitere Aktionsschritte und versuchen während des Gesprächs, unterschiedliche Perspektiven einzunehmen und mit Blick auf die Zukunft abzuwägen (Faix et al. 2017, 47).

Kernidee dieses Modells liegt in einer persönlichen Auseinandersetzung mit leistungsorientierten und emotionalen Stärken und Schwächen sowie in einem Einblick in Befindlichkeit und Gefühlswelt der/des Mentee/Mentees. Überzeugungen, Überlegungen und emotionale Ausdrucksformen der/des Mentee/Mentees stehen im Mittelpunkt des sozialen Gefüges und des Kontextes Mentoring. Gespräche zu Ressourcen, Selbstregulationsmechanismen, Resilienz, Potentialorientierung usw. können den Prozess der Begleitung, der auf Neuregelung und Zukunftsorientierung ausgelegt ist, prägen. Im Mittelpunkt steht also eine subjektorientierte bzw. personenspezifische Begleitform.

\section{Drei-Ebenen-Modell nach Alois Niggli}

Niggli (2005) beschreibt im Modell drei Ebenen von Gesprächsverläufen für das schulpraktische Mentoring mit unterschiedlichen inhaltlichen Ausrichtungen.

Tab. 31: Drei-Ebenen-Modell nach Niggli (Niggli 2005, 4)

\begin{tabular}{lll}
\hline Ebene des Wirkens & Ebene des Erkennens & Ebene des Sich-Selbst-Seins \\
\hline Bearbeiten konkreter Skills & $\begin{array}{l}\text { Wahrnehmen von nicht beobachtba- } \\
\text { ren Handlungsvoraussetzungen }\end{array}$ & $\begin{array}{l}\text { Auseinandersetzen mit Haltungen, } \\
\text { Zielsetzungen und Fähigkeiten }\end{array}$ \\
\hline Feedback-Gespräch & Reflexives Praxisgespräch & Coaching der Person \\
\hline
\end{tabular}


Niggli (2005) verfolgt mit diesem Modell einen entwicklungsorientierten Ansatz, indem Mentor/innen den Prozess der individuellen Professionalisierung bei Studierenden durch gesprächsführende Elemente unterstützen und begleiten. Ziel ist die Umsetzung der besprochenen Inhalte auf der Handlungsebene durch die/den Mentee. Auf der ersten Ebene wird durch ein Feedback-Gespräch nach Absolvierung einer Unterrichtseinheit versucht, das weitere pädagogische Handeln zu verbessern. Im reflexiven Praxisgespräch erfolgt ein Austausch über theoretisches Wissen zu Handlungen und Klärung der jeweiligen Situation. Auf der dritten Gesprächsebene werden Normen und Werte diskutiert sowie die persönliche Berufsorientierung thematisiert. In der Diskussion werden Überlegungen angestellt, wie diese in der Unterrichtsgestaltung deutlich werden (können) (Ittel et al. 2009, 200).

Kernidee dieses Modells ist die Fokussierung von Beratungsgesprächen auf Unterrichtshandlungen, Hintergrundwissen zum Unterrichtsgeschehen und auf Bereiche der Werteorientierung und des individuellen Selbst-Seins.

\section{ALACT-Modell nach Korthagen}

Das ALACT-Modell ist ein Reflexionsphasenmodell, das dazu dient, in einen Reflexionszyklus einzusteigen. Korthagen (1985) stellt die Phasen (Tabelle 32) in einem Kreismodell dar.

Tab. 32: Phasen im ALACT-Modell nach Korthagen (1985) (Korthagen et al. 2005, 49)

\begin{tabular}{|c|c|c|c|c|}
\hline Action & $\begin{array}{l}\text { Looking back } \\
\text { on the action }\end{array}$ & $\begin{array}{l}\text { Awareness of } \\
\text { essential aspects }\end{array}$ & $\begin{array}{l}\text { Creating alternative } \\
\text { methods of action }\end{array}$ & Trial \\
\hline
\end{tabular}

Ausgangs- und Endpunkt im Reflexionsprozess ist das Handeln der Person (Action und Trial). Beim Rückblick auf die Handlung (Looking back on the action) kommt es zu einem Abgleichen der Zielvorstellungen mit tatsächlich Erreichtem, wobei mögliche Schwierigkeitsstufen oder Hindernisse in Betracht gezogen werden sollten. Für die Phase 2 geben Korthagen et al. (2005) folgende Fragestellungen vor:

"What was the context? What did you want? What did you do? What were you thinking? How did you feel? What did the pupils want? What did the pupils do? What were the pupils thinking? How did the pupils feel?" (Korthagen et al. 2005, 50)

In einer weiteren Phase erfolgt die Achtsamkeit auf zusätzliche wesentliche Aspekte (Awareness). In der vierten Phase werden Handlungsfolgen entwickelt (Creating alternative methods of action), die anschließend ausprobiert werden (Trial). Der Kreislauf beginnt abermals mit der Handlung (Korthagen et al. 2010, 531). Im Wesentlichen geht es in diesem Phasenmodell darum, Handlungen auszuführen, Handlungsschritte retrospektiv nachzuvollziehen, mögliche Ereignisse oder Vorkommnisse vorschauend miteinzubeziehen und Handlungen zielorientiert zu gestalten, um verbesserte Handlungen zu setzen. Crasborn et al. (2014) sind der Meinung, dass solche Schritte in der Begleitung erlernt und angewendet werden sollen (Crasborn et al. 2014, 382). Wie bei Schön (1983) steht in diesem Modell das Handeln der Person und nicht die Person selbst im Mittelpunkt des Reflexionsprozesses. Wenn dieses Modell auf schulpraktisches Mentoring übertragen wird, dann kann Folgendes beschrieben werden: Die/Der Mentee konzentriert sich auf das Handeln und wird durch Anleitung und Begleitung zur/zum reflektierenden Praktiker/in (Schön 1983) geführt. Die/Der Mentor/in übernimmt in ihrer/seiner 
Rolle die Aufgabe der Moderation und strukturiert den Prozess, forciert den Perspektivenwechsel und den gemeinsamen Austausch und verfolgt „die kollaborative Entwicklung neuer Ideen“ (Krause 2019, 89).

Kernidee dieses Modells ist der Ausbau der Reflexionsfähigkeit, indem Handlungen reflektiert und analysiert werden und eine Verbesserung durch einen reflexiven Zugang bei neuerlicher Setzung von Handlungen erreicht werden. Ziel ist, in einen Prozess der Professionsentwicklung einzutreten.

Die beschriebenen Modelle sind eine kleine Auswahl mit unterschiedlichen Schwerpunktsetzungen. Sie wurden deshalb ausgewählt, weil sie einen proaktiven und entwicklungsorientierten Handlungsplan in den Mittelpunkt des Prozesses stellen. Für die Mentor/in-Mentee-Beziehung ist wichtig, wie die beteiligten Personen den Prozess interaktiv gestalten und wie die Beziehungskultur gelebt wird. Die Modelle halten auch vor Augen, wieviel Handlungsspielraum für Beteiligte möglich ist. Das Ansprechen von Stärken und Schwächen, die Formulierung von Zielvorstellungen, das kritische Rückwärtsschauen und Betrachten von Handlungen, eine kritische Selbstreflexion setzen ein Sich-Öffnen der/dem anderen gegenüber und eine Vertrauensbasis voraus. Aus institutioneller Sicht kommt hier das Kriterium der „richtigen“ Auswahl der/des Mentorin/Mentors ins Spiel. Aus personeller Sicht sind Persönlichkeitsmerkmale wie Empathiefähigkeit, Vertrauenswürdigkeit, positive Lebenseinstellung, aber auch Fähigkeiten wie Kommunikation, Beratung und Mediation oder Kompetenzen wie Reflexionsfähigkeit, Selbstorganisation und Selbstregulation zur Erfüllung der Aufgabe als Mentorin/Mentors notwendig.

Nachfolgend wird nun auf die Bedeutung der Beziehungsstruktur im Mentoring eingegangen. Mentor/innen und Mentee bilden für einen gewissen Zeitabschnitt eine professionelle Arbeitsgemeinschaft. Was sollte am Beginn und während des Aufeinandertreffens beachtet werden? Was trägt zu einer stabilen Beziehung bei? Was sind die Kriterien einer guten Beziehung?

\subsubsection{Beziehungsstruktur Mentor/in und Mentee}

Die Beziehungsstruktur zwischen Mentor/in und Mentee ist sehr komplex. Das Beziehungsgefüge ist in einen sozialen Kontext eingebunden und agiert im Umfeld Schule und Ausbildungsinstitution. Die Unterkapitel Mentor/in-Mentee-Beziehungen als Kernelement, MatchingProzess, Intra- und Intersubjektivität, wertebasierte Gesprächsführung sowie person-zentrieter Ansatz und Verschränkung zur Neurowissenschaft werden nun beschrieben und Forschungsbefunde dazu dargelegt und diskutiert.

Tab. 33: Auflistung der im folgenden ausgeführten Unterkapitel

\begin{tabular}{ll}
\hline Beziehungsstruktur Mentor/in und Mentee & Kurzbeschreibung \\
\hline Mentor/in-Mentee-Beziehungen als Kernelement & Forschungsbefunde zu Mentor/in-Mentee-Beziehungen \\
Matching-Prozess & Optimierung bei Tandembildung \\
Intra- und Intersubjektivität & Sich und das Gegenüber in der Person wahrnehmen \\
Wertebasierte Gesprächsführung & Wirkprinzipien wertebasierter Gesprächsführung \\
Person-zentrierter Ansatz und Verschränkung zur & Aktivierung der neuropsychologischen und psychosozia- \\
Neurowissenschaft & len Ebene bei Beziehungen \\
\hline
\end{tabular}


Die Kurzbeschreibungen weisen darauf hin, dass das Thema Beziehungsstruktur in der vorliegenden Arbeit sehr unterschiedlich thematisiert wird. Die Überlegungen und vorgestellten Forschungsbefunde in den einzelnen Unterkapitelen verdeutlichen letztendlich, dass Begegnung immer mit Selbstreflexion und Werthaltung der/des Mentorin/Mentors bzw. der/des Mentee/ Mentees einhergeht. Die Ausführungen beginnen mit dem Unterkapitel Mentor/in-MenteeBeziehungen als Kernelement.

\section{Mentor/in-Mentee-Beziehungen als Kernelement}

Während eines schulpraktischen Mentoringprozesses arbeiten Mentor/in und Mentee eng zusammen. Forschungsbefunde erkennen den Mehrwert einer guten Mentor/in-Mentee-Beziehung in der gegenseitigen Förderung der persönlichen und beruflichen Entwicklung (Hudson 2013). Autor/innen wie Bayer et al. (2015) bezeichnen Mentor/in-Mentee-Beziehungen sogar als Kernelement von Mentoring (Bayer et al. 2015, 4). Für Hudson (2013) startet sie ab dem Zeitpunkt, in dem die/der Mentor/in die/den Mentee in ihrer/seiner Klasse aufnimmt und akzeptiert (Hudson 2013,2). Das neu entstandene soziale Gefüge arbeitet dann zusammen und es entwickeln sich „professional-personal relationships“ (Hudson 2013, 2).

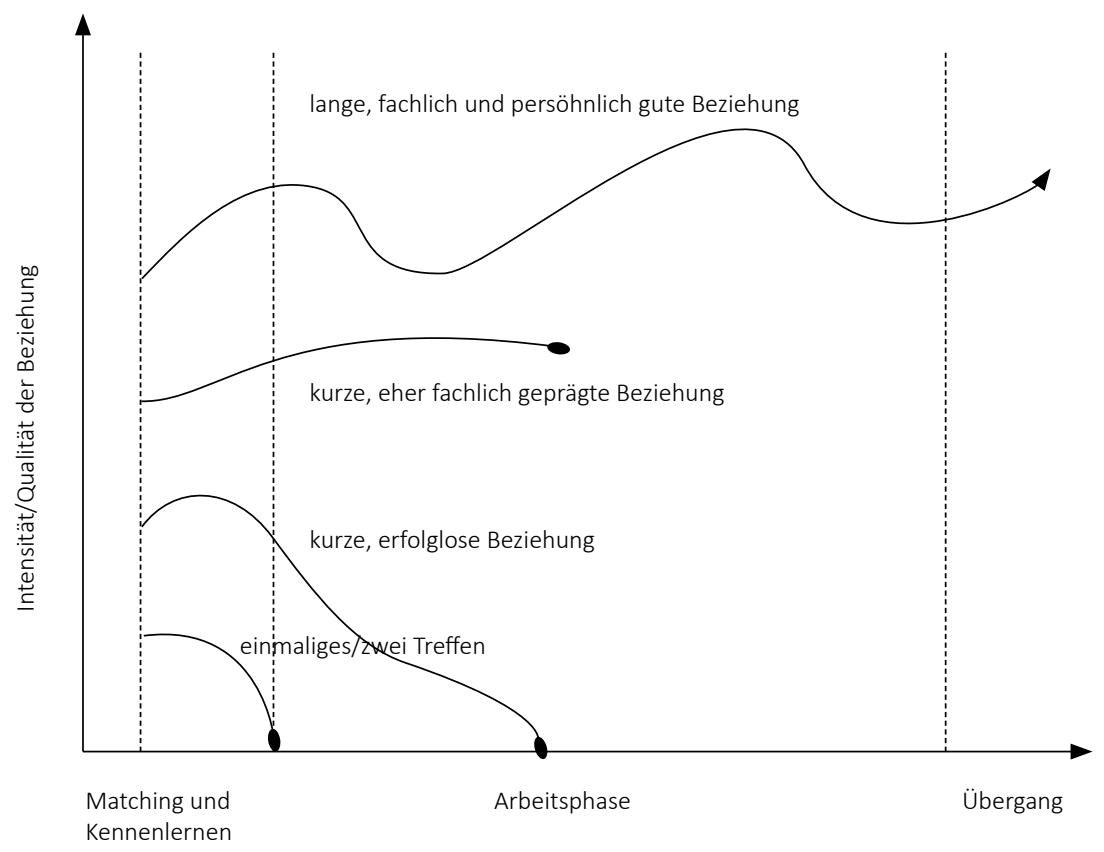

Abb. 21: Typische Verläufe von Mentoring-Beziehungen (Pflaum 2017, 253)

Forschungsbefunde nach Pflaum (2017) beschreiben in der Mentoring-Beziehung drei gegebene Phasen - Matchingphase, Arbeitsphase und eventuell Übergangsphase - und vier Verlaufsformen (Pflaum 2017, 252f). Die Länge der Arbeitsphasen (Abbildung 21) strukturieren sich Teams je nach Interaktion und Kommunikation selbst (Meixner et al. 2004, 245; Holt et al. 2002; Pflaum 2017, 64). Die Qualität bzw. Intensität der Beziehungen werden von unterschiedlichen Faktoren bestimmt: von den Persönlichkeiten und deren Fähigkeiten, Kenntnissen und 
Einstellungen, vom Kontext, in dem die Personen eingebunden sind und die Paarung von Mentor/in und Mentee (Hudson 2013, 3). Funktionierende Mentor/in-Mentee-Beziehungen besprechen nicht nur spezifische berufliche und fachliche Themen. Sie unterhalten sich auch über persönliche Fragestellungen. Pflaum (2017) kategorisiert diese Beziehungen als lange, fachlich und persönlich gute Beziehung. Wenn Teams bereits nach dem ersten (Matching und Kennenlernen) oder zweiten Treffen (in der Arbeitsphase) zerbrechen, dann wohl aus dem Grund, weil „die Chemie“ zwischen den Partner/innen nicht stimmt.

"Mentoring is distinguished from other types of relationships by the roles or functions played by the mentor." (Jacobi 1991, 511) Jacobi (1991) macht die/den Mentor/in primär für die Beziehungsgestaltung verantwortlich und räumt ein, dass deren/dessen Einstellung zu Rolle und Funktion entscheidend für die Prozessentwicklung ist (Jacobi 1991, 511). Andere Autor/innen beschreiben persönlichkeitsprägende Determinanten als wesentliche Gelingensbedingungen einer erfolgreichen Mentoring-Beziehung. So weist Niehoff (2006) darauf hin, dass Persönlichkeitsfaktoren wie Offenheit, Extrovertiertheit und Gewissenhaftigkeit von Mentor/innen idealer für Mentee sind (Niehoff 2006, 321). Turban et al. (2007) behaupten, dass die Big-FivePersönlichkeitsmerkmale - Extraversion, Offenheit, Verträglichkeit, Gewissenhaftigkeit, Neurotizismus - starken Einfluss auf die Ausprägungsform des Mentorings haben, wenngleich die Kategorienfestlegung für sie zu gering und Forschungsbefunde noch ausständig sind (Turban et al. 2007, 25). Daher schlagen sie für Untersuchungen die Einbeziehung weiterer Faktoren „wie Empathie, ,Learning Goal Orientation', Selbstbewusstsein und Reflexionsfähigkeit“ vor (Pflaum 2017, 80). Hudson (2013) ergänzt die Persönlichkeitsmerkmale mit Reflexionsbereitschaft, Unterstützungs- und Gesprächsbereitschaft, Fähigkeit des aktiven Zuhörens, positive Grundeinstellung und Vertrauen in die/den Mentee (Hudson 2013, 5).

Cherniss (2007) diskutiert das Konzept der emotionalen Intelligenz als bedeutendes Merkmal in der Mentoring-Beziehung. Garringer (2010) erklärt schulpraktisches Mentoring als gelungen, wenn sich die/der Mentor/in auf die Zielbilder konzentriert: Bemühungen um Verbundenheit, Verbesserung des Selbstwertgefühls und der Selbstwirksamkeit und Möglichkeit zur persönlichen und beruflichen Entfaltung in der Begleitung mit der/dem Mentee (Garringer 2010, 4). In Untersuchungen wird meist Augenmerk auf Verhaltensweisen der/des Mentorin/ Mentors gelegt.

Forschungsbefunde zum Verhalten von Mentee in Beziehungen bilden ein Desideratum. Olian et al. (1988) beschreiben zwei wesentliche Dimensionen für Mentee im Beziehungsgefüge:

"Job and career benefits through informations and external brokering provided by the mentor, and psychological benefits from the emotional support and friendship obtained within the relationship." (Olian et al. 1988, 19; Jacobi 1991, 510)

Nach Maynard (2000) muss die/der Mentee lernen, "how to manage mentors to ensure maximum benefits (Maynard, 2000)" (Hudson 2013, 4). Hudson (2013) ist sich sicher, dass eine gute Beziehung für beide Seiten fruchtbringend ist. "Undoubtedly, forming positive and productive relationships also requires mentees to exhibit desirable attributes and practices." (Hudson 2013,20 )

In der Mentoring-Forschung werden Beziehungsstrukturen und Ausprägungsformen meist retroperspektiv betrachtet. Johnson et al. (2010) und Fuge (2016) schlagen aus diesem Grund einen begleitenden Forschungssupport zur Erhebung von dynamischen und prozesshaften Entwicklungen vor (Johnson et al. 2010, 63; Fuge 2016, 74). Dem Beginn einer Mentoring-Bezie- 
hung wird eine wesentliche Bedeutung zugeschrieben. Es startet mit dem Aufeinandertreffen und Begegnen zweier Persönlichkeiten mit unterschiedlichen Charakteren aus unterschiedlichen Lebensumständen mit unterschiedlichen Lebensgeschichten. Aus diesem Grund wird folgend die Bedeutung des Matching-Prozesses diskutiert und werden Kennenlernformen vorgestellt. Leider findet der Matching-Prozess im Alltag des schulpraktischen Mentorings noch kaum Anwendung.

\section{Matching-Prozess}

Die Organisationsstrukturen von Praktikumsplätzen sind meist so angelegt, dass die Wahlmöglichkeit einer/eines Mentorin/Mentors nicht gegeben ist. Studierende sind somit in einer gewissen Abhängigkeitsposition (Haasen 2001; Fuge 2016).

„Erfahrungsgemäß schleppen sich Tandems, in denen es nicht funktioniert, über die Zeit. Sie äußern ihre Unzufriedenheit nicht und versuchen, nach außen, dem Tandempartner (sic!) und oft auch sich selbst gegenüber den Schein zu wahren." (Schmid et al. 2011, 88f)

Demzufolge könnte der Matching-Prozess nach dem Motto „Welche Partner/innen passen optimal zusammen?" eine besondere Gewichtung erlangen. Matching bedeutet in diesem Zusammenhang das Zusammenstellen von Tandems oder Gruppen im Mentoringprozess, um im Vorhinein „optimale“ Strukturen für die Zusammenarbeit zu schaffen. Tabelle 34 veranschaulicht einen Überblick zu Matching-Verfahren und zeigt jeweils die Vor- und Nachteile auf:

Tab. 34: Überblick zu Matching-Verfahren (Schmid et al. 2011, 86f; in Anlehnung an Fuge 2016, 46)

\begin{tabular}{|c|c|c|}
\hline $\begin{array}{l}\text { Matching- } \\
\text { verfahren }\end{array}$ & Vorteile & Nachteile \\
\hline $\begin{array}{l}\text { Externes } \\
\text { Matching }\end{array}$ & $\begin{array}{l}\text { Zusammenstellung durch die Organisation } \\
\text { Abstimmungsmöglichkeit von Zielen zum } \\
\text { Mentee } \\
\text { Geringer Zeitaufwand für Mentor/in und } \\
\text { Mentee } \\
\text { Möglichkeit zum Wechseln einer/eines } \\
\text { Partnerin/Partners (ge)geben }\end{array}$ & Gefahr des Mismatching \\
\hline $\begin{array}{l}\text { Selbst- } \\
\text { gesteuertes } \\
\text { Matching }\end{array}$ & $\begin{array}{l}\text { Einfluss auf die Auswahl der/des Partnerin/ } \\
\text { Partners } \\
\text { Berücksichtigung von Kriterien für die } \\
\text { Auswahl } \\
\text { Motivation und Engagement der Beteiligten }\end{array}$ & $\begin{array}{l}\text { Hoher Zeit- und Koordinationsaufwand vor } \\
\text { allem für (begehrte) Mentor/innen } \\
\text { Mangel an verfügbaren Mentor/innen } \\
\text { Problematik der Aufösung bei misslingender } \\
\text { Prozessgestaltung }\end{array}$ \\
\hline $\begin{array}{l}\text { Zufällige } \\
\text { Zuordnung }\end{array}$ & $\begin{array}{l}\text { Geringer Organisations-/ } \\
\text { Koordinationsaufwand }\end{array}$ & Gefahr des Mismatching \\
\hline
\end{tabular}

Die Autor/innen (Schmid et al. 2011; Fuge 2016) unterscheiden externes und selbstgesteuertes Matching und beschreiben ein Verfahren für eine zufällige Zuordnung. Organisationen haben bei der Zusammenstellung aufgrund von verschiedenen Anschauungen unterschiedliche Vorgehensweisen. Beim schulpraktischen Mentoring ist wohl eine Abwechslung des Einsatzes der Verfahren für Mentee sehr hilfreich. 
Fletcher (1998) beschreibt ein Modell der Zusammenstellung von Tandems, indem Mentor/innen Videopräsentationen anfertigten, in denen sie ihre Schule und das Vorhaben der Gestaltung der Beziehung vorstellten. Die Mentee konnten sich auf dieser Basis die/den Partner/in aussuchen. Die/Der Mentor/innen waren einem gewissen Wettbewerb ausgesetzt, die/der Mentee wählten ihre Begleiter/innen, von denen sie glaubten, dass sie gut zusammenarbeiten könnten (Fletcher 1998, 116).

Ein weiteres Beispiel zum Matching-Vorhaben erwähnt Fletcher (1998) durch das Beisein einer dritten Person beim ersten Zusammentreffen zwischen Mentor/in und Mentee. Diese erhält die Aufgabe der Analysefähigkeit der Bedürfnisse und das Hervorheben von Übereinstimmungen der Beteiligten. Sie koordiniert und analysiert das Erstgespräch und anschließend kommt es zur Entscheidung der Teambildung (Fletcher 1998, 116). Schmid et al. (2011) empfehlen keine blind-date Veranstaltungen zu initiieren, sondern raten zur Möglichkeit des informellen Kennenlernens der zugewiesenen Partner/innen über einige Wochen. In ungezwungenen und durch Eigeninitiative gestalteten Treffen sollten Erwartungen und Ziele geklärt werden und von beiden Seiten sollte eruiert werden, ob berufliche, persönliche und zwischenmenschliche Komponenten einem gemeinsamen Arbeitsprozess dienlich sind. Die endgültige Zusammensetzung der Tandems bzw. die Aufösung nach dem informellen Kennenlernen übernimmt eine dritte Person mit besonders sprachlicher und sensitiver Behutsamkeit (Schmid et al. 2011, 90f).

\section{Intra- und Intersubjektivität}

Im Mentoringprozess agieren (meist zwei) unterschiedliche Personen miteinander, stehen in Beziehung zueinander und tauschen sich aus. Im anthropologischen Verständnis wird dies als dialogisches Prinzip betitelt.

„,Der Mensch wird am Du zum Ich“ (Buber 1954). Buber spricht vom ,Zwischen` als dem Raum, welcher schon vor der Differenzierung von Ich und Du (1954/1984) besteht. Die Psychoanalyse spricht von relationalen Prozessen und Intersubjektivität." (Kämmerer 2016,8)

Professionelle Zusammenarbeit zwischen Personen bedeutet eine Sensibilisierung für Lebensgeschichten. Nach Flusser (1990) ist die Anerkennung der eigenen Lebensgeschichte und die des Gegenübers besonders wichtig. Flusser (1990) meint „der Mensch (ist) nicht nur ein Objekt, sondern auch ein Subjekt, das man nur versteht, wenn man es anerkennt (statt es erkennen zu wollen).“ (Flusser 1990, 84) Daraus folgernd lässt sich der Schluss ziehen, dass in der Begegnung mit der/dem anderen wie im Mentoring „neben professionellem Wissen und Können bewusste Werthaltungen notwendig sind.“ (Jansen 2011, 26) Handeln basiert auf Basis unterschiedlicher Motive, „die ihre Grundlage in den biografisch vermittelten Werthaltungen der Professionellen haben (z.B. Helfermotive, Bearbeitung eigener biografischer Erfahrungen, Übertragung eigener Vorstellungen ... usw.).“ (Jansen 2011, 27) Für die Arbeit als Mentor/in ergeben sich daraus die Notwendigkeiten, biografisch erworbene Werthaltungen in einem Reflexionsprozess zu überdenken und für sich zu erkennen, welche im Sinne einer Beziehungsqualität neu entwickelt werden sollten. In der dialogorientierten Auseinandersetzung sollen dann Haltungen der/des Mentorin/Mentors wie Wertschätzung, Nähe, Respekt, Distanz und Empathiefähigkeit spürbar sein. Passend dazu kann auch das Riemann-Schley Modell mit den bipolaren Spannungsfeldern Nähe und Distanz sowie Dauer und Wechsel als Grundstrebungen im kommunikativen Prozess erwähnt werden (Schley et al. 1989, 343).

Einen weiteren Aspekt der intra- und intersubjektiven Betrachtung stellt das Konstrukt Selbstkonzept dar. 
„Seit Ende des 19. Jahrhunderts hat James (1890/1950) die Vorstellung eines dichotomen Ichs geprägt, das sich aus Subjekt (,I') und Objekt (,Me') zusammensetzt. Während dabei das , I' der Akteur der eigenen Handlungen und des eigenen Wissens ist, wird das , $\mathrm{Me}^{\prime}$ als Produkt des Akteurs betrachtet und entspricht der gegenwärtigen Auffassung des Selbstkonzepts.“ (Cimeli 2012, 1)

Nach Shavelson et al. (1976) ist Selbstkonzept die Wahrnehmung der eigenen Person mit sich zuschreibenden und beurteilenden Komponenten (Shavelson et al. 1976, 407f). Erfahrungswerte im Umgang mit Personen und Umwelt prägen den Auffassungsgehalt zur eigenen Person (Langkamp 2018, 11f). Bezogen auf schulpraktisches Mentoring kommt demnach der Gestaltung der Lernumgebung, Beziehungskultur und Feedbackkultur eine hohe Bedeutung zu. So ist die Etablierung einer lernförderlichen Umgebung wohl mit positiven Einstellungen zu sich selbst und zum System verbunden. Gleichzeitig wirft es auch die Fragen auf, wie Mentee positiven Einfluss auf ihre Leistungen nehmen und wie sie sich im Praktikum stärken können. Forschungsbefunde zu Mentoring und Selbstkonzept von Mentor/innen bzw. Mentee bilden ein Desideratum. Diesbezüglich würde sich ein Forschungsfeld ergeben.

Bedeutung erlangt im Rahmen der Intra- und Intersubjektivität auch die Forschung zur Biografiearbeit mit der Verknotung von Vergangenem, Gegenwärtigem und Zukünftigem. Als Voraussetzung zur Biografiearbeit hat eine Fachgruppe Mindeststandards für Zuverlässigkeit, Vertraulichkeit, Sensitivität und Reflexivität als Rahmenbedingungen entwickelt, an denen man sich in Ansätzen auch im Mentoringprozess orientieren kann (Jansen 2011, 28f). Für Jansen (2011) gilt die Arbeit an der eigenen Biografie in Form von intensiven Reflexionsprozessen als wichtige Komponente in jedem Bildungsprozess (Jansen 2011,22). Da

„jeder Teilnehmer (sic!) seine eigene Geschichte in die Dyade mitbringt und sich selbst als Mittelpunkt der eigenen Erfahrung und Initiative erlebt, kann für jeden Partner (sic!) der Dyade potentiell immer etwas Neues entstehen, da die Einflussnahme zwischen beiden hin- und herfließt." (Teichholz 2007, 265)

Die Begegnung mit der/dem anderen und die Entstehung von Neuem bewirken eine emotionale Veränderung. Sie erhält durch Öffnen von Raum, Einlassen in Beziehungstiefe und -intensität Sinn und Bedeutung (Kämmerer 2016, 11). Es ist anzunehmen,

„dass es ein zutiefst menschliches Bedürfnis ist, dem Leben einen sinnhaften Bezug (einen Bedeutungsfaden) zu geben, sich selbst dabei als lebendigen Gestalter (sic!) der eigenen Lebensgeschichte zu erleben und damit Identität unter den Bedingungen von Kontinuität und Diskontinuität zu konstituieren - kollektiv gebunden und doch individuell verschieden." (Jansen 2011,21)

Mentor/innen sehen oftmals die „neue“ Aufgabe als sinnstiftend ergänzend zu ihrer Tätigkeit als Lehrperson. Welche Vorstellungen und Umsetzungen die Player im Mentoringprozess im Hinblick auf zeitliche Ausgestaltung, Intensität, Beziehungstiefe und das „Zwischen“ (Buber 1984) haben und welchen Weg sie bestreiten möchten (Kämmerer 2016, 13), entscheidet wohl jedes Tandem für sich. Tatsache ist, dass die Beziehungsstruktur Mentor/in und Mentee eine Reziprozität im Diskurs miteinschließt. „Das Erleben im ,Hier und Jetzt' wird von zentraler Bedeutung “(Wellhöfer 2018, 135), um es mit den Worten von Rogers (Vertreter der Humanistischen Psychologie) auszudrücken, und im Idealfall mündet sie in den Weg der Selbstaktualisierung (Wellhöfer 2018, 136f).

Awaya (2003) beschreibt Mentoring schließlich als Reise, bei der in der Begegnung vor allem die zwischenmenschlichen Aspekte wie Vertrauen, Unterstützung, Empathie rund um die Profession im Vordergrund stehen: 
"Mentoring is conceived as journey that describes a unique relationship between mentor and student teacher. The journey involves the building of an equal relationship characterized by trust, the sharing of expertise, moral support, and knowing when to help and when to sit back." (Awaya et al. 2003, 45)

Hansman (2003) spricht davon, dass so manche Mentoring-Beziehung zum lebensveränderten Ereignis gezählt werden kann (Hansman 2003, 14). Sie kann zu einem wichtigen Lebensabschnitt zählen, wenn Aktivierung von Ressourcen, Bereitschaft zur aktiven Gestaltung von Lernsettings und Selbstreflexion möglich sind und Abgrenzung oder Selbstzweifel als Identitätsentwicklung gesehen werden (Hölzle 2011,77f).

\section{Wertebasierte Gesprächsführung}

Wie gestalten sich nun Dialoge auf Basis einer wertebasierten Gesprächsführung im Mentoringprozess? Was zeigen Untersuchungsbefunde dazu auf? Was wird unter wertebasierter Gesprächsführung verstanden? Die Fragen werden im Folgenden diskutiert.

Mentor/innen übernehmen mit 69 Prozent Sprechanteil im Gesprächsverlauf die Führung (Hughes 1998; Hennissen et al. 2008), Gespräche dauern in der Regel fünf bis 60 Minuten (Edwards et al. 1996; Hennissen et al. 2008, 176), ausgebildete Mentor/innen verringern ihre Sprechzeit im Durchschnitt auf 19 Prozent der Gesprächsführung. Probleme werden dann angesprochen, wenn sie gerade in der vorangegangenen Übungseinheit aufgetreten sind (Geldens et al. 2005). Mentor/innen mit Ausbildungsqualifikation stellen vermehrt Beobachtungen an, rekonstruieren im Gespräch das Unterrichtshandeln (Evertson et al. 2001) und analysieren Leistungen (Harrison et al. 2005). Harrison et al. (2005) zeigten auf,

"that trained mentor teachers were better able to guide prospective teachers to analyse their teaching performance by clarifying their actions ("deconstructing practice") and allowed them to think of new ways of dealing with situations ("constructing practice")" (Hennissen et al. 2008, 176).

Untersuchungsbefunde zum Dialogverlauf gibt es meist aus Sicht der Mentor/innen. Hierbei fällt auf, dass Anleitungen am Beginn und am Ende des Gesprächs bezogen auf den Unterricht, Fragen zu einem Thema, ein einseitiges Feedback (Feiman-Nemser et al. 1992; Ben-Peretz et al. 1991) und Vorschläge (Wang et al. 2004) den Inhalt des Gesprächs bestimmen. Aus den dargestellten Befunden lässt sich erkennen, dass Mentoring-Dialoge immer Gespräche mit einem Rückblick und einer Vorschau im Sinne einer Qualitätssteigerung sind (Hennissen et al. 2008, 176). Hudson (2013) schlägt aufgrund seiner Untersuchungsbefunde am Beginn des Mentoringprozesses die gegenseitige Klärung der Erwartungen und Erwartungshaltungen von Mentor/in und Mentee als Erfolgsgeheimnis vor (Hudson 2013, 18f).

Nun stellt sich die Frage, wie Gesprächsverläufe gestaltet sind. Wie begegnen sich prozessbeteiligte Personen in ihrer Reziprozität? Wie werden Inhalte in der zwischenmenschlichen Interaktion sprachlich verdeutlicht? Welche individuellen Wertvorstellungen liegen dem Dialog zugrunde?

Wertschätzung, Empathie und Kongruenz nach Rogers (2009) (Person-zentrierter Ansatz) bilden die Basis und „die Säule, in Form einer spezifischen Haltung im Miteinander - in der Gestaltung einer professionellen Beziehung." (Hellwig 2016, VIII) Die Handhabung der drei Variablen können als wesentliche Prinzipien eine prozesshafte Begleitung unterstützen und zum Ausbau der Aktualisierungstendenz beitragen. Diese ,ist das übergeordnete Sinn- und Entwicklungsprinzip menschlichen Verhaltens und Erlebens, das Streben nach Selbst-Entfaltung der eigenen Ressourcen." (Rogers 2009, 26f) Damit die Kreativität (Rogers) gelingt, ist es wichtig - wie in diesem Fall bezogen auf den Mentee - Raum zur Entfaltung zu bieten. Durch eine 
wertebasierte Gesprächsführung „werden die positiven Bedingungen für eine flexible Persönlichkeitsentwicklung bereitet und eine Person darin unterstützt, auf ihre Potentiale zugreifen zu können." (Hellwig 2016, 4)

Im Folgenden werden sechs Bedingungen in Form von Wirkprinzipien wertebasierter Gesprächsführung in Anlehnung an Rogers (2009) thematisiert (Tabelle 35) und Fragestellungen zum schulpraktischen Mentoring angeführt (Tabelle 36):

Tab. 35: Wirkprinzipien wertebasierter Gesprächsführung (Rogers 2009, 46f; Hellwig 2016, 13f)

\begin{tabular}{ll}
\hline Bedingungen & Inhalt(e) \\
\hline 1. Verbindung herstellen: & - Wahrnehmen der/des Gesprächspartnerin/-partners \\
In-Kontakt-Kommen & - Ungeteilte Aufmerksamkeit für die/den anderen \\
& - Das Jetzt und Hier bewusst erleben \\
\hline 2. Inkongruenz: & - Überdenken des Rollenverhaltens \\
eigene Unstimmigkeit erspüren & - Erkennen von Spannungszuständen und Analysieren des Verhaltens \\
& - Klären von Bedarfen und Wertvorstellungen \\
& - Ansprechen von möglichen Diskrepanzen \\
\hline 3. Kongruenz: & - Im Einklang-sein mit Fühlen, Denken und Handeln \\
Einklang finden & - Überprüfen der eigenen Stimmigkeit und der des Gegenübers \\
& - Ansprechen bei Unstimmigkeiten \\
& - Erkunden der kritischen zwischenmenschlichen Situation \\
\hline 4. Wertschätzung: & - Überdenken der eigenen Werthaltungen als Maßstab \\
bedingungslos und positiv & - Akzeptieren und Respektieren von anderen Sichtweisen \\
& - Beschreiben von Verhalten auf beobachtbare Merkmalen hin \\
& - Spüren einer Kongruenz mit seinem Gegenüber \\
\hline 5. Empathie: & - Verstehen der emotionalen Beteiligung \\
Einfühlung erfahren & - Verstehen von empathischem Verhalten \\
& - Zuhören und Zusehen (wollen) \\
& - Bewusstmachen des eigenen Fühlens, Denkens und Handelns \\
\hline 6. Erreichen: & - Erkennen der Selbstaktualisierung \\
Resonanz spüren & - Überprüfen der Erreichbarkeit beim Gegenüber \\
& - Hinterfragen des Beziehungsangebotes \\
& - Wahrnehmen der Merkmale eines förderlichen Klimas \\
\hline
\end{tabular}

Wertebasierte Gesprächsführung darf nicht als Technik, sondern muss als Haltung verstanden werden (Hellwig 2016, 43). Hellwig (2016) gibt für Führungskräfte in Form von Fragestellungen Denkanstöße, die im schulpraktischen Mentoringprozess ebenso Anwendung finden können. Der angeführte Fragenkatalog (Tabelle 36) ist auf Grundlage von Hellwig (2016, 15f) und durch ergänzende Fragen zum schulpraktischen Mentoring zusammengestellt worden.

Tab. 36: Fragestellungen von Mentor/innen zu Wirkprinzipien wertebasierter Gesprächsführung (Basierend auf Rogers 2009, 46f; Hellwig 2016, 13f)

\begin{tabular}{ll}
\hline Bedingungen & Fragestellungen \\
\hline $\begin{array}{c}\text { 1. Verbindung herstellen: } \\
\text { In-Kontakt-Kommen }\end{array}$ & Wie schaffe ich Raum und Zeit für die innerliche Kontaktaufnahme mit meinem \\
& Kommen?
\end{tabular}




\begin{tabular}{ll}
\hline Bedingungen & Fragestellungen \\
\hline $\begin{array}{l}\text { 2. Inkongruenz: } \\
\text { eigene Unstimmigkeit } \\
\text { erspüren }\end{array}$ & $\begin{array}{l}\text { Wie geht es mir in der Rolle und Funktion der/des Mentorin/Mentors? Gibt es } \\
\text { Situationen und Handlungen, die mit meinem Inneren nicht übereinstimmen? } \\
\text { Wie empfindsam ist mein Gegenüber? Wie geht es mir im Kontakt mit der } \\
\text { Person? }\end{array}$ \\
\hline $\begin{array}{l}\text { 3. Kongruenz: } \\
\text { Einklang finden }\end{array}$ & $\begin{array}{l}\text { Was höre, sehe und fühle ich im Umgang mit dieser Person? Kann ich mein } \\
\text { Gegenüber annehmen? Unterscheide ich zwischen objektiv nachvollziehbarer } \\
\text { Wahrnehmung und eigener Interpretation? Was sollte ich ansprechen? }\end{array}$ \\
\hline $\begin{array}{ll}\text { 4. Wertschätzung: } \\
\text { bedingungslos und } \\
\text { positiv }\end{array}$ & $\begin{array}{l}\text { Bin ich ehrlich mit meinem Gegenüber? Achte ich sie/ihn als Person? Sind meine } \\
\text { Werte aus heutiger, aktueller Sicht (noch) gültig? Bin ich an meinem Gegenüber }\end{array}$ \\
\hline 5. Empathie: & $\begin{array}{l}\text { Höre ich meinem Gegenüber zu? Erkenne ich das Besondere am anderen an? } \\
\text { Einfühlung erfahren } \\
\text { Inwieweit kann ich, gedanklich und gefühlsmäßig, das fühlen, was ich der/dem }\end{array}$ \\
\hline Mentee mitgeben möchte? Wird Raum für Neues gegeben?
\end{tabular}

Der Person-zentrierte Ansatz (Rogers) zielt auf Selbstaktualisierung, die in einem optimalen verständnisvollen Umfeld aktiviert und ausgelebt werden kann. Sie „unterstützt die Auffassung, daß (sic!) erlebnisorientiertes Lernen genauso wichtig ist wie kognitives Lernen.“ (Rogers et al. 1991, 231) In den Schulpraktika lenkt die/der Mentor/in durch ihre/seine Art der Prozessgestaltung das Vorgehen. Für ihr/sein Handeln benötigt sie/er neben einer humanistischen Haltung Empathiefähigkeit, Selbstreflexionsfähigkeit, Verständnis für individuelle und kontextuelle Bedingungsfaktoren, Methodenkenntnisse zur Gesprächsführung und den Blick für aktuelle Situationen (Hellwig 2016, 39f).

Das Thema pädagogische Beziehungen wird auch in der Neurowissenschaft diskutiert und in Verbindung mit dem Person-zentierten Ansatz gesehen. Folgend ergeben sich dazu Überlegungen, die auf die Bedeutung der Verzahnung neurobiologischer und psychologischer Ebenen bei Begegnungen hinweisen. Ein Schaubild dokumentiert die wesentlichen Veränderungen in unterschiedlichen Bereichen.

\section{Person-zentierter Ansatz und Verschränkung zur Neurowissenschaft}

Gegenwärtige neurowissenschaftliche Befunde sehen einen Zusammenhang zwischen Personzentriertem Ansatz (Rogers) und Konzepten der Neurowissenschaft (Kunze-Pletat 2019, 342). Beispielhaft wird Lux (2014) angeführt. Der Autor beschreibt dazu den „Kontaktkreis“ (Lux 2014, 184) und zeichnet eine reziproke, dynamische Verschränkung von einer/einem Pädagogin/Pädagogen und einer/einem Lernenden/ $\mathrm{m}$ in neurobiologischer und psychosozialer Perspektive auf (Lux 2014, 184). Angesichts der Tatsache, dass es sich bei dem beschriebenen Modell um eine positive Einflussnahme des Verhaltens und Erlebens durch soziale Interaktionen handelt, soll im schulpraktischen Mentoring eine verstärkte Sichtweise auf emotionale-psychosoziale Bedingungen gelegt werden. Die folgende Abbildung (Abbildung 22) verdeutlicht die Wechselwirkung auf neurobiologischer und psychosozialer Ebene und zeigt mit Pfeildarstellung die Zunahme von Aktivierungen, Ausschüttungen und Verbesserungen auf: 


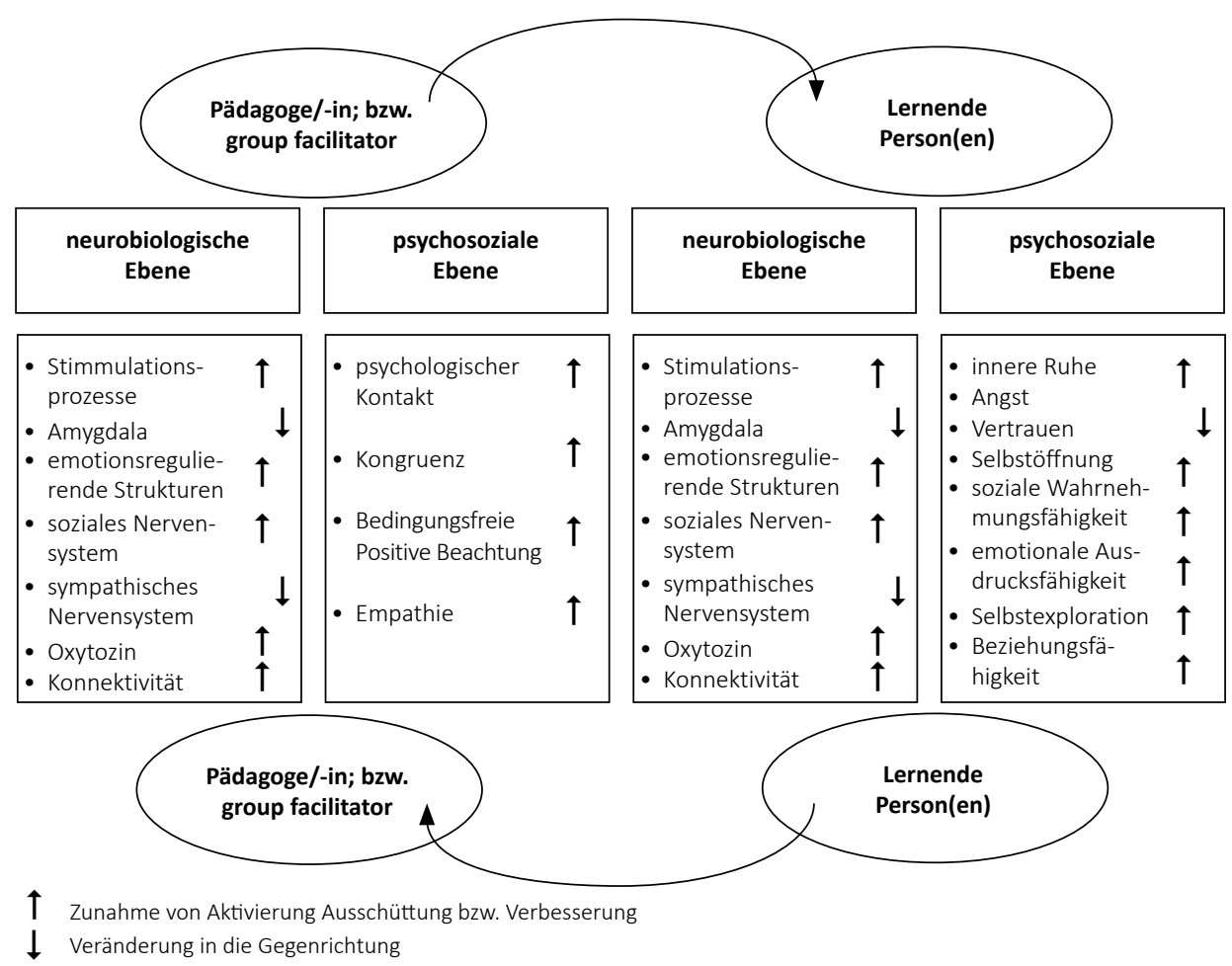

Abb. 22: Der Kreislauf des Kontakts (Lux 2014, 184; Kunze-Pletat 2019, 375)

Bei einer Bedingungsfreien Positiven Beachtung (BPB) von Kongruenz und Empathie für die/ den andere/ $\mathrm{n}$ in einer geleiteten Lernumgebung wird das Bindungshormon Oxytozin ausgeschüttet. Es „findet sich in der Blutbahn und im zentralen Nervensystem und hat einen angstund stressreduzierenden sowie einen gesundheitsförderlichen Effekt" (Lux 2014, 183; KunzePletat 2019, 350). Jede pädagogische Beziehung ist von reziproken, interaktiven Prozessen begleitet (Kunze-Pletat 2019, 375) und zielt auf einen Lernprozess. Hierbei sind Vertrauen, Kongruenz und Empathie Grundhaltungen, um nachhaltiges Lernen zu ermöglichen (KunzePletat 2019, 374). Das Beispiel zeigt auf, wie die Bedeutung der Beziehungsebene zwischen Mentor/in und Mentee noch diskutiert werden könnte.

Zusammenfassend kann gesagt werden: Wie am Beginn des Kapitels erwähnt, ist die Beziehungsstruktur zwischen Mentor/in und Mentee sehr komplex. Von Autor/innen wird die Mentor/in-Mentee-Beziehung oftmals als Kernelement von Mentoring (Bayer et al. 2015, 4) und lebensveränderndes Ereignis (Hansman 2003, 14) bezeichnet. Die Intensität und Qualität zwischen Mentor/in und Mentee hängt ab von deren/dessen beidseitiger Wertvorstellungen, Beachtung der Intra- und Intersubjektivität, Wertschätzung und Akzeptanz der eigenen und der im Prozess anvertrauten Person, Dialogbereitschaft und -fähigkeit sowie Empathiefähigkeit. Förderlich sind sicherlich auch die Annahme und Zuversicht, dass durch „volles Vertrauen in 
persönliches Wachstum“ (Rogers et al. 1991, 232) eine Persönlichkeits- und Professionsentwicklung begünstigt wird.

Wie kann nun eine personale Lern- und Entwicklungsförderung im schulpraktischen Mentoring angelegt werden? Dazu werden im folgenden Kapitel Überlegungen angestellt.

\subsubsection{Personale Lern- und Entwicklungsförderung}

Die Überschrift dieses Kapitels weist darauf hin, dass die Lern- und Entwicklungsförderung auf Basis der Subjektivität vollzogen werden soll, was in der vorliegenden Arbeit primär vor allem auf die/den Mentee und sekundär auf die/den Mentor/in ausgelegt wird. Ein Zitat von Erpenbeck et al. (2018) unterstreicht das Anliegen:

„Die neue Lernkultur als eine Selbstentwicklungskultur ist nicht auf eine Wissensvermittlung (die einen Widerspruch in sich darstellt), sondern auf einen Wissensaufbau, auf Kompetenzentwicklung und Werteentwicklung fühlender, denkender und handelnder Menschen gerichtet." (Erpenbeck et al. 2018,181$)$

Wissensaufbau, Kompetenzentwicklung und Werteentwicklung stehen im Mittelpunkt der Lernkultur, bezogen auf die jeweilige Individualität einer Person. In Bezug auf Mentoring werden für die Wissensaneignung Lern- und Erfahrungsgelegenheiten geschaffen sowie person- und situationsspezifische Reflexionsphasen im Hinblick auf die Profession iniitiert. Durch mentorielle Begleitung wie in den Pädagogisch-Praktischen Studien ist es möglich, einen Persönlichkeitsansatz zu forcieren und subjektorientiertes Mentoring aufzubauen. Ausgehend von den berufsbiografischen Erlebnissen und Erfahrungen „muss neben wissenschaftsgestütztem Basiswissen auch die Grundlage für eine kritisch-analytische Begegnung mit der Praxis geschaffen werden." (Reintjes et al. 2018, 10) Die/Der Mentor/in leitet Reflexionsphasen und geht der Frage nach, „wie aus Praxiserfahrungen Lernprozesse“ (Hascher 2012, 122) werden können und wodurch der Professionalisierungsprozess handlungsleitend und kritisch-reflexiv unterstützt werden kann.

Im nun folgenden Unterkapitel werden exemplarisch Ansätze aus den Bildungswissenschaftlichen Grundlagen, der Pädagogik und Psychologie vorgestellt, die sich mit unterschiedlichen Aspekten von Lern- und Entwicklungsförderung befassen (Tabelle 37).

Tab. 37: Auflistung der im folgenden ausgeführten Unterkapitel

\begin{tabular}{|c|c|}
\hline Personale Lern- und Entwicklungsförderung & Kurzbeschreibung \\
\hline Lernen als bildende Erfahrung & Lernen durch Erfahrung und als Erfahrung \\
\hline $\begin{array}{l}\text { Reflexion am Beispiel des Zwiebelmodells nach } \\
\text { Korthagen et al. }\end{array}$ & Reflexionsmodell zur Persönlichkeitsentwicklung \\
\hline Professionsspezifische Entwicklungsaufgaben & $\begin{array}{l}\text { Konzept zur Persönlichkeits- und Professions- } \\
\text { entwicklung }\end{array}$ \\
\hline $\begin{array}{l}\text { Bedürfnis- bzw. typenorientierte und/ } \\
\text { oder kontextsensible Begleitung }\end{array}$ & $\begin{array}{l}\text { Exemplarische Ansätze für eine personenspezifische } \\
\text { Begleitung }\end{array}$ \\
\hline Empowerment & Konzept für individuelle Begleitung in Schulpraktika \\
\hline Selbstwirksamkeitserwartung & $\begin{array}{l}\text { Konzept der Überzeugung als Bewältigungs- } \\
\text { mechanismus }\end{array}$ \\
\hline
\end{tabular}


Die Auflistung der Unterkapitel und Kurzbeschreibungen weisen darauf hin, dass Modelle wie das Zwiebelmodell und Konzepte zur Entwicklung der Selbst- und Sozialkompetenz im Mittelpunkt der Beschreibungen zur personalen Lern- und Entwicklungsförderung stehen. Ausgangspunkt sind Überlegungen zum Thema Lernen als bildende Erfahrung und damit verbunden der Ansatz, dass „Lernen durch Erfahrung und als Erfahrung“ (Buck 2019, VIII) geschieht. Im Anschluss werden Modelle und Konzepte für eine personale Lern- und Entwicklungsförderung diskutiert.

\section{Lernen als bildende Erfahrung}

Mentoring wird oftmals als Lernbeziehung bezeichnet (Höher 2014, 44). Wie können Tandems nun ihre Fähigkeiten ausbauen und ihre Persönlichkeit weiterbilden? Die Ansätze der Lerntheorien (wie Behaviorismus, Kognitivismus, Konstruktivismus) sind inhaltlich sehr unterschiedlich. Den Definitionen der Theorien ist gemein, dass es sich beim Lernen um eine Aneignung und/oder Veränderung von Verhaltensweisen einer Person aufgrund von Übung oder Erfahrung handelt (Stangl 2019). Nach Lankau et al. (2007) fehlen allerdings empirische Befunde zu Lerntheorien in Verbindung mit Mentoring (Lankau et al. 2007, 95). Die Fragen: Wie können Dispositionen im Mentoring verstärkt werden? Wie können Erfahrungen im Lernprozess nachhaltig genützt werden? Welchen Einfluss spielen Mentor/innen beim direkten Lernen? u.ä. bleiben aus Sicht der Forschung unbeantwortet. Arnold (2009) und Schüßler (2012) weisen auch auf die Bedeutung der emotional-motivationalen Lernstrategien hin und wünschen sich, dass diese verstärkt in der Forschung zum Thema Mentoring betrachtet werden (Schüßler 2012, 173; Arnold 2009).

Edelmann (2000) unterteilt Lerntheorien in eine dualisierte Form mit Außensteuerung und Innensteuerung und setzt Erfahrungsbildung als zentrales Merkmal in den Mittelpunkt des Lernprozesses (Edelmann 2000, 279). Bei der Außensteuerung wird durch vorangestellte oder nachfolgende Reize das Lernen beeinflusst. So könnte eine Mentoring-Beziehung zum Verhaltensaufbau oder - abbau beitragen. Mit Hilfe der Innensteuerung strukturiert sich der Mensch Prozesse und erwirbt ein Sach- und/oder Handlungswissen für Prozesse (Edelmann 2000, 279). Die/Der Lernende baut sich somit ein Problemlösedenken auf und setzt sich im Idealfall mit entwicklungsorientierten Lernstrategien (Boyatzis 2007, 451) auseinander. Nach Edelmann (2019) wird im Bereich Lernen „die Forderung erhoben, zukünftig häufiger ein (relativ) selbstgesteuertes, kooperatives, problemlösendes, in authentischen Lernsituationen stattfindendes und lebenslanges Lernen (Erwachsenenbildung) zu initiieren." (Edelmann 2019) Diese Forderung passt auch zum Mentoring mit der Intention der Lern- und Entwicklungsförderung. Grundlage zur Umsetzung sind - wie Kram (1985) bereits betonte - Mentoring-Beziehungen, „die von Unabhängigkeit, Gegenseitigkeit und einem offenen Verständnis und einer Reflexionsbereitschaft über Werte, Identität und Selbstwert gekennzeichnet sind.“ (Höher 2014, 364) In einer solchen Lernumgebung werden nach Dewey (1986) Erfahrungen gemacht, die die Person und „die Qualität der folgenden Erfahrungen“ (Dewey 1986, 284) durch Wechselwirkung mit der Umwelt beeinflussen. Den Beginn bzw. das In-Gang-Setzen eines Entwicklungsprozesses sieht Dewey (1986) in „der Phantasie, jener in der Innenwelt des Subjekts entworfenen Gestaltbildungen einer Problemsituation und ihrer möglichen Lösungen, die sich am Widerstand einer Sache entzünden." (Combe et al. 2007, 52)

Was könnte nun Widerstand im Mentoring bedeuten? Dazu müssen sich Beteiligte die Fragen stellen, welche Subjektkonstitutionen vorhanden sind, was auslösende Ereignisse, Widerstände, 
irritierende Erlebnisse und/oder Beunruhigungen (Combe et al. 2007, 50) im Mentoring sein könnten und wie damit als Mentor/in umgegangen werden soll? Combe et al. (2007) beruhigt mit den Worten:

„Erfahrungsräume sind Räume der Konfrontation und Artikulation. Erst durch Artikulation, durch Umformulierung der Beunruhigung in eine Frage kann diese Teile eines reflektierenden Prozesses werden, in dem, wie in einer Art Forschungsprozess, Hypothese und Annahmen in ihrer Geltung überprüft und Ideen und Optionen hinsichtlich ihrer Realisierungsmöglichkeit bedacht werden." (Combe et al. $2007,50)$

Bezogen auf das schulpraktische Mentoring bedeutet es, sich auf einen Erfahrungsprozess einzulassen und das Subjekt in seiner Person wahrzunehmen und in einen dialogorientierten Austausch zu gehen. Erfahrungsbildung geschieht im Wahrnehmen, Tun, Erleben und in der gemeinsamen reflexiven Auseinandersetzung (Höher 2014, 63). Jede/r Lernende wird nach „Abschluß (sic!) des Lernprozesses sich anders verhalten, anders denken, anders wollen, anders handeln.“ (Edelmann 2019) Mentoring ist im Rahmen der Erfahrungsbildung ein persönlichkeitsverändernder Prozess, bei dem es darum geht, im Sinne der aktiven Wortbedeutung Erfahrungen zuzulassen, Erfahrungen zu sammeln und zu erproben und im Sinne der passiven Wortbedeutung sich auf einen Erfahrungsaustausch einzulassen (Combe et al. 2007, 10). Die Erfahrungswelten werden dann in einem Reflexionsteil gesammelt und geordnet. Ausgangspunkt für Denkanstöße könnte das Zwiebelmodell nach Korthagen et al. (2005) sein, das im Folgenden vorgestellt wird.

Reflexion am Beispiel des Zwiebelmodells nach Korthagen et al. (2005)

Praxiskonzepte in der Lehrer/innenausbildung kennzeichnen sich durch unterschiedliche Gestaltungsansätze. Ihnen gemeinsam ist, neben der „berufseinführenden Professionalisierungsfunktion“ (Fischer et al. 2018, 160) bzw. der „Dimension der schulischen Unterstützung“" (Bach et al. 2018, 198) auch die „theoretisch-reflexive Professionalisierungsfunktion“ (Fischer et al. 2018, 160f) bzw. die „Unterstützung bei universitären Leistungsanforderungen" (Bach et al. 2018, 200) zu forcieren. Eine theoretisch-reflexive Auseinandersetzung ist bei Studierenden meist wenig vorhanden (Fischer et al. 2018, 161). Aber Theorie-Praxis-Verschränkung und Ausbau von Reflexionsprozessen gelten als allgemeiner Trend in der Lehrer/ innenausbildung. Ausgehend vom Ansatz des Problemlöseprozesses und des reflektierten Denkens nach Dewey (1951), dem Ansatz des reflektierten Denkprozesses der reflectionin-action und der reflection-on-action nach Schön (1983) und mit der Forderung der Berücksichtigung des wissenschaftlichen Wissens beim Reflexionsprozess nach Zeichner et al. (1987) „ist die bewusste und zielgerichtete Auseinandersetzung mit dem eigenen Denken und Handeln, den eigenen Überzeugungen und kontextuellen Bedingungen zu einem zentralen Prinzip der schulpraktischen Professionalisierung geworden" (Reintjes et al. 2018, 10) und somit ein wesentliches Aufgabenfeld für Mentor/innen. Korthagen (2005) lieferte mit seinem ALACT-Modell (1985) zunächst eine Modellstruktur für einen Reflexionsprozess (siehe Kapitel I/2.4.4.) und entwickelte dann das Zwiebelmodell. Die übereinander gelagerten Schichten repräsentieren den Fokus der Reflexionen einer Persönlichkeit. Korthagen et al. (2005) positionieren in die innere Ebene die berufliche Identität und in den innersten Kern die individuelle Mission (Abbildung 23). 


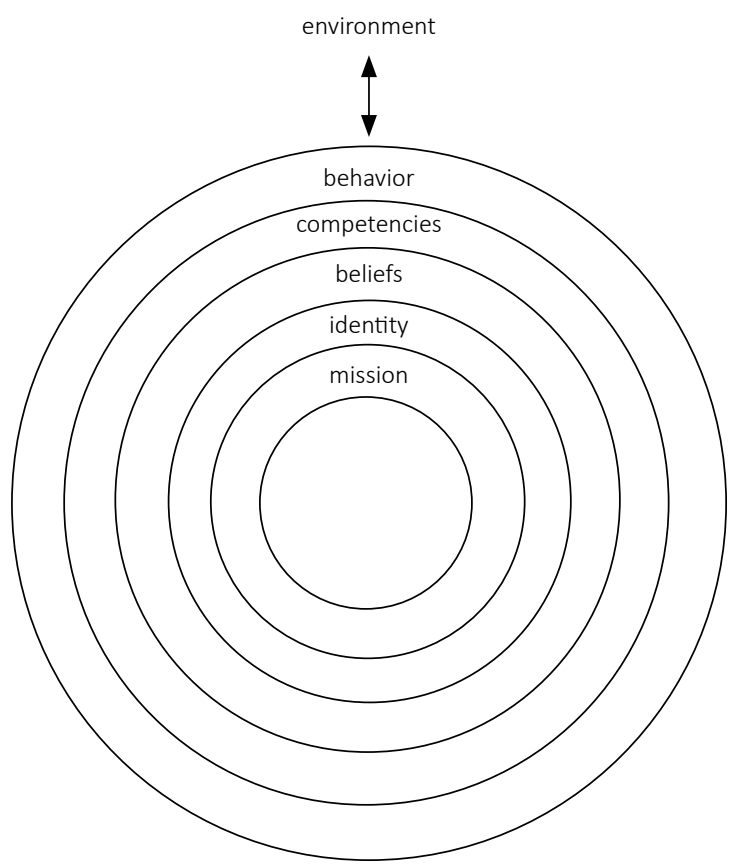

Abb. 23: Zwiebelmodell nach Korthagen et al. (Korthagen et al. 2005, 54)

Die Idee hinter dem Modell ist, „that the inner levels determine the way an individual functions on the outer levels, but that there is also a reverse influence (from outside to inside).“ (Korthagen et al. 2005, 53) Der Reflexionsprozess zielt auf Analyse des Verhaltens, Aktivierung der im Kern verwurzelten Qualitäten einer Persönlichkeit und Ausschöpfung in der Handlungsumsetzung. Mentoring kann unterstützend wirken, vorausgesetzt, dass Mentor/innen Ansätze der Positiven Psychologie unterstützen und "the attention to such core qualities in people“ (Korthagen et al. 2005, 55f) auf Prozesse lenken. Folgende Leitfragen könnten im Begleitungsprozess als Instrument Anwendung finden (Tabelle 38):

Tab. 38: Leitfragen zum Zwiebelmodell (Adaptiert nach Kernquadrat 2019)

\begin{tabular}{ll}
\hline Levels & Fragestellungen zur vertiefenden Reflexion \\
\hline Mission & Wofür tust du das? \\
Identity & Wie siehst du dich selbst? Wie siehst du deine professionelle Rolle? \\
Beliefs & Woran glaubst du? \\
Competencies & Was kannst du? \\
Behaviour & Was tust du? \\
Environment & Was begegnet dir? Womit hast du es zu tun? \\
\hline
\end{tabular}


Die exemplarischen W-Fragen verdeutlichen, dass Wertschätzung, Anerkennung, die Bereitschaft, stärken- und ressourcenorientiert der/dem anderen zu begegnen, sowie die Bereitschaft zur Förderung der Selbstreflexion bei der Anwendung dieses Modells wesentliche Voraussetzungen sind. Vor allem Fragen zur Mission und Identität können zu Widerstand und Irritation (Dewey 1986) führen, zu Momenten, in denen Erfahrungsbildung basiert und/oder aktiviert wird. Die Fragen richten sich direkt an die Person - an die/den Mentee. Sie/Er bestimmt den Fortgang des Prozesses, das Sich-Einlassen auf den Prozess und die Tiefenstruktur.

Das genannte Zwiebelmodell ist ein Beispiel für die mögliche In-Gang-Setzung eines Selbstreflexionsprozesses bzw. Unterstützung dessen. Im Folgenden werden weitere Unterstützungsmaßnahmen erwähnt und diskutiert - allen voran professionsspezifische Entwicklungsaufgaben.

\section{Professionsspezifische Entwicklungsaufgaben}

Die Bildungsgangforschung beschäftigt sich mit Fragen zum objektiven und subjektiven Bildungsgang, zur Kompetenz- und Identitätsentwicklung sowie mit dem Konzept der Entwicklungsaufgaben (Trautmann 2004, 7). Für die Theorie der subjektiven Entwicklungsaufgaben bilden die Referenztheorien von Erik Erikson (Stufenmodell der psychosozialen Entwicklung) und Robert James Havighurst (Konzept der Entwicklungsaufgaben) eine Grundlage im Sinne der Bearbeitung von Lernaufgaben im Laufe des Lebens. Laut Havighurst (1953) bedarf es bei der Entstehung von Entwicklungsaufgaben eines Zusammenspiels aus äußeren und als notwendig zu bearbeitenden inneren Komponenten (Trautmann 2004, 23). In Zusammenhang mit schulischem Lernen empfiehlt Havighurst (1953) Lehrpersonen, auf den individuellen Entwicklungsstand der Schulkinder Rücksicht zu nehmen und darauf einzugehen, indem personalisierte Aufgabenstellungen vorgelegt werden (Trautmann 2004, 30). Wie kann diese Empfehlung im Hinblick auf den Professionalisierungsprozess Studierender in der Lehrer/innenausbildung im Rahmen der Pädagogisch-Praktischen Studien umgelegt werden? Dazu ist es wichtig, dass Ausbildner/innen und/oder Mentor/innen Professionalisierungskonzepte und begleitende Unterstützungstheorien kennen. Verschiedene Autor/innen (Oerter et al. 2002; Hericks 2006; Keller-Schneider 2010) betrachteten in ihren Forschungen das Sozialisationsfeld und den Berufseinstieg von Lehrpersonen. Kraler (2009, 2012), Ostermann (2015) und Köffler (2015) haben mittels Rekonstruktion von Studierendeninterviews und reflexiver Berichte professionsspezifische Entwicklungsaufgaben der Ausbildungsphase identifiziert.

„Ergebnisse der Untersuchung zeigten, dass Lehramtsstudierende im Rahmen ihrer Ausbildung den Fokus stark auf sich selbst, die Bearbeitung von Aufgaben richten, die im Zusammenhang mit der Entwicklung ihrer Person, dem Umgang mit lebensweltlichen Herausforderungen des Studienkontextes sowie des Erwachsenwerdens im Allgemeinen stehen." (Köffler 2015, 192)

Die Forderung ist nun, dass in der Ausbildung Räume „geschaffen werden, welche Studierenden die Möglichkeit eröffnen, persönliche Lernaufgaben zu erkennen und zu bearbeiten.“ (Ostermann 2015, 185) Mentor/innen können in den Praktika einen wesentlichen Beitrag dazu leisten. 
Tab. 39: Entwicklungsaufgaben nach Ostermann $(2015,153)$ und Köffler $(2015,193)$ und die professionsspezifischen Anforderungen

\begin{tabular}{|c|c|c|}
\hline Ent & twicklungsaufgabe & Professionsspezifische Anforderungen \\
\hline & Reflexion & $\begin{array}{l}\text { Bereitschaft zur kritischen Auseinandersetzung mit der eigenen Lernbiografie, Ausbil- } \\
\text { dungsinhalten und pädagogischem Handeln; }\end{array}$ \\
\hline & $\begin{array}{l}\text { Perspektiven- } \\
\text { wechsel }\end{array}$ & Bewusstes Wahrnehmen von Schule und Unterricht aus der Sicht einer Lehrperson; \\
\hline 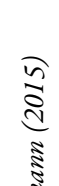 & $\begin{array}{l}\text { Schüler/innen- } \\
\text { zentrierung }\end{array}$ & $\begin{array}{l}\text { Bereitschaft zur Gestaltung des Unterrichts nach den Bedürfnissen und Möglichkei- } \\
\text { ten der Schüler/innen; } \\
\text { Ermöglichen von selbständigen Lernerfahrungen für Schüler/innen; } \\
\text { Entwickeln von Methoden zur individuellen Förderung von Schüler/innen; } \\
\text { Schaffen einer angenehmen Lernatmosphäre; }\end{array}$ \\
\hline 离 & Vermittlung & $\begin{array}{l}\text { Entwickeln einer vielfältigen Wissens- und Wertevermittlung unter Einbeziehung } \\
\text { unterschiedlicher Sozialformen und Lernmaterialien; }\end{array}$ \\
\hline$z$ & $\begin{array}{l}\text { Verhaltens- } \\
\text { kontrolle }\end{array}$ & $\begin{array}{l}\text { Erkennen der Bedeutung einer störungsfreien Lernatmosphäre und Entwickeln von } \\
\text { Strategien für einen angemessenen Umgang mit Konflikten; }\end{array}$ \\
\hline & Sicl & $\begin{array}{l}\text { Bereitschaft zur Vertiefung in unterschiedlichen Wissensbereichen und Aufbau von } \\
\text { Selbstbewusstsein im pädagogischen Handlungsfeld; }\end{array}$ \\
\hline & $\begin{array}{l}\text { Selbstverar } \\
\text { lichkeit }\end{array}$ & $\begin{array}{l}\text { Aufbau von Selbstdisziplin, -organisation und -interesse sowie Auseinandersetzen mit } \\
\text { der eigenen Psychohygiene; }\end{array}$ \\
\hline & Res & $\begin{array}{l}\text { Bereitschaft, sich unter Zuhilfenahme persönlicher Ressourcen an neue Begebenhei- } \\
\text { ten anzupassen und diesen mit Gelassenheit und Optimismus entgegenzutreten, ohne } \\
\text { ein fortwährendes Gefühl der Überforderung zu entwickeln; }\end{array}$ \\
\hline$\frac{\pi}{2}$ & Selbstkonz & $\begin{array}{l}\text { Bewusstwerden individueller Schwächen, Talente und Bedürfnisse; Vertrauen in } \\
\text { eigene Fähigkeiten erhöhen; Erkennen und Nachgehen beruflicher Wünsche und } \\
\text { Interessen; }\end{array}$ \\
\hline$\overbrace{}^{2}$ & Autonomie & $\begin{array}{l}\text { Bereitschaft zur Verselbständigung im Sinne der Entwicklung einer individuellen } \\
\text { Dynamik der Lebensgestaltung, die sich nur gering an Vorstellungen von Bezugsper- } \\
\text { sonen orientiert; }\end{array}$ \\
\hline & Fehlertoleranz & $\begin{array}{l}\text { Entwicklung eines konstruktiven, verständnisvollen und lösungsorientierten Umgangs } \\
\text { mit eigenen Fehlern und Defiziten sowie Schwächen anderer; }\end{array}$ \\
\hline & $\overline{\text { Kom }_{1}}$ & $\begin{array}{l}\text { Iindernissen des Studienalltags offensiv und } \\
\text { ohne Gefühle massiver Hilflosigkeit zu verspüren; }\end{array}$ \\
\hline
\end{tabular}

Zur inhaltlichen Präzisierung der Merkmale wurden in einer Arbeitsgruppe der Privaten Pädagogischen Hochschule Edith Stein Fragestellungen zu den sieben professionsspezifischen Entwicklungsaufgaben nach Ostermann (2015) formuliert, die nun für Aktant/innen und so auch für Mentor/innen zur Verfügung stehen. In den semesterweisen Praktika des Bachelorstudiums Primarstufe bekommen Studierende die Aufgabe, pro Praktikum jeweils zwei Entwicklungsaufgaben näher zu betrachten, ihr pädagogisches Handeln durch vorgegebene oder frei gewählte Fragestellungen zu reflektieren und sich mit den berufsfeldbezogenen Themen auseinanderzusetzen. Mentor/innen kennen das Konzept der Entwicklungsaufgaben mit den Fragestellungen und unterstützen den Reflexionsprozess während der Zeit der Begleitung. Tabelle 40 stellt die rekonstruierten Entwicklungsaufgaben nach Ostermann (2015) mit exemplarischen berufsfeldbezogenen Fragestellungen zur fokussierten Reflexion dar: 
Tab. 40: Professionsspezifische Entwicklungsaufgaben (Ostermann 2015, 153) mit Fragestellungen (KPH Edith Stein 2020)

\section{Professionsspezifische Entwicklungsaufgaben mit Fragestellungen}

\begin{tabular}{|c|c|}
\hline Reflexion & $\begin{array}{l}\text { - Welchen Einfluss hat meine Lernbiografie auf das pädagogische Handeln? } \\
\text { - Welche Rückschlüsse können aus Beobachtungen von Schüler/innen geschlossen werden? } \\
\text { - Welche pädagogischen Theorien können aufgrund der Beobachtungen reflektiert werden? } \\
\text { - Wie gehe ich mit Kritik um? } \\
\text { - Welche Kriterien beeinflussen die eigene Unterrichtstätigkeit? usw. }\end{array}$ \\
\hline $\begin{array}{l}\text { Perspektiven- } \\
\text { wechsel }\end{array}$ & $\begin{array}{l}\text { - Wodurch nehme ich mich selbst als Lehrperson wahr? } \\
\text { - Bei welchen Gelegenheiten nehme ich mich als Schüler/in bzw. als Lehrer/in wahr? } \\
\text { - Was hat mich in der eigenen Schulzeit fasziniert und warum? } \\
\text { - Wie lässt sich eine bestimmte Unterrichtssituation aus dem Blickwinkel einer Schülerin } \\
\text { oder eines Schülers und aus dem Blickwinkel einer Lehrperson beschreiben? usw. }\end{array}$ \\
\hline $\begin{array}{l}\text { Schüler/innen- } \\
\text { zentrierung }\end{array}$ & $\begin{array}{l}\text { - Wie können selbständige Lernerfahrungen für Schüler/innen geschaffen werden? } \\
\text { - Wie können Schüler/innen individuell gefördert werden? } \\
\text { - Wie stärke ich Kinder in ihren kommunikativen Fähigkeiten, in ihrer gedanklichen Akti- } \\
\text { vität, Ansichten zu entwickeln, Meinungen zu vertreten, Fragen zu stellen? } \\
\text { - Wie verlaufen Kommunikation und Interaktion zwischen Lehrpersonen und Schülerin- } \\
\text { nen und Schülern bzw. zwischen Schüler/innen? } \\
\text { - Wie werden Ideen und Bedürfnisse der Kinder berücksichtigt? usw. }\end{array}$ \\
\hline Vermittlung & $\begin{array}{l}\text { - Inwiefern beeinflussen eingesetzte Medien den Lernprozess? } \\
\text { - Wie sieht Unterricht aus, in dem möglichst viel und intensiv gelernt wird? } \\
\text { - Wie werden die Stärken der einzelnen Schüler/innen berücksichtigt? } \\
\text { - Welche konkreten Planungsschritte (Phasen, Methode, Sozialformen, ...) waren für die } \\
\text { Durchführung des Unterrichts hilfreich? } \\
\text { - Wie begründe ich die gewählte Unterrichtsmethode mit dem Lernerfolg der Schüler/ } \\
\text { innen? usw. }\end{array}$ \\
\hline $\begin{array}{l}\text { Verhaltens- } \\
\text { kontrolle }\end{array}$ & $\begin{array}{l}\text { - Wie drücken sich Wertschätzung und Achtung im Unterricht, in der Schule, im Umgang } \\
\text { miteinander aus? } \\
\text { - Was tun Schüler/innen konkret, wenn sie meiner Wahrnehmung nach den Unterricht stören? } \\
\text { - Welche Arten von Störungen nehme ich wahr (Lernschwierigkeiten, Lernaufgaben, } \\
\text { Beziehung, Arbeitsfähigkeit, Umfeld, schwelende Konflikte, ...)? } \\
\text { - Was braucht Unterricht, damit möglichst störungsarm gelernt werden kann? } \\
\text { - Welche möglichen Lösungsstrategien kenne ich, habe ich schon erprobt, würde ich gerne } \\
\text { erproben? Welche bieten sich an? } \\
\text { - Welche schulinternen und schulexternen Unterstützungen würde ich einsetzen? usw. }\end{array}$ \\
\hline Sicherheit & $\begin{array}{l}\text { - Wie schätze ich mich in Bezug auf Selbstbewusstsein im pädagogischen Handlungsfeld } \\
\text { selbst ein bzw. deckt sich diese Selbstwahrnehmung mit der Wahrnehmung einer/eines } \\
\text { Beobachterin/Beobachters? } \\
\text { - Wie schätze ich meine Expertise in den einzelnen Unterrichtsfächern ein? In welchen } \\
\text { Bereichen sehe ich Verbesserungspotential? } \\
\text { - Wie gelingt es mir, das Interesse der Schüler/innen an meinem Unterricht zu wecken, } \\
\text { damit generatives Lernen ermöglicht wird? } \\
\text { - Welche Aspekte tragen dazu bei, Sicherheit im pädagogischen Handlungsfeld zu erlangen? } \\
\text { - Habe ich ein professionelles, selbstsicheres Auftreten und Durchsetzungsvermögen, ein } \\
\text { hohes Maß an Belastbarkeit und Ausgeglichenheit und die Fähigkeit, eine Klasse leiten } \\
\text { zu können? Auf welcher Basis begründe ich meine Annahmen? } \\
\text { - Wie kommuniziere ich mit Schülerinnen und Schülern in unterschiedlichsten Situatio- } \\
\text { nen? Wie gebe ich Feedback? usw. }\end{array}$ \\
\hline
\end{tabular}




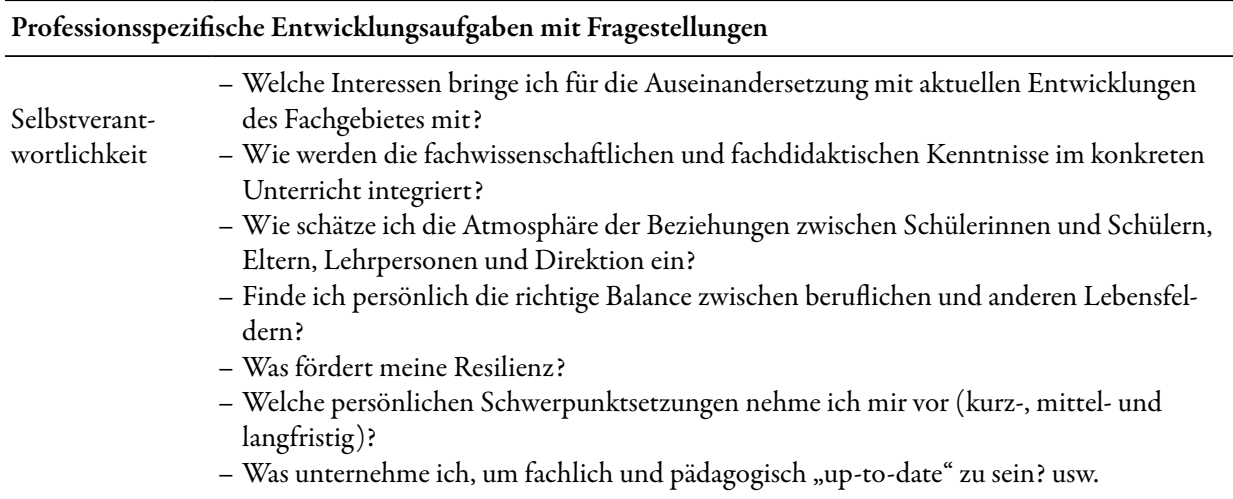

Durch Bearbeitung der Fragestellungen wird angenommen, dass sich neben dem Aufbau einer reflexiven forschenden Haltung und Professionsbewusstsein „der Blick eines Menschen für das Mögliche weitet." (Hericks 2004, 126) So gesehen tragen sie wesentlich zur Selbst- und Persönlichkeitsentwicklung und zur Entwicklung des Professionsverständnisses bei. Mentor/innen unterstützen und können ein Teil der Umsetzung des Konzepts subjektiver Entwicklungsaufgaben mit dem Hintergrund der subjektiven Lerntheorie werden. Gewinnbringend für Mentor/ innen ist es, wenn sie die Fragestellungen nicht nur an Mentee richten, sondern wenn sie selbst an Antworten feilen und in einen intra- und interpersonellen Dialog treten. Aktuell liegen keine Befunde vor, wie sich die Bearbeitung von professionsspezifischen Entwicklungsaufgaben mit Hilfe von Mentor/innen bzw. die Bedachtnahme auf Entwicklungsbedürfnisse der Studierenden während der Praktika auf den Professionalisierungsprozess auswirken. Möglicherweise breitet sich diesbezüglich ein Forschungsfeld aus.

Der folgende Abschnitt stellt weitere personenspezifische Ansätze im Rahmen von Mentoring vor. Mentee agieren sehr unterschiedlich und es kann im Aufgabenbereich und in der Verantwortung der Mentor/innen gesehen werden, darauf bestmöglichst zu reagieren und Aktionen zu setzen.

\section{Bedürfnis- bzw. typenorientierte und/oder kontextsensible Begleitung}

Im Folgenden werden exemplarische Konzepte und Beispiele für eine bedürfnis- bzw. typenorientierte und kontextsensible Begleitung diskutiert, indem das Subjekt - wie in diesem Fall die/der Mentee - mit unterschiedlichen Verhaltens- und Erlebensmustern als Person im Mittelpunkt steht.

In Anlehnung an Maslows Bedürfnispyramide (1943), wonach menschliche Bedürfnisse und Motivationen (physiologische Bedürfnisse, Sicherheitsbedürfnisse, soziale Bedürfnisse, Individualbedürfnisse, Bedürfnis nach Selbstverwirklichung) beschrieben werden, und an Forschungsergebnisse von Blömeke (2006) und Hascher (2012), die Studierendensichten zu den praktischen Ausbildungsphasen erhoben haben, beschreibt Dreer (2018) das Konzept der bedürfnisorientierten Begleitung. Hierbei geht es vor allem darum, Bedürfnisse der Mentee zu erkennen, sie systematisch auf dem Weg zur Professionalisierung zu unterstützen und Eigenständigkeit zu fördern (Dreer 2018, 19). In Tabelle 41 wird die inhaltliche und personenbezogene Rahmung des Konzepts beschrieben: 
Tab. 41: Vier Bedürfnisbereiche der bedürfnisorientierten Begleitung (Dreer 2018, 19)

„Das Bedürfnis nach Einführung in den Schulalltag umfasst:

- einen festen Platz in einer als angemessenen eingeschätzten Schule sowie

- eine räumliche und organisationsbezogene Orientierung (Verständnis von schulischen Arbeitsstrukturen, Zuständigkeiten und Machtbeziehungen).

Das Bedürfnis nach Einbindung in die Lehrer- und Schülerschaft (sic!) umfasst:

- als professionelle Kollegin bzw. professioneller Kollege wertgeschätzt und respektiert zu werden sowie

- als relevante Bezugsperson von den Schülerinnen und Schülern wahrgenommen werden.

Das Bedürfnis nach Selbsterprobung umfasst:

- angemessenen Herausforderungen beim Vorbereiten, Planen, Durchführen und Auswerten von Unterricht erfolgreich zu begegnen sowie

- beim Vorbereiten, Planen, Durchführen und Auswerten von Unterricht über Wahlmöglichkeiten zu verfügen und sich als Initiator (sic!) der eigenen Handlung zu fühlen.

Das Bedürfnis nach Selbstverwirklichung umfasst:

- das eigene Potenzial in Bezug auf die Aufgaben des Lehrerberufs (sic!) im Rahmen der schulpraktischen Ausbildung voll auszuschöpfen." (Dreer 2018, 19)

Der Ansatz von Dreer (2018) zeigt auf, wie Mentor/innen Studierende systematisch in das Sozialisationsfeld einführen können und durch schrittweise Eingliederung und Beachtung der Bedürfnisse hierbei auch auf die psychische Gesundheit der Studierenden achten (Dreer 2018, 11).

Košinár (2018) verweist in ihrer Untersuchung $(\mathrm{n}=14)$ auf die Bedeutung der Typologien (Tabelle 42) bei Studierenden (Selbstverwirklichung, Vermeidung, Entwicklung, Bewährung) und unterscheidet demzufolge Studierende, die sich im Praktikum entfalten möchten (Entfaltungsraum), die nur Erwartungen erfüllen wollen (Bewertungsraum), die darauf bedacht sind, Herausforderungen zu bestehen (Entwicklungsraum) und die, die sich bewähren wollen, aber berufsbezogene Defizite erkennen (Bewährungsraum) (Košinár 2018, 72f).

Tab. 42: Typologie der Anforderungsdeutung und -bearbeitung Studierender (Košinár 2018, 72; Košinár et al. 2018, 169)

\begin{tabular}{lllll}
\hline $\begin{array}{l}\text { Typus } \\
\begin{array}{l}\text { Vergleichs- } \\
\text { dimensionen }\end{array}\end{array}$ & $\begin{array}{l}\text { Selbstverwirkli- } \\
\text { chung }(\mathrm{N}=\mathbf{4})\end{array}$ & $\begin{array}{l}\text { Vermeidung } \\
(\mathrm{N}=\mathbf{2})\end{array}$ & $\begin{array}{l}\text { Entwicklung } \\
(\mathrm{N}=\mathbf{4})\end{array}$ & $\begin{array}{l}\text { Bewährung } \\
(\mathrm{N}=\mathbf{4})\end{array}$ \\
\hline $\begin{array}{l}\text { Bedeutung der } \\
\text { Praktika }\end{array}$ & $\begin{array}{l}\text { Entfaltungs- } \\
\text { raum }\end{array}$ & Bewertungsraum & $\begin{array}{l}\text { Entwicklungs- } \\
\text { raum }\end{array}$ & $\begin{array}{l}\text { Bewährungs- } \\
\text { raum }\end{array}$ \\
\hline $\begin{array}{l}\text { Konstituierung und } \\
\text { Bearbeitung von } \\
\text { Anforderungen }\end{array}$ & $\begin{array}{l}\text { Sich Gestaltungs- } \\
\text { raum nehmen }\end{array}$ & $\begin{array}{l}\text { Von außen herange- } \\
\text { tragene Erwartun- } \\
\text { gen erfüllen }\end{array}$ & $\begin{array}{l}\text { Berufsbezogene } \\
\text { Herausforderungen } \\
\text { meistern }\end{array}$ & $\begin{array}{l}\text { Berufsbezogene De- } \\
\text { fizite aufarbeiten }\end{array}$ \\
\hline $\begin{array}{l}\text { Rolle der Praxis- } \\
\text { lehrperson }\end{array}$ & $\begin{array}{l}\text { Ermöglichende } \\
\text { oder Verhindernde }\end{array}$ & $\begin{array}{l}\text { Bewertungs- } \\
\text { instanz }\end{array}$ & $\begin{array}{l}\text { Beratende im Ent- } \\
\text { wicklungsprozess }\end{array}$ & $\begin{array}{l}\text { Einschätzende des } \\
\text { Entwick- } \\
\text { lungsbedarfs }\end{array}$ \\
\hline
\end{tabular}


Nolle (2012) publizierte eine weitere Untersuchung zur Typologie von Studierenden $(n=308)$ im Lehramtsstudium. Hierbei erfasste er vier Gruppen von Studierenden, die sich in den Merkmalen psychosoziale Kompetenzen und Lernorientierung unterscheiden (Nolle 2012, 69). Mit psychosozialer Lernorientierung wird eine persönliche Haltung zur Förderung der eigenen Entwicklung bezeichnet. Es ist anzunehmen, dass Studierende mit hoher psychosozialer Lernorientierung gerne in soziale Interaktion treten und sie als Chance der Weiter- und Lernentwicklung erkennen (Nolle 2012, 68). Die folgende Kurzbeschreibung (Tabelle 43) ist eine Darlegung der Extremwerte. In der Realität sind Annäherungen der Personenmerkmale erkennbar (Nolle 2012, 73).

Tab. 43: Typisierende Beschreibung der Gruppen-Charakteristika (Nolle 2012, $73 \mathrm{f}$ )

\begin{tabular}{|c|c|c|c|}
\hline $\begin{array}{l}\text { Hohe aktuelle } \\
\text { psychosoziale } \\
\text { Kompetenz und hohe } \\
\text { Lernorientierung }\end{array}$ & $\begin{array}{l}\text { Niedrige aktuelle } \\
\text { psychosoziale } \\
\text { Kompetenz und hohe } \\
\text { Lernorientierung }\end{array}$ & $\begin{array}{l}\text { Hohe aktuelle } \\
\text { psychosoziale } \\
\text { Kompetenz und niedrige } \\
\text { Lernorientierung }\end{array}$ & $\begin{array}{l}\text { Niedrige aktuelle } \\
\text { psychosoziale } \\
\text { Kompetenz und niedrige } \\
\text { Lernorientierung }\end{array}$ \\
\hline $\begin{array}{l}\text { treten geschickt in Kom- } \\
\text { munikation und Interakti- } \\
\text { on, beschreiben Gefühls- } \\
\text { zustände, hohe Motivation } \\
\text { zur Weiterentwicklung, } \\
\text { Offenheit für Beratung, } \\
\text { günstige personale und } \\
\text { soziale Ressourcen }\end{array}$ & $\begin{array}{l}\text { Schwierigkeiten bei } \\
\text { der Beschreibung von } \\
\text { Emotionen, erkennen die } \\
\text { Lernfähigkeit in diesem } \\
\text { Bereich, Offenheit für } \\
\text { Beratung, hoher Arbeits- } \\
\text { einsatz, Engagement }\end{array}$ & $\begin{array}{l}\text { mittlere Ausstattung an } \\
\text { personalen und sozialen } \\
\text { Ressourcen, Vorerfahrun- } \\
\text { gen mit der Arbeit von } \\
\text { Kindern, Ausbildung we- } \\
\text { nig Bedeutung, reduzierte } \\
\text { Motivation zur Vertiefung } \\
\text { von Inhalten }\end{array}$ & $\begin{array}{l}\text { mangelnde personale und } \\
\text { soziale Ressourcen, wenig } \\
\text { Engagement, fehlende } \\
\text { Beziehungsarbeit zu Schü- } \\
\text { lerinnen und Schülern, } \\
\text { ablehnende Haltung zu } \\
\text { Reflexion und Beratung }\end{array}$ \\
\hline
\end{tabular}

Der Ansatz der Typologien veranlasst Mentor/innen, subjektive Empfindsamkeiten beim Gegenüber zu erkennen, zu deuten und im Umgang miteinander personenangepasste Verhaltensweisen anzuwenden. Vielleicht erklären sich so manche Spannungsfelder zwischen Mentor/ innen und Mentee auch dadurch, dass Empfindsamkeiten und demzufolge Erwartungshaltungen in der Begleitung zu wenig Beachtung finden (Košinár 2018, 74). Hudson (2013) schlägt am Beginn des Mentoringprozesses vor, über Erwartungen zu sprechen (Hudson 2013, 18f). Nach dem typologischen Erklärungsansatz von Košinár (2018) müsste jede/jeder Mentor/in am Beginn wohl folgende Fragen stellen: Welchen Raum nimmt für dich (Mentee) das Praktikum ein? Welche Rolle spiele ich als Mentor/in in diesem Raum für dich? o.̈. Wenn man sich den Ansatz von Nolle (2012) ansieht, lauten die Fragestellungen: Welche Bedeutung hat das Praktikum für dich? Warum möchtest du Lehrer/in werden? Wie stellst du dir die Arbeit mit Kindern vor? Was möchtest du an dir weiterentwickeln? Welche Rolle spiele ich für dich in der Begleitung? Durch die beidseitige Auseinandersetzung mit diesen oder ähnlichen Fragestellungen könnte ein sensibles Herangehen am Beginn des Mentoringprozesses gewährleistet werden. Hofmann (2019) schlägt prinzipiell eine kontextsensible Begleitung vor, damit Potentiale der Studierenden ausgeschöpft werden. Seiner Ansicht nach sollen Mentor/innen im Bereich Selbstkompetenz ausgebildet werden und einen diagnostischen Blick für Studierende entwickeln (Hofmann 2019, 6f). Auch der Einsatz von Potentialanalysen oder Erhebungsinstrumenten zur Selbstreflexion und Selbsteinsicht, wie sie am Beginn des Studiums oftmals durchgeführt werden (Trojer 2018, 191f), ist hierbei eine Möglichkeit und kann als Gesprächsbasis dienen. Darin werden dann Themen zur Berufsmotivation, zur Selbstwirksamkeitserwartung, zur psychosozialen Lernorientierung, zur Sozial- und Selbstkompetenz und zur berufsbezogenen Überzeugung angesprochen. Hofmann (2019) erweitert die zentrale Frage an Mentee im 
Rahmen von Mentoring, die lautet „Was brauchst du von mir als Mentor/in?“, mit der Forderung, dass Mentor/innen Wege und Methoden zur Entwicklungsunterstützung und -begleitung kennen sollen und anwenden können (Hofmann 2019, 7).

Die vier beschriebenen Ansätze zur Gestaltung eines personenspezifischen Mentoringprozesses wurden in den letzten Jahren publiziert. Dazu gibt es ein weiteres Konzept, das Konzept des Empowerments, das in Publikationen immer wieder auch in Verbindung mit Schulpraktika und somit indirekt mit Mentoring gebracht wird. Eine Erklärung folgt im folgenden Abschnitt.

\section{Empowerment}

Die Ansätze zum Konzept des Empowerments (englisch „empowerment“ Ermächtigung, Übertragung von Verantwortung) entstammen der Klinischen Psychologie sowie der Arbeits- und Organisationspsychologie. Masschelein et al. (2006) definieren innerhalb des Konstrukts Empowerment drei bedeutende Faktoren:

- „Analyse von Bedürfnissen und Interessen, an denen Empowerment ansetzen müsste und auf welche die Personen aufbauen sollten;

- Mobilisierung von Ressourcen und Möglichkeiten, d.h. der kontinuierliche Erwerb von Fähigkeiten und Fertigkeiten, um Menschen darin zu stärken, mit einem ständig wechselnden Kontext umzugehen;

- Aufbau echter Partnerschaften (nicht top-down-Beziehungen), um Entwicklungsprozesse des sozialen Individuums anregen zu können." (Masschelein et al. 2006, 311; Arnold et al. $2011,128)$

Selbstkontrolle, Selbstreflexion, Selbstwirksamkeitserleben und Nutzen der eigenen und sozialen Ressourcen sind somit wesentliche Elemente für die Gestaltung des individuellen Lernprozesses. Arnold et al. (2011) widmen sich in ihrer Publikation der Einbeziehung des Konzepts Empowerment in Schulpraktika. Die Autor/innen betonen unterschiedliche Eingangsbedingungen und Verschiedenheiten von Studienmotivationsaspekten bei Studierenden und plädieren daher im Hinblick auf eine „studierendenspezifische(n) Lernprozessorientierung“ (Arnold et al. 2011, 147) auf eine Differenzierung in der Begleitung und Aufgabenstellung. Die Basis für die Gestaltung einer individuellen Betreuung bilden: individuelle Lernstandserhebungen, Beachtung des bisherigen und des zukünftigen Entwicklungsverlaufs, Berücksichtigung emotionalen Lernens und Erlebens, Wissen um Emotionsregulatoren, Optimierung der Lerngelegenheiten durch Rückmeldemoderationen wie Feedbackschlaufen und Möglichkeit eines wechselseitigen interaktionalen Erfahrungsaustausches (Arnold et al. 2011, 148f). Zielorientierung des personalisierten und biografiegestützten Empowermentansatzes ist die „Stärkung der Autonomie, der Handlungspotenziale und Ressourcen der Studierenden." (Arnold et al. 2011, 138) Mentoringprozesse, die den Fokus auf ein verständnisvolles, vertrauenerweckendes, entwicklungsorientiertes und ko-konstruktives Miteinander legen, sind kompatibel mit dem Ansatz des Empowerments (Arnold et al. 2011, 186). Eine theoretische Fundierung zum Ansatz hat Kram (1985) mit den Mentoring-Funktionen bereits geliefert.

\section{Selbstwirksamkeitserwartung}

Das Konzept der Selbstwirksamkeitserwartung (SWE) wurde vom Psychologen Bandura (1977) entwickelt und ist ein Teilbereich der Motivationsforschung. Eine Person mit einer hohen Selbstwirksamkeitserwartung ist davon überzeugt, Einfluss auf Situationen und Begebenheiten in unterschiedlichsten Bereichen des Lebens und Alltags zu nehmen und Handlungsprozesse positiv mitgestalten zu können. 
„Die berufsbezogene Selbstwirksamkeitserwartung beschreibt die subjektive Überzeugung, neue oder schwierige Anforderungen im Berufsleben aufgrund der eigenen Kompetenz bewältigen zu können (Schwarzer \& Jerusalem, 2002)““ (Böhnert et al. 2018, 85)

Erfolgreiches Lehrer/innenhandeln wird in Verbindung mit dem Konstrukt Selbstwirksamkeitserwartung gesetzt (Klassen et al. 2011). Studien der Lehrer/innenforschung zeigen zu Lehrpersonen mit hoher Selbstwirksamkeit folgende Korrespondenzen im Vergleich zur Gruppe mit geringer Selbstwirksamkeit auf (Böhnert et al. 2018, 86f):

Tab. 44: Korrespondenz hohe Lehrer/innenselbstwirksamkeit mit verschiedenen Variablen (Basierend auf Böhnert et al. 2018, 86f)

\begin{tabular}{|c|c|c|}
\hline Forschungsbereich & Ergebnis/se & Autor/innen \\
\hline Berufszufriedenheit & Hoch & Caprara et al. 2006; Klassen et al. 2010 \\
\hline Stress und Burnout & Geringe Anzeichen & Klassen et al. 2010; Zee et al. 2016 \\
\hline $\begin{array}{l}\text { Kooperation mit Kolleginnen } \\
\text { und Kollegen }\end{array}$ & Hohe Gewilltheit & Henson et al. 2001 \\
\hline Classroom-Management & Positiv ausgeprägt & Künsting et al. 2016, Zee et al. 2016 \\
\hline Schüler/innenverhalten & $\begin{array}{l}\text { Guter Umgang auch } \\
\text { bei Problemen }\end{array}$ & Zee et al. 2016 \\
\hline Lernzielorientierung & $\begin{array}{l}\text { Stark ausgeprägt; } \\
\text { entwickeln Strukturen }\end{array}$ & $\begin{array}{l}\text { Künsting et al. 2016, Zee et al. 2016; } \\
\text { Cho et al. } 2016\end{array}$ \\
\hline \multicolumn{3}{|l|}{ Cho et al. 2016; } \\
\hline $\begin{array}{l}\text { Umgang mit Lernenden } \\
\text { mit hohem Förderbedarf }\end{array}$ & $\begin{array}{l}\text { Hohe Kompetenzen; } \\
\text { Hohes Wohlbefinden; }\end{array}$ & Zee et al. 2016 \\
\hline
\end{tabular}

Empirische Befunde für eine evidenzbasierte Analyse zur Entwicklung der Lehrer/innenselbstwirksamkeitserwartung in der Zeit des Studiums, in den Praxisphasen oder durch die Begleitung von Mentor/innen stellen ein Desiderat dar (Pfitzner-Eden 2016; Böhnert et al. 2018). Wenn Ergebnisse zu Studien bei Studierenden vorliegen, dann sind sie uneinheitlich in der Aussagekraft: Zunahme der Lehrer/innenselbstwirksamkeit in Praxiszeiten (Klassen et al. 2014), keine Zunahme in Praxisphasen (Schulte et al. 2008), Abnahme der Lehrer/innenselbstwirksamkeit in Praxisphasen (Garvis et al. 2012) und Induktionsphase (Woolfolk Hoy et al. 2005). In diesem Zusammenhang stellen sich Fragen nach Auswahl der Mentor/innen und Bedeutung der Mentor/innen für den schulpraktischen Mentoringprozess im Lehramtsstudium. Sollten nur Mentor/innen ausgewählt werden, die nach eigener Einschätzung eine hohe Lehrer/innenelbstwirksamkeitserwartung angeben? Welchen Einfluss haben Mentor/innen mit hoher Lehrer/innenselbstwirksamkeitserwartung auf Studierende? Welche Begleitungskriterien sind für Mentor/innen mit hoher Lehrer/innenselbstwirksamkeitserwartung von Bedeutung? Welche Strategien können Studierende bei Mentor/innen mit hoher Lehrer/innenselbstwirksamkeitserwartung erfahren? Solche und ähnliche Fragen könnten den Rahmen für Forschungsansätze bieten und Befunde zur berufsbezogenen Selbstwirksamkeitserwartung diskutieren. Im Rahmen des schulpraktischen Mentorings wären die Erkenntnisse sicherlich gewinnbringend für das reflexive Handeln der Mentor/innen bzw. bei der Erstellung von Ausbildungsprogrammen für Mentor/innen. 
Zusammenfassend kann gesagt werden: Das Kapitel behandelt die personale Lern- und Entwicklungsförderung im schulpraktischen Mentoring. Es wurden Konzepte und Modelle zur Entwicklung der Selbst- und Sozialkompetenz durch unterschiedliche Begleit- und Reflexionsansätze - wie Zwiebelmodell, professionsspezifische Entwicklungsaufgaben, bedürfnis- bzw. typenorientierte und/oder kontextsensible Begleitung, Empowerment und Selbstwirksamkeit - diskutiert und angeführt. Forschungsbefunde zur Wirksamkeitsüberprüfung in Bezug auf Mentoring sind bei allen ausständig.

Den Ausgangspunkt des Kapitels bildet der Abschnitt Lernen als bildende Erfahrung aufgrund der Überlegungen nach Hascher (2012), „wie aus Praxiserfahrungen Lernprozesse werden“ (Hascher 2012, 122) können. Die im Abschnitt beschriebenen Ausführungen unterstreichen die Ansichten von Buck (2019): „Lernen wird ,nicht nur als Folge der Erfahrung angesehen, sondern die Erfahrung muss auch umgekehrt ,als eine Folge des Lernens' aufgefasst werden." (Buck 2019, X) Antworten, wie im Mentoringprozess Erfahrungen subjektorientiert angekurbelt werden können, werden gegenwärtig durch Aussagen zur Schaffung von Entwicklungsräumen und dem Anleiten einer reflexiv-forschenden Haltung für Fragen in das Sozialisationsfeld Schule gegeben. Erpenbeck et al. (2018) liefern noch eine Erweiterung des Gedankenganges zum Lernprozess. "Jeder Entwicklungsprozess ist auch ein Lernprozess. Aber nicht jeder Lernprozess ist auch ein Entwicklungsprozess." (Erpenbeck et al. 2018, 181)

Demnach ist die zentrale Frage im schulpraktischen Mentoring: Wie können Studierende durch Lernerfahrungen in den Schulpraktika Entwicklungsprozesse in Gang setzen? Qualitätsvolles schulpraktisches Mentoring und eine bedürfnis- bzw. typenorientierte und/oder kontextsensible Begleitung in den Pädagogisch-Praktischen Studien sind eine Variante und ein sinnvoller Weg.

Am Ende des Kapitels Mentoringprozess in der Lehrer/innenausbildung bietet es sich als Resümee der vielen Überlegungen an, eine eigene Definition zum schulpraktischen Mentoringprozess zu formulieren:

Der schulpraktische Mentoringprozess als professionsspezifische Lern- und Entwicklungskultur zielt nicht auf Meisterlehre und Werkstattlernen, sondern auf einen individuellen und wertebasierten, stärken- und ressourcenorientierten Begleitungsprozess. Eine Auseinandersetzung mit situationsspezifischen Professionalisierungsmaßnahmen für Mentee umschließt im reziproken Zusammenspiel auch die Professions(weiter)entwicklung für jede/jeden Mentor/in.

Die Definition betont das gemeinsame Lern- und Entwicklungsvorhaben und unterstreicht personenspezifisches Mentoring ausgerichtet auf einen Professionalisierungsprozess. Wie der Begleitungsprozess durch mentor/innenseitige Steuerung unterstützt werden kann, wird im Modell des Transformationalen Mentorings dargelegt. Dazu werden im Vorfeld Ansätze zu Leadership und Transformationaler Führung erläutert.

\subsection{Mentoring und Transformation}

Mentoring und Transformation und dessen Bedeutuung wird in Verbindung mit Führung und mit Leadership sowie Transformationaler Führung diskutiert und konzentriert sich in erster Linie auf die lehrseitige Perspektive. Dabei wird zunächst die Definition nach Ziegler (2009) über Mentoring noch einmal aufgegriffen und als Grundlage für weitere Gedankengänge zum Transformationalen Mentoring verwendet: 
„Mentoring ist eine zeitlich relativ stabile dyadische Beziehung zwischen einem/einer erfahrenen MentorIn (sic!) und seinem/r/ihrem/r weniger erfahrenen Mentee. Sie ist durch gegenseitiges Vertrauen und Wohlwollen geprägt, ihr Ziel ist die Förderung des Lernens und der Entwicklung sowie das Vorankommen des/der Mentees.“ (Ziegler 2009, 11)

Ziegler (2009) hat eine Definition von Mentoring vorgegeben, die in vielen Publikationen zitiert wird. Eine Grundvoraussetzung zum Mentoring ist die Bereitschaft der/des Mentor/in, in eine verantwortungsvolle und sinnstiftende Mentoring-Beziehung einzutreten. Es geht nicht darum, Erfahrungen, die die/der Mentor/in (einst) gemacht hat, 1:1 weiterzugeben. Erlebte Erfahrungen bilden lediglich einen Anstoß für Diskussionen und Reflexionsprozesse in der Dyade. Wenn man den Begleitungsprozess aus der Perspektive der/des Mentorin/Mentors betrachtet, dann geht es primär darum, dass sie/er eine Führungsrolle übernimmt und den Stein für Lern- und Entwicklungsprozesse - gerecht ist natürlich für die/den Mentee als auch für die/ den Mentor/in als ständig Lernende/r - ins Rollen bringt. Die/Der Mentor/in führt, begleitet, unterstützt, beobachtet, analysiert, reflektiert - sie/er nimmt bewusst oder unbewusst, bedacht oder weniger bedacht eine Führungsrolle ein.

Wie der Diskurs um Führung und im Besonderen Transformationale Führung in die Praxis des schulpraktischen Mentorings übersetzt werden kann, wurde bisher als Forschungsansatz nicht sonderlich behandelt. Dieses Kapitel soll einen kurzen Einblick in die Thematik geben. Dazu werden nun die Begriffe Führung und Leadership sowie Transformationale Führung erläutert, um in weiterer Folge Transformationales Mentoring als Begleitform der schulischen Anteile näher zu beleuchten (Tabelle 45).

Tab. 45: Auflistung der im folgenden ausgeführten Unterkapitel

\begin{tabular}{ll}
\hline Mentoring und Transformation & Kurzbeschreibung \\
\hline Führung und Leadership & Beachtung von Subjektivität und Multiperspektivität in der Führungsrolle \\
Transformationale Führung & Konzept eines Führungsstils mit Zulassen von Veränderungen \\
Transformationales Mentoring als & $\begin{array}{l}\text { Sozialer Prozess mit Zieldimensionen und Innovationen im schulprak- } \\
\text { tischen Kontext }\end{array}$ \\
\hline
\end{tabular}

Die Kurzbeschreibungen der nun angeführten Abschnitte weisen darauf hin, dass zur Erklärung des Konzepts Transformationales Mentoring zunächst Begrifflichkeiten und Ansätze der Führungsrolle und des Führungsstils mittels Literaturrecherchen ausgeleuchtet werden.

\section{Führung und Leadership}

Der Begriff Führung oder Führen ist unterschiedlich konnotiert. Es ist ein „multidimensionales Konstrukt, welches insbesondere von den verfolgten Führungsansätzen und -theorien und den darin eingeschlossenen impliziten Annahmen - wie insbesondere Menschenbildern - abhängt." (von Au 2016, 5) In der Literatur werden „personen-, verhaltens- und situationsorientierte Ansätze" (von Au 2016, 6) unterschieden. Eine einheitliche Definition ist schon aufgrund der mehrperspektivischen Betrachtungsweise der unterschiedlichen Wissenschaften kaum möglich. Am ehesten wird die Bezeichnung „zielbezogene Einflussnahme“ (von Rosenstiel 2014, 3 ) verwendet. Nach Pinnow $(2005,38)$ stehen beim systemischen Führen „das Zusammenspiel und die wechselseitigen Einflüsse der Personen oder Themenbereiche im Vordergrund." (von 
Au 2016, 5) Somit wird suggeriert, dass „Führung als Rolle oder (...) als sozialer Prozess gekennzeichnet" (Werther 2016, 172) ist.

In unserer modernen technologisierten und globalisierten Wirtschafts- und Arbeitswelt wird neuerdings statt von Management von „Leadership“, „neuer Autorität“ (Baumann-Habersack 2017,8) oder von „New Leadership“ (von Au 2016, 13) gesprochen. Erfolgreiche Unternehmen bauen in der sich rasant veränderten Welt auf bottom-up Strategien und setzen auf eine „zieldienliche Zusammenarbeit" (Baumann-Habersack 2017, 92). Dennoch braucht es in offen geführten Strukturen auch Personen, die führen. Führen (leadern) bedeutet, „Einfluss zu nehmen auf sich, die Organisation und die darin tätigen Menschen." (Finckler 2017, 184) Um Prozesse zu gestalten und Transformationen zu entwickeln, braucht es eine „visionäre, motivierende, inspirierende und innovative" (Schley et al. 2011, 276) Führungshaltung. Wie kann sie aufgebaut und/oder entwickelt werden? Blane (2017), Consultingberater von Firmen wie Sony Pictures oder Starbucks usw., weist in seiner Publikation auf die Bedeutung der Führungsrolle hin und lenkt hierbei die Aufmerksamkeit auf eine Auseinandersetzung mit dem eigenen Selbstbild, auf Selbstverantwortung und Selbstrespekt hin (Blane 2017, 246). Seine Empfehlung lautet:

"What the people whom you lead want most of all is to trust you, respect you, and have a meaningful personal connection with you so they can come along side of you and create something noteworthy and transformational. They want that from you, but you cannot give that to them unless you trust, respect, and have a personal connection with yourself." (Blane 2017,246)

Führungspositionen benötigen somit Raum und Zeit, um sich mit Fragen im Sinne der philosophischen Anthropologie auseinanderzusetzen. In der Selbstreflexion sollen Wertvorstellungen und Überzeugungen aufgegriffen und analysiert werden, indem die Person ihre/,seine Innenperspektive (Subjektivität) mit der Außenperspektive von Beobachtern (sic!) abgleicht (Multiperspektivität).“ (Finckler 2017, 74) Baumann-Habersack (2017) verstärkt diese Ansicht und fügt hinzu, dass Führungspersönlichkeiten sich neben der individuellen Ebene auch mit der kulturellen und strukturellen Ebene auseinandersetzen und identifizieren müssen (BaumannHabersack 2017, 118). Die individuelle Ebene schließt ein Wissen um die eigene Persönlichkeitsstruktur ein, die Bereitschaft zur reflektierten Betrachtungsweise der eigenen Person und der Biografie, eine Auseinandersetzung mit der eigenen Werthaltung, Kenntnisse aus dem Bereich der emotionalen Intelligenz und eine Kompetenz zu Konflikten und Bewältigungsstrategien (Baumann-Habersack 2017, 119). Die kulturelle Ebene wirft einen Blick auf das soziale Gefüge, das interaktionistische Zusammenspiel und die Haltung in der Begegnung (BaumannHabersack 2017, 138f). Auf der strukturellen Ebene ist auf die Schaffung eines kollaborativen Handlungsrahmens und auf die Optimierung der Rahmenbedingungen zu achten (BaumannHabersack 2017, 145f).

Grint (2010) beschreibt Leadership als ein heuristisches Modell, das im Wesentlichen vier Ansatzpunkte vertritt: Leadership als Position für Leaders, Leadership als Person, Leadership als Resultat, Leadership als Prozess (Grint 2010, 4f). Die Komplexität in der Durchführung zeigt sich sowohl für die/den Führer/in als auch für die/den Geführten, die/der eine unverzichtbare Rolle einnimmt, denn "without followers, you cannot be a leader." (Grint 2010, 13f) Die Kernaufgaben von Leadership sind die Möglichkeit einer inter- und intrapersonellen Weiterentwicklung auf Evidenzbasierung und ein offener Umgang mit Fehlerkultur (Grint 2010, 67), die Identitätsfindung sowie der Aufbau einer visionär geprägten Unternehmenskultur (Finckler 2017, 89; 184). Diese Aufgaben sollten auch im schulpraktischen Mentoring Anwendung finden können: Möglichkeit(en) der Weiterentwicklung, Erfahrungsbildung und Umgang mit Fehlern, Identitätsfindung und Verwirklichung berufsfeldbezogener Visionen. 
Im folgenden Abschnitt wird der Blick auf die Bedeutung visionärer Momente gelegt - auf Transformationale Führung.

\section{Transformationale Führung}

Die von Burns (1978) entwickelte und von Bass et al. (1994) weiterentwickelte Theorie der Transformationalen Führung beruht im Wesentlichen auf „Einflussnahme und Motivation“ (Pelz 2016, 96). Nach von Au (2016) basiert die Theorie

„auf der Annahme, dass Führung einen Prozess darstellt, der Führende und Geführte (sic!) verändert, sie „transformiert“ und so einerseits zu erhöhter Produktivität, aber andererseits auch zu einem moralischen Verhalten führt." (von Au 2016,22)

\section{$\mathrm{Zu}$ dieser sinnstiftenden und werteorientierten Theorie}

„gehört die Erfüllung der Vorbildfunktion durch Führungskräfte, die Entwicklung individueller Stärken und Talente von Mitarbeitern (sic!), die Anregung zu mehr Eigeninitiative und kreativer Problemlösung sowie die Vermittlung von sinnvollen, attraktiven Zielen und Entwicklungsperspektiven." (Pelz 2016,96)

Bass et al. (1994) beschreiben zur Theorie der Transformationalen Führung die vier Dimensionen: Inspirational Motivation, Idealized Influence, Intellectual Stimulation und Individualizes Consideration als Charakteristika. Folgend werden Erklärungen und Interpretationen angeführt (Tabelle 46):

Tab. 46: Dimensionen und Beschreibung der Transformationalen Führung (Wenddorf 2018, 48; von Au 2016, 23; Pelz 2016, 96)

\begin{tabular}{ll}
\hline Dimensionen & Beschreibung \\
\hline \multirow{2}{*}{ Inspirational } & - Formulieren von anspruchsvollen, attraktiven Zielen \\
Motivation & - Aufzeigen von Visionen \\
& - Sinngebende Haltung gegenüber Zielen und Aufgaben \\
& - Zuversicht in Gruppe und Team zur Erreichung der Ziele \\
\hline \multirow{3}{*}{ Idealized Influence } & - Authentische Führungsperson mit hohen ethischen und moralischen Standards \\
& - Vertrauensvoller, respektvoller Umgang \\
& - Verlässlichkeit, Glaubwürdigkeit, Begeisterungsfähigkeit \\
& - Blick auf Gesamtinteresse vor den persönlichen Zielen und Vorteilen \\
& - Vorbildfunktion und Vorbildverhalten \\
& - Charismatische Wirkung \\
\hline \multirow{3}{*}{ Intellectual } & - Fördern der Kreativität, Innovation und Eigenständigkeit zur Problemlösung \\
Stimulation & - Fördern der Reflexionsfähigkeit \\
\hline & - Raum und Zeit für kritisches Hinterfragen \\
Individualized & - Beachten der individuellen Stärken, Schwächen und Erwartungen beim Gegenüber \\
Consideration & - Fördern der Persönlichkeit und des Potentials \\
& - Loben und positive Verstärkung \\
& - Einbeziehen in Entscheidungsprozesse \\
& - Übertragen von verantwortungsvollen Aufgaben \\
& - Ermutigen zur Eigenverantwortlichkeit \\
\hline
\end{tabular}


Alle vier Dimensionen verfolgen eine intra- und interpersonelle Perspektive in der Begleitung, wenn es darum geht, Potentiale zu erkennen, auszuschöpfen und zu stärken. Pelz (2016) führt Studien zur Transformationalen Führung an. Die Ergebnisse des MLQ-Fragebogens (Multifactor Leadership Questionnaire) zeigen eine hohe Zufriedenheit, hohe Motivation, Teamgeist, hohes Verantwortungsbewusstsein und Kreativität der/des Geführten durch Transformationale Führung (Pelz 2016, 97). Beim Ansatz der Transformationalen Führung stehen „Empowerment, Förderung und Lernen der Geführten (sic!) im Vordergrund.“ (von Au 2016, 24)

Die Frage ist, wie dieser Ansatz auf schulpraktisches Mentoring übertragen werden kann. Im Sinne einer Transformationalen Führung und nach dem Konzept von Bass et al. (1994) sollten Mentor/innen eine positive Lebenseinstellung mit Zukunftshoffnung und Sinngebung ausstrahlen (Inspirational Motivation), offene Werthaltung und Wertkultur leben (Idealized Influence), Kritik zulassen (Intellectual Stimulation) und Individualität anerkennen (Individualized Consideration). Im Gegenzug werden Mentee ermutigt, eigenverantwortlich zu handeln und Aufgaben zu lösen (Individualized Consideration). Persönlichkeit, Kreativität und Reflexionsfähigkeit werden gefördert (Intellectual Stimulation), Mentee erleben ein authentisches Umfeld mit verantwortungsvollen, begeisterten und persönlichkeitsgestärkten Mentor/innen (Idealized Influence) und Mentee erhalten Anregungen, werden in Entscheidungen eingebunden und können Ziele verfolgen (Inspirational Motivation).

Welche konkreten Maßnahmen bei der Umsetzung dieser Gedankengänge bzw. des Konzepts nach Bass et al. (1994) erforderlich sind, dazu soll Transformationales Mentoring als Begleitform der Schulpraktika Einblick geben.

\section{Transformationales Mentoring als Begleitform der schulischen Anteile}

In Rückbindung an die Definition nach Ziegler (2009) sowie die oben genannten Darstellungen zu Führung und Transformationaler Führung stellen sich für Transformationales Mentoring im schulpraktischen Begleitungskontext zwei wesentliche Fragestellungen:

(1) Wie können normativ-curriculare Vorgaben von den Beteiligten (Mentor/innen und Mentee) im Sinne eines Transformationalen Mentorings aufgefasst werden und wie erfolgt die Umsetzung?

(2) Welchen Charakteristika entspricht ein lern- und entwicklungsorientierter Prozess im Transformationalen Mentoring?

(1) Die Ziele nach Ziegler (2009) im Mentoringprozess sind „die Förderung des Lernens und der Entwicklung sowie das Vorankommen des/der Mentees.“ (Ziegler 2009, 11) Diese beruhen auf einem wechselseitigen Austauschprozess mit allen Akteurinnen/Akteuren mit Betonung auf Wechselseitigkeit, die auf mehreren Ebenen stattfinden kann und soll. Im Folgenden werden Erklärungen zur strukturellen und organisatorischen Ebene sowie zur individuellen und kulturellen Ebene nach einem Vorschlag von Baumann-Habersack (2017) angeführt, um die erste Frage zu beantworten.

Die strukturelle und organisatorische Ebene verlangt von Mentor/innen und Mentee unterschiedliche Aufgabenstellungen in den Praktika. Hierbei stellt sich die Frage, ob durch bestimmte Vorgaben Visionen im Sinne eines Transformationalen Mentorings entstehen können. Institutionen haben auf Basis der Theoriebasierung Zielorientierungen und Anforderungsprofile für Schulpraktika entwickelt. Idealerweise ist eine Beteiligung der Mentor/innen bei der Ausarbeitung der normativ-curricularen Vorgaben zur intensiven Verständigung wünschenswert. Die frühzeitige Einbindung in den Gestaltungsprozess bedingt eine kritisch-reflexive Auseinandersetzung mit Modellen, Konzepten, Theorien, Zielen, 
Inhalten und Schwerpunktsetzungen und eine berufsspezifische Identifikation. Schulpraktika bieten Raum und Zeit, theoriegeleitete Konzepte und Modelle im pädagogischen Handlungsfeld auszuprobieren und visionäre Strukturen zu erproben. In der Literatur wird vielfach vom Begehen eines „Third-Space“ (Burch et al. 2013) gesprochen.

"Third-Space working suggests coming out of our normal working environment (school or university) and into a neutral 'Third-Space' to design, develop and deliver teacher education with jointly shared understanding and vision." (Burch et al. 2013, 57f)

Beide Partner/innen - Mentor/in und Mentee - sollen mit normativ-curricularen Vorgaben vertraut sein, um in den gemeinsamen „Third-Space“ (Burch et al. 2013, 57f) eintauchen zu können und Visionen im Schulalltag umzusetzen. Außerdem soll innerhalb der Aufgabenstellung genug Handlungsspielraum möglich sein, damit Visionen umgesetzt und attraktive Ziele verfolgt werden können.

Die Soziologie verwendet für die Einbeziehung von strukturellen und organisatorischen Sichtweisen den systemtheoretischen Ansatz. Die Betrachtungsweise richtet sich auf ganzheitliche und teilbedingte Aspekte, Eigenschaften und Strukturen des Systems. „Systemisch denkende Führungspersönlichkeiten erkennen sich und ihre Geführten als zum jeweiligen System zugehörig." (von Au 2016, 26) Verhaltensweisen und Handlungen zielen auf das System ab und konstruieren letztendlich Identität (von Au 2016, 26). In Zusammenhang mit schulpraktischem Mentoring bedeuten diese Denkfiguren, dass Mentor/innen das System Schule öffnen und für Mentee eine systembedingte Vertrauensbasis geschaffen wird, indem Entfaltung und Entwicklung möglich sind. Im Sinne des Transformationalen Mentorings kommt zur Öffnung des Systems Schule die Bereitschaft, Neues zuzulassen und auf Veränderungen positiv zu reagieren.

Auf individueller und kultureller Ebene bedarf es einer kritischen Reflexion als Mentor/ in und als Person. Die Mentoring-Funktion ist eine Erweiterung zur Profession Lehrer/in und verlangt besondere Führungskompetenzen. Ausbildung und Weiterbildung sollen zum Selbstverständnis für Mentor/innen gehören, denn es braucht Verständnis und Einsicht, dass Lern- und Entwicklungsprozesse auch für Mentor/innen niemals endend sind. Pelz (2016) schlägt für die Arbeit als Führungskraft - und im Rahmen der vorliegenden Arbeit betrifft es Mentor/innen - eine klare Auseinandersetzung mit dem Selbst- und Fremdbild vor, indem ein individuell abgestimmter Entwicklungsplan seitens der Führungskraft (Mentor/in) erstellt wird. Darin sind kurzfristige Verhaltensänderungen, die sofort umgesetzt werden, und langfristige Überlegungen, die zur persönlichen und beruflichen Reife beitragen, schriftlich aufgelistet. Die Zieldimensionen im persönlichen Entwicklungsplan werden dann mit den jeweiligen Verantwortlichen z.B. der Ausbildungsinstitutionen besprochen. Im Grunde handelt es sich bei der Verhaltensänderung „um einen kontinuierlichen Verbesserungsprozess." (Pelz 2016, 109f)

Nach den angeführten Überlegungen könnte die Beantwortung der ersten Frage dieses Abschnittes folgend lauten: Zunächst ist es wichtig, dass die Beteiligten normativ-curriculare Vorgaben kennen, ideal wäre, wenn Mentor/innen in die Konzeption eingebunden sind bzw. waren. Normativ-curriculure Vorgaben haben in den meisten Fällen, wie z.B. in den Pädagogisch-Praktischen Studien Rahmencharakter. Sie bieten Handlungsspielraum und Möglichkeiten, Veränderungen zuzulassen. Vor dem Hintergrund einer Transformation steht aber auch die Absicht, dass Zieldimensionen im Mentoring angedacht werden und Handlungsschritte vollzogen werden wollen. Dazu braucht es eine kritisch-reflexive Hal- 
tung in der Mentoring-Funktion. Transformationales Mentoring wird demnach als ein Prozess verstanden, der unterschiedliche Ebenen auf dem Weg zur Umsetzung beleuchtet und bereit ist Veränderungen und Weiterentwicklungen zuzulassen.

(2) Damit Lern- und Entwicklungserfahrungen im Transformationalen Mentoring möglich sind, benötigen Mentor/innen Kenntnisse über sich selbst und andere - eine Reflexionsfähigkeit über das eigene Selbst- und Fremdbild, um einen zielbezogenen Prozess führen und gestalten zu können. Instrument für Mentor/innen zum Transformationalen Mentoring im schulpraktischen Kontext könnte die Erweiterung des MLQ-Fragebogens (Multifactor Leadership Que-stionnaire) nach Bass et al. (1994) sein, und zwar das „Gießener Inventar der Transformationalen Führungskompetenzen“, das Items zu sieben Kategorien beinhaltet: Identifikation (Vorbild und Vertrauen), Inspiration (Motivieren), Stimulation (Anregen zur Problemlösung), Förderung, Kommunikation und Fairness, Unternehmerische Haltung und Umsetzungsstärke (Pelz 2016, 99f).

Aus den angeführten Betrachtungsweisen ergeben sich Überlegungen für mögliche Kategorienbildungen zu Führungsverhalten im Transformationalen Mentoring im schulpraktischen Kontext. Dabei wird von der Annahme ausgegangen, dass durch Selbstreflexion und Wissen um Selbstkompetenz Mentor/innen gestärkt in einen Mentoringprozess hineingehen und die Führungsrolle vorbildlich übernehmen, aber auch bereit sind, Veränderungen zuzulassen und Zieldimensionen neu anzudenken. Tabelle 47 benennt rekonstruierte Kategorien aus den besagten Modellen durch die Autorin dieser Arbeit und setzt sie in Zusammenhang mit Transformationalem Mentoring. Die angeführten konzipierten Fragestellungen dienen als Unterstützung zur Selbstreflexion für Mentor/innen.

Tab. 47: Bereiche und Fragehorizonte zum Transformationalen Mentoring im schulpraktischen Mentoring für Mentor/innen

\begin{tabular}{ll}
\hline Kategorien & Fragen zur kritischen Auseinandersetzung \\
\hline Motivation und Identität & Warum will ich Mentor/in sein? Welchen Sinn hat für mich die Begleitungstätigkeit? \\
\hline Kontextbedingungen & $\begin{array}{l}\text { In welchem Kontext bin ich beim Mentoring eingebettet? Wie kann ich visionäres } \\
\text { Denken und Handeln unterstützen? }\end{array}$ \\
\hline Rolle und Funktion & $\begin{array}{l}\text { Wie definiere ich meine Rolle in der Begleitung? Wie habe ich Rollen und Funkti- } \\
\text { onen bisher im Mentoring erlebt? }\end{array}$ \\
\hline Werthaltung & Welche Werte vertrete ich? Und warum? \\
\hline Persönlichkeitsmerkmale & Welche Stärken und Schwächen bringe ich mit? \\
\hline Beziehung & Was bedeutet für mich Beziehungskultur im Begleitungsprozess? \\
\hline Kommunikation und & $\begin{array}{l}\text { Welche Kompetenzen bringe ich zu Kommunikation und Mediation mit? Wie } \\
\text { gehe ich mit Fehlerkultur um? }\end{array}$ \\
\hline Erwartungen & $\begin{array}{l}\text { Welche Erwartungen werden an mich gestellt? Welche Erwartungen habe ich im } \\
\text { Begleitungsprozess? }\end{array}$ \\
\hline Zieldimensionen & $\begin{array}{l}\text { Was sind meine Zieldimensionen in der Begleitung? Welche Schritte muss ich } \\
\text { einsetzen, damit ich eine Zieldimension erreiche? Wann habe ich die/eine Zieldi- } \\
\text { mension erfolgreich erreicht? }\end{array}$ \\
\hline Verhaltensänderung & $\begin{array}{l}\text { Lasse ich Verhaltensänderungen zu? Wie erkenne ich, dass sich Verhalten verändert } \\
\text { hat? Wann ist für mich der Entwicklungsprozess erfolgreich? }\end{array}$ \\
\hline
\end{tabular}


Die Fragen bieten also zunächst Anlass zur Selbstreflexion. Eine inhaltliche Vertiefung sollte das Ziel verfolgen, Entwicklungs- und Verbesserungsstrategien im Mentoringprozess unter Einbeziehung der Rahmenbedingungen zu vereinbaren und zu konkretisieren. Die Kunst eines gelingenden Prozesses liegt wohl darin, motiviert an den Prozess heranzugehen, die personellen Besonderheiten der/des Einzelnen zu respektieren, Freude und Sinn im gegenseitigen Lernen zu erkennen, Gemeinsamkeiten hervorzuheben, Differenzkategorien wahr- und anzunehmen, Werthaltungen zu vertreten und Zieldimensionen zu planen und zu verfolgen. Der kritische Blick auf Subjektivität der Führungspersönlichkeit und Multiperspektivität im Prozess sollen schließlich zu inspirierender Motivation, idealisiertem Einfluss, intellektueller Anregung und individueller Betrachtung (vier Dimensionen nach Bass et al. 1994) führen und den Entwicklungsprozess bei Mentor/in und Mentee in Gang setzen und Gestaltungsveränderungen zulassen. Und: "Luckily for leaders, that need not be that difficult because it depends not upon rational analysis of 'the facts' but upon an emotional and often unconscious response." (Grint 2010, 80) In der Selbstreflexion wird das eigene Handeln, Denken und Fühlen hinterfragt und der Weg für Neues angebahnt und geöffnet.

Kraler et al. (2012) bieten zum Aspekt Veränderung das Modell Change of pattern (Kraler et al. 2012a, 92) an. Die Autoren schlagen für die Lehrer/innenausbildung einen Musterwechsel und eine Verschiebung von best practice zu next practice vor. Der Grundgedanke, dass das Ziel nicht das Hervorheben von „best practice“ und „more of the same“ (Kraler et al. 2012, 93) Beispiele sind, sondern das Einläuten einer next-practice-Entwicklung vollzogen werden soll, kann auch in Zusammenhang mit der Betreuung und Begleitung von Mentee gesehen und in einem $\mathrm{Zu}$ sammenhang mit Transformationalem Mentoring betrachtet werden (Abbildung 24).

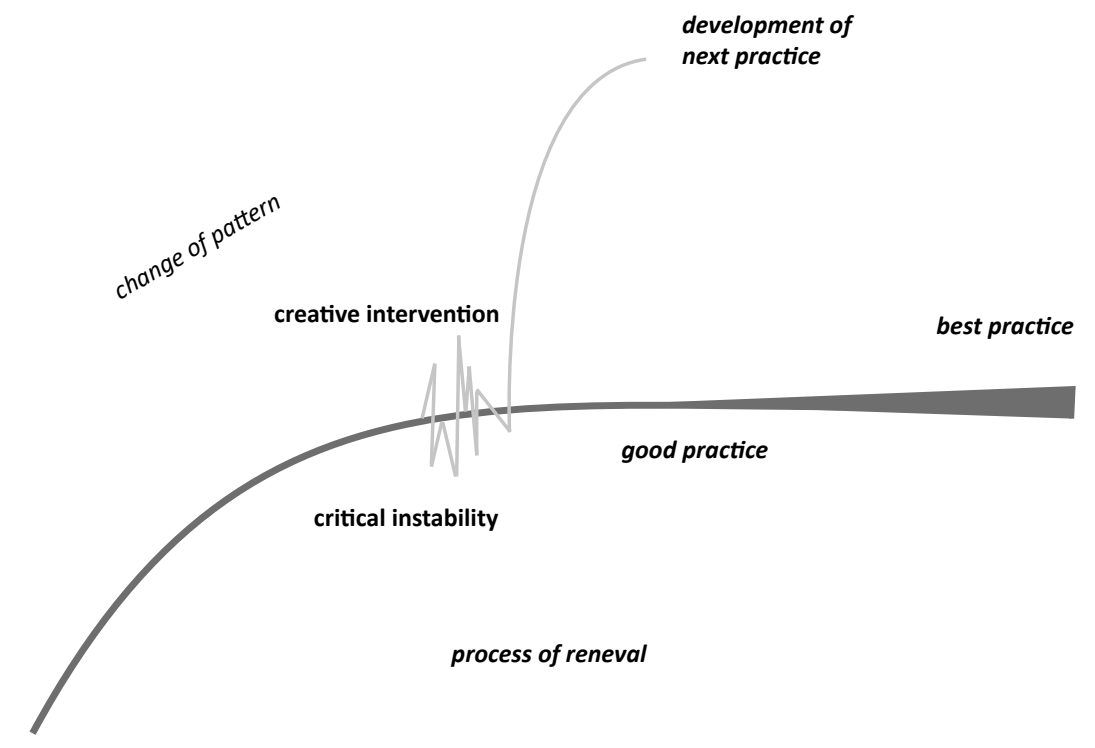

Abb. 24: Musterwechsel (Kraler et al. 2012a, 92)

"For new patterns to emerge, critical incidents or interventions are necessary to enable the opening of the next practice perspective (Kruse, 2004).” (Kraler et al. 2012, 92) Wie kritische Vorfälle oder Instabilitäten aussehen können, divergiert in jedem sozialen Gefüge und im sozialen 
System. „In der Instabilität verringert sich in Systemen zwar vorübergehend die Handlungsfähigkeit, aber die Anpassungsfähigkeit ist erhöht, und sie werden kreativ." (Kruse 2004, 61) So ist anzunehmen, dass Visionen umgesetzt werden und die Lernkurve sich anhebt und nicht stagnierend weiterschreitet (Kraler et al. 2012, 92f).

Zum Transformationalen Mentoring ergeben sich noch zwei wesentliche Überlegungen im Hinblick auf Mentee: Welche Rolle haben Geführte? Nehmen sie überhaupt eine Rolle ein? Nach Ziegler (2009) ist Mentoring eine „dyadische Beziehung“ (Ziegler 2009, 11) und beide Partner/innen nehmen - wie Grint (2010) behauptet - eine Rolle und eine Funktion ein: beide würden ohne einander nicht existieren (Grint 2010, 111). Bezogen auf schulpraktisches Mentoring ist die Rolle der/des Geführten noch wenig erforscht. In Anlehnung an Modelle zur Identitätsfindung und Vorstellungen zu selbstreflexiven Prozessen sowie nach dem „Gießener Inventar der Transformationalen Führungskompetenzen“ (Bass et al. 1994) werden im Folgenden die zehn Kategorien, die bereits für Mentor/innen beschrieben wurden, wieder verwendet und exemplarische Fragestellungen bezogen auf Mentee entwickelt (Tabelle 48). Es wird dabei darauf geachtet, ob die Kategorien für Mentor/innen und Mentee Gültigkeit finden und in der Selbstreflexion Anwendung finden können.

Tab. 48: Bereiche und Fragehorizonte zum Transformationalen Mentoring im schulpraktischen Mentoring für Mentee

\begin{tabular}{ll}
\hline Kategorien & Fragen zur kritischen Auseinandersetzung \\
\hline Motivation und Identität & $\begin{array}{l}\text { Bin ich motiviert in einen Begleitungsprozess zu treten? Wie kann ich meine } \\
\text { Identität herausstreichen? }\end{array}$ \\
\hline Kontextbedingungen & In welchem Kontext bin ich beim Mentoring eingebettet? \\
\hline Rolle und Funktion & $\begin{array}{l}\text { Wie definiere ich meine Rolle in der Begleitung? Welche Rollen und Funktionen } \\
\text { sind mir im Mentoring wichtig? }\end{array}$ \\
\hline Werthaltung & Welche Werte vertrete ich? Und warum? \\
\hline Persönlichkeitsmerkmale & $\begin{array}{l}\text { Welche Stärken und Schwächen bringe ich mit? } \\
\text { Wie möchte ich als Person wahrgenommen werden? }\end{array}$ \\
\hline Beziehung & $\begin{array}{l}\text { Was bedeutet für mich Beziehungskultur im Begleitungsprozess? Wie möchte ich } \\
\text { Beziehung erleben? Wie werde ich Beziehung gestalten? Welche Kriterien gelten } \\
\text { für mich für eine dyadische Beziehungen, in denen ich die/der Mentee bin? }\end{array}$ \\
\hline Kommunikation & $\begin{array}{l}\text { Welche Kompetenzen bringe ich zu Kommunikation mit? Wie begegne ich } \\
\text { meinem Gegenüber in der Kommunikation? Wie äußere ich Sachverhalte? }\end{array}$ \\
\hline Erwartungen & $\begin{array}{l}\text { Welche persönlichen Erwartungen habe ich? Welche Erwartungen habe ich im } \\
\text { Begleitungsprozess? Welche Erwartungen werden an mich gestellt? }\end{array}$ \\
\hline Zieldimensionen & $\begin{array}{l}\text { Was sind meine Zieldimensionen? Welche Schritte muss ich einsetzen, damit ich } \\
\text { eine Zieldimension erreiche? Wann habe ich die/eine Zieldimension erfolgreich } \\
\text { erreicht? Wie kann mich meine/mein Mentor/in unterstützen? }\end{array}$ \\
\hline Verhaltensänderung & $\begin{array}{l}\text { Lasse ich Verhaltensänderungen zu? Wie gehe ich mit Kritik um? Wie erkenne } \\
\text { ich, dass sich Verhalten verändert hat? Wann ist für mich der Entwicklungspro- } \\
\text { zess erfolgreich? }\end{array}$ \\
\hline
\end{tabular}

Die exemplarischen Fragen sollen Hilfestellungen für die Positionierung der eigenen Person als Mentee sein und Struktur im Verlauf des Prozesses bieten, indem eine kritische Auseinandersetzung und Analyse erfolgt. Wie bei Mentor/innen gilt, dass der kritische Blick auf Sub- 
jektivität und Multiperspektivität auf Personen und Systeme sich bereichernd für Lern- und Entwicklungsprozesse auswirken kann. Die/Der Geführte nimmt im Mentoring ebenso eine Rolle ein. Selbstreflexion und Selbsteinschätzung sind Grundsteine zu Kreativität, Innovation und Identifikation.

Die zehn angeführten Kategorien zeigen, dass zu den jeweiligen Kategorien Fragestellungen aus dem Blickwinkel der/des Mentorin/Mentors und der/des Mentee/Mentees formuliert werden können. Lediglich bei der Kategorie Kommunikation und Mediation ist anzunehmen, dass diese Kategorienbeziehung auf Mentor/innen ausgelegt werden muss. Für Mentee würde die Kategorie Kommunikation zutreffend sein. Abbildung 25 veranschaulicht noch einmal die zehn rekonstruierten Kategorien und setzt sie in Beziehung zum Transformationalen Mentoring - als Vorschlag für einen selbstreflexiven Prozess.

\begin{tabular}{|c|c|c|c|}
\hline Motivation und Identität & Rolle und Funktion & Werterhaltung & Beziehung \\
\hline Persöhnlichkeitsmerkmale & \multicolumn{2}{|c|}{ Transformationales Mentoring } & $\begin{array}{l}\text { Kommunikation } \\
\text { (und Meditation) }\end{array}$ \\
\hline Kontextbedingungen & Erwartungen & Verhaltensänderung & Zieldimension \\
\hline
\end{tabular}

Abb. 25: Rekonstruierte Kategorien zum Transformationalen Mentoring

Die zentrale Frage zu jeder der zehn Kategorien sowohl aus Sicht der/des Mentorin/Mentors als auch aus Sicht der/des Mentee/Mentees muss im Rahmen Transformationalen Mentorings lauten: Welche Schritte kann/soll/muss/darf ich setzen, damit ein sozialer Prozess und ein Fortschritt im Sinne einer Lern- und Erfahrungsentwicklung zu verzeichnen sind?

Zusammenfassend soll hier noch einmal auf die eingangs zweite gestellte Frage eingegangen werden: Welchen Charakteristika entspricht ein lern- und entwicklungsorientierter Prozess im Transformationalen Mentoring? Hier wird die Annahme - analog zur Theorie der Transformationalen Führung (Burns 1978; Bass et al. 1994) - vertreten, dass die/der Mentor/in in der Rolle als Führende/r wesentlichen Einfluss auf das schulpraktische Mentoring nimmt. Daher wird der Fokus zunächst auf eine reflexive Auseinandersetzung der eigenen Person, der Mentoring-Rolle und somit der Mentoring-Identität gelegt. Bedeutend für einen gelingenden wechselseitigen Lern- und Entwicklungsprozess ist aber auch, dass sich die/der Mentee mit dem eigenen Selbst- und Fremdbild und der Mentee-Rolle beschäftigt. Es kann nun als Aufgabe der/des Mentorin/Mentors angesehen werden, Studierende bzw. Mentee dahingehend zu animieren bzw. zu begleiten, um in diesen gewünschten Reflexionsprozess einzutreten. Vielleicht ist auch die bewusste Verankerung von kritischen Instabilitäten notwendig, um den Entwicklungsraum einer neuen bzw. nächsten Praxisperspektive (Kruse 2004; Kraler et al. 2012) zu öffnen.

Transformationales Mentoring unter Einbeziehung der diskutierten Konzepte zu Führung und Leadership sowie Transformationaler Führung im schulpraktischen Kontext bedeutet demnach, dass die/der Mentor/in bereit ist, die subjektiven Einstellungen und Werthaltungen zu reflektieren, Führung bzw. Verantwortung für einen Prozess zu übernehmen und eine „zieldienliche Zusammenarbeit“ (Baumann-Habersack 2017, 92) zu forcieren. Damit wird eine positiv 
konnotierte „zielbezogene Einflussnahme“ (von Rosenstiel 2014, 3) auf die/den anvertraute/n Mentee verbunden. Im Sinne von Grint (2010) kann somit Transformationales Mentoring als Position für Mentor/innen als auch als sozialer Prozess (Grint 2010, 4f) angesehen werden. Entscheidend im Begleitungsprozess sind systemische, intra- und interpersonelle Betrachtungsweisen und Reflexionsprozesse zu etablieren, Transformationsabsichten zu verwirklichen, Entwicklung an der Person und im System zuzulassen (Hauser 2013, 285; von Au 2016, 21) und Veränderungen, Weiterentwicklungen und sinnstiftende Innovationen als Möglichkeit für produktives Gestalten zu erkennen. Dann wird Transformationales Mentoring eine Win-WinSituation für Akteurinnen und Akteure, denn in Anlehnung an Sokrates gilt: „Mentoring is about sharing wisdom - a two-way street that benefits both." (Sokrates)

\subsection{Zwischenfazit}

Das Aufgabenfeld von Mentor/innen besteht darin, Mentee in ihrem/seinem Motivationsvorhaben zu stärken, das Potential hervorzuheben und auf die Entwicklung der/des Mentee/Mentees zu achten. Diese Anschauungen decken sich mit Kriterien der Mentoringkonzepte in den unterschiedlichen Kontexten, wenn es darum geht, Personalentwicklung und Personalförderung voranzutreiben. Gerade auch in der Lehrer/innenausbildung erhält Begleitungs- und Betreuungstätigkeit im schulischen wie im hochschulischen Feld eine hohe Gewichtung.

Begleitende Lehrveranstaltungen zu Praktika mit reflexiv-forschenden Anteilen und der Möglichkeit eines erfahrungsbasierten Austausches prägen das heutige Ausbildungskonzept. Vermehrte Anteile Pädagogisch-Praktischer Studien, in denen Studierende das Berufsfeld Schule in unterschiedlichen Facetten kennenlernen können, sind fixer Bestandteil der Curricula der Pädagogischen Hochschulen und Universitäten in Österreich. Deren Verankerung beruht auf einer gesetzlichen Grundlage (BMB 2013, 124. Bundesgesetzblatt, Bundeskanzleramt 2013). In den Schulpraktika werden erfahrene Lehrpersonen in der Funktion der/des Mentorin/Mentors Studierenden zur Seite gestellt. Sie/Er erhält die Aufgabe, Studierende in den schulischen Lerngelegenheiten zu begleiten und zu unterstützen. Ausbildungsinstitutionen und Partnerschulen sind somit aufgefordert, zu kooperieren und in Austausch zu gehen.

Schulpraktisches Mentoring hat in der österreichischen Lehrer/innenausbildung lange Tradition. Sogenannte Besuchsschullehrpersonen, Übungsschullehrpersonen, Praxisausbildner/ innen, Betreuungslehrer/innen, Praxislehrpersonen standen und stehen als Expert/innen den angehenden Lehrpersonen in unterschiedlichen Ausbildungsphasen zur Verfügung. Die Meisterlehre und das Belehrtwerden - gepaart mit guten Ratschlägen, Vorgaben zielorientierter Strategiemuster und erfolgversprechenden Aussichten („So geht es! So hat es immer funktioniert!“) - sollen abgewendet werden. Vielmehr fordern Ausbildungsinstitutionen prozess- und förderungsorientierte Ansätze (Faix et al. 2017, 36), die zum reflektierten Handeln und Bilden von subjektiven Theorien führen (Klement et al. 1996, 16; Brenn et al. 1996, 24f).

Der Mentoringprozess ist durch Individuen gestaltet, die unterschiedliche Lebens- und Berufserfahrungen, ein differenziertes Professionsverständnis, subjektive Wertvorstellungen, Einstellungen und Haltungen, kulturelle Gepflogenheiten mit sich bringen und im besten Fall auf normativ-curriculare Konzepte, Vorgaben und Anforderungen, Personen und deren Kontext eingehen.

Mentor/innen haben unterschiedliche persönliche Zugänge und Auffassungen in der Ausübung ihrer Mentoring-Funktion - wie Bewältigungs-Funktion, Vorbild-Funktion und/oder Unter- 
stützungs-Funktion. Den Hintergrund dieser Mentoring-Funktionen bilden unterschiedliche theoretische Konzepte - wie das Konzept der Entwicklungsaufgaben, das Kognitionsmodell, Lernen am Modell oder das Konzept der sozialen Unterstützung (Fuge 2016, 76). Formale Standardisierungen sowohl zu Pädagogisch-Praktischen Studien als auch zu Mentoringprogrammen im schulpraktischen Kontext fehlen allerdings in Österreich bis heute.

"A differentiated mentoring curriculum would be flexible with a vision and clear aims for achieving state and national teaching standards appropriate to the mentee's level of proximal development." (Hudson 2013b, 32)

Im internationalen Kontext zum schulpraktischen Mentoring wird die Notwendigkeit von Standards immer wieder betont (Sachs 2003, 175f).

Strukturen und Zielvorstellungen innerhalb der Pädagogisch-Praktischen Studien und des Mentorings sind regional und institutionell unterschiedlich. In Publikationen werden zu den schulischen Anteilen oftmals Ziele erwähnt, wie primärer Ausbau und Forcierung der Reflexionsfähigkeit, Förderung der professionsspezifischen Entwicklung und Eigenständigkeit im pädagogischen Handeln (Arnold et al. 2011, 62f), Mitbestimmung des Prozesses durch Lernende sowie gemeinsamer Diskurs zu pädagogischen lernprozessbezogenen Tätigkeitsfeldern „(Komplexität über forschungsmethodische Zugänge erkunden, pädagogische Handlungssituationen planen, pädagogische Handlungssituationen durchführen, Theorien auf Situationen beziehen, mit Situationen analytisch-reflexiv umgehen)“ (Doll et al. 2018, 40). Befunde zeigen, dass sich Mentor/innen meist auf schulische Angelegenheiten im Umgang mit den Mentee konzentrieren (Bach et al. 2018, 198). Aus Sicht der Ausbildungsinstitutionen lässt diese Erkenntnis wiederum den Ruf laut werden, Mentor/innen in den Ausbildungsprozess an der Hochschule zu integrieren und mit Ausbildner/innen und Ausbildnern in einen Austausch zu Konzepten, Theorien und Modellen zu gehen.

Die Absolvierung der Ausbildung zur Praxislehrperson und/oder Betreuungslehrperson in Form eines Hochschullehrganges im Umfang von 6 bis 10 ECTS-AP reichte bisher als Erfordernis für die Ausübung der Tätigkeit. Aufgrund eines quantitativen Mangels an Mentor/ innen wurden auch Lehrpersonen beauftragt, die keine Ausbildung vorweisen können. Diese Praxislehrpersonen wurden auf Empfehlung der Direktionen zur Ausübung vorgeschlagen. Seitens des Bildungsministeriums liegt nun ein Empfehlungsschreiben (2019) zur Konzeption von Mentoringhochschullehrgängen vor. Darin wird auf die „Mischung aus forschungs- und theorie-basierter Wissensvermittlung, gemeinsamer Analyse- und Theoriebildung, praktischen Übungen und Arbeiten an Fallbeispielen aus der eigenen Praxis“ (Empfehlungsschreiben BMBWF 2019) im Umfang von 15, 30 bzw. 90 ECTS-AP hingewiesen. Es bleibt abzuwarten, wie Konzepte in den einzelnen Verbünden umgesetzt werden, wie Pädagogische Hochschulen in Kooperation mit den Universitäten den Konzeptualisierungsprozess verstehen und wie Lehrpersonen und angehende Mentor/innen dieses Ausbildungsformat annehmen.

Das Forschungsfeld zum schulpraktischen Mentoring ist im deutschsprachigen Raum überschaubar. Befunde sind meist in Zusammenhang mit Schulpraktika oder den in Deutschland neu etablierten Praxissemestern zu sehen (Reintjes et al. 2018, 11; König et al. 2011). Untersuchungsdesigns beschäftigen sich vor allem mit der Wirkungsweise von schulpraktischem Mentoring (König et al. 2018; Hobson 2009), prozessbegleitenden Formaten (Reintjes et al. 2018; Schüssler et al. 2017) und/oder der Erfolgsgenerierung (Hobson et al. 2009). Was versteht man unter Erfolg? Welche Prozesse sind im schulpraktischen Mentoring erfolgreich und generalisierbar? Wie sollen Beziehungen aussehen, damit erfolgreiches Lernen und Entwickeln mög- 
lich ist? Solche und ähnliche Fragen bleiben letztendlich auch in der Forschung ergebnisoffen. Jacobi (1991) meint dazu:

"The result of this definitional vagueness is a continued lack of clarity about the antecedents, outcomes, characteristics, and mediators of mentoring relationships despite a growing body of empirical research." (Jacobi 1991, 505)

Konzepte und Modelle zu Mentoring weisen auf einen personenbezogenen und beziehungsorientierten Ansatz zur Förderung und Unterstützung hin. Hierbei zu erwähnen sind die Modelle wie das GROW-Modell nach Whitmore (Faix et al. 2017), 4R-Modell nach Logan (Faix et al. 2017), Drei-Ebenen-Modell nach Niggli (Niggli 2005), ALACT-Modell nach Korthagen (Korthagen et al. 2005) usw., in denen Phasen in der Begleitung und ein möglicher Weg zur Prozessbegleitung beschrieben werden. Hobson et al. (2009) gehen davon aus,

„dass kein allgemeingültiges Mentoring-Modell für alle Mentees effektiv sei, sondern flexibles Eingehen auf die jeweiligen Bedürfnisse und Erfordernisse der Mentees entscheidend sei. Dies bedeutet jedoch nicht beliebige Vielfalt des Mentorings, sondern dass verschiedene Strategien und Stile bei Mentees je nach professioneller Entwicklungsphase, Persönlichkeit, Kontext zur Anwendung kommen sollen.“ (Brandau et al. 2018, 208)

Autor/innen wie Fletcher (1998), Schmid et al. (2011) und Fuge (2016) schlagen im Vorfeld der Zusammenarbeit sogenannte Matching-Prozesse vor, in denen Mentor/innen und Mentee die Gelegenheit bekommen, sich näher kennenzulernen und Vorstellungen von Arbeitsauffassung und Arbeitsverläufen besprechen. Nach dieser Erstbegegnung und dem gegenseitigen Kennenlernen wird die Entscheidung über eine Teambildung auf beiden Seiten getroffen. MatchingProzesse bieten vor allem auch die Möglichkeit, Erwartungshaltungen zu versprachlichen und zu verdeutlichen. Jede/Jeder Mentor/in kann in der Mentoring-Funktion davon ausgehen, dass sie/er mit Rollenerwartungen konfrontiert ist. Wie Hudson (2013) in seiner mixed-methodStudie dokumentiert, haben auch Mentor/innen Erwartungen an Studierende:

"enthusiasm for teaching, being personable for relationship building (not just with the mentor but also with students, staff and parents), displaying commitment to children and their learning, being a lifelong learning, having the ability to reflect on constructive feedback, having resilience, and taking responsibility for their learning." (Hudson 2013, 5)

Für Hudson (2013) ist es besonders wichtig, dass am Beginn des Mentoringprozesses die gegenseitigen Erwartungen geklärt werden.

Ein Blick in die Geschichte zeigt, dass beim schulpraktischen Mentoring lange davon ausgegangen wurde, dass es sich bei der Begleitform um einen expertisetheoretischen Ansatz handelt. Neuzeitige Befunde beschreiben den Prozess als wechselseitige Bereicherung und als gemeinsames Fortschreiten im professionsspezifischen Feld. Im Idealfall „entsteht ein Wechselspiel zwischen Rahmenbedingungen, Rollenverantwortung und einer Unterstützung der Entwicklung des individuellen Soseins." (Hellwig 2016, 40) Garvey (2000) veranschaulicht in einem Modell mit den Achsen Kompetenz und Erfahrung unterschiedliche Herangehensweisen (Abbildung 26). Die erste Abbildung verdeutlicht das Herantasten des Mentee (Protégé) an die/den Mentor/in und das gleichbleibende Kontinuum beider Beteiligten in der Kompetenzentwicklung. Die zweite Abbildung zeigt das gemeinsame Voranschreiten von Mentor/in und Mentee (Protégé) in der Kompetenzentwicklung durch den gemeinsamen Erfahrungsaustausch auf (Garvey 2000, 9). 

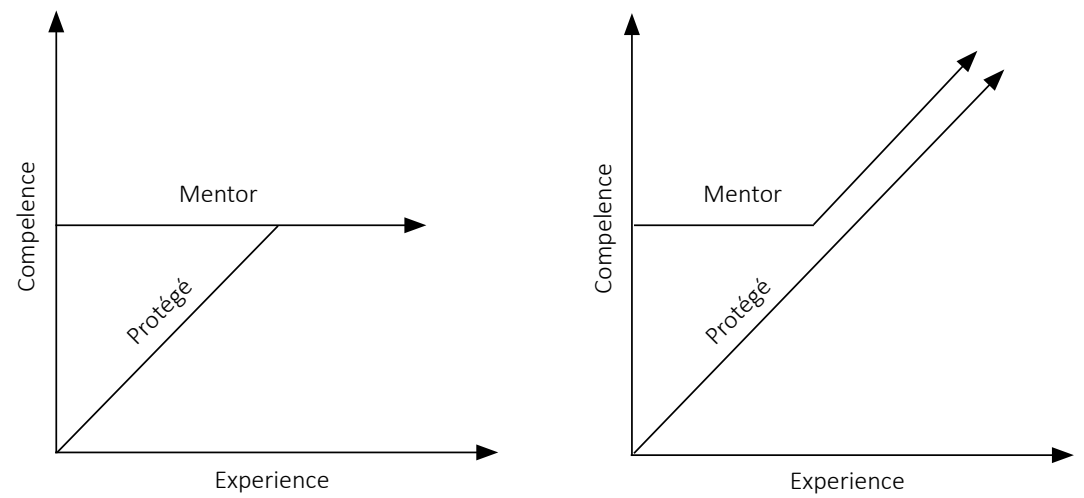

Abb. 26: Kompetenz-Erfahrungs-Modell im wechselseitigen Austausch (Garvey 2000, 9)

Ein lern- und entwicklungsförderndes Umfeld bedarf nach Rogers (2009) der Wertschätzung, Empathie und Kongruenz (Rogers 2009, 46f). Die Ausgestaltung dieser drei Komponenten im Prozessgeschehen können durch wertschätzenden Umgang, verantwortliche Gestaltung und Mitbestimmung des Lernprozesses, Vertrauen in die Fähigkeit, Lösungen für Problemstellungen zu finden (Deweys 1986), zum Ausbau der Selbstaktualisierungstendenz beitragen (Rogers et al. 1991, 231). Entfaltung zum Selbst und zur Kreativität (Rogers) werden in jenen Mentoringsettings, wie z.B. durch personen- bzw. subjektorientierte (Rogers 2009), bedürfnisorientierte (Dreer 2018), typenorientierte (Košinár 2018; Nolle 2012) und/oder kontextsensible (Hofmann 2019) Begleitung unterstützt, die das Subjekt mit seinen Ausprägungsmustern in das Zentrum stellen und in einen Dialog treten. Was bewirkt Kreativität?

„Die Hauptquelle der Kreativität scheint die gleiche Tendenz zu haben, die wir als tiefste heilende Kraft in der Psychotherapie entdecken - die Tendenz des Menschen, sich selbst zu verwirklichen, seine Potentialitäten zu entfalten. Ich verstehe darunter den richtungsgebenden Impuls, der in allem organischen und menschlichen Leben evident wird - der Impuls, sich auszudehnen, sich zu erweitern, zu entwickeln, zu reifen - die Tendenz, alle Fähigkeiten des Organismus auszudrücken, und zwar in einem Maße, dass eine solche Aktivierung den Organismus oder das Selbst fördert. Diese Tendenz ist die primäre Motivation zu Kreativität, durch die der Organismus in seinem Streben, in umfassender Weise er selbst zu sein, neue Beziehungen mit der Umwelt aufnimmt." (Rogers 1990, 2007, 240f)

An der Privaten Pädagogischen Hochschule Edith Stein wird in den Pädagogisch-Praktischen Studien das Konzept der Entwicklungsaufgaben (Havighurst 1953; Trautmann 2004; Hericks 2006; Keller-Schneider 2010; Kraler 2012; Ostermann 2015; Köffler 2015) in den Mentoringprozess miteinbezogen. Studierende bearbeiten pro Praktikum je zwei Entwicklungsaufgaben und besprechen sich mit der/dem Mentor/in. Dazu liegen für jede rekonstruierte Entwicklungsaufgabe (Ostermann 2015) exemplarische Fragen als Hilfestellung zur Reflexionstätigkeit (KPH Edith Stein 2020) vor. Das Beziehungsgefüge Mentor/in und Studierende/r vertieft sich in personenspezifischem stärken- und lösungsorientiertem pädagogischem Denken und Handeln. Miteinbezogen werden auch Konzepte wie Empowerment (Arnold et al. 2011) und Selbstwirksamkeitserwartung (Bandura 1977).

Eine wesentliche Frage bleibt jedoch in diesem Zusammenhang bestehen. Welche zentrale Rolle spielt die/der Mentor/in in ihrer/seiner Funktion, damit Selbstverantwortung, Selbstbestim- 
mung und Selbstwirksamkeit erlebt werden können? Autor/innen wie Blane (2017), Finckler (2017) und Baumann-Habersack (2017) betonen die Notwendigkeit der Identitätsfindung von Mentor/innen auf persönlicher, kultureller und situationsspezifischer Ebene (Blane 2017, 246; Finckler 2017, 74; Baumann-Habersack 2017, 145f). Burns (1978) und Bass et al. (1994) ergänzen mit der Theorie zur Transformationalen Führung. Hierbei wird die Annahme getätigt, dass durch Selbstreflexionsprozesse der Mentor/innen und durch eine multiperspektivische Betrachtungsweise inspirierende Motivation, idealisierter Einfluss, intellektuelle Anregung und positive individuelle Betrachtung im Mentoringprozess stattfinden (Bass et al. 1994 in Wenddorf 2018, 48; von Au 2016, 23; Pelz 2016, 96). Aufbauend auf diesen Überlegungen wurde Transformationales Mentoring diskutiert.

Transformationales Mentoring wird in der vorliegenden Arbeit als Konzept einer proaktiven und positiv beeinflussenden Begleitform verstanden. Mentor/innen setzen sich selbstkritisch mit ihrer Mentoring-Funktion und insbesondere mit der Führungsrolle auseinander und steigen in einen sozialen Prozess ein. Dieser ist durch Selbstverantwortung und Selbstrespekt seitens der Mentor/innen und Mentee gekennzeichnet und auf zielorientierte innovative Zusammenarbeit ausgelegt. In der Arbeit werden Fragehorizonte zu zehn Kategorien aus Sicht der Mentor/in und des Mentee diskutiert (Kapitel I/2.5). Das Gesamtbild vermittelt den Eindruck, dass es beim Transformationalen Mentoring darauf ankommt, dass Erwartungshaltungen und Zieldimensionen kommuniziert werden, subjektive Fragestellungen zu Motivation, Identität, Werthaltung, Beziehung, Persönlichkeitsmerkmal und Verhaltensänderung Berücksichtigung finden, Rollendefinitionen und Rollenfunktionen geklärt sind, Kommunikationsstrategien vorhanden und transparent sind und durch multiperspektivische Herangehensweise Schule bzw. Lehrer/innenausbildung innovativ und visionär gestaltend betrachtet werden.

Als Quintessenz kann - auch bezugnehmend zu den unterschiedlichen Zugängen (siehe Kapitel I/2.4) der Arbeit - festgestellt werden:

Schulpraktische Mentoringprozesse gehen im Idealfall mit einer Lern- und Entwicklungskultur einher, die nicht auf Meisterlehre und Werkstattlernen (historiografischer und systemischer Zugang) ausgerichtet sind, sondern auf einen personifizierten, wertebasierten, stärken- und ressourcenorientierten Begleitungsprozess für denkende, handelnde und fühlende (Erpenbeck et al. 2018, 181) Mentee und professionsorientierte Mentor/innen (personalisierter Zugang). Im Mittelpunkt stehen das gemeinsame Agieren in den Praktika im Rahmen des Systems Schule und der Lehrer/innenausbildung (systemischer Zugang), das Einläuten und Umsetzen themenbezogener Veränderungen aufgrund reflexiver Erfahrungsbasierung und das Einnehmen eines positiven prospektiven Blickes.

Das Kapitel Grundlagen verdeutlichte den theoretisch-konzeptionellen Rahmen zu Mentoring und insbesondere zu schulpraktischem Mentoring. In der empirischen Studie sollen nun Gelingensbedingungen zum schulpraktischen Mentoring erfasst und dokumentiert werden. 


\section{Empirischer Zugang}

Im empirischen Teil ist aufbauend auf die Darstellung und Analyse von theoretischen Konzepten zum Mentoringprozess das Ziel, im angelegten Forschungsdesign die subjektive Wahrnehmung der Gelingensprozesse aus Sicht der Mentor/innen sowie der Studierenden zu erfassen, zu beschreiben und ein Modell bzw. Modelle für das schulpraktische Mentoring zu entwickeln. Grundlage des Forschungsdesigns bildet die empirische Sozialforschung. In ihr werden Lebenswelten des Sozialgefüges beobachtet, dokumentiert und analysiert,

„um (...) zur Weiterentwicklung von Theorien beizutragen. Auch wenn diese Forschungsprozesse als „empirisch“, d.h. als „auf Erfahrung beruhend“ bezeichnet werden, gehen sie von Theorien aus und tragen zu ihnen bei. Sie tun das, indem sie angeleitet durch Theorien die soziale Realität beobachten und aus den Beobachtungen theoretische Schlüsse ziehen." (Gläser et al. 2010, 24)

In diesem Forschungsvorhaben wurden Interviews mit Lehrpersonen, die auch als Mentor/ innen fungieren, und Studierenden geführt und mittels der qualitativen Methode Grounded Theory analysiert. Wichtig dabei ist für die/den Forscher/in, mit dem Forschungsgegenstand in Beziehung zu treten und Einsicht in Sichtweisen, Deutungsmuster und Denkfiguren durch den Handlungskontext zu erhalten (Eickelpasch 1982, 17; Kromrey 2006, 547). Im Laufe des Forschungsprozesses wurden Kodierparadigmata, Kernkategorien und Modelle entwickelt. Eine Theoriegenerierung, wie die Grounded Theory vorschlägt, hat sich im Laufe des Forschungsprozesses nicht ergeben.

Im Folgenden wird die Forschungsfrage vorgestellt. Anschließend erfolgt eine Beschreibung des methodologischen sowie methodischen Zugangs und die Erläuterung der genauen Verlaufsschritte der Datenanalyse mit dem Ziel der Bildung von Kernkategorien. Die detaillierten Beschreibungen zu ursächlichen Bedingungen, Handlungsstrategien, Kontext und intervenierenden Bedingungen, Konsequenzen und Phänomenen der Untersuchung im Rahmen der Grounded Theory zeigen ein Bild von grundlegenden und kollektiven Themen zum schulpraktischen Mentoring(-prozess) auf. Ausgehend von weiteren Rekonstruktionen und Interpretationen der Ergebnisse werden Befunde des Forschungsprozesses am Ende des Kapitels diskutiert. Hierbei wurden Gelingensbedingungen zu schulpraktischen Mentoringprozessen aus Metakernkategorien, Gelingensbedingungen aufgrund kontextueller Rahmung und Gelingensbedingungen aus den schulpraktischen Mentoring-Funktionen abgeleitet. Die rekonstruierten Modelle erklären die Bedeutung des Selbstreflexionsprozesses der/des Mentorin/Mentors bzw. beschreiben mögliche Dimensionen zum mentoringinduzierten Wirkungsmodell. 


\section{Forschungsfrage}

In der neuen Lehrer/innenausbildung wird schulpraktisches Mentoring als Struktur in den Pädagogisch-Praktischen Studien verankert. Die institutionelle Umsetzung ist durch die Partnerschulen der Universitäten und Pädagogischen Hochschulen gegeben. Mentor/innen unterstützen hierbei Studierende bei der Konzeption und Gestaltung von Lernarrangements, reflektieren theoriebasierend mit ihnen pädagogisches Handeln, diskutieren Grundfragen zu bildungswissenschaftlichen, fachwissenschaftlichen und fachdidaktischen Modellen und Konzepten und setzen sich mit Forschungsfragen und subjektiven Theorien auseinander. Lehrpersonen wirken als Mentor/innen. Ihnen kommt eine große Aufgabe bei der Begleitung der Studierenden auf dem Weg zur Professionalisierung zu. Sie stellen das Lernfeld Klasse zur Verfügung und unterstützen den Transfer wissenschaftlicher Erkenntnisse in pädagogische Praxis.

In der vorliegenden Untersuchung werden Sichtweisen, Erwartungen und Erfahrungen seitens der Mentor/innen als auch der Studierenden erhoben. Das Forschungsdesign der Arbeit wurde durch eine qualitative Erhebung durch Expert/inneninterviews bei Mentor/innen und Studierenden realisiert.

Die zentrale Forschungsfrage lautet:

Welche Gelingensbedingungen lassen sich im Mentoringprozess der Lehramtsausbildung rekonstruieren bzw. identifizieren?

Davon werden spezifizierend folgende Detailfragen abgeleitet und im Rahmen der Arbeit behandelt:

- Welche Gelingensbedingungen lassen sich aus Sicht der Mentor/innen rekonstruieren bzw. identifizieren?

- Welche Gelingensbedingungen lassen sich aus Sicht der Studierenden rekonstruieren bzw. identifizieren?

Die Fragestellungen richteten sich an Lehrpersonen, die sowohl Mentoringprozesse gestalten (als Mentor/innen) als auch während ihrer Ausbildungszeit als Studierende diese Prozesse erlebt haben sowie an Lehramtsstudierende, die vor dem Abschluss ihrer Ausbildung standen und die vielfältige Formen der Unterstützung durch Mentor/innen erfahren hatten. Um zu Befunden zu gelangen, wurden in der Feldforschung qualitative Daten anhand von Interviews erhoben. Zur Auswertung der Daten wurde die Methode der Grounded Theory herangezogen. Sie ermöglicht die strukturierte Rekonstruktion und Modellbildung des Feldes aus dem Datenmaterial. Weitere Befunde ergeben sich aus der Integration der Ergebnisse mit Literaturrecherchen. Diese wiederum werden zum einen mit bestehenden Theorien und Forschungsergebnissen in Beziehung gesetzt, und zum anderen können sie Ausgangspunkt für weitere Untersuchungen sein.

Was den Forschungsansatz der vorliegenden Arbeit auszeichnet, ist die Einbeziehung der Lehrenden-bzw. Mentor/innenperspektive und der Studierenden- bzw. Menteeperspektive in einem gemeinsamen Forschungsprojekt. Gegenwärtige Untersuchungen konzentrieren sich verstärkt auf mentor/innenseitige Aspekte, wie Prozessstrukturen im Verlauf des Mentorings, Motivlage oder Einstellungen der Mentor/innen (Magg-Schwarzbäcker 2014; Fuge 2016; Pflaum 2017; Reintjes et al. 2018). Des Weiteren liegen Forschungsschwerpunkte zur Wirkungsweise schul- 
praktischen Mentorings verbunden mit der Lernwirksamkeit in den Schulpraktika vor (Crisp 2010; Bach 2013; König et al. 2018).

Die Untersuchung konzentriert sich auf die Rekonstruktion von Gelingensbedingungen - also Bedingungen bzw. Voraussetzungen, die für die Gestaltung eines schulpraktischen Mentoringprozesses bedeutend sind. Die Benennung solcher könnte auch darauf hinweisen, wie unterschiedlich von den Beteiligten an den Prozess herangegangen wird und welche Handlungsnotwendigkeiten am Beginn, während und am Ende des Prozesses aus Sicht der Mentor/innen und aus Sicht der Mentee beachtet werden sollten. Vor allem Ausbildungsinstitutionen könnten aufgrund der Erkenntnisse der vorliegenden Untersuchung Rückschlüsse für Ausbildungskonzepte ziehen. Schließlich hat schulpraktisches Mentoring eine hohe Bedeutung. In der gegenwärtigen Literatur wird Mentoring mit einer Lern- und Entwicklungsmöglichkeit verbunden, indem Raum und Zeit für Entfaltung sowie Aufbau von Fähigkeiten und Kompetenzen gegeben werden. Dazu gilt es Bedingungen zu identifizieren, die bei Beachtung und Umsetzung auf eine Erfolgsgenerierung im schulpraktischen Mentoring hindeuten.

Der strukturierte Rekonstruktionsprozess - beginnend mit dem methodologischen Zugang und der Datenerhebung - wird im folgenden Kapitel genau beschrieben und dokumentiert. 


\section{Methodologischer Zugang und Datenerhebung}

Empirisches Arbeiten verlangt von der/dem Forscher/in eine objektive Beschreibung und Klassifizierung von Phänomenen der Gegenstandswelt und das Finden von möglichst allgemeingültigen Regeln durch Erklärung der Ereignisse (Kromrey 2006, 22). Hierbei beobachtet man die soziale Welt, bezieht sich auf bereits vorhandene Kenntnisse und Theorien und entwickelt diese weiter (Gläser et al. 2010, 24). In der vorliegenden Studie wurde bei der Datenerhebung das Expert/inneninterview herangezogen. „Als Experte (sic!) wird interviewt, wer sich durch eine ,institutionalisierte Kompetenz zur Konstruktion von Wirklichkeit' (Hitzler/Honer/Maeder 1994) auszeichnet.“ (Meuser et al. 2011, 57) Die/Der Expertin/Experte ist in einem Funktionskontext eingebunden und verfügt über „spezialisiertes Sonderwissen“ (Meuser et al. 2011, 57), das in einem rekonstruierenden Prozess erhoben wird. Bogner et al. (2002) definieren Expert/ innen und deren Wissen wie folgt:

„Der Experte (sic!) verfügt über technisches, Prozess- und Deutungswissen, das sich auf sein spezifisches professionelles oder berufliches Handlungsfeld bezieht. Insofern besteht das Expertenwissen (sic!) nicht allein aus systematisiertem, reflexiv zugänglichen Fach-oder Sonderwissen, sondern es weist zu großen Teilen den Charakter vom Praxis- oder Handlungswissen auf, in das verschiedene und durchaus disparate Handlungsmaxime und individuelle Entscheidungsregeln, kollektive Orientierungen und soziale Deutungsmuster einfließen." (Bogner et al. 2002, 46)

Nach der Untersuchung einer geringen Anzahl von Samples erfolgt im Auswertungsprozess eine Reduktion der Datenmenge (Gläser et al. 2010, 26f). Hierbei standen immer wieder folgende Fragen im Mittelpunkt des Interesses wie: Was ist mit diesen Aussagen gemeint? Was steckt hinter einzelnen Aussagen? Welche Gelingensbedingungen könnten noch erwähnt sein? Somit wird ersichtlich, dass das Prinzip der induktiven Herangehensweise durchbrochen und der Ablauf des Prozesses in einen „zunehmend deduktiven Umgang mit Text und Kategorien (Kombination von Induktion und Deduktion)“ (Flick 2016, 400) verschoben wird. Die Methode Grounded Theory wurde in diesem Forschungsprojekt aufgrund der Intention einer Modellbildung verwendet.

\subsection{Expert/inneninterviews als Ausgangsbasis}

Für die Bearbeitung der Aussagen der Interviewpartner/innen sind zunächst eine Reihe von methodischen Schritten und Vorüberlegungen nötig. Zunächst wurden Forschungsfrage, Forschungsgegenstand und Wahl der Erklärungsstrategie in den theoretischen Vorüberlegungen festgelegt (Gläser et al. 2010, 35). Weiters wurden die nächsten zu vollziehenden Schritte bis zum Importieren der Daten organisiert. Es wurde beschlossen, einen Interviewleitfaden zu konzipieren und Expert/inneninterviews mittels Interviewleitfaden zu führen (Kruse 2006).

Das Konzipieren des Interviewleitfadens zielt darauf ab, dass Expert/innen als Repräsentant/ innen für Handlungs- und Sichtweisen die Möglichkeit haben, erzählgenerierend zu agieren und dass sie durch eventuelle Nachfragen verstärkt in die Thematik hingeführt werden. Durch die Wahl der Expert/innen wird bereits auf eine systematische Erweiterung des Wissens über den Untersuchungsgegenstand abgezielt. Mittels Probeinterviews wurden zunächst zwei Interviewpartner/innen ausgewählt, Daten analysiert und erste Ergebnisse festgehalten. Eine Präzisierung der Fragestellungen des Interviewleitfadens wurde vorgenommen und eventuelle Nach- 
fragen vorbereitet. Im weiteren Verlauf wurde nun die Gruppe der Interviewpartner/innen vergrößert, um eine Modifizierung oder Bestätigung der bisherigen Aussagen zu erhalten (Häder 2010, 175). Die Interviews dauerten in der Regel fünfzehn bis fünfundzwanzig Minuten in einer entspannten und wohlwollenden Atmosphäre. Die Fragestellungen waren so gestaltet, dass Antworten nach jeweiligem Interessens-, Erfahrungs- und Wissensstand geäußert werden können. Durch diesen Grad der Offenheit entspricht das Leitfadeninterview dem Prinzip der Offenheit (Gläser et al. 2010, 115).

Die Interviews mit den Lehrpersonen $(n=12)$ erfolgten im Juni 2017 jeweils am Schulstandort der jeweiligen Lehrperson. Hierbei wurden drei Lehrpersonen aus dem Bereich Volksschule (Primarstufe), vier Lehrpersonen aus dem Bereich Neue Mittelschule (Sekundarstufe I) und fünf Lehrpersonen aus dem Bereich Allgemeinbildende Höhere Schule (Sekundarstufe) ausgewählt. Die Expert/innen bringen divergente Erfahrungen mit, haben Einblick in unterschiedliche Schultypen und absolvierten unterschiedliche Ausbildungsformen.

Die Aufzeichnung der Interviews mit Studierenden $(n=12)$ wurden in den Räumlichkeiten der Universität Innsbruck und der Pädagogischen Hochschule Edith Stein mit dem Hochschulstandort Stams gemacht. Die Studierenden waren zum Zeitpunkt der Befragung (Juni 2017) am Ende ihres Studiums an der Pädagogischen Hochschule oder der Universität. Als Interviewpartner/innen standen jeweils drei Lehramtsstudierende Volksschule bzw. Neue Mittelschule und sechs Lehramtsstudierende Allgemeinbildende bzw. Berufsbildende Höhere oder Mittlere Schule zur Verfügung. Auch wurden im Hinblick auf eine repräsentative Stichprobe Studierende aus unterschiedlichen Ausbildungsformen zur Untersuchung herangezogen, denn dadurch erschließen sich divergierende Erfahrungswelten.

Bei der Zusammenstellung des Leitfadens wurde darauf geachtet, dass neben den Prinzipien der Offenheit und des Verstehens die vier Anforderungen nach Hopf $(1978,99 f f)$ - wie Reichweite, Spezifität, Tiefe und personaler Kontext - erfüllt werden. Die Anforderung Reichweite meint, der/dem Interviewpartner/in die Möglichkeit zum Erzählen und nicht nur zum Abfragen von Handlungen zu geben. Spezifität zielt darauf ab, bestimmte Reaktionen aufgrund der Darstellung der Inhalte der/des Interviewpartnerin/Interviewpartners verstehen zu lernen und dann diese Inhalte im Forschungsprozess herauszuarbeiten. Fragen, die die Anforderungen Tiefe und personaler Kontext berücksichtigen, lassen der/dem Interviewpartner/in Raum für gefühls-, wissens-, persönlichkeits- oder wertbezogene Kontextdarstellungen (Gläser et al. 2010, 116 in Anlehnung an Hopf 1978, 99ff). Vor dem Hintergrund dieser Prinzipien wurden folgende Ausgangsfragen für die Interviews gewählt bzw. folgende Nachfragen überlegt (Tabelle 49):

Tab. 49: Interviewleitfaden Mentor/innen und Studierende

\begin{tabular}{lll}
\hline Frage & Fragenkatalog Lehrende & Fragenkatalog Studierende \\
\hline 1 & Welchen Stellenwert/Welche Bedeutung nehmen & Welchen Stellenwert/Welche Bedeutung nehmen \\
& $\begin{array}{l}\text { für Sie persönlich die schulpraktischen Anteile in } \\
\text { der Lehramtsausbildung ein? Und warum haben }\end{array}$ & $\begin{array}{l}\text { für Sie persönlich die schulpraktischen Anteile in } \\
\text { Ihrer Lehramtsausbildung ein? Und warum haben } \\
\text { sie diese Bedeutung? }\end{array}$ \\
\hline 2 & Welche Aufgabenbereiche sind für Sie von zentra- & Wie sollte Ihrer Meinung und Erfahrung nach die \\
& ler Bedeutung? Und warum? & $\begin{array}{l}\text { Einbettung der schulpraktischen Anteile während } \\
\text { der Ausbildung erfolgen? }\end{array}$ \\
\hline
\end{tabular}




\begin{tabular}{|c|c|c|}
\hline Frage & Fragenkatalog Lehrende & Fragenkatalog Studierende \\
\hline 3 & $\begin{array}{l}\text { Warum stellen Sie sich als Mentor/in zur Verfü- } \\
\text { gung? }\end{array}$ & $\begin{array}{l}\text { Was wünschen Sie sich von den Praxisbegleitperso- } \\
\text { nen? Wie sollten diese sein? }\end{array}$ \\
\hline 4 & $\begin{array}{l}\text { Welche besondere Verantwortung sehen Sie für } \\
\text { sich in dem Zeitraum der Begleitung? }\end{array}$ & $\begin{array}{l}\text { Was ist für Sie relevant dafür, dass Sie ein Prakti- } \\
\text { kum im Nachhinein als optimal einschätzen? }\end{array}$ \\
\hline 5 & $\begin{array}{l}\text { Woran machen Sie fest, dass Studierende im Ver- } \\
\text { lauf des Praktikums Entwicklungen durchgemacht } \\
\text { haben? Wie sehen Sie im Praktikum das Verhältnis } \\
\text { zwischen Ihnen und der/dem Studierenden? }\end{array}$ & $\begin{array}{l}\text { Welche Erfahrungen haben Sie im Praktikum mit } \\
\text { Ihren Praxisbegleitpersonen gemacht? Welches } \\
\text { Verhältnis würden Sie sich idealerweise zu Ihrer } \\
\text { Praxisbegleitperson wünschen? }\end{array}$ \\
\hline 6 & $\begin{array}{l}\text { Wenn Sie sich selbst einmal in die Person der } \\
\text { Studierenden hineinzuversetzen versuchen, wie } \\
\text { glauben Sie geht es denen mit den Praktika? Was } \\
\text { haben Studierende von Praktika? }\end{array}$ & $\begin{array}{l}\text { Wie glauben Sie, geht es ihr/ihm mit Praktikantin- } \\
\text { nen? Wie glauben Sie, können Praxisbegleitperso- } \\
\text { nen von der Praktikumssituation mit Studierenden } \\
\text { profitieren? }\end{array}$ \\
\hline 7 & $\begin{array}{l}\text { Angenommen Sie würden an einer Schaltstelle } \\
\text { im System sitzen und könnten eine Innovation } \\
\text { durchsetzen, was würden Sie hier hinsichtlich der } \\
\text { Begleitung von Praktika verändern? Was ist das } \\
\text { zentrale Anliegen? }\end{array}$ & $\begin{array}{l}\text { Angenommen Sie könnten bei der Wahl von Pra- } \\
\text { xisbegleitpersonen etwas verändern, was würden } \\
\text { Sie dann machen? }\end{array}$ \\
\hline 8 & Möchten Sie noch etwas ergänzen? & Möchten Sie noch etwas ergänzen? \\
\hline
\end{tabular}

Der Interviewleitfaden für Lehrende und Studierende wurde nach ähnlichem Aufbau zusammengestellt. Einleitungsfragen, personalisierte und inhaltliche Fragen, Simulationsfragen (Patton 2002) und offene Fragen charakterisieren den Leitfaden. Die unterschiedlich typisierten Fragen wurden so gestellt, dass die Antworten erzählanregenden Charakter aufweisen - vorausgesetzt, die/der Interviewpartner/in verfolgt ebenso dieses Ziel.

Die beiden Einleitungsfragen bzw. Nachfragen wurden gewählt, da sie am Beginn des Gesprächsverlaufes in die Thematik des Mentoringprozesses führen und gezielt auf die inhaltliche Ebene lenken. Sie bieten auch Raum und Gelegenheit, über persönliche Erfahrungen zu berichten. Offene Fragestellungen sind eine Orientierungshilfe für die/den Interviewer/ in zum freien Äußern (Ullrich 1999, 436f). Durch unterschiedliche Ausbildungsabschlüsse (Universitätsabschluss, Akademieabschluss) der Befragten ergeben sich auch verschiedene Sichtweisen in Bezug auf Strukturen und Aufgabenbereiche von Praktika. Gläser et al. (2010) bezeichnen diese Art der Fragen als „Anwärmfragen“ (Gläser et al. 2010, 146f), da sie vom/von der Gesprächspartner/in leicht zu beantworten sind und sich ein natürlicher Gesprächsverlauf ergibt (Gläser et al. 2010, 146f). Frage drei und vier als personalisierte Fragen spiegeln die persönlichen Meinungen der Mentor/innen bzw. Studierenden wider. Die weiteren drei Fragestellungen zeigen das Empathieverhalten und geben somit Einblick in das Wahrnehmungsempfinden der befragten Personen. Frage sieben als Simulationsfrage (Patton 2002) zielt auf curriculumsentwicklungs- und organisationstechnische (Kraler 2017, 200) sowie begleitungsrelevante Vorschläge und Maßnahmensetzungen im Sinne der Nachhaltigkeit von Praktika. Die Fragestellungen sind so formuliert, dass sie auffordern, möglichst erzählend und begründet zu antworten und der Interviewleitfaden zeigt auch auf, dass die Interviewerin von der/dem Expertin/Experten Anregungen und Ideen erhalten will (Gläser et al. 2010, 126). Mentor/innen und Studierende haben Gelegenheit „sich selbstbewusst und aktiv-mitgestaltend in diese Diskussion“ (Terhart 2013, 80) der Qualitätsentwicklung einzubringen. Die letzte Frage wird thematisch als offene Frage gestaltet, damit den Interviewpartner/innen 
die Möglichkeit eingeräumt wird, sich noch einmal frei zu äußern und nicht angesprochene Themen zu artikulieren.

Nach Einspielung der Mediendatei in das System f4 transkript Edu Version v6.2.4. erfolgte die Transkription. Hierbei wurden Transkriptionsregeln in Anlehnung an Kallmeyer et al. (Kallmeyer et al. 1976 in Mayring 2016, 92) verwendet. Im Anschluss an die Transkription wurden die schriftlichen Aufzeichnungen auf Rechtschreibung korrigiert, anonymisiert und in einem Worddokument in Windows gespeichert. Damit der Kodierungsprozess einsetzen kann, wurden zunächst die zwölf Lehrpersonen-Interviewdokumente und im weiteren Verlauf die zwölf Studierenden-Interviewdokumente in das MAXQDA 12 Programm hochgeladen. Diese Software ermöglicht auf computergestützte Weise, Daten und Texte qualitativ mit Hilfe von Codes und Memos zu analysieren. Das Programm bietet auch das Werkzeug für einen quantitativen Analyseteil. Im Folgenden werden statistische Daten der Interviewpartner/innen vorgestellt.

\subsection{Eckdaten der Stichprobe}

Nach jedem Interview wurden von den Interviewpartner/innen statistische Daten mittels einer schriftlichen Befragung erhoben. Die untersuchten Fälle wurden nach dem Prinzip des selektiven Samplings ausgewählt. Die Personen weisen unterschiedliche soziodemografische Merkmale wie Studiengang, Ausbildungsgrad, Geschlecht, Alter, Wohnort, Fächerwahl und soziale Herkunft als Hintergrund auf. Insgesamt wurden 24 Interviews - davon zwölf mit Lehrpersonen und zwölf mit Studierenden - geführt. Das Alter der Interviewpartner/innen liegt zwischen 21 und 62 Jahren. Sie leben in kleinen Orten, Märkten und eine Interviewpartnerin in einer Stadt. Bis auf eine Lehrperson mit Abschluss Studienberechtigungsprüfung haben alle Befragten die Hochschulreife (Matura) an einer Allgemeinbildenden Höheren Schule (AHS) wie Gymnasium (BRG oder BORG), an einer Berufsbildenden Höheren Schule (BHS) wie Handelsakademie (HAK) oder an der Höheren Bundeslehranstalt für Wirtschaftliche Berufe (HLW) absolviert. Die Interviewpartner/innen haben unterschiedliche Fächerkombinationen in der Ausbildung gewählt. Die Volksschullehrpersonen bzw. Studierenden des Studienganges Volksschule werden in allen Unterrichtsgegenständen der Volksschule ausgebildet und geben daher keine spezifische Fächerwahl an. Die Befragten haben außerdem verschiedene zusätzliche berufliche Ausbildungen absolviert und sind auch teilweise nebenberuflich beschäftigt. Die Lehrpersonen weisen eine Berufserfahrung von sieben bis 39 Jahren vor. Acht der zwölf Studierenden schlossen ihr Studium im Jahr der Befragung $2017 \mathrm{ab}$, die anderen vier im Jahr 2018. Die Anzahl der Geschwister liegt zwischen null und vier. Fast die Hälfte der Eltern haben die Hochschulreife. Die Distanz zwischen Wohnort und Dienstort bzw. Studienort ist bei den Lehrpersonen eher gering, bei den Studierenden teilweise beträchtlich hoch. In Tabelle 50 sind sämtliche soziodemografischen Daten aller Interviewpartner/innen enthalten. 
Tab. 50: Statistische Daten der Interviewpartner/innen ( $\mathrm{L}=$ Lehrpersonen 1-12; $\mathrm{S}=$ Studierende 13-24)

\begin{tabular}{|c|c|c|c|c|c|c|c|c|}
\hline 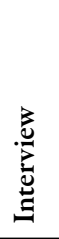 & 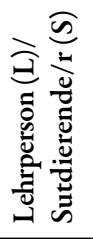 & 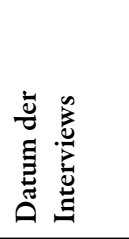 & 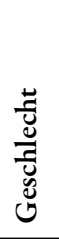 & $\frac{\dot{\Xi}}{4}$ & 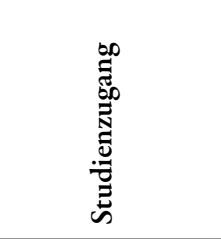 & 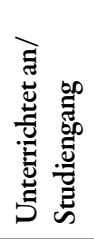 & 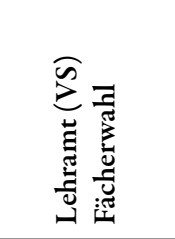 & 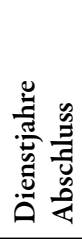 \\
\hline 1 & $\mathrm{~L}$ & $06 / 2017$ & $\mathrm{M}$ & 40 & BRG 1992 & AHS & $\mathrm{M} / \mathrm{PH}$ & 18 \\
\hline 2 & $\mathrm{~L}$ & $06 / 2017$ & $\mathrm{M}$ & 51 & BORG 1984 & AHS & $\mathrm{BS} / \mathrm{GS}$ & 25 \\
\hline 3 & $\mathrm{~L}$ & $06 / 2017$ & W & 34 & HAK 2002 & AHS & Ital/BU & 7 \\
\hline 4 & $\mathrm{~L}$ & $06 / 2017$ & W & 62 & BORG 1974 & AHS & D/REL & 37 \\
\hline 5 & $\mathrm{~L}$ & $06 / 2017$ & $\mathrm{~W}$ & 41 & BRG 1994 & AHS & $\mathrm{E} / \mathrm{Ital}$ & 17 \\
\hline 6 & $\mathrm{~L}$ & $06 / 2017$ & $\mathrm{M}$ & 53 & BRG 1983 & NMS & $\mathrm{D} / \mathrm{GS}$ & 27 \\
\hline 7 & $\mathrm{~L}$ & $06 / 2017$ & W & 42 & HAK 1994 & NMS & D/BU/Ital & 20 \\
\hline 8 & $\mathrm{~L}$ & $06 / 2017$ & W & 58 & $\begin{array}{l}\text { Studienberechti- } \\
\text { gung } 1978\end{array}$ & NMS & $\begin{array}{c}\text { D/GS/WE/ } \\
\text { EH/INF }\end{array}$ & 39 \\
\hline 9 & $\mathrm{~L}$ & $06 / 2017$ & W & 45 & HAK 1991 & VS & VS & 23 \\
\hline 10 & $\mathrm{~L}$ & $06 / 2017$ & $\mathrm{M}$ & 42 & HAK 1994 & VS & VS & 16 \\
\hline 11 & $\mathrm{~L}$ & $06 / 2017$ & $\mathrm{M}$ & 53 & BRG 1982 & VS & VS & 31 \\
\hline 12 & $\mathrm{~L}$ & $06 / 2017$ & W & 52 & BRG 1983 & VS & VS & 29 \\
\hline 13 & $S$ & $06 / 2017$ & $\mathrm{~W}$ & 30 & HAK 2007 & UNI & RUSS/GW & 2018 \\
\hline 14 & $S$ & $06 / 2017$ & $\mathrm{M}$ & 24 & HAK 2013 & UNI & INF/GW & 2018 \\
\hline 15 & $S$ & $06 / 2017$ & W & 24 & HLW 2012 & UNI & REL/GS & 2018 \\
\hline 16 & $S$ & $06 / 2017$ & W & 25 & BRG 2011 & UNI & $\mathrm{E} / \mathrm{GS}$ & 2018 \\
\hline 17 & $S$ & $06 / 2017$ & W & 33 & HLW 2003 & UNI & $\mathrm{E} / \mathrm{GS}$ & 2017 \\
\hline 18 & $S$ & $06 / 2017$ & W & 23 & BRG 2011 & UNI & $\mathrm{D} / \mathrm{E}$ & 2017 \\
\hline 19 & $S$ & $06 / 2017$ & $\mathrm{M}$ & 26 & BORG 2011 & NMS & $\mathrm{E} / \mathrm{BU}$ & 2017 \\
\hline 20 & $S$ & $06 / 2017$ & $\mathrm{M}$ & 24 & BORG 2011 & NMS & $\mathrm{M} / \mathrm{PC}$ & 2017 \\
\hline 21 & $S$ & $06 / 2017$ & W & 22 & BORG 2014 & NMS & $\mathrm{M} / \mathrm{ME}$ & 2017 \\
\hline 22 & $S$ & $06 / 2017$ & W & 24 & HLW 2012 & VS & VS & 2017 \\
\hline 23 & $S$ & $06 / 2017$ & $\mathrm{M}$ & 23 & BORG 2012 & VS & VS & 2017 \\
\hline 24 & $S$ & $06 / 2017$ & W & 21 & BORG 2014 & VS & VS & 2017 \\
\hline
\end{tabular}




\begin{tabular}{|c|c|c|c|c|c|c|}
\hline 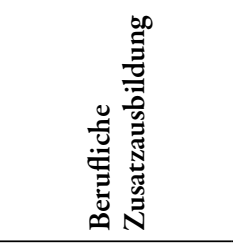 & 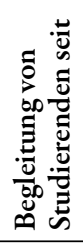 & 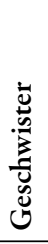 & 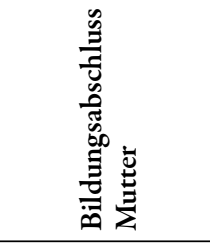 & 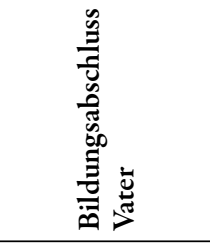 & 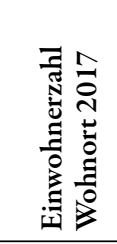 & 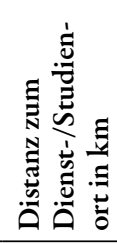 \\
\hline Schilehrer & 2009 & 1 & Päd. Akademie & Päd. Akademie & 3500 & 25 \\
\hline Trainerausbildung & 2015 & 2 & Lehrabschluss & Lehrabschluss & 3700 & 21 \\
\hline Lernbegleiterin & 2017 & 1 & Lehrabschluss & Lehrabschluss & 1000 & 7 \\
\hline $\begin{array}{l}\text { MAS für Sprach- } \\
\text { und Kommunika- } \\
\text { tionsstörungen }\end{array}$ & 2001 & 2 & Pflichtschule & Pflichtschule & 1200 & 11 \\
\hline- & 2013 & 1 & Hochschulreife & Hochschulreife & 1500 & 30 \\
\hline- & 2006 & 3 & Pflichtschule & Pflichtschule & 3000 & 4 \\
\hline- & 2010 & 2 & Pflichtschule & Päd. Akademie & 1500 & 10 \\
\hline- & 2007 & 2 & Pflichtschule & Pflichtschule & 2500 & 1 \\
\hline Notfallsanitäterin & 2009 & 1 & Lehrabschluss & Lehrabschluss & 10000 & 5 \\
\hline- & 2011 & 2 & Pflichtschule & Pflichtschule & 15400 & 31 \\
\hline Milizoffizier & 2013 & 2 & $\begin{array}{l}\text { Lehrerbildungs- } \\
\text { anstalt (LBA) }\end{array}$ & LBA & 1800 & 8 \\
\hline- & 2015 & 1 & LBA & LBA & 3000 & 25 \\
\hline Trainerausbildung & - & 1 & Lehrabschluss & Lehrabschluss & 260 & 220 \\
\hline- & - & 3 & Hochschulreife & Päd. Akademie & 7000 & 200 \\
\hline Tourismus & - & 3 & Universität & Universität & 5300 & 65 \\
\hline- & - & 4 & Staatsexamen & Staatsexamen & 124580 & 1 \\
\hline Management & - & 2 & Päd. Akademie & Universität & 2000 & 300 \\
\hline- & - & 0 & Lehrabschluss & Lehrabschluss & 16660 & 128 \\
\hline Rettungssanitäter & - & 0 & Pflichtschule & Pflichtschule & 700 & 40 \\
\hline Tennislehrer & - & 2 & Pflichtschule & Lehrabschluss & 4983 & 34 \\
\hline- & - & 4 & Hochschulreife & Lehrabschluss & 10500 & 20 \\
\hline Buchhaltung & - & 3 & Pflichtschule & Hochschulreife & 1200 & 57 \\
\hline Schilehrer & - & 1 & Päd. Akademie & Päd. Akademie & 2353 & 63 \\
\hline- & - & 1 & Hochschulreife & Hochschulreife & 1050 & 35 \\
\hline
\end{tabular}

Im Weiteren werden die soziodemografischen Merkmale näher beschrieben. Die Übersichten und Abbildungen enthalten eine Gesamtübersicht jedes Merkmales über die Interviewpartner/innen und/oder eine jeweilige Aufstellung zur Gruppe der Lehrpersonen bzw. der Studierenden. 


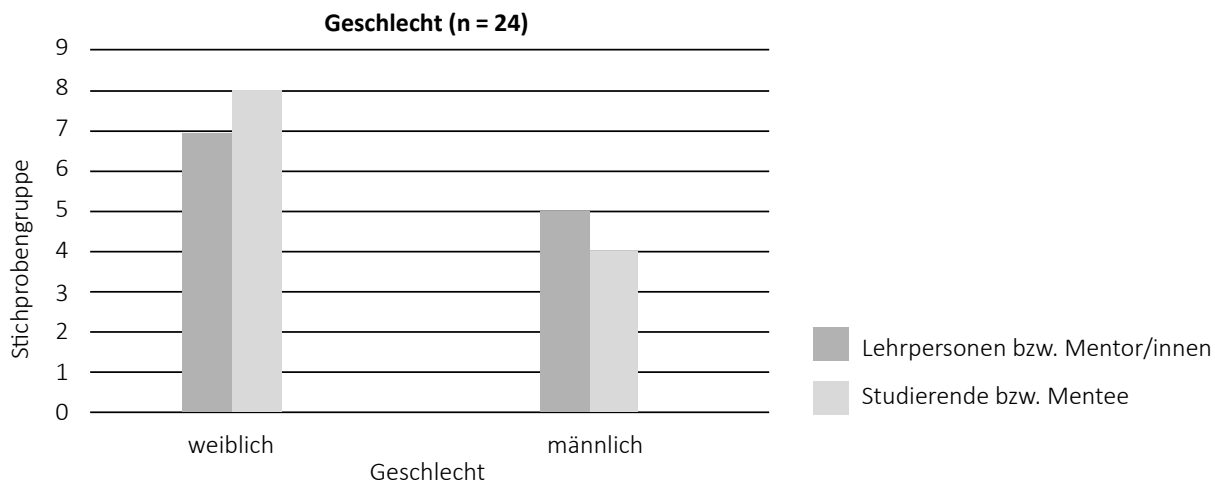

Abb. 27: Geschlecht der Interviewpartner/innen $(\mathrm{n}=24)$

Die Interviews wurden mit sieben weiblichen und fünf männlichen Lehrpersonen (L) sowie mit acht weiblichen und vier männlichen Studierenden (S) (Abbildung 27) geführt. Von den zwölf Lehrpersonen unterrichten drei weibliche und zwei männliche in einer Allgemeinbildenden Höheren Schule (AHS), ein männlicher und zwei weibliche in einer Neuen Mittelschule (NMS) und zwei weibliche und zwei männliche in einer Volksschule (VS). Bei den zwölf befragten Studierenden absolvierten fünf weibliche und ein männlicher das Lehramtsstudium an einer Universität, eine weibliche und zwei männliche den (damaligen) Studiengang Neue Mittelschule und zwei weibliche und ein männlicher den Studiengang Volksschule an einer Pädagogischen Hochschule.

Abbildung 28 zeigt das Alter der einzelnen Befragten. Das Alter der Lehrpersonen liegt zwischen 34 und 62 Jahren, der Durchschnitt beträgt 47,8 Jahre (obere strichlierte Linie) und der Median 51 Jahre. Dieser erhöht somit den Mittelwert um 3,2 Jahre.

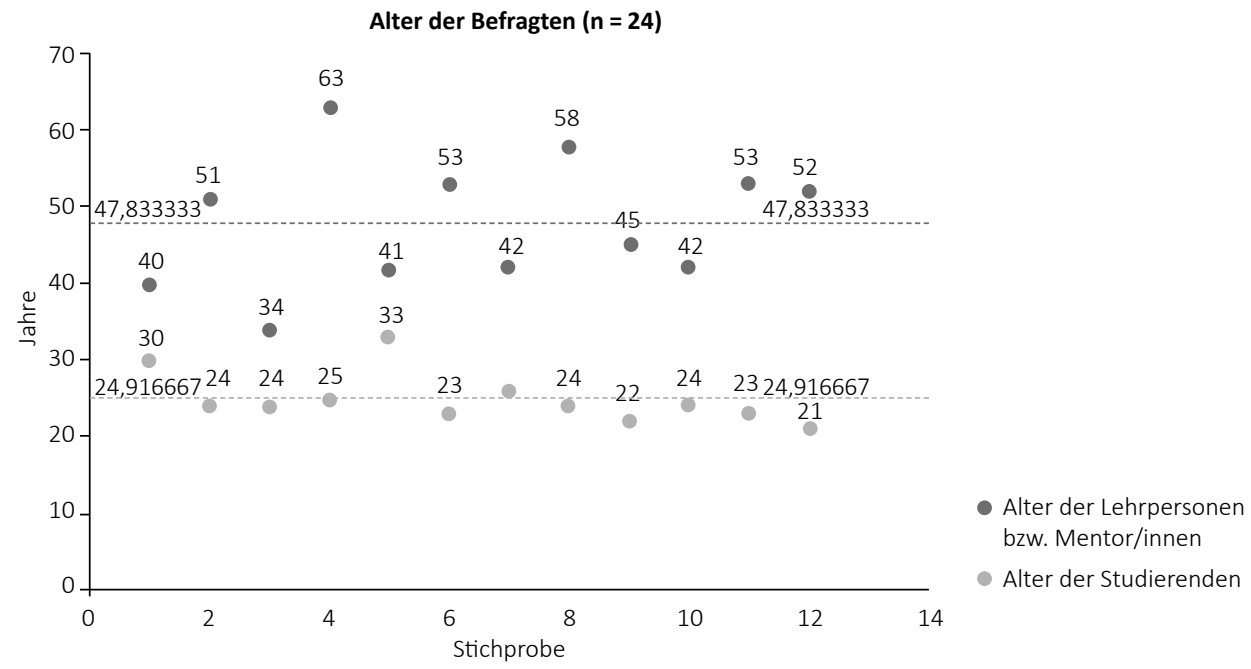

Abb. 28: Alter der Interviewpartner/innen mit strichlierten Mittelwertslinien Mentor/innen bzw. Studierende $(\mathrm{n}=24)$ 
Das Alter der Studierenden am Ende der Studienzeit liegt zwischen 21 und 33 Jahren. Der Mittelwert beträgt 24,9 Jahre (untere strichlierte Linie) und der Median 24 Jahre. Acht der zwölf befragten Studierenden geben an, ihr Studium im Jahr der Befragung 2017 zu beenden, die vier restlichen geben als Ende des Lehramtsstudiums 2018 an. Für sechs der zwölf befragten Studierenden verlief der Weg nach dem Erwerb der Hochschulreife nicht direkt in das Lehramtsstudium.

Zum Vergleich mit der Datenanalyse der Studierendeninterviews werden statische Daten von Lehramtsstudienanfängerinnen und -anfängern angeführt (Sozialerhebung Österreich 2015), die folgende Aussagen unterstreichen:

Tab. 51: Statistische Daten der Lehramtsstudienanfänger/innen (2015) in Österreich (Sozialerhebung Österreich, 2015)

\begin{tabular}{lccccccc}
\hline Lehramt & Anzahl & Anteil & $\begin{array}{c}\text { Frauenan- } \\
\text { teil }\end{array}$ & $\begin{array}{c}\text { Anteil } \\
\geq 26 \text { Jahre }\end{array}$ & $\begin{array}{c}\varnothing \\
\text { Alter }\end{array}$ & $\begin{array}{c}\text { Alter } \\
\text { Frauen }\end{array}$ & $\begin{array}{c}\text { Alter } \\
\text { Männer }\end{array}$ \\
\hline Universität & 2835 & $5 \%$ & $66 \%$ & $4 \%$ & $20,0 \mathrm{~J}$. & $19,7 \mathrm{~J}$. & $20,6 \mathrm{~J}$. \\
Volksschule & 2050 & $3,6 \%$ & $90 \%$ & $20 \%$ & $22,9 \mathrm{~J}$. & $22,6 \mathrm{~J}$. & $25,5 \mathrm{~J}$. \\
Neue Mittelschule & 1389 & $2,4 \%$ & $68 \%$ & $33 \%$ & $25,1 \mathrm{~J}$. & $24,6 \mathrm{~J}$. & $26,0 \mathrm{~J}$. \\
\hline
\end{tabular}

Ein hoher Anteil der Lehramtsstudierenden ist weiblich. Im Lehramt Volksschule beträgt der Anteil 90 Prozent. Auch an der Universität und für das (damalige) Lehramt Neue Mittelschule liegen die Frauenanteile bei 66 bzw. 68 Prozent. Lehramtsstudierende an der Universität sind in der Regel am Beginn ihres Studiums um das 20. Lebensjahr. Studierende für das Lehramt Volksschule bzw. Neue Mittelschule sind bei Studienbeginn im Durchschnitt zwei bis fünf Jahre älter als die Studierenden der Universität. Die Vermutung liegt nahe, dass diese Gruppe der Studierenden bereits eine andere Tätigkeit bzw. ein anderes Studium vor dem eigentlichen Lehramtsstudium ausgeführt hat. Ähnliche Daten liegen von den skandinavischen Staaten vor. Auch dort wird vermutet, dass die erstsemestrigen Lehramtsstudierenden vor dem Studium eine andere Tätigkeit ausgeübt haben (Werler 2014, 112).

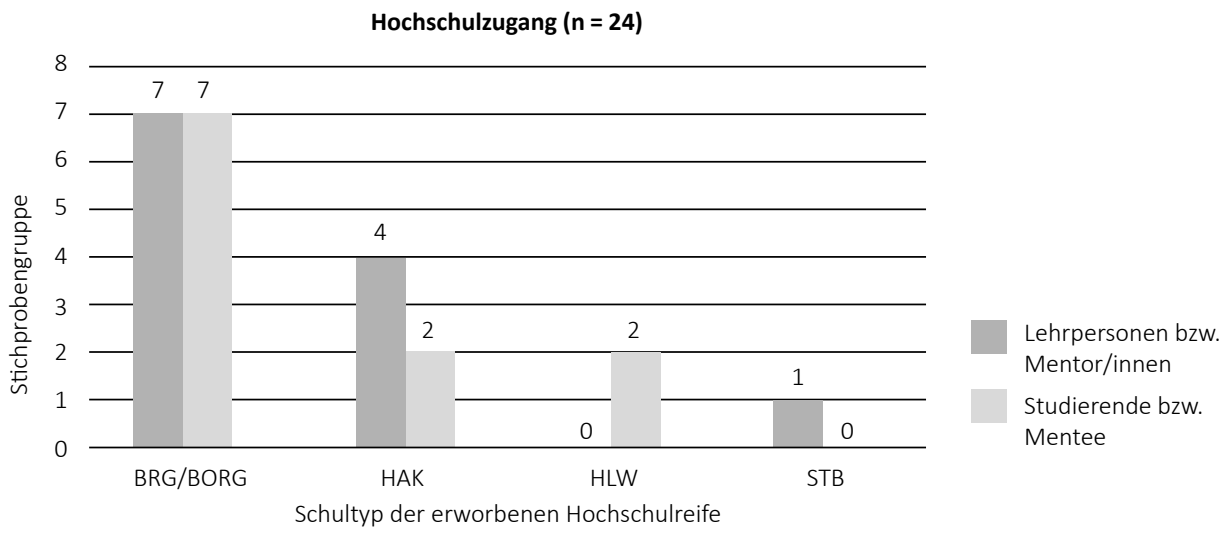

Abb. 29: Hochschulzugang der Interviewpartner/innen $(n=24)$ 
Von den 12 befragten Lehrpersonen hat eine Lehrperson (4,2\%) die Studienberechtigungsprüfung (STB) als Voraussetzung zum Studienzugang absolviert. 58,3 Prozent der Befragten erwarben die Voraussetzung für den Hochschulzugang über die Matura in einer vierjährigen Allgemeinbildenden Höhere Schule (AHS) wie Bundesrealgymnasium (BRG) bzw. Bundesoberstufenrealgymnasium (BORG) und 37,5 Prozent über eine fünfährige Berufsbildenden Höheren Schule (BHS) wie Handelsakademie (HAK) oder Höhere Lehranstalt für wirtschaftliche Berufe (HLW) (Abbildung 30).

42,8 Prozent der Absolvent/innen einer Allgemeinbildenden Höheren Schule (AHS) und 55,6 Prozent einer Berufsbildenden Höheren Schule (BHS) gingen nach der Matura an die Universität. Je 28,6 Prozent der Absolvent/innen einer Allgemeinbildenden Höheren Schule (AHS) absolvierten das Lehramt Neue Mittelschule (NMS) oder Volksschule (VS). 33,3 Prozent der Absolvent/innen einer Berufsbildenden Höheren Schule (BHS) streben das Lehramt Volksschule (VS) und 11,1 Prozent das Lehramt Neue Mittelschule (NMS) an. Eine Lehrperson wählte das Lehramt Neue Mittelschule (NMS) über die Studienberechtigungsprüfung (STB). Weiters geht hervor, dass 7 von 12 der befragten Studierenden nicht im unmittelbaren Anschluss das Lehramtsstudium begonnen haben, sondern dass sie vorher noch eine andere berufliche Tätigkeit im Durchschnitt von 7 Jahren ausübten bzw. ein anderes Studium im Durchschnitt von 2,2 Jahren absolvierten. Ostermann (2015) kommt zu einem ähnlichen Datenprofil. 19 von 30 Untersuchten einer Pädagogischen Hochschule haben vor dem Lehramtsstudienbeginn eine andere Ausbildungsstätte aufgesucht bzw. einen Beruf ausgeübt (Ostermann 2015, 65). Eine Begründung der Übereinstimmung der Aussagen der beiden Studien zu dieser Sachlage ist auf den gleichen Untersuchungsraum zurückzuführen.

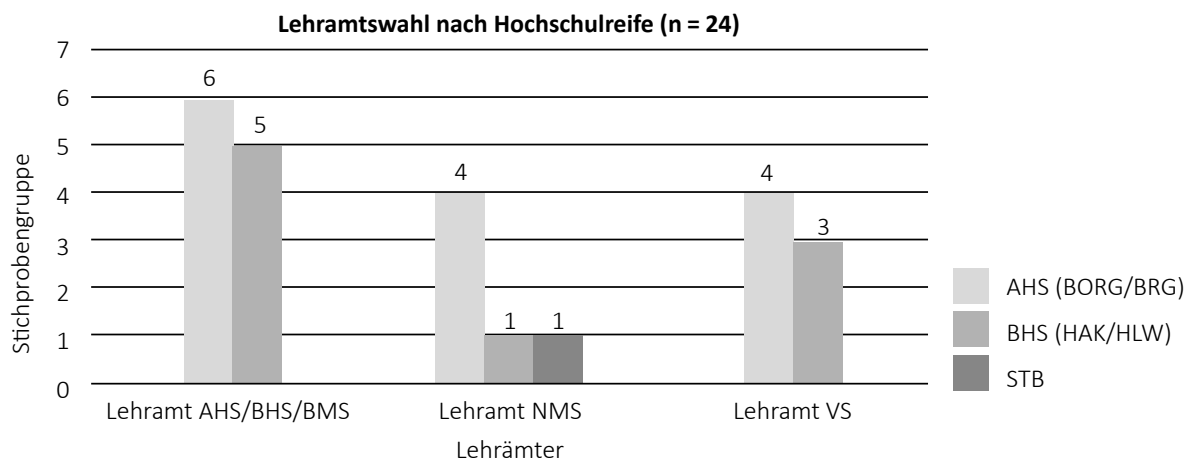

Abb. 30: Lehramtswahl nach Hochschulreife der Interviewpartner/innen $(n=24)$

Für die Studie wurden Interviewpartner/innen ausgewählt, die unterschiedliche Ausbildungsformen und verschiedene Fächerkombinationen gewählt haben. Vier Lehrpersonen und drei Studierende absolvierten das Lehramt Volksschule. Diese Personengruppe hatte alle Unterrichtsgegenstände der Volksschule in der Ausbildung. Die Auflistung der Fächerkombinationen zeigt auf: Drei Lehrpersonen und drei Studierende wählten das Unterrichtsfach Geschichte und Sozialkunde, vier Lehrpersonen und eine Studierende Deutsch, eine Lehrperson und vier Studierende Englisch, eine Lehrperson und zwei Studierende Mathematik, drei Lehrpersonen und drei Studierende Italienisch, zwei Lehrpersonen und ein Studierender Biologie und Um- 
weltkunde, zwei Studierende Geographie und Wirtschaftskunde, eine Lehrperson und eine Studierende Katholische Religion, eine Lehrperson und ein Studierender Informatik, eine Studierende Russisch, eine Lehrperson Physik, ein Studierender Physik und Chemie, eine Lehrperson Bewegung und Sport, eine Lehrperson Werkerziehung, eine Studierende Musikerziehung und eine Lehrperson das Unterrichtsfach Ernährung und Haushalt.

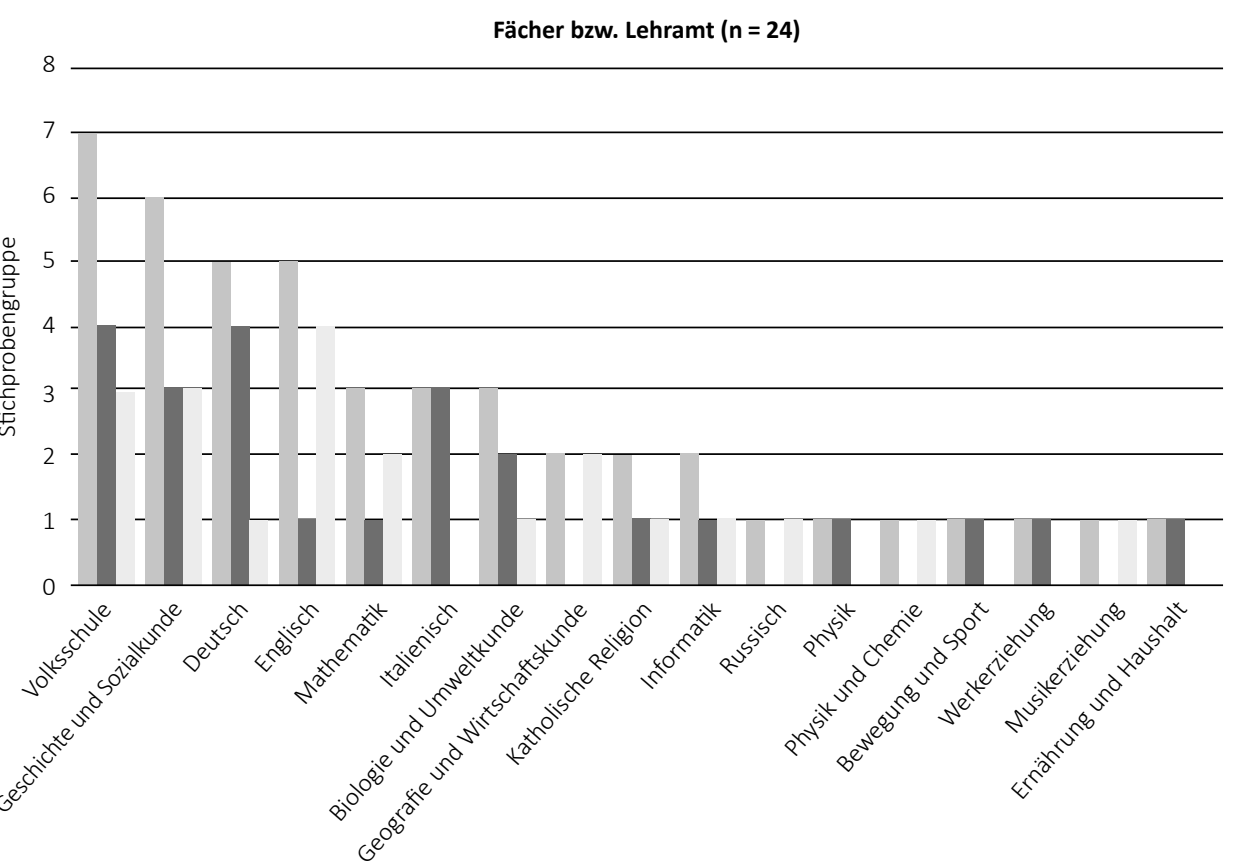

Abb. 31: Fächerwahl bzw. Lehramtswahl (Volksschule) der Interviewpartner/innen $(\mathrm{n}=24)$

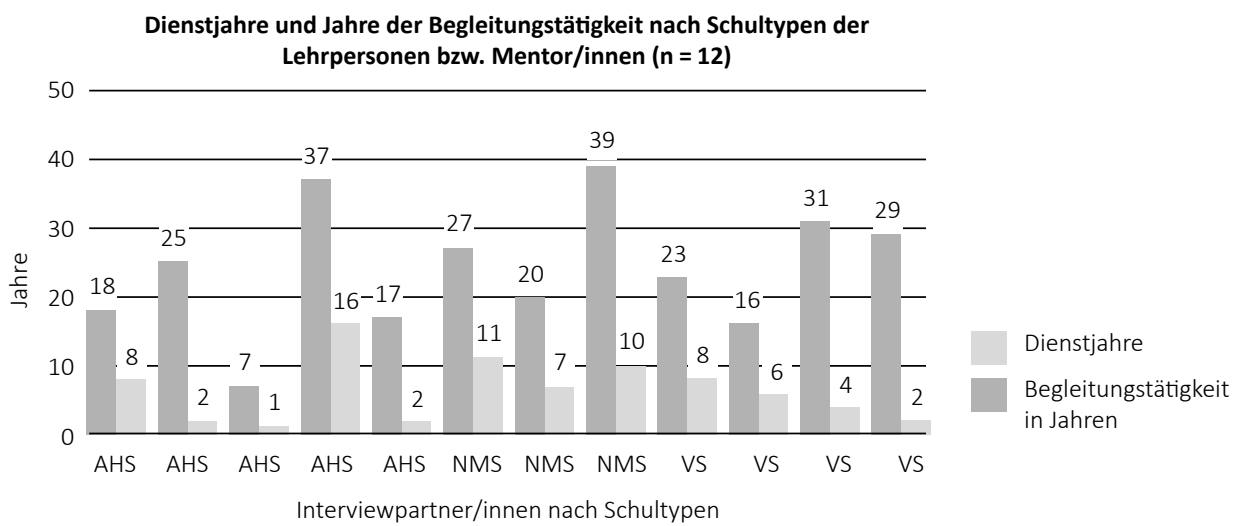

Abb. 32: Dienstjahre und Jahre der Begleitungstätigkeit nach Schultypen der Lehrpersoen bzw. Mentor/innen $(n=12)$ 
Die Angaben über die Dienstjahre der Lehrpersonen zeigen, dass die Befragten zum Zeitpunkt der Befragung zwischen 7 und 39 Jahre im Dienst tätig sind. Der Mittelwert liegt bei 24 Jahren. Auf die Frage nach der Dauer der Mentor/innentätigkeit gaben die Lehrpersonen 1 bis 16 Jahre an. Der Mittelwert beträgt 6,4 Jahre. Es wurden Lehrpersonen befragt, die im Durchschnitt nach 17,6 Dienstjahren mit der Mentor/innentätigkeit begonnen haben.

Geschwisteranzahl $(n=24)$

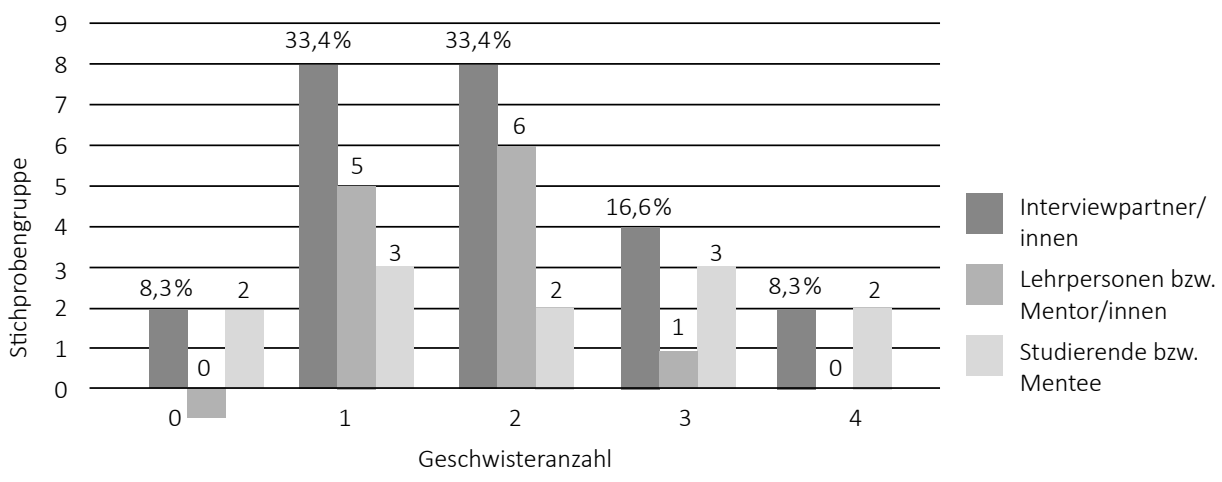

Abb. 33: Geschwisteranzahl der Interviewpartner/innen $(n=24)$

Je zwei Studierende sind Einzelkinder (8,3\%), haben zwei oder vier Geschwister (8,3\%). Je drei Studierende haben ein Geschwisterteil oder noch drei Geschwister. Die befragten Lehrpersonen haben meist noch ein oder zwei Geschwisterteile, eine Lehrperson hat drei Geschwister. Die Ergebnisse aller Befragten zeigen bei der Geschwisterkonstellation auf, dass je 33,4 Prozent ein oder zwei Geschwisteranteile haben. Die Stellung in der Geschwisterreihe wurde in dieser Untersuchung nicht erhoben.

Nach Untersuchungen von Kraler (2009) ist der frühe Sozialkontakt zu Geschwistern ein Faktor, der dazu beiträgt, das Berufsmotiv Lehrerin bzw. Lehrer als lebensgeschichtliche Verankerung festzulegen (Kraler 2009). In seiner Studie untersuchte der Autor auch die Stellung der Geschwisterreihe - angegeben in Prozenten. Tabelle 52 dokumentiert Ergebnisse zu Geschwisterkonstellationen nach Kraler (2009):

Tab. 52: Geschwisterkonstellationen (Kraler 2009)

\begin{tabular}{lccccc}
\hline Geschwisterkonstellationen & $\mathbf{0}$ & $\mathbf{1}$ & $\mathbf{2}$ & $\mathbf{3}$ & $\mathbf{4}$ \\
\hline Anzahl Geschwister in \% & $5,4 \%$ & $41,6 \%$ & $23,8 \%$ & $20,5 \%$ & $4,7 \%$ \\
\hline Stellung in Geschwisterreihe in \% & & $40,6 \%$ & $32,4 \%$ & $12,4 \%$ & $6,5 \%$ \\
\hline
\end{tabular}

Nach Kraler (2009) haben Lehramtsstudierende $(n=185)$ mit 42 Prozent noch ein Geschwisterteil, 24 Prozent zwei Geschwister und 21 Prozent drei Geschwister, nur 5 Prozent sind Einzelkinder oder haben vier Geschwister. Lehramtsstudierende sind meist Erstgeborene (41 Prozent) oder Zweitgeborene (32 Prozent) und sind aus lebensbiografischer Perspektive gewöhnt, Verantwortung und Aufgaben zu übernehmen (Kraler 2009). 
Auf die Frage nach der beruflichen Zusatzausbildung geben 13 (54,2\%) von 24 der Befragten die Absolvierung einer weiteren Ausbildung an - davon 6 bei den Lehrpersonen (50\%) und 7 bei den Studierenden (58,3\%). Hierbei fällt auf, dass 7 der 13 Personen (53,8\%) eine besondere Form der Lehrtätigkeit, wie Schilehrer, Tennislehrer, Trainerausbildung, Lernbegleiterin und Master für Sprach- und Kommunikationsstörungen, absolviert haben. 6 der 13 Personen $(46,2 \%)$ nennen Berufsausbildungen wie Notfallsanitäter, Rettungssanitäter, Milizoffizier, Buchhaltung oder im Bereich Tourismus und Management. Die Auflistung der Berufsausbildungen zeigt, dass mehr als die Hälfte der befragten Lehrpersonen und Lehramtsstudierenden eine andere Ausbildung erworben haben, und sie somit die Möglichkeit hätten, den Beruf zu wechseln.

Tabelle 53 verdeutlicht den Bildungsgrad der Eltern der Interviewpartner/innen:

Tab. 53: Bildungsgrad der Eltern der Interviewpartner/innen $(\mathrm{n}=24)$

\begin{tabular}{|c|c|c|c|c|c|c|c|}
\hline Eltern & $\begin{array}{l}\text { Pflicht- } \\
\text { schule }\end{array}$ & $\begin{array}{c}\text { Lehr- } \\
\text { abschluss }\end{array}$ & Matura & $\begin{array}{l}\text { Lehrerbil- } \\
\text { dungsanstalt } \\
\text { (LBA) }\end{array}$ & $\begin{array}{l}\text { Pädago- } \\
\text { gische } \\
\text { Akademie }\end{array}$ & $\begin{array}{l}\text { Univer- } \\
\text { sität }\end{array}$ & Lehrer/in \\
\hline Mütter Lehrpersonen & $5(41,7 \%)$ & $3(25,0 \%)$ & $1 \quad(8,3 \%)$ & $2(16,7 \%)$ & $1(8,3 \%)$ & 0 & $3(25,0 \%)$ \\
\hline Väter Lehrpersonen & $4(33,3 \%)$ & $3(25,0 \%)$ & $1 \quad(8,3 \%)$ & $2(16,7 \%)$ & $2(16,7 \%)$ & 0 & $4(33,3 \%)$ \\
\hline Mütter Studierende & $3(25,0 \%)$ & $2(16,7 \%)$ & $3(25,0 \%)$ & 0 & $2(16,7 \%)$ & $2(16,7 \%)$ & $4(33,3 \%)$ \\
\hline Väter Studierende & $1(8,3 \%)$ & $4(33,3 \%)$ & $2(16,7 \%)$ & 0 & $2(16,7 \%)$ & $3(25 \%)$ & $4(33,3 \%)$ \\
\hline Mütter Alle Befragten & $8(33,3 \%)$ & $5(20,8 \%)$ & $4(16,7 \%)$ & $2(8,3 \%)$ & $3(12,5 \%)$ & $2(8,3 \%)$ & $7(29,2 \%)$ \\
\hline Väter Alle Befragten & $5(20,8 \%)$ & $7(29,2 \%)$ & $3(12,5 \%)$ & $2(8,3 \%)$ & $4(16,7 \%)$ & $3(12,5 \%)$ & $8(33,3 \%)$ \\
\hline
\end{tabular}

Aus Tabelle 53 über den Bildungsabschluss der Eltern der Befragten $(\mathrm{n}=24)$ ist zu erkennen, dass bei den Studierenden weniger Elternpaare mit Pflichtschulabschluss (25\% und 8,3\% zu $41,7 \%$ und 33,3\%) und mehr mit Matura- (25\% und 16,7\% zu 8,3\% und 8,3\%) und Universitätsabschluss (16,7\% und $25 \% \mathrm{zu} 0 \%$ ) als bei den Elternpaaren der Lehrpersonen sind. Es kommt zu einem Berufsanstieg beim Generationswechsel. Fasst man die Gruppe der befragten Lehrpersonen und die Gruppe der befragten Studierenden zusammen, so stellt sich heraus, dass 45,8 Prozent der Mütter (13 von 24 Müttern) und 50 Prozent der Väter (12 von 24 Vätern) die Matura absolviert haben. Den Beruf Lehrer/in übten und üben bei den Müttern fast ein Drittel $(29,2 \%)$ und bei den Vätern genau ein Drittel $(33,3 \%)$ der Befragten aus. Vier von fünf Elternteile der Studierenden mit Universitätsabschluss sind Lehrpersonen. Eder et al. (2011) kommen zu ähnlichen Zahlen. Bei $30 \%$ der Studienanfänger/innen hat ein Elternteil die Profession Lehrer/in (Eder et al. 2011, 213). Es zeigt sich also, dass ein sehr hoher Anteil den gleichen Beruf wie ihre Eltern wählen - vielleicht weil sie meinen, dieses Berufsbild schon sehr gut zu kennen und damit vertraut zu sein (Kraler 2009). Aus dieser Denkfigur könnte man eine Berufsvererbung annehmen.

Die folgende Abbildung veranschaulicht durch das Aufzeigen der Einwohner/innenzahl die Struktur des Lebensraumes der Interviewpartner/innen (Abbildung 34): 


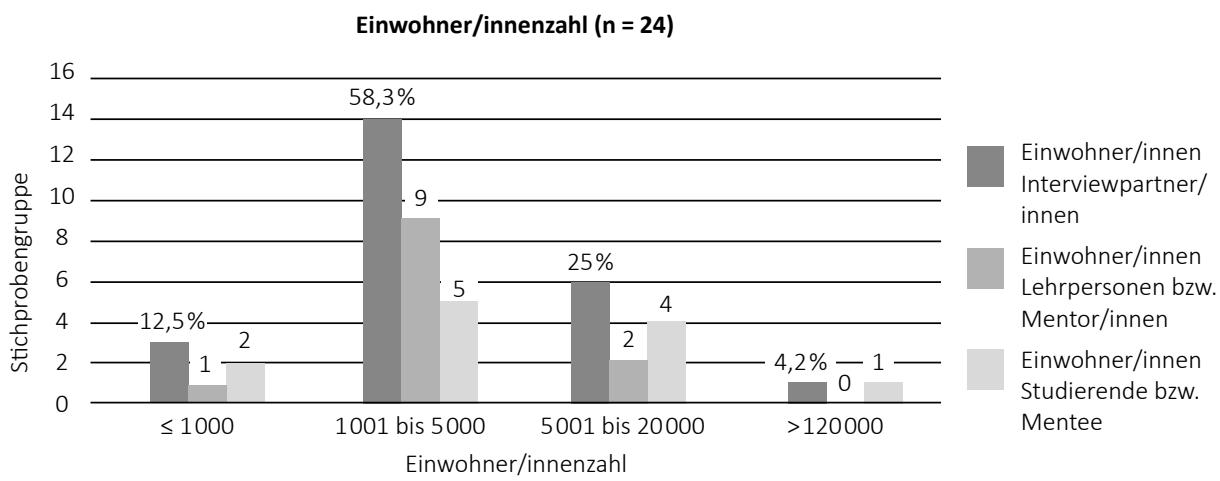

Abb. 34: Einwohner/innenzahl des Wohnortes der Interviewpartner/innen $(n=24)$

70,8 Prozent der Befragten (17 von 24) lebt in Dörfern bis 5000 Einwohner/innen. Lediglich zwei Lehrpersonen und vier Studierende leben in einem Ort bzw. einer Marktgemeinde von 5001 bis 20000 Einwohner/innen und eine Studierende lebt in einer Stadt über 120000 Einwohner/innen. Die Interviewpartner/innen kommen somit aus ländlichen Regionen. Die Studierenden verbringen die meiste Zeit an ihrem Wohnort, zum Studieren pendeln sie.

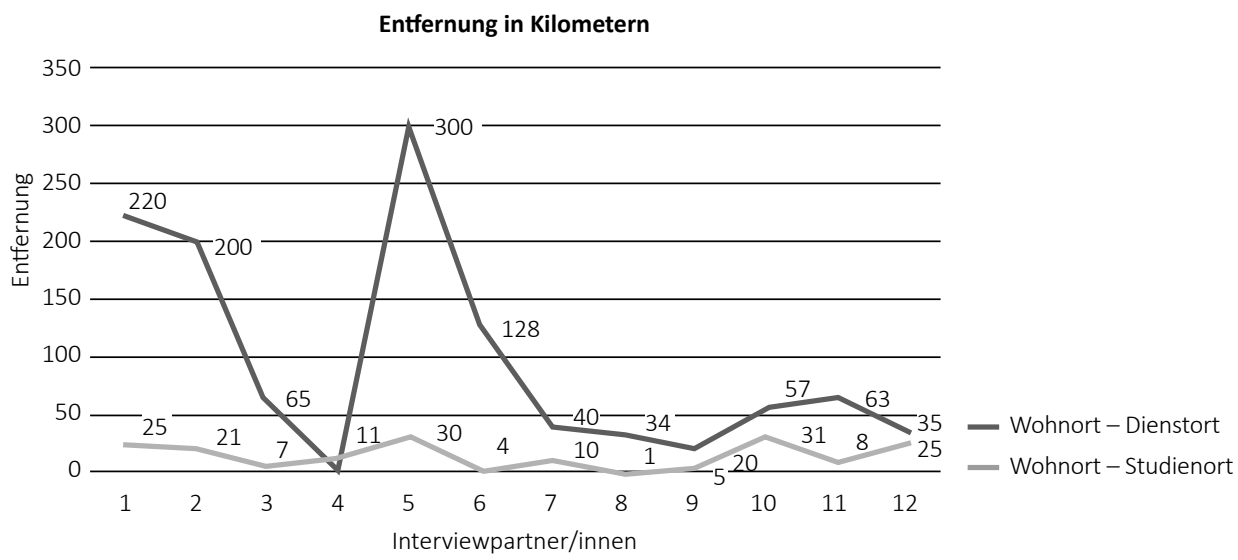

Abb. 35: Entfernung Wohnort zu Dienstort/Studienort in Kilometern der Interviewpartner/innen $(\mathrm{n}=24)$

Die Entfernung vom Wohnort zum Dienstort liegt bei den Lehrpersonen zwischen 1 und 31 Kilometer und im Durchschnitt bei 14,8 Kilometern. Jene Lehrpersonen, die an einer Allgemeinbildenden Höheren Schule unterrichten, haben im Durchschnitt 18,8 Kilometer Distanz, hingegen Lehrpersonen der Neuen Mittelschule nur 5 Kilometer und Lehrpersonen an Volksschulen 17,2 Kilometer. Die Studierenden überwinden eine größere Entfernung - vom Wohnort zum Studienort sind es bei dieser Gruppe der Befragten zwischen 1 und 300 Kilometer und im Durchschnitt 96,9 Kilometer. Es zeigt sich auch, dass Studierende an den Universitäten 
im Durchschnitt 152,3 Kilometer und Studierende an Pädagogischen Hochschulen nur 41,5 Kilometer zurücklegen. Die Studierenden der Pädagogischen Hochschule leben in Dörfern in der Nähe der Hochschule (die Hochschule befindet sich 38 Kilometer westlich von Innsbruck). Es wird verdeutlicht, dass Lehrpersonen in der Nähe des Wohnortes ihren Dienstort haben und eine Vermutung wird angedacht, dass Studierende den Berufswunsch verwirklichen, da die Ausübung der Tätigkeit als Lehrer/in in der Nähe bzw. im Wohnort möglich ist.

Die Befragten sind allesamt Subjekte, die im Kontext des schulpraktischen Mentoringprozesses aktiv sind bzw. Formen von Begleitung erlebt haben. Die befragten Lehrpersonen haben unterschiedlich lange Erfahrungszeiträume in der Begleitungstätigkeit. Die befragten Studierenden standen am Ende des Studiums und konnten auf unterschiedliche Betreuungsformen zurückblicken. Nach der Darstellung der statistischen Daten der Interviewpartner/innen wird im Folgenden die methodische Herangehensweise diskutiert. Um zu einem umfangreichen Datenmaterial zu kommen, wurde die Anforderung nach Wolff (2012) berücksichtigt,

„sich selbst im Verhältnis zum Feld so zu positionieren, dass die sachlichen, zeitlichen und sozialen Rahmenbedingungen für eine sachgerechte Durchführung der geplanten Forschungsarbeit gewährleistet oder zumindest seiner entsprechenden Handlungsmöglichkeiten nicht entscheidend eingeschränkt sind.“ (Wolff 2012, 336)

Unter Bedachtnahme dieser Hinweise lieferten die durchgeführten Interviews ein umfassendes Datenmaterial. Das gewonnene Textmaterial wurde nach dem Verfahren der Grounded Theory nach Strauss und Corbin (1996) analysiert.

Im Folgenden wird der methodische Zugang vorgestellt und erläutert, bevor die Untersuchungsergebnisse dokumentiert werden. 


\section{Methodischer Zugang}

Der Grounded-Theory-Ansatz geht auf die beiden Medizinsoziologen Anselm Strauss und Barney G. Glaser zurück. In Feldforschungsarbeiten zur Organisation von Krankenhausarbeit stellten die Forscher 1967 ihr Konzept vor. Die Namensbezeichnung der Forschungsmethode drückt die Arbeitsweise aus, „die eng am vorgefunden Material arbeitet bzw. in den Daten verankert (grounded) ist." (Bortz et al. 2006, 332) Die methodologische Herangehensweise zielt auf die Generierung einer induktiv abgeleiteten, gegenstandsverankerten Theorie ab.

„Eine Theorie auf Grundlage von Daten zu generieren, heißt, dass die meisten Hypothesen und Konzepte nicht nur aus den Daten stammen, sondern im Laufe der Forschung systematisch mit Bezug auf die Daten ausgearbeitet werden. Theorie zu generieren, ist ein Prozess." (Glaser et al. 2005, 15)

Für die prozessgeleitete Forschungsarbeit ergeben sich nach Strauss (1991) zwei Konsequenzen (Strübing 2014, 10): Arbeitsorganisation und Wechselbeziehung (Strauss 1991b, 34f). Diese Prinzipien werden auch in der Arbeit angewandt und in der weiteren Beschreibung wird auf die strukturelle Herangehensweise eingegangen, denn eine systematische Verfahrensreihe zur Erschließung des Phänomens wird von den Entwicklern nicht suggeriert. Autor/innen wie Flick (2005, 2016), Lamneck (2005), Przyborski et al. (2008, 2014), Strübing (2004, 2014), Heiser (2018), Breuer et al. (2018) haben sich mit der Komplexität der Arbeitsorganisation beschäftigt und mögliche Arbeitsschritte diskutiert. Strauss (1991b) betont die „zeitliche Parallelität und wechselseitige funktionale Abhängigkeit der Prozesse von Datenerhebung, -analyse und Theoriebildung" (Strübing 2014, 10) als maßgebend und als Orientierung für den Arbeitsprozess. Die sequentielle Analyse verlangt somit stets eine Verzahnung. Der Aspekt der Auswahl an Konzepten und Kategorien ist in der Arbeitsorganisation entscheidend für die Weiterentwicklung und eine Herausforderung für Forscher/innen.

Die zweite Konsequenz ist das Eingehen einer Wechselbeziehung zwischen dem Forschungsgegenstand und der/dem Forscher/in. Der/Dem Forscher/in muss es gelingen, Fragen an das Datenmaterial zu stellen, Vergleiche anzustellen und sich auf die Suche nach neuen Begrifflichkeiten, Konzepten und Kategorien zu machen (Strauss et al. 1996, 18). Hierbei ist eine gewisse Subjektivität im Forschungsprozess mitverankert - das ist auch in dieser Studie anzunehmen. Strübing (2014) lehnt sich in seiner Erklärung an die Worte von Strauss (1991b) an, indem dieser zusammenfassend erklärt:

„Wenn Forschung Arbeit ist und Arbeit als dialektisches Wechselverhältnis zwischen Subjekt und Objekt aufgefasst wird, dann muss das Resultat des Prozesses, die erarbeitete Theorie, immer auch ein subjektiv geprägtes Produkt sein." (Strübing 2014, 12)

Für den Arbeitsprozess mit Grounded Theory ist es nach epistemologischer Positionierung unabdingbar, gewisse Ebenen/Arbeitsschritte/Elemente/Hilfsmittel/Verfahren/Analyseprozesse (Begriffe je nach Literaturrecherche) zu berücksichtigen, um eine Theorie bzw. wie in dieser Studie ein Modell zu konzipieren. Autor/innen verwenden für die Erklärung des Ablaufes der Auswertung in ihren Publikationen unterschiedliche Bezeichnungen der Analyseschritte und Reihenabfolgen. Die unterschiedliche Herangehensweise an die Ausgestaltung des Datenmaterials innerhalb der Methode Grounded Theory bedarf einer genauen Recherche. Im Allgemeinen zeigt sie: 
„Der Forschungsprozess der Grounded Theory ist nicht linear organisiert. Datenerhebung, Kodieren und das Schreiben theoretischer Memos begleiten den gesamten Forschungsprozess und stehen in enger Relation zueinander. Dadurch werden immer wieder Rückgriffe auf Daten und frühere Kodes und ggfs. (sic!) auch neue Erhebungen angestoßen." (Przyborski et al. 2014, 209)

Das Erhebungsverfahren zur Entwicklung eines Modells bzw. einer Theorie ist nicht hierarchisch und linear, sondern unterliegt einer zirkulären Herangehensweise (Kuckartz 2010, 79). Breuer (2010) zeigt in seiner Abbildung (Abbildung 36) die Verwobenheit und Verzahnung der einzelnen Analyseschritte auf (Breuer et al. 2018, 257). Der Autor betont die drei Kodierungsverfahren: Offenes Kodieren, Axiales Kodieren, Selektives Kodieren und verknüpft es mit Operationsvorgängen. Ein Verdichtungsprozess wird beim Sortieren 1. Ordnung in Form der Konzeption eines Kodierparadigmas und beim Sortieren 2. Ordnung durch die Strukturgebung innerhalb der Kernkategorie realisiert.

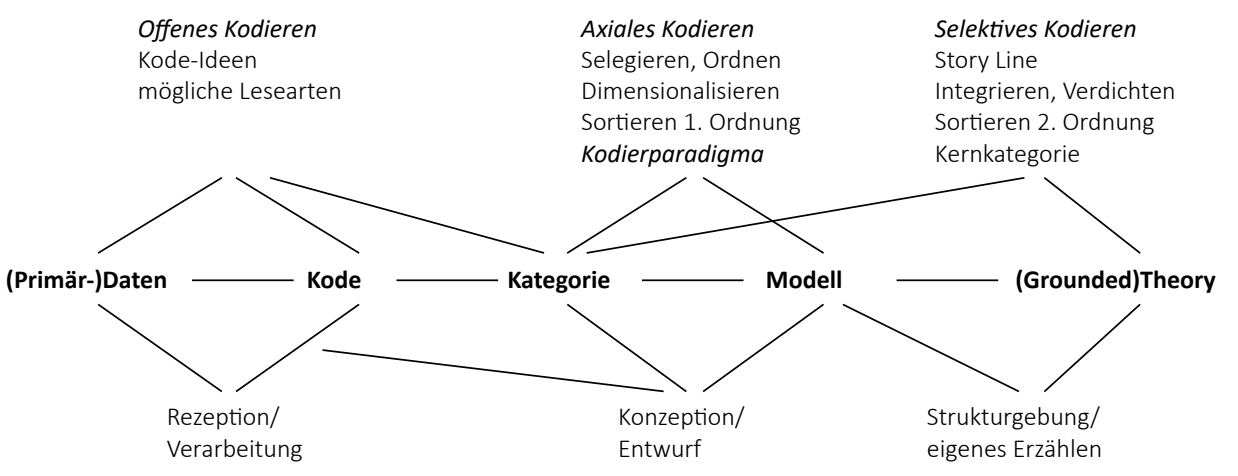

Abb. 36: Kodier-Prozeduren in systematisierter Anordnung (Breuer 2010, 76 in Breuer et al. 2018, 257)

Für die vorliegende Studie wurden zu den drei Kodierungsverfahren folgende Operationsebenen vorgenommen (Abbildung 37):

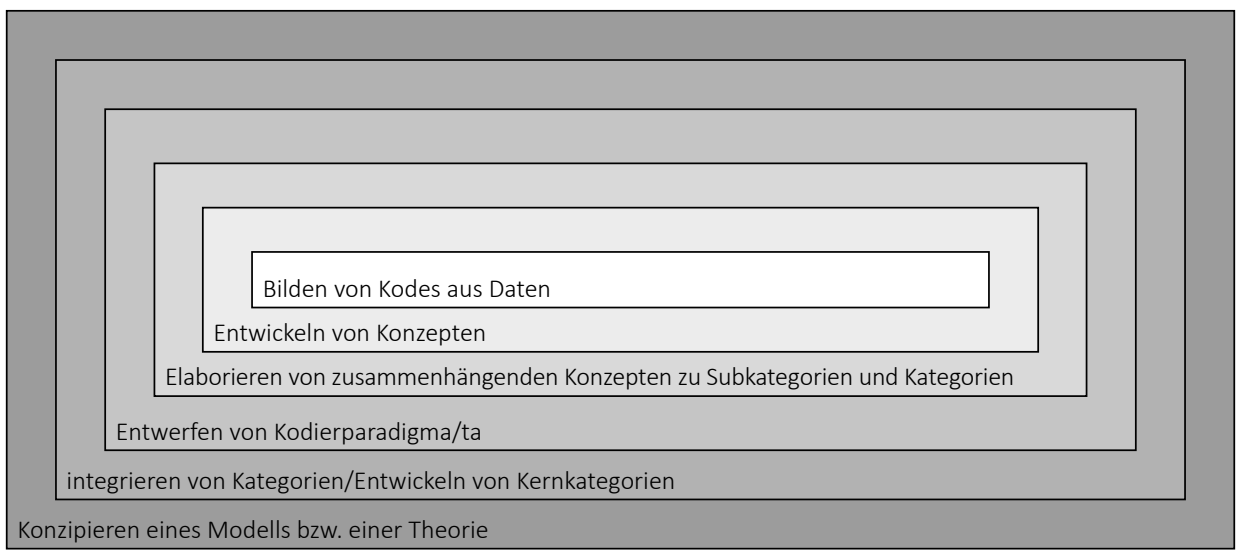

Abb. 37: Ebenen der Modell- bzw. Theoriegenerierung (Basierend auf Wieser 2013, 125) 
Die sechs beschriebenen Operationsmöglichkeiten wurden systematisch angewandt: Bilden von Kodes aus Daten, Entwickeln von Konzepten, Elaborieren von zusammenhängenden Konzepten zu Subkategorien und Kategorien, Entwerfen von Kodierparadigmata, Integrieren von Kategorien und Entwickeln von Kernkategorien, Konzipieren eines Modells bzw. einer Theorie. Im Folgenden werden die Ebenen, beginnend mit Bilden von Kodes aus Daten, näher erläutert und bereits mit ersten Ergebnissen sowohl aus Sicht der Lehrenden bzw. Mentor/innen als auch der Studierenden untermauert.

\section{Bilden von Kodes aus Daten}

Den Beginn des Arbeitsprozesses bildet die Analyse von Daten. Daten sind zunächst durch den Transkriptionsprozess der Interviews in schriftlicher Form vorhanden. Es handelt sich wie bei dieser Untersuchung um Daten aus dem Feld: Beobachtungen, Beschreibungen, Erinnerungen, Erfahrungswerte, Vergleiche, Metaphern und/oder Sichtweisen, die Lehrpersonen und Studierende von sich geben. Zur Kodierung braucht es nach Strauss et al. (1996) eine theoretische Sensibilität. „Theoretische Sensibilität bezieht sich auf die Fähigkeit, Einsichten zu haben, den Daten Bedeutung zu verleihen, die Fähigkeit zu verstehen und das Wichtige vom Unwichtigen zu trennen." (Strauss et al. 1996, 25) Als Instrument zum Aufbrechen des Datenmaterials nennen die Entwickler die zwei Verfahren: Fragestellung und Anstellen von Vergleichen (Strauss et al. 1996, 44). Ziel ist, Einblicke in das Datenmaterial zu gewinnen, ohne die inhaltliche Interpretation durch die/den Forschende/n vorwegzunehmen.

Die Entwickler nennen den ersten Prozess im analytischen Verfahren auch offenes Kodieren des Datenmaterials. „Das offene Codieren ist als Prozess des Aufbrechens, Untersuchens, Vergleichens, Konzeptualisierens und Kategorisierens von Daten definiert." (Kuckartz 2010, 75) In der Studie wurden einzelne Wörter markiert, einzelne Wörter oder Wortgruppen zu Sinneinheiten zusammengefasst, um dann als In-vivo-Kodes oder Konstrukte in MAXQDA verankert zu werden (Flick 2016, 388). Bei den Lehrpersoneninterviews wurden Fragen: Wer? Was? Wie? Wann? Wie lange? Wo? Wie viel? Wie stark? Warum? Wozu? Womit? an das Datenmaterial gestellt, wie z.B.: Wer begleitet Studierende in den Praktika? Was ist den Lehrpersonen im Begleitungsprozess ein Anliegen? Wie sollten Praktika nach Meinung der Lehrperson gestaltet sein? Wann und warum hat die Lehrperson begonnen, Studierende zu begleiten? Wie lange sollten Praktika dauern und warum? Welche Erfahrungen haben die Lehrpersonen in der Zeit der Ausbildungspraktika gemacht? Wie viele Praktika sollten Studierenden angeboten werden? Wie stark ist die Beziehung zum Studierenden in der Zeit der Praktika? Warum wollen Lehrpersonen Studierende in den Praktika begleiten und unterstützen? Wozu dienen Praktika und welche Aufgabe haben Mentor/ innen? Womit sollte sich ein/e Mentor/in unbedingt beschäftigen, wenn sie/er Studierende betreut? Diese Fragestellungen u.a. zielen darauf, ein tiefes Textverständnis zu erhalten.

Auch bei den Studierendeninterviews erfolgte nach der Transkription die Kodierung. Mit unterschiedlichen Fragestellungen: Wer? Was? Wie? Wann? Wie lange? Wo? Wie viel? Wie stark? Warum? Wozu? Womit? wurde auch dieses Datenmaterial beleuchtet: Wer trägt zum Gelingen der Praktika bei? Was erwarten sich Studierende von den Praxislehrpersonen? Wie sollten Lehrpersonen die optimale Begleitung in den Praktika aus Sicht der Studierenden verstehen? Wie haben Studierende Praktika und die Begleitung grundsätzlich erlebt? Wann sollen Praktika während der Ausbildungszeit prinzipiell erfolgen? Wie lange sollten Praktika dauern? Woran ermessen Studierende den Nutzen in der Begleitung? Wie viel Handlungsspielraum hatten Studierende während der Zeit der Praktika? Wie stark gelingt es Lehrpersonen, Studierende in das Alltagsleben Schule miteinzubeziehen? Warum haben sich Praktika als Herausforderung 
erwiesen? Wozu sollten Mentor/innen herangezogen werden? Worauf sollen Praxislehrpersonen während der Begleitung achten? Welche Maßnahmen würden Studierende setzen? Welche Ansprüche haben Studierende an Praxislehrpersonen?

Bei den Lehrpersoneninterviews ergaben sich 718 Kodes und bei den Studierendeninterviews 780 Kodierungen aufgrund von Wörtern, Wortgruppen und In-Vivo-Kodierungen.

\section{Entwickeln von Konzepten}

„Ein Konzept ist ein Etikett, das einzelnen Ereignissen zugeordnet wird.“ (Strauss et al. 1996, 43) Das folgende Beispiel verdeutlicht den analytischen Prozessverlauf beim offenen Kodieren aus einem Lehrpersoneninterview (L 01) dieser Untersuchung (Abbildung 38):

\begin{tabular}{|c|c|c|}
\hline Interviewausschnitt & Kodes & Konzept/e \\
\hline $\begin{array}{l}\text { „Ich habe einfach von meinen Praktikumslehrern sehr } \\
\text { viel Freiheit bekommen }\end{array}$ & Erhalt von Freiheit & $\begin{array}{l}\text { Erhalt von } \\
\text { Unterstützung }\end{array}$ \\
\hline $\begin{array}{l}\text { Die haben mich schon unter Anführungszeichen } \\
\text { kontrolliert aber so, dass es mir und den Klassen kaum } \\
\text { aufgefallen sind. Sozusagen sie sind in den Wolken } \\
\text { oberhalb geschwebt, da waren die noch nicht da. }\end{array}$ & $\begin{array}{l}\text { Unsichtbare Kontrolle } \\
\text { der Praxislehrperson }\end{array}$ & Form der Unterstützung \\
\hline $\begin{array}{l}\text { Sie haben die Information doch bekommen, was sie } \\
\text { gebraucht haben, um mich dann in gewissen Punkten } \\
\text { festzumachen: Das passt so nicht, das musst du dir noch } \\
\text { einmal anschauen. }\end{array}$ & $\begin{array}{l}\text { Erhalt von } \\
\text { Informationen; Kritische } \\
\text { Rückmeldungen }\end{array}$ & $\begin{array}{l}\text { Erhalt von } \\
\text { Unterstützung; Form } \\
\text { der Unterstützung }\end{array}$ \\
\hline $\begin{array}{l}\text { Ich habe da gelernt, dass es wirklich wohltuend ist, } \\
\text { wertschätzend; aber auch konstruktiv kritisch beurteilt } \\
\text { zu werden. }\end{array}$ & $\begin{array}{l}\text { Wertschätzender } \\
\text { Umgang; konstruktive } \\
\text { Begleitung }\end{array}$ & $\begin{array}{l}\text { Verhältnis Lehrperson } \\
\text { und Studierende/r; } \\
\text { Form der Unterstützung }\end{array}$ \\
\hline $\begin{array}{l}\text { Und es war in den ersten Monaten wirklich eine gute } \\
\text { Erfahrung, Fortschritte an mir selbst zu sehen. }\end{array}$ & $\begin{array}{l}\text { Erkennen der eigenen } \\
\text { Fortschritte }\end{array}$ & Positiver Prozessverlauf \\
\hline $\begin{array}{l}\text { Weil du natürlich, wenn du das erste Jahr oder erste Mal } \\
\text { in die Klasse reingehst als Lehrer, dann zittern einmal } \\
\text { gleich bei } 95 \text { Prozent der Studenten die Knie - auch bei } \\
\text { mir war es natürlich so, war vollkommen klar. }\end{array}$ & $\begin{array}{l}\text { Zittern der Knie; } \\
\text { Unsicherheit als } \\
\text { Studierende/r }\end{array}$ & $\begin{array}{l}\text { Auftreten von } \\
\text { Unsicherheiten bei } \\
\text { ersten Lehrtätigkeiten }\end{array}$ \\
\hline $\begin{array}{l}\text { Und da gab es einerseits Sicherheit, andererseits auch } \\
\text { die Freiheit, Dinge ausprobieren zu können, mit dem } \\
\text { gesamten Kontext im Hintergrund, das es auch eine } \\
\text { konstruktive und kritische Beurteilung gibt. (...) }\end{array}$ & $\begin{array}{l}\text { Erhalt von Sicherheit; } \\
\text { Freiheit zum } \\
\text { ausprobieren }\end{array}$ & $\begin{array}{l}\text { Erhalt von } \\
\text { Unterstützung }\end{array}$ \\
\hline $\begin{array}{l}\text { Und ich hoffe, dass ich das, was ich als Studierender } \\
\text { erlebt habe, auch so weitergeben kann. }\end{array}$ & $\begin{array}{l}\text { Wunsch nach } \\
\text { Weitergabe }\end{array}$ & $\begin{array}{l}\text { Ichbezogene } \\
\text { Weitergabe }\end{array}$ \\
\hline $\begin{array}{l}\text { Ich versuche auch immer Freiheiten zu geben, wo } \\
\text { es wichtig war und notwendig ist, aber schon auch } \\
\text { Grenzen zu setzen, und Kontrolle und Feedback zu } \\
\text { geben, wo es wichtig ist und auch dort einzuschreiten, } \\
\text { wo es wirklich manchmal auch Probleme gibt." }\end{array}$ & $\begin{array}{l}\text { Einräumen von } \\
\text { Freiheiten; Setzen von } \\
\text { Grenzen; Geben von } \\
\text { Feedback; Ansprechen } \\
\text { von Problemen; }\end{array}$ & $\begin{array}{l}\text { Setzen von Unterstüt- } \\
\text { zungsmaßnahmen als } \\
\text { Praxislehrperson; Ver- } \\
\text { halten bei Problemen } \\
\text { als Praxislehrperson; }\end{array}$ \\
\hline
\end{tabular}

Abb. 38: Beispiel im offenen Kodierungsprozess: Bilden von Konzepten nach der Grounded Theory (Basierend auf Przyborski et al. 2018, 214) 
Der vorgestellte transkribierte Text des Lehrpersoneninterviews wurde zunächst in Sinneinheiten bzw. Abschnitte gegliedert. Anschließend erfolgte die eigentliche Kodierung aus Datensegmenten mit folgenden Fragestellungen: Wer ist daran beteiligt? Wie wurde die Praxiszeit erlebt? Was war der/dem Studierenden in ihrer/seiner Ausbildungszeit wichtig? Wie agierte die Praxislehrperson damals? Wie interagieren Mentor/in und Mentee miteinander? usw. Hierbei entstand eine Liste von Benennungen. Nun wurden Ähnlichkeiten und Unterschiede der Kodes festgehalten und zu Konzepten gruppiert (Flick 2016, 392). Im Rahmen der Lehrpersonen- und Studierendeninterviews entstanden somit im offenen Kodierprozess unzählige Konzepte.

\section{Elaborieren von zusammenhängenden Konzepten zu Subkategorien und Kategorien}

Nach den ersten Analyseschritten im offenen Kodieren erfolgte die Zusammenfassung der Konzepte in eine Kategorie bzw. in Kategorien und bedingte eine weitere Verdichtung und Abstraktion.

„Kategorie ist für Strauss ein unabhängiges begriffliches Element einer Theorie, eine Klassifikation von Konzepten. Die Klassifikation wird erstellt, wenn Konzepte miteinander verglichen werden und sich offenbar auf ein ähnliches Phänomen beziehen. So werden die Konzepte unter einem Konzept höherer Ordnung zusammengruppiert - ein abstraktes Konzept, genannt Kategorie“. (Kuckartz 2010, 75)

Kategorien sind demnach Konzepte höherer Ordnung, die also eine Reihe von Konzepten mit den gleichen Eigenschaften integrieren (Strauss et al. 1996, 47). Das Ergebnis im axialen Kodierungsprozess der Studie ergab während des Kodierens eine weitere Gruppierung der Konzepte zu Subkategorien und dann zu Kategorien. Abbildung 39 verdeutlicht die Umsetzung:

\begin{tabular}{|c|c|c|c|}
\hline Kodes & Konzept/e & Subkategorie/n & Kategorie/n \\
\hline Erhalt der Freiheit & $\begin{array}{l}\text { Erhalt von } \\
\text { Unterstützung }\end{array}$ & Unterstützung & $\begin{array}{l}\text { Blick auf } \\
\text { Studierende }\end{array}$ \\
\hline $\begin{array}{l}\text { Unsichtbare } \\
\text { Kontrolle der } \\
\text { Praxislehrperson }\end{array}$ & $\begin{array}{l}\text { Form der } \\
\text { Unterstützung }\end{array}$ & Unterstützung & $\begin{array}{l}\text { Blick auf } \\
\text { Studierende }\end{array}$ \\
\hline $\begin{array}{l}\text { Wertschätzender } \\
\text { Umgang; konstruktive } \\
\text { Begleitung }\end{array}$ & $\begin{array}{l}\text { Verhältnis } \\
\text { Lehrperson und } \\
\text { Studierende/r }\end{array}$ & $\begin{array}{l}\text { Beziehungs- } \\
\text { geflechte }\end{array}$ & $\begin{array}{l}\text { Blick auf } \\
\text { Studierende }\end{array}$ \\
\hline $\begin{array}{l}\text { Erkennen der eigenen } \\
\text { Fortschritte }\end{array}$ & $\begin{array}{l}\text { Positiver } \\
\text { Prozessverlauf }\end{array}$ & $\begin{array}{l}\text { Entwicklungs- } \\
\text { potential }\end{array}$ & $\begin{array}{l}\text { Blick auf } \\
\text { Studierende }\end{array}$ \\
\hline $\begin{array}{l}\text { Zittern der Knie; } \\
\text { Unsicherheit als } \\
\text { Studierende/r }\end{array}$ & $\begin{array}{l}\text { Auftreten von } \\
\text { Unsicherheiten bei } \\
\text { ersten Lehrtätigkeiten }\end{array}$ & Befindlichkeit & $\begin{array}{l}\text { Blick auf } \\
\text { Studierende }\end{array}$ \\
\hline $\begin{array}{l}\text { Wunsch nach } \\
\text { Weitergabe }\end{array}$ & $\begin{array}{l}\text { Ichbezogene } \\
\text { Weitergabe }\end{array}$ & Motivation & $\begin{array}{l}\text { Blick auf Lehrperson/ } \\
\text { Sich selber }\end{array}$ \\
\hline $\begin{array}{l}\text { Einräumen von } \\
\text { Freiheiten; Setzen von } \\
\text { Grenzen; } \\
\text { Geben von Feedback; } \\
\text { Ansprechen von } \\
\text { Problemen }\end{array}$ & $\begin{array}{l}\text { Setzen von } \\
\text { Unterstützungs- } \\
\text { maßnahmen als } \\
\text { Praxislehrperson }\end{array}$ & $\begin{array}{l}\text { Unterstützung } \\
\text { Einstellungen }\end{array}$ & $\begin{array}{l}\text { Blick auf } \\
\text { Studierende } \\
\text { Blick auf } \\
\text { Lehrperson/Sich selber }\end{array}$ \\
\hline
\end{tabular}

Abb. 39: Beispiel im axialen Kodierungsprozess: Erstellen von Kategorien nach der Grounded Theory 
Wie sich also im weiteren Kodierungsprozess herausstellte, wurde nach der Bildung der vermeintlichen Kategorien, diese sind in der Abbildung 39 schon als Subkategorien ausgewiesen, eine höherwertige Kategorisierung vorgenommen. Demzufolge gestaltete sich die Abfolge: Kodes, Konzepte, Subkategorien und Kategorien. Somit wurde dem Forschungsgedanken und Kodierungsprozess der Grounded Theory entsprochen, denn Strauss und Corbin (1996) schreiben:

„Offenes Kodieren bricht die Daten auf und erlaubt es, einige Kategorien, deren Eigenschaften und dimensionale Ausprägungen zu identifizieren. Axiales Kodieren fügt diese Daten auf neue Art wieder zusammen, indem Verbindungen zwischen Kategorien und ihren Subkategorien ermittelt werden." (Strauss et al. 1996, 76)

Sowohl zur Studierenden- als auch Lehrendenperspektive ergaben sich im Analyseprozess jeweils eine dreiteilige Kategorienbildung mit unzähligen Konzepten (siehe Kapitel II/6.1 und II/6.2).

Nun wurden in diesem Abschnitt der Kodierung eine Art „Modellierungs-Logik“ (Breuer et al. 2018, 281) angewandt, Relationen hergestellt und versucht, kausale Wechselbeziehungen zu finden. Eigenschaften wurden herausgefiltert und in Beziehung zu dimensionalen Ausprägungen gestellt. Das folgende Beispiel aus dem Lehrpersoneninterview zeigt die Vorgehensweise (Abbildung 40):

\begin{tabular}{|c|c|c|}
\hline Kategorie & Eigenschaften & Dimensionale Ausprägung (pro Ereignis) \\
\hline \multirow{8}{*}{ Form der Unterstützung } & Häufigkeit & oft --.------ nie \\
\hline & Ausmaß & 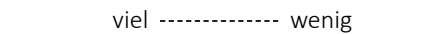 \\
\hline & Intensität & 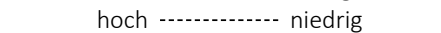 \\
\hline & Dauer & lang --.--.-- kurz \\
\hline & Art und Weise & wohlwollend --.--- hemmend \\
\hline & Funktion & wichtig ---1---- unwichtig \\
\hline & Bedeutung & hoch ---.-- niedrig \\
\hline & Zielangabe & einsichtig --.-- intransparent \\
\hline
\end{tabular}

Abb. 40: Beispiel des Dimensionalisierens in der Grounded Theory (Basierend auf Strauss et al. 1996, 53)

Zur Kategorie Form der Unterstützung wurden wiederum Konzepte und Eigenschaften zusammengefasst und mit den dimensionalen Ausprägungen in Relation gesetzt. Das Dimensionalisieren von Eigenschaften ist ein wichtiger Schritt beim offenen Kodieren, denn „es ist wichtig, Eigenschaften und Dimensionen zu erkennen und systematisch zu entwickeln, weil sie die Grundlage bilden, um Beziehungen zwischen Kategorien und Subkategorien - und später auch Hauptkategorien - herauszuarbeiten.“ (Strauss et al. 1996, 51) Diese „dimensional analysis“ (Schatzmann 1991) wird nach Schatzmann (1991) als eine multiperspektivische Analyse und Interpretation der Daten gesehen. Die methodische Frage beim Dimensionalisieren richtet sich vor allem darauf, „was hier alles involviert ist." (Schatzmann 1991, 310) Im Laufe der vorliegenden Arbeit werden eine Reihe von Eigenschaften und dimensionale Ausprägungen angeführt. 
Bei der Integration von Konzepten und bei der Entwicklung von Kategorien wird ein weiteres Element wie das Schreiben von Memos eingesetzt (Heiser 2018, 215). Das Schreiben eines Memos - in Form von Kode-Notizen (Darstellung der Ergebnisse) und Planungs-Notizen (Weiterzuverfolgende Ideen) - soll im Auswertungsprozess einen weiteren Überblick verschaffen, zum Nachdenken anregen und helfen, Konzepte weiterzuentwickeln (Yankov 2009, 14). Memos wurden im axialen und selektiven Kodierungsprozess geschrieben. Anbei ein Beispiel aus den Lehrpersoneninterviews (Abbildung 41):

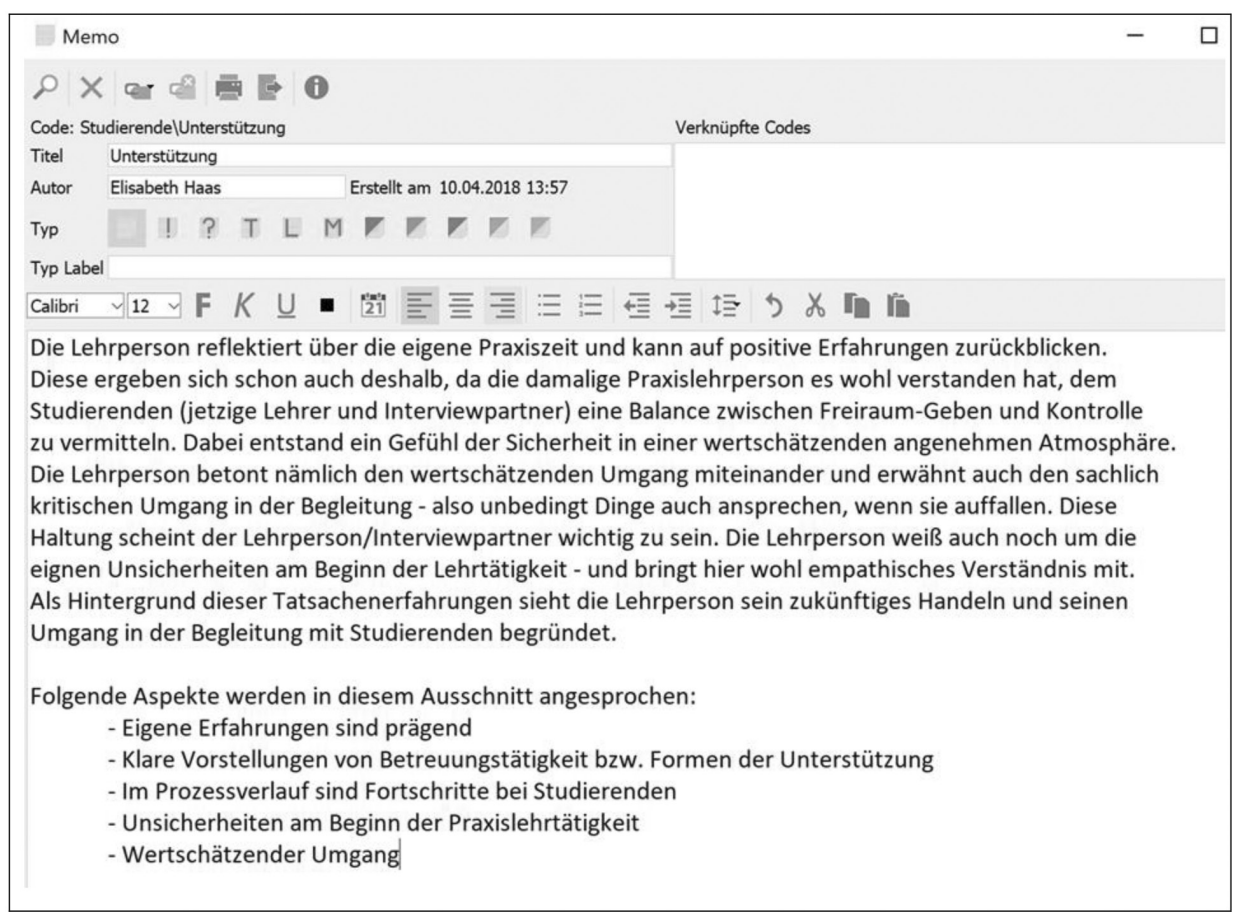

Abb. 41: Beispiel eines Memos zur vorliegenden Untersuchung mit Hilfe von MAXQDA

Aus Kodes, Konzepten, Eigenschaften und dimensionalen Ausprägungen wurde ein Text verfasst und - wie hier im Beispiel ersichtlich - eine Kurzzusammenfassung geschrieben. Die Nutzung von Memos (in Abbildung 42 als Vierecke markiert) zur Integration und Verbindung von Konzepten und zur Entwicklung von Subkategorien bzw. Kategorien kann als intensiver Aufgabenbereich im Kodierungsprozess verstanden werden. Abbildung 42 deutet mit den Fünfecken Konzepte an. Ein Übereinanderlegen symbolisiert die Integration und die Verbindung der Konzepte. Dabei werden Memos verfasst und eine Brücke hergestellt. Der Zusammenschluss mehrerer Konzepte (Fünfecke) verdeutlicht die Entwicklung von Kategorien - wie in der Abbildung veranschaulicht: 

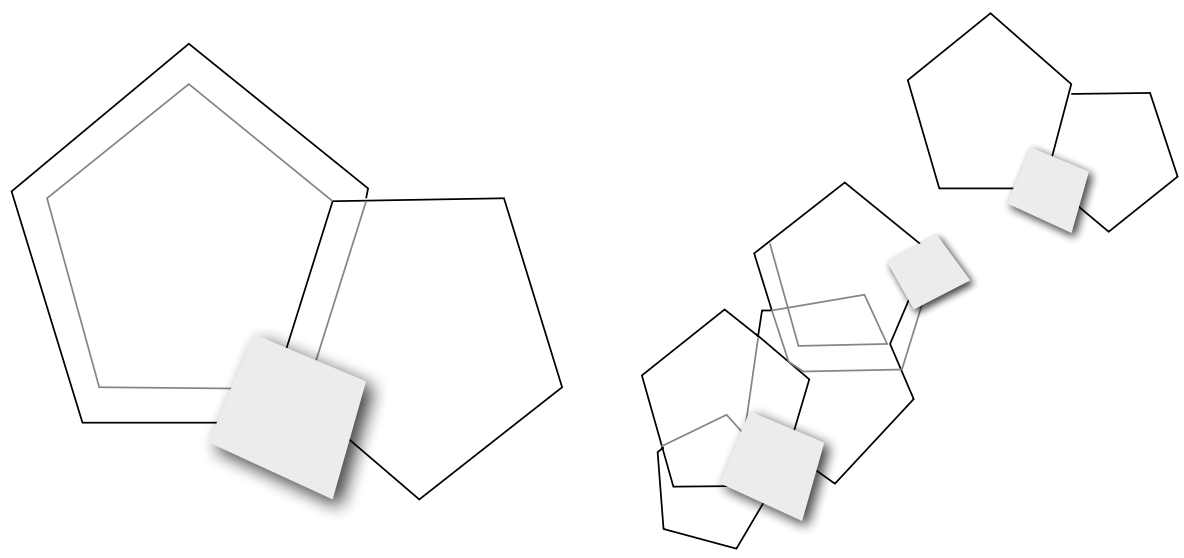

Abb. 42: Nutzung von Memos zur Integration und Verbindung von Konzepten und zur Entwicklung von Kategorien (Wieser 2013, 129)

Memos oder auch Diagramme sind für die/den Forscher/in Instrumente zur reflexiven Auseinandersetzung. Breuer et al. (2018) schlagen zum Verfassen von Memos und/oder Diagrammen eine Reihe von inhaltsbezogenen Beispielen vor, wie zum Forschungsthema, zu den disziplinären Präkonzepten, zur persönlichen Berührung mit dem Thema, zum Forschungsfeld und den Feldmitgliedern, zu operativen Planungen, zu ethischen Fragen, zu Erlebnissen in der Forschungsinteraktion, zu Kodierideen, zur sprachlichen Benennung von Konzepten, zu Diagrammen, zur Modellbildung, zur Einordnung in die Welt vorgängiger Forschungsergebnisse usw. (Breuer et al. 2018, 177f). Die Inhalte der Memos der vorliegenden Arbeit sind meist zusammengefasste Berichte zur unmittelbaren Mentor/innentätigkeit, zu Gedankenkonstrukten der Ausbildungsinstitutionen, zu erfahrenen Begleitmaßnahmen, zu lebensbiografischen Erlebnissen im Umfeld Schule und Äußerungen von Wunschvorstellungen im Mentoringprozess.

\section{Entwerfen von Kodierparadigmata}

Im Laufe des Kodierungsprozesses

„kommen als Hilfsmittel der Systematisierung bestimmte gegenstandsunspezifische, verallgemeinertmetatheoretische Vorstellungen ins Spiel. Nach den kanonischen Lehrbüchern der Strauss- bzw. Strauss/Corbin-Linie sind hier das Paradigmatische Modell bzw. das Kodierparadigma sowie die Bedingungs-Konsequenzen-Matrix zu nennen (Strauss et al. 1991, 75ff, 132ff; Corbin et al. 2015, 156ff, 163ff).“ (Breuer et al. 2018, 281)

Glaser (1978) schlägt auch die Kodierfamilie (Glaser 1978, 75-82; Böhm 2015, 481) vor. In der vorliegenden Arbeit wurden Kodierparadigmata als Modell ausgearbeitet. Bei den Abstraktionsprozessen handelt es sich um die Verdeutlichung der „Mittel-Zweck-, Ursache-Wirkungs-, zeitlichen oder räumlichen Beziehungen“ (Flick 2016, 395) zwischen den Kategorien, Subkate- 
gorien und Konzepten (Flick 2016, 393f) - wohl ein Sortier- und Ordnungsschema auf einer weiteren Ebene. Böhm (2015) schlägt als Visualisierung und Transparenz die Verankerung der Anordnungen um das Phänomen gruppiert in folgender Form vor: ursächliche Bedingungen, Kontext und intervenierende Bedingungen, Handlungsstrategien und Konsequenzen (Böhm 2015, 479).

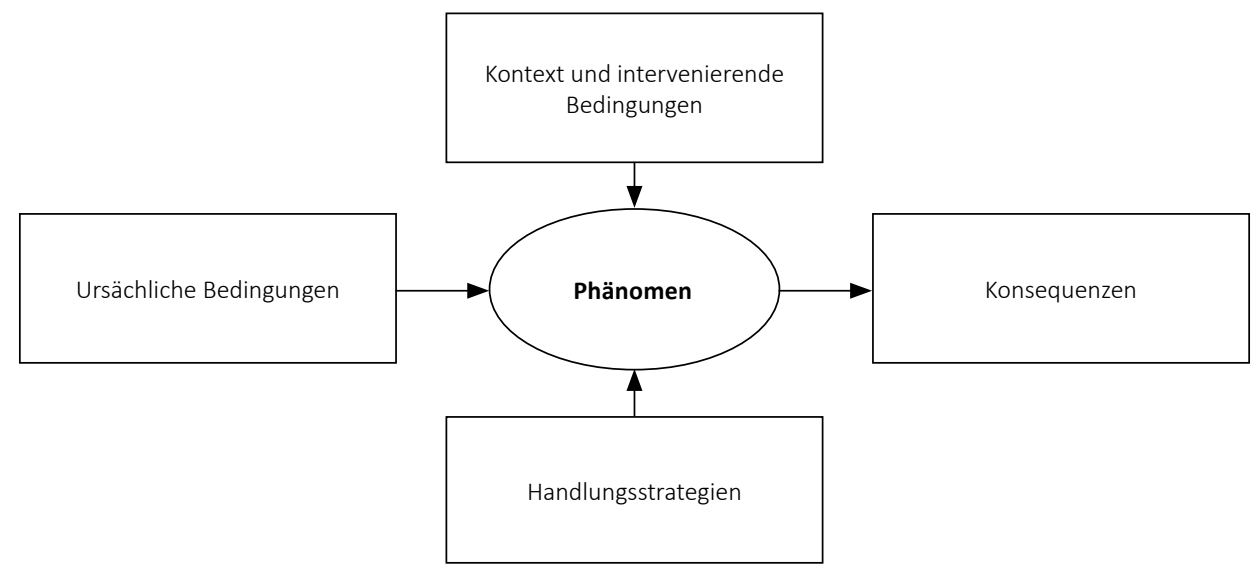

Abb. 43: Kodierparadigma (Böhm 2015, 479)

Im heuristischen Analysemodell (Heiser 2018, 231) steht das Phänomen im Zentrum. Es verdeutlicht ein Ereignis oder einen Zustand, auf das bzw. auf den Handlungen gerichtet sind und/ oder worauf eine Reihe von Kategorien und Konzepten beruhen. Andere Kategorien weisen auf die ursächliche Bedingung hin, die dann zum Phänomen führt. Unter Kontextbedingungen und intervenierenden Bedingungen werden Kategoriegruppierungen in Zusammenhang mit strukturellen Bedingungen aufgeschlüsselt (Flick 2016, 394; Heiser 2018, 232). Für Strauss et al. (1996) sind die Konsequenzen auf der einen Seite Handlungen, die aufgrund des Phänomens etabliert werden, oder auf der anderen Seite Maßnahmensetzungen, die in späterer Folge wiederum zu einer Bedingung werden (Strauss et al. 1996, 85). Im Laufe des Kodierungsprozesses entstanden bei der vorliegenden Arbeit zwei Kodierparadigmata bei beiden Interviewgruppen.

\section{Integrieren von Kategorien und Entwickeln von Kernkategorien}

Durch die Subsumierung aus der im axialen Kodierungsprozess gewonnenen Subkategorien und Kategorien wird bzw. werden die Kernkategorie bzw. die Kernkategorien erstellt.

„Die Kernkategorie ist ein umfassender und abstrakter Begriff, durch den in kurzen Worten benannt wird, was nach Ansicht des Autors (sic!) das hauptsächliche Thema oder Ergebnis der Studie ist. Wenn ein solches zentrales Konzept festgelegt ist, werden die übrigen Kategorien durch erklärende Ausführungen dazu ins Verhältnis gesetzt." (Corbin et al. 2015, 199f; in Übersetzung von Breuer et al. 2018, 286)

Im vorliegenden Forschungsprozess entstanden die Überlegungen, aus den vier Kodierparadigmata eine Kernkategorie zu entwickeln. Nach eingehender Analyse wurde es aber als sinnvoller erachtet, je eine Kernkategorie pro Interviewgruppe zu entwickeln, da die Aussagekraft dadurch intensiviert werden kann und „eine konzeptuelle Repräsentativität“ (Strübing 2014, 31) für beide Stichprobengruppen gegeben ist. 


\section{Konzipieren eines Modells bzw. einer Theorie}

Nachdem Prozesse des Kodierens, des Dimensionalisierens, des Samplings, des Verdichtens und des Kategorisierens angewandt wurden, erfolgt die Generierung eines Modells bzw. einer Theorie, welche aus Konzepten besteht, die interpretiert und in Beziehung gesetzt werden können. Die konzeptuellen Bezeichnungen stammen aus den Daten (Strauss et al. 1996, 13f). Somit spricht Strauss et al. (1996) von einer gegenstandsbezogenen bzw. bereichsbezogenen Theorie, „die die Kriterien für ,gute' Forschung erfüllt: Signifikanz, Vereinbarkeit von Theorie und Beobachtung, Verallgemeinerbarkeit, Reproduzierbarkeit, Präzision, Regelgeleitetheit und Verifizierbarkeit." (Strauss et al. 1996, 18) In dieser Untersuchung wurden zwei Modelle mittels Grounded Theory herausgearbeitet:

- 1. ATM-Modell: Analyse Transformationales Mentoring im berufsfeldbezogenen Wirkungskontext

- 2. MIW-Modell: Mentoringinduziertes Wirkungsmodell zum schulpraktischen Mentoringprozess

Die Konzeption der Modelle gestaltete sich vor dem Hintergrund der Konzepte, Subkategorien, Kategorien, Kodierparadigmata, Kernkategorien und somit aus Daten aus den Interviews. Die Rekonstruktionen werden in weiteren Kapiteln dargelegt.

Zusammenfassend kann gesagt werden: Die Methode der Grounded Theory verlangt von der/ dem Forscher/in eine zeitintensive und inhaltsausschöpfende Auseinandersetzung mit dem qualitativ erhobenen Datenmaterial, um zu einer „konzeptuellen Repräsentativität“ (Strübing 2014, 32) zu gelangen.

Im folgenden Kapitel werden die Befunde der qualitativen Analyse - basierend auf den Leitideen des Kodierprozesses nach den dreistufigen Kodierschritten: offenes Kodieren, axiales Kodieren und selektives Kodieren sowie dem Dimensionalisieren - vorgestellt und theoretische Sättigung in den Kodierparadigmata und Kernkategorien der Untersuchung getroffen. 


\section{Untersuchungsergebnisse}

Wie im vorhergehenden Kapitel beschrieben, wurde der Kodierungsablauf nach den Vorgaben zur Grounded Theory getätigt: offenes Kodieren, axiales Kodieren und selektives Kodieren. Dabei wurden nach dem Transkriptions- und Kodierungsprozess Konzepte, Subkategorien und Kategorien erstellt. „Die verschiedenen Stufen der Datenanalyse, d.h. die Datensammlung, Kodierung, Kategorienbildung, Hypothesen- und Theorieentwicklung, laufen gleichzeitig ab und unterstützen sich wechselseitig im Verlauf des Forschungsprozesses." (Lamnek et al. 2016, 111f) Mit der Datenanalyse werden die durch die Interviews gewonnenen qualitativen Daten einer ständigen Überprüfung unterzogen (Lamnek et al. 2016, 108). Es obliegt demnach der/dem Forscher/in, eine Balance zwischen frühzeitiger und sinnvoller Festlegung der Konzepte zu finden sowie den Zeitpunkt für den Abschluss der Dateninterpretation festzulegen. Die „Theoriebildung soll auf der Basis vielfältiger Kategorien erfolgen, die auf möglichst vielen Niveaus konzeptioneller und hypothetischer Generalisierung zusammengefasst sein sollen." (Lamnek et al. 2016, 113) In der folgenden Darstellung der Ergebnisse der Lehrpersonen- und Studierendeninterviews werden die Verfahren bzw. Operationsebenen in zeitlicher Sequenzierung angeführt. Tabelle 54 schlüsselt die von Strauss et al. (1996) entwickelten Kodierungsschritte und die im Prozess des theoretischen Kodierens angewandten Operationsverfahren auf (Tabelle 54):

Tab. 54: Operationsebenen zum theoretischen Kodieren

\begin{tabular}{ll}
\hline Theoretisches Kodieren nach Strauss et al. (1996) & Integrierte Operationsebenen in der vorliegenden Arbeit \\
\hline Offenes Kodieren & Bilden von Kodes aus Daten \\
& Entwickeln von Konzepten \\
\hline \multirow{3}{*}{ Axiales Kodieren } & Elaborieren von zusammenhängenden Konzepten \\
& zu Subkategorien und/oder Kategorien \\
& Entwerfen von Kodierparadigma/ta \\
\hline \multirow{3}{*}{ Selektives Kodieren } & Integrieren von Kategorien \\
& Entwickeln von Kernkategorie/n \\
& Konzipieren eines Modells bzw. einer Theorie \\
\hline
\end{tabular}

Das Aufzeigen der Operationsebenen dient lediglich der Übersichtlichkeit in Bezug auf Ergebnisdarstellung und Interpretationsvorgang, denn „diese Prozeduren sollten weder als klar voneinander trennbare Vorgehensweisen noch als zeitlich eindeutig getrennte Phasen des Prozesses (miss-)verstanden werden." (Flick 2016, 387) Die Analyse und Ergebnisdarstellung beginnt mit den Interviews der Lehrpersonen, die als Mentor/innen tätig sind und im weiteren Verlauf der Arbeit so bezeichnet werden.

\subsection{Stichprobe Mentor/innen}

Ausgehend vom Leitfadeninterview war das Ziel des Analyseprozesses die Erstellung von Kernkategorien sowie die Zusammenfassung der Konzepte und Kategorien, die die Entwicklung eines Modells forcieren und die die empirische Realität abbilden sollen. Als Forschungsfeld für die qualitative Analyse wurden Lehrpersonen $(n=12)$ in Form eines Expert/inneninterviews 
ausgewählt, die bereits seit mehreren Jahren Studierende in der Ausbildungsphase begleiten und in unterschiedlichem persönlichen Anspruchsniveau mit der Universität und/oder der Pädagogischen Hochschule kooperieren.

\section{Offenes Kodieren}

Beim offenen Kodieren der zwölf Interviews mit Mentor/innen ergaben sich 718 Kodierungen (Kodes) und Memos, welche „Ergebnisse des tatsächlichen Kodierens einschließlich theoretisch sensibilisierender und zusammenfassender Notizen" (Strauss et al. 1996, 192) beinhalten. Ziele des extensiven Analysierens der Daten beim offenen Kodieren sind die ersten Schritte der Theoretisierung und Generalisierung von Konzepten (Przyborski et al. 2014, 213). So verbergen sich hinter dem Konzept Signale der Studierenden wahrnehmen die Kodes: Signale beachten, Signale der Studierenden aufnehmen, Signale erkennen, Handeln nach einem Signal der Studierenden, Einschreiten nur nach einem Signal.

\section{Axiales Kodieren}

Folgende Fragestellungen richteten sich im axialen Kodierungsprozess an das Datenmaterial: Was sagen die Konzepte aus? Ergibt sich eine Relation oder eine Beziehung zwischen den Konzepten? Können sie zu Subkategorien und/oder Kategorien zusammengefasst werden? Auf welcher Ebene schauen sich Mentor/innen Praktika und Begleitung an? Und schließlich: Worüber berichten Mentor/innen eigentlich? Worauf werfen Mentor/innen ihren Blick? Man kann wohl annehmen, dass die weiteren Beschreibungen Gedankenfiguren sind, die apriorisch erschlossen worden sind (Breuer et al. 2018, 281). Die genauere Differenzierung ergab, dass Mentor/innen ihren Blick auf den strukturellen Rahmen, auf die eigene Person und auf Studierende richten (Abbildung 44). Abbildung 44 zeigt die lineare dreiteilige Kategorienbildung auf:

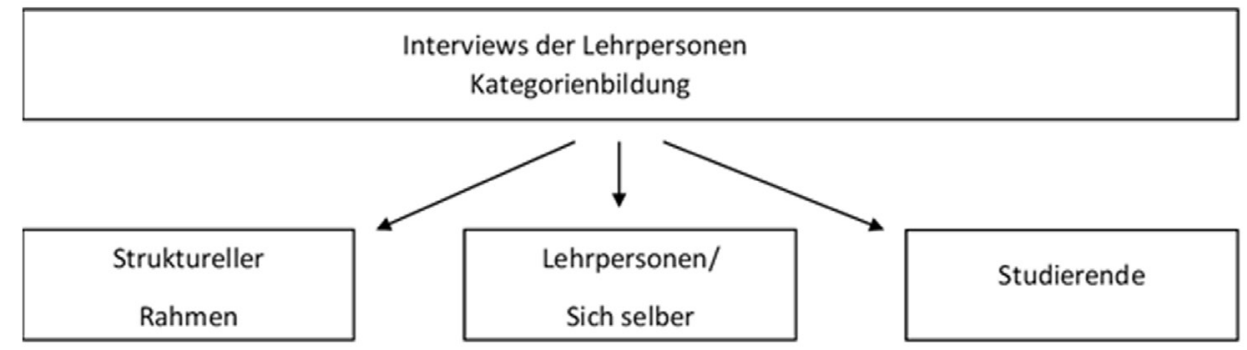

Abb. 44: Binnendifferenzierung bei Lehrpersoneninterviews $(n=12)$

Jede der angeführten Kategorie (Struktureller Rahmen, Lehrpersonen/Sich selber, Studierende) umfasst thematische Zusammenhänge mit Aussagen in den Subkategorien und Konzepten, die in einem ersten Kodierungsprozess generiert wurden. Im Sinne der Entwickler der Grounded Theory ist es nicht üblich, quantitative Darstellungen und Berechnungen darzulegen. Computergestützte Auswertungsprogramme - wie im weiteren Beispiel durch die Verwendung von MAXQDA-Programmen - ermöglichen es, Übersichten zu Kodierungen zu erstellen und quantitative Erhebungen aufzuzeigen (Kuckartz 2010, 83). Daher wird diese Möglichkeit genützt.

Die Häufigkeiten der Kodierungen innerhalb der dreiteiligen Kategorienbildung mit ihren Subkategorien werden in der angeführten Abbildung (Abbildung 45) in Form einer visuellen Darstellung durch den Code-Matrix-Browser von MAXQDA veranschaulicht. Die Größe der 
Knoten bezieht sich auf die relative Häufigkeit der Kodierungen. Die Summe (SUM...) gibt die Anzahl der Kodierungen an. Die Anzahl wird im anschließenden Teil der Beschreibung in Klammer angegeben.

\begin{tabular}{|c|c|c|}
\hline Codesystem & Lehrpersonen & SUM... \\
\hline$\checkmark$ Struktureller Rahmen & & 0 \\
\hline$>$ Bedeutung der Praktika & घ & 47 \\
\hline$>$ Aufgabenbereiche der Praktika & घ & 46 \\
\hline$>$ Art der Praktika & - & 30 \\
\hline$>$ Vernetzung mit Ausbildungsinstitution & - & 18 \\
\hline$>$ Vorschläge für Innovationen & - & 14 \\
\hline$\checkmark$ Lehrpersonen/Sich selber & & 0 \\
\hline$>\bullet$ Motivation & $\square$ & 110 \\
\hline$>$ Einstellungen & $\square$ & 106 \\
\hline$>\cdot$ Verantwortungsbewusstsein & 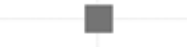 & 80 \\
\hline$\checkmark$ Studierende & & 0 \\
\hline$>$ Beziehungsgeflechte & $\mathbf{\square}$ & 62 \\
\hline$>$ Möglichkeit einer Probeidentifikation & घ & 61 \\
\hline$>\odot$ Unterstützung & - & 56 \\
\hline$>$ Befindlichkeit der Studierenden & - & 51 \\
\hline$>$ Entwicklungspotential der Studierenden & - & 37 \\
\hline$\sum$ SUMME & 718 & 718 \\
\hline
\end{tabular}

Abb. 45: Kategorien und Subkategorien der Kodes aus den Lehrpersoneninterviews ( $\mathrm{n}=12)$

Die einzelnen Kategorien werden nun näher beschrieben und die folgenden Abbildungen zeigen die Subkategorien zur jeweiligen Kategorie und die Anzahl der Kodierungen pro Interviewpartner/in (Lehrperson 01, usw.) in den Spalten bzw. die Anzahl der gesamten Subkategorien in den Zeilen. Durch die Visualisierung der Kodierungen anhand des Gewichtsfilters mittels Code-Matrix-Browser in MAXQDA lässt sich erkennen, welche Personen zu den einzelnen Themen viele oder nur wenige Kodierungen aufzeigen und wie die Gewichtung der einzelnen Subkategorie aussieht.

Struktureller

Rahmen

Unter der Kategorie Struktureller Rahmen (155 Kodierungen) werden all jene Subkategorien untergeordnet, die Bezüge zum Stellenwert von Praktika, zu Rahmenbedingungen der Praktika und mögliche Innovationen bei Praktika bzw. bei der Begleitung von Studierenden innerhalb des Ausbildungssystems nehmen. Daraus ergaben sich folgende Subkategorien: Bedeutung der Praktika, Aufgabenbereiche der Praktika, Art der Praktika, Vernetzung zum Ausbildungssystem und Innovationen zur Ausbildungsinstitution. Aussagen zur Bedeutung der Praktika (47) und zu den Aufgabenbereichen der Praktika (46) sind innerhalb der Kategorie Struktureller Rahmen am häufigsten vorhanden. Nur wenige Kodierungen weisen die Subkategorien Vernetzung mit Ausbildungsinstitution (18) und Vorschläge für Innovationen (14) auf. 
Die Aufschlüsselung zur Kategorie Struktureller Rahmen mit Bedeutung der Praktika, Aufgabenbereiche der Praktika, Art der Praktika, Vernetzung mit Ausbildungsinstitution, Vorschläge für Innovationen als Subkategorien zeigt folgende Verteilung (Abbildung 46):

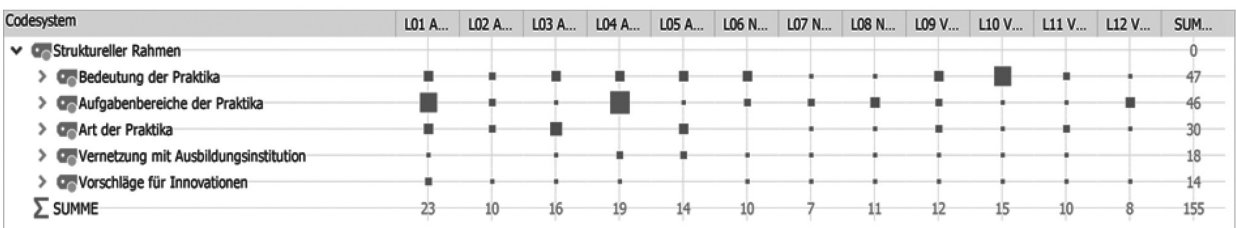

Abb. 46: Kategorie Struktureller Rahmen bei Lehrpersonenkodes

Auffallend hierbei ist, dass zur Bedeutung der Praktika (47 Kodierungen) und zu den Aufgabenbereichen der Praktika (46 Kodierungen) die meisten Aussagen getroffen werden. Es kommen wenige Vorschläge zu Innovationen zu den Praktika (14 Kodierungen). Inhaltlich äußern Mentor/innen hierbei die Einräumung der Möglichkeit zur Begegnung der Schulrealität während der Ausbildung als sehr notwendig.

Zwei Mentor/innen (L01, L04) mit Lehrbefähigung an einem Gymnasium tätigen vermehrt Aussagen zu den Aufgabenbereichen der Praktika. Sie sprechen vor allem von der Wichtigkeit der Umsetzung des Fachwissens in den Schulalltag, über die Möglichkeit, Teamteaching ausprobieren zu können und die Anwendung der Methodenvielfalt während der Zeit der Praktika. Für L10 sind Praktikumserfahrungen in der Schule und am Beginn des Studiums sehr wichtig. Der Interviewpartner spricht vor allem den Hospitations- und Reflexionsphasen eine hohe Bedeutung zu. Die Interviewpartner/innen L07 und L11 äußern sich insgesamt im Vergleich zu den anderen Interviewpartner/innen zur Kategorie Struktureller Rahmen nur sehr bedingt ( 7 bzw. 8 Kodierungen), die Interviewpartner/innen L01 und L04 mit 23 bzw. 19 Kodierungen überdurchschnittlich oft.

Lehrpersonen/

Sich selber

Unter der Kategorie Lehrpersonen/Sich selber (296 Kodierungen) werden Aussagen gebündelt, in denen die/der Mentor/in ihren/seinen Blick auf sich selbst, ihr/sein Tun und Handeln in der Rolle als Lehrperson und in der Funktion als Begleiter/in gerichtet hatte. Innerhalb dieser Kategorie sind die persönliche Bedeutung der Funktion Mentor/in, Ziel- und Wertvorstellungen sowie Begründungen des pädagogischen Handelns im eigenen Setting verankert. Die Kategorie wurde unterteilt in Motivation (110), persönliche Einstellungen (106) und Verantwortungsbewusstsein (80). Die Anzahl der Häufigkeiten zeigen an, dass die Kategorie Lehrpersonen/Sich selber bei der Dreiteilung der Kategorienbildung am stärksten gewichtet ist.

Die Kategorie Lehrpersonen/Sich selber zeigt die Verteilung mit den Subkategorien Motivation, Einstellungen, Verantwortungsbewusstsein auf(Abbildung 47):

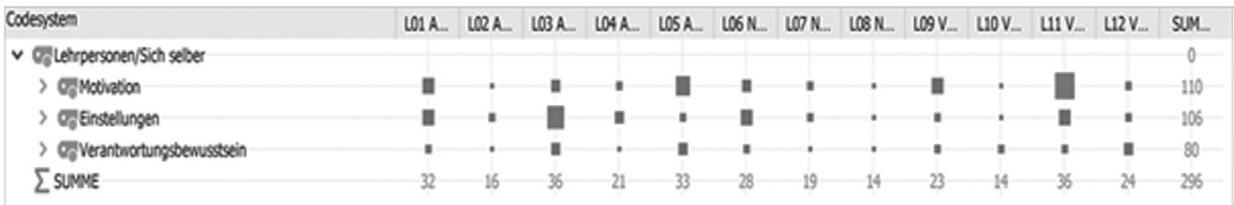

Abb. 47: Kategorie Lehrpersonen/Sich selber bei Lehrpersonenkodes 
Jede/r Mentor/in tätigt unterschiedlich häufige Aussagen zum Beweggrund und Motiv, Studierende begleiten zu wollen. Der Faktor Motivation ist ein wichtiger Aspekt bei der Tätigkeitsausübung und hat in der oben angeführten Kategorie mit der Subkategorie Motivation (110 Kodierungen) die meisten Kodierungen. Lehrpersonen nennen ichbezogene, systembezogene und student/innenbezogene Motive. Die Interviewpartner/innen L05 und L11 vereinen in den Aussagen die Bedeutung aller drei Motive für sich und erkennen für sich einen erfüllenden Aufgabenbereich im Lehrberuf.

Auch die Subkategorie Einstellungen hat bei den meisten Mentor/innen eine hohe Gewichtung. Hierbei betonen die Stichprobengruppe, wie sie sich das professionelle Verhältnis mit Studierenden vorstellen und wie sie den Umgang auf Basis subjektiver Verhaltensmuster gestalten. An Beispielen zeigen sie die Sichtweise der Beziehungsebene Mentor/in zu Student/in auf. Verantwortungsbewusstsein im Begleitungsprozess nimmt für alle Mentor/innen einen besonderen Stellenwert bei der Tätigkeitsausübung ein: Verantwortung für sich selber und der Schulumgebung gegenüber, Verantwortung der/dem Studierenden gegenüber und Verantwortung in der Systementwicklung Lehrprofession.

Interviewpartnerin L03 geht thematisch in den Aussagen intensiv darauf ein, dass das Belassen der Persönlichkeit ihres Gegenübers während der Zeit der Begleitung für sie die größte Bedeutung hat. Authentizität sollte ihrer Meinung nach von Beginn der Ausbildung der Professionsentwicklung einen Stellenwert haben. Besonders die Interviewpartner/innen L03 und L11 richten ihren Blick auf sich selber. Die Interviewpartner/innen L08 und L10 haben wenige Aussagen zur Kategorie Lehrpersonen/Sich selber getätigt.

Studierende

Unter der Kategorie Studierende (267 Kodierungen) sind all jene Aussagen enthalten, in denen die Mentor/innen sich mit der/dem Mentee auseinandergesetzt haben und den Blick auf die/den anderen innerhalb der Begleitung zur Professionalisierung werfen. Sie enthalten auch Kodierungen, wo sich Mentor/innen zurückerinnern, in die Zeit, in der sie Studierende waren und aus dieser Perspektive erzählen. Sie nehmen die Sichtweise der/des Studierenden ein und versetzen sich in die Rolle der/des Studierenden. Mentor/innen sprechen hierbei das Beziehungsgeflecht (62) während der Zeit der Begleitung, die Möglichkeit einer Probeidentifikation (61) und den Unterstützungssupport (56) an. Sie achten auch auf die Befindlichkeit der Studierenden (51) während der Praxiszeit und erkennen das Entwicklungspotential der Studierenden (37). Die Kategorie Studierende ist nach dem Dreiteilungsschema am zweitstärksten gewichtet.

Die Kategorie Studierende weist die fünf Subkategorien auf: Beziehungsgeflechte, Möglichkeit einer Probeidentifikation, Unterstützung, Befindlichkeit der Studierenden und Entwicklungspotential der Studierenden mit folgender Verteilung (Abbildung 48):

\begin{tabular}{|c|c|c|c|c|c|c|c|c|c|c|c|c|c|}
\hline codesytem & LO1A. & LORA & $103 A$ & LOAA... & LOSA. & $106 \mathrm{~N}$ & $100 \mathrm{~N}$. & LOBN. & $100 \mathrm{~V}_{\text {... }}$ & L10 V. & LII V... & $412 V_{\ldots}$ & SU. \\
\hline$\checkmark$ C्तStuderende & & & & & & & & & & & & & 0 \\
\hline$>$ CgBresehungspeflechte & 뭄 & - & * & - & . & - & - & . & " & a & - & $\mathbf{n}$ & 62 \\
\hline > Co Untertitang & - & - & & , & ' & , & , & , & " & , & , & 口 & s6 \\
\hline$>$ Cgerinalichket des Shudierenden & • & & n & $:$ & * & " & $\cdot$ & $\cdot$ & - & $=$ & $\approx$ & " & 51 \\
\hline
\end{tabular}

Abb. 48: Kategorie Studierende bei Lehrpersonenkodes 
37 bis 62 Kodierungen sind innerhalb der Subkategorien. Die geringsten Kodierungen hat die Subkategorie Entwicklungspotential der Studierenden (37), am höchsten die Subkategorien Beziehungsgeflecht (62) und die Möglichkeit einer Probeidentifikation (61). Auffallend ist, dass sich keine hohen Gewichtungen bei allen Subkategorien ergeben. Interviewpartner L01 diskutiert in der Subkategorie Möglichkeit einer Probeidentifikation die Thematik des Perspektivenwechsels und deren Wichtigkeit, dadurch miteinhergehend die Erleichterung der Berufswahlentscheidung durch Praktikumsphasen und weiters den Theorie-Praxis-Transfer. L01 betont auch die unterschiedlichen Beziehungsgeflechte, die in Praktikumsphasen entstehen. Für ihn ist der Aufbau einer Beziehung zur/zum Schüler/in durch Studierende sehr wichtig. Interviewpartnerin L12 bezieht in der Subkategorie Unterstützung ihre Form der Begleitung auf sachliche und zwischenmenschliche Bereiche und betont das Selbständig-werden-Lassen der/des Studierenden. Bei den Interviewpartner/innen L01 und L12 zeigen sich innerhalb der Kategorie Studierende die meisten Kodierungen, bei den Interviewpartner/innen L02 und L09 die wenigsten.

In Abbildung 49 werden die Kodierungsverteilungen pro Interviewpartner/in $(n=12)$ nach dem Durchschnittswert jeder Kategorie veranschaulicht. Das Schaudiagramm (Abbildung 49) gibt Aufschluss über die Quantifizierung der Kodierungen der einzelnen Kategorien bei jeder/ jedem Interviewpartner/in, gemessen am Durchschnittswert der jeweiligen Kategorie.

\section{Durchschnittskodierungsverteilung pro Interviewpartner/in der Lehrpersonen bzw. Mentor/innen $(\mathbf{n}=\mathbf{1 2})$}

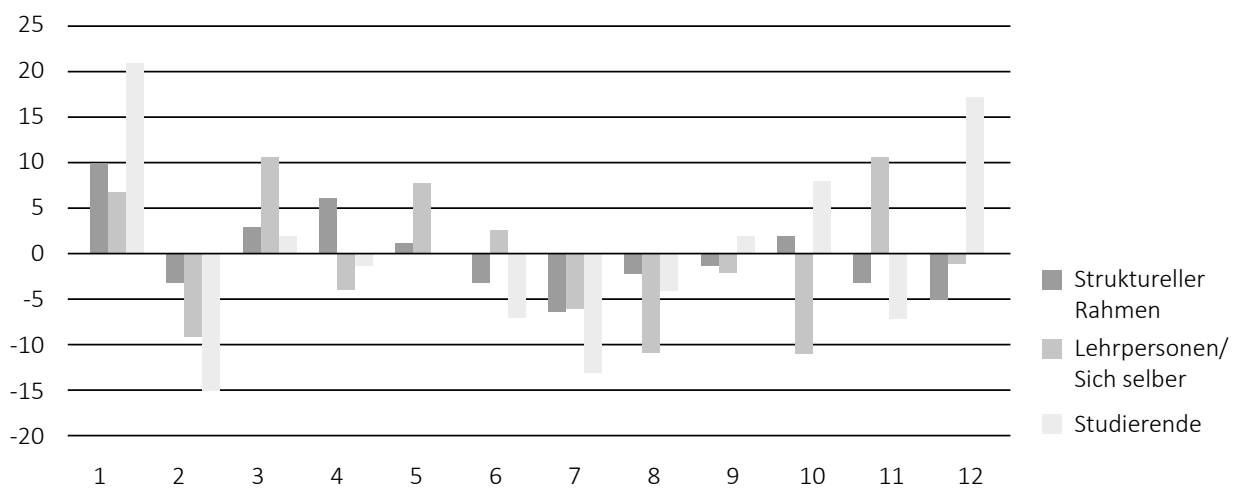

Abb. 49: Durchschnittskodierungsverteilung pro Interviewpartner/in der Lehrpersonen bzw. Mentor/innen $(n=12)$

Alle Interviewpartner/innen äußern sich mit Aussagen zu den Kategorien Struktureller Rahmen, Lehrpersonen/Sich selber und Studierende. L05 erreicht bei der Kategorie Studierende genau den Durchschnittswert 22, daher ergeben sich keine Werte für eine Säulendarstellung. Die Interviewpartner/innen L01 und L03 äußern sich überdurchschnittlich häufig bei allen drei Kategorien und erhalten somit dreimal positive Werte bei der Diagrammdarstellung. Der Durchschnittswert pro Interviewpartner/in der Kategorie Struktureller Rahmen, gemessen an der Varianz, der 155 Kodierungen liegt bei 12,9 Kodierungen (aufgerundet auf 13 Kodierungen). Auffallend ist, dass sich vier von fünf Mentor/innen der Allgemeinbildenden Höheren Schulen zur Kategorie Struktureller Rahmen äußern, aus dem Pflichtschulbereich hingegen nur eine Mentorin von sieben. In dieser Kategorie gibt es die positiven Spitzenwerte bei den Inter- 
viewpartner/innen L01 und L04; L07 und L12 beziehen sich in ihren Aussagen unterdurchschnittlich weniger zu dieser Kategorie.

Der Durchschnittswert pro Interviewpartner/in bei der Kategorie Lehrpersonen/Sich selber gemessen an der Summe der 296 Kodierungen liegt bei 24,6 Kodierungen (aufgerundet auf 25 Kodierungen). Drei von fünf Mentor/innen der Allgemeinbildenden Höheren Schule richten ihren Blick häufiger auf die Kategorie Lehrpersonen/Sich selber, bei den Mentor/innen der Neuen Mittelschule sind es eine von drei und bei der Volksschule ebenfalls eine von vier. Den höchsten positiven Wert in dieser Kategorie, gemessen am Durchschnittswert, nehmen die Interviewpartner/innen L03 und L11 ein, den niedrigsten L08 und L10.

Der Durchschnittswert pro Interviewpartner/in, gemessen an der Summe aller 267 Kodierungen, liegt bei der Kategorie Studierende bei 22,25 Kodierungen (abgerundet auf 22 Kodierungen). Zwei von fünf Mentor/innen der Allgemeinbildenden Höheren Schule gehen in der Kategorie Studierende auf deren Situation während der Zeit des Praktikums ein, bei den Mentor/ innen der Neuen Mittelschule liegen die Werte unter dem Durchschnittswert. Auffallend ist auch, dass drei von vier Mentor/innen aus dem Volksschulbereich überdurchschnittlich häufig den Blick auf Studierende werfen. In dieser Kategorie gibt es die positiven Spitzenwerte, gemessen vom Durchschnittswert, bei den Interviewpartner/innen L01 und L12; L02 und L07 nehmen unterdurchschnittlich Bezug zu den Studierenden.

Im Folgenden wird nun ein weiterer Verdichtungsprozess der Konzepte, Subkategorien und Kategorien durch das Entwerfen von Kodierparadigmata verdeutlicht.

\section{Entwerfen von Kodierparadigmata}

Beim axialen Kodieren geht es auch darum, die Kategorien und Subkategorien in Beziehung zu setzen. Die methodologischen Überlegungen wechseln hierbei zwischen induktiven und deduktiven Zugängen (Strauss et al. 1996, 92). Sie gehen von der Spezifität der Bedingungen und der Kontextdaten aus den Kodierungen aus und konzentrieren sich auf die Erstellung des Phänomens. Die Konsequenzen sind dann die Schlussfolgerungen aus dem Phänomen und den Handlungen (Strauss et al. 1996, 85). Das Kodierparadigma gilt als ein Modell für eine „handlungstheoretisch fundierte Heuristik, anhand derer sich die Zusammenhänge zwischen Kategorien darstellen lassen." (Heiser 2018, 231)

Der Blick richtete sich auf die Vielfalt der Konzepte, Subkategorien bzw. Kategorien und es stellten sich allgemeine und konkrete Fragen zur Forschung: Wie kommt dieses Ergebnis bzw. dieses Phänomen zustande? Wann tritt das Ergebnis auf? Welche Ereignisse sind bzw. welches Ergebnis ist markant? Welche Bedingungen sind maßgeblich? Was sind die Vorbedingungen? Wie gehen die Mentor/innen mit diesem Ergebnis um? Worauf beziehen Mentor/innen sich in ihren Erklärungen? Was sind die Konsequenzen dieses Ergebnisses? (in Anlehnung an Strübing 2014, 25) In Anlehnung an Böhm (2015) entstand folgende Abbildung (Abbildung 50) zur Visualisierung und Transparenz und zur Darstellung der Zusammenhänge - das Kodierparadigma (Strauss et al. 1996, 75ff). Pfeile symbolisieren eine Bedeutung und weisen einen hypothetischen apriorischen Charakter auf (Breuer et al. 2018, 281). Das Phänomen ist „die zentrale Idee, das Ereignis, Geschehnis, der Vorfall, auf den eine Reihe von Handlungen oder Interaktionen gerichtet ist." (Strauss et al. 1996, 75) Im Fall der Interviews mit den Mentor/innen ergaben sich zwei Kodierparadigmata und somit zwei Phänomene, die im Folgenden näher erläutert werden.

Praktika (Lehrendenperspektive): Das Phänomen Praktika (Lehrendenperspektive) kann wiederum als Kategorie gedeutet werden, die sich um die ursächlichen Bedingungen entwickelt, wie Erfahrungen eigener Ausbildungszeit durch Begleitung und Bedeutung der Theorie-Praxis-Verschränkung, um die Kontextbedingung Bedingungsfaktoren zu Praktika (als Ist-Bereich) und um 
die Handlungsstrategie Auseinandersetzung mit dem Ausbildungskonzept (als Soll-Bereich). Bedingungen und Strategien werden im Kontext eingesetzt und reagieren auf das Phänomen. Diese Handlungsabfolgen ziehen eine Konsequenz mit sich - im Phänomen Praktika (Lehrendenperspektive) ergibt sich die Konsequenz Verantwortungsbewusstsein für Begleitung.

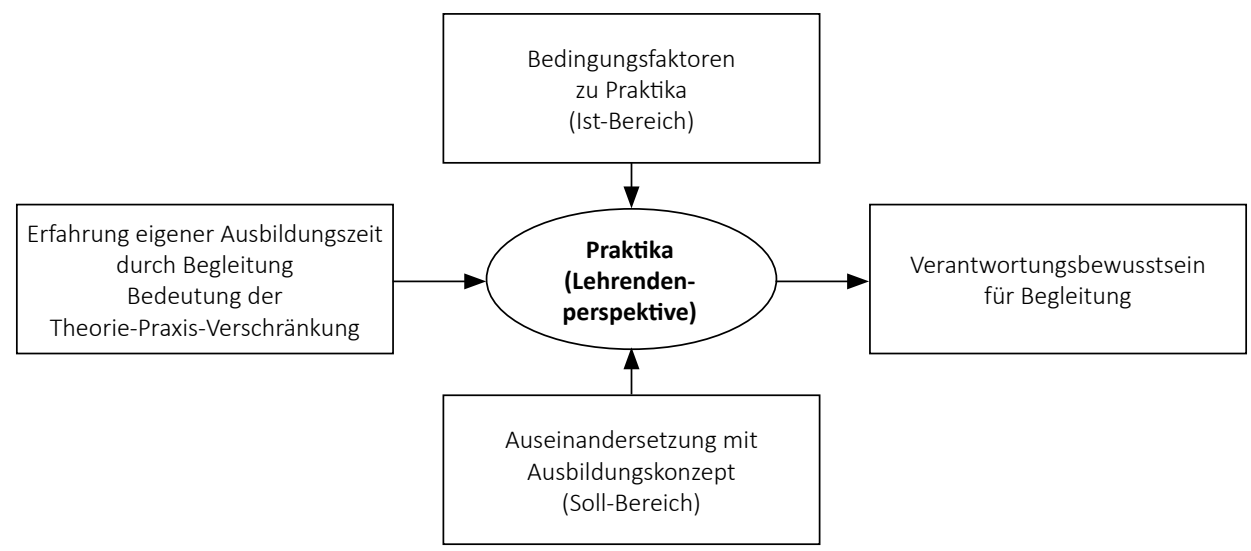

Abb. 50: Kodierparadigma 1 aus Lehrpersoneninterviews

Auf die Frage „Was trägt zum Zustandekommen des Phänomens bei?“ (Strübing 2014, 25) ergeben sich zwei ursächliche Bedingungen: Erfahrungen eigener Ausbildungszeit durch Begleitung und Bedeutung von Theorie-Praxis-Verschränkung. Die ursächlichen Bedingungen besitzen „Attribute und Charakteristika" (Strauss et al. 1996, 45) und lassen sich als dimensionale Ausprägungen beschreiben und führen schließlich zum Phänomen Praktika (Lehrendenperspektive) (Abbildung 50). Abbildung 51 beschreibt die Eigenschaften und dimensionalen Ausprägungen der ursächlichen Bedingungen Erfahrungen eigener Ausbildungszeit durch Begleitung und Bedeutung von Theorie-Praxis-Verschränkung bezogen auf das Phänomen Praktika (Lehrendenperspektive):

Ursächliche Bedingungen

Erfahrungen eigener Ausbildungszeit durch Begleitung:

Bedeutung von Theorie-Praxis-Verschränkung

\section{Eigenschaften}

Persönlichkeitsbeziehung

Prägung

Unterstützung

Seelischer Beistand

Umgang

Lernen von Praxislehrperson

Stellenwert/Bedeutung

Anwendungsmöglichkeit von Theorie

Verzahnung von Theorie und Praxis

Erfahrungen in Praxis machen

Transfermöglichkeiten schaffen

Freiräume zum Ausprobieren

Hilfestellung geben

Reflexionsprozess

\section{Phänomen}

Praktika (Lehrendenperspektive)

\begin{aligned} & \multicolumn{2}{c}{ Dimensionale Ausprägung } \\ & prägend nicht prägend \\ & positiv negativ \\ & fördernd hemmend \\ & aufbauend nicht aufbauend \\ & vertrauensvoll nicht vertrauensvoll \\ & viel wenig \\ & hoch niedrig \\ & zeitnah zeitfern \\ & ermöglichen nicht ermöglichen \\ & gleich später \\ & offen ablehnend \\ & offen geschlossen/eng \\ & bejahend verneinend \\ & intensiv nicht intensiv \end{aligned}

Abb. 51: Eigenschaften und Dimensionen des Phänomens Praktika (Lehrendenperspektive) 
Erfahrungen eigener Ausbildungszeit durch Begleitung enthalten wesentliche Eigenschaften und werden auf einem Kontinuum beschrieben. Als Charakteristika für Erfahrungen eigener Ausbildungszeit durch Begleitung zeigten sich Persönlichkeitsbeziehung zur Lehrperson, Prägungsfaktor, Unterstützung, seelischer Beistand, Umgang im kommunikativen Prozess und Lernen von der Praxislehrperson. Hierbei fällt auf, dass im Rückblick der emotionale und sachliche Prozess in der Begleitung zum Tragen kommt. Die Arbeitsphilosophie in der Begleitung der/ des Ausführenden erhält je nach Ausprägungsgrad eine besondere Bedeutung für den Mentee. Das Selbsterlebte als ehemalige/ $r$ Studierende/ $r$ ist ein wesentlicher Teil in der Berufsbiografie der Mentor/innen und spiegelt sich im jetzigen Begleitungssetting wider.

Die ursächliche Bedingung Bedeutung von Theorie-Praxis-Verschränkung erschließt sich aus den Konzepten: Stellenwert/Bedeutung der Praktika, Anwendungsmöglichkeiten von Theorie, Verzahnung von Theorie und Praxis, Erfahrungen in Praxis machen, Transfermöglichkeiten schaffen, Freiräume zum Ausprobieren, Hilfestellung geben, Reflexionsprozess. Die Möglichkeit zur Transformation von Theorie und Praxis ist den Mentor/innen und war den jetzigen Lehrpersonen schon während der eigenen Ausbildungszeit sehr wichtig. Bei den Eigenschaften der Bedeutung der Theorie-Praxis-Verschränkung besteht wiederum eine enge Verknüpfung zur inhaltlichen Art und Intensität der Begleitung der Praktika. Im Optimalfall sind Mentor/innen bemüht, offene Gestaltungsräume zu schaffen, in denen ein Erproben von Handlungen stattfinden kann. Sie geben Hilfestellung, wenn nötig, und tauchen gemeinsam in einen konstruktiven Reflexionsprozess ein. Je nach dimensionalem Kontinuum der Eigenschaften wird das Verantwortungsbewusstsein für Begleitung als hochgradige oder niederschwellige Handlungsaufgabe für das eigene Tun als Mentor/in und/oder das Unterstützen der Studierenden angesehen.

Im Kodierungsprozess der Lehrpersoneninterviews wurde ein weiteres Kodierparadigma mit Phänomen herausgefiltert.

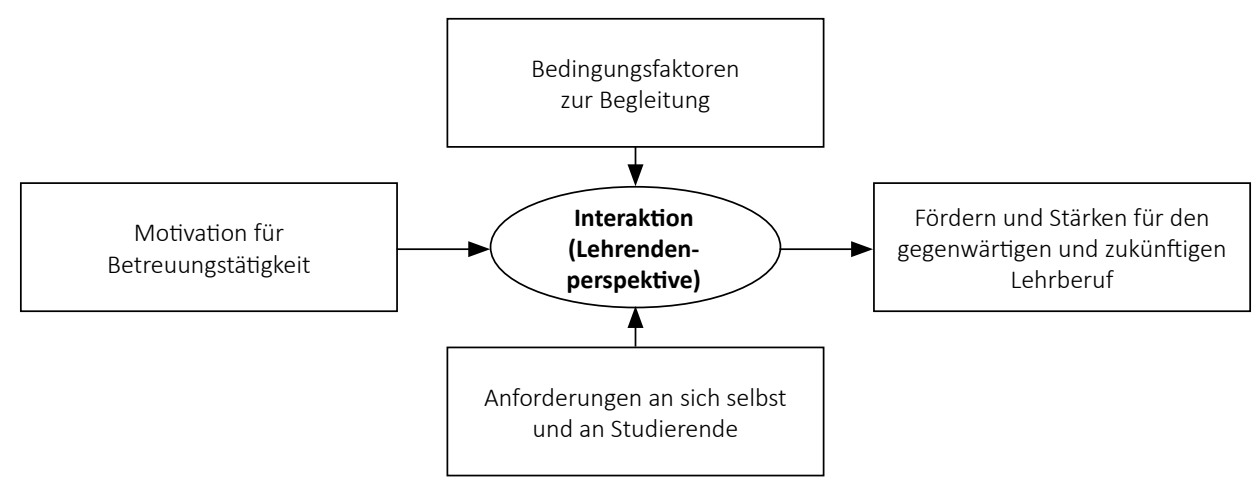

Abb. 52: Kodierparadigma 2 aus Lehrpersoneninterviews

Interaktion (Lehrendenperspektive): Das Phänomen Interaktion (Lehrendenperspektive) geht von der ursächlichen Bedingung der Motivation für Betreuungstätigkeit aus. Als Kontextbedingung für die Tätigkeit werden die Bedingungsfaktoren zur Begleitung genannt. Diese Bedingungsfaktoren umschließen den Anforderungskatalog der schulischen und konzeptionellen Anteile der Ausbildungsinstitutionen. Auf die Frage „Wie gehen die Akteure [sic!] mit dem Phänomen um?“ (Strübing 2014, 25) werden die Handlungsstrategien beleuchtet. Im Fall des Phänomens Interaktion (Lehrendenperspektive) setzen Mentor/innen bewusst Anforderungen an sich selbst und an 
Studierende als Handlungsstrategie und gestalten dadurch die Interaktion. Als Konsequenz ergibt sich ein Fördern und Stärken für den gegenwärtigen und zukünftigen Lehrberuf. Diese Konsequenz kann als Summe der einzelnen empirischen Vorkommnisse in Zusammenhang mit der Wechselbeziehung Mentor/in versus Studierende/r gesehen werden. Mentor/innen erachten es auch als notwendig, sich selbst in der Profession zu fördern und zu stärken, indem sie bewusst in diese Wechselbeziehung gehen und dadurch einen Austausch mit in Ausbildung stehenden Personen haben. Das Phänomen Interaktion (Lehrendenperspektive) ist wesentlich von der ursächlichen Bedingung der Motivation für Betreuungstätigkeit geprägt. In den Ausführungen betonen die Mentor/innen die unterschiedlichen Beweggründe: Ichbezogene Motivation, Student/innenbezogene Motivation oder Systembezogene Motivation. Die unterschiedlichen Motive für die Betreuungstätigkeit sind mit unterschiedlichen Intentionen verbunden. Abbildung 53 beschreibt die Eigenschaften und dimensionalen Ausprägungen der ursächlichen Bedingungen: Motivation für Betreuungstätigkeit zum Phänomen Interaktion (Lehrendenperspektive):

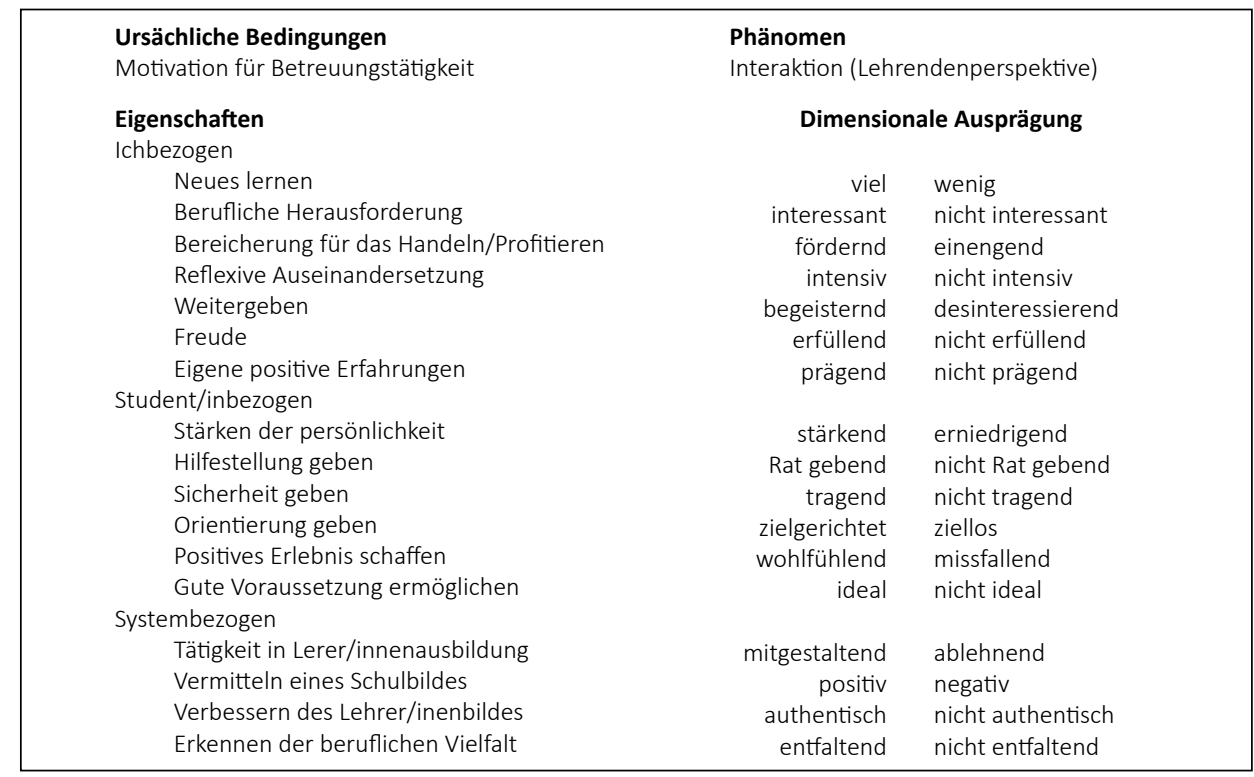

Abb. 53: Eigenschaften und Dimensionen des Phänomens Interaktion (Lehrendenperspektive)

Ichbezogene Motivation hat im Idealfall die eigene professionelle Weiterentwicklung (Neues lernen, berufliche Herausforderung, Bereicherung für das eigene Handeln) und den Wunsch und das Bedürfnis nach Weitergabe der pädagogischen Fähigkeiten (Weitergeben) im Fokus. Weitere wesentliche Aspekte sind die Freude an der Tätigkeit und die eigenen positiven Erfahrungen während der Studienzeit. Die Student/innenbezogene Motivation umschließt Elemente der Wertschätzung und Unterstützung der/dem anderen gegenüber (Stärken der Persönlichkeit, Hilfestellung geben, Sicherheit geben, Orientierung geben, Positives Erlebnis schaffen) und bereitet das schulische Umfeld student/innenfreundlich vor, indem ein möglichst ideales pädagogisches Handeln möglich ist. Als Charakteristika für die Systembezogene Motivation können die Tätigkeit in der Lehrer/innenausbildung, das Vermitteln eines positiven Schulbil- 
des, das Verbessern des Lehrer/innenbildes und das Erkennen der beruflichen Vielfalt genannt werden. Je nach dimensionaler Ausprägung dieser Eigenschaften wird das Fördern und Stärken für den gegenwärtigen und zukünftigen Lehrberuf intensiver oder weniger intensiv betrieben. Nach diesem zweiten theoretischen Kodierungsgang wurde im Untersuchungsverlauf ein weiterer Verdichtungsprozess im Sinne des selektiven Kodierens getätigt.

\section{Selektives Kodieren mit Kernkategorie}

Das Prinzip des selektiven Kodierens besteht in der Integration der herausgearbeiteten Kategorien (Heiser 2018, 234) und mündet in die Entwicklung einer Kernkategorie (Strauss et al. 1996, 94). Im Mittelpunkt steht das zentrale Phänomen, das bei Strauss und Corbin als Fall verstanden wird (Flick 2016, 397). Es beginnt ein Überarbeitungsprozess der Kodierungen - es vollzieht sich ein analytischer Perspektivenwechsel der bisherigen Kodierung auf eine einheitliche Ebene (Strübing 2014, 18) und mündet in die Bildung einer Kernkategorie (Abbildung 54).

Zusammenarbeit: Das zentrale Phänomen bzw. der Fall nach Integration aller Konzepte und Kategorien in Causa Lehrpersoneninterviews zum Thema Praxis und Interaktion im schulpraktischen Mentoring heißt Zusammenarbeit. Mentor/innen sind bereit, in eine Wechselbeziehung mit Studierenden der Ausbildung und der Ausbildungsinstitution zu treten und das eigene und das studentische Handeln reflexiv auf unterschiedlichen Ebenen zu betrachten. Hierbei kann dieses begleitende Handeln als Herausforderung oder Bereicherung für sich und den Mentee gesehen werden. In einem späteren Abschnitt werden zu diesen ursächlichen Bedingungen Eigenschaften und dimensionale Ausprägungen dokumentiert. Beeinflussend für die Zusammenarbeit gestalten sich personale Ausbildungs- und Berufserfahrungen sowie inhaltliche, organisatorische und strukturelle Bedingungsfaktoren. Die Ausprägung und Bearbeitung dieser Faktoren sind für das Gelingen oder Misslingen der Gestaltung des Interaktionsprozesses entscheidend. Als optimale Handlungsstrategie wird ein Reflexionsprozess mit dem Ausbildungskonzept und der eigenen Lehrtätigkeit sowie mit Themen der Interaktion und Kommunikation angesehen. Gerade diese persönliche Auseinandersetzung mündet im Optimalfall in die Konsequenz der Bereitschaft zur mentoriellen Betreuung und Weiterentwicklung der eigenen Profession bzw. Weiterentwicklung in der Profession.

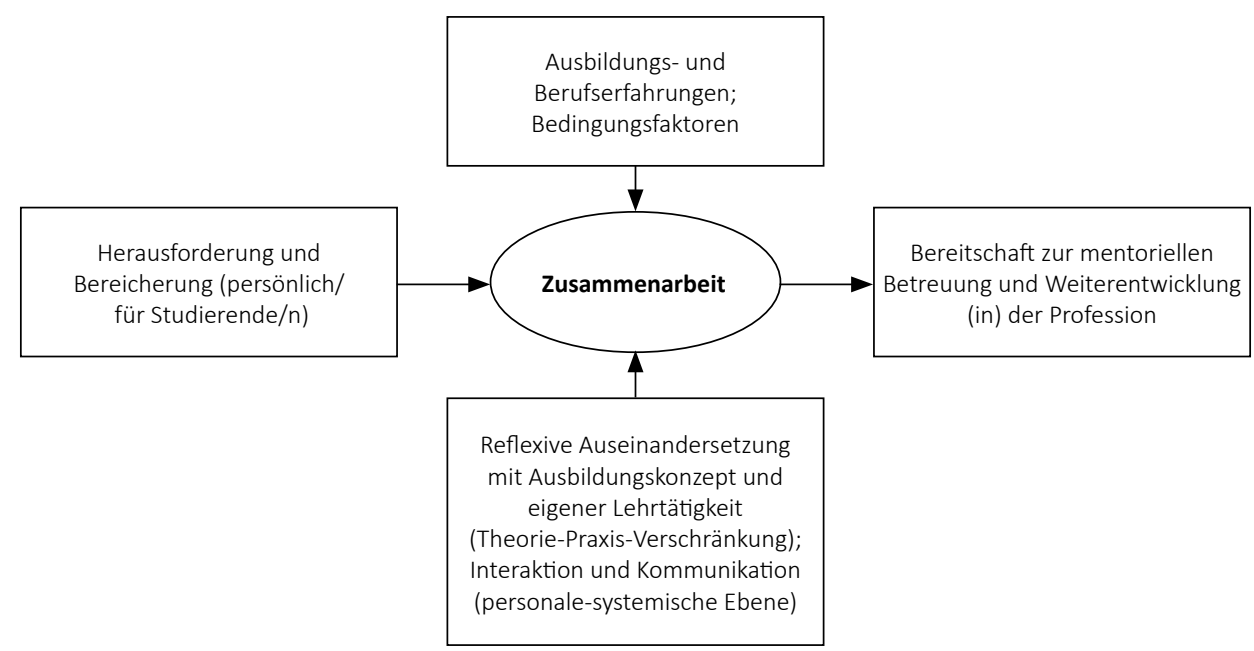

Abb. 54: Kernkategorie Mentor/innen 
Die Fragestellungen bei den ursächlichen Bedingungen heißen: Warum ist die Zusammenarbeit für Mentor/innen aus deren Sicht eine Herausforderung und eine Bereicherung? Die Gründe gehen aus den konzeptuellen Repräsentanten der Kodierung hervor. Hierbei werden persönliche Herausforderungen und persönlich bereichernde Aufgabenbereiche der Mentor/ innen genannt. Abbildung 55 beschreibt die Eigenschaften und dimensionalen Ausprägungen der ursächlichen Bedingungen bezogen auf die/den Mentor/in:

\section{Ursächliche Bedingungen \\ Herausforderung und Bereicherung \\ (persönlich/für Studierende/n)}

Eigenschaften (Persönlich)
Pädagog/innenausbildung
Zusammenarbeit mit Ausbildungsinstitution
Berufliche Aufgabe
Tätigkeitsbereich
Eigeninteresse
Art und Dauer der Praktika
Stärken der Persönlichkeit
Kommunikationsebene
Verantwortung
Freiräume
Reflexionsphasen
Folgewirkungen
Problemsituationen
Konfliktmanagement
Art und Dauer des Einsatzes

\section{Phänomen}

Zusammenarbeit

\begin{aligned} & \multicolumn{2}{c}{ Dimensionale Ausprägung } \\ & dynamisch statisch \\ & gegeben nicht vorhanden \\ & einbringend ablehnend \\ & freudvoll missfallend \\ & gewinnbringend nicht lernend \\ & beziehungsfördernd nicht \\ & forcierend hemmend \\ & komplementär hierarchisch \\ & einlassend ablehnend \\ & gebend bewahrend \\ & intensiv oberflächlich \\ & beteiligt unbeteiligt \\ & lösbar belastend \\ & erfahren unerfahren \\ & intensiv oberflächlich \end{aligned}

Abb. 55: Eigenschaften (Persönlich) und Dimensionen zum Phänomen Zusammenabeit in der Kernkategorie

Als Charakteristika zeigten für Mentor/innen systemisch-strukturelle (Pädagog/innenausbildung, Zusammenarbeit mit Ausbildungsinstitution, Art und Dauer der Praktika), motivationale (Berufliche Aufgabe, Eigeninteresse) und intrapersonale (Verantwortung, Freiräume geben, Reflexionsphasen, Folgewirkungen, Problemsituationen, Konfliktmanagement, Art und Dauer des Einsatzes) sowie interpersonale (Stärken der Persönlichkeit, Kommunikationsebene, Freiräume geben, Folgewirkungen) Gründe.

Mentor/innen erkennen auch, dass die Zusammenarbeit für Studierende eine Herausforderung oder eine Bereicherung ist. Die nachstehende Fragestellung wird mit der Auflistung der Eigenschaften und dimensionalen Ausprägungen (Abbildung 56) beantwortet. Sie lautet: Warum ist die Zusammenarbeit für die/den Studierende/n aus Sicht der Mentor/innen eine Herausforderung oder Bereicherung?

Ob die Zusammenarbeit eine Herausforderung und/oder eine Bereicherung ist, sehen Mentor/ innen durch das Gelingen oder Misslingen der Identifikation der Studierenden mit den Feldern Professionalität und Schule. Es ergeben sich Wenn-Dann-Beziehungen. Wenn Studierenden der Perspektivenwechsel gelingt - das Rollenverständnis, das Kennenlernen des Schulalltags, der Umgang mit Schülerinnen und Schülern, das Umsetzen des Fachwissens, das Auseinandersetzen mit Fachdidaktiken, das Einsetzen der Methodenvielfalt, das Teamteaching -, dann ist die Zusammenarbeit eine Bereicherung. Die Konzepte Sammeln von Erfahrung, Berufswahl und Lernen in der Praxis sind abhängig von der Einstellung der Studierenden und prägen somit die Zusammenarbeit. 
Ursächliche Bedingungen

Herausforderung und Bereicherung

(persönlich/für Studierende/n)

Eigenschaften (Persönlich)
Perspektivenwechsel
Rollenverständnis
Kennenlernen des Schualltags
Umgang mit Schüler/innen
Umsetzen des Fachwissens
Auseinandersetzen mit Fachdidaktik
Methodenvielfalt
Teamteaching
Sammeln von Erfahrung
Art und Dauer der praktika
Berufswahl
Veränderung der Schulsituation
Lernen in der Praxis
Seelische befindlichkeit
Kritikfähigkeit
Lösungsansätze bei problemen
Entwicklungspotential

Phänomen

Zusammenarbeit

$\begin{aligned} & \text { Dimensionale Ausprägung } \\ & \text { gelingend } \text { nicht gelingend } \\ & \text { vorhanden } \text { entstehend } \\ & \text { gewollt } \text { ablehnend } \\ & \text { wertschätzend } \text { missfallend } \\ & \text { schwer } \text { leicht } \\ & \text { schüler/innenorientiert } \text { nicht } \\ & \text { wissend } \text { nicht wissend } \\ & \text { bejahend } \text { ablehnend } \\ & \text { eigenständig } \text { nicht eigenständig } \\ & \text { intensiv } \text { nicht intensiv } \\ & \text { klar gegeben } \text { zweifelnd } \\ & \text { schnell } \text { langsam } \\ & \text { intensiv } \text { nicht intensiv } \\ & \text { ausgeglichen } \text { angespannt/nervös } \\ & \text { annehmend } \text { ablehnend } \\ & \text { vorhanden } \text { nicht vorhanden } \\ & \text { vorhanden } \text { weckend/schlafend }\end{aligned}$

Abb. 56: Eigenschaften (Für Studierende/n) und Dimensionen zum Phänomen Zusammenarbeit in der Kernkategorie

Lehrpersonen wissen in der Interaktion, dass die Praxiszeit eine arbeits- und zeitintensive Ausbildungsphase für Studierende ist. Sie weisen auf die Veränderungen der Schulsituationen hin und sehen es als einen herausfordernden und/oder bereichernden Aspekt.

Lehrpersonen nehmen Art und Dauer der Praktika als einen weiteren Faktor für Herausforderung und Bereicherung an. Persönlichkeitsstrukturen bei Studierenden, wie seelische Befindlichkeit, Kritikfähigkeit und die grundsätzliche Bereitschaft des Findens von Lösungsansätzen bei Problemen oder das Vorhandensein des Entwicklungspotentials nehmen Einfluss auf die Interaktion.

Nach den Ausführungen der Lehrpersoneninterviews folgt die strukturierte Ergebnisdarstellung und Analyse aus den Studierendeninterviews.

\subsection{Stichprobe Studierende}

In Anlehnung an die Interviews mit den Mentor/innen $(n=12)$ wurde auch die Sichtweise der Studierenden $(\mathrm{n}=12)$ zu Praktika und Begleitung für die rekonstruierende Untersuchung der vorliegenden Arbeit als wesentlich angesehen.

„Um Interviewpartner (sic!) auswählen zu können, muss man sich zunächst vergegenwärtigen, wer über die für die angestrebte Rekonstruktion notwendigen Informationen verfügt. Alle notwendigen Informationen zu beschaffen bedeutet meist, mehrere Akteure (sic!) zu befragen, die aufgrund ihrer spezifischen Stellung in dem zu rekonstruierenden Prozess jeweils über andere Informationen verfügen. (...) Deshalb ist es ratsam, Informationen über einen Sachverhalt von mehreren Interviewpartnern (sic!) einzuholen, also zu triangulieren." (Gläser et al. 2010, 117) 
Es erfolgte eine Befragung von 12 Studierenden, wobei alle Studierenden am Ende ihres Studiums waren und somit Erfahrungswerte in den Aussagen aus der Zeit der Praktika während des Studiums einbringen konnten. Insgesamt wurden sechs mehr oder weniger zufällig ausgewählte Studierende mit nahendem Abschluss des Universitätsstudiums und sechs persönlich ausgewählte Studierende mit nahendem Abschluss an der Pädagogischen Hochschule interviewt. Studierende bezeichnen in den Interviews ihre Mentor/innen als Praxislehrpersonen - die geläufige und offizielle Bezeichnung im Interviewzeitraum.

\section{Offenes Kodieren}

Beim offenen Kodieren der Studierendeninterviews ergaben sich 780 Kodierungen. Folgende Kodes bzw. Konzepte haben Mehrfachnennungen (Anzahl in Klammer): konstruktives Feedback (26), Erfahrungsbericht (23), Bezug zu Klasse/Schülerinnen und Schülern (16), Theorie-Praxis-Bezug (15), Lehrer/innenerfahrung als Lerngewinn (14), Tagespraktika (14), längere Praktika (14), Stellenwert (14), ausprobieren können (10), Beziehung zur Praxislehrperson (10).

\section{Axiales Kodieren}

Nach dem Prozess des offenen Kodierens und der Gewinnung von Kodes, Konzepten und Subkategorien aus den Studierendeninterviews setzte der Prozess des axialen Kodierens ein. Ähnlich wie bei den Lehrpersoneninterviews ergab sich im Laufe der Kodierung und Konzeptentwicklung eine dreiteilige Kategorienbildung (Abbildung 57) der insgesamt 780 Kodierungen. Studierende richteten im Gespräch ihren Blick auf den strukturellen Rahmen, auf die Erfahrungen im Handlungsfeld Praktika und Begleitung und auf die Erwartungen in den Praktika.

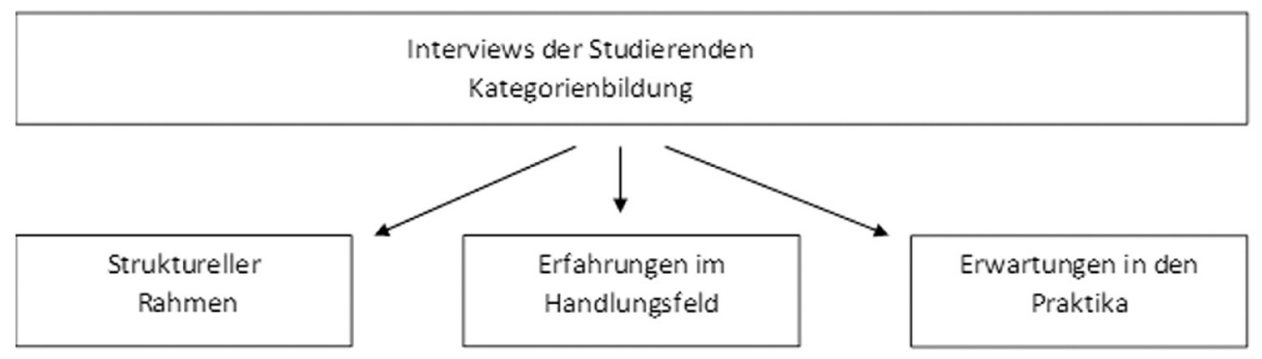

Abb. 57: Binnendifferenzierung bei Studierendeninterviews $(n=12)$

Bei der Generierung ergaben sich innerhalb dieser drei Kategorien themenbezogene Subkategorien, die in der folgenden Abbildung (Abbildung 58) mit Hilfe des Code-Matrix-Browsers dargestellt werden. Die Größe der Knoten bezieht sich auf die relative Häufigkeit der Kodierungen. Die Summe gibt die Anzahl der Kodierungen an. 


\begin{tabular}{|c|c|c|c|}
\hline \multicolumn{2}{|c|}{ Codesystem } & \multirow[t]{2}{*}{ Studierende } & \multirow{2}{*}{$\begin{array}{c}\text { SUMME } \\
0\end{array}$} \\
\hline$\checkmark$ & Struktureller Rahmen & & \\
\hline & $>$ Dauer der Praktika & $\square$ & 70 \\
\hline & $>$ Kennenlernen des Schulalltags & - & 25 \\
\hline & $>$ Lehrer/innenpersönlichkeit & $\cdot$ & 19 \\
\hline & $>$ Sinnhaftigkeit & $\cdot$ & 15 \\
\hline & $>$ Inhaltliche Begleitung & $\cdot$ & 15 \\
\hline & $>$ Vorschläge zu Praxiskonzept & - & 9 \\
\hline$\checkmark$ & Erfahrungen & & 0 \\
\hline & $>$ Ideenfindung für Praxislehrperson & $\square$ & 67 \\
\hline & $>$ Bedeutung von Praktika & 口 & 56 \\
\hline & $>$ Kritischer Erfahrungsbericht & $\mathbf{\square}$ & 54 \\
\hline & $>$ Lernzuwachs für Studierende & - & 36 \\
\hline & $>$ Unterstützung durch Praxislehrperson & $=$ & 36 \\
\hline & $>$ Beziehungsstruktur zur Praxislehrperson & - & 35 \\
\hline & $>$ Zufriedenstellung nach Praktika & - & 21 \\
\hline & $>$ Positiver Erfahrungsbericht zu Praktika & $\cdot$ & 21 \\
\hline & $>$ Betreuungstätigkeit: Grenzgang für Praxislehrperson & $\cdot$ & 15 \\
\hline$\checkmark$ & Erwartungen & & 0 \\
\hline & $>$ Beziehung zur Praxislehrperson & & 105 \\
\hline & $>$ Professionalität & 7 & 88 \\
\hline & $>$ Konstruktives Feedback & 口 & 45 \\
\hline & $>$ Theorie-Praxis Bezug & 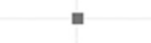 & 35 \\
\hline & $>$ Bedeutung von Praktika & $\cdot$ & 13 \\
\hline & $\sum$ SUMME & 780 & 780 \\
\hline
\end{tabular}

Abb. 58: Kategorien und Subkategorien der Kodes aus Studierendeninterviews $(n=12)$

Im Folgenden werden die Inhalte der Kategorien beschrieben. Die nachstehenden Abbildungen veranschaulichen anhand des Gewichtsfilters im Code-Matrix-Browser von MAXQDA die jeweiligen Kodierungen pro Zeile und Spalte der Interviewpartner/in.

Struktureller

Rahmen

Die Kategorie Struktureller Rahmen (152 Kodierungen) enthält Subkategorien, die sich auf inhaltliche und organisatorische Aspekte der Bedeutung und Etablierung von Praktika beziehen. Es wird darauf hingewiesen, dass Praxislehrpersonen eine wichtige Rolle spielen und eine professionelle inhaltliche Begleitung im schulischen Anteil der Ausbildungsphase gegeben sein muss. Studierende erwähnten im Interview auch einige Vorschläge zu Praxiskonzepten. Somit ergeben sich unter der Kategorie Struktureller Rahmen folgende Subkategorien: Dauer von Praktika, Sinnhaftigkeit, Kennenlernen des Schulalltags, Bedeutung der Lehrer/innenpersönlichkeit und Inhaltliche Begleitung zu den Praktika sowie Vorschläge zu den Praxiskonzepten. Die Subkategorie Dauer der Praktika (70) weist die häufigsten Kodes auf. Die Subkategorie Vorschläge zu Praxiskonzept (9) hingegen hat am wenigsten Kodierungen. Zu betonen ist auch, dass Studierende neben dem Wunsch und der ersichtlichen Notwendigkeit des Kennenlernens des Schulalltages (25) während der Ausbildung auch erwähnen, dass der Auswahl der Lehrer/innenpersönlichkeit (19) für die Begleitung innerhalb der Struktur eine hohe Bedeutung zukommt. 
Anbei die Aufschlüsselung zur Kategorie Struktureller Rahmen (Abbildung 59):

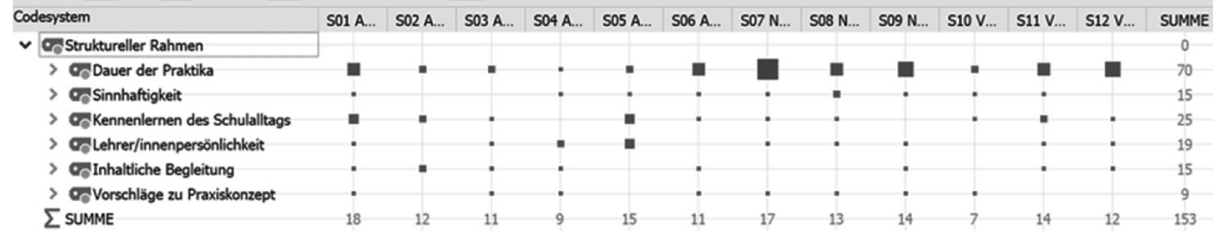

Abb. 59: Kategorie Struktureller Rahmen bei Studierendenkodes

Auffallend bei der Kategorie Struktureller Rahmen (153 Kodierungen) ist, dass die Subkategorie Dauer der Praktika (70) die höchste Zahl aller Subkategorien hat und dass sich vor allem fünf von sechs Studierenden (S07 N, S08 N, S09 N, S11 V, S12 V) mit Lehramtsabschluss Volksschule (V) und Neue Mittelschule (N) verstärkt dazu äußern. Bei Studierenden der Universität (A) sind es nur zwei von sechs (S02 A, S06 A). In den Aussagen geht es aus Sicht der Studierenden darum, dass es regelmäßige Praxiszeiten während des Studiums geben soll, am besten in jedem Semester und dass Studierende auch längere Praxisphasen an der Schule bevorzugen also sogenannte Blockpraktikumsformen. Die neun Vorschläge zu Praxiskonzepten (9) sind als relative Häufigkeit bescheiden. Die Vorschläge konzentrieren sich auf strukturell organisatorische Rahmenbedingungen, indem Studierende den Praktikumsplatz sich zunächst einmal anschauen möchten, ob er für sie passend ist bzw. den Studierenden die Möglichkeit einzuräumen, den Platz auch während der Praktikumszeiten wechseln zu können, wenn es Schwierigkeiten gibt. Die Interviewpartner/innen S04 und S10 äußern sich im Vergleich zu den anderen Interviewpartner/innen zur Kategorie Struktureller Rahmen nur sehr bedingt (9 bzw. 7 Kodierungen), die Interviewpartner/innen S01 und S07 mit 18 bzw. 17 Kodierungen überdurchschnittlich oft.

Erfahrungen im Handlungsfeld

Die Kategorie Erfahrungen im Handlungsfeld (341 Kodierungen) dokumentiert aus Sicht der Studierenden Erlebtes zu den Praktika und zur Begleitung durch Mentor/innen. In ihren Aussagen richten sie den Blick auf Intrapersonales wie Lernzuwachs für Studierende (36) und Zufriedenstellung nach Praktika (21), auf Interpersonales wie Ideenfindung für Praxislehrperson (67), Beziehungsstruktur zur Praxislehrperson (35), Unterstützung durch Praxislehrperson (36), Betreuungstätigkeit: ein Grenzgang für Praxislehrperson (15) sowie Systemisches wie Bedeutung von Praktika (56), Positiver (21) und Kritischer Erfahrungsbericht (54) zu Praktika und Praxislehrpersonen. Die thematischen Aspekte beziehen sich vor allem auf die strukturelle Ebene der Praktika sowie auf die inter- und intrapersonale Ebene im Verlauf der Praktika. Die Subkategorie Ideenfindung für Praxislehrperson (67) hat die meisten Kodes. Studierende sehen in der Tätigkeitsausübung einen Mehrgewinn für Praxislehrpersonen, erwähnen aber auch die Herausforderungen für Praxislehrpersonen - zusammengefasst in der Subkategorie Betreuungstätigkeit: Grenzgang für Praxislehrpersonen (15). Die zweithäufigste Anzahl der Kodierungen hat die Subkategorie Bedeutung der Praktika (56). Einen hohen Anteil an Aussagen nehmen auch die kritischen Stellungnahmen zu Erfahrungen zu den Praktika und zu Praxislehrpersonen (54) ein. Studierende dokumentieren vor allem ein Desinteresse in der inhaltlichen professionellen Begleitung von Praxislehrpersonen. 
Die Kategorie Erfahrungen zeigt folgende Verteilung (Abbildung 60):

\begin{tabular}{|c|c|c|c|c|c|c|c|c|c|c|c|c|c|}
\hline Codesystem & S01 A... & SO2A... & S03 A... & S04 A... & S05 A... & S06 A... & SO7 N... & S08 N... & SO9 N... & $\mathrm{S} 10 \mathrm{~V} . .$. & S11 V... & S12 V... & \multirow{2}{*}{$\begin{array}{c}\text { SUMME } \\
0\end{array}$} \\
\hline$\vee$ ब्ठ Erfahrungen & & & & & & & & & & & & & \\
\hline$>\sigma_{\sigma}$ Bedeutung von Praktika & a & : & $\mathbf{\square}$ & - & a & a & - & a & 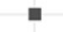 & a & - & a & 56 \\
\hline$>$ ФंLernzuwachs für Studierende & $=$ & $\square$ & & - & - & - & & - & 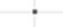 & & - & $=$ & 36 \\
\hline$>\Phi_{\text {ZZufriedenstellung nach Praktika }}$ & - & & & & - & & & & & - & 口 & - & 21 \\
\hline$>\mathscr{\sigma}$ Ideenfindung für Praxislehrperson & $=$ & $\mathbf{\square}$ & $=$ & $=$ & - & a & & $\mathbf{\square}$ & L & $=$ & $=$ & $=$ & 67 \\
\hline$>\mathscr{C}_{\text {Beziehungsstruktur zur Praxislehrp }}$ & - & - & - & - & - & 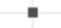 & & - & $\dot{\theta}$ & $\mathbf{a}$ & - & - & 35 \\
\hline$>\Phi_{\sigma}$ Unterstützung durch Praxislehrpers & $=$ & & & $=$ & - & - & & - & & - & - & a & 36 \\
\hline$>\mathscr{C}_{\text {Betreuungstätigkeit ein Grenzgang }}$ & - & & & & - & & - & - & - & - & - & - & 15 \\
\hline$>\mathscr{\sigma}_{\boldsymbol{\sigma}}$ (Positiver) Erfahrungsbericht zu $\mathrm{Pr}$ & $\cdot$ & & i & $=$ & & - & - & $\dot{2}$ & - & • & - & $=$ & 21 \\
\hline$>\Phi_{\sigma}$ Kritischer Erfahrungsbericht über $\mathrm{F}$ & $\mathbf{a}$ & $\mathbf{a}$ & 口 & $\square$ & $\mathbf{a}$ & - & - & 口 & - & - & - & & 54 \\
\hline$\sum$ SUMME & 28 & 30 & 22 & 28 & 27 & 25 & 45 & 26 & 34 & 24 & 29 & 23 & 341 \\
\hline
\end{tabular}

Abb. 60: Kategorie Erfahrungen bei Studierendenkodes

Die Kategorie Erfahrungen (341 Kodierungen) weist neun Subkategorien auf. Hierbei geben Studierende Erlebtes wieder. Alle interviewten Studierenden äußern sich zur Ideenfindung für Praxislehrpersonen (67). Darin behaupten sie, dass Praxislehrpersonen einen großen Nutzen aus den methodisch-didaktischen Umsetzungsprogrammen der Studierenden und vor allem durch den Einsatz der digitalen Medien ziehen können. Auffallend ist hierbei, dass der Gewichtsfilter bei Studierenden mit Lehramt Neue Mittelschule (N) in diesem Bereich eine höhere Anzeige hat als bei Studierenden der Universität (A) oder mit Lehramt Volksschule (V). Ebenso treffen alle Befragten Aussagen zur Bedeutung der Praktika (56) und zur Beziehungsstruktur zur Praxislehrperson (35). Kritische Anmerkungen (54) zu den Abläufen der Praktika bzw. zur Praxislehrperson treffen vor allem Studierende der Universität. Studierende mit Lehramt Volksschule (V) oder Neue Mittelschule (N) erwähnen in der Subkategorie Betreuungstätigkeit: Grenzgang für Praxislehrperson (15) auch, dass sie glauben, dass die Betreuungstätigkeit stressig ist, dass es eine Überwindung braucht, seine Klasse jemandem anderen zu überlassen und dass für die Betreuung Erfahrung notwendig ist, damit man in den verschiedenen Situationen professionell reagieren kann. S07 hat mit Abstand viele Kodierungen (45) zur Kategorie Erfahrung.

Erwartungen

in den Praktika

Unter der Kategorie Erwartungen (286 Kodierungen) werden in den Kodes Wünsche, Vorschläge und Impulse der Studierenden zu Praktika und Begleitung angeordnet. Studierenden ist es besonders wichtig, eine Beziehung zur Praxislehrperson (105) aufzubauen. Sie erwarten sich von der Lehrperson eine Professionalität (88) in der Begleitung. Besonders am Herzen liegen Studierenden auch das konstruktive Feedback (45), um sich weiterentwickeln zu können, sowie die Theorie-Praxis-Verschränkung (35). Am wenigsten Kodierungen in dieser Kategorie nimmt die Subkategorie Bedeutung der Praktika (13) ein. Die Kategorie Erwartungen weist fünf Subkategorien mit folgender Verteilung auf (Abbildung 61):

\begin{tabular}{|c|c|c|c|c|c|c|c|c|c|c|c|c|c|}
\hline Codesystem & S01 A... & S02 A ... & S03 A. & S04 A & S05 A ... & S06 A... & $\mathrm{S} 07 \mathrm{~N}$... & S08 N. & SO9 N... & $\mathrm{S} 10 \mathrm{~V}$ & $\mathrm{~S} 11 \mathrm{~V} \ldots$ & $\mathrm{S} 12 \mathrm{~V}$ & SUMME \\
\hline$\checkmark \odot$ Erwartungen & & & & & & & & & & & & & 0 \\
\hline > Beziehung zur Praxislehrperson & 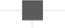 & - & $\mathbf{a}$ & $\square$ & - & $\mathbf{\square}$ & - & $\square$ & $\square$ & $\mathbf{\square}$ & 口 & $\mathbf{\square}$ & 105 \\
\hline$>$ CProfessionalität & $\mathbf{\square}$ & $\mathbf{a}$ & $\mathbf{\square}$ & $\mathbf{a}$ & $\mathbf{\square}$ & - & - & - & - & - & - & $\mathbf{\square}$ & 88 \\
\hline$>\sigma^{-}$Theorie-Praxis Bezug & & - & - & $\square$ & - & $\cdot$ & - & - & & - & - & $\cdot$ & 35 \\
\hline > $ণ$ Bedeutung von Praktika & $\cdot$ & & & - & $\cdot$ & - & & & & & $=$ & - & 13 \\
\hline$\sum$ SUMME & 27 & 33 & 25 & 36 & 26 & 17 & 20 & 22 & 17 & 15 & 23 & 25 & 286 \\
\hline
\end{tabular}

Abb. 61: Kategorie Erwartungen bei Studierendenkodes 
Die Subkategorie Beziehung zur Praxislehrperson (105) hat die meisten Kodierungen, die wenigsten die Subkategorie Bedeutung von Praktika (13). Studierende haben eine klare Vorstellung darüber, wie die Beziehung zur Praxislehrperson auf zwischenmenschlicher und kommunikativer Ebene auszusehen hat. Studierende haben auch eine Erwartungshaltung zur Professionalität (88) der Lehrperson. Es sind Kodierungen und Konzepte, die Aussagen zur Rolle und Aufgabe der Praxislehrperson aus Sicht der Studierenden enthalten. Besonders wertvoll erachten Studierende auch, wenn sie ein konstruktives Feedback (45) von der Praxislehrperson entgegennehmen. Auffallend ist auch, dass Themen zur Begleitung und zum Agieren im Mentoringprozess (Beziehung zur Praxislehrperson, Professionalität, Konstruktives Feedback) häufiger als Erwartungen angesprochen werden als Themen zur Ausbildung (Theorie-Praxis-Bezug, Bedeutung von Praktika). Bei den Interviewpartner/innen S02 und S04 zeigen sich innerhalb der Kategorie Erwartungen die meisten Kodierungen, bei den Interviewpartner/innen S06, S09 und S10 die wenigsten.

In der folgenden Abbildung (Abbildung 62) werden die Kodierungsverteilungen pro Interviewpartner/in $(\mathrm{n}=12)$ nach dem Durchschnittswert jeder Kategorie veranschaulicht. Das Schaudiagramm gibt Aufschluss über die Quantifizierung der Kodierungen der einzelnen Kategorien bei jeder/jedem Interviewpartner/in, gemessen am Durchschnittswert der jeweiligen Kategorie.

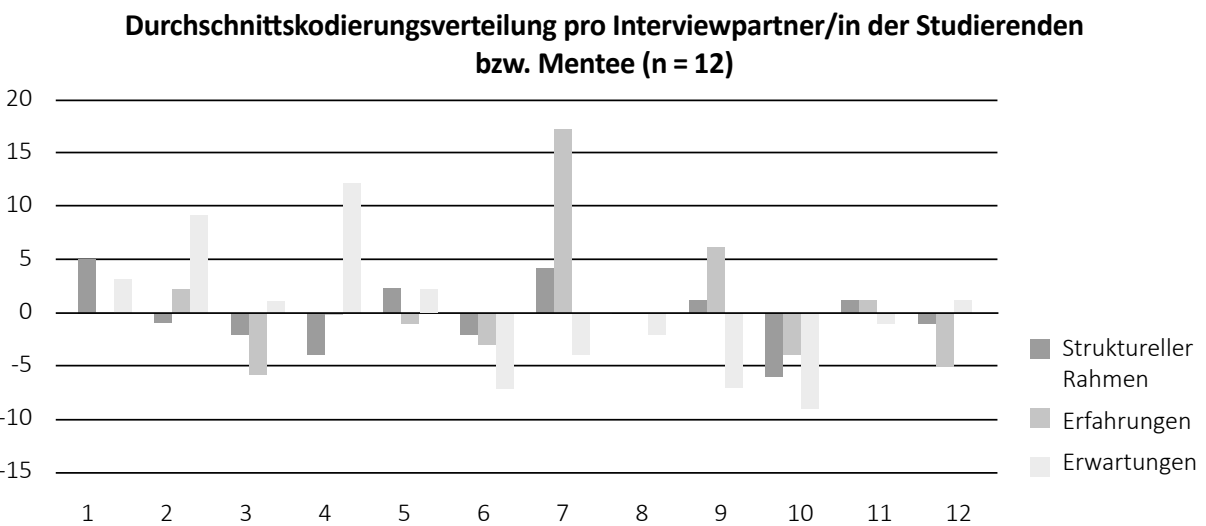

Abb. 62: Durchschnittskodierverteilungen pro Interviewpartner/in der Studierenden bzw. Mentee $(\mathrm{n}=12)$

Alle Interviewpartner/innen äußern sich mit Aussagen zu den Kategorien Struktureller Rahmen, Erfahrungen und Erwartungen. S01 und S04 erreichen bei der Kategorie Erfahrungen genau den Durchschnittswert 28 und S08 den Durchschnittswert 13 bei der Kategorie Struktureller Rahmen, daher ergeben sich in diesem Fall keine Säulendarstellungen.

Der Durchschnittswert pro Interviewpartner/in der Kategorie Struktureller Rahmen gemessen an der Summe der 153 Kodierungen liegt bei 12,75 Kodierungen (aufgerundet auf 13 Kodierungen), bei der Kategorie Erfahrungen bei 28,4 (abgerundet auf 28 Kodierungen) und bei der Kategorie Erwartungen bei 23,8 Kodierungen (aufgerundet auf 24 Kodierungen). Auffallend ist, dass sich fünf von sechs Studierenden (S01 bis S06) der Universität überdurchschnittlich häufig zu den Erwartungen äußern, während bei den Studierenden mit Lehramt zum Pflichtschulbereich die Säulen bei fünf von sechs unter dem Durchschnitt liegen. Herausragend ist bei S07 die hohe Abweichung vom Durchschnittswert bei der Kategorie Erfahrungen. SO7 hat in der Begleitung negative Erfahrungen gemacht und im Interview den Unmut dazu geäußert. 
Im Folgenden werden die zwei Kodierparadigmata vorgestellt und diskutiert.

\section{Entwerfen von Kodierparadigmata}

Konzepte und Kategorien sammeln sich im Untersuchungsprozess an und im axialen Kodierungsprozess wird versucht, eine Integration zu finden. Fragestellungen sollen helfen, eine theoretische Sättigung zu erreichen. Bei den Kodierungen der Studierendeninterviews wurden die gleichen Fragen wie bei den Kodierungen der Lehrpersoneninterviews gestellt: Wann tritt das Ergebnis auf? Welche Ereignisse sind bzw. welches Ergebnis ist markant? Welche Bedingungen sind maßgeblich? Was sind die Vorbedingungen? Wie gehen Studierende mit diesem Ergebnis um? Worauf beziehen sich Studierende in ihren Erklärungen? Was sind die Konsequenzen dieses Ergebnisses? (in Anlehnung an Strübing 2014, 25). Abbildung 63 verdeutlicht die Zusammenhänge. Aus den Interviews mit den Studierenden und nach dem Kodierungsprozess ergeben sich zwei Kodierparadigmata mit unterschiedlichen Phänomenen.

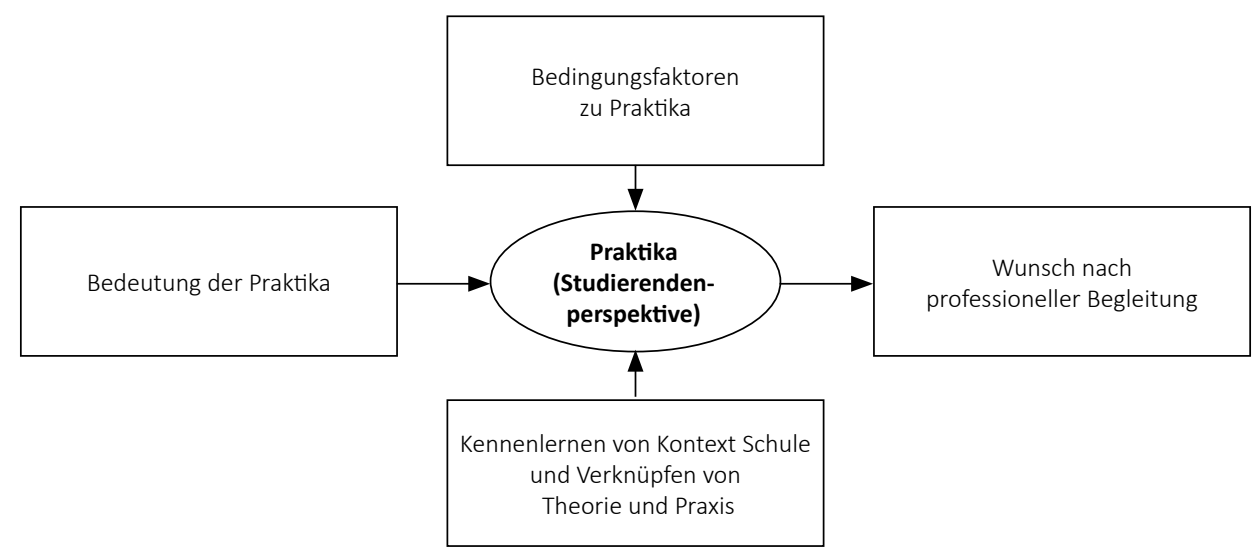

Abb. 63: Kodierparadigma 1 aus Studierendeninterviews

Praktika (Studierendenperspektive): Das erste Kodierparadigma mit dem Phänomen Praktika (Studierendenperspektive) hat als ursächliche Bedingung Bedeutung der Praktika. Bedingungsfaktoren zu Praktika sind im Rahmen der Kontextbedingungen und intervenierenden Bedingungen wesentlich, die die formalen und organisatorischen Kriterien von Struktur und Ablauf der Praktika vorgeben. Die Handlungsstrategie zeichnet das Kennenlernen im Kontext Schule und das Verknüpfen von Theorie und Praxis. Die Fragenstellungen: Wie wollen Studierende den Schulalltag kennenlernen? Wie gehen Studierende an Praktika heran? Welche Erwartungen haben Studierende in den Praktika? Wie gelingt Studierenden die Verknüpfung von Theorie und Praxis? u.ä. führten zur Konsequenz eines Wunsches nach professioneller Begleitung. Abbildung 64 beschreibt die Eigenschaften und dimensionalen Ausprägungen der ursächlichen Bedingung Bedeutung der Praktika zum Phänomen Praktika (Studierendenperspektive). Die ursächliche Bedingung Bedeutung der Praktika umfasst die Konzepte Sinnhaftigkeit/Stellenwert/Nutzen der Praktika, Berufswahlentscheidung, Theorie-Praxis-Bezug, Gewinnen von Einblick, Erfahren der Lehrer/innenperspektive, Zeit zum Üben, persönlicher Lerngewinn, persönlicher Lernfortschritt, Bezug zu Schülerinnen und Schülern, Gewinnen von Selbstvertrauen, Ausprobieren der Lehrer/innenpersönlichkeit, Begleitung und Unterstützung durch 
Praxislehrperson. Studierende erleben Praktika als sehr bedeutend, wenn sie einerseits in einen Lernprozess einsteigen und Schulalltag erleben können und andererseits, wenn die Begleitung durch die Praxislehrperson als unterstützend erfahren wird.

\begin{tabular}{|lcr|}
\hline Ursächliche Bedingungen & \multicolumn{2}{c|}{ Phänomen } \\
Bedeutung der Praktika & Praktika (Studierendenperspektive) \\
Eigenschaften & Dimensionale Ausprägung \\
Sinnhaftigkeit/Stellenwert/Nutzen & hoch & niedrig \\
Berufswahlentscheidung & früh & spät \\
Theorie-Praxis-Bezug & verschränkend & isolierend \\
Gewinnen von Einblick & intensiv & mäßig \\
Erfahrungen der Lehrer/innenperspektive & ausreichend & unzureichend \\
Zeit zum Üben & viel & wenig \\
Persönlicher Lerngewinn & hoch & niedrig \\
Persönlicher Lernfortschritt & hoch & niedrig \\
Bezug zu Schüler/innen & gegeben & nicht vorhanden \\
Gewinnen von Selbstvertrauen & möglich & nicht möglich \\
Ausprobieren der Lehrer/innenpersönlichkeit & gegeben & nicht gegeben \\
Begleitung durch Praxislehrperson & fördernd & destruktiv \\
Unterstützung durch Praxislehrperson & fördernd & destruktiv \\
\hline
\end{tabular}

Abb. 64: Eigenschaften und Dimensionen zum Phänomen Praktika (Studierendenperspektive)

Erfahrungslernen in den Praktika mit Begleitung: Das zweite Kodierparadigma umschließt das Phänomen Erfahrungslernen in den Praktika mit Begleitung. Das Phänomen Erfahrungslernen in den Praktika mit Begleitung kann wiederum als Kategorie gesehen werden, die sich um die ursächlichen Bedingungen Aktions- und Entfaltungsmöglichkeiten (persönlich/durch Praxislehrperson) und um die Kontextbedingungen Lehrer/innenpersönlichkeit, Beziehungsgeflecht, Rollendynamik, Bedingungen der Praktika entwickelt. Unterstützung durch erfahrene Praxislehrpersonen und kompetenzorientiertes Vorbereiten auf den Lehrberuf als Handlungsstrategien stehen in Beziehung zum Phänomen. Als Konsequenz ergibt sich somit klares Wissen um Rolle und Aufgabe in der Begleitung.

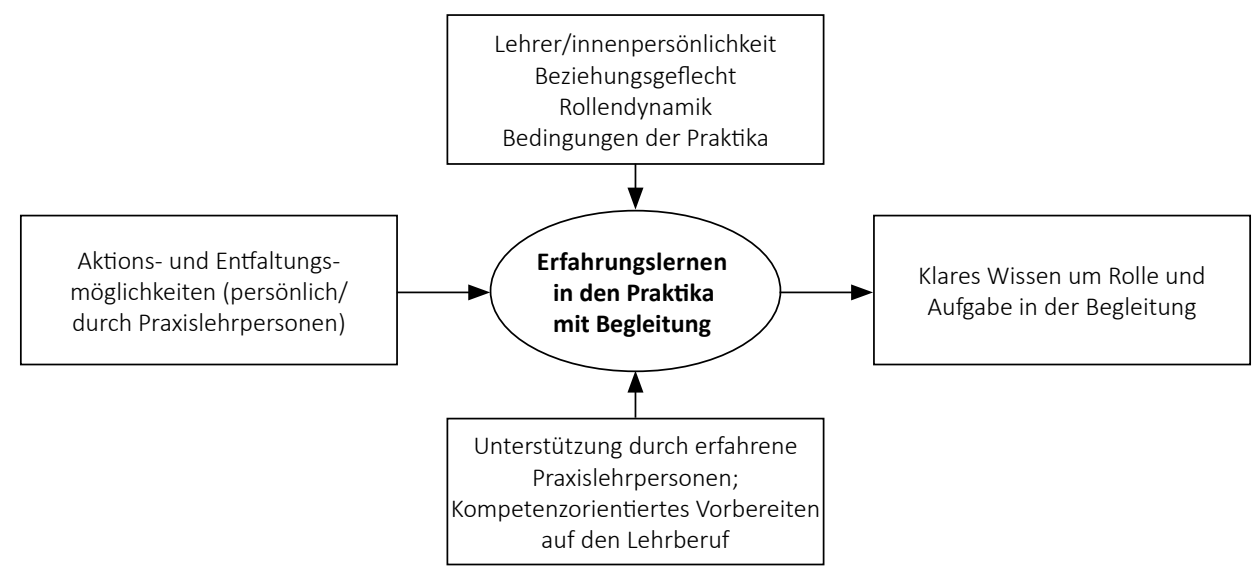

Abb. 65: Kodierparadigma 2 aus Studierendeninterviews 
Abbildung 66 führt die Eigenschaften und Dimensionen zur ursächlichen Bedingung Aktionsund Entfaltungsmöglichkeiten (persönlich/durch Praxislehrpersonen) an.

Ursächliche Bedingungen

Aktions- und Entfaltungsmöglichkeiten

(persönlich/durch Praxislehrpersonen)

\section{Eigenschaften}

Pädagogisches Handeln

Zeit zum Üben

Methoden- und Sozialformgestaltung

Vernetzung von Theorie und Praxis

Sammeln von Erfahrung

Selbstständigkeit

Potentialentfaltung

Erkennen von Veränderung/Fortschritt

Selbstvertrauen

Integration im Schulablauf/-alltag

Beziehungsebene

Beziehungskultur

Kommunikationsverhalten

Freiräume innerhalb einer Struktur

Geben von Feedback

Begleitende Reflexionseinheiten

Zeitressource in der Begleitung

\section{Phänomen}

Erfahrungslernen in den Praktika mit Begleitung

\begin{aligned} & \multicolumn{2}{c}{ Dimensionale Ausprägung } \\ & gewinnbringend nicht \\ & gegeben verwehrt \\ & ausschöpfend beengt \\ & realisierend nicht umsetzbar \\ & handelnd reflexiv nicht möglich \\ & ermöglichen abwehrend \\ & ausprobierend hemmend \\ & kontinuierlich gleichbleibend \\ & aufbauend destruktiv \\ & vorhanden nicht gegeben \\ & ebenbürtig hierarchisch \\ & wertschätzend nicht wertschätzend \\ & gesprächsbereit zögernd \\ & offen geschlossen \\ & konstruktiv destruktiv \\ & fordernd einengend \\ & viel wenig \\ & \hline\end{aligned}

Abb. 66: Eigenschaften und Dimensionen zum Phänomen Erfahrungslernen in den Praktika mit Begleitung (Studierendenperspektive)

Die ursächliche Bedingung zeigt auf, dass Studierende während der Zeit der Praktika die Möglichkeit erhalten wollen, in einer Schule/in einer Klasse/mit Schülerinnen und Schülern in Aktion zu treten und sich entfalten zu können. Dieser Wunsch ist sehr stark auch an die Bedeutung der Praktika geknüpft. Den Studierenden geht es hierbei darum, das Handwerkszeug aktional zu erproben (Pädagogisches Handeln, Zeit zum Üben, Methoden- und Sozialformgestaltung, Vernetzung Theorie und Praxis, Sammeln von Erfahrung) und sich in der persönlichen Perspektive als Lehrer/in zu entfalten (Selbständigkeit, Potentialentfaltung, Erkennen von Veränderung/Fortschritt, Selbstvertrauen). Im Aktions- und Entfaltungsprozess fühlten sich Studierende sehr stark vom Gutdünken der Lehrpersonen abhängig. Kodierungen weisen durch die konzeptuellen Repräsentanten, wie Integration im Schulablauf/-alltag, Beziehungsebene, Beziehungskultur, Kommunikationsverhalten, Freiräume innerhalb einer Struktur, Geben von Feedback, Begleitende Reflexionseinheiten und Zeitressource in der Begleitung darauf hin. Je nach Ausprägung der Dimensionen wird das Phänomen beeinflusst.

\section{Selektives Kodieren mit Kernkategorie}

In diesem Abschnitt des Kodierungsprozesses erfolgt die Zusammenschau der zwei Kodierparadigmata.

Lernen in den Praktika mit Begleitung: In der Kernkategorie gestaltete sich das Phänomen Lernen in den Praktika mit Begleitung heraus. Hierbei wirkt die ursächliche Bedingung Wunsch nach Entfaltungsmöglichkeit durch Begleitung auf das Phänomen ein. 


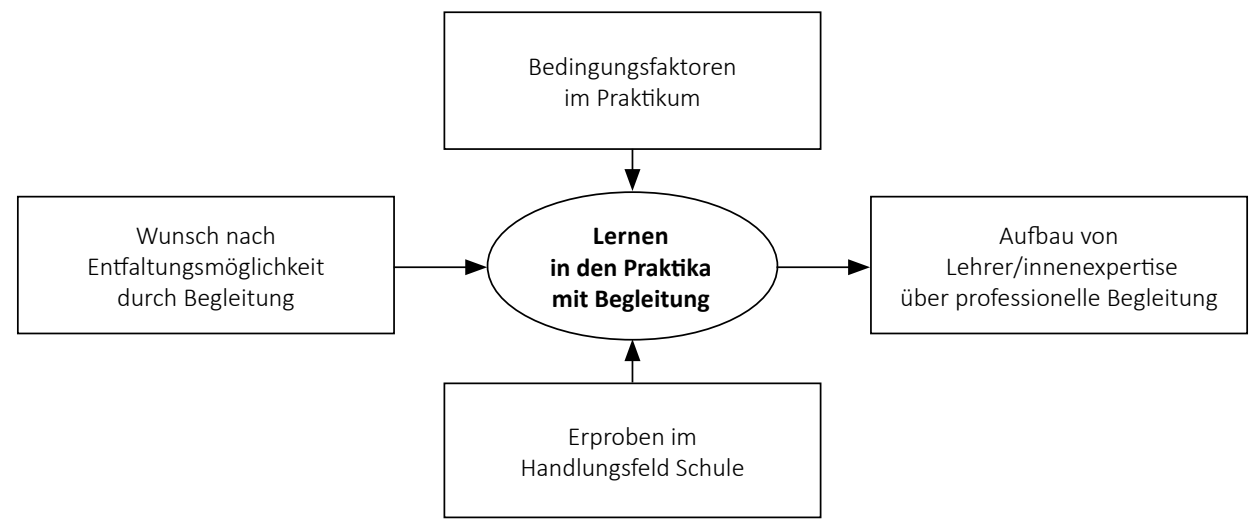

Abb. 67: Kernkategorie Studierende bzw. Mentee

Die ursächliche Bedingung hat Eigenschaften, mit denen sich Dimensionen eines Phänomens erklären lassen wie in Abbildung 68 angeführt. Die Kontextbedingung Bedingungsfaktoren im Praktikum stellt

„den besonderen Satz von Bedingungen dar, innerhalb dessen die Handlungs- und Interaktionsstrategien stattfinden, um ein spezifisches Phänomen zu bewältigen, damit umzugehen, es auszuführen und darauf zu reagieren." (Strauss et al. 1996, 80f) „Diese Bedingungen wirken entweder fördernd oder einengend auf die Handlungs- und interaktionalen Strategien ein.“ (Strauss et al. 1996, 82)

Die Kontextbedingungen Bedingungsfaktoren im Praktikum in der Kernkategorie weisen aus Sicht der Studierenden darauf hin, dass es Bedingungsfaktoren im Praktikum und im Mentoring braucht, die es ermöglichen, dass Studierende sich zielorientiert und zweckgerichtet im Handlungsfeld Schule erproben können. Als Konsequenz kann dann der Aufbau von Lehrer/innenexpertise angesehen werden, wobei eine professionsspezifische Begleitung maßgeblich beteiligt ist/sein muss.

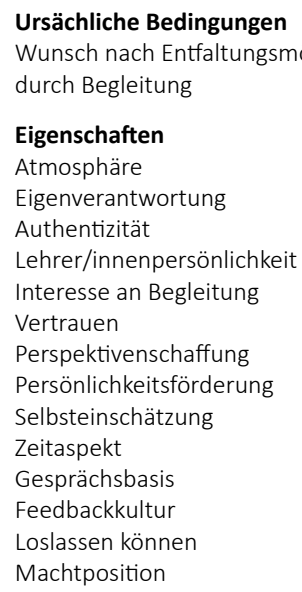

\section{Phänomen}

Lernen in den Praktika mit Begleitung

\begin{aligned} & \multicolumn{2}{c}{ Dimensionale Ausprägung } \\ & wohlfühlend missempfindend \\ & überlassend nicht überlassen \\ & bewahrend ablehnend \\ & unterschiedlich gleich/ähnlich \\ & vorhanden nicht vorhanden \\ & schenkend nicht schenkend \\ & eröffnend ablehnend \\ & achtend ignorierend \\ & einfordernd ignorierend \\ & gebend missachtend \\ & ebenbürtig hierarchisch \\ & konstruktiv destruktiv \\ & vorhanden nicht vorhanden \\ & ausspielend nicht vorhanden \end{aligned}

Abb. 68: Eigenschaften und Dimensionen des Phänomens Lernen in den Praktika mit Begleitung (Studierendenperspektive) 
Bei der ursächlichen Bedingung Wunsch nach Entfaltungsmöglichkeit durch Begleitung stellen sich die Fragen: Was ist den Studierenden wichtig, damit sie sich entfalten können? Wie sollte die/der Mentor/in im Idealfall aus Sicht der Studierenden reagieren, damit eine Entfaltung möglich ist? Welche Eigenschaften sind zur Entfaltung ideal? Die konzeptuellen Repräsentanten weisen auf intra- und interpersonelle Aspekte hin. Studierende schätzen eine wohlfühlende Atmosphäre, in der sie eigenverantwortlich und authentisch handeln können. Für Studierende ist es eine Bereicherung, wenn sie im Laufe der Praktika unterschiedliche Lehrer/innenpersönlichkeiten kennenlernen dürfen. Im Optimalfall sollen diese Lehrpersonen Interesse an der Begleitung haben, den Studierenden Vertrauen schenken, neue Perspektiven eröffnen, Selbsteinschätzung zulassen und sie in der Persönlichkeitsentwicklung stärken. Studierende äußern sich positiv zu Begleitungsprozessen, wenn Lehrpersonen genügend Zeitressourcen haben, einen wertschätzenden Kommunikationsstil pflegen und in der Lage sind, konstruktives Feedback zu geben. Die Art und der Grad der Entfaltungsmöglichkeit ist weiters sehr stark von der Ausprägung der Dimensionen Loslassen können und Machtposition der Lehrpersonen geprägt.

Im Kapitel Untersuchungsergebnisse wurden die systematische Arbeitsorganisation (Strauss 1991b, 34f) und die Wechselbeziehung (Strauss 1991b, 34f) von der Datenerhebung bis zur Datenanalyse mit der Darstellung der Kernkategorien und den subjektiven Sichtweisen zur vorliegenden Untersuchung dokumentiert. Im Folgenden werden weitere Rekonstruktionsschritte unternommen und Interpretationen zu Ergebnissen vorgenommen. 


\section{Interpretation der Befunde}

Die herausgearbeiteten Konzepte, Subkategorien und Kategorien, welche den ursächlichen Bedingungen, Handlungsstrategien, Kontext und intervenierenden Bedingungen, Konsequenzen und Phänomenen zugeordnet wurden, wurden in einem weiteren Schritt aufgebrochen, auf einer weiteren höheren Ordnung verdichtet, mit Bereichen der Kodierparadigmata und Kernkategorien abgeglichen, um zu einem Modell im Rahmen des schulpraktischen Mentoringprozesses zu gelangen. Hierbei bilden die Begrifflichkeiten des handlungstheoretisch-fundierten heuristischen Kodierparadigmas bzw. Kernkategorie-Modells der Grounded Theory die Basis für die methodische Herangehensweise (Abbildung 69).

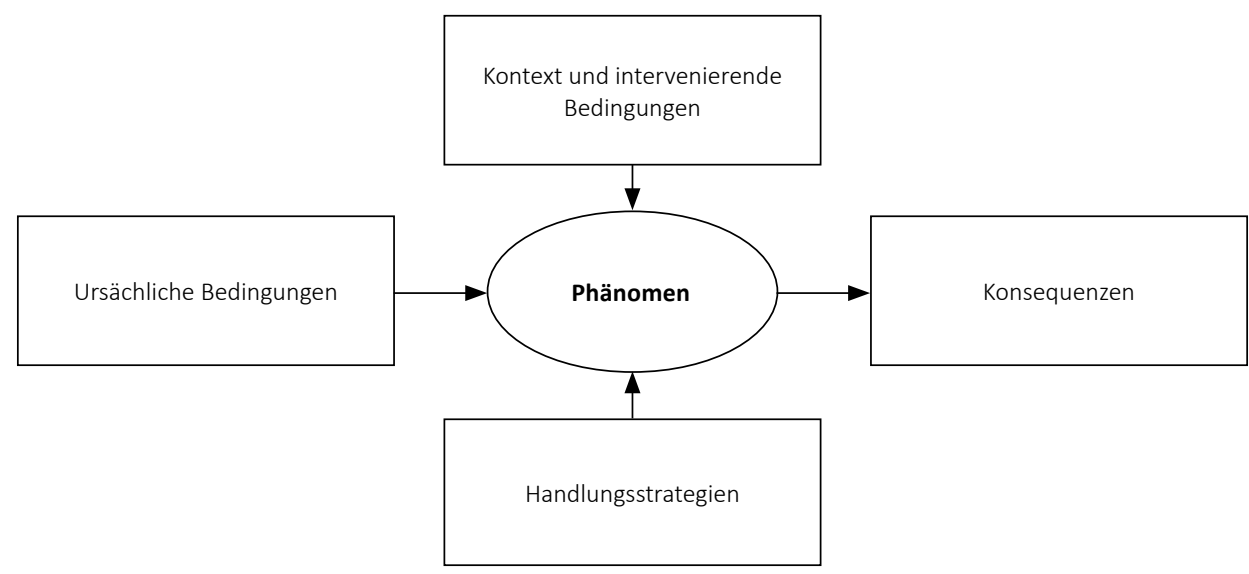

Abb. 69: Kodierparadigma (Böhm 2015, 479)

Ausgangspunkt der Interpretation ist die inhaltliche Betrachtung der Beschreibung der gewonnenen Phänomene aus den Interviews beider Interviewgruppen. Die weitere Analyse erstreckt sich über die ursächlichen Bedingungen, die Handlungsstrategien, die intervenierenden Bedingungen und schließlich hin zu den Konsequenzen. Hierbei werden sowohl Lehrendenperspektive als auch Studierendenperspektive dargelegt und, wenn möglich, Inhalte miteinander verglichen, gegenübergestellt und/oder die konzeptuellen Repräsentanten näher beleuchtet. Die Absicht besteht darin, treffende Aussagen zum schulpraktischen Mentoringprozess herauszukristallisieren und letztendlich ein Modell bzw. Modelle abzuleiten.

In den Ausführungen werden fünf Bereiche in folgender Reihenfolge betrachtet:

1. Interpretation der Phänomene

2. Interpretation der ursächlichen Bedingungen

3. Interpretation der Handlungsstrategien

4. Interpretation des Kontextes und der intervenierenden Bedingungen

5. Interpretation der Konsequenzen 
Die Aufschlüsselung dieser Bereiche verdeutlicht, dass als Ausgangsbasis für die Interpretation die Ansätze der Grounded Theory weiterverfolgt und Aussagen verdichtet werden - beginnend mit den Phänomenen der vorliegenden Untersuchung.

\subsection{Interpretation der Phänomene}

Auf Basis aller Einzelkategorien wurden Kodierparadigmata und Kernkategorien herausgearbeitet, die es ermöglichen, Konzepte und Kategorien sowie deren Dimensionen zu verbinden und zu integrieren. Im Zentrum jedes Paradigmas steht das Phänomen. Im Untersuchungsprozess mit Datenerhebung und ständiger Auswertung in Richtung Sampling fasst die Aussage des Phänomens die Zuschreibungen der anderen Bereiche des Zusammenhangsmodells zusammen. Anbei ist die Benennung der Phänomene aus Kodierparadigmata und Kernkategorien der Analyse mit der Beschreibung weiterer Rekonstruktionen angeführt (Tabelle 55).

Tab. 55: Professionsentwicklung aus dem Rekonstruktionsprozess der Phänomene

\begin{tabular}{|c|c|c|c|c|}
\hline Ebenen & \multicolumn{2}{|c|}{$\begin{array}{l}\text { Lehrendenperspektive } \\
\text { Mentor/innenperspektive }\end{array}$} & \multicolumn{2}{|c|}{$\begin{array}{l}\text { Studierendenperspektive } \\
\text { Menteeperspektive }\end{array}$} \\
\hline $\begin{array}{l}\text { Phänomen }(\mathrm{e}) \\
\text { Kodierparadigma }\end{array}$ & Praktika & Interaktion & Praktika & $\begin{array}{c}\text { Erfahrungslernen } \\
\text { in den Praktika } \\
\text { mit Begleitung }\end{array}$ \\
\hline $\begin{array}{l}\text { Phänomen(e) } \\
\text { Kernkategorie }\end{array}$ & \multicolumn{2}{|c|}{ Zusammenarbeit } & \multicolumn{2}{|c|}{$\begin{array}{l}\text { Lernen in den Praktika } \\
\text { mit Begleitung }\end{array}$} \\
\hline Rekonstruktion & \multicolumn{2}{|c|}{$\begin{array}{c}\mathbf{V} \\
\text { Professions(weiter)entwicklung }\end{array}$} & \multicolumn{2}{|c|}{$\begin{array}{c}\boldsymbol{\nabla} \\
\text { Professionsentwicklung }\end{array}$} \\
\hline Conclusio & \multicolumn{4}{|c|}{ Professionsentwicklung } \\
\hline
\end{tabular}

Die ersten Kodierparadigmata nehmen in den Phänomenen Praktika, Interaktion und Erfahrungslernen in den Praktika mit Begleitung in den Fokus. Im selektiven Kodierungsverlauf und nach dem Sortieren 2. Ordnung (Breuer et al. 2018, 257) kristallisierten sich das Phänomen Lernen in den Praktika mit Begleitung in der Kernkategorie der Studierendeninterviews und das Phänomen Zusammenarbeit in der Kernkategorie der Lehrpersoneninterviews heraus.

Da Lernen und Zusammenarbeit zentrale Themen im Mentoringprozess zu sein scheinen, stellte sich die Frage, wie die Phänomene zusammenhängend betrachtet werden können. Um die inhaltliche Analyse vornehmen zu können, erfolgte wiederum ein Aufbrechen des Datenmaterials und Analysieren durch Fragestellung und Anstellen von Vergleichen (Strauss et al. 1996, 44). Fragen zu den Kategorien der Lehrpersoneninterviews lauteten: Warum wollen Mentor/innen zusammenarbeiten? Unter welchen Umständen sind Lehrpersonen bereit, Studierende im Praktikum zu begleiten? Welche Bedingungen stellen Lehrpersonen an die Zusammenarbeit? Wovon hängt die Zusammenarbeit ab? Welche Ansprüche haben Lehrpersonen an sich, an die/ den andere/ $\mathrm{n}$ und an das System? Welchen Mehrwert sehen Lehrpersonen in einer Zusammenarbeit? 
Fragen an Kodierungen der Studierendeninterviews waren: Was wollen Studierende lernen? Was verstehen sie unter dem Begriff Lernen? Welche Erwartungen haben Studierende an sich, an die Begleitungsperson und das System im Rahmen des Lernprozesses? Was brauchen Studierende, damit sie lernen können?

Nach Aufbrechen der Fragen wird in den Phänomenen der Kodierparadigmata als auch der Kernkategorien der Lehrenden- und Studierendenperspektive Professionsentwicklung als Dreh- und Angelpunkt gesehen. Zu bemerken ist allerdings, dass Lehrpersonen und Studierende im Bereich der Professionsentwicklung unterschiedliche Intentionen im Mentoringprozess verfolgen. Abbildung 70 verdeutlicht den theoretischen Bezugsrahmen zum Konstrukt Professionsentwicklung aus Sicht der Lehrenden und Studierenden (Abbildung 70):

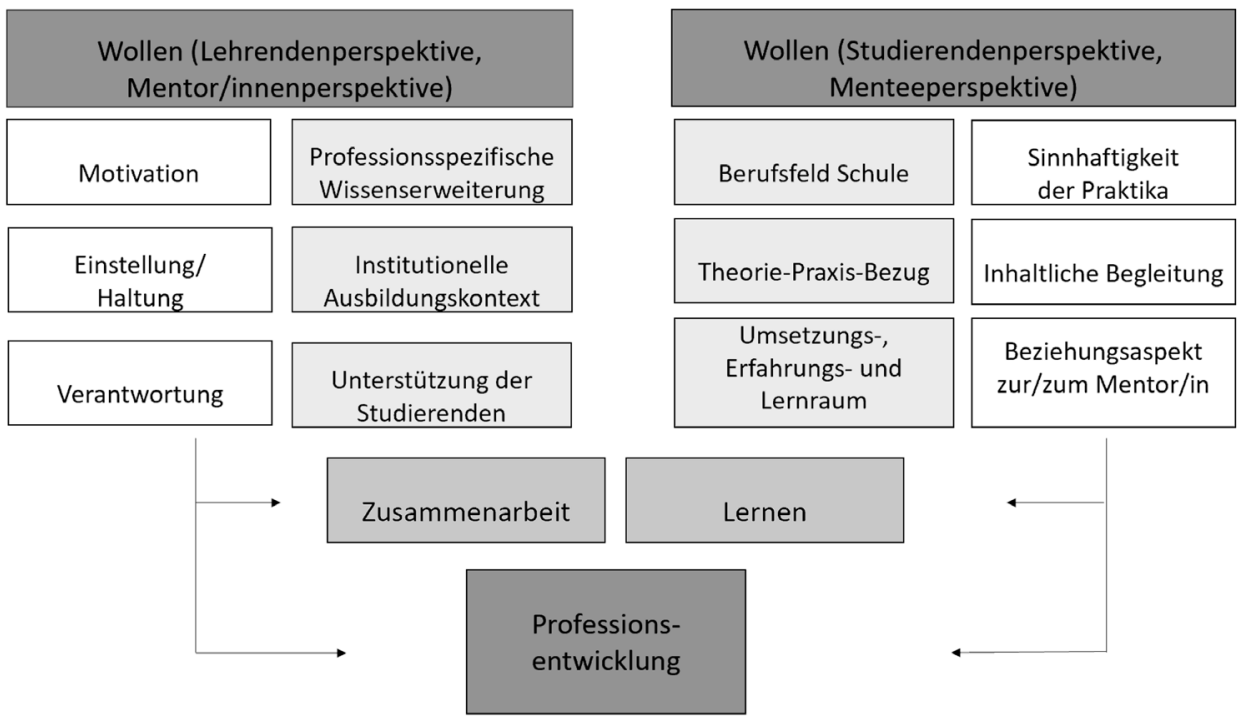

Abb. 70: Rekonstruktion aus den Phänomenen

Studierendenperspektive bzw. Menteeperspektive: Studierende sehen den Mehrwert beim Lernen darin, das Berufsfeld Schule kennenzulernen und sich darin aktiv zu bewegen, eine Theorie-Praxis-Verknüpfung herzustellen und unterschiedliche professionsspezifische Kompetenzen im Umsetzungs-, Erfahrungs- und Lernraum (er)lernen zu können und für sich einen professionsspezifischen „Gewinn“ verbuchen zu können. Bei Studierenden steht zum Phänomen Lernen ein „individuelles Wollen“ (Kraler 2012b) im Vordergrund.

Das Berufsfeld Schule ermöglicht Studierenden, sich mit dem Berufswunsch auseinanderzusetzen, das System Schule mit den vielen Facetten kennenzulernen, einen strukturierten Schulablauf zu gestalten, die Entwicklung der Schüler/innen über einen Zeitraum zu beobachten und eine Beziehung zu den Schülerinnen und Schülern aufzubauen. Es gibt ihnen die Möglichkeit, Neues zu erfahren und Neues einzubringen, Handlungsmuster sowie unterschiedliche Sozialformen und Methoden auszuprobieren. Eine Studierende unterstreicht die Behauptungen mit der Aussage: „Ich finde es wichtig, dass es diese schulpraktischen Anteile gibt, da man da in die Schulen kommt und den Tagesablauf und den Schulrhythmus kennenlernt." (S01; 15-18) 
Den Theorie-Praxis-Bezug sehen Studierende als ein wesentliches Lernkriterium. „Diesen Bogen zu spannen zwischen Theorie und Praxis war eigentlich die größte Erfahrung, die größte Schwierigkeit" (L02; 244-246), meint ein Interviewpartner. Oftmals sprechen Studierende von einer Kluft zwischen Lehre an der Hochschule bzw. Universität und Gegebenheiten an der Schule. Ein Verständnis über die Sinnhaftigkeit von so manchen bildungswissenschaftlichen, fachwissenschaftlichen und fachdidaktischen Ausbildungsinhalten zeigt sich erst im direkten Kontakt mit Schülerinnen und Schülern bzw. Lehrpersonen. An dieser Stelle wird vermutet, dass in diesem Zusammenhang von einem „Rezeptlernen“ die Rede ist. Studierende würden gerne viele Hospitationen an unterschiedlichen Schulen wahrnehmen und infolge dessen sich mit der Begleitperson über Konzepte, Modelle, Strukturen und Abläufe unterhalten. Die Einsicht in die Notwendigkeit von reflexiven Gedanken wächst, wenn es darum geht, lernprozessbezogene professionsspezifische Tätigkeiten (Planungen konzipieren, Lernsettings gestalten, ...) in der Handlungsausübung zu erwerben und zu festigen. Es eröffnet sich eine Bereitschaft zum reflexiven Diskurs.

Die Elemente Umsetzungs-, Erfahrungs- und Lernraum verdeutlichen die Ansicht, dass ein persönlicher Bildungsnutzen hinter dem Phänomen Lernen und der Absicht der Professionsentwicklung bei Studierenden steht.

„Wenn ich das Gefühl habe, ich gehe raus aus dem Praktikum und ich kann etwas mitnehmen. Ich habe das Gefühl, ich habe Erfahrungen gesammelt, habe aber auch das Gefühl, okay, ich weiß hier gibt es Handlungsbedarf. Hier muss ich was verändern oder sollte ich was verändern. Und dann bekomme ich die Möglichkeit, dass ich Veränderungen herbeiführen kann." (S05; 840-845)

Die drei eingangs erwähnten Komponenten Berufsfeld Schule, Theorie-Praxis-Bezug, Umsetzungs-, Erfahrungs- und Lernraum unterstreichen aus Sicht der Studierenden die Sinnhaftigkeit und Notwendigkeit von Praktika während der Ausbildungszeit. Schulische Anteile haben einen ausgesprochen hohen Stellenwert. Als bedeutende Kriterien gelten der enorme Lernzuwachs im Lehrberuf und die persönliche Reifung (Auftreten, Selbstvertrauen).

Wichtig für den Lernprozess ist Studierenden eine inhaltliche Begleitung sowohl durch Mentor/innen als auch durch begleitende Lehrveranstaltungen. Das konstruktive reflexive Feedback wird als Mittel der Einsicht- und Erfahrungsgewinnung gedeutet. Lernen in den Praktika wird weiters aus Sicht der Studierenden mit dem Beziehungsaspekt zur/zum Mentor/in in Verbindung gesetzt. Zu diesem Aspekt gehen Studierende in eine hohe Erwartungshaltung. In der persönlichen Wahrnehmung korrelieren sie oftmals ein erfolgreiches Praktikum mit einer/einem verständnisvollen/verständnisvollen Mentor/in.

Lehrendenperspektive bzw. Mentor/innenperspektive: Die Zusammenschau der konzeptuellen Repräsentanten aus den Lehrpersoneninterviews zeigt zum Phänomen Zusammenarbeit, dass Mentor/innen sich auf einen Mentoringprozess einlassen, da ihnen professionsspezifische Wissenserweiterung, Eintreten in den institutionellen Ausbildungskontext und Unterstützung von Studierenden sehr wichtig sind. Diese drei Aspekte erklären das ,individuelle Wollen“ (Kraler 2013a) zum Mentoringprozess. Die professionsspezifische Wissenserweiterung wird mit Kategorien und Konzepten, wie Möglichkeit Neues von den Studierenden zu lernen, Begleitung aus Eigeninteresse am Fach und an der Materie, eigene Lernbereicherung und eigener professionsspezifischer Profit deklariert. Lehrpersonen geben auch an, sich auf institutionelle Ausbildungskontexte einlassen zu wollen. In ihrer Rolle fungieren sie hierbei sowohl als Lernende/ $r$ als auch als Brückenbauer/innen. In der Lernposition gibt es Gedankenfiguren 
wie: auf dem neuesten Stand zu sein, das eigene Handeln stärker überdenken, selbst wieder Lernende/r zu sein, eigene Reflexionskompetenz zu stärken. Die Brückenbauer/in-Funktion zeigt sich in den Kategorien Herausstreichen der Bedeutung der Theorie-Praxis-Verschränkung, Schaffen eines Realitätsbezuges und Möglichkeit zum handlungsorientierten Umsetzen des Fachwissens. Ein dritter Aspekt, der Lehrpersonen zur Zusammenarbeit veranlasst, ist die Unterstützung der Studierenden. Lehrpersonen wollen den Studierenden die Möglichkeit des Perspektivenwechsels einräumen, bei Fragen der Berufswahlentscheidung zur Seite stehen, in der Zeit der Praktika ein Gefühl der Sicherheit geben, Freiräume zur Gestaltung von Lernsettings zulassen. Bei genauer Betrachtung der drei Aspekte professionsspezifische Wissenserweiterung, Eintreten in den institutionellen Ausbildungskontext und Unterstützung von Studierenden ergibt sich ein Zusammenhang zur Themenzentrierten Interaktion mit Ich $\mathrm{Du}$ - Sache - Umfeld (Globe).

Voraussetzung für die Bereitschaft der Zusammenarbeit und der Begleitungstätigkeit sind persönliche Motivation, Aspekte der Einstellung/Haltung sowie ein Verantwortungsbewusstsein. Die Beweggründe zur Motivation sind ichbezogen, systembezogen und student/innenbezogen. (Eine konkrete inhaltliche Darstellung der Dimensionen findet sich im Kapitel II/6.1)

Die Kategorie Einstellung/Haltung bezieht sich in den Aussagen der Lehrpersonen in erster Linie auf beziehungs- und kommunikationsorientierte Elemente und unterliegt intrapersonalen Sichtweisen der Mentor/in. Als zentrale Fragestellungen für jede/n Mentor/in kristallisierten sich heraus: Wie behandle ich eine Person? Wie bewerte ich eine Person? Lehrpersonen klassifizieren das Verhältnis zur/zum Studierenden mit den Worten Arbeitsverhältnis, Vertrauensverhältnis, partnerschaftliches Verhältnis bzw. Ausbildungsverhältnis oder umschreiben die Begleitsituation mit Arbeiten mit gleichgestellten Kolleg/innen, Arbeiten im Team bzw. Aufbauen einer Arbeitskolleg/innenschaft. Hinter all diesen Begrifflichkeiten verbergen sich Einstellungen und Haltungen gegenüber einer Person. Lehrpersonen ist bewusst, dass Studierende auf einer anderen Ebene im Berufsfeld Schule stehen. Ein starkes Hierarchiegefälle sollte nach Meinung der Lehrpersonen im Mentoringprozess jedoch nicht spürbar sein. Ein Unterschied wird vielleicht aufgrund des Expertiseansatzes dennoch gegeben sein. Ein Interviewpartner meinte, dass er sich in der Mentor-Funktion als Kapitän sieht, der das Schiff letztendlich steuert (L10; Zeile 2030). Auf Kommunikationsebene betonen Lehrpersonen sowohl die komplementäre - im Sinne einer ergänzenden - als auch die symmetrische Ebene. Bezogen auf den komplementären Ansatz liegt die Vermutung nahe, dass wiederum der Expertiseansatz aufgrund von Berufserfahrung, Fachwissen, Rollenverständnis, Ausbildungsmodus greift. Einstellungen/Haltungen zeigen sich im Weiteren auch bei Aussagen zu Lehrer/innentugenden wie Verlässlichkeit, Pünktlichkeit, Höflichkeit, Umgänglichkeit, Arbeitsfleiß, Sauberkeit, Gepflegtheit usw. Die Interviewpartner/innen nennen negative Beispiele und räumen ihrerseits wenig Toleranz ein. Die Bereitschaft zur Mentor/innentätigkeit hängt auch davon ab, ob und wieviel Erziehungsarbeit in diese Richtung bei Studierenden geleistet werden muss. Es ergibt sich die Sichtweise, dass Mentor/innen Fragestellungen am Beginn der Tätigkeit beantworten werden müssen. Diese könnten wie folgt lauten: Welche Einstellungen/Haltungen habe ich und zeige ich nach außen? Welche sind mir wichtig und fordere ich ein? Wie tolerant bin ich meiner/meinem Nächsten gegenüber?

Mit der Kategorie Verantwortungsbewusstsein verdeutlichen Mentor/innen, dass die Tätigkeit mit einer hohen Verantwortung - vorausgesetzt bei entsprechendem Einsatz - einhergeht. Sie treten am Beginn auch in einen Abwägungsprozess zwischen Beachtung persönlicher Ressourcen bzw. professionsspezifischer Kenntnisse zur Aufgabenerfüllung und Bereitschaft der Über- 
nahme von Verantwortung ein. Überlegungen sind: Habe ich genug Raum und Zeit, die Aufgabe zu übernehmen? Bin ich der Situation gewachsen? Kann ich Studierenden weiterhelfen? Was lernen Studierende mit mir und bei mir? Wie kommuniziere ich richtig? Wie kann ich Studierende fördern? Was tue ich mit „Problemstudierenden“? Gerade diese Frage beschäftigt Mentor/innen und veranschaulicht, dass während der Begleitungstätigkeit Verantwortung in diesem Sinne gezeigt werden muss, indem Probleme angesprochen und Lösungswege überlegt werden müssen. Es ist im Bewusstsein der Interviewpartner/innen, dass Entscheidungen über das erfolgreiche oder nicht erfolgreiche Bestehen eines Praktikums immer auch mit Konsequenzen verbunden sind. Dazu meint ein Interviewpartner (L01):

„Die Verantwortung hat die/der Mentor/in, dass sie/er, wenn sie/er tatsächlich problematische Bereiche feststellt, dass sie/er diese auch klar anspricht. Es hilft nichts mit einer, ja, übertriebenen Höflichkeit oder übertriebenen Rücksichtnahme mit den Studierenden umzugehen. Sie müssen auf Probleme hingewiesen werden, nicht in einer herabwürdigenden Art und Weise, aber doch in einer deutlichen Sprache." (L01; Zeile 92-97)

Das Ansprechen von Problemen zeigt sich als ein weiterer Aspekt im Rahmen der Bereitschaft zur Verantwortung. Die drei Komponenten Motivation, Einstellung/Haltung sowie vorhandenes Verantwortungsbewusstsein bilden letztendlich das Fundament für die Bereitschaft, die Aufgaben der/des Mentorin/Mentors zu übernehmen.

Nach erfolgter Beschreibung des Rekonstruktionsprozesses zu den Phänomenen Zusammenarbeit und Lernen in den Praktika ergibt sich eine weitere Fragestellung: Wie kann Professionsentwicklung im Hinblick auf diese Phänomene interpretiert werden? Zeigt sich in der phänomenalen Beschreibung ein mögliches Spannungsfeld?

Der Rekonstruktionsprozess der Phänomene verdeutlicht, dass beide Interviewgruppen schulpraktische Mentoringprozesse auf Professionsentwicklung ausrichten (Abbildung 71).

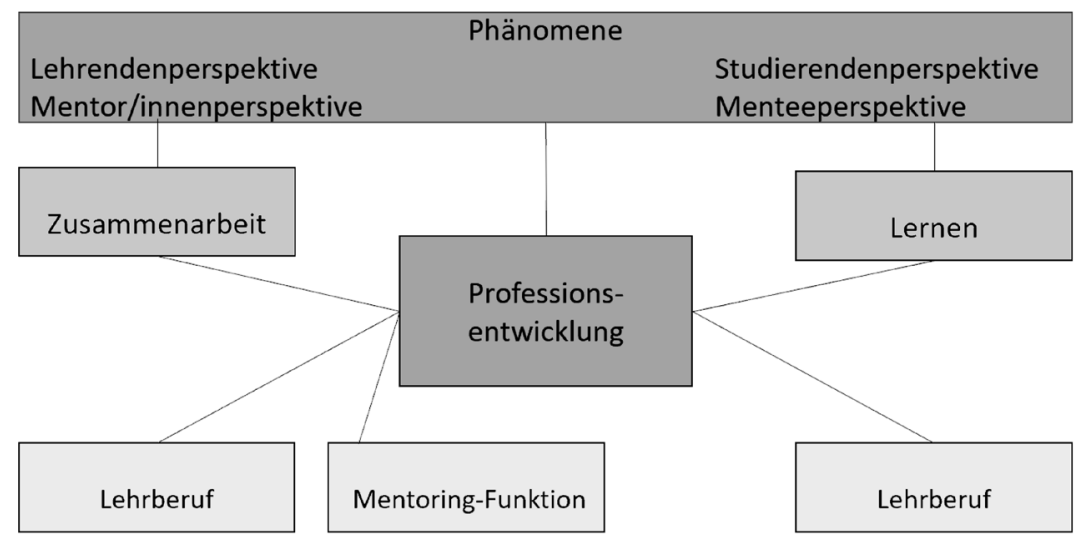

Abb. 71: Analyse zur Professionsentwicklung

Die Befunde zeigen auf, dass Lehrpersonen sich durch die Zusammenarbeit mit Studierenden und dem Ausbildungskontext sowohl im Lehrberuf als auch in der Mentoring-Funktion (weiter)entwickeln wollen. Die Mentoring-Funktion stellt sich als neues Aufgaben- und vielleicht auch im weiteren Sinn als Professionsfeld dar. Studierende konzentrieren sich im schulprakti- 
schen Mentoringprozess auf die Professionsentwicklung Lehrberuf. Ein mögliches Spannungsfeld im Mentoringprozess zum Phänomen Professionsentwicklung kann allerdings nicht dokumentiert werden.

Im Folgenden erfolgt die präzise Darstellung der Spezifizierung des Phänomens Professionsentwicklung durch weitere vier Felder: ursächliche Bedingungen, Handlungsstrategien, Kontext und intervenierende Bedingungen und Konsequenzen.

\subsection{Interpretation der ursächlichen Bedingungen}

Ursächliche Bedingungen sind stark an die Frage des Zustandekommens des Phänomens (Strübing 2014, 25) gekoppelt. Tabelle 56 fasst die ursächlichen Bedingungen der Kodierparadigmata und Kernkategorien zusammen und leitet in einem zweischrittigen Rekonstruktionsprozess Inhalts- und Beziehungsaspekte als ursächliche Bedingungen für das schulpraktische Mentoring ab. Ursächliche Bedingungen sind in weiterer Folge auch als „Auslöser für Handlungen“ (Heiser 2018, 232) zu verstehen.

Tab. 56: Inhalts- und Beziehungsaspekte aus dem Rekonstruktionsprozess der ursächlichen Bedingungen

\begin{tabular}{|c|c|c|c|c|}
\hline Ebenen & \multicolumn{2}{|c|}{$\begin{array}{l}\text { Lehrendenperspektive } \\
\text { Mentor/innenperspektive }\end{array}$} & \multicolumn{2}{|c|}{$\begin{array}{l}\text { Studierendenperspektive } \\
\text { Menteeperspektive }\end{array}$} \\
\hline $\begin{array}{l}\text { Phänomen }(\mathrm{e}) \\
\text { Kodierparadigma }\end{array}$ & Praktika & Interaktion & Praktika & $\begin{array}{l}\text { Erfahrungslernen } \\
\text { in den Praktika } \\
\text { mit Begleitung }\end{array}$ \\
\hline $\begin{array}{l}\text { Ursächliche } \\
\text { Bedingungen } \\
\text { Kodierparadigma }\end{array}$ & $\begin{array}{l}\text { Erfahrungen eige- } \\
\text { ner Ausbildungszeit } \\
\text { durch Begleitung; } \\
\text { Bedeutung der } \\
\text { Theorie-Praxis- } \\
\text { Verschränkung }\end{array}$ & $\begin{array}{l}\text { Motivation für } \\
\text { Betreuungs- } \\
\text { tätigkeit }\end{array}$ & $\begin{array}{l}\text { Bedeutung der } \\
\text { Praktika }\end{array}$ & $\begin{array}{l}\text { Aktions- und } \\
\text { Entfaltungs- } \\
\text { möglichkeiten } \\
\text { (persönlich/durch } \\
\text { Praxislehrpersonen) }\end{array}$ \\
\hline Rekonstruktion 1 & $\begin{array}{c} \\
\text { Inhaltsaspekt }\end{array}$ & $\begin{array}{c} \\
\text { Inhaltsaspekt } \\
\text { Beziehungsaspekt }\end{array}$ & $\begin{array}{c} \\
\text { Inhaltsaspekt }\end{array}$ & $\begin{array}{c} \\
\text { Inhaltsaspekt }\end{array}$ \\
\hline $\begin{array}{l}\text { Phänomen }(\mathrm{e}) \\
\text { Kernkategorie }\end{array}$ & \multicolumn{2}{|c|}{ Zusammenarbeit } & \multicolumn{2}{|c|}{$\begin{array}{l}\text { Lernen in den Praktika } \\
\text { mit Begleitung }\end{array}$} \\
\hline $\begin{array}{l}\text { Ursächliche } \\
\text { Bedingungen } \\
\text { Kernkategorie }\end{array}$ & \multicolumn{2}{|c|}{$\begin{array}{l}\text { Herausforderung und Bereicherung } \\
\text { (persönlich/für Studierende/n) }\end{array}$} & \multicolumn{2}{|c|}{$\begin{array}{c}\text { Wunsch nach Entfaltungsmöglichkeit } \\
\text { durch Begleitung }\end{array}$} \\
\hline Rekonstruktion 2 & \multicolumn{2}{|c|}{ Inhaltsaspekt und Beziehungsaspekt } & \multicolumn{2}{|c|}{ Inhaltsaspekt und Beziehungsaspekt } \\
\hline Conclusio & \multicolumn{4}{|c|}{$\begin{array}{l}\text { Inhalts- und Beziehungsaspekte als ursächliche Bedingungen } \\
\text { des schulpraktischen Mentorings }\end{array}$} \\
\hline
\end{tabular}


Studierendenperspektive bzw. Menteeperspektive: Für Studierende bieten Praktika Raum und Zeit, um ,in Aktion treten“ zu können. Der Inhaltsaspekt steht hierbei im Vordergrund. Das Phänomen Lernen in den Praktika mit Begleitung in den Kernkategorien verdeutlicht, dass Studierende den Fokus auf den Inhaltsaspekt richten, wohlwissend, dass der Beziehungsaspekt in den Praktika für sie eine große Rolle spielt. Im Sinne von Watzlawick ist ersichtlich, dass jede Kommunikation einen Inhalts- und Beziehungsaspekt hat. Studierende sehen sich in den Praktika in einer gewissen Abhängigkeit von der Begleitperson. Es wird von Studierenden der Schluss gezogen, dass Unstimmigkeiten im pädagogischen Handeln sehr schnell auf die Beziehungsebene übertragen werden können. Daher kommt im Bereich Konsequenzen seitens der Studierenden die Forderung nach Professionalität seitens der Mentor/innen.

Lehrendenperspektive bzw. Mentor/innenperspektive: Positive und negative Erfahrungen wie Erfahrungen im Schulalltag, gelungener oder misslungener Theorie-Praxis-Bezug, Möglichkeit oder Negieren des Ausprobierens in den Praktika während der eigenen Ausbildungszeit sind Begründungsfaktoren der ursächlichen Bedingungen bei Lehrpersonen. Weiters erkennen sie in der Tätigkeit als Mentor/in eine neue Aufgabenfunktion, die für sie in der ursächlichen Bedingung der Kernkategorien eine Herausforderung und eine Bereicherung darstellen. Der Gedanke, Neues zu lernen, sich in der Materie auf dem kontextbezogenem Feld vertiefen und sich in Fragen zu aktuellen professionsspezifischen Sichtweisen mit Expert/innen der Ausbildungsinstitutionen und/oder den Studierenden austauschen zu können, dokumentiert die Bedeutung des Inhalts- und Beziehungsaspektes seitens der Mentor/ innen. Dies wird mit folgender Aussage belegt:

„Es ist immer ganz interessant und ich hole mir auch immer meine Inputs und neue Ideen selber - von den Materialien, die die Studenten herrichten oder einfach wie sie unterrichten, wie sie ein Sachthema aufarbeiten. Das ist für mich schon total interessant und es ist auch interessant, wenn sie offene Unterrichtsstunden halten oder wie sie es machen. Also für mich ist das sehr fruchtbar - wahrscheinlich auf beiden Seiten. Es bringt frischen Wind hinein in mein Denken und Handeln." (L12; 2500-2503)

Aus den Konzepten, Kategorien und Paradigmata können Inhalts- und Beziehungsaspekte als Befunde zu den ursächlichen Bedingungen des schulpraktischen Mentorings extrahiert werden, die auf Seite der Mentor/innen und auf Seite der Studierenden noch einmal näher beleuchtet werden (Abbildung 72).

Lehrende forcieren verstärkt den Inhaltsaspekt (neues Aufgabenfeld, Motivation, eigene Lernbiografie, hohe Bedeutung der Theorie-Praxis-Verschränkung) und eher zweitrangig den Beziehungsaspekt (eigene Lernbiografie) als ursächliche Bedingungen. Die Motive Lerngelegenheit Praktika, individuelle Lern- und Entwicklungsmöglichkeit, Selbstwirksamkeitserprobung seitens der Studierenden betonen den Inhaltsaspekt als ursächliche Bedingung. Der Beziehungsaspekt tritt nur in den Vordergrund, wenn es darum geht, im Praktikum, das als Ort für Lerngelegenheiten gesehen wird, unterstützt und begleitet zu werden. Vorrangig ist für Studierende in der Zeit der Praktika der Inhaltsaspekt vor dem Beziehungsaspekt. 


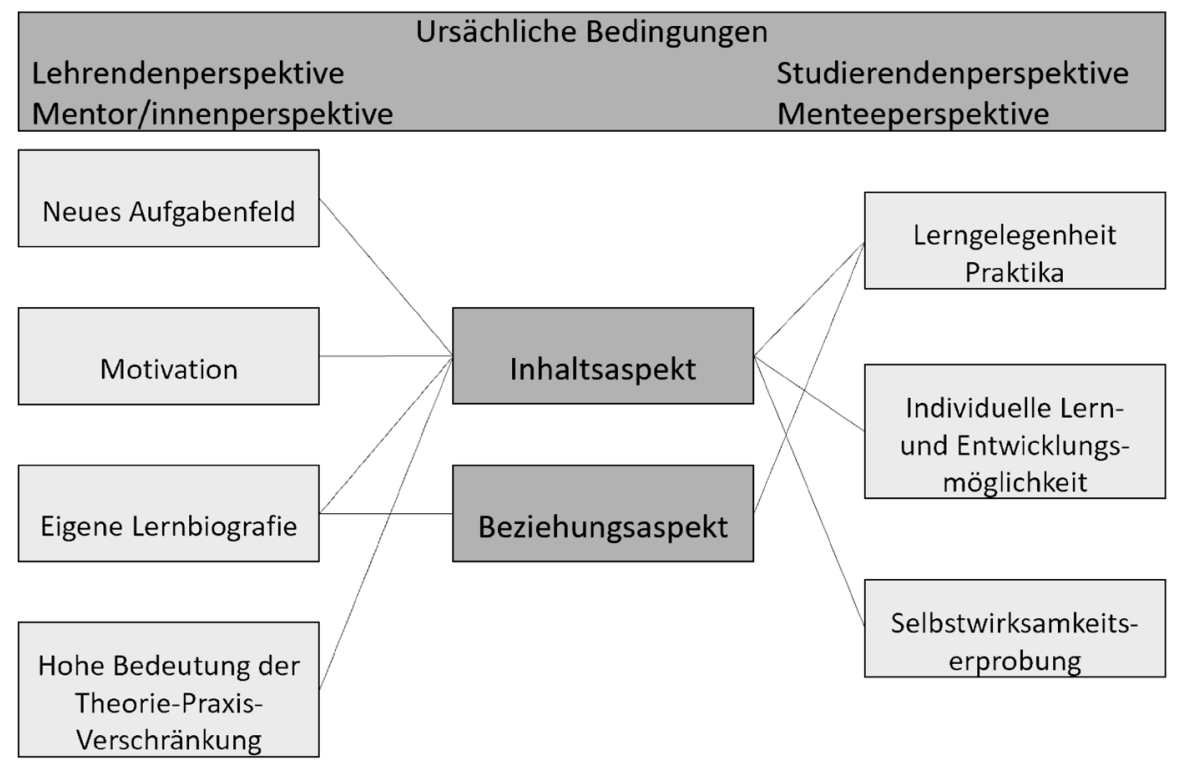

Abb. 72: Analyse zu ursächlichen Bedingungen

Insgesamt zeigen die Befunde auf, dass Mentor/innen sich mit kommunikationstheoretischen Modellen, psychoanalytischen Phänomenen wie Prägung und Übertragung stark auseinandersetzen müssten. Außerdem eröffnen sich weitere Fragestellungen: Welche Inhalte möchte mein/e mir anvertraute/r Mentee kennenlernen? Was erwarte ich mir bei der Umsetzung dieser Inhalte? Wie kann ich die/den Mentee auf der Inhaltsebene unterstützen und begleiten? Unter welchem Aspekt laufen Kommunikationsprozesse zwischen Mentor/in und Mentee ab? Die Fragestellungen lassen Überlegungen zu Ausbildungskonzepten für Mentor/innen und zur Bedeutung eines personenspezifischen Mentorings aufkommen.

\subsection{Interpretation der Handlungsstrategien}

Phänomene und ursächliche Bedingungen führen im Sinne der Interpretation im Kodierparadigma zu Handlungsstrategien. Daraus ergeben sich Fragestellungen wie: Welches Handeln könnte gemeint sein (Heiser 2018, 232) und wie wird es in Verbindung zum Phänomen und den anderen Komponenten verstanden? (Breuer et al. 2018, 288)

Die zusammenfassende inhaltliche Darstellung der Phänomene und Handlungsstrategien aus den Kodierparadigmata und Kernkategorien der Lehrenden- und Studierendenperspektive (Tabelle 57) veranlasste zunächst einen zweischrittigen Rekonstruktionsprozess und in weiterer Folge einen weiteren Verdichtungsschritt zur Metarekonstruktion, um zu einer Conclusio zu gelangen. Die Metarekonstruktion ergab sich aus Rekonstruktionsebene 1 und 2. 
Tab. 57: Kontextbezogener und personenspezifischer Lernprozess aus dem Rekonstruktionsprozess der Handlungsstrategien

\begin{tabular}{|c|c|c|c|c|}
\hline Ebenen & \multicolumn{2}{|c|}{$\begin{array}{l}\text { Lehrendenperspektive } \\
\text { Mentor/innenperspektive }\end{array}$} & \multicolumn{2}{|c|}{$\begin{array}{l}\text { Studierendenperspektive } \\
\text { Menteeperspektive }\end{array}$} \\
\hline $\begin{array}{l}\text { Phänomen }(\mathrm{e}) \\
\text { Kodierparadigma }\end{array}$ & Praktika & Interaktion & Praktika & $\begin{array}{l}\text { Erfahrungslernen } \\
\text { in den Praktika } \\
\text { mit Begleitung }\end{array}$ \\
\hline $\begin{array}{l}\text { Handlungs- } \\
\text { strategie }(\mathrm{n}) \\
\text { Kodierparadigma }\end{array}$ & $\begin{array}{l}\text { Auseinander- } \\
\text { setzung mit Aus- } \\
\text { bildungskonzept }\end{array}$ & $\begin{array}{l}\text { Anforderungen } \\
\text { an sich selbst } \\
\text { und an } \\
\text { Studierende }\end{array}$ & $\begin{array}{l}\text { Kennenlernen } \\
\text { von Kontext } \\
\text { Schule und Ver- } \\
\text { knüpfen von Theo- } \\
\text { rie und Praxis } \\
\end{array}$ & $\begin{array}{l}\text { Unterstützung } \\
\text { durch erfahrene } \\
\text { Praxislehrpersonen; } \\
\text { Kompetenzorien- } \\
\text { tiertes Vorbereiten } \\
\text { auf den Lehrberuf } \\
\text { _ }\end{array}$ \\
\hline Rekonstruktion 1 & $\begin{array}{c}\boldsymbol{V} \\
\begin{array}{c}\text { Systemisch-integrati- } \\
\text { ver Zugang }\end{array}\end{array}$ & Wertehaltung & $\begin{array}{l}\text { Verbindung } \\
\text { Studium und } \\
\text { Handlungsfeld } \\
\text { Schule }\end{array}$ & $\begin{array}{c} \\
\text { Begleitende } \\
\text { Unterstützung; } \\
\text { Vorbereiten auf } \\
\text { Beruf }\end{array}$ \\
\hline $\begin{array}{l}\text { Phänomen }(\mathrm{e}) \\
\text { Kernkategorie }\end{array}$ & \multicolumn{2}{|c|}{ Zusammenarbeit } & \multicolumn{2}{|c|}{$\begin{array}{l}\text { Lernen in den Praktika } \\
\text { mit Begleitung }\end{array}$} \\
\hline $\begin{array}{l}\text { Handlungs- } \\
\text { strategie(n) } \\
\text { Kernkategorie }\end{array}$ & \multicolumn{2}{|c|}{$\begin{array}{l}\text { Reflexive Auseinandersetzung } \\
\text { mit Ausbildungskonzept und eigener } \\
\text { Lehrtätigkeit; } \\
\text { Interaktion und Kommunikation }\end{array}$} & \multicolumn{2}{|c|}{$\begin{array}{l}\text { Erproben im Handlungsfeld } \\
\text { Schule }\end{array}$} \\
\hline Rekonstruktion 2 & $\begin{array}{r}\text { Lernfokus: Hinter } \\
\text { Austan } \\
\text { kommunikationsl }\end{array}$ & $\begin{array}{l}\text { fragen, Profitieren, } \\
\text { uschen, } \\
\text { basiertes Begleiten }\end{array}$ & $\begin{array}{r}\text { Lern } \\
\text { Ausprobiere } \\
\text { Weitere }\end{array}$ & $\begin{array}{l}\text { fokus: } \\
\text { n, Erproben, } \\
\text { ntwickeln }\end{array}$ \\
\hline Metarekonstruktion & $\begin{array}{l}\text { Lernen in der } \\
\text { aus der Bezie }\end{array}$ & $\begin{array}{l}\text { Beziehung und } \\
\text { ehung heraus }\end{array}$ & $\begin{array}{l}\text { Lernen als erfa } \\
\quad \text { prospekti }\end{array}$ & $\begin{array}{l}\text { hrungsbasierter } \\
\text { er Prozess }\end{array}$ \\
\hline Conclusio & & $\begin{array}{l}\text { Kontextbezogener u } \\
\text { Lernprozess im schu }\end{array}$ & $\begin{array}{l}\text { d personenspezifische } \\
\text { raktischen Mentorin }\end{array}$ & \\
\hline
\end{tabular}

Studierendenperspektive bzw. Menteeperspektive: Aus Sicht der Studierenden werden Handlungsstrategien zum Phänomen Praktika so angelegt, dass eine Verbindung zwischen Studium und Handlungsfeld Schule stattfindet. Im Lernort Schule können sie den Kontext Schule kennenlernen und eine Verknüpfung von Theorie und Praxis auf handlungsbasierter Ebene realisieren. Dazu ist es für Studierende hilfreich, Unterstützung von Mentor/innen zu erhalten, um sich auf den Lehrberuf vorbereiten zu können. Auch Studierende setzen Handlungsstrategien, um in einen Lernprozess eintauchen zu können. Der Lernfokus der Studierenden liegt jedoch auf dem Aspekt des Ausprobierens und Erprobens sowie der Möglichkeit des Weiterentwickelns. Auf Grundlage einer Verdichtung auf der Ebene der Metarekonstruktion lässt sich erkennen, dass 
Studierende beim Ausführen von Handlungsstrategien in einen erfahrungsbasierten prospektiven Lernprozess treten. Ein Interviewpartner meint dazu:

„Ich glaube ein Praktikum ist optimal, wenn ich etwas dazugelernt habe, wenn ich neue Erfahrungen negative als auch positive - habe lernen dürfen." (S07; 1256-1258)

Lehrendenperspektive bzw. Mentor/innenperspektive: Mentor/innen erkennen im Blick auf Handlungsstrategien in Verbindung mit dem Phänomen Praktika die Notwendigkeit eines systematisch-integrativen Zuganges. Sie sehen es als ihre Pflicht, sich mit den institutionell geforderten normativen Anforderungen und Ausbildungskonzepten auseinanderzusetzen.

Das Zusammenspiel von Phänomen Zusammenarbeit und den Handlungsstrategien Anforderungen an sich selbst und an Studierende verdeutlicht weiters die Notwendigkeit der Reflexion über Werthaltungen. Mentor/innen treten vor allem in der Rolle der Lehrperson auf und setzen Richtlinien z.B. zur Vorbildfunktion, zum Kommunikationsstil oder in Bezug auf Verhaltensweisen im Umgang miteinander. Meist erklären und erläutern sie ihre Werthaltungen durch normative Ansprüche im System und am System.

Mentor/innen sind sich bewusst, dass sie bei der Umsetzung von Handlungsstrategien im schulpraktischen Mentoring in einen Lernprozess gehen. Dieser wird von ihnen als ein reflexives und kommunikationsbasiertes Auseinandersetzen in einem umfassenden themenbezogenen Kontext gedeutet. Der Lernfokus besteht im Hinterfragen, Profitieren, Austauschen und im kommunikationsbasierten Begleiten. Ein Beispiel verdeutlicht die Denkfigur:

„Wie ist mir das Umsetzen meiner Sichtweisen im Laufe der Jahre gelungen oder was ist nicht gelungen? Einfach auch sagen, einfach mich selber ein bisschen hinterfragen. Funktioniert das eigentlich schon so, wie ich das mache oder muss ich mich im Zuge der Betreuung auch einmal ändern? Also nicht sagen, das hat 30 Jahre so funktioniert, das lasse ich so, sondern wie kann ich mich Erneuern oder neue Ideen einbauen, dass ich ganz persönlich für mich weiterkomme." (L11; 2157-2163)

Darauf aufbauend resultiert ein weiterer Verdichtungsprozess in Richtung Metarekonstruktion. Die Verdichtung der Daten zeigt auf, dass Lehrpersonen zum Phänomen Zusammenarbeit Handlungsstrategien setzen, die darauf zielen, in einen Lernprozess einzutreten und ein Lernen in der Beziehung zu Systemen und Personen und ein Lernen aus der Beziehung heraus forcieren.

Welche resümierende/n Aussage/n kann/können nun nach Rekonstruktion und Metarekonstruktion zu den Handlungsstrategien in Angleichung mit den Phänomenen gemacht werden? Wie gestalten Mentor/innen und Studierende Handlungsstrategien? Was steckt dahinter?

Mit dem Setzen von Handlungsstrategien zielen Mentor/innen und Studierende auf einen kontextbezogenen und personenspezifischen Lernprozess im schulpraktischen Mentoring. Beide Interviewgruppen veranlassen gezielte Handlungsstrategien und gelangen hierbei in einen Lernprozess. Zweckmäßigkeit und Absicht werden von Lehrenden und Studierenden jedoch inhaltlich unterschiedlich betrachtet. Während Mentor/innen in erster Linie den Beziehungscharakter als Lernmoment ausnützen und für sich damit professionsspezifische „Erfolge“ erzielen, sehen Studierende Lernen als einen erfahrungsbasierten prospektiven Prozess. Als gemeinsame Ebene wird ein kontextbezogener und personenspezifischer Lernprozess in Zusammenhang mit den Handlungsstrategien angenommen. Das erklärt sich, da beide Interviewgruppen Handlungsstrategien im Rahmen des Kontextes verorten und in einer aktiven Beziehung zueinanderstehen. 


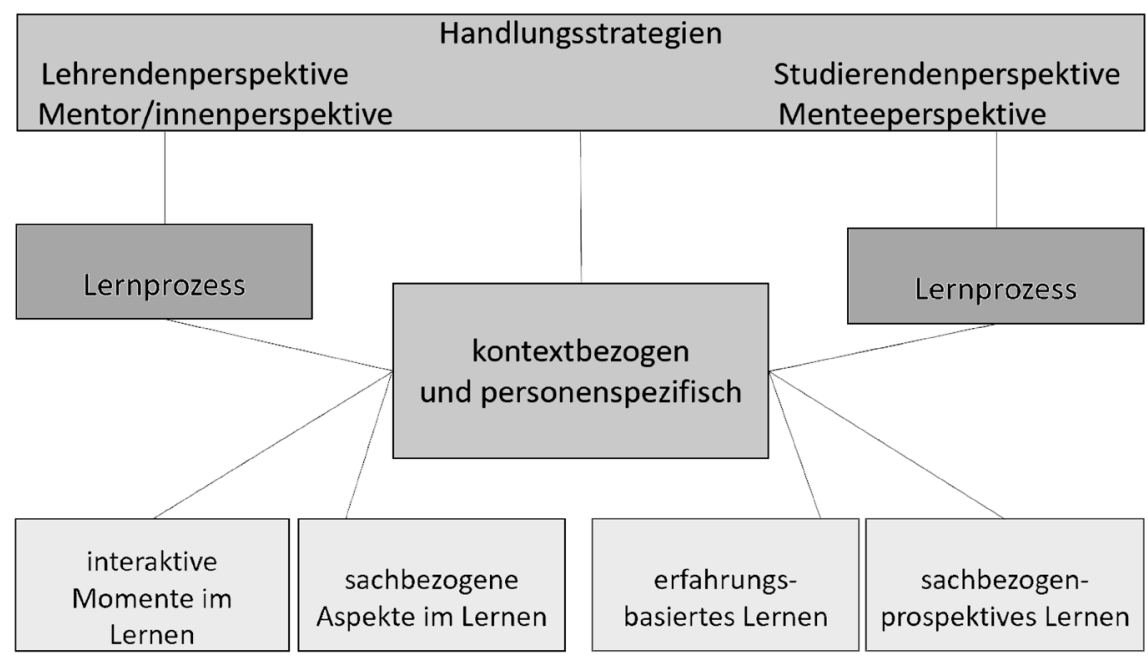

Abb. 73: Analyse zu Handlungsstrategien

Zusammenfassend können die eingangs gestellten Fragen: Welches Handeln könnte gemeint sein (Heiser 2018, 232)? Wie wird es in Verbindung zum Phänomen bzw. zu den Phänomenen und den anderen Komponenten verstanden? folgendermaßen beantwortet werden:

Die Befunde verdeutlichen (Abbildung 73), dass aus Sicht der Mentor/innen das Setzen von Handlungsstrategien in Zusammenhang mit dem Phänomen Zusammenarbeit auf einen Lernprozess zielt, der sich innerhalb kontextbezogener und personenspezifischer Gestaltungswelten abspielt. Vorrangig sind interaktive Momente auf Basis eines dialogorientierten Miteinanders und kommunikativen Austausches. Sachbezogene Aspekte im Lernprozesse verdeutlichen die Notwendigkeit, sich mit institutionellen Rahmenkonzepten und normativen Vorgaben zu beschäftigen.

Studierende richten ihre Handlungsstrategien vor allem auf kontextbezogene Inhalte zum Lehrberuf aus und treten mit dem Erproben im Handlungsfeld Schule in einen erfahrungsbasierten, sachbezogen-prospektiven Lernprozess ein. Hierbei gehen sie meist nach folgenden Schritten vor: planen - konzipieren - umsetzen - dokumentieren und reflektieren - innovieren.

So unterschiedlich sich der Lernprozess beider Interviewgruppen auch gestaltet, das Handeln der Akteurinnen/Akteure - Mentor/in und Studierende/ $\mathrm{r}$ - legt den Fokus auf Professionsentwicklung.

Aus den angeführten Erklärungen entsteht hierbei die Notwendigkeit, dass für Mentor/innen Inhalte der Ausbildungskonzepte transparent sind. Weiters sollen Werthaltungen dargelegt, offen diskutiert und besprochen werden. Was ist das Gemeinsame? Was ist das Trennende? Wo können sie sich wechselseitig unterstützen? Diese Fragestellungen vereinfachen die Interaktion, wenn sie geklärt werden, da Richtlinien und Regeln klargelegt wurden.

Letztendlich werden von beiden Interviewgruppen - Mentor/innen und Studierenden - professionsspezifische Handlungsstrategien im schulpraktischen Mentoringprozess gefordert und erwartet. Wenn aufgrund der Handlungsstrategien ein Lernprozess in Gang gesetzt wird, der auf einen Entwicklungsprozess zielt, erfüllt dies wohl Kriterien eines gelingenden Mentoringprozesses. Studierende beurteilen ihr Praktikum oftmals als erfolgreich, wenn sie durch Ausprobieren und Erproben von pädagogisch-praktischen Handlungen einen Lerngewinn erzielen und einen Fortschritt entdecken konnten. 


\subsection{Interpretation des Kontextes und der intervenierenden Bedingungen}

„Unter ,Kontextbedingungen` werden (...) diejenigen strukturellen Bedingungen subsummiert, die das untersuchte Phänomen rahmen - etwa örtliche, zeitliche oder biografische Rahmenbedingungen." (Heiser 2018, 232) Ergänzend zu Heisers Vorschlag (2018) werden in dieser Untersuchung noch normative und personale Bedingungen angeführt (Tabelle 58). Unter den intervenierenden Bedingungen werden generelle Vorbedingungen für Strategien erwähnt (Strübing 2014, 25). In einem zweischrittigen Rekonstruktionsprozess zu Kontext und intervenierenden Bedingungen wird auf die Bedeutung praktikumsspezifischer (curriculare, inhaltliche, normative, strukturelle bzw. organisatorische) sowie beziehungsorientierter personaler Bedingungen im schulpraktischen Mentoring hingewiesen.

Tab. 58: Praktikumsspezifische und beziehungsorientierte Bedingungen aus dem Rekonstruktionsprozess des Kontextes und der intervenierenden Bedingungen

\begin{tabular}{|c|c|c|c|c|}
\hline Ebenen & \multicolumn{2}{|c|}{$\begin{array}{l}\text { Lehrendenperspektive } \\
\text { Mentor/innenperspektive }\end{array}$} & \multicolumn{2}{|c|}{$\begin{array}{l}\text { Studierendenperspektive } \\
\text { Menteeperspektive }\end{array}$} \\
\hline $\begin{array}{l}\text { Phänomen }(\mathrm{e}) \\
\text { Kodierparadigma }\end{array}$ & Praktika & Interaktion & Praktika & $\begin{array}{l}\text { Erfahrungslernen } \\
\text { in den Praktika } \\
\text { mit Begleitung }\end{array}$ \\
\hline $\begin{array}{l}\text { Kontext und } \\
\text { intervenierende } \\
\text { Bedingungen } \\
\text { Kodierparadigma }\end{array}$ & $\begin{array}{l}\text { Bedingungs- } \\
\text { faktoren } \\
\text { zu Praktika } \\
\text { (Ist-Bereich) }\end{array}$ & $\begin{array}{l}\text { Bedingungs- } \\
\text { faktoren } \\
\text { zur Begleitung }\end{array}$ & $\begin{array}{l}\text { Bedingungs- } \\
\text { faktoren } \\
\text { zu Praktika }\end{array}$ & $\begin{array}{l}\text { Lehrer/innenper- } \\
\text { sönlichkeit; } \\
\text { Beziehungsgeflecht; } \\
\text { Rollendynamik; } \\
\text { Bedingungen der } \\
\text { Praktika }\end{array}$ \\
\hline Rekonstruktion 1 & $\begin{array}{c}\downarrow \\
\text { Curriculare } \\
\text { Anforderungen } \\
\text { an Praktika }\end{array}$ & $\begin{array}{c}\downarrow \\
\text { Mentoring- } \\
\text { Wissen }\end{array}$ & $\begin{array}{l}1 \\
\text { Curriculare } \\
\text { Anforderungen } \\
\text { an Praktika }\end{array}$ & $\begin{array}{c} \\
\text { Kompetentes, } \\
\text { beziehungs- } \\
\text { orientiertes } \\
\text { Mentoring }\end{array}$ \\
\hline $\begin{array}{l}\text { Phänomen }(\mathrm{e}) \\
\text { Kernkategorie }\end{array}$ & \multicolumn{2}{|c|}{ Zusammenarbeit } & \multicolumn{2}{|c|}{$\begin{array}{l}\text { Lernen in den Praktika } \\
\text { mit Begleitung }\end{array}$} \\
\hline $\begin{array}{l}\text { Kontext und } \\
\text { intervenierende } \\
\text { Bedingungen } \\
\text { Kernkategorie }\end{array}$ & \multicolumn{2}{|c|}{$\begin{array}{l}\text { Ausbildungs- und Berufserfahrungen; } \\
\text { Bedingungsfaktoren }\end{array}$} & \multicolumn{2}{|c|}{$\begin{array}{l}\text { Bedingungsfaktoren } \\
\text { im Praktikum }\end{array}$} \\
\hline Rekonstruktion 2 & \multicolumn{2}{|c|}{$\begin{array}{l}\text { Berufserfahrung und Wissen } \\
\text { in der Mentoring-Funktion }\end{array}$} & \multicolumn{2}{|c|}{$\begin{array}{l}\text { Kontextgeprägtes Handeln } \\
\text { im Praktikum }\end{array}$} \\
\hline Conclusio & \multicolumn{4}{|c|}{$\begin{array}{l}\text { Praktikumsspezifische und beziehungsorientierte Bedingungen } \\
\text { als Kontext und intervenierende Bedingungen des } \\
\text { schulpraktischen Mentorings }\end{array}$} \\
\hline
\end{tabular}


Studierendenperspektive bzw. Menteeperspektive: Studierende brauchen im Kontext zum Phänomen Praktika ein Wissen zu curricularen Anforderungen im Praktikum. Hierbei sind vor allem normative Vorgaben von Bedeutung. Diese zählen oftmals als wesentliche Elemente im Praktikum. Quantitative Angaben zu Hospitations- und zu haltenden Unterrichtseinheiten, Besprechungsstunden und Seitenangaben des Dokumentations- und Reflexionsteils werden als Beispiele genannt. Studierende erwarten sich beim Phänomen Erfahrungslernen in den Praktika mit Begleitung die Beachtung ihrer affektiv-emotionalen Bedingungslagen durch Mentor/innen. In dieser Erwartungshaltung zeigen Studierende auf, dass Mentor/innen je nach Beziehungsqualität, Beziehungskultur und Sensibilität Erfahrungslernen bedeutend unterstützen könnten, wenn sie Stimmungssituationen wahrnehmen, erkennen und darauf adäquat reagieren würden. Studierende sind im Kontext Mentoring auf Mentor/innen angewiesen und erleben geglückte und misslungene Rollendynamiken, an denen sie so manchen Lern- und Entwicklungsfortschritt festmachen. Im Kontext wird der Beziehungsaspekt zur/zum Mentor/in für Studierende sehr wichtig. Sie verlangen und fordern eine Lehrer/innenpersönlichkeit, die für die Aufgabenstellung als Mentor/in bereit und gewillt ist und die auch Aufgaben einer Lehrperson nach Ansicht der Studierenden professionell erfüllt. Studierende gehen davon aus, dass Bedingungsfaktoren zur Abhaltung von Praktika seitens institutioneller Vorgaben für Mentor/innen transparent und klar geregelt sind und sie als Unterstützer/in, wenn nötig und gewünscht, fungieren. Resümierend kann gesagt werden, dass sich Studierende ein kompetentes, beziehungsorientiertes Mentoring erwarten - dann ist für sie ein kontextgeprägtes Handeln im Praktikum möglich.

Lehrendenperspektive bzw. Mentor/innenperspektive: Mentor/innen brauchen zum Phänomen Praktika ein Verständnis und ein Wissen zu curricularen Anforderungen der Praktika, um die Tätigkeit kompetent ausführen zu können. Zum Phänomen Interaktion wollen Mentor/ innen auch mit Faktoren der Begleitung wie Kommunikationsstrategien vertraut sein. In der Rekonstruktion ergibt sich daher, dass Mentor/innen aus ihrer Sicht ein Mentoring-Wissen zur Ausübung benötigen. Im Kontext zum Phänomen Zusammenarbeit spielen Ausbildungs- und Berufserfahrungen sowie Bedingungsfaktoren zu Praktika und Begleitung eine zentrale Rolle. Sie veranlassen Mentor/innen zu Handlungsweisen und zum Handlungsvollzug.

„Also die Verzahnung wäre für mich das ganz Wichtigste (...). Dass wir als Praktiker einmal im Jahr oder zweimal im Jahr - würde sich jeder bereit erklären - Kontakt aufnimmt, mit ganz konkreten Fragen oder auch ohne Programm. Also nur so Kontakt aufnimmt: was stellt ihr euch vor? Also ein bisschen mehr Kontaktaufnahme noch zwischen PHs und Schule - das wäre für mich, das würde mich sehr interessieren." (L11; 2270-2275)

Daraus lässt sich ableiten, dass im Kontext Berufserfahrung ein Wissen in der Mentoring-Funktion hilfreich ist, um im Kontext Prozesse mit Mentee gestalten zu können.

In der Zusammenschau des Kontextes und der intervenierenden Bedingungen ergibt sich aus Lehrenden- und Studierendenperspektive die Notwendigkeit der Beachtung praktikumsspezifischer sowie beziehungsorientierter Bedingungen im schulpraktischen Mentoring. Die folgende Abbildung analysiert noch einmal die wesentlichen Faktoren aus Sicht der Interviewgruppen und zeigt Verbindungen zwischen den Kriterien auf (Abbildung 74).

Die Analyse aus Kontext und intervenierenden Bedingungen macht deutlich, dass praktikumsspezifische und beziehungsorientierte Bedingungen den Rahmen bilden. Hierbei leiten sich für die Interviewgruppen verschiedene Fragestellungen ab. Lehrpersonen und Mentor/innen suchen Antworten zu folgenden Fragen: Welche Bedingungsfaktoren gibt es für mich auf institutioneller, curricularer, struktureller und organisatorischer Ebene? Mit welcher Haltung begegne 
ich Studierenden in der Begleitung? Was sind meine persönlichen Anforderungen und Erwartungen an Studierende? Was habe ich in meiner Ausbildung und bisherigen Berufslaufbahn erlebt? Was ist mir in der Begleitung und somit in der Mentoring-Funktion wichtig?

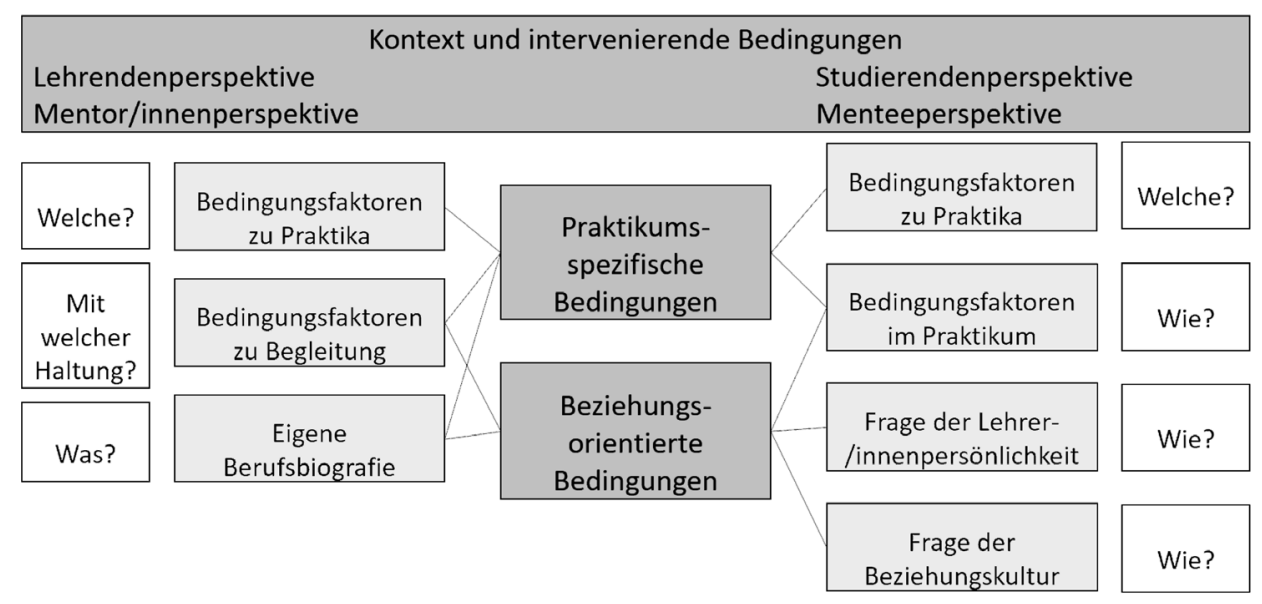

Abb. 74: Analyse zu Kontext und intervenierenden Bedingungen

Mentor/innen im schulpraktischen Mentoringprozess konzentrieren sich auf Fragestellungen, die sich auf eine curriculare und strukturelle Ebene sowie auf Intra- und Intersubjektivität im Lehrberuf und im Rahmen der Mentoring-Funktion beziehen. Studierende betrachten ebenso die strukturelle Ebene und in weiterer Folge vor allem die Intersubjektivität im Begleitungsprozess. Fragestellungen der Studierenden sind in der Mehrheit als Modalsätze formuliert: Welche institutionellen, normativ-curricularen und organisatorischen Anforderungen (Bedingungsfaktoren) ergeben sich für mich als Studierende/r zu Praktika und im Praktikum? Wie gestalten sich für mich persönlich Bedingungsfaktoren im Praktikum? Wie agiert die/der Mentor/in aus meiner Sicht im Lehrberuf? Wie erlebe ich meine/n Mentor/in als Lehrer/innenpersönlichkeit? Wie erlebe ich die Beziehung zur/zum Mentor/in? Wie erwarte ich mir, wahrgenommen zu werden? Wie wird die Beziehung seitens der Mentor/in gestaltet?

Aus dem Rekonstruktionsprozess kann geschlossen werden, dass beim Mentoringprozess eine Klärung praktikumsspezifischer Bedingungsfaktoren von beiden Interviewgruppen als notwendig erachtet wird. Mentor/innen benötigen außerdem Transparenz für Bedingungsfaktoren zur Begleitung und somit ein Mentoring-Wissen. Institutionen der jeweiligen Ausbildungen sind gefordert, Kontakt mit Mentor/innen zu halten, sie in Konzeptentwicklungen zu integrieren und im Sinne der Qualitätssicherung in Austausch zu gehen. Anforderungen, Aufgabenbereiche, Prüfungsmodalitäten usw. sind vor Beginn des Begleitungsprozesses abzuklären und als festgelegte Formalitäten bekannt zu geben. Für Studierende ist besonders auch die Beachtung psychosozialer und personal-emotionaler Bedingungen durch Mentor/innen essentiell. Mit der Darstellung des Kontextes und der intervenierenden Bedingungen wird bewusst, dass Studierende in der Begleitsituation ein Abhängigkeitsgefühl verspüren und bei ihnen dadurch Unsicherheitsfaktoren vorhanden sind. Die Befunde der Inhalte zum Kontext und den intervenierenden Bedingungen, die als Sach- und Situationszusammenhänge im schulpraktischen Mentoring verstanden werden, sind für das Phänomen Professionsentwicklung schließlich mittragend. 


\subsection{Interpretation der Konsequenzen}

„Aus Handlungen ergeben sich Folgen, die wir dem Feld ,Konsequenzen ' zuordnen“. (Heiser 2018, 232) Im Folgenden werden die Konsequenzen der Kodierparadigmata und Kernkategorien näher beleuchtet und in Anlehnung an das Konzept von Kram (1985) analysiert. Tabelle 59 verweist auf Phänomene und Konsequenzen aus den Kodierparadigmata und Kernkategorien und veranschaulicht den zweischrittigen Rekonstruktionsprozess. Nach Aufstellung der Professionsspezifischen Funktionen als Lehrperson, Mentoring-Funktionen, Rollenfunktionen, Psychosozialen und personal-emotionalen Aspekten, Forderung an die Mentoring-Rolle, Aufbau professionsspezifischer Kompetenzfunktionen über die Verfügbarkeit eines Rollenmodells ergibt sich der Rückschluss der Notwendigkeit schulpraktischer Mentoring-Funktionen im Mentoringprozess. Anbei erfolgt die Darstellung der Rekonstruktionsschritte und deren Beschreibung (Tabelle 59):

Tab. 59: Schulpraktische Mentoring-Funktionen aus dem Rekonstruktionsprozess der Konsequenzen

\begin{tabular}{|c|c|c|c|c|}
\hline Ebenen & \multicolumn{2}{|c|}{$\begin{array}{l}\text { Lehrendenperspektive } \\
\text { Mentor/innenperspektive }\end{array}$} & \multicolumn{2}{|c|}{$\begin{array}{l}\text { Studierendenperspektive } \\
\text { Menteeperspektive }\end{array}$} \\
\hline $\begin{array}{l}\text { Phänomen }(\mathrm{e}) \\
\text { Kodierparadigma }\end{array}$ & Praktika & Interaktion & Praktika & $\begin{array}{l}\text { Erfahrungslernen } \\
\text { in den Praktika } \\
\text { mit Begleitung }\end{array}$ \\
\hline $\begin{array}{l}\text { Konsequenz(en) } \\
\text { Kodierparadigma }\end{array}$ & $\begin{array}{l}\text { Verantwortungs- } \\
\text { bewusstsein für } \\
\text { Begleitung }\end{array}$ & $\begin{array}{l}\text { Fördern und Stärken } \\
\text { für den gegen- } \\
\text { wärtigen und zu- } \\
\text { künftigen Lehrberuf }\end{array}$ & $\begin{array}{l}\text { Wunsch } \\
\text { nach } \\
\text { professioneller } \\
\text { Begleitung }\end{array}$ & $\begin{array}{l}\text { Klares Wissen } \\
\text { um Rolle und } \\
\text { Aufgabe in der } \\
\text { Begleitung }\end{array}$ \\
\hline Rekonstruktion 1 & $\underset{\text { Rollenfunktionen }}{\downarrow}$ & $\begin{array}{l}\mathbf{\nabla} \\
\text { Professions- } \\
\text { spezifische } \\
\text { Funktionen }\end{array}$ & $\begin{array}{c}\mathbf{V} \\
\text { Psychosoziale und } \\
\text { personal-emotionale } \\
\text { Funktionen }\end{array}$ & $\begin{array}{c} \\
\text { Forderung an die } \\
\text { Mentoring-Rolle }\end{array}$ \\
\hline $\begin{array}{l}\text { Phänomen }(\mathrm{e}) \\
\text { Kernkategorie }\end{array}$ & \multicolumn{2}{|c|}{ Zusammenarbeit } & \multicolumn{2}{|c|}{$\begin{array}{l}\text { Lernen in den Praktika } \\
\text { mit Begleitung }\end{array}$} \\
\hline $\begin{array}{l}\text { Konsequenz(en) } \\
\text { Kernkategorie }\end{array}$ & \multicolumn{2}{|c|}{$\begin{array}{l}\text { Bereitschaft zur } \\
\text { mentoriellen Betreuung und Weiterent- } \\
\text { wicklung (in) der Profession }\end{array}$} & \multicolumn{2}{|c|}{$\begin{array}{l}\text { Aufbau von } \\
\text { Lehrer/innenexpertise über } \\
\text { professionelle Begleitung }\end{array}$} \\
\hline Rekonstruktion 2 & Mentorin & -Funktionen & $\begin{array}{l}A \nu \\
\text { professionsspezifische } \\
\text { über Verfügbarkei }\end{array}$ & $\begin{array}{l}\text { Kau } \\
\text { Kompetenzfunktionen } \\
\text { eines Rollenmodells }\end{array}$ \\
\hline Conclusio & \multicolumn{4}{|c|}{ Schulpraktische Mentoring-Funktionen } \\
\hline
\end{tabular}

Studierendenperspektive bzw. Menteeperspektive: Die Konsequenz zum Phänomen Praktika offeriert den Wunsch der Studierenden nach professioneller Begleitung. Hierbei sollen vor allem psychosoziale und personal-emotionale Aspekte im Begleitungsprozess berücksichtigt wer- 
den. Im zweiten Kodierparadigma der Studierendenperspektive konnte die Konsequenz Klares Wissen um Rolle und Aufgabe der Begleitung herausgearbeitet werden. Normativ-curriculare Anforderungen und Ansprüche sowie subjektive Deutungen sollen im Wissen und in der Handlungsweise der Mentor/innen verankert bzw. für Studierende transparent und offenkundig sein. Die Beschreibung dieser Konsequenz resümiert im Rekonstruktionsprozess in eine Forderung an die Mentoring-Rolle. Die Konsequenz in der Kernkategorie Aufbau von Lehrer/innenexpertise über professionelle Begleitung zum Phänomen Lernen in den Praktika mit Begleitung verdeutlicht den Wunsch der Studierenden nach Gewährleistung des Aufbaus professionsspezifischer Kompetenzfunktionen über die Verfügbarkeit eines Rollenmodells. Studierende fordern von der Begleitperson ein Verhalten ein, das Mentor/innen über eine Rolle realisieren können.

Lehrendenperspektive bzw. Mentor/innenperspektive: Die Konsequenz zum Phänomen Praktika aus der Lehrendenperspektive lautet Verantwortungsbewusstsein für Begleitung. Mentor/ innen sind in der Mentoring-Funktion gewillt und bereit, Verantwortung zu übernehmen und betrachten diesen Aspekt als besonders notwendig. Verantwortung als abstraktes Prinzip wird im Rekonstruktionsprozess der Rollenfunktion zugeschrieben. Die Konsequenzen Fördern und Stärken für den gegenwärtigen und zukünftigen Lehrberuf sind professionsspezifischen Funktionen zuzuordnen. Mentor/innen sehen durch die Begleitungstätigkeit einen Mehrgewinn für das eigene pädagogische Handeln und bieten in der Funktion der/des Mentorin/Mentors der/ dem Mentee Unterstützung, Sicherheit und den Raum eines lernorientierten Umfeldes an.

„Diese Studierenden, die während der Ausbildung kommen, die sind oft sehr eifrig und wollen Sachen ausprobieren. Da stelle ich dann gerne meine Expertisen oder auch meine Zeit zur Verfügung, natürlich auch den Rahmen oder auch die Klassen, in denen das gut funktionieren wird. Schließlich ist es auch für mich eine Bereicherung." (L05; 973-977)

Die Konsequenz Bereitschaft zur mentoriellen Betreuung und Weiterentwicklung (in) der Profession weist auf die Bedeutung von Mentoring-Funktionen hin, welche sich im zweiten Schritt des Rekonstruktionsprozesses herauskristallisierten.

Summierend aus den Konsequenzen und Rekonstruktionsschritten lassen sich Schulpraktische Mentoring-Funktionen ableiten, die unterschiedliche inhaltliche Elemente in den Mittelpunkt stellen (Abbildung 75).

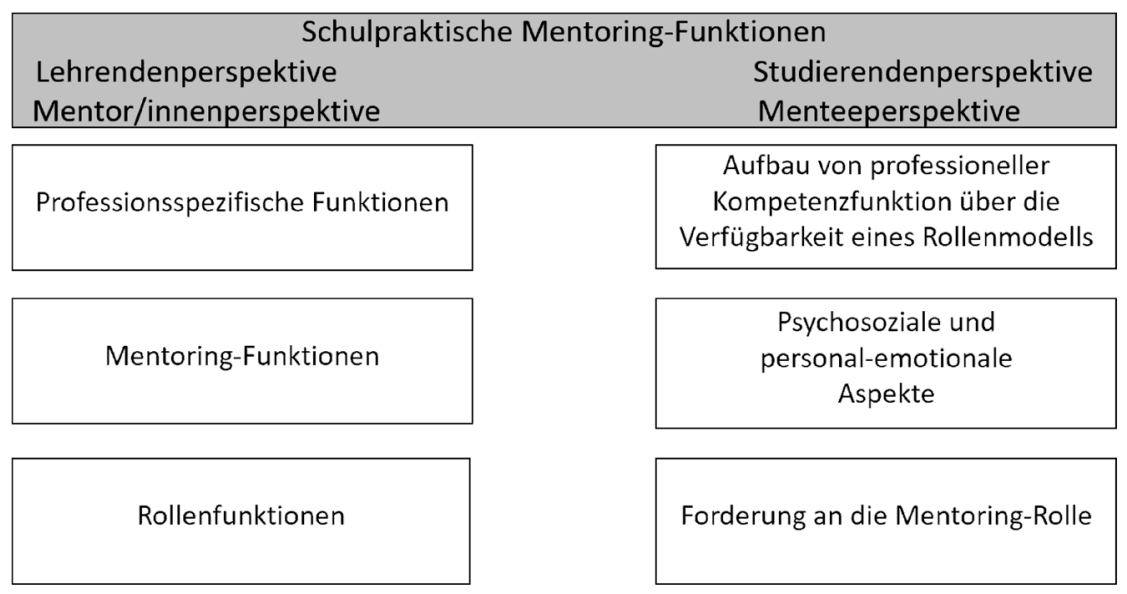

Abb. 75: Analyse zu Konsequenzen in Form von Schulpraktischen Mentoring-Funktionen 
Die Inhalte zu Rollenfunktionen, professionsspezifische Funktionen, Mentoring-Funktionen, psychosoziale und personal-emotionale Aspekte, Forderung an die Mentoring-Rolle, Aufbau professionsspezifischer Kompetenzfunktionen über die Verfügbarkeit eines Rollenmodells verweisen auf subjektive Deutungsmuster, Anforderungsstrukturen, Ermöglichungs- und Erwartungshaltungen. In Tabelle 60 und Tabelle 61 werden erneut die Schulpraktischen Mentoring-Funktionen aus Lehrenden- und Studierendenperspektive und Kategorien aufgelistet. In einem weiteren Rekonstruktionsprozess wurden die angeführten Kategorien thematisch zusammengefasst und verdichtet und auf eine weitere Metaebene gehoben. In diesem Zusammenhang kann von Metakategorien der schulpraktischen Mentoring-Funktionen gesprochen werden.

Studierende fordern Rollenfunktionen der Begleitpersonen ein, wünschen sich die Beachtung psychosozialer und emotionaler Aspekte und sind für sich am Aufbau von professionellen Kompetenzfunktionen über die Verfügbarkeit eines Rollenmodells im schulpraktischen Mentoringprozess interessiert.

Tab. 60: Kategorien und Metakategorien zu Schulpraktischen Mentoring-Funktionen aus Studierendenperspektive bzw. Menteeperspektive

\begin{tabular}{|c|c|c|}
\hline $\begin{array}{l}\text { Mentoring-Funktionen } \\
\text { in den Schulpraktika }\end{array}$ & Kategorien & Metakategorien \\
\hline \multicolumn{3}{|c|}{ Studierendenperspektive bzw. Menteeperspektive } \\
\hline \multirow{3}{*}{$\begin{array}{l}\text { Aufbau professions- } \\
\text { spezifischer } \\
\text { Kompetenzfunktionen } \\
\text { über Verfügbarkeit eines } \\
\text { Rollenmodells }\end{array}$} & $\begin{array}{l}\text { Stellenwert der Praktika } \\
\text { Kennenlernen des Systems Schule } \\
\text { Einbindung in das System Schule } \\
\text { Theorie-Praxis-Bezug }\end{array}$ & $\begin{array}{l}\text { Praktikum als berufliches } \\
\text { Lernfeld }\end{array}$ \\
\hline & $\begin{array}{l}\text { Lern- und Erfahrungsprozess } \\
\text { Entwicklungsprozess } \\
\text { Reflexionsprozess }\end{array}$ & $\begin{array}{l}\text { Professionsspezifische } \\
\text { Selbstwirksamkeit }\end{array}$ \\
\hline & $\begin{array}{l}\text { Beziehungsstruktur } \\
\text { Wunsch nach Authentizität } \\
\text { Bereichernder Austausch } \\
\text { Kommunikationsverhalten }\end{array}$ & $\begin{array}{l}\text { Kommunikation und } \\
\text { Interaktion }\end{array}$ \\
\hline $\begin{array}{l}\text { Psychosoziale und } \\
\text { personal-emotionale } \\
\text { Aspekte }\end{array}$ & $\begin{array}{l}\text { Dauer und Art der Praktika } \\
\text { Herausforderung } \\
\text { Belastung } \\
\text { Feedbackkultur } \\
\text { Ebenenbetrachtung } \\
\text { Beziehungsverhältnis } \\
\text { Vertrauensaspekt } \\
\text { Distanz und Nähe }\end{array}$ & $\begin{array}{l}\text { Individuelle psychosoziale } \\
\text { Erfahrungen und Kompetenz- } \\
\text { entwicklung im Praktikum }\end{array}$ \\
\hline \multirow[t]{3}{*}{$\begin{array}{l}\text { Forderung an die } \\
\text { Mentoring-Rolle }\end{array}$} & $\begin{array}{l}\text { Engagement und Motivation } \\
\text { Interesse der/des Mentorin/Mentors } \\
\text { Vorhandene Zeitressource } \\
\text { Auswahlverfahren für Mentor/in } \\
\end{array}$ & Mentoring-Organisation \\
\hline & $\begin{array}{l}\text { Vorhandenes Rollenverständnis } \\
\text { Rollenbild } \\
\text { Wechselseitiger Lernprozess } \\
\text { Tätigkeit als Herausforderung/Belastung }\end{array}$ & Mentoring-Verständnis \\
\hline & $\begin{array}{l}\text { Professionalität als Lehrperson } \\
\text { Lehrer/innenpersönlichkeit }\end{array}$ & Mentoring-Professionalität \\
\hline
\end{tabular}


Praktikum als berufliches Lernfeld: Aus Studierendenperspektive haben Praktika einen hohen Stellenwert. Die Öffnung des Berufsfeldes Schule während der Zeit der Ausbildung ermöglicht ein Kennenlernen des Systems und gleichzeitig bereits eine Einbindung ins System. Die allgemeine Befürwortung einer vermehrten Anzahl an Praktika und die Tendenz für Praktika mit längerer Verweildauer an Schulen gehen mit Argumenten zur Effizienz der Selbstwirksamkeit sowie Bedeutung des Aufbaus eines Bezugs zur Klasse und Schülerinnen und Schülern einher.

Professionsspezifische Selbstwirksamkeit: Studierende betrachten Praktika als Mehrgewinn, wenn sie sich im Handlungsfeld erproben können, Neues einbringen dürfen, Vorschläge zur Verbesserung und konstruktives Feedback erhalten, bei sich selbst im Laufe der Zeit einen Fortschritt erkennen und einen Lernzuwachs feststellen können. Sie geben auch an, dass die Anleitung zu Reflexionsprozessen hilfreich, wenn auch aufgrund des Schreibausmaßes oft mühsam und hartnäckig ist. Das Eintauchen in einen Lern- und Entwicklungsprozess wird als Ziel verfolgt, wenn personenbezogene Unterstützung und Begleitung als notwendig erachtet werden. Letztendlich dienen Praktika in Bezug auf professionsspezifische Selbstwirksamkeit zur Gewinnung von Sicherheit, zum Erleben von Erfolgen und Bestätigungen im pädagogischen Handeln und dazu, eigenverantwortliches Handeln auszuprobieren.

Kommunikation und Interaktion: Studierende fühlen sich in einer gewissen Abhängigkeitsposition zu ihrer/ihrem Mentor/in. Sie berichten im Rückblick ihrer absolvierten Praktika sowohl von positiven Erlebnissen als auch negativen Erfahrungen. Gewünscht wird ein bereichernder Austausch, ein regelmäßiges konstruktives Feedback und nicht nur das Hören des Standardsatzes „Ja, passt schon!“. Weiters soll Positives herausgestrichen werden. In der Interaktion wird ein ehrliches miteinander Umgehen und der verstärkte Wunsch, als Studierende/r authentisch sein und bleiben zu können, geäußert.

Individuelle psychosoziale Erfahrungen und Kompetenzentwicklung im Praktikum: Studierende sind in Zusammenhang mit schulpraktischen Mentoring-Funktionen darauf bedacht, dass Mentor/innen psychosoziale und personal-emotionale Aspekte berücksichtigen. Je nach Art und Dauer der Praktika sind Studierende emotionalen Belastungen und Herausforderungen ausgesetzt. Hierbei treten unterschiedliche psychische Belastungssymptome auf. In dem gewünschten persönlichen und kollegialen Vertrauensverhältnis mit der/dem Mentor/in ist es Studierenden möglich, Schwierigkeiten und Probleme anzusprechen. Die Erwartungshaltung der Studierenden ist, dass Mentor/innen in ihrer Rollenfunktion adäquat auf sie eingehen. Die Kunst für Mentor/innen ist es, das Verhalten Distanz und Nähe in Abstimmung mit dem Gegenüber situationsgebunden einzusetzen. Sind für Studierende optimale psychosoziale Rahmenbedingungen geschaffen und ist ein Klima des Wohlwollens vorhanden, dann wünschen sich Studierende, ihnen ein selbständiges Erfahrungslernen zu ermöglichen und nur zu unterstützen, falls es notwendig ist.

Mentoring-Mission: Studierende nennen in ihrer Erwartungshaltung und meist aus leidvollen negativen Erfahrungsberichten vier wesentliche Kriterien zur Mentoring-Mission: Motivation, Interesse, Engagement und Zeitressource.

Mentoring-Organisation: Für Studierende ist das Auswahlverfahren der Mentor/innen von zentraler Bedeutung. Besonders bei Anstellung von Mentor/innen sollte ihrer Meinung nach auf professionsspezifische Kriterien wie Fachwissen, pädagogische Haltung und Führung, Einsatz von „zeitgemäßer“ Pädagogik sowie auf den Nachweis der Absolvierung einer Mentor/innenausbildung geachtet werden. Weiters sollten Mentor/innen eine positive Grundeinstellung zum System Schule mitbringen, motiviert und interessiert für den Umgang mit Lernenden sein und vor Beauftragung ein Motivationsschreiben verfassen müssen. Studierende schlagen die 
Einrichtung einer unabhängigen Beschwerdestelle vor, sollte es zu Problemen in der Betreuung durch Mentor/innen kommen. Einige Aussagen gingen auch dahin, dass die Kombination Mentor/in und Mentee auf Beziehungsebene wohl nicht immer dem Optimalfall entspreche und die Möglichkeit eines Wechsels gewährleistet werden sollte.

Mentoring-Verständnis: Studierende fordern, dass Mentor/innen ein Rollenverständnis mitbringen und in ihrer/seiner Mentoring-Rolle gefestigt sind. Sie betonen, dass durch Mentoring ein wechselseitiger Lernprozess entsteht. Nicht selten beobachteten Studierende, dass Mentor/ innen Konzepte und Methoden in Anlehnung an studentisches Handeln in ihr eigenes Handlungsrepertoire aufnahmen. Studierende erfassen den zeitlichen und persönlichen Mehraufwand der Mentor/innen, der sich durch die Betreuungstätigkeit ergibt. Manche Mentor/innen wirken gestresst, genervt bzw. überfordert.

Mentoring-Professionalität: In der Erwartungshaltung gehen Studierende von einem Expertiseansatz in der Mentoring-Funktion aus. Vorhandenes Wissen um praktikumsspezifische Anforderungen, Kenntnisse über Beziehungsaspekte und Inhaltsaspekte in der Begleitung sowie Fähigkeit zum Aufbau einer stärkenorientierten Beziehungskultur sind Kriterien, die Mentor/ innen in den Augen der Studierenden haben sollten. Studierende fordern in der Rollenfunktion der/des Mentorin/Mentors Lehrer/innenpersönlichkeiten mit einem professionellen Verständnis in der Ausübung des Lehrberufs.

Eine Erklärung der Inhalte der Kategorien und Metakategorien in Zusammenhang mit den Schulpraktischen Mentoring-Funktionen aus Lehrendenperspekivte wird im Weiteren angeführt. Lehrpersonen übernehmen mit der Aufgabe Mentor/in aus ihrer Sicht professionsspezifische Funktionen sowohl als Lehrperson, Mentoring-Funktionen als auch Rollenfunktionen.

Tab. 61: Kategorien und Metakategorien zu Schulpraktischen Mentoring-Funktionen aus Lehrendenperspektive bzw. Mentor/innenperspektive

\begin{tabular}{|c|c|c|}
\hline $\begin{array}{l}\text { Mentoring-Funktionen } \\
\text { in den Schulpraktika }\end{array}$ & Kategorien & Metakategorien \\
\hline \multicolumn{3}{|c|}{ Lehrendenperspektive bzw. Mentor/innenperspektive } \\
\hline \multirow[t]{2}{*}{$\begin{array}{l}\text { Professionsspezifische } \\
\text { Funktionen als } \\
\text { Lehrperson }\end{array}$} & $\begin{array}{l}\text { Zusammenarbeit mit Institutionen } \\
\text { Ausbildungsmodus Neu } \\
\text { Theorie-Praxis-Verschränkung }\end{array}$ & $\begin{array}{l}\text { Professionsspezifische } \\
\text { Zusammenarbeit und Integration }\end{array}$ \\
\hline & Wechselseitiger Lern- und Reflexionsprozess & Gegenstandsbezogener Austausch \\
\hline \multirow{3}{*}{ Mentoring-Funktion } & $\begin{array}{l}\text { Übernahme einer Aufgabe } \\
\text { Entdeckung beruflicher Vielfalt } \\
\text { Ansehenhebung des Lehrberufs } \\
\text { Problembetrachtung als Aufgabe }\end{array}$ & Selbstkonzept Mentor/in \\
\hline & $\begin{array}{l}\text { Öffnen des Systems Schule } \\
\text { Möglichkeit der Probeidentifikation } \\
\text { Umgang mit Schulkindern } \\
\text { Entwicklungspotentialentfaltung }\end{array}$ & $\begin{array}{l}\text { Verständnis für Identifikations- } \\
\text { prozess hin zum Lehrberuf }\end{array}$ \\
\hline & $\begin{array}{l}\text { Feedbackkultur } \\
\text { Beziehungskultur } \\
\text { Unterstützungsgeleitetes Begleiten } \\
\text { Kommunikativer Austausch } \\
\text { Wahrnehmen der Persönlichkeitsstruktur und } \\
\text { psychischen Grundstimmung }\end{array}$ & Psychosozialer Austausch \\
\hline
\end{tabular}




\begin{tabular}{|c|c|c|}
\hline $\begin{array}{l}\text { Mentoring-Funktionen } \\
\text { in den Schulpraktika }\end{array}$ & Kategorien & Metakategorien \\
\hline \multirow{3}{*}{ Rollenfunktionen } & $\begin{array}{l}\text { Aspekte der Motivation } \\
\text { Aufgabe mit Verantwortung } \\
\text { Vorbildfunktion } \\
\text { Eigenerfahrungen }\end{array}$ & Mentoring-Mission \\
\hline & $\begin{array}{l}\text { Rollenverständnis } \\
\text { Persönliche Ressourcen } \\
\text { Persönlicher Reflexionsprozess } \\
\text { Problembetrachtung } \\
\text { Stellenwert, Dauer und Inhalt der Praktika }\end{array}$ & Mentoring-Identität \\
\hline & $\begin{array}{l}\text { Empathiefähigkeit } \\
\text { Kommunikative Grundhaltung } \\
\text { Beziehungsgestaltung } \\
\text { Ebenenbetrachtung }\end{array}$ & Psychosoziale Moment(e) \\
\hline
\end{tabular}

Professionsspezifische Zusammenarbeit und Integration: Das Ausbildungskonzept hat sich durch Reformprozesse im Zuge von Pädagog/innenbildung NEU (siehe Kapitel I/1.1.2) stark verändert. Mentor/innen sind interessiert an der Zusammenarbeit mit Institutionen, wollen Strukturen und Konzepte kennen- und verstehenlernen. Ein wesentlicher Aspekt für die eigene Professionsentwicklung ist die Bedeutsamkeit der Theorie-Praxis-Verschränkung. „Up-to-datezu sein" als Schlagwort kennzeichnet das Interesse an der Aneignung von Wissen über die aktuellen bildungswissenschaftlichen, fachwissenschaftlichen und fachdidaktischen Erkenntnisse, die seitens der tertiären Ausbildungsinstitution vermittelt werden und in den schulischen Anteilen erfahrbar gemacht werden können.

Gegenstandsbezogener Austausch: Lehrpersonen lernen in den Praktika durch Impulse der Vertreter/innen der Ausbildungsinstitutionen und Studierenden. In Folge reflektieren sie durch äußere Anregungen bewusster eigene Handlungen, überlegen sich Unterrichtskonzeptionen und pädagogische Handlungen im Vorfeld intensiver und treten somit verstärkt in eine forschende Haltung ein. Lern- und Reflexionsanstöße durch den gegenstandsbezogenen Austausch aktivieren den Lern- und Entwicklungsprozess der Lehrperson.

Selbstkonzept Mentor/in: Für die Ausübung der Mentoring-Funktion braucht es Bereitschaft zur Übernahme der Aufgabe und Überzeugung, Eigenschaften und Fähigkeiten mitzubringen, um diese Aufgabe zu erfüllen. Mentor/innen entdecken durch Begleitungstätigkeit für sich ein erweitertes Aufgabenfeld innerhalb des Lehrberufs. Sie kommen auch in ein Kettenwirkungsdenken, wenn sie glauben, dass sie durch Sichtbarwerden der benötigten Qualifikationen im Lehrberuf dazu beitragen können, das Ansehen des Lehrberufs zu heben. Mentor/innen sind auch gewillt, sich mit Problemsituationen auseinanderzusetzen. Sie betrachten dies sogar als eine Aufgabe im Rahmen ihrer Tätigkeit.

Verständnis für Identifikationsprozess hin zum Lehrberuf: Mentor/innen bieten den Lernund Erfahrungsraum Schule für Studierende an, indem sie das System Schule für sie öffnen und Möglichkeiten zur Probeidentifikation im Rahmen pädagogisch-praktischer Handlungsausübungen und Reflexionseinheiten schaffen. Es ist ihnen auch ein Anliegen, dass Studierende Lerngelegenheiten der Schüler/innen beobachten, Lernstand evaluieren und Lernen fördern können und somit in einen Austausch mit ihnen kommen. Ein Hauptanliegen in der Ausübung der Mentoring-Funktion und zum Verständnis für einen Identifikationsprozess hin zum Lehrberuf besteht darin, zur Entwicklungspotentialentfaltung beitragen zu können, wie Konflikt- 
management, Selbständigkeit, Auftreten, Selbstbewusstsein, Selbsteinschätzung, Reflexionsfähigkeit.

Psychosozialer Austausch: Unter diesen Aspekt fallen Fragen der Feedbackkultur und der Gestaltung der Beziehungskultur innerhalb der Mentoring-Funktion. Absichtserklärungen deuten darauf hin, dass Mentor/innen ein unterstützungsgeleitetes Begleiten mit Schaffen von entgegenkommenden Voraussetzungen, Zeit nehmen für Gespräche, Freiräume geben bevorzugen und gerne in einen kommunikativen Austausch mit angehenden Lehrpersonen kommen wollen. Ein Anliegen der Studierenden ist es, dass psychosoziale und personal-emotionale Aspekte wahrgenommen werden. Mentor/innen beschreiben unterschiedliche Persönlichkeitsstrukturen und nehmen psychische Grundstimmungen wie Ängste, Stress, Freude, Unsicherheit, Nervosität, Überforderung der Studierenden zwar wahr, jedoch beschreiben kein Konzept, keine Kategorie oder Subkategorie der Untersuchung, wie Mentor/innen konkret damit umgehen. Sie bleiben hierbei eher in einer Wahrnehmungsposition und äußern keine konkreten Handlungsstrategien. Vielleicht weil sie auch dazu nicht gefragt wurden.

Mentoring-Mission: Die Mentoring-Mission kann der Rollenfunktion zugeordnet werden. Die zentralen Fragen zur Mentoring-Mission sind: Warum übe ich die Mentoring-Funktion aus? Was sind meine Motive dafür? Mentor/innen geben ichbezogene, student/innenbezogene und systembezogene Motivationsgründe zur Erklärung an. Miteinhergehend sind Bereitschaft der Aufgabenverantwortung und Vorbildseinwollen oder sich persönlich in einer Vorbildfunktion zu sehen. Häufig spielen positiv oder negativ erlebte Eigenerfahrungen bei der Entscheidung der Übernahme der Rollenfunktion Mentor/in mit.

Mentoring-Identität: Zur Mentoring-Identität gehören Klärung des Rollenverständnisses, Beachtung der persönlichen Ressourcen im Sinne der Psychohygiene, Bereitschaft, in einen Reflexionsprozess zu treten, Wissen um die Tatsache der Auseinandersetzung möglicher Problembereiche und Kenntnisse der normativ-curricularen Vorgaben.

Psychosoziale Moment(e): Auch innerhalb der Rollenfunktion ist eine Beachtung der psychosozialen Komponente wichtig. Aus Sicht der Lehrpersonen braucht es für die Ausübung der Tätigkeit Empathiefähigkeit und eine kommunikative Grundhaltung, klare Vorstellungen zur Beziehungsgestaltung und Ebenenbetrachtung bzw. des Hierarchieverständnisses. In der Rollenfunktion Mentor/in erhält der Beziehungsaspekt einen hohen Stellenwert.

Die genannten und beschriebenen Metakategorien der Schulpraktischen Mentoring-Funktionen erlauben einen weiteren Vertiefungsprozess und eine Gegenüberstellung der Perspektiven der Interviewgruppen - nachzulesen im folgenden Kapitel unter Befund 3.

In diesem Kapitel wurden Phänomene, ursächliche Bedingungen, Handlungsstrategien, Kontexte und intervenierende Bedingungen sowie Konsequenzen aus den Kodierparadigmata und Kernkategorien der Untersuchung diskutiert und weiteren Rekonstruktionsprozessen unterworfen. Hierbei wurden Professionsentwicklung, Inhalts- und Beziehungsaspekte, kontextbezogener und personenspezifischer Lernprozess, praktikumsspezifische und beziehungsorientierte Bedingungen als wesentliche Elemente im schulpraktischen Mentoringprozess identifiziert. Im folgenden Kapitel geht es um die Integrationsleistung der einzelnen genannten Elemente innerhalb der paradigmatischen Positionierungen aus den Kodierparadigmata und Kernkategorien und das Konzipieren von Zusammenhangsmodellen. 


\section{Integration und Kontextualisierung der Befunde}

Im Kapitel Integration und Kontextualisierung der Befunde werden die bisher dokumentierten Ergebnisse auf einer weiteren Metaebene zusammengeführt und die Forschungsfrage und binnendifferenzierten Fragestellungen zu Gelingensbedingungen im schulpraktischen Mentoringprozess mit weiteren Befunden in Beziehung und in Verbindung mit bestehenden Konzepten und Modellen gesetzt. Weiters werden ein Modell zu Analyse Transformationales Mentoring (ATM-Modell) und das Mentoringinduzierte Wirkungsmodell zum schulpraktischen Mentoringprozess (MIW-Modell) als Befunde der vorliegenden Studie vorgestellt und diskutiert. Durch das weitere Verdichten und Sortieren der bisherigen Befunde der vorliegenden Studie erfolgt somit eine weitere „Strukturgebung und ein eigenes Erzählen“ (Breuer et a. 2018, 257). In den folgenden Ausführungen werden fünf Befunde, aufbauend auf den bisherigen Befunddarstellungen und durch die Verdichtung im Rahmen weiterer Rekonstruktionsprozesse, systematisch vorgestellt.

1. Befund 1: Gelingensbedingungen schulpraktischer Mentoringprozesse im Rahmen der Metakernkategorie

2. Befund 2: Gelingensbedingungen schulpraktischer Mentoringprozesse in kontextueller Rahmung

3. Befund 3: Gelingensbedingungen schulpraktischer Mentoringprozesse durch MentoringFunktionen

4. Befund 4: ATM-Modell - Analyse Transformationales Mentoring im berufsfeldbezogenen Wirkungskontext

5. Befund 5: MIW-Modell - Mentoringinduziertes Wirkungsmodell zum schulpraktischen Mentoringprozess

\subsection{Befund 1: Gelingensbedingungen schulpraktischer Mentoringprozesse im Rahmen der Metakernkategorie}

Die vorliegende empirische Untersuchung brachte je zwei Kodierparadigmata und je eine Kernkategorie aus Mentee- und Mentor/innenperspektive im Kodierungsprozess mittels Grounded Theory hervor. Paradigmata und Kernkategorien gaben Anlass für weitere Rekonstruktionen und Metarekonstruktionen, die schließlich zu einer Metakernkategorie führten (Tabelle 62):

Tab. 62: Anzahl der Paradigmata und Kernkategorien aus den Interviews

\begin{tabular}{llll}
\hline $\begin{array}{l}\text { Menteeperspektive bzw. } \\
\text { Studierendenperspektive }\end{array}$ & \multicolumn{2}{l}{$\begin{array}{l}\text { Mentor/innenperspektive bzw. } \\
\text { Lehrendenperspektive }\end{array}$} \\
\hline & Kernkategorie 1 & Metakernkategorie 1 & \\
& & \multicolumn{2}{c}{ Kernkategorie 1 } \\
Kodierparadigma 1 & Kodierparadigma 2 & Kodierparadigma 1 & Kodierparadigma 2 \\
\hline
\end{tabular}

Die Metakernkategorie erfasst Kategorien aus ursächlichen Bedingungen, Handlungsstrategien, Kontext und intervenierenden Bedingungen, Konsequenzen und Phänomenen in der Tiefenstruktur. Sie ist Ergebnis eines tiefenstrukturierten Rekonstruktionsprozesses, indem 
„Arbeitsorganisation und Wechselbeziehung“(Strauss 1991b, 34f) immer im Mittelpunkt des prozessgeleiteten Forschungsansatzes standen. Schrittweise wurden in den vorhergehenden Kapiteln die Arbeitsprozesse von der Datenerhebung bis zur Datenanalyse dargestellt und erläutert. Mit Wechselbeziehung verbindet Strauss (1991b) die Interaktion der/des Forscherin/ Forschers mit dem Datenmaterial. Bezogen auf diese Aspekte sei noch einmal die Forschungsfrage wiederholt, bevor die Metakernkategorie vorgestellt wird: Welche Gelingensbedingungen lassen sich im Mentoringprozess der Lehramtsausbildung rekonstruieren bzw. identifizieren? Das im Anschluss angeführte Modell veranschaulicht die Kernaussagen zu unterschiedlichen Bereichen im Rahmen der Metakernkategorie (Abbildung 76).

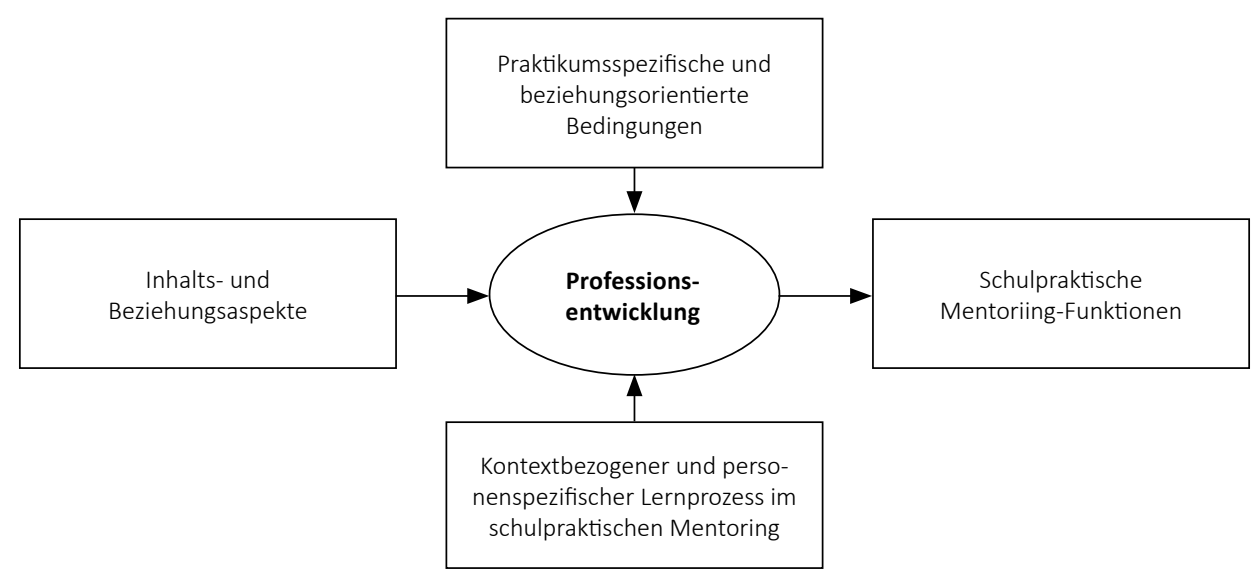

Abb. 76: Metakernkategorie zum schulpraktischen Mentoringprozess aus den Rekonstruktionsschritten der Mentor/ innen und Studierendenperspektiven

Aufgrund des Forschungsganges mit Entwicklung einer Metakernkategorie lassen sich transparente Aussagen zu Gelingensbedingungen im schulpraktischen Mentoringprozess zusammenfassen. Auslöser für Zustandekommen ursächlicher Bedingungen, Kontext von Mentoringprozessen in der Lehramtsausbildung, notwendige Handlungsstrategien im Mentoringprozess und die daraus resultierenden Konsequenzen umrahmen das Phänomen. Das Phänomen Professionsentwicklung bildet den Kern von schulpraktischen Mentoringprozessen und kam im Rahmen der theoretischen Sättigung zustande. Erklärungen zu Gelingensbedingungen lassen sich aus den Daten der Anordnungen und durch „das In-Beziehung-Setzen aller Hauptkategorien zur Kernkategorie und untereinander“ (Strauss et al. 1996, 117) erschließen. Im Folgenden werden alle fünf Bereiche erläutert und die Forschungsfrage beantwortet.

Inhalts- und Beziehungsaspekte als ursächliche Bedingungen: Ausgangsmotive für schulpraktisches Mentoring sind bei Mentor/innen und Mentee primär auf der Inhalts- und sekundär auf der Beziehungsebene begründet. Die Möglichkeit des Aneignens von professionsspezifischem Wissen durch das neue Aufgabenfeld bzw. durch aktives, handlungsorientiertes Lernen in den Schulpraktika, die Möglichkeit der Weitergabe von Erfahrungswerten im Berufsfeld Schule und der Einbeziehung theoretischer Konzepte in den Schulalltag, die Möglichkeit des Erprobens und Ausprobierens, um Lern- und Entwicklungsprozesse in Gang zu setzen und in der professionsspezifischen Selbstwirksamkeit gestärkt zu werden, zeigen beispielhaft, dass der Inhaltsaspekt mit Ermöglichungsgründen und Erwartungen der beiden Interviewgruppen ver- 
bunden wird. Der Beziehungsaspekt als ursächliche Bedingung kommt bei Mentor/innen zu tragen, wenn sie von eigenen Ausbildungserfahrungen mit Mentor/innen und der Vorstellung von Mentoring berichten. Dann werden Vergleiche mit dem jetzigen Handeln im Mentoring gezogen und ähnlich ablaufende Verhaltensweisen in der momentanen Begleitung oder Ablehnung von erlebten Prozessgestaltungen diskutiert. Für Mentee kommt der Beziehungsaspekt in den Vordergrund, wenn es darum geht, wie Lerngelegenheiten in den Praktika durch Mentor/ innen vollzogen werden sollten. Studierende neigen dann dazu, das Mentoring auf die Lerngelegenheit zu verkürzen, und der für das Mentoring bedeutende Reflexionsprozess scheint außer Acht zu geraten. Resümierend kann zu den ursächlichen Bedingungen gesagt werden:

Um gelingende Prozesse zu gestalten, sind motivierte Mentor/innen aufgefordert, Mentee Lern- und Erfahrungsräume zur Verfügung zu stellen, die wechselseitige Lern- und Entwicklungschancen im professionsspezifischen Feld bieten. Der Reflexionsprozess muss verstärkt in den Blick des Mentorings aufgenommen werden.

Praktikumsspezifische und beziehungsorientierte Bedingungen als Kontext und intervenierende Bedingungen: Im Rahmen der Schulpraktika brauchen Mentor/innen und Mentee ein Wissen über Bedingungsfaktoren zu Praktika. Dazu ist eine enge Zusammenarbeit mit Ausbildungsinstitution bzw. Ausbildungsinstitutionen gerade in Zeiten der Umstrukturierung der Lehrer/innenausbildung notwendig (Kapitel I/1.1.2). Strukturelle und organisatorische Rahmenbedingungen sollten transparent sein und im Vorfeld mit allen Beteiligten kommuniziert werden. Für Mentor/innen ist neben dem Wissen zu praktikumsspezifischen Bedingungen auch die reflexive Auseinandersetzung mit Bedingungsfaktoren zu Begleitung notwendig, was wiederum für die Etablierung von Mentoring-Ausbildungskonzepten und Fortbildungsformaten spricht. Rollen- und Funktionsdefinitionen, Auseinandersetzung mit Ermöglichungs- und Erwartungshaltungen, supervidierte Fallanalysen sowie ein Wissen zu Mentoring-Funktionen im Begleitungsprozess könnten hierbei Inhalte sein. Für Mentee ist in Zusammenhang mit dem Kontext die Beziehung zur/zum Mentor/in essentiell. Sie erwarten sich ein kompetentes beziehungsorientiertes Mentoring in den Schulpraktika und möchten in einen Begleitungskontext kommen, in dem Respekt, Vertrauen, Akzeptanz und Authentizität gelebte Wirklichkeiten sind. Gewünscht wird ein Wahrnehmen der Person, der Persönlichkeit, von Fähigkeiten, Fertigkeiten und Kompetenzen. Resümierend kann gesagt werden:

Mentor/innen brauchen Ausbildungs- und Fortbildungsformate mit informativen und reflexiven Anteilen zu praktikumsspezifischen und beziehungsorientierten Bedingungsfaktoren und entwickeln eine stetige reflexive Haltung in der Betreuungstätigkeit. Mentee erwerben sich im Vorfeld jedes Praktikums ein Wissen über praktikumsspezifische Anforderungen und brauchen Offenheit in unterschiedlichen Schulsettings und in der Begegnung mit verschiedenen Lehrer/innenpersönlichkeiten.

Kontextbezogener und personenspezifischer Lernprozess im schulpraktischen Mentoring als Handlungsstrategien: Die Umsetzung von forschungsgeleiteten Konzepten und Theorien, Reflexionen pädagogischen Denkens und Handelns und das Eintreten in einen erfahrungsbasierten Austausch im Berufsfeld Schule prägen das gemeinsame Setting, bedingen Handlungsstrategien und schließlich das Phänomen Professionsentwicklung. Im Rahmen des schulpraktischen Mentorings treten beide Partner/innen in einen Lernprozess ein. Die Klärung von Rahmenbedingungen, Darlegung von Wert- und Erwartungshaltungen sowie eine positive Einstellung zum Lernen begünstigen diesen Lernprozess. Resümierend kann gesagt werden: 
Lernen ist im Mentoring ein individuell angelegter Prozess mit unterschiedlichen Absichten. Im Zusammenspiel der Tandems ist es wesentlich, dass ein Begriffsverständnis für den gemeinsamen Kontext, Offenlegung von Erwartungen und ein Wissen personenspezifischer Besonderheiten sowie Transparenz in der Gestaltung der gemeinsamen Settings vorhanden bzw. gewährleistet sind. Der Lern- und Entwicklungsprozess kann als das verbindende Gemeinsame im Mentoring gesehen werden.

Schulpraktische Mentoring-Funktionen als Konsequenzen: Aus den Rekonstruktionsprozessen der Interviewdaten der Mentor/innen und Studierenden ergeben sich Schulpraktische Mentoring-Funktionen wie professionsspezifische Funktionen als Lehrperson, MentoringFunktionen, Rollenfunktionen, psychosoziale und personal-emotionale Aspekte, Forderung an die Mentoring-Rolle und der Aufbau professionsspezifischer Kompetenzfunktionen über die Verfügbarkeit eines Rollenmodells. Mentor/innen sind gewillt, Verantwortung zu übernehmen und erklären ihre Bereitschaft zur mentoriellen Betreuung. Sie erkennen durch persönliche Weiterentwicklung (in) der Profession einen Mehrwert im Aufgabenfeld. Im Gegenzug fordern und erwarten Mentee Verhaltensweisen von Mentor/innen, die durch ein Rollenverständnis und eine Mentoring-Professionalität umgesetzt werden können. Letztendlich beharren Studierende auf einem persönlichen Bildungsnutzen. Sie wollen professionsspezifische Kompetenzen mithilfe des Mentorings aufbauen. Die angeführte Argumentation zeigt die Notwendigkeit der Klärung von Rollen und Funktionen beider Partner/innen im Mentoring. Deshalb wurden in einem weiteren Verdichtungsprozess die rekonstruierten Mentoring-Funktionen analysiert. Die Beschreibung erfolgt im Kapitel II/8.3. Resümierend kann gesagt werden:

Im schulpraktischen Mentoringprozess braucht es eine Klärung zur Mentoring-Funktion, eine Mentoring-Professionalität und eine Rollenidentität beider Partner/innen.

Professionsentwicklung als Phänomen: „Das zentrale Phänomen ist das Herzstück des Integrations-Prozesses (sic!). (Strauss et al. 1996, 101) Im Mentoring zielen beide Partner/innen - Mentor/innen und Mentee - auf Professionsentwicklung. Für Mentor/innen ergibt sich die Möglichkeit der Entwicklung aufgrund von Zusammenarbeit mit Mentee, Ausbildungsinstitutionen und durch situationsbedingte Anstöße zur Reflexion subjektiver Theorien und professionsspezifischer Konzepte. Mentor/innen wollen sich in ihrer Mentoring-Funktion entwickeln und professionell agieren. Mentee bewerten den Lernort Praktikum als Möglichkeit des Eintauchens in das unmittelbare Sozialisationsfeld Schule und als Chance zur Professionsentwicklung. Praktika ermöglichen ihnen in erster Linie ein individuelles, themenbezogenes, erfahrungsbasiertes Lernen in der Ausbildung und ein Aneignen von professionsspezifischem Wissen. Resümierend kann gesagt werden:

Professionsentwicklung wird zum „individuellen Wollen“ (Kraler 2013a) der Partner/innen, Mentoring unterstützt und fördert diese Intention.

Die Ausführungen zur Metakernkategorie heben wesentliche Faktoren im Sinne eines gelingenden prozess- und förderungsorientierten Ansatzes (Faix et al. 2017, 36) im Mentoring mit dem Phänomen Professionsentwicklung hervor. In der Grobgliedrigkeit kann die Beachtung schulpraktischer Mentoring-Funktionen genannt werden (siehe Kapitel II/7.5). In der Feinabstimmung sind es Aspekte, wie kontextuelle Abstimmung und Eingliederung im Mentoring, Schaffung von Freiräumen für lernprozessbezogene Tätigkeiten, Möglichkeit der Probeidentifikation, Beachtung von Beziehungskultur und Persönlichkeitsmerkmalen, Etablierung von Ausbildungsformaten für Mentor/innen (siehe Kapitel II/6.1). 
Eine Übereinstimmung mit Forschungsbefunden ergibt sich vor allem im Bereich der kontextuellen Beachtung (Hobson et al. 2009), bei Freiräumen geben (Duffield 2006), Ausbildung von Mentor/innen (Hobson et al. 2009) und Beachtung von Persönlichkeitsmerkmalen (Rogers 2009; Nolle 2012; Dreer 2018; Košinár 2018; Hofmann 2019) innerhalb des Mentorings, welche im theoretischen Teil beschrieben worden sind.

In der folgenden Befunddarstellung wird die Einbettung des schulpraktischen Mentoringprozesses in den Kontext näher betrachtet und es werden Gelingensbedingungen erläutert.

\subsection{Befund 2: Gelingensbedingungen schulpraktischer Mentoringprozesse im Kontext}

In Anlehnung an das Schichtmodell (siehe Kapitel I/2.4.6) nach Korthagen et al. (2005), der im innersten Kern die zu reflektierenden Konstrukte Mission und Identität positioniert und bei den weiteren Umrahmungen (beliefs, competencies, behaviour, enviroment) für einen stetigen Reflexionsprozess im Sinne einer Verhaltensanalyse mit Handlungsfolgen plädiert (Korthagen et al. 2005, 55f), kann das nachstehend angeführte Mentoring-Modell ebenso als dynamisches Bedingungsgefüge angesehen werden (Abbildung 77). Hierbei werden die aus der Untersuchung rekonstruierten Phänomene des Mentoringprozesses sowie personelle, strukturelle, bildungspolitische und historiografische Bedingungen in eine Wechselbeziehung gestellt. Die kontextuelle Einbindung des schulpraktischen Mentoringprozesses wurde bereits in Kapitel I/2.4.2 diskutiert.

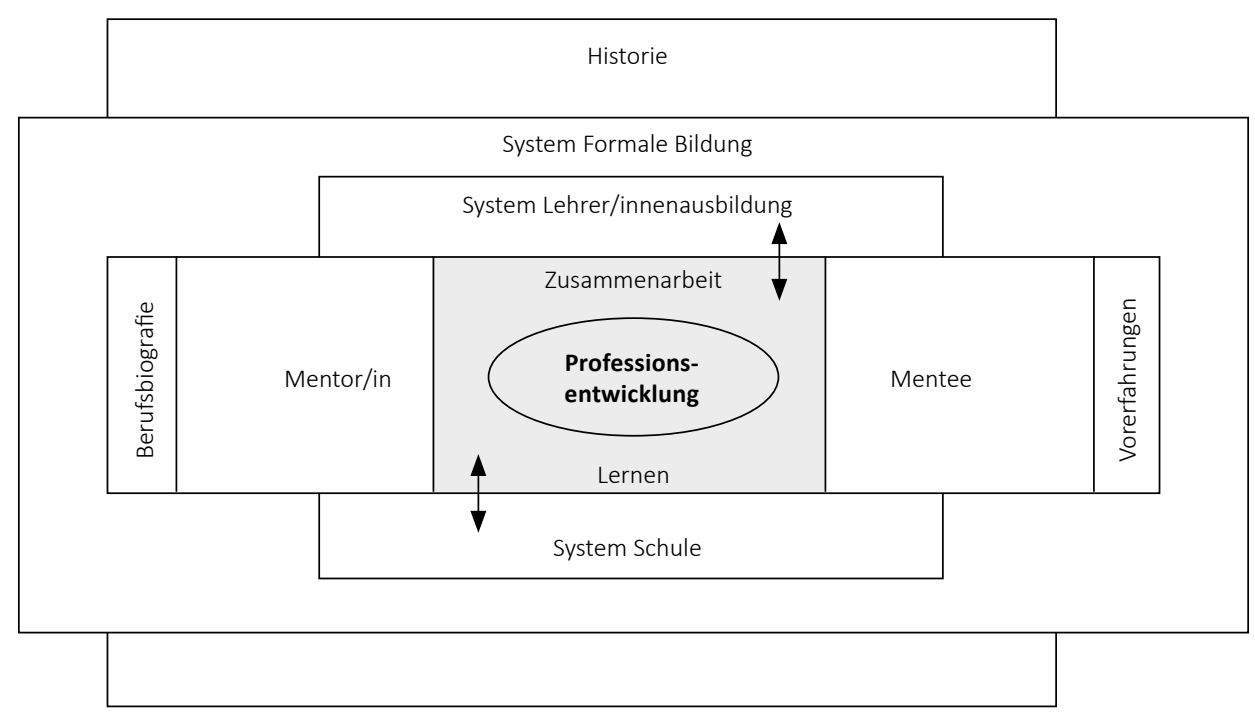

Abb. 77: Mentoring-Modell mit Einbeziehung der Phänomene und Einbindung der Personen und Systeme

Bei näherer Betrachtung des Modells ist ersichtlich, dass die von Strauss et al. (1996) entwickelte "Conditional Matrix“ (Strauss et al. 1996, 132f) die theoretische Grundlage darstellt. Die Autoren differenzieren hierbei den Kontext und die intervenierenden Bedingungen der Paradig- 
mata in einen erweiterten strukturellen Rahmen und betonen, dass sich ein „kontinuierliche(r) Übergang von interaktionalen Face-to-Face-Situationen zu meso- und makrosozialen Strukturmomenten und zurück“ (Strübing 2014, 28) zeigt. „Damit soll verdeutlicht werden, dass die $\mathrm{zu}$ analysierenden Phänomene nicht nur von intervenierenden Bedingungen auf verschiedenen Ebenen gerahmt werden, sondern ihrerseits zur Reproduktion eben dieser Bedingungen ihren Beitrag leisten." (Strübing 2014, 28) Eine Reproduktion wird auch hier beschrieben.

Mentoringprozess mit Mentor/in und Mentee: In der Untersuchung wurden praktikumsspezifische und beziehungsorientierte Bedingungen als Kontext sowie intervenierende Bedingungen erhoben, die auf das Phänomen der Metakernkategorien Professionsentwicklung und auf die Phänomene der Kernkategorien Zusammenarbeit und Lernen wirken. Als Akteurinnen/Akteure im Mentoringprozess fungieren die/der Lehrer/in in der Rolle als Mentor/in und die/der Studierende in der Rolle als Mentee. Mentor/innen werden als Expert/innen (Arnold et al. 2011, 13) gesehen, erledigen berufsfeldbezogene Aufgabenstellungen in der Vorbildfunktion (Faix et al. 2017, 36), haben sich Handlungsmuster im Laufe des berufsbiografischen Entwicklungsprozesses (Richter 2011; Cramer 2012, 34) aufgebaut und sind damit „als Rollenmodell prädestiniert“ (Fuge 2016, 33). Nach einem Matching-Verfahren (Schmid et al. 2011, 86f; Fuge 2016, 46) treten Mentor/in und Mentee in einen Prozess ein und bilden eine ,zeitlich stabile dyadische Beziehung“ (Ziegler 2009, 11). Die Eingliederung der Mentee in das Beziehungsgefüge und Berufsfeld Schule sehen Autor/innen als Aufgabe von Mentor/innen (Crisp 2010; Hobson et al. 2009; König et al. 2018). Bei einem gelingenden Verlauf kommt es nach Einzelstudienbefunden zur Steigerung des Selbstvertrauens, Stärkung des Selbstkonzepts, Anreicherung von Unterrichtskompetenzen und zu einem gemäßigteren Beanspruchungserleben (König et al. 2018, 44; Hobson et al. 2009, 210). In der Beziehungskultur sind wechselseitiges Vertrauen und ein respektvoller Umgang miteinander die Basisindikatoren für gelingende Zusammenarbeit (Hudson 2013, 17). In dieser Untersuchung kann aufgezeigt werden, dass aus Studierendenperspektive kein Mentee-Rollenverständnis und keine Mentee-Identität vorhanden sind. Mentee gehen in eine Erwartungshaltung, bewerten und beurteilen das Rollenverhalten der Mentor/innen, wünschen sich bzw. fordern ein Mentoring-Verständnis und eine Mentoring-Professionalität des Gegenübers. Hennissen et al. (2011) beschreiben ähnliche Befunde, die die Forderung nach emotionaler Unterstützung und Aufgabenbewältigung seitens der Mentee dokumentieren (Hennissen et al. 2011, 1050). Ansonsten fehlen weitreichende Untersuchungen zum Verhalten von Mentee im Kontext schulpraktischer Mentoring-Beziehungen (Garringer 2010, 4). Der Mentoringprozess ist mit bestimmten Erwartungshaltungen (Schmid et al. 2011; Wellhöfer 2018,31) und Ermöglichungshaltungen verbunden, die es im Austausch offen zu legen und zu vergegenwärtigen gilt.

System Schule: Die Einbettung des Mentoringprozesses findet in erster Linie im unmittelbaren Berufsfeld Schule statt. Forschungen zum Wirkungsgrad der Schüler/innen auf den Mentoringprozess bilden ein Desideratum. Auch in Zusammenhang mit Wirksamkeit der Schulpraktika bei Studierenden wird die Einbeziehung der Schüler/innen in Forschungssupports meist außer Acht gelassen. Direktionen stehen der Studierendenbegleitung seitens des Kollegiums positiv gegenüber, wohlwissend, dass ein Aufbrechen von personellen und institutionellen Gegebenheiten durch eine reflexive Haltung möglich ist (Ittel et al. 2009, 203; Norman et al. 2005, 695). Schulische Strukturen und Settings sind im Allgemeinen sehr divergierend. Mentor/innen erhalten im Sinne des Zusammenarbeitens die Aufgabe der Einführung in das System Schule und der Maßnahmensetzung zur Zurechtfindung, um in einen Lernprozess einsteigen zu können. System Lehrer/innenausbildung: Schulpraktika sind in den Pädagogisch-Praktischen Studien der Curricula der neuen Lehramtsausbildung im Rahmen von Pädagog/innenbildung NEU in- 
tegriert und sind Teil der Bildungsforschung. Vor allem Fragen der berufsfeldbezogenen Orientierung, Lernwirksamkeit, Kompetenzerweiterung, Etablierung eines prozessorientierten Erprobungsformates zum Ausbau einer forschend reflexiven Haltung (Topsch 2004b, 484f; Herzmann et al. 2016, 155; Leonhard et al. 2018, 13f) und zum Stellenwert der Praktika (Cramer 2012; Bach 2013; Ostermann 2015) rahmen das Forschungsfeld. Im Idealfall sind Mentor/innen in Ausbildungskonzepte und Forschungsvorhaben integriert, da sie als Bindeglied agieren und durch Betreuung der Studierenden einen wichtigen Part im Ausbildungssetting übernehmen. Untersuchungsergebnisse zeigen, dass nicht Länge und Dauer von Praktika entscheidend sind, sondern die Qualität der Lernprozesse (Gröschner et al. 2015) und Lernerfolge im Praktikum (Dieck et al. 2009; Müller 2010). Erfolge und Nutzen von Praktika sind sehr stark mit den Fragen verbunden, wie theoriegeleitete Reflexionsteile aufbereitet werden bzw. was im Reflexionsteil von Studierenden in den begleitenden Lehrveranstaltungen verlangt wird (Hascher 2012; Arnold et al. 2014).

System Formale Bildung und Historie: Das formale Bildungssystem Österreichs ist in den europäischen Hochschulraum eingebunden. Entwicklungen sind hinsichtlich historischer und gesellschaftspolitischer Zugänge zu betrachten, so auch aktuelle Veränderungen im Mentoring aufgrund der Neukonzeptionen von Lehrer/innenausbildung und Schulsettings wie durch Bildungsdirektionen und Schulentwicklungsprogramme.

Die Strukturierung des angeführten Mentoring-Modells (Abbildung 77) erinnert auch an das psychologisch-pädagogische Verfahren zur optimalen Gestaltung von Gruppenprozessen nach Ruth Cohn (1997) - die Themenzentrierte Interaktion (TZI). Sie beschreibt im VierfaktorenModell das wechselseitige Bedingungsgefüge mit Individuum (Ich), Gruppe (Wir), Aufgabe (Es) und Umfeld (Globe) und betont hierbei die Bedeutung der beziehungsorientierten, inhaltlichen und kommunikativen Aspekte (Oehmann et al. 2019, 127). Legt man das Modell auf Mentoring um, so entspricht die Komponente Individuum der/dem Mentorin/Mentor bzw. der/dem Mentee, die Komponente Gruppe ebenso der/dem Mentorin/Mentor bzw. der/dem Mentee, die Komponente Aufgabe der Professionsentwicklung und die Komponente Umfeld den formalen Bildungsinstitutionen. In Anlehnung an Cohn (1997) können exemplarische Fragestellungen für Beteiligte im Mentoringprozess heißen: Wer bin ich? Welche Bedürfnisse, Interessen und Ziele habe ich im Prozess? Wie sehe ich meinen Teil in der Beziehung? Wie verstehe ich den Mentoringprozess? Wie bin ich in den Prozess eingebunden? Wie kann ich an die Aufgabe(n) herangehen? Welche Schritte sind bei der Umsetzung notwendig? Welcher Kontext beeinflusst das Beziehungsgefüge? Nach Cohn (1997) vollzieht der Mensch Entwicklungs- und Veränderungsprozesse, wenn er im Austausch mit anderen agiert und als Subjekt wahrgenommen wird (Cohn 1997, 155). Der Ansatz verdeutlicht die Bedeutung von Selbstreflexionsprozessen, die in Befund 4 zur Sprache kommen.

Resümierend kann angeführt werden:

Mentee und Mentor/innen brauchen im wechselseitigen Zusammenspiel einen Blick auf ihr jeweiliges Rollenverständnis und auf ihre Rollenidentität - Professionalität wird von beiden in der Zusammenarbeit verlangt und bildet wohl die Basis für Professionsentwicklung innerhalb der Systeme Schule und Lehrer/innenausbildung.

In diesem Sinn gilt es, in einem kontinuierlichen Lern- und Entwicklungsprozess eine kritischreflexive Haltung zu fördern. Mentor/innen müssen dazu verstärkt in den Ausbildungskontext miteinbezogen werden. Sie müssen sich mit den Rahmenbedingungen und aktuellen Herausforderungen der Mentee auseinandersetzen. Mentee müssen lernen, ihre Rolle wahrzunehmen. Zudem stellen sich Wie-Fragen, wie Direktionen sowie Kolleginnen und Kollegen an den Part- 
nerschulen in den Mentoringprozess eingebunden werden können und wie die einzelne Schule durch Inputs von außen profitiert. Schließlich könnte sich daraus ein spannendes Forschungshaben entwickeln, das die Auswirkungen schulpraktischen Mentorings aus der Perspektive von Schülerinnen und Schülern untersucht und entsprechende didaktische Folgerungen aufzeigt. Die folgende Befunddarstellung bezieht sich auf die Metakategorien in den rekonstruierten Konsequenzen.

\subsection{Befund 3: Gelingensbedingungen schulpraktischer Mentoringprozesse durch Mentoring-Funktionen}

Der folgende Abschnitt diskutiert die thematischen Teilfragen zur eigentlichen Forschungsfrage und wirft noch einmal einen Blick auf die Aussagen der Lehrenden- und Studierendenperspektive. Die Fragen lauten:

- Welche Gelingensbedingungen lassen sich aus Sicht der Mentor/innen rekonstruieren bzw. identifizieren?

- Welche Gelingensbedingungen lassen sich aus Sicht der Studierenden rekonstruieren bzw. identifizieren?

Die Antworten werden aufgrund eines mehrschrittigen Rekonstruktionsprozesses der Interviewdaten mittels Grounded Theory gegeben. Die Verortung ist im Rahmen der Konsequenzen als Schulpraktische Mentoring-Funktionen in der Metakernkategorie. Metakategorien bilden die Kernaussagen der Gelingensbedingungen. Hintergrund bildet das Modell von Kram (1985) mit den rekonstruierten Funktionen aus 18 Interviews. In dieser Untersuchung kategorisierte sie Mentoring-Funktionen in Psychosoziale Funktionen und Karrierefunktionen.

In der vorliegenden Untersuchung wurde ein differenzierter Blick auf Kategorien geworfen und es wurden sechs Schulpraktische Mentoring-Funktionen erhoben (Professionsspezifische Funktion, Mentoring-Funktionen, Rollenfunktionen, Aufbau professionsspezifischer Kompetenzfunktionen über die Verfügbarkeit eines Rollenmodells, Psychosoziale und personal-emotionale Aspekte, Forderung an die Mentoring-Rolle). Tabelle 63 dokumentiert innerhalb der Funktionen die Metakategorien und listet im Weiteren die mehrmals genannten Anforderungen, Subkategorien und Kategorien und demzufolge Aussagen der Interviewpartner/innen auf. Bei der Erstellung der Tabelle wurde auf eine thematische Gegenüberstellung der Gelingensbedinungen - wie in Tabelle 63 angeführt - mit den Metakategorien geachtet (z.B. Psychosoziale Moment(e) und psychosozialer Austausch - Individuelle psychosoziale Erfahrungen), wenn sich inhaltliche Überschneidungen ergaben. Ansonsten darf es als reine Anordnung betrachtet werden.

Tab. 63: Gesamtübersicht der rekonstruierten Gelingensbedingungen schulpraktischer Mentoringprozesse aus Sicht der Mentor/innen und Studierenden bzw. Mentee

Gelingensbedingungen schulpraktischer Mentoringprozesse unter Beachtung der Metakategorien der Schulpraktischen Mentoring-Funktionen

\begin{tabular}{lc}
\hline Mentor/innen & Studierende bzw. Mentee \\
\hline - Professionsspezifische Funktionen & - Aufbau professionsspezifischer Kompetenz- \\
- Mentoring-Funktionen & funktionen über die Verfügbarkeit eines \\
- Rollenfunktionen & Rollenmodells \\
& - Psychosoziale und personal-emotionale Aspekte \\
& - Forderung an die Mentoring-Rolle \\
\hline
\end{tabular}


Gelingensbedingungen schulpraktischer Mentoringprozesse unter Beachtung der Metakategorien der Schulpraktischen Mentoring-Funktionen

\begin{tabular}{|c|c|}
\hline Mentor/innen & Studierende bzw. Mentee \\
\hline $\begin{array}{l}\text { Professionsspezifische Zusammenarbeit und } \\
\text { Integration } \\
\text { - Kennenlernen des Ausbildungsmodus } \\
\text { - Auseinandersetzung mit theoriegeleiteten Aus- } \\
\text { bildungskonzepten } \\
\text { - Begegnung mit Vertreter/innen der Ausbil- } \\
\text { dungsinstitutionen }\end{array}$ & $\begin{array}{l}\text { Praktikum als berufliches Lernfeld } \\
\text { - Kennenlernen des Systems Schule } \\
\text { - Einbindung in das System Schule } \\
\text { - Möglichkeit zum Beziehungsaufbau zur Klasse } \\
\text { bzw. zu Schülerinnen und Schülern } \\
\text { - Herstellung eines Theorie-Praxis-Bezuges } \\
\text { - Gewährung eines Sicherheitsaspektes }\end{array}$ \\
\hline $\begin{array}{l}\text { Verständnis für Identifikationsprozess hin zum } \\
\text { Lehrberuf } \\
\text { - Öffnung des Systems Schule } \\
\text { - Schaffung der Möglichkeiten zur Probeidentifi- } \\
\text { kation und Entwicklungspotentialentfaltung }\end{array}$ & $\begin{array}{l}\text { Professionsspezifische Selbstwirksamkeit und } \\
\text { Kompetenzentwicklung im Praktikum } \\
\text { - Möglichkeit des Eintretens in einen Lern- und } \\
\text { Erfahrungsprozess } \\
\text { - Schaffung von Erfolgserlebnissen } \\
\text { - Eigenverantwortliches Handeln } \\
\text { - Feedbackkultur und Erfahrungslernen } \\
\text { - Gemeinsamer Reflexionsprozess }\end{array}$ \\
\hline $\begin{array}{l}\text { Gegenstandsbezogener Austausch } \\
\text { - Konstruktiver lernprozessorientierter Erfah- } \\
\text { rungsaustausch mit Vertreter/innen der Ausbil- } \\
\text { dungsinstitutionen und Studierenden } \\
\text { - Eintreten in einen intensiven Lern- und } \\
\text { Reflexionsprozess }\end{array}$ & $\begin{array}{l}\text { Kommunikation und Interaktion } \\
\text { - Konstruktive Feedbackkultur } \\
\text { - Wunsch nach Authentizität } \\
\text { - Beleuchten der Abhängigkeitsposition } \\
\text { - Ehrlichkeit im Umgang }\end{array}$ \\
\hline $\begin{array}{l}\text { Mentoring-Mission } \\
\text { - Klärung der Motivationsgründe } \\
\text { - Reflexion der Eigenerfahrungen }\end{array}$ & $\begin{array}{l}\text { Mentoring-Mission } \\
\text { - Motivation, Engagement, Interesse } \\
\text { - Vorhandene Zeitressource }\end{array}$ \\
\hline $\begin{array}{l}\text { Mentoring-Identität } \\
\text { - Klärung des Rollenverständnisses } \\
\text { - Reflexionsbereitschaft } \\
\text { - Verantwortung bei Problemen } \\
\text { - Beachtung der eigenen Psychohygiene }\end{array}$ & $\begin{array}{l}\text { Mentoring-Verständnis und Mentoring-Organi- } \\
\text { sation } \\
\text { - Klärung des Rollenverständnisses } \\
\text { - Entwicklung eines persönlichen Rollenbildpro- } \\
\quad \text { fils } \\
\text { - Kriterien für Auswahlverfahren }\end{array}$ \\
\hline $\begin{array}{l}\text { Selbstkonzept Mentor/in } \\
\text { - Persönliche positive Einschätzung zur Aufgaben- } \\
\text { erfüllung aufgrund von Persönlichkeitsstruktu- } \\
\text { ren und Fähigkeiten } \\
\text { - Grundsätzliche Systembejahung } \\
\text { - Lösungsorientierter Ansatz bei Problemen } \\
\end{array}$ & $\begin{array}{l}\text { Mentoring-Professionalität } \\
\text { - Reflexion zur Annahme des Expertiseansatzes } \\
\text { und der Professionalität im Lehrberuf } \\
\text { - Auseinandersetzung mit Professionalität in der } \\
\text { Mentoring-Funktion }\end{array}$ \\
\hline $\begin{array}{l}\text { Psychosoziale Moment(e) und psychosozialer Aus- } \\
\text { tausch } \\
\text { - Auseinandersetzung mit Beziehungskultur und } \\
\text { Fragen der Gestaltung der Beziehungskultur } \\
\text { - Kenntnisse zu psychosozialen und personal-emo- } \\
\text { tionalen Aspekten sowie Einsicht in Wirkungs- } \\
\text { weise im Umgang } \\
\text { - Klärung der Frage der Verantwortung im Rah- } \\
\text { men der Mentoring-Funktion } \\
\end{array}$ & $\begin{array}{l}\text { Individuelle psychosoziale Erfahrungen } \\
\text { - Beobachten psychischen Verhaltens } \\
\text { - Erkennen von Belastungssymptomen } \\
\text { - Aufbau eines Vertrauensverhältnisses } \\
\text { - Distanz und Nähe }\end{array}$ \\
\hline
\end{tabular}


Aus der Lehrendenperspektive in der Rolle als Mentor/innen ergeben sich zu den Professionsspezifischen Funktionen, Mentoring-Funktionen und Rollenfunktionen folgende darin beschriebene Gelingensbedingungen: Professionsspezifische Zusammenarbeit und Integration, Verständnis für Identifikationsprozess hin zum Lehrberuf, Gegenstandsbezogener Austausch, Mentoring-Mission, Mentoring-Identität, Selbstkonzept Mentor/in, Psychosoziale Moment(e) und psychosozialer Austausch. Die in Tabelle 63 angeführten Unterpunkte weisen auf eine mögliche inhaltliche Verständigung hin. Eine Ausdifferenzierung wurde bereits in Kapitel II/7.5 vorgenommen.

Aus der Studierendenperspektive in der Rolle als Mentee kommen in den Schulpraktischen Mentoring-Funktionen die Bereiche Aufbau professionsspezifischer Kompetenzfunktionen über die Verfügbarkeit eines Rollenmodells, Psychosoziale und personal-emotionale Aspekte und Forderungen an die Mentoring-Rolle zum Tragen. Die daraus rekonstruierten Gelingensbedingungen lauten: Praktikum als berufliches Lernfeld, Professionsspezifische Selbstwirksamkeit, Kommunikation und Interaktion, Mentoring-Mission, Mentoring-Verständnis und Mentoring-Organisation, Mentoring-Professionalität, Individuelle psychosoziale Erfahrungen und Kompetenzentwicklung im Praktikum. Auch die in Tabelle 63 angeführten Kategorien dokumentieren die inhaltliche Schwerpunktsetzung jeder Bedingung. Wiederum finden sich genauere Beschreibungen bereits im Kapitel II/7.5.

Im Folgenden wurden die Metakategorien der schulpraktischen Mentoring-Funktionen bzw. die Gelingensbedingungen noch einmal inhaltlich systematisch zusammengefasst und auf Ähnlichkeiten bzw. Differenzen der beiden Interviewpartner/innen überprüft. Somit ergab sich ein weiterer Rekonstruktionsschritt und eine weitere Arbeitsorganisation. Inhaltliche Überschneidungen von Gelingensbedingungen werden als gemeinsames Wollen im schulpraktischen Mentoring bezeichnet (Tabelle 64).

Tab. 64: Gemeinsames Wollen im schulpraktischen Mentoring

\begin{tabular}{|l|l|l|l|l|l|}
\hline Mentor/in & $\begin{array}{l}\text { Mentoring- } \\
\text { Mission }\end{array}$ & $\begin{array}{l}\text { Mentoring- } \\
\text { Identitität } \\
\begin{array}{l}\text { Wemeinsames } \\
\text { Mentoring }\end{array}\end{array}$ & $\begin{array}{l}\text { Selbstkonzept } \\
\text { Mentor/in }\end{array}$ & $\begin{array}{l}\text { Gegenstands- } \\
\text { bezogener } \\
\text { Austausch }\end{array}$ & $\begin{array}{l}\text { Psychosoziale } \\
\text { Moment(e); } \\
\text { Psychosozialer } \\
\text { Austausch }\end{array}$ \\
\cline { 2 - 6 } Mentee & Mission & $\begin{array}{l}\text { Mentoring- } \\
\text { Verständnis } \\
\text { Mentoring- } \\
\text { Organisation }\end{array}$ & $\begin{array}{l}\text { Mentoring- } \\
\text { Professionalität }\end{array}$ & $\begin{array}{l}\text { Kommunikation } \\
\text { und Interaktion }\end{array}$ & $\begin{array}{l}\text { Individuelle } \\
\text { psychosoziale } \\
\text { Erfahrung }\end{array}$ \\
\hline
\end{tabular}

Dazu zählen Mentoring-Mission, Mentoring-Identität bzw. Mentoring-Verständnis und Mentoring-Organisation, Selbstkonzept Mentor/in und Mentoring-Professionalität, Gegenstandsbezogener Austausch bzw. Kommunikation und Interaktion, Psychosoziale(s) Moment(e), Psychosozialer Austausch und Individuelle psychosoziale Erfahrung. Alle Metakategorien wurden bereits im Kapitel II/7.5 genauer beschrieben. Aufgrund von differenzierten Aussagen der Mentor/innen und Mentee ergeben sich ein mentor/inbezogenes und menteebezogenes Wollen. Hintergrund bildet das Modell von Kraler (2013a) mit dem rekonstruierten ,individuellen Wollen“ (Kraler 2013a). Tabelle 65 stellt einen Überblick über gemeinsame und divergierende rekonstruierte Gelingensbedingungen aus den Interviews der beiden Interviewpartner/innen 
dar und zieht ein Gesamtresümee. Für Mentor/innen zeigt sich als Gelingensbedingung ein professionsspezifisches Mentoring unter Berücksichtigung funktionsbezogener und emotionaler Faktoren, für Mentee eine mentoringinduzierte Selbstwirksamkeits- und Professionsentwicklung.

Tab. 65: Gemeinsames, mentor/inbezogenes und menteebezogenes Wollen im schulpraktischen Mentoring

\begin{tabular}{|c|c|c|c|c|c|}
\hline $\begin{array}{l}\text { Conclusio } \\
\text { Mentor/in }\end{array}$ & \multicolumn{5}{|c|}{$\begin{array}{l}\text { Professionsspezifisches Mentoring unter Berücksichtigung } \\
\text { funktionsbezogener und emotionaler Faktoren }\end{array}$} \\
\hline $\begin{array}{l}\text { Mentor/in- } \\
\text { bezogenes Wollen }\end{array}$ & \multicolumn{5}{|c|}{$\begin{array}{l}\text { Professionsspezifische Zusammenarbeit und Integration; } \\
\text { Verständnis für Identifikationsprozess hin zum Lehrberuf; }\end{array}$} \\
\hline \multirow{2}{*}{$\begin{array}{l}\text { Mentor/in } \\
\text { Gemeinsames } \\
\text { Wollen im } \\
\text { Mentoring }\end{array}$} & $\begin{array}{l}\text { Mentoring- } \\
\text { Mission }\end{array}$ & $\begin{array}{l}\text { Mentoring- } \\
\text { Identitität }\end{array}$ & $\begin{array}{l}\text { Selbstkonzept } \\
\text { Mentor/in }\end{array}$ & $\begin{array}{l}\text { Gegenstands- } \\
\text { bezogener } \\
\text { Austausch }\end{array}$ & \begin{tabular}{|l} 
Psychosoziale \\
Moment(e); \\
Psychosozialer \\
Austausch
\end{tabular} \\
\hline & $\begin{array}{l}\text { Mentoring- } \\
\text { Mission }\end{array}$ & $\begin{array}{l}\text { Mentoring- } \\
\text { Verständnis } \\
\text { Mentoring- } \\
\text { Organisation }\end{array}$ & $\begin{array}{l}\text { Mentoring- } \\
\text { Professionalität }\end{array}$ & $\begin{array}{l}\text { Kommuni- } \\
\text { kation und } \\
\text { Interaktion }\end{array}$ & $\begin{array}{l}\text { Individuelle } \\
\text { psychosoziale } \\
\text { Erfahrung }\end{array}$ \\
\hline $\begin{array}{l}\text { Menteebezogenes } \\
\text { Wollen }\end{array}$ & \multicolumn{5}{|c|}{$\begin{array}{l}\text { Praktikum als berufliches Lernfeld; } \\
\text { Professionsspezifische Selbstwirksamkeit; }\end{array}$} \\
\hline $\begin{array}{l}\text { Conclusio } \\
\text { Mentee }\end{array}$ & $\downarrow$ & nduzierte & rirksamkeits & Profession & cklung \\
\hline
\end{tabular}

Gemeinsames Wollen von Mentor/innen und Mentee im schulpraktischen Mentoringprozess: Mentor/innen und Mentee vereinen viele Sichtweisen und Aspekte im schulpraktischen Mentoring - sowohl in der Ermöglichungsposition als auch in der Erwartungshaltung. Die Mentoring-Mission soll ausdrücken, dass es klare Motive zur Ausübung der Tätigkeit seitens der/des Mentorin/Mentors braucht, um ein Betreuungsverhältnis einzugehen. Mentee fordern Mentor/innen mit hoher Motivation, Bereitschaft, Engagement, Zeitressourcen und Interesse an der Betreuung. Für beide Interviewgruppen ist Mentoring-Mission ein wichtiges Kriterium im Mentoringprozess. Unter Mentoring-Identität und Mentoring-Verständnis werden Begrifflichkeiten wie Rollenbild, Rollenverständnis und Rollenfunktion diskutiert. Mentor/innen zeigen in der Mentoring-Identität Bereitschaft zur Selbstreflexion, zur Anleitung von Reflexionsprozessen und zur Übernahme von Verantwortung bei Problemsituationen. Dazu fehlen konkrete Instrumente bzw. Standards für die Umsetzung. Mentee wünschen sich Feedback von Mentor/innen. Sie erwarten sich das Einnehmen eines Rollenbildes seitens der Mentor/innen ohne Bedachtnahme, dass sie als Mentee auch eine Rolle und Funktion haben und diese transparent legen sollen.

Wichtig sind für Mentee die Mentoring-Organisation und dazu vor allem Kriterien zum Auswahlverfahren von Mentor/innen. Im Rahmen des Selbstkonzepts Mentor/in zeigen Mentor/ innen Bereitschaft zur Ausübung der Tätigkeit und schätzen ihre Kompetenzen, Fähigkeiten und Ressourcen weitgehend als ausreichend für die Aufgabe ein. Mentee fordern diese Merkma- 
le in der Mentoring-Professionalität ein. Sie gehen von einem Expertiseansatz aus. Der gegenstandsbezogene Austausch, die Kommunikation und Interaktion dienen für beide Interviewgruppen dazu, in einen Lern- und Erfahrungsprozess gehen zu können. Die Basis bildet der Beziehungsaspekt. Wertschätzender Umgang, Authentizität und Feedbackkultur sind wichtige Säulen gelingender Mentoringprozesse. Ein weiterer Aspekt für das gemeinsame Wollen stellt das psychosoziale Moment, der psychosoziale Austausch oder die individuelle psychosoziale Erfahrung dar. Mentor/innen sind sich bewusst, dass sie in der Beziehung mit Empathiefähigkeit und emotionaler Behutsamkeit auf Mentee eingehen sollen. Mentee wiederum fordern eine sorgfältige Bedachtnahme und ein Verständnis für Herausforderungen und Belastungen sowie das Erkennen von Belastungssymptomen. Instrumente und konkrete Handhabungen innerhalb der Mentoringprozesse fehlen. Zusammenfassend kann zum gemeinsamen Wollen von Mentor/innen und Mentee im schulpraktischen Mentoringprozess gesagt werden:

Mentor/innen und Mentee heben eindeutige Motivgründe wie Selbstverständnis für Mentoring-Identität, Mentoring-Professionalität, Mentoring-Mission und einen professionsspezifischen Lern- und Erfahrungsaustausch auf dialogorientierter Basis hervor. Als Aspekte des Gemeinsamen sehen sie das Wahrnehmen von praktikums- und personenspezifischen Herausforderungen im schulpraktischen Mentoringprozess.

Mentor/inbezogenes Wollen im schulpraktischen Mentoringprozess: Mentor/innen zählen professionsspezifische Zusammenarbeit und Integration sowie ein Verständnis für den Identifikationsprozess hin zum Lehrberuf als wesentliche Gelingensbedingungen auf. Mit dem erstgenannten Aspekt drücken sie die Notwendigkeit der Vernetzung mit Ausbildungsinstitutionen, theoriegeleiteten Konzepten und Modellen auch im Sinne der eigenen Professionsentwicklung (siehe Kapitel II/7.1) aus. Dieser Aspekt gilt als Anforderung und vielleicht auch als Herausforderung in der Lehrer/innenausbildung. Das Ergebnis ergibt sich wohl aufgrund der derzeitigen Reformen in der Lehrer/innenausbildung in Österreich.

Der zweitgenannte Aspekt verdeutlicht die Bereitschaft und Notwendigkeit der Öffnung des Systems Schule und die Möglichkeit zur Probeidentifikation und Entwicklungspotentialentfaltung für Mentee. Das bedingt die Auseinandersetzung mit persönlichen Verhaltensmustern, mit Fragen der Regelgeleitetheit und des Führungsstils. Es fordert auch ein reflektiertes Bild von Rollenverständnis (Schmid et al. 2011, 39) und begleitungsunterstützenden Formen und Formaten (Arnold et al. 2011; Ostermann 2015; Köffler 2015; Dreer 2018; Košinár 2018; Hofmann 2019).

Die reflexive Betrachtung des eigenen Handelns, der Austausch mit Ausbildungsinstitutionen und dem Gegenüber zu Fragen der affektiv-motivationalen Befindlichkeit sind in Zusammenhang mit dem mentor/inbezogenen Wollen wichtige Gelingenskomponenten.

Professionsspezifisches Mentoring unter Berücksichtigung funktionsbezogener und emotionaler Faktoren: ist die Kernaussage der rekonstruierten Gelingensbedingungen aus Sicht der Mentor/innen.

Mentor/innen sind bereit in ein verantwortungsvolles professionsspezifisches Mentoring einzutreten. Wesentlich ist dabei die Beachtung folgender Faktoren: Entwicklung der Profession, Fördern und Stärken für den gegenwärtigen und zukünftigen Lehrberuf, Schaffung von Lernund Entwicklungsmöglichkeiten, unterstützungsgeleitetes Begleiten und Wahrnehmen der Persönlichkeitsstruktur und psychischen Grundstimmung. 
Durch die Mentor/innentätigkeit kommt es vielfach zu einer Revitalisation der eigenen Lehrtätigkeit (Moor et al. 2005) und zu einem Bedürfnis nach eigener professionsspezifischer berufsbiografischer Entwicklung (Terhart 2001). An dieser Stelle ergeben sich für Mentor/innen die Fragen: Wie kann man die Entwicklung des anderen positiv beeinflussen? Wie kann man Stärken des anderen fördern? (Pelz 2016, 96). Nach Schweer (2004) kann unterstützungsgeleitetes Begleiten nur umgesetzt werden, wenn eine Vertrauenskomponente vorhanden ist (Schweer 2004, 280f). Hudson (2013) stellt die Phänomene Respekt und Vertrauen in das Zentrum der Kommunikation (Hudson 2013, 16). Das Wahrnehmen und Erkennen von psychosozialen Belastungen bedingt Instrumente für den Umgang, die letztendlich für das schulpraktische Mentoring fehlen. Ein gelingender Prozess eines professionsspezifischen Mentorings unter Berücksichtigung funktionsbezogener und emotionaler Faktoren kann im Sinne der Transformationalen Führung dann gegeben sein, wenn Dimensionen wie Inspirational Motivation, Idealized Influence, Intellectual Stimulation und Individualized Consideration (Bass et al. 1994; Wenddorf 2018, 48; von Au 2016, 23; Pelz 2016, 96) Beachtung finden (siehe Kapitel I/2.5).

Menteebezogenes Wollen im schulpraktischen Mentoringprozess: Die zwei Gelingensbedingungen des menteebezogenen Wollens sind: Praktikum als berufliches Lernfeld und professionsspezifische Selbstwirksamkeit. Darin kommt zum Ausdruck, dass Mentee schulische Anteile in der Zeit der Ausbildung als besonderen Mehrwert erachten und darin die Möglichkeit der Erfahrungsbildung und Identifikation mit dem zukünftigen Lehrberuf sehen. Diese Befunde decken sich mit Untersuchungen von Girmes (2004, 18), Beyer et al. (2006), Arnold et al. (2014, 244), Ostermann (2015), Leonhard et al. $(2018,12)$ usw.

Erproben, Erkunden und selbsttätiges pädagogisch praktisches Handeln sowie zur Überzeugung gelangen, Handlungsprozesse positiv gestalten zu können und Einfluss auf professionsspezifische Situationen ausüben zu können, zählen zu den Gelingensbedingungen menteebezogenen Wollens im schulpraktischen Mentoringprozess.

Mentoringinduzierte Selbstwirksamkeits- und Professionsentwicklung: wird als Kernaussage der rekonstruierten Gelingensbedingungen aus Sicht der Mentee herangezogen. Mentee haben eine Erwartungshaltung, eine Vorstellung von Mentoring und Rollenfunktionen sowie Aufgabenbereichen der Mentor/innen. Bereits die Aussagen in den Phänomenen der Kodierparadigmata und Kernkategorien weisen auf die hohe Bedeutung der Praktika während der Zeit der Ausbildung hin. Sie betonen weiters, dass Praktika zum Erfahrungslernen und Aufbau von Lehrer/innenexpertise dienen. Mentee tauchen in einen erfahrungsbasierten und sachbezogenen prospektiven Lernprozess ein, der als Ziel Selbstwirksamkeits- und Professionsentwicklung verfolgt. Die Rolle und Funktion von Mentee wird in Untersuchungen in Zusammenhang mit schulpraktischen Mentoringprozessen kaum beleuchtet. Ein mögliches Forschungsfeld würde sich hierzu ergeben.

Zusammenfassend kann gesagt werden:

Mentor/innen sollen begleiten, beraten und unterstützen, wenn nötig, und andernfalls Freiräume und Entfaltungsmöglichkeiten schaffen. Mentee konzentrieren sich im Prozess in erster Linie auf den persönlichen Bildungsnutzen. Sie verstehen unter Selbstwirksamkeits- und Professionsentwicklung den Erwerb von Kompetenzen, Fähigkeiten und Fertigkeiten und sind bedacht, dass dadurch Bewältigungsstrategien verinnerlicht werden, Ressourcenstärkung und Sicherheit im pädagogischen und praktischen Handeln eintreten. 
Auf Basis der Befunde aus den bisherigen rekonstruierten Gelingensbedingungen beider Interviewgruppen lautet die Definition zum schulpraktischen Mentoring resümierend:

Schulpraktisches Mentoring ist als professionsspezifischer Lern- und Entwicklungsprozess zu verstehen, der Aspekte gemeinsamen, mentor/inbezogenen und menteebezogenen Wollens umschließt und unter Berücksichtigung funktionsbezogener und emotionaler Faktoren von denkenden, handelnden, empfindsamen Mentor/innen und Mentee auf Professions- und Selbstwirksamkeitsentwicklung zielt.

Wie die Gestaltung des Lern- und Entwicklungsprozesses aus Sicht der/des Mentor/in aussehen kann, liefert im Folgenden das Modell zum Transformationalen Mentoring im berufsfeldbezogenen Wirkungskontext (ATM-Modell).

\subsection{Befund 4: ATM-Modell - Analyse Transformationales Mentoring im berufsfeldbezogenen Wirkungskontext}

Im Kapitel I/2.5 wird das Konzept Transformationales Mentoring in Anlehnung an Grint (2010) als Position der/des Mentorin/Mentors als auch als sozialer Prozess diskutiert. Aus den rekonstruierten Schulpraktischen Mentoring-Funktionen, den Gelingensbedingungen und Metakategorien und in Anlehnung an das „Gießener Inventar der Transformationalen Führungskompetenzen" (Identifikation, Inspiration, Stimulation, Förderung, Kommunikation, Fairness, Unternehmerische Haltung, Umsetzungsstärke) (Pelz 2016, 99f) erschließt sich im Folgenden das Modell Analyse Transformationales Mentoring im berufsfeldbezogenen Wirkungskontext (Abbildung 78). Als Bezeichnung wurde ATM-Modell (Erstbuchstaben: ATM für Analyse Transformationales Mentoring) gewählt. Es besteht aus drei Komponenten und insgesamt neun Ereignisfeldern im Kontext des schulpraktischen Mentorings.

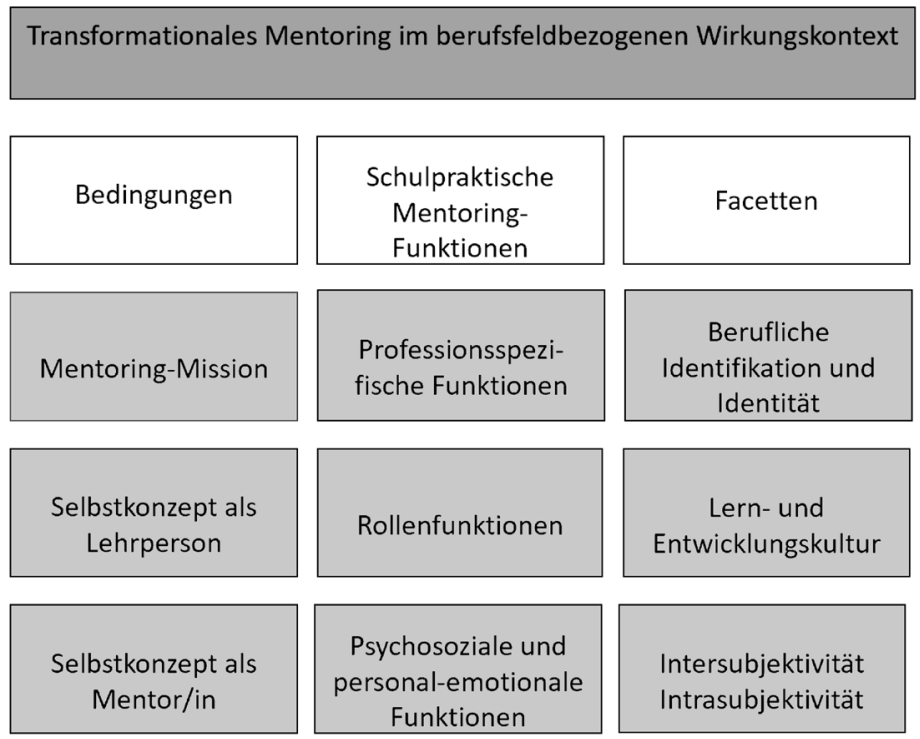

Abb. 78: Modell: Analyse Transformationales Mentoring im berufsfeldbezogenen Wirkungskontext (ATM-Modell) 
Im Kontext eingebunden sind die drei Komponenten Bedingungen, Schulpraktische MentoringFunktionen und Facetten. Die Bedingungen beziehen sich auf den selbstreflexiven Bereich der/des Mentorin/Mentors mit den Komponenten Mentoring-Mission, Selbstkonzept als Lehrperson und Selbstkonzept als Mentor/in. Im Vordergrund steht die Wahrnehmung der eigenen Person mit sich zuschreibenden und beurteilenden Komponenten (Shavelson 1976, 407f), mit der Beschreibung von personenbezogenen Stärken und Schwächen sowie das Bewusstwerden von Interessen und Bedürfnissen (Köffler 2015, 193). Eine intrapersonale reflexive Betrachtungsweise soll Klarheit zu Motiven, Verhaltensweisen, Einstellungen, Werthaltungen und pädagogischen Fragestellungen geben, um zu einem Wissen sowie einer Festlegung und Interpretation subjektbezogenen Verhaltens als Lehrperson und als Mentor/in zu kommen. Ziele sind die Erhöhung des Vertrauens in eigene Fähigkeiten und Fertigkeiten und eine reflektierte und persönliche Standpunktsetzung.

Die Schulpraktischen Mentoring-Funktionen umfassen den funktionalen Tätigkeitsbereich und Wissensaspekte zu professionsspezifischen Funktionen, Rollenfunktionen, psychosozialen und personal-emotionalen Aspekten. In der Auseinandersetzung geht es im Wesentlichen um Themen zu Anforderungsfunktion, Rollenverständnis und sozialer Unterstützung.

In den Ergebnisfeldern der Facetten werden Überlegungen zum personenbezogenen/partner/ innenbezogenen und systemischen Aufgabenbereich angestellt. Die Facetten lauten: berufliche Identifikation und Identität, Lern- und Entwicklungskultur, Intra- und Intersubjektivität und die Inhalte und Überlegungen berücksichtigen systemische Faktoren und zu integrierende Beziehungsfaktoren im Sinne einer Professionsentwicklung.

Ziel des vorgestellten ATM-Modells ist es, durch Reflexionsprozesse der/des Mentorin/Mentors sowohl subjektive Befindlichkeiten wahrzunehmen und in Frage zu stellen, als auch subjektive Standpunkte zu vertreten und einen multiperspektivischen Ansatz zu verfolgen, um Offenheit im Mentoringprozess zu gewährleisten und Entwicklungen zu forcieren. Kernelemente bilden demnach „Aufmerksamkeit bzw. Achtsamkeit“ (von Au 2016, 34), „Einflussnahme und Motivation“ (Pelz 2016, 96), „gegenseitiges Vertrauen und Wohlwollen“ (Ziegler 2009, 11) und eine „visionäre, motivierende, inspirierende und innovative“ (Schley et al. 2011, 276) Haltung. Von $\mathrm{Au}(2016)$ ist überzeugt:

„Der Fokus auf Aufmerksamkeit bzw. Achtsamkeit (...) entwickelt sich zu einer bedeutenden Erfolgsgröße im Bereich Leadership, da es nachweislich eine Persönlichkeitsentwicklung anstößt. (...) Eine achtsame Führung beginnt mit einer reflektierten Selbstführung (...), die unterschiedlich weiter entwickelt werden kann. (...) Zugleich bleibt der Fokus auf Achtsamkeit in einer hektischen und von Störungen geprägten Arbeitswelt eine immer größere Herausforderung." (von Au 2016, 34)

Die „reflektierte(n) Selbstführung“ (von Au 2016,34) wird als Kriterium in den nun weiteren Ausführungen der Arbeit herangezogen. Auf Basis der Ergebnisfelder zur Analyse Transformationales Mentoring im berufsfeldbezogenen Wirkungskontext (ATM-Modell) und bezogen auf Kategorien der Gelingensbedingungen (Metakategorien der Schulpraktischen MentoringFunktionen) werden folgend exemplarische Fragestellungen thematisch zu einer wahlweisen Kategorie formuliert und das Modell somit auf Brauchbarkeit und Gültigkeit überprüft. Es wurden die Kategorien: Persönliche positive Einschätzung zur Aufgabenerfüllung aufgrund von Persönlichkeitsstrukturen und Fähigkeiten, Eintreten in einen intensiven Lern- und Reflexionsprozess sowie Erkennen von Belastungssymptomen gewählt (Tabelle 66, 67, 68). Beim ersten Beispiel mit der Kategorie Persönliche positive Einschätzung zur Aufgabenerfüllung aufgrund von Persönlichkeitsstrukturen und Fähigkeiten hinterfragt die/der Mentorin/Mentor vor allem die persönliche Stimmungslage (Tabelle 66). 
Tab. 66: Analyse Transformationales Mentoring im berufsfeldbezogenen Wirkungskontext in Bezug zur Kategorie: Persönliche positive Einschätzung zur Aufgabenerfüllung aufgrund von Persönlichkeitsstrukturen und Fähigkeiten

\begin{tabular}{ll}
\hline Ergebnisfelder & $\begin{array}{l}\text { Exemplarische Fragestellungen zur Kategorie: } \\
\text { Persönliche positive Einschätzung zur Aufgabenerfüllung aufgrund von Persönlich- } \\
\text { keitsstrukturen und Fähigkeiten }\end{array}$ \\
\hline Mentoring-Mission & Bin ich für die Aufgabenerfüllung bereit? Bin ich grundsätzlich positiv dazu gestimmt? \\
\hline $\begin{array}{l}\text { Selbstkonzept } \\
\text { als Lehrperson }\end{array}$ & $\begin{array}{l}\text { Habe ich eine persönliche positive und reale Einschätzung in Bezug auf meine Un- } \\
\text { terrichtstätigkeit und meinen Aufgabenbereich als Lehrperson? Welche Fähigkeiten, } \\
\text { Fertigkeiten und Persönlichkeitsstrukturen unterstützen mein berufliches Handeln und } \\
\text { Denken? }\end{array}$ \\
\hline $\begin{array}{ll}\text { Selbstkonzept } \\
\text { als Mentor/in }\end{array}$ & $\begin{array}{l}\text { Habe ich eine positive und reale Einschätzung meiner Fähigkeiten und Kompetenzen } \\
\text { zur Erfüllung der Aufgabe als Mentor/in? Welche meiner Persönlichkeitsstrukturen und }\end{array}$ \\
& Fähigkeiten könnten sich positiv auf mein Gegenüber auswirken? \\
\hline $\begin{array}{l}\text { Professionsspezifi- } \\
\text { sche Funktionen }\end{array}$ & $\begin{array}{l}\text { Bin ich bereit, mit Ausbildungsinstitutionen in einen Austausch zu gehen? Ist ein wech- } \\
\text { selseitiger Lern- und Reflexionsprozess von einer grundsätzlichen positiven Stimmung } \\
\text { geprägt? }\end{array}$ \\
\hline Rollenfunktionen & Habe ich eine positive Einstellung zu meiner Rolle als Mentor/in? \\
\hline $\begin{array}{l}\text { Psychosoziale und } \\
\text { emotionale Aspekte }\end{array}$ & $\begin{array}{l}\text { Wie ist mein Menschenbild? Welche Werte und Haltungen vertrete ich innerhalb der } \\
\text { Mentoring-Funktion? }\end{array}$ \\
\hline $\begin{array}{l}\text { Berufliche Identifi- } \\
\text { kation und Identität }\end{array}$ & $\begin{array}{l}\text { Bin ich mir meiner eigenen beruflichen Identifikation und Identität bewusst? Wie wird } \\
\text { berufle Identifikation und Identität im Wirkungsfeld verstanden? Wie kann ein }\end{array}$ \\
\hline $\begin{array}{l}\text { Prozess gestaltet sein, indem berufliche Identifikation und Identität vollzogen wird? } \\
\text { Entwicklungskultur }\end{array}$ & $\begin{array}{l}\text { Bin ich an einer Lern- und Entwicklungskultur interessiert? } \\
\text { Lass ich die/den andere/n in seinem Aufgabenbereich wachsen? }\end{array}$ \\
\hline $\begin{array}{l}\text { Inter- und } \\
\text { Intrasubjektivität }\end{array}$ & $\begin{array}{l}\text { Habe ich eine positive Einstellung zu mir und meiner/meinem Mentee? Bemühe ich } \\
\text { mich um die/den anderen? }\end{array}$ \\
\hline
\end{tabular}

Beim zweiten Beispiel mit der Kategorie Eintreten in einen intensiven Lern- und Reflexionsprozess wird verdeutlicht, dass die Fragestellungen auf das Handeln der/des Mentorin/Mentors bezogen sind, um für sich zu erkennen, ob sie/er einen Lern- und Reflexionsprozess zulässt.

Tab. 67: Analyse Transformationales Mentoring im berufsfeldbezogenen Wirkungskontext in Bezug zur Kategorie: Eintreten in einen intensiven Lern- und Reflexionsprozess

\begin{tabular}{ll}
\hline Ergebnisfelder & $\begin{array}{l}\text { Exemplarische Fragestellungen zur Kategorie: } \\
\text { Eintreten in einen intensiven Lern- und Reflexionsprozess }\end{array}$ \\
\hline Mentoring-Mission & $\begin{array}{l}\text { Was will ich erreichen? Bin ich bereit, in einen Lern- und Reflexionsprozess zu } \\
\text { treten? }\end{array}$ \\
\hline $\begin{array}{l}\text { Selbstkonzept } \\
\text { als Lehrperson }\end{array}$ & $\begin{array}{l}\text { Entspreche ich in meiner Lehrtätigkeit meiner eigenen Vorstellung von Lehrper- } \\
\text { son? Entspricht das, was ich tue, meinem Konzept von Lehrperson-Sein? }\end{array}$ \\
\hline $\begin{array}{l}\text { Selbstkonzept } \\
\text { als Mentor/in }\end{array}$ & Entspricht mein Handeln dem, was ich als Aufgabe im Mentoring sehe? \\
\hline $\begin{array}{l}\text { Professionsspezifische } \\
\text { Funktionen }\end{array}$ & $\begin{array}{l}\text { Entspricht mein Handeln (und Denken) den professionsspezifischen Theorien } \\
\text { und Konzepten? Sind meine Vorstellungen und mein Tun im Rahmen der } \\
\text { Mentoring-Funktion geeignet? }\end{array}$ \\
\hline
\end{tabular}




\begin{tabular}{ll}
\hline Rollenfunktionen & $\begin{array}{l}\text { Welche Position nehme ich im Mentoring ein? Bin ich authentisch in dieser } \\
\text { Funktion? }\end{array}$ \\
\hline $\begin{array}{l}\text { Psychosoziale und } \\
\text { emotionale Aspekte }\end{array}$ & $\begin{array}{l}\text { Wie geht es mir? Wie fühle ich mich? Was brauche ich, damit ich auf andere } \\
\text { eingehen kann? Wie nehme ich andere wahr? }\end{array}$ \\
\hline $\begin{array}{l}\text { Berufliche Identifikation } \\
\text { und Identiät }\end{array}$ & $\begin{array}{l}\text { Will ich berufliche Identifikation und Identität? Welches situationsgerechte } \\
\text { Handeln wird von mir erwartet, damit berufliche Identifikation und Identität } \\
\text { beim anderen wirken kann? }\end{array}$ \\
\hline Lern- und & $\begin{array}{l}\text { Ist mein Handeln zur Erlangung von neuen Erkenntnissen offen? } \\
\text { Entwicklungskultur }\end{array}$ \\
$\begin{array}{l}\text { Wie kann ich eine Lern- und Entwicklungskultur aktiv gestalten? Welche Rah- } \\
\text { menbedingungen sind notwendig, damit ein Lernprozess möglich ist? }\end{array}$ \\
\hline Inter- und & Ist das aktuelle Mentoring fördernd für Zusammenarbeit? \\
Intrasubjektivität & Findet ein gemeinsamer Lern- und Entwicklungsprozess statt? \\
\hline
\end{tabular}

Das dritte Beispiel mit der Kategorie Erkennen von Belastungssymptomen zeigt auf, dass die Fragestellungen auf das Wahrnehmen von emotionalen Faktoren gelegt werden, um nachzudenken, wie man damit umgehen kann.

Tab. 68: Analyse Transformationales Mentoring im berufsfeldbezogenen Wirkungskontext in Bezug zur Kategorie: Erkennen von Belastungssymptomen

\begin{tabular}{ll}
\hline Ergebnisfelder & $\begin{array}{l}\text { Exemplarische Fragestellungen zur Kategorie: } \\
\text { Erkennen von Belastungssymptomen }\end{array}$ \\
\hline Mentoring-Mission & $\begin{array}{l}\text { Bin ich bereit, mich auch mit unvorhergesehenen Herausforderungen des schul- } \\
\text { praktischen Mentorings auseinander zu setzen? }\end{array}$ \\
\hline $\begin{array}{l}\text { Selbstkonzept } \\
\text { als Lehrperson }\end{array}$ & $\begin{array}{l}\text { Gibt es für mich besondere Herausforderungen im Schulalltag und im beruflichen } \\
\text { Umfeld? Wie gehe ich mit ihnen um? Wie ist mein aktuelles Belastungserleben? }\end{array}$ \\
\hline $\begin{array}{l}\text { Selbstkonzept } \\
\text { als Mentor/in }\end{array}$ & $\begin{array}{l}\text { Welche Einstellung und Haltung habe ich, wenn in der Betreuungssituation } \\
\text { verstärkt (belastende) affektiv-motivationale Faktoren auftreten? Wie reagiere } \\
\text { ich aktiv auf solche? }\end{array}$ \\
\hline $\begin{array}{l}\text { Professionsspezifische } \\
\text { Funktionen }\end{array}$ & $\begin{array}{l}\text { Ist mir Achtsamkeit in der Begleitung in Bezug auf Belastungssymptome ein } \\
\text { wichtiges Anliegen? Wo sind meine Grenzen im Betreuungsverhältnis? }\end{array}$ \\
\hline Rollenfunktionen & $\begin{array}{l}\text { Bin ich bereit, diagnostische Instrumente, Modelle und Konzepte kennenzuler- } \\
\text { nen und mir anzueignen, um eine personenspezifische und beziehungsorientier- } \\
\text { te Begleitung zu ermöglichen? }\end{array}$ \\
\hline $\begin{array}{l}\text { Psychosoziale und } \\
\text { emotionale Aspekte }\end{array}$ & $\begin{array}{l}\text { Wie erfolgt meine Auseinandersetzung mit der eigenen Psychohygiene? Nehme } \\
\text { ich überhaupt und wenn ja, auf welche Weise nehme ich die unterschiedlichen } \\
\text { Repräsentanten emotionaler Prozesse wahr? }\end{array}$ \\
\hline $\begin{array}{l}\text { Berufliche Identifikation } \\
\text { und Identität }\end{array}$ & $\begin{array}{l}\text { Welche selbstregulativen Maßnahmen sind mir im Berufsleben wichtig? Wie } \\
\text { gelingt es mir, Selbstdisziplin und Selbstorganisation sinnvoll aufzubauen? }\end{array}$ \\
\hline $\begin{array}{l}\text { Lern- und } \\
\text { Entwicklungskultur }\end{array}$ & $\begin{array}{l}\text { Wie kann ich situationsgerecht Ansätze zu den Konzepten Empowerment, Selbst- } \\
\text { wirksamkeit und Entwicklungsaufgaben im Mentoring wirksam umsetzen? }\end{array}$ \\
\hline $\begin{array}{l}\text { Inter- und } \\
\text { Intrasubjektivität }\end{array}$ & $\begin{array}{l}\text { Wie ist das Beziehungsverhältnis zwischen mir und der/dem Mentee? Werden } \\
\text { beidseitig Emotionen in ihrem Kontext wahrgenommen und respektiert? Wel- } \\
\text { che Regulationsfaktoren beeinflussen und unterstützen mein Erleben? }\end{array}$ \\
\hline
\end{tabular}


Das angeführte ATM-Modell mit seinen Ereignisfeldern und die drei Analysebeispiele mit Fragenkatalogen zu unterschiedlichen Kategorien erklären, dass Mentoring zunächst ein personales Geschehen ist, das eine reflexiv-forschende Haltung und reflektiertes Handeln der/des Mentorin/Mentors notwendig machen, damit Transformationen überhaupt erst möglich sind. Lernen und Entwickeln basieren aufgrund von Verhaltensaktionen der Personen und verdeutlichen sich in Prozessen. Beziehungsstrukturen, wie im Mentoring, sind im Idealfall auf personenspezifische Förderung und Stärkung ausgelegt und münden in einen gemeinsamen Erfahrungsraum (Stangl 2019; Cohn 1997, 155). Das Zusammenspiel von Innensteuerung und Außensteuerung (Edelmann 2000, 279) entscheidet schließlich über einen gelingenden schulpraktischen Mentoringprozess.

Nach dem vorliegenden ATM-Modell ist Transformationales Mentoring in Ereignisfeldern von Bedingungen, Schulpraktischen Mentoring-Funktionen und Facetten eingebunden und im berufsfeldbezogenem Kontext ausgewiesen. Nur die Vernetzung und Interaktion der Ereignisfelder deuten auf gelingende Bedingungen im schulpraktischen Mentoringprozess. Abbildung 79 veranschaulicht die Vernetzung und Verschränkung.

\begin{tabular}{|c|c|c|c|}
\hline Berufsfeldbezogener Wirkungskontext \\
Mentoring-Mission & $\begin{array}{c}\text { Professionsspezifische } \\
\text { Funktion }\end{array}$ & Rollenfunktion & $\begin{array}{c}\text { Psychosoziale und } \\
\text { personal-emotionale } \\
\text { Funktion }\end{array}$ \\
\hline $\begin{array}{c}\text { Selbstkonzept als } \\
\text { Lehrperson }\end{array}$ & Transformationales Mentoring & $\begin{array}{c}\text { Inter- und } \\
\text { Intrasubjektivität }\end{array}$ \\
\hline $\begin{array}{c}\text { Selbstkonzept als } \\
\text { Mentor/in }\end{array}$ & $\begin{array}{r}\text { Lntwicklungskultur } \\
\text { Lern- und }\end{array}$ & $\begin{array}{c}\text { Berufliche Identifikation } \\
\text { und Identität }\end{array}$ & \begin{tabular}{c} 
Intion \\
\hline
\end{tabular}
\end{tabular}

Abb. 79: Transformationales Mentoring unter Vernetzung der Ereignisfelder im berufsfeldbezogenen Wirkungskontext (ATM-Modell)

Das ATM-Modell berücksichtigt im berufsfeldbezogenen Wirkungskontext strukturelle und organisatorische Ebenen (siehe Kapitel I/2.5) so wie es auch der systemtheoretische Ansatz vorschlägt, in dem Aspekte, Eigenschaften und Strukturen des Systems in Konzeption, Gestaltung und Durchführung miteinbezogen werden (von Au 2016, 26f). Im Transformationalen Mentoring versteht man darunter die Einbeziehung der Systeme Schule, Lehrer/innenausbildung und Formale Bildung. Die neun Ergebnisfelder des ATM-Modells mit Vorschlägen zum Selbstreflexionsprozess beziehen sich auch auf die individuelle und kulturelle Ebene (siehe Kapitel I/2.5). Auseinandersetzungen mit dem Selbst- und Fremdbild sowie konstruktive Gedankengänge könnten von Mentor/innen in einem Entwicklungsplan festgehalten und in Folge Zieldimensionen formuliert werden (Pelz 2016, 109f) - ein Vorschlag für einen vertiefenden Selbstreflexionsprozess.

Die rekonstruierten neun Ergebnisfelder im berufsfeldbezogenen Wirkungskontext des Mentorings sowie die angeführten Beispiele mit ihren Fragestellungen geben einen Überblick über Selbstreflexionsprozesse und werden im Folgenden mit mentoringinduziertem Wollen verbunden. 
Wie das Zusammenspiel des ATM-Modells mit den Gelingensbedingungen schulpraktischer Mentoringprozesse aus den Konsequenzen im Rahmen der Schulpraktischen Mentoring-Funktionen erfolgen könnte, veranschaulicht das Mentoringinduzierte Wirkungsmodell (MIWModell).

\subsection{Befund 5: MIW-Modell - Mentoringinduziertes Wirkungsmodell zum schulpraktischen Mentoringprozess}

Die vorgestellten Befunde bedingen in einem weiteren Rekonstruktionsprozess eine Verbindung und Verschränkung auf der Ebene des mentoringinduzierten, mentor/inbezogenen, gemeinsamen und menteebezogenen Wollens. Das nachfolgende Mentoringinduzierte Wirkungsmodell zum schulpraktischen Mentoringprozess - kurz MIW-Modell (Erstbuchstaben: MIW für Mentoringinduzierte Wirkungsmodell) - verdeutlicht das Zusammenspiel der erwähnten Komponenten und vollzieht sich in der mentoringinduzierten Wirkung, die im Optimalfall auf Professions- und Selbstwirksamkeitsentwicklung zielt.

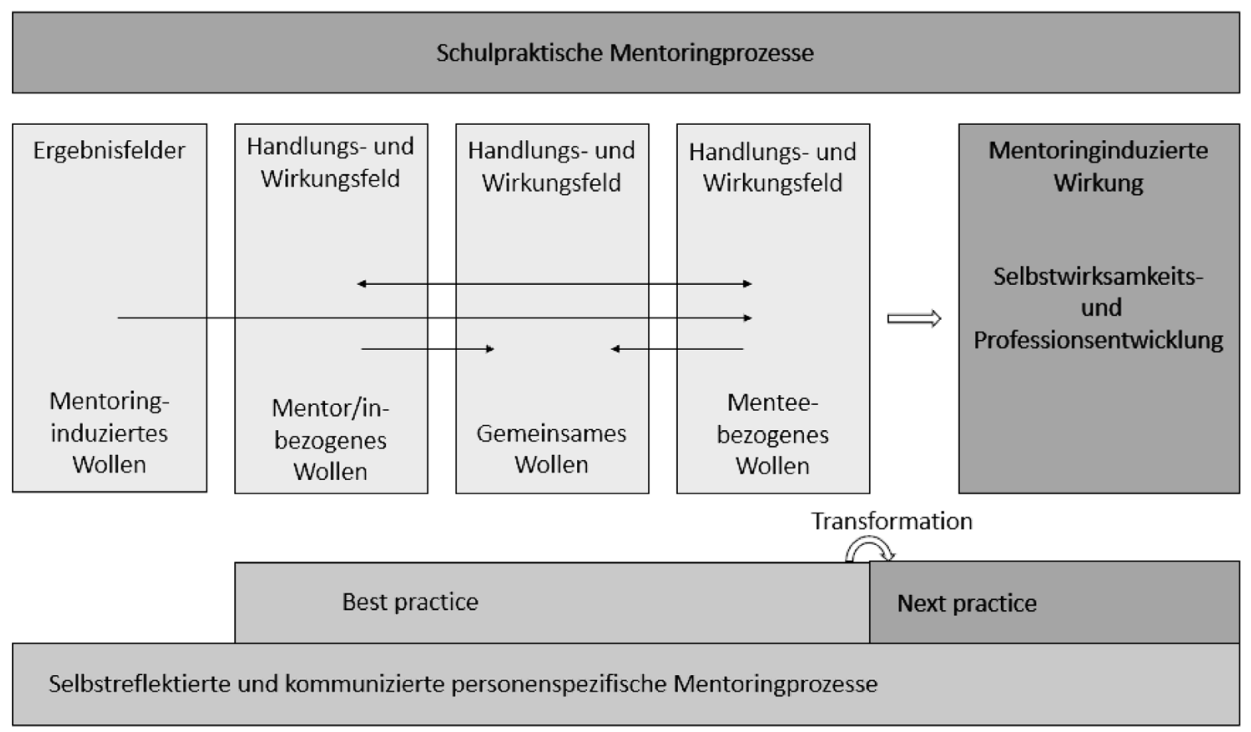

Abb. 80: Mentoringinduzierte Wirkung im schulpraktischen Mentoringprozess (MIW-Modell)

Schulpraktisches Mentoring gestaltet sich als ein zwischen handelnder $/ \mathrm{m}$, denkender $/ \mathrm{m}$, fühlender/m Mentor/in und Mentee selbstreflektierter und im Idealfall kommunizierter personenspezifischer Mentoringprozess. Den Ausgang bildet das mentoringinduzierte Wollen mit hohem Anteil an Selbstreflexion (siehe Ergebnisfelder). Mentor/innen beachten Aspekte der Bedingungen, Schulpraktischen Mentoring-Funktionen und Facetten in ihrem Reflexionsprozess. Sie festigen damit ihre Mentoring-Funktion und Mentoring-Identität und klären die Bedeutung von Führung und Leadership für sich persönlich. Ihre/Seine Überlegungen wirken auf das Handlungs- und Wirkungsfeld im schulpraktischen Mentoringprozess. Dieses Handlungs- und Wirkungsfeld gestalten Mentor/innen und Mentee in einer wechselseitigen Bezie- 
hung. Hierbei treffen Vorstellungen, Werthaltungen, Ermöglichungen und Erwartungen - wie im Rahmen des mentor/inbezogenen, gemeinsamen, menteebezogenen Wollens beschrieben aufeinander. Die Beteiligten treten in einen sozialen Prozess ein, indem Selbstrespekt und Selbstverantwortung zum Tragen kommen muss. Ein gelingender Prozess wird davon abhängig sein, ob eine Balance zwischen mentor/inbezogenem und menteebezogenem Wollen gefunden wird und das gemeinsame Wollen in den Vordergrund rückt. Im Handlungs- und Wirkungsfeld ergeben sich Chancen und Herausforderungen.

Im Kontext des schulpraktischen Mentoringprozesses ist eine Transformation von best-practice zu next-practice möglich und wünschenswert. Eine veränderte Praxisperspektive kann - im Sinne des beschriebenen Ansatzes zum Transformationalen Mentoring - vor dem Hintergrund einer selbstkritischen, proaktiven und prospektiven Beziehungsform im Rahmen des sozialen Prozesses gelingen. Der Prozess zielt im Sinne einer mentoringinduzierten Wirkung auf Selbstwirksamkeits- und Professionsentwicklung denkender, handelnder, empfindsamer Mentee und wie aus der vorliegenden Untersuchung erhoben auch auf die Selbstwirksamkeits- und Professionsentwicklung der Mentor/innen im Sozialisationsfeld Schule ab. Die Selbstwirksamkeits- und Professionsentwicklung könnten als Resultat eines gelingenden Prozesses verstanden werden. Nach der Darlegung der Erkenntnisse kann als Rahmung des schulpraktischen Mentoringprozesses ein professions-, praktikums- und personenspezifisches Mentoring Bedeutung erlangen. Denn es gilt:

Ziel ist, im Rahmen mentoringinduzierter Wirkung förderliche Lern- und Entwicklungsbedingungen zu schaffen, Entwicklungen seitens der Mentee als auch der Mentor/innen zuzulassen und darauf zu achten, dass reflektierte Kompetenzen, Fähigkeiten und Fertigkeiten angeeignet und vertieft werden, damit Anforderungen in der Profession bewältigt werden können.

Im Kapitel Integration und Kontextualisierung der Befunde wurden weitere verdichtete Befunde dokumentiert und diskutiert. Unter Einbeziehung der vorgestellten Darstellungen über rekonstruierte Gelingensbedingungen schulpraktischer Mentoringprozesse in der Lehrer/innenausbildung kann zusammenfassend festgestellt werden:

Schulpraktische Mentoringprozesse - unter Einbeziehung von Kriterien und Aspekten einer lern- und entwicklungsorientierten Beziehung zwischen Mentor/in und Mentee und Metakategorien der Schulpraktischen Mentoring-Funktionen - fördern eine reflexive, proaktive, transformationsgeleitete, subjektorientierte und professionsspezifische Haltung. Diese bildet die Grundlage für einen erhöhten mentoringinduzierten Wirkungsgrad zur Selbstwirksamkeits- und Professionsentwicklung.

Die Erklärung umschließt die Notwendigkeit der Beachtung der Beziehungs- und Inhaltsaspekte, die auf einen fördernden personenspezifischen Lern- und Entwicklungsprozess ausgelegt und aufgrund der Anforderungsprofile im Kontext der Lehrer/innenausbildung strukturell verankert sind.

Basierend auf den grundlegenden Überlegungen aus Kapitel Grundlagen und den empirischen Befunden wird im letzten Kapitel ein Resümee gezogen, die Grenzen der Untersuchung diskutiert und im Rahmen des Ausblicks Vorschläge für Gestaltung von Mentoringausbildungsformaten und Forschungsvorhaben angeführt. 


\section{Resümee und Ausblick}

Ziel der vorliegenden Arbeit ist die Identifikation und Rekonstruktion von Gelingensbedingungen schulpraktischer Mentoringprozesse. Die Fragestellung zur empirischen Untersuchung erleichterte es, Abstand von hypothesenüberprüfenden Verfahren zu nehmen und sich methodologisch auf eine konzeptuelle Verknüpfung von Datenerhebung und Datenauswertung wie bei der Grounded Theory zu konzentrieren. Im Analyseprozess erfolgte eine systematische Verdichtung der Aussagen, wie im Kapitel II der Arbeit verdeutlicht.

Den Ausgangspunkt bildeten Leitfadeninterviews, in denen die Sichtweisen von Lehrpersonen $(\mathrm{n}=12)$, die mit der Aufgabe und Funktion des Mentorings betraut sind, sowie von Studierenden $(n=12)$, die am Ende ihrer Ausbildungszeit standen, erhoben wurden. Anlassstiftend für die Untersuchung sind mehrere Faktoren: Neustrukturierung der Lehrer/innenausbildung im Rahmen von Pädagog/innenbildung NEU in Österreich, Einbeziehung und der hohe Stellenwert PädagogischPraktischer Studien in den Curricula der Pädagogischen Hochschulen und Universitäten, Wichtigkeit der Einbeziehung von Mentor/innen als Brückenbauer/innen zwischen Schule und Institutionen in das Ausbildungskonzept sowie Empfehlungen seitens des Bildungsministeriums und hohes Interesse der Institutionen, die Aus-, Fort- und Weiterbildung von Mentor/innen zu forcieren. Als Expert/innen wurden Personen ausgewählt, bei denen man annehmen kann, Informationen zur Forschungsfrage zu bekommen (Misoch 2015, 186). Außerdem sollten Repräsentativität und Generalisierung berücksichtigt werden. Es wird versucht, eine „theoretische Sättigung“(Strauss 1991, 21) aufgrund der bedeutsamen Unterschiede im Feld zu erreichen (Przyborski et al. 2014, 182).

„Mit Sättigung ist der Punkt im Verlauf der Analyse gemeint, an dem zusätzliches Material und weitere Auswertungen keine neuen Eigenschaften der Kategorie mehr erbringen und auch zu keiner relevanten Verfeinerung des Wissens um diese Kategorie mehr beiträgt." (Strübing 2014, 32)

Die transkribierten und kodierten Daten wurden mittels Grounded Theory ausgewertet. In der Grounded Theory bezeichnet man den Prozess der Auswahl der zu untersuchenden Personen oder Ereignisse (Datenerhebung) und die Auswahl jener Konzepte und Kategorien, die im Zuge der Datenanalyse weiterentwickelt werden, als „Theoretisches Sampling" (Glaser et al. 1998, 53). Die Auswahlkriterien bzw. die Samplingschritte ergeben sich in jeder Operationsebene z.B. durch Fragestellungen. Das Verfahren zielt darauf, durch wiederkehrende und kontrollierende Steuerung Kriterien der Theoretischen Sättigung - dem Nichtauffinden neuer Eigenschaften - zu erfüllen. Schlussendlich obliegt es der/dem Forscher/in, Entscheidungen zur Sättigung zu treffen: den Zustand des endgültigen Festlegens von empirisch zu belegenden Kategorien und Phänomenen (Strübing 2014, 29f).

In diesem abschließenden Teil der vorliegenden Arbeit werden die Grenzen der Arbeit thematisiert, bevor Ergebnisse vor dem Hintergrund der Forschungsfrage zusammengefasst und Empfehlungen für Ausbildungsmodelle aufgezeigt werden.

\section{Grenzen der Arbeit}

Jede wissenschaftliche Arbeit soll in einer kritischen Reflexion im Blick auf Grenzen der Untersuchung analysiert werden. Aber gerade für die qualitative Sozialforschung stellt sich oftmals 
die Frage, welche Kriterien besonders relevant sind. Dazu könnte der Vorschlag Lüders (2011) aufgegriffen werden, „den Weg für verfahrens- und gegenstandsbezogene Kriterien zu öffnen.“ (Lüders 2011,82) Dabei muss angemerkt werden, dass es für die Grounded Theory kaum explizite Kriterien gibt (Strübing 2014, 81f).

Das vorliegende Forschungsdesign ist auf Erhebung der Daten durch Expert/inneninterviews und analytische Identifizierung des Datenmaterials aufgrund der Ausführung von „bestimmten Operationen“" (Straus 1991b, 33) gekennzeichnet, um über Abstraktion und Strukturierung zu einer nächsthöheren Ebene zu gelangen.

Die Frage der Generalisierbarkeit ist mit dem eindimensionalen Untersuchungsverfahren und der Auswahl der Samples verknüpft und bedeutet so im Vorhinein eine bestimmte Begrenztheit. Eine quantitativ aufbauende Studie auf Erkenntnissen dieser Untersuchung würde die Aussagen verifizieren bzw. falsifizieren, eine numerische Darstellung der Sichtweisen aufzeigen und den Grad der Generalisierbarkeit erhöhen (Bortz et al. 2006, 336). Aber das am Beginn der Untersuchung zwar zweiphasig angelegte Untersuchungsmodell (qualitative und dann quantitative Methode) wurde im Laufe der Arbeit aufgrund der Datenfülle und systematischen Vorgehensweise auf eine eindimensionale Untersuchung abgeändert, da es den Rahmen der vorliegenden Arbeit gesprengt hätte. Die Ergebnisse dieser Studie können aber Ausgangspunkt weiterer quantitativer Forschungsvorhaben sein.

Bei der Auswahl der Expert/innen vollzog sich ein Selektionsprozess. Die Erhebung wurde zwar unmittelbar im so genannten Feld gemacht, dennoch haben sich Mentor/innen für Interviews zur Verfügung gestellt, die seit Jahren bereits eine Begleitungstätigkeit ausüben und den Eindruck erwecken, dass sie mit Freude die Mentoring-Funktion ausüben. Bei den Mentee kamen Personen zu Wort, die im Laufe ihres Studiums als engagierte und als stets sich kritisch äußernde Studierende aufgefallen sind. Die Wahl der Samples fiel auf eine kleine Stichprobengröße von Mentor/innen und Studierende, wohnhaft und eingebettet in einem geografisch ländlichen und relativ homogenen Bezugsrahmen. Dieser Umstand zeigt einen weiteren Aspekt der Limitation der Generalisierbarkeit im Rahmen dieser Untersuchung auf. Betont darf aber werden, dass das Untersuchungsdesign die Perspektiven der/des Mentorin/Mentors und der Mentee berücksichtigt.

Die Nachvollziehbarkeit der Rekonstruktionsschritte ist wohl aufgrund der differenzierten und transparenten Analyse gegeben, wenngleich die Objektivität der Aussagen (Bortz et al. 2006, 326; Häder 2010, 108) aufgrund der intersubjektiv geprägten Herangehensweise und der Nähe der Forscherin zum Feld (Heiser 2018, 47f) kritisch betrachtet werden könnte. Aus diesem Grund wurde bei der Erstellung der Kodierparadigmata, Kernkategorien, Metakernkategorie und Modelle im Laufe der Rekonstruktion und Interpretation immer wieder auf Konzepte, Subkategorien bzw. Kategorien verwiesen. So kann in den unterschiedlichen Rekonstruktionsverfahren auf eine konzeptuelle Repräsentativität (Strübing 2014, 83) zurückgegriffen werden. Außerdem wurden Zitate als Belege angeführt, um eine empirische Verankerung (Heiser 2018, 49) zu verdeutlichen. Durch diese Vorgehensweise kann von einer Transparenz bei der Analyse und Rekonstruktion ausgegangen werden (Heiser 2018, 273). Des Weiteren fanden während des Analyseprozesses zwei Teamsitzungen statt. Nach den Teilarbeitsprozessen - vor allem nach der Konzeption der Meilensteine wie Kodierparadigmata, Kernkategorien, Metakernkategorie und Modelle - wurde mit dem Betreuer zeitintensiv reflektiert. Damit wird dokumentiert, dass eine Reflexion zur Subjektivität stattgefunden hat (Heiser 2018, 49) und nach Steinke (2013) kann davon ausgegangen werden, dass eine theoretische Kohärenz (Steinke 2013, 329f) nachgewiesen werden kann, da die Arbeitsschritte einem „kontinuierlichen Überprüfungsprozess“ (Strübing 2014, 82) - auch in der Interaktion mit anderen Forscherinnen und Forschern - unterzogen wurden. Unterstützung in der Datenanalyse bildeten neben den Kodierungsverfahren die Methode des Fragenstellens an das 
Material und das Schreiben von Memos. Diese Verfahrenselemente ließen der Forscherin einen gewissen Spielraum, erforderten mitunter einen kreativen und flexiblen Zugang (Heiser 2018, 46). Wichtig war und ist dabei, „offen zu bleiben für neue und überraschende Erkenntnisse." (Heiser 2018, 46) Die Frage, ob sich bei einer Wiederholung dieser Untersuchung die gleichen Phänomene und Modelle herauskristallisieren würden, bleibt offen. Generell sind Corbin et al. $(1990,424)$ der Meinung, „dass eine buchstäbliche Wiederholbarkeit der Studie mit identischen Ergebnissen faktisch ausscheidet, weil die Herstellung identischer Ausgangsbedingungen für die erneute Untersuchung nicht zu leisten sei.“ (Strübing 2014, 81)

Ein Folgeprojekt zu Gelingensbedingungen schulpraktischer Mentoringprozesse mit einer quantitativen Testanalyse und einer hohen Anzahl an Proband/innen würde nicht nur eine Streuung der Messwerte ergeben, sondern auch die Relevanz der vorgestellten Befunde verdeutlichen. Es könnte auch zur Validierung und Weiterentwicklung der qualitativen Befunde beitragen. Die Forschungsfragen dazu könnten lauten: Wie schätzen Studierende die Bedeutung der Gelingensbedingungen ein? Wie schätzen Mentor/innen die Bedeutung der Gelingensbedingungen ein? Ein solches Forschungsvorhaben wäre sicher eine lohnende Aufgabe im Blick auf eine weitere Qualitätsentwicklung in der Pädagog/innenbildung. Weitere Vorschläge für künftige Folgeprojekte finden sich im Abschnitt Zusammenschau der empirischen Befunde und Ausblick.

Auf eine grundsätzliche Problematik dieser Arbeit wird noch kurz hingewiesen: Aufgrund der Struktur und der gewählten Methode dieser Forschungsarbeit lassen sich gewisse Wiederholungen nicht vermeiden. Auf diese Weise treten aber die beschriebenen Phänomene umso deutlicher hervor und unterstreichen deren Bedeutung.

\section{Zusammenschau der empirischen Befunde und Ausblick}

Aus Sicht der Ausbildungsinstitutionen gilt wissenschaftlich fundiertes, forschungsgeleitetes Wissen als Basis für professionelles pädagogisches Handeln im Lehrberuf (Herzmann et al. 2016, 20). Aneignung und Erwerb des theoriebegründeten Professionswissens „(z.B. Unterrichtstheorie und Didaktik, empirische Lehr-Lern-Forschung, pädagogische Diagnostik)“ (Herzmann et al. 2016, 20) sind seitens ausbildungsinstitutioneller Sicht auf eine bestimmte Altersgruppe bzw. Schulstufen (Primarstufe und Sekundarstufe) und in der Sekundarstufenausbildung auf Fächer bzw. Fach und Spezialisierung (wie Inklusive Pädagogik, Medienpädagogik) bezogen. Hintergrund dieses Konzeptionsgedankens ist die Umsetzung und Sicherung einer theoretischen bildungswissenschaftlichen, fachwissenschaftlichen, fachdidaktischen und pädagogischen Fundierung in der jeweiligen beruflichen Qualifikation (Horstkemper 2004, 471). Im Idealfall werden Bereiche und Inhalte auf normativ-curricularer bzw. formell-institutioneller Ebene abgestimmt und in Beziehung gesetzt, und zwar so, dass eine Verschränkung oder Köharenz stattfinden kann.

„Kohärenz beschreibt eine sinnhafte Verknüpfung von Strukturen, Inhalten und Phasen in der Lehrerbildung (sic!). Kohärente Lehr-Lern-Gelegenheiten stellen systematische Bezüge her, welche es den Lernenden ermöglichen, diese Strukturen, Inhalte und Phasen als zusammenhängend und sinnhaft zu erleben." (Hellmann 2019, 9)

Ziel ist es, dass Studierende während der Zeit der Ausbildung die Verknüpfung der unterschiedlichen Elemente wahrnehmen und sinnstiftend und situationsspezifisch auf individueller Ebene im Sinne der Relationierung agieren können (Cramer 2020, 270).

Im Rahmen dieses gegenwärtigen Ausbildungskonzepts sind Pädagogisch-Praktische Studien und Schulpraktika ein fester Bestandteil jeder modernen Lehrer/innenausbildung. Innerhalb der beruflichen Professionalisierung wird den schulpraktischen Anteilen „eine ,Brückenfunk- 
tion' in der Vermittlung, zwischen den universitären Theoriestudien und der späteren Berufspraxis' (Arnold et al. 2011, 90) zugeschrieben." (Fischer et al. 2018, 152)

Die Einbeziehung und Berücksichtigung schulpraktischer Anteile während der Zeit der Ausbildung kommen den Wünschen und Forderungen der Lehramtsstudierenden entgegen. Studien zeigen auf, dass Studierende besonders Wert darauf legen, Einblick in den beruflichen Alltag und die Möglichkeit des Unterrichtens zu bekommen (Hascher 2007; Bach 2013, 106). Praktika werden von ihnen häufig als „Herzstück“ (Hascher 2006, 130), „Kernstück“ (Arnold et al. 2011, 9), „Highlight der Ausbildung“ (Ostermann 2015) wahrgenommen. Hierbei sind jedoch nicht Länge und Dauer von Praktika entscheidend, sondern die Qualität der Lernprozesse (Gröschner et al. 2015) und Lernerfolge im Praktikum (Dieck et al. 2009; Müller 2010). Erfolge und Nutzen von Praktika sind sehr stark mit den Fragen verbunden, wie der theoriegeleitete Reflexionsteil aufbereitet wird bzw. was im Reflexionsteil von Studierenden in den begleitenden Lehrveranstaltungen verlangt wird (Hascher 2012; Arnold et al. 2014). Der Begleitung in den Praktika und professionellen Vor- und Nachbereitung in den schulischen und hochschulischen Anteilen kommt demnach eine besondere Bedeutung zu (Dehne et al. 2018, 109, Hesse et al. 2020) - schulpraktisches Mentoring bekommt eine Schlüsselfunktion.

Schulpraktisches Mentoring ist in den Curricula der Lehrer/innenausbildung strukturell verankert und vollzieht sich institutionell in den Partnerschulen der Pädagogischen Hochschulen und Universitäten. Es besteht ein allgemeines Übereinkommen, dass Mentoring einen hohen Stellenwert hat (Hobson 2009; König et al. 2018). Es wird angenommen, dass die Beziehungs- und Betreuungsqualität von Mentor/innen mit der Wirksamkeit von Schulpraktika korreliert (Wilson et al. 2001; Abel et al. 2007; Hascher et al. 2012). Führt man - wie oben angeführt - das Bild der Brücke weiter, dann könnte man Mentor/innen als weitere Brückenpfeiler im Sinne der Professionalisierung sehen. Ergebnisse der vorliegenden Arbeit verdeutlichen, dass im schulpraktischen Mentoring Personen - Mentee und Mentor/in - mit unterschiedlichen Vorstellungen, Erwartungen, Werthaltungen und Intentionen im institutionellen Kontext Lehrer/innenausbildung und Schule einander begegnen. Nach der Grounded Theory wurden Phänomene in den Kernkategorien und der Metakernkategorie aus Studierenden- und Lehrendenperspektive rekonstruiert (Abbildung 81).

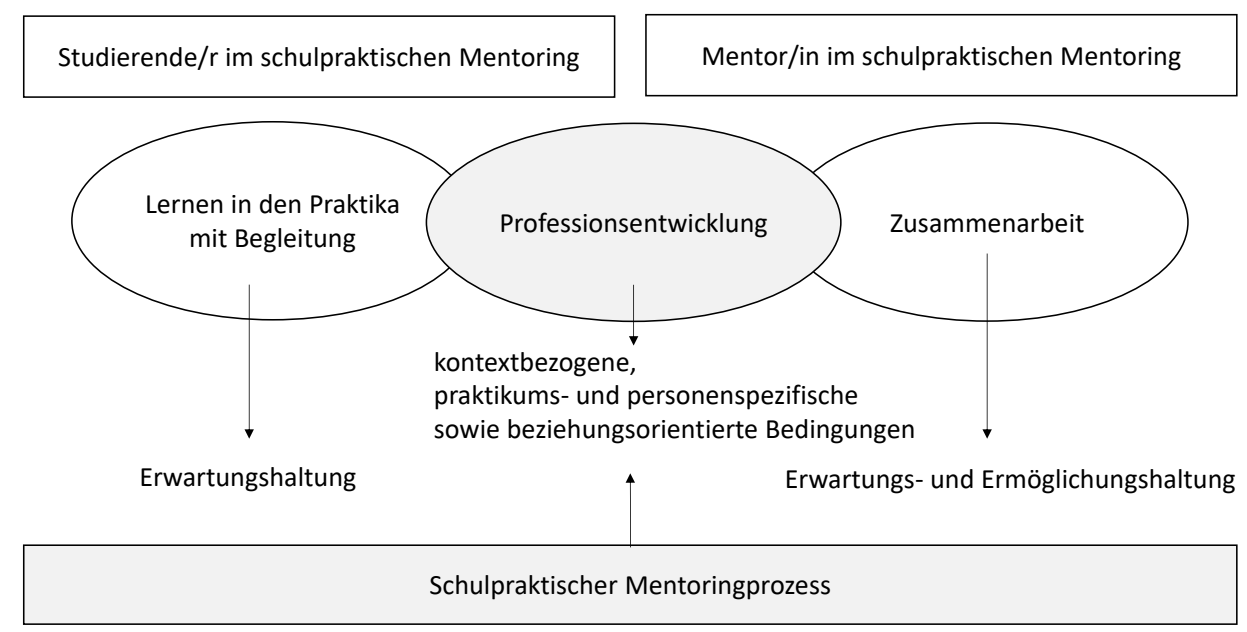

Abb. 81: Forschungsbefunde 
Für Mentee stehen das Phänomen Lernen in den Praktika mit Begleitung und für Mentor/innen das Phänomen Zusammenarbeit im Fokus. Mentee verspüren in der ursächlichen Bedingung den Wunsch nach Entfaltungsmöglichkeit. Somit treten sie im Mentoringprozess und in ihrer Rolle als Mentee in eine Erwartungshaltung ein. Auch Mentor/innen haben subjektive Erwartungen an Studierende, das System und sich selbst, sobald sie bereit und motiviert sind, die Funktion auszuüben. Die Untersuchungsgruppe zeigt auf, dass sie im Speziellen erkennen, dass sie Studierenden im System Schule, Klasse und Unterricht einen Lern- und Erfahrungsraum durch Konzeption und Durchführung unterschiedlicher Gestaltungsformen ermöglichen bzw. ermöglichen können, und dabei auch für sich selbst Erkenntnisse, Chancen und Perspektiven eröffnen. Bei näherer Betrachtung dieser beiden Phänomene aus den Kernkategorien kristallisiert sich heraus, dass beide Stichprobengruppen schulpraktisches Mentoring als Möglichkeit der Professionsentwicklung für sich verwenden bzw. erkennen. Damit Lern- und Entwicklungsprozesse zur Profession innerhalb des Mentorings vollzogen werden können, bedarf es der Etablierung von kontextbezogenen und personenspezifischen Lern- und Erfahrungsräumen und der Berücksichtigung praktikumsspezifischer und beziehungsorientierter Bedingungen. Die Begegnungen fordern demzufolge auf, sich auf einen Lern- und Reflexionsprozess einzulassen.

In der vorliegenden Untersuchung wurden Schulpraktische Mentoring-Funktionen mit Hilfe der Grounded Theory rekonstruiert und Gelingensbedingungen dazu dokumentiert. Aus Sicht der Mentor/innen ergeben sich Überlegungen zu Professionsspezifischen Funktionen, Mentoring-Funktionen und Rollenfunktionen. Mentee heben die Wichtigkeit von Aufbau professionsspezifischer Kompetenzfunktionen über die Verfügbarkeit eines Rollenmodells, Psychosoziale und personal-emotionale Aspekte und Forderungen an die Mentoring-Rolle hervor. Die Verständigung dieser rekonstruierten Schulpraktischen Mentoring-Funktionen mündet in Erklärungen des gemeinsamen, mentor/inbezogenen und menteebezogenen Wollens. Fragestellungen wie: Was möchte ich? Was möchtest du? Was möchten wir? Was gibt uns der Kontext vor und was ist im Kontext möglich? sind somit Teil der Aktionen und Handlungen im schulpraktischen Mentoring. Dabei legen Mentee einen gelingenden Prozess auf mentoringinduzierte Selbstwirksamkeits- und Professionsentwicklung aus, Mentor/innen fokussieren ein professionsspezifisches Mentoring unter Berücksichtigung funktionsbezogener und emotionaler Faktoren. Diese Erkenntnisse legen den Schluss nahe, dass im schulpraktischen Mentoring ein praktikums- und personenspezifisches Mentoring eingefordert werden muss.

Weiters wurden neun Ergebnisfelder rekonstruiert, die die Grundlage für Selbstreflexionsprozesse für Mentor/innen bilden können und in der Beschreibung des ATM-Modells (Analyse Transformationales Mentoring im berufsfeldbezogenen Wirkungskontext) dokumentiert sind. Hierbei werden Fragestellungen wie: Warum übernehme ich Mentor/innentätigkeit? Was ist mir als Lehrperson und Mentor/in wichtig? Was bedeutet für mich berufliche Identität? Wie gestalte ich Mentoringprozesse? Was beachte ich in der zwischenmenschlichen Beziehung? in den Mittelpunkt gestellt. Die selbstreflektierte Beantwortung und Umsetzung in konkreten Handlungsstrategien werden als wesentliche Komponenten für eine gelingende Begleitung angesehen. Eine reflektierte persönliche Standpunktsetzung der/des Mentorin/Mentors untermauert letztlich die Bereitschaft mentoringinduzierten Wollens, das ausdrückt, dass Mentor/innen sowohl auf der Persönlichkeits- als auch auf der Inhalts- und Beziehungsebene gewillt sind, in einen proaktiven und prospektiven Prozess und Austausch zu gehen. Aufbauend auf diesen neun Ergebnisfeldern könnte sich zwischen Mentor/ in und Mentee eine Lernkultur entfalten, die im Sinne des Transformationalen Mentorings darauf ausgelegt ist, für Neues offen zu sein, einen Musterwechsel zuzulassen und ein next-practice Beispiel zu implementieren bzw. entstehen zu lassen. Transformationales Mentoring vertritt die Ansicht, dass Mentor/innen mit ihrem Führungsverhalten eine bedeutende Rolle einnehmen. Durch selbst- 
reflektierte Haltung in der Begleitform werden Räume geöffnet und Ideen entfacht, um in einen wirkungsvollen sozialen Prozess einzutreten, Innovationen und Entwicklungen voranzutreiben.

Die Anleitung zu Selbstreflexionsprozessen - ausgehend von den neun Ergebnisfeldern - findet auch im MIW-Modell (Mentoringinduziertes Wirkungsmodell zum schulpraktischen Mentoringprozess) ihre Verortung. In diesem Modell werden zudem Aspekte des gemeinsamen, mentor/inbezogenen und menteebezogenen Wollens innerhalb des Handlungs- und Wirkungsfeldes in Beziehung gesetzt. Von einem gelingenden Prozess kann dann ausgegangen werden, wenn durch selbstreflektierte und kommunizierte personenspezifische Vorgehensweisen Transformationen möglich sind und eine mentoringinduzierte Wirkung im Rahmen von Selbstwirksamkeits- und Professionsentwicklung erzielt wird.

Ein mögliche Definition könnte resümierend lauten:

Schulpraktisches Mentoring ist als professionsspezifischer Lern- und Entwicklungsprozess zu verstehen, der Aspekte gemeinsamen, mentor/inbezogenen und menteebezogenen Wollens umschließt und unter Berücksichtigung funktionsbezogener und emotionaler Faktoren von denkenden, handelnden, empfindsamen Mentor/innen und Mentee auf Professions- und Selbstwirksamkeitsentwicklung zielt.

Mehrfach wurde in der vorliegenden Arbeit betont, dass Ausbildungen und Qualifikationen für Mentor/innen gefordert werden, die inhaltliche Standards ausweisen. Diese fehlen derzeit für Mentoring und Mentoringprogramme in Österreich. Seitens des Bildungsministeriums werden Empfehlungen für Ausbildungsformate zum Hochschullehrgang Mentoring (15 bzw. 30 ECTSAP) bzw. Hochschullehrgang mit Masterabschluss Mentoring: Berufseinstieg professionell begleiten (90 ECTS-AP) gegeben (BMBWF 2019).

Gemeinsam mit Vertreter/innen der Universität Innsbruck, Fakultät für Lehrer/innenbildung und Vertreter/innen der Privaten Pädagogischen Hochschule Edith Stein wurde ein Curriculum zum Hochschullehrgang Mentoring im Ausmaß von 30 ECTS-Anrechnungspunkten konzipiert. Dieser wird ab dem Wintersemester 2020/21 angeboten. Die wesentlichen Schwerpunkte sind:

„1) Reflektieren des personalisierten Erfahrungsraumes zum professionsspezifischen Bearbeiten der Schulpraktika und Unterrichtstätigkeit

2) Erwerben eines relevanten rechtlichen-beratungspädagogischen-fachdidaktischen Wissens für die Arbeit als Mentor/in

3) Entwickeln einer Beratungshaltung für Studierende bzw. Berufseinsteiger/innen

4) Erlernen und Einüben von Instrumenten für Coaching, Beratung, Supervision und Mentoring sowie Mediationsverfahren

5) Erleben und Verstehen der Ausbildung zur/zum Mentor/in als Erweiterung des eigenen professionellen Selbstverständnisses

6) Bewusster Umgang mit ressourcenorientierten, entwicklungsförderlichen, personalisierten wertund deutungsfreien Erfahrungsräumen im Kontext eines doppelt dichotomen Systems (Hierarchie, Wissen)“ (HLG Mentoring Curriuculum 2020, 5; Homepage KPH Edith Stein)

Die Inhalte des Curriculums zielen auf eine reflexive Auseinandersetzung mit der persönlichen professionellen Berufsbiografie, auf Entwicklung und Rollenverständnis von Beratungskompetenzen und Beratungshaltungen, Aneignung forschungsbasierter Konzepte und Modelle zu Pädagogisch-Praktischen Studien, Beratungsformen (Coaching, Mentoring, Supervision, Beratung und Mediation) und professionsspezifische Aufgabenbereiche im Mentoring, Reflexion und Integration professionsspezifischer Mentoring-Funktionen sowie auf Etablierung einer Community of Practice (HLG Mentoring Curriuculum 2020, 6f; Homepage KPH Edith 
Stein). Mit diesem Beispiel wird ein Modell vorgestellt, wie Pädagogische Hochschulen Fortbildungen im Rahmen des Mentorings (HLG Mentoring 30 ECTS-Anrechnungspunkte) konzipieren und in Kooperation mit der Universität gestalten. Ziel ist, im Interesse der Institutionen und Studierenden Mentoring zu professionalisieren und die spezifische Qualifizierung der Mentor/innen durch Mentor/innenausbildungen zu erhöhen.

Durch die in der vorliegenden Studie gewonnenen Erkenntnisse können für die Konzeption von Mentoring-Qualifikationsgprogrammen folgende Bedingungen bzw. Qualitätskriterien z.B. im Sinne eines Rahmencurriculums vorgeschlagen werden:

Entwickeln eines reflexiven, proaktiven, forschungs- und transformationsgeleiteten, systemisch-integrativen, intra- und interpersonalen professionsspezifischen Ansatzes

Bereits im selektiven Kodierungsschritt (Kapitel II/6) wird ansatzweise auf die Forderung dieses Ansatzes hingewiesen, der durch weitere Verdichtungsschritte (Kapitel II/7 und II/8) zu dieser klaren Aussage führt. Dort erwähnen die Interviewgruppen im Rahmen der dreiteiligen Kategorienbildung, dass Mentor/innen im Rahmen des schulpraktischen Mentorings auf strukturelle Rahmenbedingungen, auf Lehrpersonen/Sich selber und auf Studierende achten, während Mentee zunächst strukturelle Rahmenbedingungen in den Fokus nehmen und im Weiteren in den positiven und negativen dargestellten Erfahrungsberichten sowie in der Erwartungshaltung zum schulpraktischen Mentoringprozess die Elemente des Ansatzes wie reflexiv, proaktiv, intra- und interpersonal, professionsspezifisch verdeutlichen. Das Zusammenspiel der Kategorien unterstreicht letztlich die Bedeutung gelingender Prozesse und bildet den Hintergrund des geforderten Ansatzes für Ausbildungsformate.

In einem weiteren Untersuchungsschritt würden sich auf Basis der vorliegenden Untersuchungsergebnisse Definitionen und Kriterien für Standards bei Mentoringprogrammen herausbilden. Eine Evaluierung von bereits bestehenden Fortbildungsprogrammen für Mentor/innen nach den erwähnten Determinanten könnte ebenso Gegenstand von Untersuchungen sein.

Institutionen und Formate von Ausbildungsprogrammen schaffen die Möglichkeit der Vernetzung innerhalb der Mentor/innen - im Idealfall entstehen Netzwerk-Beziehungen im schulpraktischen Mentoring. Es könnten, wie vielfach gegenwärtig in Schulentwicklungskonzepten gefordet und im Rahmen der Clusterbildungen forciert, auch professionelle Lerngemeinschaften - sogenannte Communities of practice (Brown 2019) - im Rahmen des schulpraktischen Mentorings entstehen, wie Abbildung 82 grafisch verdeutlicht (Abbildung 82):

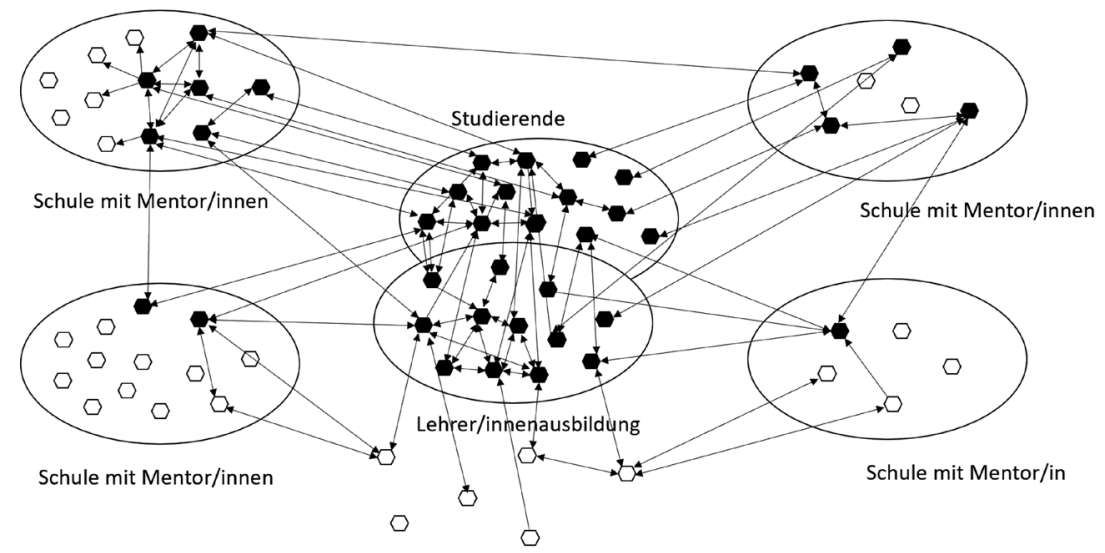

Abb. 82: Netzwerk-Beziehungen im schulpraktischen Mentoring (Adaptiert nach Brown 2019) 
Ausbildner/innen, Schulleitungen, Mentor/innen und Studierende (dunkle Punkte in der Abbildung) agieren im Rahmen Pädagogisch-Praktischer Studien auf vielfältigste Art und Weise in den gemeinsamen Systemen Lehrer/innenausbildung und Schule bzw. im ThirdSpace (Burch et al. 2013, 57f) und kooperieren im schulpraktischen Mentoring auf dialogorientierter Basis im Sinne eines lern- und entwicklungsfördernden Umfeldes. Durch Begleitungs- und Betreuungstätigkeit entstehen nach Möglichkeit innerhalb und außerhalb des schulischen Umfeldes unterschiedlich intensiv gefärbte professionsspezifische NetzwerkBeziehungen mit divergierenden Zieldimensionen. Austausch und Zusammenarbeit könnten Chancen für alle Beteiligten sein, forschungsorientierte Interventionen und/oder Lehr- und Lernaktivitäten in Schulen zu verbessern bzw. weiterzuentwickeln (Brown 2019, 5f). Die Anleitung zu Netzwerk-Beziehungen im Bereich des schulpraktischen Mentorings könnte eine Herausforderung für Institutionen in nächster Zeit sein. Forschungsdesigns dazu sollten mitgedacht werden.

Anregungen zu weiteren Untersuchungen in Zusammenhang mit schulpraktischem Mentoring ergeben sich durch gesellschaftliche Veränderungen, gesellschaftliche Diversität und Spezifität. Lehrer/innenausbildung reagiert in Konzepten und Programmen bereits darauf, da Diversität in Schulen schon längst Realität geworden ist. Auch innerhalb des schulpraktischen Mentorings muss zukünftig auf Phänomene wie Diversität sowie Geschlechts-, Domänen- und Schulformenspezifität geachtet werden. Empirische Befunde zu domänenspezifischen Sichtweisen aus dem anglo-amerikanischen Sprachraum zeigen auf, dass „Lehrkräfte und Dozenten (sic!) unterschiedlicher Fachgebiete durchaus über unterschiedliche LehrLern-Vorstellungen verfügen." (Seifried et al. 2009, 89) So dokumentiert eine Studie das unterschiedliche Planungsverhalten von Lehrpersonen in den Unterrichtsfächern Mathematik, Deutsch und Chemie. Hier stellt sich die Frage, was solche Ergebnisse für das schulprakische Mentoring bedeuten. Eine Befundlandschaft zur Domänenspezifität im Lehrberuf als auch im schulpraktischen Mentoring stellen im Weiteren ein Desiderat dar (Seifried et al. 2009, 90).

Hinreichend wenig erforscht ist auch, wie sich die geschlechtsspezifische Sozialisation des Lehrberufs auf Altersgruppe, den Lehr-Lernzyklus (Seifried et al. 2009, 90f) und somit auch auf das schulpraktische Mentoring auswirkt. Dazu ergeben sich also eine Bandbreite an Forschungsthemen im Kontext des schulpraktischen Mentorings und der Lehrer/innenausbildung.

\section{Dimensionen der Pädagogisch-Praktischen Studien}

Mentoring in der Lehrer/innenbildung ist vielschichtig. In der vorliegenden Arbeit wurde versucht, wichtige Aspekte des Mentorings bezogen auf aktuellste Entwicklungen in der Lehrer/ innenbildung differenziert darzustellen und zu erarbeiten. Professionalisierung lebt von und entwickelt sich in der Verschränkung grundlegend theoretischer Überlegungen sowie im Feld gemachter Erfahrungen. Im Kontext der Erkenntnisse aus der vorliegenden Untersuchung lassen sich resümierend und den Band abschließend folgende sechs mentoringbezogene Dimensionen des praxisbezogenen Ausbildungsfeldes resümierend als Entwicklungs- und Diskussionsmatrix identifizieren: normativ-curriculare Dimension, aufgabenspezifische Dimension, begleitungsspezifische Dimension, Zeitdimension, Ortsdimension, personale Dimension. 


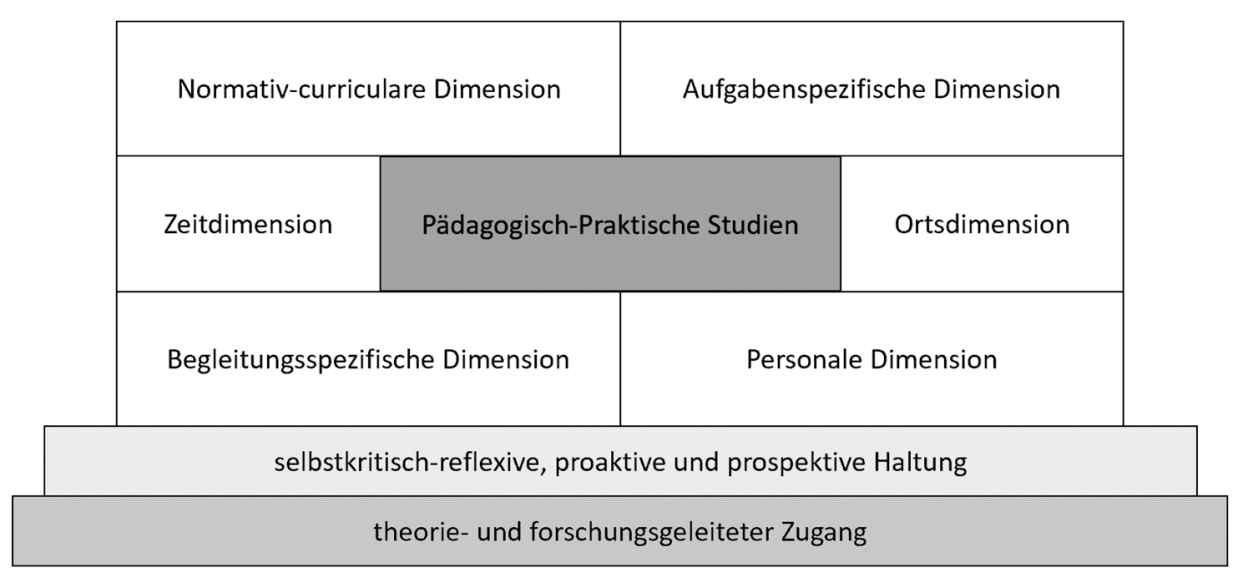

Abb. 83: Dimensionen der Pädagogisch-Praktischen Studien

Das Fundament sowohl in den schulischen als auch hochschulischen Anteilen der PädagogischPraktischen Studien bilden ein theorie- und forschungsgeleiteter Zugang und die Ausgestaltung einer selbstkritisch-reflexiven, proaktiven und prospektiven Haltung. Im Folgenden werden die Dimensionen im Sinne eines abschließendes Ausblickes kurz erläutert.

(1) Normativ-curriculare Dimension

Pädagogisch-Praktische Studien sind auf gesetzlicher Basis in den Curricula der Lehramtsstudien verankert. Sie sind integrierter Bestandteil in den Bildungswissenschaftlichen Grundlagen und Fachwissenschaften bzw. Fachdidaktiken. Die Strukturierung erfolgt in hochschulische und schulische Anteile mit unterschiedlichen Anforderungen und der Zielsetzung hinsichtlich einer Verschränkung bzw. Kohärenz von theoretisch-inhaltlichreflexiven und handlungsorientiert-praktischen Kompetenzen. Das Ausmaß der Anteile und der ECTS-Anrechnungspunkte sind in den Curricula der Bachelor- und Masterstudien institutionssituativ festgelegt.

(2) Aufgabenspezifische Dimension

$\mathrm{Zu}$ den wesentlichen Aufgabenbereichen der Pädagogisch-Praktischen Studien zählen die Auseinandersetzung mit grundlegenden und vertiefenden Professionalisierungskonzepten und -modellen sowohl auf unterschiedlichen kognitiven Reflexionsebenen als auch in den Handlungsbereichen basierend auf theoretischer Fundierung. Die Handlungsbereiche sind für Studierende verbunden mit Unterrichten, Erziehen, Beurteilen, Innovieren (KMK Modell 2004) und Beobachten und münden im Idealfall in die Ebenen Reflektieren und Forschen. In den Lehrveranstaltungen der Pädagogisch-Praktischen Studien wird auf die Bedeutung einer selbstkritisch-reflexiven und forschenden Haltung hingewiesen und es wird Raum und Zeit für den Aufbau dieser Kompetenz verankert. In der Zeit der Ausbildung lernen Studierende hierbei Methoden und Verfahren kennen, die sie befähigen, das Handlungs- und Sozialisationsfeld Schule zu erfassen, schrittweise zu gestalten und kritisch auch im Sinne gesellschaftlicher Veränderungen und Entwicklungen zu beleuchten. 
(3) Begleitungsspezifische Dimension

Praktika zeichnen sich in der historiografischen Weiterentwicklung innerhalb der Lehramtsausbildung dadurch aus, dass diese im Rahmen der Pädagogisch-Praktischen Studien durch Lehrveranstaltung an der Hochschule begleitet und Studierende in der Ausbildungszeit aufgrund der Vor- und Nachbereitungen unterstützt werden. Theoretische Fundierungen, wie z.B. Habituskonzept (Bourdieu 1997), Entwicklungsaufgaben (Hericks 2004) usw., bilden Begleitkonzepte und Grundlage für Anforderungsprofile in der konkreten strukturellen Umsetzung der Praktika und damit in den begleitenden Lehrveranstaltungen. Schriftliche Aufzeichnungen der Studierenden in Form von Entwicklungsportfolios, Praktikumsberichten oder Lernjournals u.ä. veranschaulichen den Grad der Auseinandersetzung mit professionsspezifischen Anforderungen. Eine weitere bestehende Realisierung innerhalb der begleitungsrelevanten Dimension ist die Einbeziehung der Mentor/innen der Partnerschulen in das Ausbildungskonzept. Sie agieren unterstützend und prozessorientiert im Sinne unterrichtlichen, gestalterisch-pädagogischen Handelns und Reflektierens auf dialogorientierter lernförderlicher Basis. Mentoring wird als erfolgsversprechendes Instrument der Begleitung angesehen. Gegenwärtige Ausbildungsprogramme für Mentor/ innen zielen auch auf deren Weiterentwicklung professionsspezifischer Aufgabenfelder und auf einen Kenntnisgewinn über kommunikationsadäquate professionelle Begleitstrategien.

(4) Zeitdimension

Das Ausmaß der ECTS-Anrechnungspunkte und die zeitliche Anordnung der Pädagogisch-Praktischen Studien in den jeweiligen Semestern der Lehramtsstudien variieren auf nationaler Ebene. Hierbei spielen institutionelle Rahmenbedingungen und Sichtweisen eine bedeutende Rolle. Praktika mit ihren schulischen Verknüpfungen werden in unterschiedlichen Formen angeboten - als Tagespraktikum, geblocktes Tagespraktikum oder Blockpraktikum. Aufgabenspezifische Anforderungen sind mit der jeweiligen Organisationsform verbunden. Die Einbindung der Studierenden in längere zeitliche Praxisphasen bedingt meist auch die Übernahme von vermehrten Aufgabenfeldern. Die Dauer der Praktika wird mit der einherzugehenden Qualität von Begleitung diskutiert. Praktika und begleitende Lehrveranstaltungen am Beginn des Studiums - in der sogenannten Studieneingangsphase - zielen auf die Auseinandersetzung mit subjektiven Theorien, auf einen Perspektiven- bzw. Rollenwechsel sowie auf die Berufswahlentscheidung.

(5) Ortsdimension

Normativ-curriculare und aufgabenspezifische Anforderungen in der Beschreibung der Curricula weisen auf die Relevanz der Auseinandersetzung mit schulart- und standortspezifischen Begebenheiten im Rahmen der Ausbildung hin. In der Umsetzung werden den Studierenden durch die Einbindung in vielfältige Schulalltagssituationen somit Erfahrungsräume eröffnet und professionsspezifische Herausforderungen vor Augen geführt, die wiederum als Grundlage für Reflexionsprozesse exemplarisch aufbereitet werden können. Es besteht auch die Möglichkeit, neue Strukturen und Denkfiguren kennenzulernen und das Handlungsfeld Schule und Unterricht in einem vielfältigen Spektrum zu erfassen.

(6) Personale Dimension

Pädagogisch-Praktische Studien bieten durch die Kleingruppenstruktur in den begleitenden Lehrveranstaltungen und in den Praktika die Chance und Möglichkeit, auf Anliegen, Wünsche, Emotionen und Anregungen von Studierenden einzugehen und ihnen auf individueller und ressourcenfördernder Ebene zu begegnen. Im Sinne des Professionalisierungsprozesses der Studierenden sollen Interaktionen aller Beteiligten gewährleistet und Lehr- und Lernansätze sinnstiftend auf die jeweilige Person bezogen werden. Lehrveran- 
staltungsleiter/innen haben ebenso die Möglichkeit, durch individuelle Schwerpunktsetzungen ihre Stärken und Ressourcen einzubringen. Mentor/innen nehmen innerhalb der personalen Dimension im Weiteren eine bedeutende Rolle ein - wie in der vorliegenden Arbeit bereits mehrmals betont.

Die Dimensionen der Pädagogisch-Praktischen Studien können als Grundlage für Qualitätsmaßnahmenprogramme herangezogen werden. Letztendlich ist abzuwarten, wie sich die Pädagogisch-Praktischen Studien und schulpraktisches Mentoring im Kontext Lehrer/innenausbildung und im System Schule in den nächsten Jahren weiterentwickelt, welche Ausbildungskonzepte für Mentor/innen etabliert werden und ob ein praktikums- und personenspezifisches Mentoring einen Beitrag für ein qualitätsvolles Lehrer/innenhandeln leistet. 



\section{Verzeichnisse}

\section{Abbildungsverzeichnis}

Abb. 1: Fünf-Themenbereichs-Konzept Lehrer/innenbildung WEST (Curriculumhandbuch 2016) ......

Abb. 2: Bestimmungsversuche des Verhältnisses von wissenschaftlichem und handlungsbezogenem Wissen (Dewe et al. 1990; Herzmann et al. 2016, 152) ...................................

Abb. 3: Prozessmodell forschenden Lernens (Wildt 2005, 187; Herzmann et al. 2016, 162) .............

Abb. 4: Zusammenspiel von Theoriewissen, Erfahrungswissen, Handlungskompetenz (Meyer 2004, 137;

Standop et al. 2015, 252) ................................................

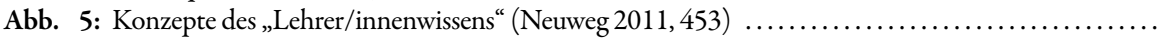

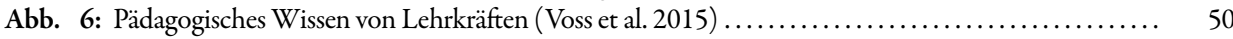

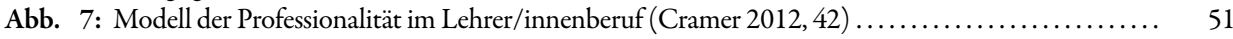

Abb. 8: Ausbildungsvorgaben und professionsspezifische Entwicklungsaufgaben (Ostermann 2015, 184) . 52

Abb. 9: Mentoring in der Lehrer/innenausbildung aus dem Kontext wirtschaftlicher Felder ........... 61

Abb. 10: Mentoring in der Lehrer/innenausbildung zur Stärkung affektiv-motivationaler und

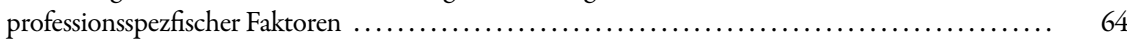

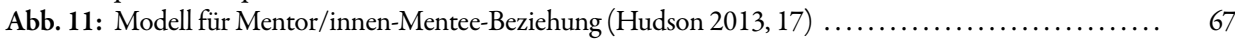

Abb. 12: Lehrpersönlichkeit der Mentor/innen, Selbstwirksamkeit als Mentor/in, Selbstreflexion,

Vorstellungen der Mentor/innen über den Lehrberuf (Reintjes et al. 2018, 174) ............. 68

Abb. 13: Übersicht über Nutzen von Mentor/innen und Mentee (Pflaum 2017, 271) ................ 70

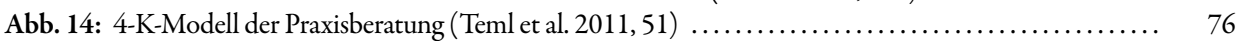

Abb. 15: Studienarchitektur zum Hochschullehrgang für Mentor/innen (BMBWF 2019) ............ 78

Abb. 16: Mentoring-Modell unter Einbindung der Personen und Systeme

(Adaptiert nach Oettler 2009, 36) ......................................... 80

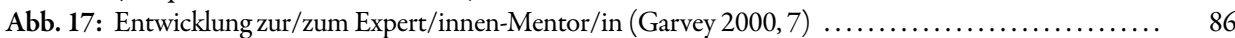

Abb. 18: Anforderungen an Funktion, Rolle und Mensch (Graf et al. 2017, 48) .................... 91

Abb. 19: The MERID-Modell (Hennissen et al. 2008, 177) ............................... 93

Abb. 20: Vorgehensmodell im Mentoring nach Mentus-Modell (Graf et al. 2017, 85) . . . . . . . . . . . 97

Abb. 21: Typische Verläufe von Mentoring-Beziehungen (Pflaum 2017,253) ................... 104

Abb. 22: Der Kreislauf des Kontakts (Lux 2014, 184; Kunze-Pletat 2019, 375) . ................... 111

Abb. 23: Zwiebelmodell nach Korthagen et al. (Korthagen et al. 2005, 54) ..................... 115

Abb. 24: Musterwechsel (Kraler et al. 2012a, 92) ........................................ 131

Abb. 25: Rekonstruierte Kategorien zum Transformationalen Mentoring ...................... 133

Abb. 26: Kompetenz-Erfahrungs-Modell im wechselseitigen Austausch (Garvey 2000, 9) . ............ 137

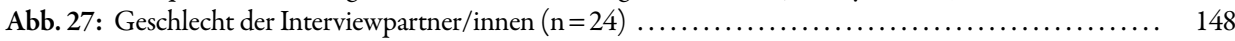

Abb. 28: Alter der Interviewpartner/innen mit strichlierten Mittelwertslinien Mentor/innen bzw.

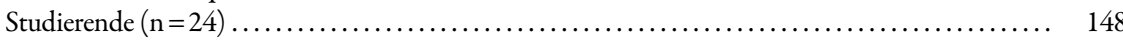

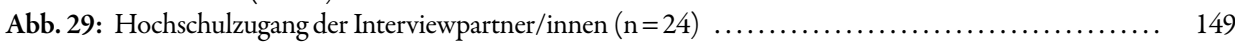

Abb. 30: Lehramtswahl nach Hochschulreife der Interviewpartner/innen $(n=24) \ldots \ldots \ldots \ldots \ldots \ldots \ldots . \quad 150$

Abb. 31: Fächerwahl bzw. Lehramtswahl (Volksschule) der Interviewpartner/innen $(n=24) \ldots \ldots \ldots \ldots \ldots . .151$

Abb. 32: Dienstjahre und Jahre der Begleitungstätigkeit nach Schultypen der Lehrpersoen bzw.

Mentor/innen $(\mathrm{n}=12)$

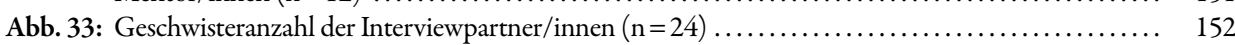

Abb. 34: Einwohner/innenzahl des Wohnortes der Interviewpartner/innen $(n=24) \ldots \ldots \ldots \ldots \ldots \ldots \ldots \ldots \ldots \ldots$

Abb. 35: Entfernung Wohnort zu Dienstort/Studienort in Kilometern der Interviewpartner/innen $(n=24)$. 154

Abb. 36: Kodier-Prozeduren in systematisierter Anordnung (Breuer 2010, 76 in Breuer et al. 2018, 257) . . 157

Abb. 37: Ebenen der Modell- bzw. Theoriegenerierung (Basierend auf Wieser 2013, 125) ............ 157

Abb. 38: Beispiel im offenen Kodierungsprozess: Bilden von Konzepten nach der Grounded Theory

(Basierend auf Przyborski et al. 2018, 214) .................................... 159

Abb. 39: Beispiel im axialen Kodierungsprozess: Erstellen von Kategorien nach der Grounded Theory . .... 160 
Abb. 40: Beispiel des Dimensionalisierens in der Grounded Theory (Basierend auf Strauss et al. 1996, 53) .. 161

Abb. 41: Beispiel eines Memos zur vorliegenden Untersuchung mit Hilfe von MAXQDA .............. 162

Abb. 42: Nutzung von Memos zur Integration und Verbindung von Konzepten und zur Entwicklung

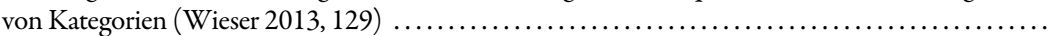

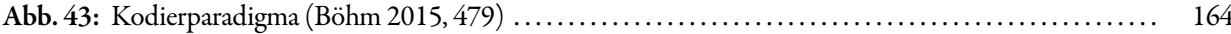

Abb. 44: Binnendifferenzierung bei Lehrpersoneninterviews $(n=12) \ldots \ldots \ldots \ldots \ldots \ldots \ldots \ldots \ldots \ldots \ldots$

Abb. 45: Kategorien und Subkategorien der Kodes aus den Lehrpersoneninterviews $(n=12) \ldots \ldots \ldots \ldots$

Abb. 46: Kategorie Struktureller Rahmen bei Lehrpersonenkodes $\ldots \ldots \ldots \ldots \ldots \ldots \ldots \ldots \ldots \ldots \ldots \ldots \ldots$

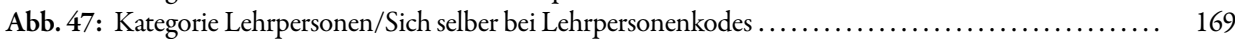

Abb. 48: Kategorie Studierende bei Lehrpersonenkodes $\ldots \ldots \ldots \ldots \ldots \ldots \ldots \ldots \ldots \ldots \ldots \ldots \ldots \ldots \ldots . \ldots$

Abb. 49: Durchschnittskodierungsverteilung pro Interviewpartner/in der Lehrpersonen bzw.

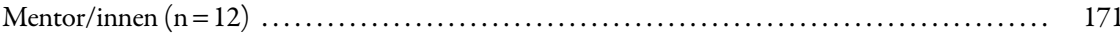

Abb. 50: Kodierparadigma 1 aus Lehrpersoneninterviews $\ldots \ldots \ldots \ldots \ldots \ldots \ldots \ldots \ldots \ldots \ldots \ldots \ldots \ldots \ldots, 173$

Abb. 51: Eigenschaften und Dimensionen des Phänomens Praktika (Lehrendenperspektive) ........... 173

Abb. 52: Kodierparadigma 2 aus Lehrpersoneninterviews $\ldots \ldots \ldots \ldots \ldots \ldots \ldots \ldots \ldots \ldots \ldots \ldots \ldots \ldots \ldots$

Abb. 53: Eigenschaften und Dimensionen des Phänomens Interaktion (Lehrendenperspektive) $\ldots \ldots \ldots \ldots \quad 175$

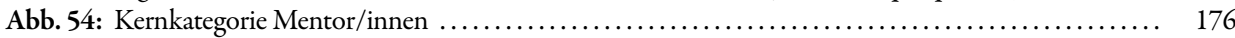

Abb. 55: Eigenschaften (Persönlich) und Dimensionen zum Phänomen Zusammenabeit in der

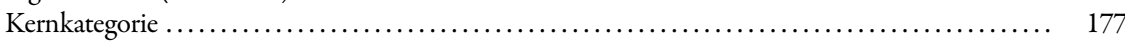

Abb. 56: Eigenschaften (Für Studierende/n) und Dimensionen zum Phänomen Zusammenarbeit

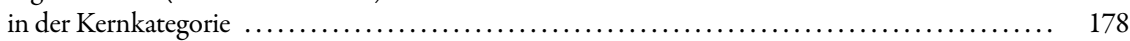

Abb. 57: Binnendifferenzierung bei Studierendeninterviews $(n=12) \ldots \ldots \ldots \ldots \ldots \ldots \ldots \ldots \ldots \ldots$

Abb. 58: Kategorien und Subkategorien der Kodes aus Studierendeninterviews $(n=12) \ldots \ldots \ldots \ldots \ldots \ldots$

Abb. 59: Kategorie Struktureller Rahmen bei Studierendenkodes $\ldots \ldots \ldots \ldots \ldots \ldots \ldots \ldots \ldots \ldots \ldots \ldots \ldots 1$

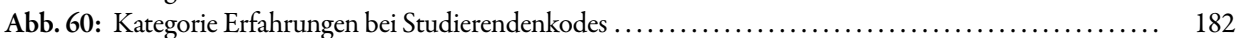

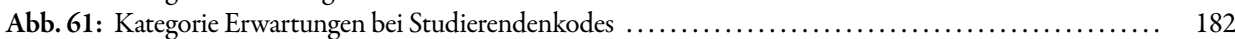

Abb. 62: Durchschnittskodierverteilungen pro Interviewpartner/in der Studierenden bzw.

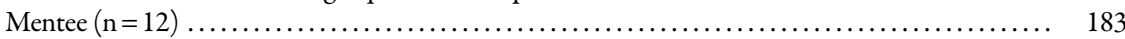

Abb. 63: Kodierparadigma 1 aus Studierendeninterviews $\ldots \ldots \ldots \ldots \ldots \ldots \ldots \ldots \ldots \ldots \ldots \ldots \ldots \ldots \ldots$

Abb. 64: Eigenschaften und Dimensionen zum Phänomen Praktika (Studierendenperspektive) $\ldots \ldots \ldots \ldots .185$

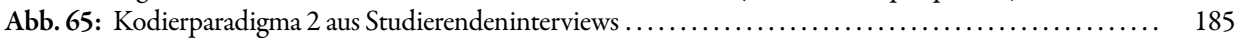

Abb. 66: Eigenschaften und Dimensionen zum Phänomen Erfahrungslernen in den Praktika mit

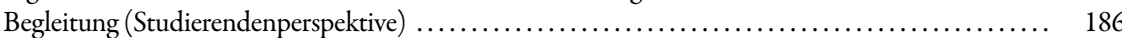

Abb. 67: Kernkategorie Studierende bzw. Mentee $\ldots \ldots \ldots \ldots \ldots \ldots \ldots \ldots \ldots \ldots \ldots \ldots \ldots \ldots \ldots \ldots \ldots \ldots \ldots$

Abb. 68: Eigenschaften und Dimensionen des Phänomens Lernen in den Praktika mit Begleitung

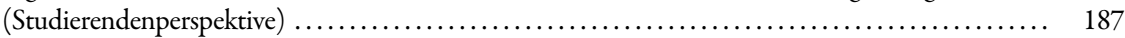

Abb. 69: Kodierparadigma $($ Böhm 2015,479$) \ldots \ldots \ldots \ldots \ldots \ldots \ldots \ldots \ldots \ldots \ldots \ldots \ldots \ldots \ldots \ldots \ldots$

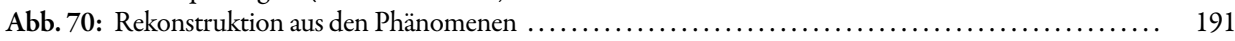

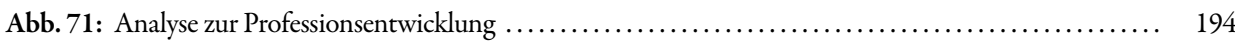

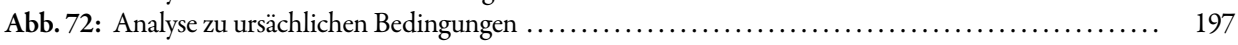

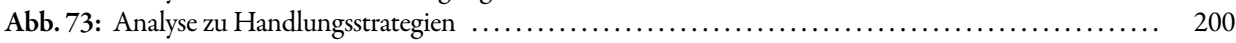

Abb. 74: Analyse zu Kontext und intervenierenden Bedingungen ......................... 203

Abb. 75: Analyse zu Konsequenzen in Form von Schulpraktischen Mentoring-Funktionen ........... 205

Abb. 76: Metakernkategorie zum schulpraktischen Mentoringprozess aus den Rekonstruktionsschritten der Mentor/innen und Studierendenperspektiven . . . . . . . . . . . . . . . . . . . . . . . . . . . .

Abb. 77: Mentoring-Modell mit Einbeziehung der Phänomene und Einbindung der Personen und

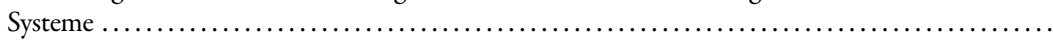

Abb. 78: Modell zum Analyse Transformationalen Mentoring im berufsfeldbezogenen Wirkungskontext

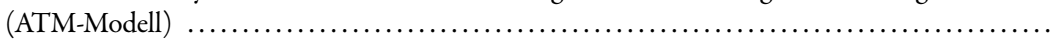

Abb. 79: Transformationales Mentoring unter Vernetzung der Ereignisfelder im berufsfeldbezogenen

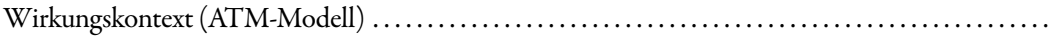

Abb. 80: Mentoringinduzierte Wirkung im schulpraktischen Mentoringprozess (MIW-Modell) ..........

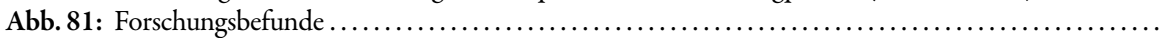

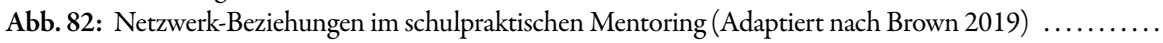

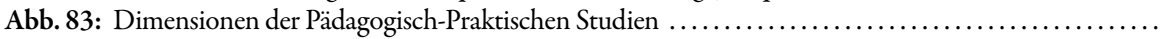




\section{Tabellenverzeichnis}

Tab. 1: Studienarchitektur Bachelor-Master-Studium Primarstufe ab dem Studienjahr 2015/16

(Curriculum Primarstufe KPH Edith Stein) ...................................

Tab. 2: Studienarchitektur Bachelor-Master-Studium Sekundarstufe Allgemeinbildung

Lehrer/innenbildung WEST ab dem Studienjahr 2015/16 (Curriculum 2016) ...............

Tab. 3: Phasenmodell der Pädagogisch-Praktischen Studien an der KPH Edith Stein

(Curriculum Primarstufe 2016)

Tab. 4: Zielsetzungen Schulpraktikum I Lehrer/innenbildung WEST (Curriculumhandbuch 2016, 38) ..

Tab. 5: Diagnoseinstrumente mit Kompetenzfacetten zur Studie (Basierend auf Mertens et al. 2018, 72f)

Tab. 6: Mittelwerte (M) und Standardabweichungen (SD) der untersuchten Kompetenzaspekte zu Beginn ( $\mathrm{t} 1$ ) und am Endes (t2) des Praxissemesters (Mertens et al. 2018, 76) ..............

Tab. 7: Subdimensionen der Skala und Kennwerte (Fischer et al. 2018, 162) ........................

Tab. 8: Funktionen und Fragestellungen (Adapiert nach Patry 2014,32) $\ldots \ldots \ldots \ldots \ldots \ldots \ldots \ldots \ldots \ldots \ldots \ldots$

Tab. 9: Begriffsdefinitionen Profession, Professionalisierung, Professionalität (Mieg 2016, 30) ...........

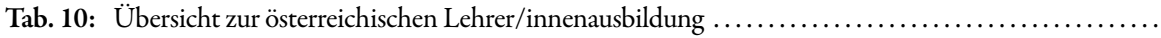

Tab. 11: Theoretische Hintergründe des Mentoring (in Anlehnung an Fuge 2016, 76) . . . . . . . . . . . .

Tab. 12: Mentoring-Funktionen nach Kram (Kram 1985, 23f; Übersetzung Magg-Schwarzbäcker 2014, 18)

Tab. 13: Hintergründe des Mentorings aus psychologischer Perspektive (Fuge 2016, 76) ..............

Tab. 14: Unterstützung in schulischen und universitären Bereichen (Bach et al. 2018, 198)

Anmerkung: Vierstufige Skala ( $1=$ nie bis $4=$ sehr oft $)$

Tab. 15: Zusammenhang der Ergebnisse der Multi-Item Multi-Trait-Analyse für das Mentoring-Inventar in der Stichprobe der Mentor/innen (N=206) (Brandau et al. 2018, 217) Anmerkung:

Der mögliche Wertebereich der Skalen beträgt $1=$ „trifft nie zu“ bis $6=$ „trifft immer zu“ $\ldots . .$. .

Tab. 16: Varimax-rotierte Faktorladungsmatrix der Faktorenanalyse zweiter Ordnung über die

Dimension des Mentoring-Inventars (Brandau et al. 2018, 221)

Tab. 17: Beschreibung der Elemente des 4 bzw. 5-K-Modells (Adaptiert nach Teml 2011, 51f;

Brenn et al. 1996, 44f; Klement et al. 1996, 193f) ....

Tab. 18: Fragestellungen zur Analysemöglichkeit zum Handlungsbereich Beobachten

(Brenn et al. 1996, 125)

Tab. 19: Empfehlung für Kompetenz- und Inhaltsbereiche zum Hochschullehrgang Mentor/innen (BMBWF 2019)

Tab. 20: Auflistung der folgenden Gestaltungsparameter und Kurzbeschreibungen zu schulpraktischem

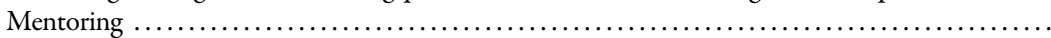

Tab. 21: Verschiedene Ansätze für die Auswahl von Mentor/innen . . . . . . . . . . . . . . . . . . . . . . .

Tab. 22: Verbindung von intendierten Lernprozessen der Studierenden in Praxisphasen und

Lernbegleitung sowie erforderlichen Kompetenzen (Schnebel 2018, 24) ...................

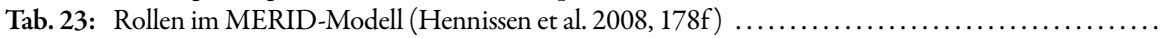

Tab. 24: Forschungsbefunde zu Rollen im MERID-Modell (Hennissen et al. 2008, 178f) ...............

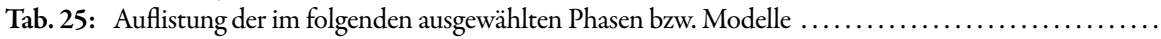

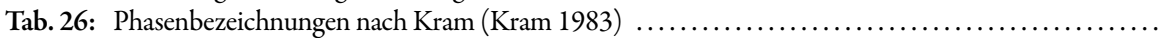

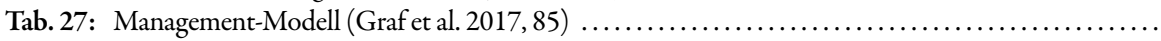

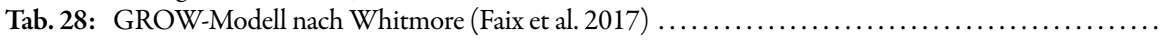

Tab. 29: Fragestellungen und Absichten zum GROW-Modell im schulpraktischen Mentoringprozess ......

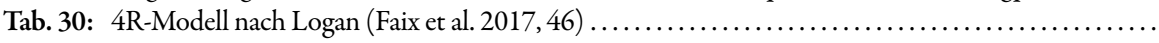

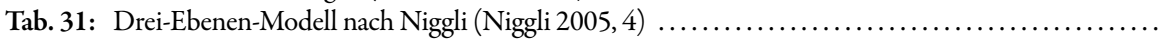

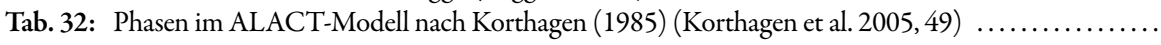

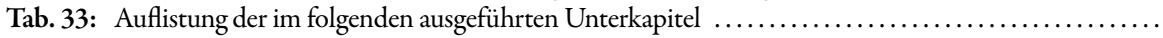

Tab. 34: Überblick zu Matching-Verfahren (Schmid et al. 2011, 86f; in Anlehnung an Fuge 2016, 46) .....

Tab. 35: Wirkprinzipien wertebasierter Gesprächsführung (Rogers 2009, 46f; Hellwig 2016, 13f) ........

Tab. 36: Fragestellungen von Mentor/innen zu Wirkprinzipien wertebasierter Gesprächsführung

(Basierend auf Rogers 2009, 46f; Hellwig 2016, 13f) .

Tab. 37: Auflistung der im folgenden ausgeführten Unterkapitel ... 
Tab. 39: Entwicklungsaufgaben nach Ostermann $(2015,153)$ und Köffler $(2015,193)$ und die

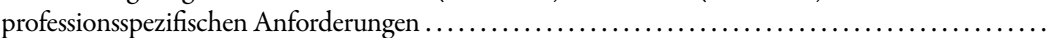

Tab. 40: Professionsspezifische Entwicklungsaufgaben (Ostermann 2015, 153) mit Fragestellungen (KPH Edith Stein 2020)

Tab. 41: Vier Bedürfnisbereiche der bedürfnisorientierten Begleitung (Dreer 2018, 19) $\ldots \ldots \ldots \ldots \ldots \ldots \quad 120$

Tab. 42: Typologie der Anforderungsdeutung und -bearbeitung Studierender (Košinár 2018, 72; Košinár et al. 2018, 169)

Tab. 43: Typisierende Beschreibung der Gruppen-Charakteristika (Nolle 2012,73f) ............... 121

Tab. 44: Korrespondenz hohe Lehrer/innenselbstwirksamkeit mit verschiedenen Variablen

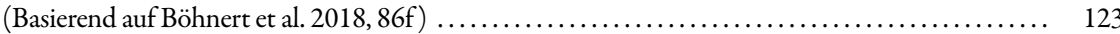

Tab. 45: Auflistung der im folgenden ausgeführten Unterkapitel $\ldots \ldots \ldots \ldots \ldots \ldots \ldots \ldots \ldots \ldots \ldots \ldots \ldots, 125$

Tab. 46: Dimensionen und Beschreibung der Transformationalen Führung (Wenddorf 2018, 48; von Au 2016, 23; Pelz 2016, 96) ........................ 12

Tab. 47: Bereiche und Fragehorizonte zum Transformationalen Mentoring im schulpraktischen

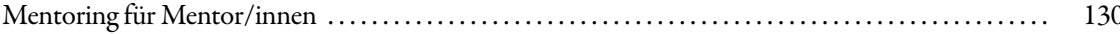

Tab. 48: Bereiche und Fragehorizonte zum Transformationalen Mentoring im schulpraktischen Mentoring für Mentee

Tab. 49: Interviewleitfaden Mentor/innen und Studierende

Tab. 50: Statistische Daten der Interviewpartner/innen ( $\mathrm{L}=$ Lehrpersonen 1-12; S = Studierende 13-24) .. 146

Tab. 51: Statistische Daten der Lehramtsstudienanfänger/innen (2015) in Österreich (Sozialerhebung Österreich, 2015)

Tab. 52: Geschwisterkonstellationen (Kraler 2009)

Tab. 53: Bildungsgrad der Eltern der Interviewpartner/innen $(n=24) \ldots \ldots \ldots \ldots \ldots \ldots \ldots \ldots \ldots \ldots \ldots \ldots$

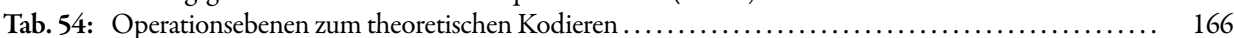

Tab. 55: Professionsentwicklung aus dem Rekonstruktionsprozess der Phänomene ................. 190

Tab. 56: Inhalts- und Beziehungsaspekte aus dem Rekonstruktionsprozess der ursächlichen Bedingungen .. 195

Tab. 57: Kontextbezogener und personenspezifischer Lernprozess aus dem Rekonstruktionsprozess

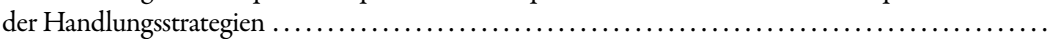

Tab. 58: Praktikumsspezifische und beziehungsorientierte Bedingungen aus dem Rekonstruktionsprozess

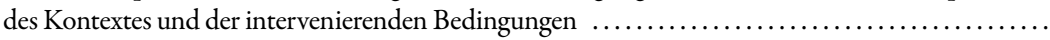

Tab. 59: Schulpraktische Mentoring-Funktionen aus dem Rekonstruktionsprozess der Konsequenzen .....

Tab. 60: Kategorien und Metakategorien zu Schulpraktischen Mentoring-Funktionen aus

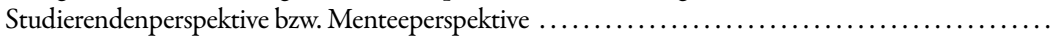

Tab. 61: Kategorien und Metakategorien zu Schulpraktischen Mentoring-Funktionen aus

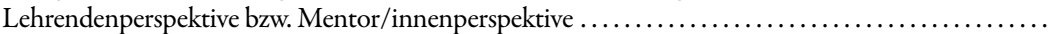

Tab. 62: Anzahl der Paradigmata und Kernkategorien aus den Interviews . . . . . . . . . . . . . . . . .

Tab. 63: Gesamtübersicht der rekonstruierten Gelingensbedingungen schulpraktischer

Mentoringprozesse aus Sicht der Mentor/innen und Studierenden bzw. Mentee ..............

Tab. 64: Gemeinsames Wollen im schulpraktischen Mentoring $\ldots \ldots \ldots \ldots \ldots \ldots \ldots \ldots \ldots \ldots \ldots \ldots \ldots$

Tab. 65: Gemeinsames, mentor/inbezogenes und menteebezogenes Wollen im schulpraktischen

Mentoring

Tab. 66: Analyse Transformationales Mentoring im berufsfeldbezogenen Wirkungskontext in Bezug zur Kategorie: Persönliche positive Einschätzung zur Aufgabenerfüllung aufgrund von Persönlichkeitsstrukturen und Fähigkeiten

b. 67: Analyse Transformationales Mentoring im berufsfeldbezogenen Wirkungskontext

Tab. 68: Analyse Transformationales Mentoring im berufsfeldbezogenen Wirkungskontext in Bezug zur Kategorie: Erkennen von Belastungssymptomen 


\section{Literaturverzeichnis}

Abbott, A. (1988). The System of Professions. An Essay on the Division of Expert Labour. Chicago: University of Chicago Press.

Abbott, A. (2005). Linked ecologies: States and universities as environments for professions. In: Sociological Theory. $23(3) .245-274$.

Abel, J., Lunkenbein, M. \& Rahm, S. (2007). Beobachtungen der Beoachter. Wahrnehmung von Verschiedenheit im Klassenzimmer. In: Journal für Lehrerinnen- und Lehrerbildung. 7. 38-45.

Allinder, R.M. (1994). The relationship between efficacy and the instructional practices of special education teachers and consultants. Teacher Education and Special Education. 17 (2). 86-95.

Altrichter, H. \& Feindt, A. (2004a): Handlungs- und Praxisforschung. In: Böhme, J., Helsper, W. (Hrsg.): Handbuch der Schulforschung. Wiesbaden: VS Verlag für Sozialwissenschaften. 417-438.

Amann, K. \& Hirschauer, S. (Hrsg.) (1997). Die Befremdung der eigenen Kultur. Frankfurt am Main: Suhrkamp Verlag.

Arnold, K.-H. (2005). Praxisorientierung in der Lehrerbildung. Schulpraktische Studien (SPS) als wissenschaftlich begründetes Lernen im Handlungsfeld Schule (unveröffentlichtes Manuskript). Hildesheim: Universität Hildesheim. Institut für Angewandte Erziehungswissenschaft und Allgemeine Didaktik.

Arnold, R. (2009). Seit wann haben Sie das? Grundlinien eines Emotionalen Konstruktivismus. Heidelberg: Carl-Auer Verlag.

Arnold, K.-H., Hascher, T., Messner, R., Patry, J.-L. \& Rahm, S. (2011). Empowerment durch Schulpraktika, Perspektiven wechseln. Bad Heilbrunn: Klinkhardt Verlag.

Arnold, K.-H., Gröschner, A. \& Hascher, T. (Hrsg.) (2014). Schulpraktika in der Lehrerbildung. Theoretische Grundlagen, Konzeptionen, Prozesse und Effekte. Münster, New York: Waxmann Verlag.

Au, C. von (2016). Wirksame und nachhaltige Führungsansätze. Leadership und Angewandte Psychologie. Wiesbaden: Springer Fachmedien.

Awaya, A., McEvan, H., Heyler, D., Linsky, S., Lum, D. \& Wakukawa, P. (2003). Mentoring as a journey. Teaching and Teacher Education. 19. 45-46.

Aymanns, P. (1995). Soziale Netzwerke und kritische Lebensereignisse. In: Ninge, R. \& Funke, W. (Hrsg.) (1995). Soziale Netze in der Praxis. Göttingen: Verlag für Angewandte Psychologie.

Bach, A. (2013). Kompetenzentwicklung im Schulpraktikum. Ausmaß und zeitliche Stabilität von Lerneffekten hochschulischer Praxisphasen. Münster: Waxmann Verlag.

Bach, A., Besa, K.S. \& Arnold, K.-H. (2014). Bedingungen von Lernprozessen in Schulpraktika: Ergebnisse aus dem Projekt ESIS (Entwicklung Studierender in Schulpraktika). In: Arnold, K.-H., Gröschner, A. \& Hascher, T. (Hrsg.) (2014). Praktika in der Lehrerbildung: Theoretische Grundlagen, Konzeptionen, Prozesse und Effekte. Münster und New York: Waxmann Verlag. 165-182.

Bach, A., Fischer, T. \& Rheinländer, K. (2018). Einstellungen von Mentorinnen und Mentoren zur Theorie- und Praxisorientierung des Lehramtsstudiums und deren Effekte auf die Betreuung von Lehramtsstudierenden im Praxissemester. In: Reintjes, Ch., Bellenberg, G. \& Brahm im, G. (2018). Mentoring und Coaching als Beitrag zur Professionalisierung angehender Lehrpersonen. Münster, New York: Waxmann Verlag. 190-205.

Bach, A. (2020). Schulpraktika: Tages- und Blockpraktika. In: Cramer, C., König, J., Rothland, M. \& Blömeke, S. (Hrsg.) (2020). Handbuch Lehrerinnen- und Lehrerbildung. Bad Heilbrunn: Klinkhardt Verlag. 621-628.

Bader, G. (2019): rückblicken_wahrnehmen_inspirieren. Religionspädagogische Perspektiven im Wandel der Zeit. Wien: LIT Verlag.

Bäuerle, S. (1989). Der gute Lehrer. Empfehlungen für den Umgang mit Schülern, Eltern und Kollegen. Stuttgart: J.B. Metzlersche Verlagsbuchhandlung.

Bandura, A. (1976). Lernen am Modell. Ansätze zu einer sozial-kognitiven Lerntheorie. Stuttgart: Klett Verlag.

Bandura, A. (1977). Self-efficacy. Toward a unifying theory of behavioral change. Psychological Review. 84 (2). 191-215.

Barlage, H., Boekhoff, I. \& Graumann, O. (2006). Theorie-Praxis-Verzahnung in einer konsekutiven Lehrerausbildung: das Hildesheimer Modell. In: Hilligus, A.H. \& Rinkens, H. (Hrsg.) (2006). Standards und Kompetenzen - neue Qualität in der Lehrerausbildung? Neue Ansätze und Erfahrungen in nationaler und internationaler Perspektive. Berlin: LIT Verlag. 135-142.

Bass, B.M. \& Avolio, B.J. (Hrsg.) (1994). Improving Organizational Effectiveness through Transformational Leadership. Thousand Oaks. CA: Sage.

Bauer, K.-O. (1998). Pädagogisches Handlungsrepertoire und professionelles Selbst von Lehrerinnen und Lehrern. In: Zeitschrift für Pädagogik. 44 (3). 343-359.

Baumann-Habersack, F.H. (2017). Mit neuer Autorität in Führung. Die Führungshaltung für das 21. Jahrhundert. Wiesbaden: Springer Fachmedien. 
Baumert, J. \& Kunter, M. (2006). Stichwort: Professionelle Kompetenz von Lehrkräften. In: Zeitschrift für Erziehungswissenschaft 9, Wiesbaden: Springer Fachmedien. 469-520.

Baumert, J. \& Kunter, M. (2011). Das Kompetenzmodell von COACTIV. In: Kunter, M., Baumer, J., Blum, W., Klusmann, U., Krauss, S. \& Neubrand, M. (Hrsg.) (2011). Professionelle Kompetenz von Lehrkräften. Ergebnisse des Forschungsprogramms COACTIV. Münster: Waxmann Verlag. 29-55.

Bayer, A., Grossman, J. \& DuBois, D. (2015). School-Based Mentoring Programs: Using Volunteers to Improve the Academic Outcomes of Underserved Students. SREE Spring. Conference Abstract Template. 1-6.

Beck, E., Baer, M., Guldimann, T., Bischoff, S., Brühwiler, C., Müller, P., Niedermann, R., Rogalla, M. \& Vogt, F. (Hrsg.) (2008). Adaptive Lehrkompetenz. Analyse und Struktur, Veränderung und Wirkung handlungssteuernden Lehrerwissens. Münster: Waxmann Verlag.

Bennack, J. (1989). Möglichkeiten und Grenzen der Schulpraktika im erziehungswissenschaftlichen Lehramtsstudium. Gerhard Steindorf zum 60. Geburtstag. Bildung und Erziehung. 42 (2). 331-346.

Bennewitz, H. (2012). Der Blick auf Lehrer/innen. In: De Boer, H. \& Reh, S. (Hrsg.) (2012). Beobachtung in der Schule - Beobachten lernen. Wiesbaden: Springer VS Verlag. 203-214.

Ben-Peretz, M. \& Rumney, S. (1991). Professional thinking in guided practice. Teaching and Teacher Education. 7 (5/6). 517-530.

Beraterkreis (2019). Abgerufen unter: https://www.beraterkreis.at/wp-content/uploads/2015/12/GROW-Modell. $\operatorname{pdf}(2019-05-26)$.

Bergmann, C. (1998). Bedingungen und Auswirkungen einer interessenentsprechenden Studienwahl. In: Abel, J. \& Tarnai, C. (Hrsg.) (1998). Pädagogisch-psychologische Interessenforschung in Studium und Beruf. Münster, New York: Waxmann Verlag.

Beyer, K., Wisbert, R., Plöger, W., Wasmuth, K-U. \& Anhalt, E. (2006). Schulpraktikum - Einführung in die theoriegeleitete Planung, Durchführung und Reflexion. Stuttgart, Baltmannsweiler: Schneider Verlag Hohengehren.

Blane, H. (2017). 7 principles of transformational leadership. Create a mindset of passion, innovation and growth. Wayne: Career Press.

Blömeke, S. (2002). Universität und Lehrerausbildung. Bad Heilbrunn: Klinkhardt Verlag.

Blömeke, S., Reinhold, P. \& Tulodziecki, R. (Hrsg.) (2004). Handbuch Lehrerbildung. Bad Heilbrunn: Klinkhardt Verlag.

Blömeke, S. (2005). Lehrerausbildung - Lehrerhandeln - Schülerleistungen: Perspektiven nationaler und internationaler empirischer Bildungsforschung. Antrittsvorlesung vom 10. Dezember 2003. Berlin: Humboldt-Universität.

Blömeke, S. (2006). „Fast fish - Loose fish“: International-vergleichende Forschung zur Wirksamkeit der Lehrerausbildung. In: Hilligus, A.H. \& Rinkens, H. (Eds.) (2006). Standards und Kompetenzen - neue Qualität in der Lehrerausbildung? Neue Ansätze und Erfahrungen in nationaler und internationaler Perspektive. Berling: LIT Verlag189-214.

Blömeke, S., Kaiser, G. \& Lehmann, R. (Hrsg.) (2008). Professionelle Kompetenz angehender Lehrerinnen und Lehrer. Wissen, Überzeugungen und Lerngelegenheiten deutscher Mathematikstudierender und -refendare - Erste Ergebnisse zur Wirksamkeit der Lehrerausbildung. Münster: Waxmann Verlag.

Blömeke, S., Bohl, Th., Haag, L., Lang-Wojtasik, G. \& Sacher, W. (2009). Handbuch Schule. Theorie - Organisation Entwicklung. Bad Heilbrunn: Klinkhardt Verlag.

BMB (2013). Bundesgesetz (2013). 124. Bundesrahmengesetz zur Einführung einer neuen Ausbildung für Pädagoginnen und Pädagogen (NR: GP XXIV RV 2348 AB 2397 S. 206. BR: 9006 AB 9012 S. 822.) Abgerufen unter: https://www. ris.bka.gv.at/Dokumente/BgblAuth/BGBLA_2013_I_124/BGBLA_2013_I_124.pdfsig (2018-12-27).

BMB (2013). Verordnung der Bundesministerin für Unterricht, Kunst und Kultur über die Curricula der Pädagogischen Hochschulen (Hochschul-Curriculaverordnung 2013, HCV 2013). BGBl. II Nr. 335/2013 vom 7.11.2013. Abgerufen unter: https://www.bmbwf.gv.at/Themen/schule/schulrecht/gvo/hcv_entwurf.html (2019-12-22).

BMB (2016). Abgerufen unter: https://www.bmb.gv.at/schulen/lehr/labneu/index.html (19-07-2016).

BMB (2016). Abgerufen unter: https://www.bmb.gv.at/schulen/recht/erk/bgbla_2013_i_124_25097.pdf?5h6we5 (19-07-2016).

BMB (2017). Abgerufen unter: https://www.bmb.gv.at/schulen/bw/ueberblick/bildungswege2017_grafik.pdf?61ys15 (2017-11-15).

BMWFM (2014). Abgerufen unter: http://wissenschaft.bmwfw.gv.at/bmwfw/studium/der-europaeische-hochschulraum-bologna-prozess/bologna-worum-gehts/(20-07-2016)

BMWFW (2016). Abgerufen unter: http://wissenschaft.bmwfw.gv.at/bmwfw/wissenschaft-hochschulen/universitaeten/paedagoginnenbildung-ne (08-08-2016).

BMBWF (2018). Bundesministerium für Bildung, Wissenschaft und Forschung. Entwicklungsverbünde. Abgerufen unter: https://bildung.bmbwf.gv.at/schulen/pbneu/ev/verbuende.html (2018-12-27).

BMBWF (2018). Abgerufen unter: https://bildung.bmbwf.gv.at/schulen/bw/abs/hs.html (2018-04-08).

BMBWF (2018). Abgerufen unter: https://bildung.bmbwf.gv.at/schulen/bw/ueberblick/bildungswege2017_grafik. pdf?6a8t76 (2018-04-08). 
BMBWF (2019). Empfehlungsschreiben zu Mentoring und Induktion. Adaptierter Rahmen und konkretisierende Vorschläge Hochschullehrgang Mentoring: Berufseinstieg professionell begleiten.

Boelhauve, U. (2005). Forschendes Lernen. Perspektiven für erziehungswissenschaftliche Praxisstudien. In: Hilligus, A. \& Rinkens, H.-D. (2005). Zentren für Lehrerbildung. Neue Wege im Bereich Praxisphasen. Münster: LIT-Verlag. 103-127.

Bogner, A. \& Menz, W. (2002). Das theoriegenerierende Experteninterview - Erkenntnisinteresse, Wissensform, Interaktion. In: Bogner, A., Litting, B. \& Menz, W. (Hrsg.) (2002). Das Experteninterview - Theorie, Methode, Anwendung. Opladen: Leske \& Budrich. 33-70.

Böhm, A. (2015): Theoretisches Codieren: Textanalyse in der Grounded Theory. In: Flick, U., von Kardorff, E. \& Steinke, I. (Hrsg.) (2015). Qualitative Forschung. Ein Handbuch. Originalausgabe, 11. Reinbek bei Hamburg: Rowohlts Enzyklopädie. Rowohlt Taschenbuch Verlag. 475-485.

Böhnert, A., Mähler, M., Klingebiel, F., Hänze, M., Kuhn, H.P. \& Lipowsky, F. (2018). Die Entwicklung der berufsspezifischen Selbstwirksamkeitserwartung von Lehramtsstudierenden in schulischen Praxisphasen - Ein Vergleich von Lehramtsstudierenden im Praxissemester mit Studierenden in einem fünfwöchigen Blockpraktikum. In: Rothland, M. \& Schaper, N. (2018). Lehrerbildung auf dem Prüfstand. 11 (1). Landau: Verlag Empirische Pädagogik. 85-108.

Bortz, J. \& Döring, N. (2006 $)$. Forschungsmethoden und Evaluation für Human- und Sozialwissenschaftler. Heidelberg: Springer Medizin Verlag.

Bourdieu, P. (1997). Die verborgenen Mechanismen der Macht. Hamburg: VS Verlag.

Boyatzis, R.E. (2007). Developing Emotional Intelligence Competencies. In: Ciarrochi, J. \& Mayer, J.D. (Eds.) (2007). Applying emotional intelligence: A practitioner's guide. Psychology Press.

Brandau, J., Studencnik, P., Schaupp, H. \& Kopp-Sixt, S. (2018). Deutschsprachiges Mentoring-Stil-Inventar mit Fokus auf die Ausbildung von Lehrpersonen. Validität eines Inventars für Stile und Ebenen des Mentorings im schulischen Unterrichtskontext. In: Reintjes, Ch., Bellenberg, G. \& Brahm im, G. (2018). Mentoring und Coaching als Beitrag zur Professionalisierung angehender Lehrpersonen. Münster, New York: Waxmann Verlag. 207-225.

Breidenstein, G. (2012). Ethnographisches Beobachten. In: De Boer, H. \& Reh, S. (2012) (Hrsg.). Beobachtung in der Schule - Beobachten lernen. Wiesbaden: Springer VS Verlag. 27-44.

Brenn, H. (1991). Hinweise zur Planung, Durchführung und Auswertung von Unterrichtsbeobachtungen. Innsbruck: Studienverlag.

Brenn, H., Buchberger, F., Eichenberger, H., Freund, J., Harb, H., Klement, K., Künz, I., Lobendanz, A. \& Teml, H. (1996). Berufspraktische Studien. Innsbruck: Studienverlag.

Breuer, F., Muckel, P. \& Dieris, B. (2018³). Reflexive Grounded Theory. Eine Einführung für die Forschungspraxis. Wiesbaden: Springer Verlag.

Bromme, R. (1992). Der Lehrer als Experte. Bern: Huber Verlag.

Bromme, R. (1997). Kompetenzen, Funktionen und unterrichtliches Handeln des Lehrers. In: Weinert, F.E. (Hrsg.) (1997). Psychologie des Unterrichts und der Schule. Enzyklopädie der Psychologie. Pädagogische Psychologie. Band 3. Göttingen: Springer Verlag. 177-212.

Bromme, R. (2008). Lehrerexpertise. In: Schneider, W. \& Hasselhorn, M. (Hrsg.) (2008). Handbuch der pädagogischen Psychologie. Göttingen: Hogrefe. 159-167.

Bromme, R. \& Haag, I. (2008). Forschung zur Lehrerpersönlichkeit. In: Helsper, W. \& Böhme, J. (Hrsg.) (2008). Handbuch der Schulforschung. Wiesbaden: VS Verlag. 803-819.

Brouwer, C.N. (2007). Alternative teacher education in the Netherlands 2000-2005. A standards-based synthesis. European Journal of Teacher Education. 30 (1).21-40.

Brown, Ch. (2019). Exploring the current context for Professional Learning Networks, the conditions for their success, and research needs moving forwards. Abgerufen unter: https://emeraldopenresearch.com/articles/1-1. und ttps://emeraldopenresearch.s3.amazonaws.com/manuscripts/14151/3577685d-0851-415f-99eb-e905aac6b84c_12904_-_chris_ brown_v2.pdf ?doi=10.12688/emeraldopenres.12904.2\&numberOfBrowsableCollections=1\&numberOfBrowsableInstitutionalCollections=0\&numberOfBrowsableGateways=6. $(2020-03-22)$.

Brühwiler, C. (1999). Die Wirkung von Motivation in der Lehrerinnen- und Lehrerbildung. Freiburg: Pädagogisches Institut der Universität Freiburg.

Bruner, J.S. (1960). The process of education. Cambridge Mass.

Buber, M. (1954). Ich und Du. In: Buber, M. (1984). Das dialogische Prinzip. Heidelberg: Lambert Schneider Verlag.

Buchberger, F. \& Riedl, J. (Hrsg.) (1987). Lehrerbildung - heute. Kommentar zum Lehrplan der Pädagogischen Akademie. Teil I. Wien: Bundesministerium für Unterricht, Kunst und Sport.

Bundesgesetz (2013). 124. Bundesrahmengesetz zur Einführung einer neuen Ausbildung für Pädagoginnen und Pädagogen (NR: GP XXIV RV 2348 AB 2397 S. 206. BR: 9006 AB 9012 S. 822.) Abgerufen unter: https://www.ris. bka.gv.at/Dokumente/BgblAuth/BGBLA_2013_I_124/BGBLA_2013_I_124.pdfsig (2018-12-27).

Bundesgesetzblatt (2013):Abgerufen unter: https://www.ris.bka.gv.at/Dokumente/BgblAuth/BGBLA_2013_I_211/ BGBLA_2013_I_211.html (2020-02-12). 
Burch, J. \& Jackson, A. (2013). Developing partnership through third space activity. Teacher Education Advancement Network Journal. 5 (2). 57-68.

Burns, J.M. (1978). Leadership. New York: Harper \& Row.

Caprara, G.V., Barbaranelli, C., Steca, P. \& Malone, P.S. (2006). Teachers' self-efficacy beliefs and determinants of job satisfaction and students' academic achievement: A study at the school level. Journal of School Psychology. 44 (6). 473-490.

Cherian, F. (2007). Learning to teach: teacher candidates reflect on the relational, conceptual and contextual influences of responsive mentorship. Canadian Journal of Education. 30 (1). 25-46.

Cherniss, C. (2007). The Role of Emotional Intelligence in the Mentoring Process. In: Ragins, B.R. \& Kram, K. E. (Hrsg.) (2007). The Handbook of Mentoring at Work. Theory, Research and Practice. London: Sage Publications. 427-446.

Cho, Y. \& Shim, S.S. (2013). Predicting teachers' achievement goals for teaching. The role of perceived school goal structure and teachers' sense of efficacy. Teaching and Teacher Education. 32. 12-21.

Cimeli, P. (2012). Das Selbstkonzept von Kindern in der Schuleingangsphase. Universität Bern. Doktorarbeit. Abgerufen unter: https://pdfs.semanticscholar.org/562b/4a8041b199236a61429021bfbc81d65be8ef.pdf. (2019-11-24).

Clarke, A., Triggs, V. \& Nielsen, W. (2014). Cooperation teacher participation in teacher education: A review of the literature. Review of Educational Research. 84 (2). 163-202.

Clutterbuck, D. (2017). 30 Years of Modern Mentoring. In: Graf, N. \& Edelkraut, F. (Hrsg.) (2017). Mentoring. Das Praxisbuch für Personalverantwortliche und Unternehmer. Wiesbaden: Springer Verlag. 309-311.

Cohn, R. (1997). Über den ganzheitlichen Ansatz der Themenzentrierten Interaktion: Integrative Therapie. 5. 252-258.

Cohn, R. (1997). Von der Psychoanalyse zur Themenzentrierten Interaktion. Von der Behandlung einzelner zu einer Pädagogik für alle. 13. Stuttgart: Klett-Cotta Verlag.

Combe, A. \& Helsper, W. (Hrsg.) (1996). Pädagogische Professionalität. Untersuchungen zum Typus pädagogischen Handelns. Frankfurt: Suhrkamp.

Combe, A. \& Helsper, W. (1996a). Einleitung. Pädagogische Professionalität. Historische Hypotheken und aktuelle Entwicklungstendenzen. In: Combe, A. \& Helsper, W. (1996b). Pädagogische Professionalität. Untersuchungen zum Typus pädagogischen Handelns. Frankfurt: Suhrkamp. 9-48.

Corbin, J. \& Strauss, A.L. (1990). Groundes Theory Research. Procedures, Canons and Evaluative Criteria. In: Zeitschrift für Soziologie. 19. Heft 6. 418-427.

Corbin, J. \& Strauss, A.L. $\left(2015^{4}\right)$. Basics of qualitative research: Techniques and prodedures for developin Grounded Theory. Thousand Oaks, CA: Sage.

Cramer, C. (2012). Entwicklung von Professionalität in der Lehrerbildung. Empirische Befunde zu Eingangsbedingungen, Prozessmerkmalen und Ausbildungserfahrungen Lehramtsstudierender. Kempten: Klinkhardt Verlag.

Cramer, C. (2020). Kohärenz und Relationierung in der Lehrerinnen- und Lehrerbildung. In: Cramer, C., König, J., Rothland, M. \& Blömeke, S. (Hrsg.) (2020). Handbuch Lehrerinnen- und Lehrerbildung. Bad Heilbrunn: Klinkhardt Verlag. 269-279.

Crasborn, F.A.J., Hennissen, P.P.M. \& Brouwer, C.N. (2005). Teacher coaches' dialogues with prospective teachers. A study of transfer of training. Paper presented at the biennial conference of the European Association for Research on Learning and Instruction.

Crasborn, F., Hennissen, P., Brouwer, N., Korthagen, F. \& Bergen, T. (2011). Exploring a two-dimensional model of mentor teacher roles in mentoring dialogues. In: Teaching and Teacher Education. 27 (2). 320-331.

Crasborn, F. \& Hennissen, P. (2014). Training mentor teachers for effective supervision: The impact of the SMART programme. In: Arnold, K-H., Gröschner, A. \& Hascher, T. (Hrsg.) (2014). Schulpraktika in der Lehrerbildung: Theoretische Grundlagen, Konzeptionen, Prozesse und Effekte. Münster: Waxmann Verlag. 377-397.

Crisp, G. (2010). The impact of mentoring on the success of community college students. The Review of Higher Education. 34 (1). 39-60.

Curriculumhandbuch (2016). Bildungswissenschaftliche Grundlagen. Bachelor. Version 0.7. Entwicklungsverbund West. Koordination: Kraler, Christian. Innsbruck: School of Education.

Curriculum Primarstufe. KPH Edith Stein (2016). Abgerufen unter: http://www.kph-es.at/fileadmin/user_upload/ pdf/Curriculum_Primarstufe_2015-05-11_-final.pdf(2016-07-28).

Curriculum Sekundarstufe. Universität Innsbruck (2016). Abgerufen unter: https://www.uibk.ac.at/fakultaeten-servicestelle/pruefungsreferate/studien/soe.html.de. (2019-12-30).

De Boer, H. \& Reh, S. (Hrsg.) (2012). Beobachtung in der Schule - Beobachten lernen. Wiesbaden: Springer VS Verlag.

Dehne, M., Klaß, S. \& Gröschner, A. (2018). Veränderung motivationaler Orientierungen im Praxissemester: Eine videobasierte Studie auf Basis der Erwartungs-Wert-Theorie. In: Rothland, M. \& Schaper, N. (2018). Lehrerbildung auf dem Prüfstand. 11 (1). Landau: Verlag Empirische Pädagogik. 109-131. 
Demographie Netzwerk. https://www.demographie-netzwerk.de/praxis/wiki/wissensokonomie/(2019-04-16).

Dewe, B., Ferchhoff, W. \& Radtke, E.-O. (1990). Die opake Wissensbasis pädagogischen Handelns. Einsichten aus der Verschränkung von Wissensverwendungsforschung und Professionalisierungstheorie. In: Alisch, L.-M., Baumert, J. \& Beck, B. (Hrsg.) (1990). Professionswissen und Professionalisierung. Braunschweig: Copy-Center Comsee. 291-320.

Dewey, J. (1951). Wie wir denken. Eine Untersuchung über die Beziehung des reflektiven Denkens zum Prozess der Erziehung. Zürich: Morgarten.

Dewey, J. (1986). Erziehung durch und für Erfahrung. Stuttgart: Klett Cotta Verlag.

Dick, M. (2016). Professionsentwicklung als Forschungs- und Handlungsfeld. In: Dick, M., Marotzki, W. \& Mieg, H. (Hrsg.) (2016). Handbuch Professionsentwicklung. Bad Heilbrunn: Klinkhardt Verlag. 9-24.

Dieck, M., Kucharz, D., Küster, O., Müller, K., Rosenberger, T. \& Schnebel, S. (2010). Kompetenzentwicklung von Lehramtsstudierenden in verlängerten Praxisphasen. Ergebnisse der wissenschaftlichen Begleitung des Modellversuchs „Praxisjahr Biberach“ durch die Pädagogische Hochschule Weingarten. In: Gehrmann, A., Hericks, U. \& Lüders, M. (Hrsg.) (2010). Bildungsstandards und Kompetenzmodelle. Beiträge zu einer aktuellen Diskussion über Schule, Lehrerbildung und Unterricht. Bad Heilbrunn: Klinkhardt Verlag. 99-110.

Döbert, H. \& Weishaupt, H. (2014). Innovative Ansätze in der Lehrerbildung im Ausland - eine thematische Einführung. In: Döbert, H., Von Kopp, B. \& Weishaupt, H. (Hrsg.) (2014). Innovative Ansätze der Lehrerbildung im Ausland. Münster, Berlin: Waxmann Verlag.

Doll, J., Jentsch, A., Meyer, D., Kaiser, G., Kaspar, K. \& König, J. (2018). Zur Nutzung schulpraktischer Lerngelegenheiten an zwei deutschen Hochschulen: Lernprozessbezogene Tätigkeiten angehender Lehrpersonen in Masterpraktika. In: Rothland, M. \& Schaper, N. (2018). Lehrerbildung auf dem Prüfstand. 11 (1). Landau: Verlag Empirische Pädagogik. 24-45.

Domović, V., Gehrmann, S., Helmchen, J., Krüger-Potratz, M. \& Petravić, A. (Hrsg.) (2013). Europäische Lehrerbildung. Annäherung an ein neues Leitbild. Berichte aus West- und Südosteuropa. Münster: Waxmann Verlag.

Dreer, B. (2018). Praxisleitfaden Lehrerausbildung. Für eine professionelle Begleitung vom Praktikum bis zum Berufseinstieg. Weinheim, Basel: Beltz Verlag.

Duffield, S. (2006). Safety net or free fall: the impact of cooperation teachers. Teacher Development. 10 (2). 167-178.

Dunn, T.G. \& Taylor, C.A. (1993). Cooperating teacher advice. Teaching and Teacher Education. 9 (4). 411-423.

Dunne, E. \& Bennett, N. (1997). Mentoring processes in school-based training. British Educational Research Journal. 23. 225-237.

Eby, L.T., Rhodes, J.E. \& Allen T.D. (2007). Definition and Evolution of Mentoring. In: Allen, T.D., Eby, L.T. (Hrsg.) (2007). The Blackwell Handbook of Mentoring. A Multiple Perspectives Approach. Malden: Blackwell. 7-20.

Edelmann, W. $\left(2000^{6}\right)$. Lernpsychologie. Weinheim: Beltz Verlag.

Edelmann, W. (2019). Lernen. Abgerufen unter: https://www.spektrum.de/lexikon/psychologie/lernen/8749 (2019-0813).

Eder, F., Dämon, K. \& Hörl, G. (2011). Das „Autonomie-Paritäts-Muster“: Vorberuflich erlerntes Stereotyp, Bewältigungsstrategie oder Ergebnis der beruflichen Sozialisation? In: Zeitschrift für Bildungsforschung. I (3). $199-217$.

Edwards, A. \& Collison, J. (1996). Mentoring and developing practice in primary schools: Supporting student teacher learning in schools. Chapter 3. Buckingham: Open University Press.

Edwards, A. \& Protheroe, L. (2004). Teaching by proxy: Understanding how mentors are positioned in partnerships. Oxford Review of Education. 30 (2). 183-197.

Eickelpasch, R. (1982). Das ethnomethodologische Programm einer „radikalen“ Soziologie. In: Zeitschrift für Soziologie. Jahrgang 11. Heft 1. Bielefeld: Lucius \& Lucius Verlagsgesellschaft. 7-27.

Eilerts, K. \& Rinkens, H.-D. (2010). Die Einführung von Standards und Kompetenzen in der Lehrerausbildung. In: Abel, J. \& Faust, G. (Hrsg.) (2010). Wirkt Lehrerbildung? Antworten aus der empirischen Forschung. Münster: Waxmann Verlag. 105-112.

Engelbrecht, H. (1986). Geschichte des österreichischen Bildungswesens. Erziehung und Un-terricht auf dem Boden Österreichs. Band 4. Von 1848 bis zum Ende der Monarchie. Wien: Österreichischer Bundesverlag GmbH.

Enzelberger, S. (2001). Sozialgeschichte des Lehrerberufs. Gesellschaftliche Stellung und Professionalisierung von Lehrerinnen und Lehrern von den Anfängen bis zur Gegenwart. Weinheim, München: Juventa Verlag.

Ericsson, K.A., Charness, N., Feltovich, P.J. \& Hoffman, R.R. (Hrsg.) (2006). The Cambridge Handbook of Expertise and Expert Performance. Cambridge.

Erpenbeck, J. \& Sauter, W. (2015). Wissen, Werte und Kompetenzen in der Mitarbeiterentwicklung. Ohne Gefühl geht in der Bildung gar nichts. Wiesbaden: Springer Fachmedien.

Erpenbeck, J. \& Sauter, W. (2018). Wertungen, Werte - Das Fieldbook für ein erfolgreiches Wertemanagement. Berlin: Springer Verlag.

Eurydice (2002). Der Lehrerberuf in Europa: Profil, Tendenzen und Anliegen. Bericht I: Lehrerausbildung und Maßnahmen für den Übergang in das Berufsleben. Allgemein bildender Sekundarbereich I. Brüssel: Eurydice. 
Evertson, C.M. \& Smithey, M.W. (2001). Mentoring effects on protégé classroom practice. Journal of Educational Research. 93 (5). 294-304.

Evetts, J. (2003). Professionalization and professionalism: explaining professional performance initiatives. In: Mieg, H.A. \& Pfadenhauer, M. (Hrsg.) (2003). Professionelle Leistung - Professional Performance: Positionen der Professionssoziologie. Konstanz: UVK. 49-69.

Fabel-Lamla, M. \& Pietsch, S. (2012). Vom Beobachten zum Handeln im Lehrberuf. Herausforderungen und Prozessstrukturen bei der Bearbeitung pädagogischer Problemsituationen. In: De Boer, H. \& Reh, S. (Hrsg.) (2012). Beobachtung in der Schule - Beobachten lernen. Wiesbaden: Springer VS Verlag. 281-300.

Faix, T. \& Wiedekind, A. (2017). Mentoring. Das Praxisbuch. Ganzheitliche Begleitung von Glaube und Leben. Neukirchen-Vluyn: Neukirchener Verlagsgesellschaft.

Fichten, W. (2010): Forschendes Lernen in der Lehrerbildung. In: Eberhardt, U. (Hrsg.) (2010). Neue Impulse in der Hochschuldidaktik. Wiesbaden: VS Verlag für Sozialwissenschaften, 127-182.

Fichten, W. (2017). Forschendes Lernen in der Lehrerbildung. In: Schüssler, R., Schönring, A., Schwier, V., Schicht, S., Gold, J. \& Weyland, U. (Hrsg.) (2017). Forschendes Lernen im Praxissemester. Zugänge, Konzepte, Erfahrungen. Bad Heilbrunn: Klinkhardt Verlag. 30-38.

Feiman-Nemser, S., Parker, M.B. \& Zeichner, K. (1992). Are mentor teachers teacher educators? Research Project. East Lansing, Michigan: The National Center for Research on Teacher Learning. 92-111.

Fenstermacher, G.D. (1994). The Knower and the Known. The Nature of Knowledge in Research on Teaching. In: Darling-Hammond, L. (Hrsg.) (1994). Review of Research in Education 20. Washington: AERA. 3-56.

Finckler, P. (2017). Transformationale Führung. Wegweiser für nachhaltigen Führungs- und Unternehmungserfolg. Berlin, Heidelberg: Springer Verlag.

Fischer, T., Bach, A. \& Rheinländer, K. (2018). Veränderung von Einstellungen zur Theorie- und Praxisorientierung des Lehramtsstudiums im Praxissemester. In: Rothland, M. \& Schaper, N. (2018). Lehrerbildung auf dem Prüfstand. 11 (1). Landau: Verlag Empirische Pädagogik. 152-167.

Flagmeyer, D., Dietze-Münnich, U. \& Strietzel, A. (2002). Schule als Studienfeld. Die ersten schulpraktischen Studien vorbereiten. Leipzig: Leizipger Universitätsverlag.

Fletcher, S. (1998). Attaining Self-actualisation Through Mentoring. European Journal of Teacher Education, 21 (1), 109-118.

Flick, U., Kardoff, E.v. \& Steinke, I. (2005). Qualitative Forschung. Ein Handbuch. Hamburg: Rowohlt Taschenbuch Verlag.

Flick, U. (20167). Qualitative Sozialforschung. Eine Einführung. Hamburg: Rowohlt Taschenbuch Verlag.

Flusser, V. (1990). Nachgeschichten. Düsseldorf: Bollmann Verlag.

Floden, R.E. (2015). Learning What Research Says About Teacher Preparation. In: Feuer, F.J., Berman, A.I. \& Atkinson, R.C. (Eds.) (2015). Past as Prologue. The National Academy of Education at 50. Members Reflect. Washington: National Academy of Education.

Floß, P. \& Rotermund, M. (2010). Das Praxissemester in Nordrhein-Westfalen. In: Krüger, A., Nakamura, Y. \& Rotermund, M. (Hrsg.) (2010). Schulentwicklung und schulpraktische Studien - Wie können Schulen und Lehrerbildung voneinander profitieren? Leipzig: Leipziger Universitätsverlag. 263-278.

Fraefel, U. \& Seel, A. (2017). Konzeptionelle Perspektiven Schulpraktischer Studien. Eine Einführung. In: Fraefel, U. \& Seel, A. (2017). Konzeptionelle Perspektiven Schulpraktischer Studien. Partnerschaftsmodelle - Praktikumskonzepte - Begleitformate. Münster: Waxmann Verlag. 7-10.

Fraefel, U. (2018). Die Wende zum kollaborativen Mentoring in Schulpraktika. Überblick zu empirischen Befunden, theoretischen Verortungen und Strategien der Transformation. In: Reintjes, Ch., Bellenberg, G. \& Brahm, G. (2018). Mentoring und Coaching als Beitrag zur Professionalisierung angehender Lehrpersonen. Münster, New York: Waxmann Verlag. 41-66.

Franke, A. \& Dahlgren, L. O. (1996). Conceptions of mentoring: An empirical study of conceptions of mentoring during the school-based teacher education. Teaching and Teacher Education. 12 (6). 627-641.

Freidson, E. (1994). Professionalism Reborn: Theory, Prophexy, and Policy. Chicago.

Freidson, E. (2001). Professionalism. The third logic. Cambridge. UK.

Freidson, E. (2003). Professionalism. The third logic. London: Polity Press.

Frey, A. (2006). Methoden und Instrumente zur Diagnose beruflicher Kompetenzen von Lehrkräften. Eine erste Standortbestimmung zu bereits publizierten Instrumenten. In: Allemann-Ghionda, Ch. \& Terhart, E. (2006). Kompetenzen und Kompetenzentwicklung von Lehrerinnen und Lehrern. Zeitschrift für Pädagogik. Beiheft 51. Weinheim: Beltz Verlag. 30-46.

Frey, A. \& Jung, C. (2011). Kompetenzmodelle und Standards in Lehrerbildung und Lehrerbedarf. In: Terhart, E., Bennewitz, H. \& Rothland, M. (Hrsg.) (2011). Handbuch der Forschung zum Lehrerberuf. Münster: Waxmann Verlag. 540-572. 
Fried, L. (2004). Polyvalenz und Professionalität. In: Blömeke, S., Reinhold, R., Tulodziecki, G. \& Wildt, J. (Hrsg.) (2004). Handbuch Lehrerbildung. Kempten: Klinkhardt Verlag.

Fuge, J. (2016). Mentoring als hochschuldidaktisches Instrument zur Gestaltung der Studieneingangsphase. Eine vergleichende Analyse verschiedener Mentoring-Formen. Detmold: Eusl-Verlagsgesellschaft GmbH.

Garringer, M. (2010). Planning a School-Based Mentoring Programm. Lessons Learned. Portland: Education Northwest. Volume 1. Issue 4. 1-5.

Garvey, D.J. (2000). Mentoring Beginning Teachers. Program Handbook. The Alberta Teachers' Association. http:// ncee.org/wp-content/uploads/2017/01/Alb-non-AV-18-ATA-Mentoring-beginning-teachers.pdf. (2019-07-12).

Garvis, S., Pendergast, D. \& Keogh, J. (2012). Changes in teacher self-efficacy in the first year of primary school teacher education study. Journal of the World Universities Forum. 5 (1). 87-96.

Gaspard, H., Dicke, A.-L., Flunger, B., Schreier, B., Häfner, I., Trautwein, U. \& Nagengast, B. (2015). More value through greater differentiation. Gender differences in value beliefs about math. Journal fo Educational Psychology. 107. 663-677.

Geldens, J.J.M., Popeijus, H., Peters, V. \& Bergen, Th. (2005). Mentoring met kwaliteit als krachtig kenmerk van werkplekomgevingen voor aanstaande leraren. Analyseresultaten van mentoringsgesprekken [The quality of mentoring as a feature of effective workplace environments for prospective teachers. Analysing results of mentoring dialogues]. Paper presented at the Onderwijs Research Dagen.

Gerhardt, U. (1971). Rollenanalyse als kritische Soziologie. Neuwied.

Girmes, R. (2004). (Sich) Aufgaben stellen. Seelze: Kallmeyer Verlag.

Glaser, B.G. (1978). Theoretical sensitivity: Advances in the methodology of grounded theory. Mill Valley. CA: Sociology Press.

Glaser, B. \& Strauss, A. (1998). Grounded Theory. Bern: Hans Huber.

Glaser, B (2005). The Grounded Theory prespective III: Theoretical Coding. Mill Valley, CA: Sociology Press.

Gläser, J. \& Laudel Grit $\left(2010^{4}\right)$. Experteninterviews und qualitative Inhaltsanalyse. Wiesbaden: Springer Fachmedien.

Gobo, G. (2004). Sampling, Representativeness and Generalizability. In: Seale, C., Gobo, G., Gubrium, J.F. \& Silverman, D. (Hrsg.) (2004). Qualitative Research Practice. London: Sage. 435-456.

Gold, B., Förster, S. \& Holodynski, M. (2013). Evaluation eines videobasierten Trainingsseminars zu Förderung der professionellen Wahrnehmung von Klassenführung im Grundschulunterricht. In: Zeitschrift für Pädagogische Psychologie. 27 (3). 141-155.

Gollwitzer, M. \& Schmitt, M. (2006). Sozialpsychologie. Weinheim: Work Book.

Gorges, J. \& Kandler, C. (2012). Adults' learning motivation: Expectancy of success, value, and the role of affective memories. Learning and Individual Differences. 22. 610-617.

Graf, N. \& Edelkraut, F. (Hrsg.) (2017). Mentoring. Das Praxisbuch für Personalverantwortli-che und Unternehmer. Wiesbaden: Springer Verlag.

Grint, K. (2010). Leadership. A very short introduction. New York: Oxford University Press.

Gröschner, A., Bauer, J., Müller, K. \& Seidel, T. (2015). Praxisphasen in der Lehrerausbildung - Eine Strukturanalyse am Beispiel des gymnasialen Lehramtsstudiums in Deutschland. In: Zeitschrift für Erziehungswissenschaft. 18 (4). $687-712$

Haas, E. \& Schneider, K. (2018). Schulpraktikum I im Lehramtsstudium der Sekundarstufe Allgemeinbildung: Erkenntnisse aus der Sicht von Studierenden. In: Fridrich, R., Mayer-Frühwirth, G., Potzmann, R., Greller, W. \& Petz, R. (Hrsg.) (2018). Forschungsperspektiven 10. Wien: LIT Verlag. KG. 47-64.

Haasen, N. (2001). Mentoring. Persönliche Karriereförderung als Erfolgskonzept. München: Heyne Verlag.

Haggarty, L. (1995). The use of content analysis to explore conversations between schoolteacher mentors and student teachers. British Educational Research Journal. 21 (2). 183-197.

Hansman, C.A. (2003). Reluctant mentors and resistant protégés: Welcome to the "real" world of mentoring. Adult Learning. 14 (1). 14-16.

Harrison, J., Lawson, T. \& Wortley, A. (2005). Mentoring the beginning teacher: Developing professional autonomy through critical reflection on practice. Reflective Practice. 6 (3). 419-441.

Hascher, T. \& Wepf, L. (2007). Lerntagebücher im Praktikum von Lehramtsstudierenden. Empirische Pädagogik. 21 (2). 101-118.

Hascher, T. (2006). Veränderungen im Praktikum - Veränderungen durch das Praktikum. Eine empirische Untersuchung zur Wirkung von schulpraktischen Studien in der Lehrerbildung. In: Allemann-Ghionda, Ch. \& Terhart, E. (2006). Kompetenzen und Kompetenzentwicklung vonLehrerinnen und Lehrern. In: Zeitschrift für Pädagogik. Beiheft 51. Weinheim: Beltz Verlag. 130-148.

Hascher, T. (2012). Lernfeld Praktikum - Evidenzbasierte Entwicklungen in der Lehrer/innenbildung. In: Zeitschrift für Bildungsforschung. 2 (2). 109-129. 
Hascher, T. \& Hagenauer, G. (2016). Openness to theory and its importance for student teachers' selfefficacy, emotions, and classroom behaviour in the practicum. International Journal of Educational Research. 77. 15-25.

Hascher, T. \& Brandenberger, C. (2017). Emotionen und Lernen im Unterricht. In: Huber, M. \& Krause, S. (Hrsg.) (2017). Bildung und Emotion. Wiesbaden: Springer Verlag. 289-310.

Hauser, B. (2013). Wo ist die Führungskraft? Management, Leadership, Shared Leadership und die Evolution der Führungsrolle. In: Landes, M. \& Steiner, E. (Hrsg.) (2013). Psychologie der Wirtschaft. Wiesbaden: Springer Verlag. 279-295.

Havighurst, R.J. (1948). Developmental Tasks and Education. New York: Reprint von 1952. David McKay Company. Inc.

Havighurst, R.J. (1953). Human Development and Education. Oxford: Longmans Green.

Hawkey, R.J. (1997). Roles, responsibilities, and relationships in mentoring: al literature review and agenda for research. Journal of Teacher Education. 48 (5). 325-335.

Hawkey, K. (1998a). Mentor pedagogy and student teacher professional development: A study of two mentoring relationships. Teaching and Teacher Education. 14 (6). 657-670.

Hawkey, K. (1998b). Consultative supervision and mentor development: An initial exploration and case study. Teachers and Teaching: theory and practice. 4 (2). 331-348.

Heiser, P. (2018). Meilensteine der qualitativen Sozialforschung. Eine Einführung entlang klassischer Studien. Wiesbaden: Springer Fachmedien.

Hellmann, K. (2019). Kohärenz in der Lehrerbildung - Theoretische Konzeptionalsierung. In: Hellmann, K., Kreutz, J., Schwichow, M. \& Zaki, K. (2020). Kohärenz in der Lehrerbildung. Wiesbaden: Springer Verlag. 9-30.

Hellwig, C. (2016). Wertebasierte Gesprächsführung. Essentials. Wiesbaden: Springer Fachmedien.

Helsper, W. (2001). Praxis und Reflexion. Die Notwendigkeit einer „doppelten Professionalisierung“ des Lehrers. In: Journal für Lehrerinnen- und Lehrerbildung. (3). 7-15.

Helsper, W. \& Tippelt, R. (2011). Pädagogische Professionalität. In: Zeitschrift für Pädagogik. Beiheft 57. Weinheim.

Helsper, W. (2014). Lehrerprofessionalität - der strukturtheoretische Professionsansatz zum Lehrerberuf. In: Terhart, E., Bennewitz, H. \& Rothland, M. (Hrsg.) (2014). Handbuch der Forschung zum Lehrerberuf. Münster: Waxmann Verlag. 216-240.

Hennissen, P., Crasborn, F., Brouwer, N., Korthagen, F. \& Bergen, T. (2008). Mapping mentor teachers' roles in mentoring dialogues. Educational Research Review 3. 168-186.

Hennissen, P., Crasborn, F., Brouwer, N., Korthagen, F. \& Bergen, T. (2011). Clarifying preservice teacher perceptions of mentor teachers' developing use of mentoring skills. Teaching and Teacher Education. 27. 1049-1058.

Henson, R.K. (2001). The effects of participation in teacher research on teacher efficacy. Teaching and Teacher Education. $17(7) .819-836$.

Hericks, U. (2004). Entwicklungsaufgaben, Habitus und Professionalisierung von Lehrerinnen und Lehrern. In: Trautmann, M. (2004): Entwicklungsaufgaben im Bildungsgang. Wiesbaden: VS Verlag für Sozialwissenschaften. 117135.

Hericks, U, (2006). Professionalisierung als Entwicklungsaufgabe. Rekonstruktion zur Berufseingangsphase von Lehrerinnen und Lehrern. Wiesbaden: Verlag für Sozialwissenschaften.

Herzmann, P. \& König, J. (2016). Lehrerberuf und Lehrerbildung. Studientexte Bildungswissenschaften. Bad Heilbrunn: Klinkhardt Verlag.

Herzmann, P. \& Liegmann, A.B. (2018). Studienprojekte im Praxissemester. Wirkungsforschung im Kontext Forschenden Lernens. In: Rothland, M. \& Schaper, N. (2018). Lehrerbildung auf dem Prüfstand. 11 (1). Landau: Verlag Empirische Pädagogik. 46-65.

Herzog, W. (1995). Reflexive Praktika in der Lehrerinnen- und Lehrerbildung. In: Beiträge zur Lehrerinnen- und Lehrerbildung. 13 (3). 253-273.

Herzog, S. (2011). Über den Berufseinstieg hinaus: Berufsbiographien und Lehrerinnen und Lehrern im Blickfeld der Forschung. In: Terhart, E., Bennewitz, H. \& Rothland, M. (Hrsg.) (2011). Handbuch der Forschung zum Lehrerberuf. Münster: Waxmann Verlag. 314-338.

Hesse, F. \& Lütgert, W. (2020). Auf die Lernbegleitung kommt es an! Konzepte und Befunde zu Praxisphasen in der Lehrerbildung. Bad Heilbrunn: Klinkhardt Verlag.

Higgins, M.C. \& Kram, K.E. (2001). Reconceptualizing Mentoring at Work: A Developmental Network Perspective. In: Academy of Management Review. 26 (2). 264-288.

Hitzler, R., Honer, A. \& Maeder, Ch. (Hrsg.) (1994). Expertenwissen. Die institutionalisierte Kompetenz zur Konstruktion von Wirklichkeit. Opladen.

Huberman, M. (1989). The professional life cycle of teachers. Teachers College Record. 106 (6). 31-57.

Hudson, P. (2013). Forming the Mentor-Mentee Relationship. Journal of Mentoring and Tutoring. Queensland. 1-25.

Hudson, P. (2013b). Strategies for mentoring pedagogical knowledge. Teachers and Teaching: Theory and Practice. DO I:10.1080/13540602.2013.770226. 1-32. 
Hughes, M. (1998). Developing the use of qualitative analysis to describe and explore the effectiveness of post-observational discourse between school-based mentors and students in teacher education. Paper presented at the European conference on educational research.

Hobson, A.J., Malderez, A., Tracey, L., Homes, M., Mitchell, N., Biddulph, M. Giannakaki, M.S., Rose, A., Pell, R.G., Roper, T., Chambers, G.N. \& Tomlinson, P.D. (2007). Newly qualified teachers' expériences of their first year of teaching: Findings from phase III of the becoming a teacher project. Nottingham: Department for Children, Schools and Families (DCSF).

Hobson, A.J., Ashby, P., Malderez, A. \& Tomlinson, P.D. (2009). Mentoring beginning teachers: What we know and what we don't. Teaching and Teacher Education. 25. 207-216.

Hofmann, F. (2019). Kontextsensibilität als wichtiges Kompetenzziel im Mentoring. Impuls beim Symposium zum Thema Mentoring. Ausbildung und Aufgabenprofil der Mentor/inn/en in der neuen Lehrer/innenbildung. Vortrag in Salzburg. (2019-01-18).

Höher, F. (2014). Vernetztes Lernen im Mentoring. Eine Studie zur nachhaltigen Wirkung und Evaluation von Mentoring. Wiesbaden: Springer Fachmedien.

Hölzle, C. \& Jansen, I. (Hrsg.) (2011). Ressourcenorientierte Biografiearbeit. Grundlagen - Zielgruppen - Kreative Methoden. Wiesbaden: Springer Fachmedien.

Holland, J.L. (1997³). Making vocational choices. A theory of vocational personalities and work environments. Odessa. Florida: Psychological Assessment Resources.

Holt, N.L., Strean, W.B. \& Bengoechea, E.G. (2002). Expanding the Teaching Games for Understanding Model: New Avenues for Future Research and Practice. Journal of Teaching in Physical Education. 21. 162-177.

Homfeld, W. (1978). Theorie und Praxis der Lehrerausbildung: Ziele und Auswirkungen der Reformdiskussion im 19. und 20. Jahrhundert. Weinheim: Beltz Verlag.

Hopf, C. (1978). Die Pseudo-Exploration - Überlegungen zur Technik qualitativer Interviews in der Sozialforschung. In: Zeitschrift für Soziologie 7.97-115.

Horstkemper, M. (2004). Erziehungswissenschaftliche Ausbildung. In: Blömeke, S., Reinhold, P., Tulodziecki, G. \& Wildt, J. (Hrsg.) (2004). Handbuch Lehrerbildung. Kempten: Klinkhardt Verlag. 461-476.

House, J.S. (1981). Work stress and social support. Reading, Mass.: Addison-Wesley.

Ilien, A. (2008). Lehrerprofession. Grundprobleme pädagogischen Handelns. Wiesbaden: VS Verlag der Sozialwissenschaften.

Ittel, A. \& Raufelder, D. (2009). Mentoring in der Schule. Professionelle Praxis und Qualitätssicherung. In: Stöger, H., Ziegler, A. \& Schminke, D. (Hrsg.) (2009). Mentoring. Theoretische Hintergründe, empirische Befunde und praktische Anwendungen. Lengerich: Pabst. 193-206.

Jackson, A. \& Burch, J. (2018). New directions for teacher education: investigating school/university partnership in an increasingly school-based context. Professional Development in Education. 45 (1). Abgerufen unter: http://insight. cumbria.ac.uk/id/eprint/3697/. (2019-11-24).

Jacobi, M. (1991). Mentoring and Undergraduate Academic Success. A Literature Review. American Educational Research. 61 (4). 505-532.

Jahnke, I. (2006). Dynamik sozialer Rollen beim Wissensmanagement. Soziotechnische Anforderungen an Communities und Organisationen. Wiesbaden: Universitätsverlag.

Jansen, I. (2011). Biografie im Kontext sozialwissenschaftlicher Forschung und im Handlungsfeld pädagogischer Biografiearbeit. In: Hölzle, C. \& Jansen, I. (Hrsg.) (2011). Ressourcenorientierte Biografiearbeit. Grundlagen - Zielgruppen - Kreative Methoden. Wiesbaden: Springer Fachmedien. 17-30.

James, W. (1890/1950). The Consciousness of Self. In: William, W. (Eds.). The principles of psychology. New York: Dover Publications.

Johnson, W.B. (2003). A framework for conceptualizing competence to mentor. Ethics \& Behavior. 13 (2). $127-151$.

Johnson, B.J., Rose, G. \& Schlosser, L.Z. (2010). Student-faculty mentoring: Theoretical and methodological issues. In: Allen, T.D. \& Eby, L.T. (Eds.) (2010). The Blackwell handbook of mentoring: a multiple perspectives approach. Malden, MA: Blackwell. 49-69.

Juen, G. (1986). Lehren - Bilden - Lehrerbildung. 150 Jahre Lehrerbildung in Zams. 1836 - 1986. Pädagogische Akademie Zams. Thaur: Kulturverlag.

Jünger, S. \& Reintjes, Ch. (2017). Lehrer/innenbildung im hybriden Raum. Anforderungen an eine kooperative Professionalisierung. Jahrbuch für Allgemeine Didaktik. 7. 102-121.

Kallmeyer, W., Schütze, F. (1976). Konversationsanalyse. Studium Linguistik. 1. 1-28.

Kämmerer, W. (2016). Auf der Suche nach dem Wort, das berührt. Psychotherapie: Praxis. Heidelberg, Berlin: Springer Verlag.

Kelle, U. \& Kluge, S. (1999). Vom Einzelfall zum Typus. Fallvergleich und Fallkontrastierung in der qualitativen Sozialforschung. Opladen: Leske und Budrich Verlag. 
Kelle, U. (2008). Die Integration qualitativer und quantitativer Methoden in der empirischen Sozialforschung - Theoretische Grundlagen und methodologische Konzepte. Wiesbaden: VS Verlag für Sozialwissenschaften.

Keller-Schneider, M. (2010). Entwicklungsaufgaben im Berufseinstieg von Lehrpersonen. Münster: Waxmann Verlag.

Kemnitz, H. (2011). Forschung zur Geschichte und Entwicklung des Lehrerberufs vom 18. Jahrhundert bis zur Gegenwart. In: Terhart, E., Bennewitz, H. \& Rothland, M. (Hrsg.) (2011). Handbuch der Forschung zum Lehrerberuf. Münster: Waxmann Verlag. 15-33.

Kernquadrat (2019). Das Zwiebelmodell von den Schichten der Persönlichkeit. Grundlage zur Reflexion des wahrnehmbaren Verhaltens eines Menschen mit Bezug zu tieferliegenden Schichten seiner Persönlichkeit. Abgerufen unter: https://kernquadrat.de/das-zwiebelmodell-der-persoenlichkeit/(2019-08-16).

Kerschensteiner, G. (1927). Die Seele des Erziehers und das Problem der Lehrerbildung. München: Oldenbourg Verlag.

Klassen, R.M. \& Chiu, M.M. (2010). Effects on teachers'self-efficacy and job satisfaction: Teacher gender, years pf experience, and job stress. Journal of Educational Psychology. 102 (3). 741-756.

Klassen, R.M., Tze, V.M., Betts, S.M. \& Gordon, K.A. (2011). Teacher efficacy research 1998-2009. Signs of progress or unfulfilled promise? Educational Psychology Review. 23 (1). 21-43.

Klassen, R.M. \& Durksen, T.L. (2014). Weekly self-efficacy and work stress during the teaching practicum: A mixed methods study. Learning an Instruction. 33. 158-169.

Klement, K. \& Teml, H. (Hrsg.) (1996). Schulpraxis reflektieren. Wege zum forschenden Lernen in der Lehrerbildung. Innsbruck: Studienverlag Ges.m.b.H..

Klement, K., Lobendanz, A. \& Teml, H. (Hrsg.) (2002). Schulpraktische Studien. Beiträge zur Qualitätsentwicklung in der Lehrerbildung unter Berücksichtigung europäischer Perspekti-ven. Innsbruck: Studienverlag.

KMK (2004). Standards für die Lehrerbildung: Bildungswissenschaften. Beschuss der Kultusministerkonferenz vom 16.12.2004. Abgerufen unter: https:/www.kmk.org/fileadmin/Dateien/veroeffentlichungen beschluesse/2004/2004_12_16-Standards-Lehrerbildung-Bildungswissenschaften.pdf (2018-08-30).

Köck, P. \& Ott, H. (1994). Wörterbuch für Erziehung und Unterricht. Donauwörth: Auer Verlag.

Köffler, N.M. (2015). Entwicklungsaufgaben im Lehramtsstudium. Bad Heilbrunn: Klinkhardt Verlag.

König, J. \& Blömeke, S. (2009). Disziplin- oder Berufsorientierung? Zur Struktur des pädagogischen Wissens angehender Lehrkräfte. Lehrerbildung auf dem Prüfstand. 2 (1). 126-147.

König, J. \& Seifert, A. (2012). Lehramtsstudierende erwerben pädagogisches Professionswissen: Ergebnisse der Längsschnittstudie LEK zur Wirksamkeit der erziehungswissenschaftlichen Lehrerausbildung. Münster: Waxmann Verlag.

König, J. \& Rothland, M. (2013). Pädagogisches Wissen und berufsspezifische Motivation am Anfang der Lehrerausbildung. Zum Verhältnis von kognitiven und nicht-kognitiven Eingangsmerkmalen von Lehramtsstudierenden. In: Zeitschrift für Pädagogik. 59 (1). Weinheim: Beltz Verlag. 43-65.

König, J., Tachtsoglou, S., Darge, K. \& Lünnemann, M. (2014). Zur Nutzung von Praxis: Modellierung und Validierung lernprozessbezogener Tätigkeiten von angehenden Lehrkräften im Rahmen ihrer schulpraktischen Ausbildung. In: Zeitschrift für Bildungsforschung. 4 (1). Salzburg: Springer Verlag. 3-22.

König, J., Rothland, M. \& Schaper, N. (Hrsg.) (2018). Learning to Practice, Learning to Reflect?. Wiesbaden: Springer Verlag.

Kopp, B. von (2014). Lehrerbildung im Ausland: Aktuelle und innovative Tendenzen der Lehrerbildung in internationaler Perspektive. In: Döbert, H., Von Kopp, B. \& Weishaupt, H. (Hrsg.) (2014). Innovative Ansätze der Lehrerbildung im Ausland. Münster, Berlin: Waxmann Verlag. 23-108.

Kordes, H. (1996). Entwicklungsaufgabe und Bildungsgang. Münster: LitGoogle Scholar.

Korthagen, F.A.J. (1985). Reflective Teaching and Preservice Teacher Education in the Netherlands. Journal of Teacher Education.

Korthagen, F.A.J. \& Vasalos, A. (2005). Levels in reflection: core reflection as a means to enhance professional growth. Journal Teachers and Teaching. Theory and practice. 11. (1). 47-71.

Korthagen, F.A.J. \& Vasalos, A. (2010). Going to the Core. Deepening Reflection by Con-necting the Person to the Profession. In Lyons, N. (Ed.). Handbook of Reflection and Reflective Inquiry. Mapping a Way of Knowing for Professional Reflective Inquiry. New York, NY: Springer Verlag. 529-552.

Košinár, J. \& Laros, A. (2018). Zwischen Einlassung und Vermeidung - Studentische Orientierungen im Umgang mit lehrberuflichen Anforderungen im Spiegel von Professionalität. In: Leonhard, T., Košinár, J. \& Reintjes, Ch. (Hrsg.) (2018). Praktiken und Orientierungen in der Lehrerbildung. Potentiale und Grenzen der Professionalisierung. Bad Heilbrunn: Klinkhardt Verlag. 157-174.

Košinár, J. (2018). Das Mentorat zwischen Individualisierung und Standardisierung - eine empirie- und theoriebasierte Konzeption. In: Reintjes, Ch., Bellenberg, G. \& Brahm im, G. (2018). Mentoring und Coaching als Beitrag zur Professionalisierung angehender Lehrpersonen. Münster, New York: Waxmann Verlag. 67-84.

Kounin, J.S. (1970). Discipline and group management in classrooms. New York: Holt, Rinehart \& Winston.

KPH Edith Stein (2020). Homepage. Abgerufen unter: www.kph-es.at. (2020-01-01). 
Kraler, Ch. (2009). Entwicklungsaufgaben in der universitären Lehrerbildung - Startverpflegung auf dem Weg zu einer lebenslangen Professionalisierung. In: Erziehung und Unterricht 159 (1/2). Wien: Österreichischer Bundesverlag Schulbuch. KG. 187-197. Abgerufen unter: www.uibk.ac.at/ils/mitarbeiter/christian-kraler/papers/kraler-entwicklungs aufgaben-e_u-2008.pdf(2018-12-04).

Kraler, Ch. \& Schratz, M. (2012a). From Best Practice to Next Practice: A Shift through Research-Based Teacher Education. http://reflectingeducation.net. Vol. 8. No. 2. December 2012. London: Institute of Education. 88-125.

Kraler, Ch. (2012b). Professionsspezifische Entwicklungsaufgaben (in) der Universitären Lehrer/innenbildung. Sammelhabilitationsschrift. Institut für LehrerInnenbildung und Schulforschung. Fakultät für Bildungswissenschaften. Universität Innsbruck.

Kraler, Ch., Reich, K. \& Fügenschuh, B. (2017). PädagogInnenbildung Neu im Verbund LehrerInnenbildung West. Eine Standortbestimmung zu Gelingensbedingungen und Herausforderungen. In: Zeitschrift für Hochschulrecht Heft 16. April 2017. Wien: Verlag Österreich. 79-86.

Kram, K.E. (1983). Phases on the mentor relationship. In: Academy of Management Journal (1983). 26 (4). 608-625.

Kram, K.E. (1985). Mentoring at work. Developmental Relationships in Organizational Life. Glenview/III: Scott Foresman and Co.

Kramis, J. (1990). Bedeutsamkeit, Effizienz, Lernklima. Grundlegende Kriterien für Unterricht und Didaktische Prinzipien. In: Beiträge der Lehrerbildung 8. 279-296.

Krause, G. (2019). Training zur Förderung von Kompetenzen für die Arbeit mit Videofeedback. In: Uhde, G. \& Thies, B. (Hrsg.) (2019). Kompetenzentwicklung im Lehramtsstudium durch professionelles Training. Braunschweig: https://doi.org/10.24355/dbbs.084-201901231126-0.

Kreis, A. (2012). Produktive Unterrichtsbesprechungen. Lernen im Dialog zwischen Mentoren und angehenden Lehrpersonen. Bern: Haupt Verlag.

Kromrey, H. (2006). Empirische Sozialforschung. Modelle und Methoden der standardisierten Datenerhebung und Datenauswertung. Stuttgart: Lucius \& Lucius Verlagsgesellschaft.

Kruse, J. (2006). Kritische Theorie und Soziale Arbeit. Ein Plädoyer für eine erneute Analyse gesellschaftlicher Widersprüche aus der Perspektive der frühen Kritischen Theorie. In: Dungs, S., Gerber, U., Schmidt, H. \& Zitt, R. (2006): Ethik und Soziale Arbeit im 21. Jahrhundert. Leipzig: Evangelisches Verlagshaus Leipzig.

Kruse, P. (2004). Next practice. Erfolgreiches Management von Instabilität. Offenbach: Gabal Verlag.

Kuckartz, U. $\left(2010^{3}\right)$. Einführung in die computergestützte Analyse qualitativer Daten. Wiesbaden: Verlag für Sozialwissenschaften.

Künsting, J., Neuber, V. \& Lipowsky, F. (2016). Teacher self-efficacy as a long-term predictor of instructional quality in the classroom. In: European Journal of Psychology of Education. 31 (3). 299-322.

Kunter, M., Klusmann, U. \& Baumert, J. (2009). Professionelle Kompetenz von Mathematik-Lehrkräften. In: ZlatkinTroitschanskaia, O., Beck, K., Sembill, D., Nickolaus, R. \& Mulder, R. (2009). Lehrprofessionalität: Bedingungen, Genese, Wirkungen und ihre Messung. Weinheim, Basel: Beltz Verlag. 153-165.

Kunter, M., Baumert, J., Blum, W., Klusmann, U., Krauss, S. \& Neubrand, M. (2011). Die pro-fessionelle Kompetenz von Lehrkräften. Ergebnisse des Forschungsprogramms COACTIV. Münster: Waxmann Verlag.

Kunter, M., Kunina-Habenicht, O., Baumert, J. Dicke, T. Holzberger, D., Lohse-Bossenz, H., Leutner, D., SchulzeStocker, F. \& Terhart, E. (2017). Bildungswissenschaftliches Wissen und professionelle Kompetenz in der Lehramtsausbildung. Ergebnisse des Projekts BilWiss. In: Gräsel, C. \& Trempler, K. (Hrsg.) (2017). Entwicklung von Professionalität pädagogischen Personals. Interdisziplinäre Betrachtungen, Befunde und Perspektiven. Wiesbaden: Springer Verlag. 37-55.

Kunze-Pletat, D. (2019). Personzentrierte Erwachsenenpädagogik Die pädagogische Beziehung als Mittelpunkt im Lehr-Lern-Prozess. Wiesbaden: Springer Fachmedien.

Kyriakides, L. (2007). Generic and differentiated Models of Educational Effectiveness. In: Townsend, Td. (Ed.). International Handbook of School Effectiveness and Improvement. Vol 1. Dordrecht. Springer Verlag. 41-56.

Lamnek, S. (2005). Qualitative Sozialforschung. (3). Weinheim: Beltz Verlag.

Lamnek, S. \& Krell, C. (2016). Qualitative Sozialforschung. (6). Weinheim: Beltz Verlag.

Langenkamp, I. (2018). Stärkung des kindlichen Selbstkonzepts. Psychologie in Bildung und Erziehung: Vom Wissen zum Handeln. Wiesbaden: Springer Fachmedien.

Lankau, M. \& Scandura, T. (2007). Mentoring as a forum for personal learning in organizations. In: Ragins, B.R. \& Kram, K.E. (2007). The Handbook of Mentoring at Work. Thousand Oaks: Sage Publications. 95-122.

Lechthaler, A. (1966). Von Lehrerbildnern, Zöglingen und Lehrern des Innsbrucker Pädagogiums und seiner Vorgänger. Zur Zweihundertjahrfeier im Jahr 1967. Innsbruck: Universitätsverlag Wagner.

Lehmann-Grube, S.K. \& Nickolaus, R. (2009). Professionalität als kognitive Disposition. In: Zlatkin-Troitschanskaia, O., Beck, K., Sembill, D., Nickolaus, R. \& Mulder, R. (2009). Lehrprofessionalität. Bedingungen, Genese, Wirkungen und ihre Messung. Weinheim, Basel: Beltz Verlag. 59-70. 
Lemmermöhle, D. \& Jahreis, D. (Hrsg.) (2003). Professionalisierung der Lehrerbildung. Perspektiven und Ansätze in internationalen Kontexten. Weinheim: Juventa Verlag.

Lenske, G., Thillmann, H., Wirth, J., Dicke, T. \& Leutner, D. (2015). Pädagogisch-psychologisches Professionswissen von Lehrkräften: Evaluation des ProwiN-Tests. In: Zeitschrift für Erziehungswissenschaft. 18 (2). 225-245.

Leonhard, T. (2018a). Das Ende von Theorie und Praxis? Versuch einer alternativen Rahmung für die Lehrerinnen und Lehrerbildung. In: Fridrich, R., Mayer-Frühwirth, G., Potzmann, R., Greller, W. \& Petz, R. (Hrsg.) (2018). Forschungsperspektiven 10. Wien: LIT Verlag. KG.

Leonhard, T. \& Herzog, S. (2018). Was Langzeitpraktika leisten (können) - empirische und konzeptionelle Erkundungen. In: Rothland, M. \& Schaper, N. (Hrsg.) (2018). Lehrerbildung auf dem Prüfstand. LbP 2018. Themenheft. Forschung zum Praxissemester in der Lehrerbildung. 11 (1). Landau: Verlag Empirische Pädagogik. 5-23.

Lipowsky. F. (2006). Auf den Lehrer kommt es an. Empirische Evidenzen für Zusammenhänge zwischen Lehrerkompetenzen, Lehrerhandeln und dem Lernen der Schüler. In: Zeitschrift für Pädagogik. 51. Weinheim: Beltz Verlag. 47-70.

Lüders, Ch. (2011). Gütekritieren. In: Bohnsack, R., Marotzki, W. \& Meuser, M. (Hrsg.) (2011). Hauptbegriffe Qualitativer Sozialforschung. Opladen \& Farmington Hills: Verlag Barbara Budrich. 80-82.

Lunkenbein, M. (2012). Beobachtungen in schulpraktischen Studien: Bamberg: University of Bamberg Press.

Lux, M. (2014). Neurowissenschaftliche Grundlagen Personzentrierter Psychotherapie. In: Person (2014). Internationale Zeitschrift für Personzentrierte und Experienzielle Psychotherapie und Beratung. (2). Wien: Facultas Verlag. $176-186$

Maag Merki, K. \& Werner, S. (2011). Erfassung und Bewertung professioneller Kompetenz von Lehrpersonen. In: Terhart, E., Bennewitz, H. \& Rothland, M. (Hrsg.) (2011). Handbuch der Forschung zum Lehrerberuf. Münster: Waxmann Verlag. 573-591.

Maag-Schwarzbäcker, M. (2014). Mentoring für Frauen an Hochschulen. Die Organisation informellen Wissenstransfers. Wiesbaden: Springer Verlag.

Mantle-Bromley, C. (2003). The status of early theories of professional development school potential. In: Guardarrama, I.N., Ramsey, J. \& Nath, J.L. (Eds.) (2003). Forging alliances in community and thought. Research in professional development schools. Greenwich: Information Age. 3-30.

Maslow, A.H. (1943). A theory of human motivation. Psychological Review, 50 (4). 370-396.

Masschelein, J. \& Quaghebeur, K. (2006). Participation making a difference? Critical analysis of the participatory claims of change, reversal, and empowerment. Interchange. 37 (4).309-331.

MAXQDA. (2013). MAXQDA 11. The Art of Data Analysis: Referenzhandbuch. Retrieved from: http://www.maxqda.de/download/manuals/MAX11_manual_ger.pdf. (2017-07-13).

MAXQDA. (2015). The Art of Data Analysis Retrieved from: http://www.maxqda.de (2017-07-13).

Maynard, T. (2000). Learning to teach or learning to manage mentors? Experiences of school-based teacher training. Mentoring and Tutoring. 8 (1). 17-30.

Mayring, Ph. $\left(2016^{6}\right)$. Einführung in die qualitative Sozialforschung. Eine Anleitung zu qualitativem Denken. Weinheim, Basel: Beltz Verlag.

McDonnell, L.M. (1995). Opportunity to learn as a research concept and a policy instrument. Educational Evaluation and Policy Analysis. 17.305-322.

Mc Kenzie, J. (1999). Scaffolding for Success. In: FNO - The Educational Technology Journal. Band 9 (4).

Meixner, J. \& Müller, K. (2004). Konstruktivismus in der Praxis: Pädagogik und Erwachsenenbildung. In: Ameln, F.v. (Hrsg.) (2004). Konstruktivismus: die Grundlagen systemischer Therapie, Beratung und Bildungsarbeit. Tübingen: Francke Verlag. 245-268.

Mertens, S. \& Schlag, S. (2018). Die Bedeutung der Berufswahlmotivation, Selbstregulation und Kompetenzselbsteinschätzung für das bildungswissenschaftliche Professionswissen und die Unterrichtswahrnehmung angehender Lehrkräfte zu Beginn und am Ende des Praxissemesters. In: Rothland, M. \& Schaper, N. (2018). Lehrerbildung auf dem Prüfstand. 11 (1). Landau: Verlag Empirische Pädagogik. 66-84.

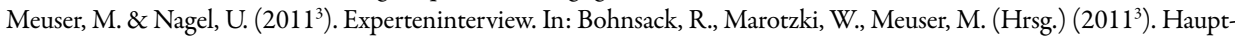
begriffe Qualitativer Sozialforschung. Regensburg: Verlag Barbara Budrich. Opladen \& Farmington Hills. 57-58.

Meyer, H. (2004). Was ist guter Unterricht? Berlin: Cornelson Scriptor Verlag.

Meyer, M. (2012). Kultur, Kompetenz und Lehrerbildung aus der Perspektive der Bildungsgangforschung. In: Kraler, Ch., Schnabel-Schüle, H., Schratz, M. \& Weyland, B. (Hrsg.) (2012). Kulturen der Lehrerbildung. Professionalisierung eines Berufsstands im Wandel. Münster: Waxmann Verlag. 155-175.

Mieg, H. \& Pfadenhauer, M. (Hrsg.) (2003). Professionelle Leistung - Professional Performance. Konstanz: UVK.

Mieg, H. (2016). Profession: Begriff, Merkmale, gesellschaftliche Bedeutung. In: Dick, M., Marotzki, W. \& Mieg, H. (2016). Handbuch Professionsentwicklung. Bad Heilbrunn: Klinkhardt Verlag. 27-40.

Misoch, S. (2015). Qualitative Interviews. Berlin, München, Boston: de Gruyter Oldenbourg Verlag. 
Moor, H., Halsey, K., Jones, M., Martin, K., Stott, A., Brown, C. \& Harland, J. (2005). Professional development for teachers early in their careers: an evaluation of the early professional development pilot scheme. Nottingham: Department for Education an Skills.

Müller, K. (2010). Das Praxisjahr in der Lehrerbildung. Empirische Befunde zur Wirksamkeit studienintegrierter Langzeitpraktika. Bad Heilbrunn: Klinkhardt Verlag.

Neuweg, G.H. (2004). Figuren der Relationierung von Lehrerwissen und Lehrerkönnen. In: Hackl, B. \& Neuweg, G.H. (Hrsg.) (2004). Zur Professionalisierung pädagogischen Handelns. Münster: LIT Verlag. 1-26.

Neuweg, G.H. (2011). Das Wissen der Wissensvermittler. Problemstellungen, Befunde und Perspektiven der Forschung zum Lehrerwissen. In: Terhart, E., Bennewitz, H. \& Rothland, M. (Hrsg.) (2011). Handbuch der Forschung zum Lehrerberuf. Münster: Waxmann Verlag. 451-477.

Neuweg, G.H. (2011). Distanz und Einlassung. Skeptische Anmerkungen zum Ideal einer Theorie-Praxis-Integration in der Lehrerbildung. Erziehungswissenschaft. Mitteilungen der Deutschen Gesellschaft für Erziehungswissenschaft. $22(43) .33-45$.

Niehoff, B.P. (2006). Personality predictors of participation as a mentor. Career Development International. 11 (4). 321-333.

Nienhaus, D., Pannatier, G. \& Töngi, C. (Hrsg.) (2005). Akademische Seilschaften. Mentoring für Frauen im Spannungsfeld individueller Förderung und Strukturveränderung. Bern, Wettingen: eFef-Verlag.

Niggli, A. (2005). Unterrichtsbesprechungen im Mentoring. Oberentfelden: Sauerländer.

Nittel, D. (2000). Von der Mission zur Profession? Stand und Perspektiven der Verberuflichung in der Erwachsenenbildung. Bielefeld: W. Bertelsmann Verlag.

Nolle, T. (2012). Psychosoziale Basiskompetenzen und Lernorientierung in der Eingangsphase des Lehramtsstudiums. Ergebnisse einer Evaluationsstudie. In: Bosse, D., Dauber, H., Döring-Seipel, E. \& Nolle, T. (Hrsg.) (2012). Professionelle Lehrerbildung im Spannungsfeld von Eignung, Ausbildung und beruflicher Kompetenz. Bad Heilbrunn: Klinkhardt Verlag. 67-80.

Norman, D.M. \& Ganser, T. (2004). A Humanistic Approach to New Teacher Mentoring. Journal of Humanistic Counseling, Education and Development 43. 129-140.

Norman, P.J. \& Feiman-Nemser, S. (2005). Mind activity in teaching and mentoring. Teaching and Teacher Education. 21. 679-697.

OECD (2010). Educating Teachers for Diversity: Meeting the Challenge. Paris: OECD Publishing.

Oehmann, K. \& Blumschein, P. (2019). Schluss mit der Donut-Pädagogik. Lebensnahe Lernaufgaben leicht gemacht. Bern: Hep-Verlag.

Oerter, R. \& Dreher, E. (2002). Jugendalter. (7). In: Oerter, R. \& Montada, L. (Hrsg.) (2002). Entwicklungspsychologie. Weinheim: Beltz Verlag. 258-318.

Oettler, J. (2009). Rolle und Selbstverständnis der Mentoren in den Schulpraktischen Studien. Entwicklung einer Qualifizierungsmaßnahme zur wissenschaftlichen Begleitung von Lehramtsstudenten. Norderstedt: Books on Demand.

Olian, J.D., Carroll, S.J., Giannantonia, C.M. \& Ferren, B.D. (1988). What do proteges look for in a mentor? Results of three experimental studies. In: Journal of Vocational Behaviour. 33. 15-37.

Ortenburger, A. (2016). Lehrer und Lehrerinnen. In: Dick, M., Marotzki W. \& Mieg, H. (Hrsg.) (2016). Handbuch Professionsentwicklung. Bad Heilbrunn: Klinkhardt Verlag. 559-566.

Ostermann, E. (2015). LehrerIn werden im Spannungsfeld subjektiver Erwartungen und objektiver Ausbildungsanforderungen. Bad Heilbrunn: Klinkhardt Verlag.

Ostermann, E. (2017). Pädagogisch-Praktische Studien im Spannungsverhältnis von wissenschaftlicher Ausbildung und Praxiserprobung. Entwurf eines Konzeptes für das Bachelor-Lehramtsstudium im Bereich der Primarstufe. Forschungsperspektiven 9. Wien: LIT Verlag. 51-68.

QSR Qualitätssicherungsrat (2014). Basisinformationen zu Pädagoginnen- und Pädagogenbildung NEU. Wien: 2014. Abgerufen unter: https://www.qsr.or.at/dokumente/1863-20140729-100721-AllgInfo_PaedBildung_Primarstufe_201406.pdf(2018-12-27).

Parlament Österreich (2018). 94. Bundesgesetz: Akademien-Studiengesetz 1999 - AStG. Abgerufen unter: https:// www.ris.bka.gv.at/Dokument.wxe?Abfrage=BgblPdf\&Dokument nummer=1999_94_1 (2018-12-27).

Parsons, T. (1939). The Professions and Social Structure. In: Social Forces 17. 457-467.

Parsons, T. (1968). Professions. In: Sills, D.L. (Ed.). International Encyclopaedia of the Social Science. Number 12. 536-547.

Patry, J.-L. (2014). Theoretische Grundlagen des Theorie-Praxis-Problems in der Lehrer/innenbildung. In: Arnold, K.H., Gröschner, A. \& Hascher, T. (Hrsg.) (2014). Schulpraktika in der Lehrerbildung. Theoretische Grundlagen, Konzeptionen, Prozesse und Effekte. Münster, New York: Waxmann Verlag. 
Patton, M. Q. (2002). Qualitative Evaluation and research Methods. Newbury Park: Sage.

Pelz, W. (2016). Transformationale Führung - Forschungsstand und Umsetzung in der Praxis. In: Au von, C. (Hrsg.) (2016). Wirksame und nachhaltige Führungsansätze. Leadership und Angewandte Psychologie. Wiesbaden: Springer Fachmedien. 93-112.

Perkhofer-Czapek, M. \& Potzmann, R. (2016). Begleiten, Beraten und Coachen. Der Lehrberuf im Wandel. Wiesbaden: Springer Fachmedien.

Pfadenhauer, M. (2003). Macht - Funktion - Leistung: Zur Korrespondenz von Eliten- und Professionstheorien. In: Mieg, H. \& Pfadenhauer, M. (Hrsg.) (2003). Professionelle Leistungen - Professional Performance. Konstanz: UVK. 71-87.

Pfitzner-Eden, F. (2016). I feel less confident so I quit? Do true changes in teacher self-efficacy predict changes in preservice teachers' intention to quit their teaching degree? Teaching an Teacher Education. 55. 240-254.

Pflaum, S. (2017). Mentoring beim Übergang vom Studium in den Beruf. Eine empirische Studie zu Erfolgsfaktoren und wahrgenommenem Nutzen. Wiesbaden: Springer Verlag.

Pflaum, S. \& Wüst, L. (2019). Der Mentoring Kompass für Unternehmen und Mentoren. Persönliche Erfahrungsberichte, Erfolgsprinzipien aus Forschung und Praxis. Wiesbaden: Springer Medienverlag.

Pinnow, D.F. (2005). Führen - Worauf es wirklich ankommt. Wiesbaden: Springer Gabler Verlag.

PISA (2012). Abgerufen unter: https://www.oecd.org/berlin/themen/PISA-2012-Zusammenfassung.pdf. (2019-12$30)$.

PISA (2015). Abgerufen unter: https://www.oecd.org/berlin/themen/pisa-studie/PISA_2015_Zusammenfassung. pdf. (2019-12-30).

Podell, S. \& Soodak, L. (1993). Teacher efficacy and bias in special education referrals. In: Journal of Educational Research. 86 (4). 247-253.

Preyer, G. (2012). Rolle, Status, Erwartungen und soziale Gruppe. Mitgliedschaftstheoretische Reinterpretation. Wiesbaden: Verlag für Sozialwissenschaften.

Prisching, M. (2008). Bildungsideologien. Ein zeitdiagnostischer Essay an der Schwelle der Wissensgesellschaften. Wiesbaden: Verlag für Sozialwissenschaften.

Przyborski, A. \& Wohlrab-Sahr, M. (2014). Qualitative Sozialforschung. Ein Arbeitsbuch. München: Oldenbourg Verlag.

Raggins, B.R. \& Kram, K.E. (2007a). The Roots and Meaning of Mentoring. In: Raggins, B.R. \& Kram, K.E. (2007). The Handbook of Mentoring at Work. Los Angeles: Sage Publ. 3-15.

Rahm, S. \& Lunkenbein, M. (2014). Anbahnung von Reflexivität im Praktikum. Empirische Befunde zur Wirkung von Beobachtungsaufgaben im Grundschulpraktikum. In: Arnold, K.-H., Gröschner, A. \& Hascher, T. (Hrsg.) (2014). Schulpraktika in der Lehrerbildung. Theoretische Grundlagen, Konzeptionen, Prozesse und Effekte. Münster, New York: Waxmann Verlag. 237-256.

Reh, S. (2004). Abschied von der Profession, von Professionalität oder vom Professionellen? Theorien und Forschung zur Lehrerprofessionalität. In: Zeitschrift für Pädagogik. 50 (3). Weinheim: Beltz Verlag. 358-372.

Reintjes, Ch. (2007). Erziehungswissenschaft - ein notwendiger Bestandteil der gymnasialen Lehrerausbildung? Münster: LIT Verlag.

Reintjes, Ch., Bäuerlein, K. \& Bellenberg, G. (2018). Professionalisierung in der einphasigen Waldorf-Lehrpersonenausbildung. Lerngelegenheiten im Schulfeld in Abhängigkeit von personalen Merkmalen der Mentorinnen und Mentoren. In: Reintjes, Ch., Bellenberg, G. \& Brahm im, G. (2018). Mentoring und Coaching als Beitrag zur Professionalisierung angehender Lehrpersonen. Münster, New York: Waxmann Verlag. 161-187.

Reinisch, H. (2009). „Lehrprofessionalität“ als theoretischer Term - Eine begriffssystematische Analyse. In: ZlatkinTroitschanskaia, O., Beck, K., Sembill, D., Nickolaus, R. \& Mulder, R. (2009). Lehrprofessionalität: Bedingungen, Genese, Wirkungen und ihre Messung. Weinheim, Basel: Beltz Verlag.

Rhodes, J.E. (2002). Stand by me: The risks and rewards of mentoring today's youth. Cambridge. MA: Harvard University Press.

Richert, V. (2006). Mentoring und lebenslanges Lernen: Individuelles Wissensmanagement im Informationszeitalter. Saarbrücken: VDM.

Richter, D. (2011). Lernen im Beruf. In: Kunter, M., Baumert, J., Blum, W. \& Neubrand, N. (Eds.) (2011). Professionelle Kompetenz von Lehrkräften. Ergebnisse des Forschungsprogramms COACTIV. Münster: Waxmann Verlag. $318-325$.

RIS (2016). Abgerufen unter: https://www.ris.bka.gv.at/Dokument.wxe?Abfrage= Bundesnormen\&Dokumentnum mer=NOR40153062 (27-06-2016).

Rogers, C.R. \& Schmid, P.F. (1991). Person-zentriert. Grundlagen von Theorie und Praxis. Mit einem kommentierten Beratungsgespräch von Carl Rogers. Mainz: Matthias-Grünewald-Verlag. 
Rogers, C.R. (1990/2007). Auf dem Wege zu einer Theorie der Kreativität. In: Petzold, H. \& Orth, I. (Hrsg.) (2007). Die neuen Kreativitätstherapien. Handbuch der Kunsttherapie. Theorie und Praxis. Band I. (4). Bielefeld: Lucius \& Lucius Verlagsgesellschaft. 237-255.

Rogers, C.R. (2009). Eine Theorie der Psychotherapie. München: Ernst Reinhardt Verlag.

Röhrle, B. (1994). Soziale Netzwerke und soziale Unterstützung. Weinheim: Beltz Verlag.

Ronfeldt, M., Farmer, S., McQueen, K. \& Grissom, J. (2015). Teacher collaboration in instructional teams and student achievement. American Educational Research Journal, 52. (3). 475-514.

Rosenstiel von, L. (2014). Grundlagen der Führung. In: Rosenstiel von, L., Regnet, E. \& Domsch, M. (Hrsg.) (2014). Führung von Mitarbeitern. Stuttgart: Schäffer-Poeschl Verlag. 3-28.

Rosenthal, G. (2005). Interpretative Sozialforschung. Eine Einführung. Weinheim, München: Juventa Verlag.

Rothland, M. (2009). Das Dilemma des Lehrerberufs sind ... die Lehrer? Anmerkungen zur persönlichkeitspsychologisch dominierten Lehrerbelastungsforschung. In: Zeitschrift der Erziehungswissenschaft. 13. 111-125.

Rothland, M. \& Terhart, E. (2010). Forschung zum Lehrerberuf. In: Tippelt, R. \& Schmidt, B. (Hrsg.) (2010). Handbuch Bildungsforschung. Wiesbaden: Verlag für Sozialwissenschaften.

Rothland, M. (2013). Beruf: Lehrer/Lehrer-Arbeitsplatz: Schule. Charakteristika der Arbeitstätigkeit und Bedingungen der Berufssituation. In: Rothland, M. (Hrsg.) (2013). Belastung und Beanspruchung im Lehrerberuf. Modelle, Befunde, Interventionen. (2). Wiesbaden: Springer Verlag. 21-39.

Rothland, M. \& Schaper, N. (Hrsg.) (2018). Lehrerbildung auf dem Prüfstand. LbP 2018. Themenheft. Forschung zum Praxissemester in der Lehrerbildung. 11 (1). Landau: Verlag Empirische Pädagogik.

Ryle, G. (1970). Begriffskonflikte. Göttingen: Vandenhoeck \& Ruprecht Verlag.

Sachs, J. (2003). Teacher Professional Standards: controlling or developing teaching? Teachers and Teaching: Theory and practice. 9. 2. Taylor and Francis Ltd.

Sader, M. (1969). Rollentheorie. In: Graumann, C.F. (Hrsg.) (1969). Handbuch der Psychologie. Band 7. Sozialpsychologie. Göttingen: Hogrefe Verlag. 204-231.

Sander, E. (2009). Beschreibung und Evaluationsergebnisse des Ada-Lovelace-Projekts. In: Stöger, H., Ziegler, A. \& Schminke, D. (Eds.) (2009). Mentoring. Theoretische Hintergründe, empirische Befunde und praktische Anwendungen. Lengerich: Pabst. 55-75.

Sandfuchs, U. (2004). Geschichte der Lehrerbildung in Deutschland. In: Blömeke, S., Reinhold, P. \& Tulodziecki, R. (Hrsg.) (2004). Handbuch Lehrerbildung. Bad Heilbrunn: Klinkhardt Verlag. 14-37.

Schatzmann, L. (1991). Dimensional Analysis: Notes on an Alternative Approach to the Grounding of Theory in Qualitative Research. In: Maines, D.R. (Hrsg.) (1991). Social Organizations and Social Processes. Essays in Honour of Anselm Strauss. New York: Aldine de Gruyter. 303-314.

Scheerens, J. (2017). Conceptualization. In: Scheerens, J. (ed.) (2017). Opportunity to Learn. Curriculum Alignment and Test Preparation. Springer International Publishing. 7-22.

Schenk, B. (2005). Bausteine einer Bildungsgangtheorie. Wiesbaden: Verlag für Sozialwissenschaften.

Schenk, B. (2012). Bausteine einer Bildungsgangtheorie. Wiesbaden: Verlag für Sozialwissenschaften.

Schiefele, U., Streblow, L. Ermgassen, U. \& Moschner, B. (2003). Lernmotivation und Lernstrategien als Bedingungen der Studienleistung. In: Zeitschrift für Pädagogische Psychologie. 17 (3/4). Göttingen: Hogrefe Verlag. 185-198.

Schley, W., Boban, I. \& Hinz, A. (1989). Integrationsklassen in Hamburger Gesamtschulen. Hamburg: Curio-Verlag Erziehung und Wissenschaft.

Schley, W. \& Schratz, M. (2011). Developing Leaders, Building Networks, Changing Schools Through System Leadership. In: Townsend, T. \& MacBeath, J. (Hrsg.) (2011). International Handbook of Leadership for Learning. 267295.

Schmid, B. \& Haasen, N. (2011). Einführung in das systemische Mentoring. Heidelberg: Auer Verlag.

Schmitz, G.S. \& Schwarzer, R. (2002). Individuelle und kollektive Selbstwirksamkeitserwartung von Lehrern. In: Zeitschrift für Pädagogik. 44. Weinheim: Beltz Verlag. 192-214.

Schnautz, B.M. (2014). The effects of school-based mentoring on student achievement for junior high school students. Submitted to the Office of Graduate and Professional Studies of Texas A\&M University.

Schnebel, S. (2018). Welche Qualifizierung brauchen Praxislehrpersonen? Überlegungen, Kenntnisse und Kompetenzen. In: journal für lehrerInnenbildung. 18 (4). Wien: Facultas Verlags- und Buchhandels AG. 22-27.

Schnebel, S. (2019). Lernbegleitung im schulischen Unterricht und in Praxisphasen der Lehrerausbildung. Studien zur Gestaltung unterschiedlicher Formen von Lernbegleitung durch Lehrpersonen. Bad Heilbrunn: Klinkhardt Verlag.

Schneider, P.B. \& Blickle, G. (2009). Mentor-Protegé-Beziehungen in Organisationen. In: Stöger, H., Ziegler, A. \& Schminke, D. (Hrsg.) (2009). Mentoring. Theoretische Hintergründe, empirische Befunde und praktische Anwendungen. Lengerich: Pabst. 139-160.

Schön, D. A. (1983). The reflective practitioner. How professionals think in action. New York NY: Basic Books. 
Schubarth, W., Speck, K., Seidel, A., Gottmann, C., Kamm, C., Kleinfeld, M. \& Krohn, M. (2012). Kompetenzentwicklung im Praxissemester: Ergebnisse einer Längsschnittanalyse zum „Potsdamer Modell der Lehrerbildung“. In: Hascher, T. \& Neuweg, G.H. (Hrsg.) (2012). Forschung zur (Wirksamkeit der) Lehrer/innen/bildung. Wien: LIT Verlag. 201-220.

Schubarth, W., Gottmann, C., \& Krohn, M. (2014). Wahrgenommene Kompetenzentwicklung im Praxissemester und dessen berufsorientierende Wirkung: Ergebnisse der ProPrax-Studie. In: Arnold, K.-H., Gröschner, A. \& Hascher, T. (Eds.) (2014). Schulpraktika in der Lehrerbildung. Theoretische Grundlagen, Konzeptionen, Prozesse und Effekte. Münster: Waxmann Verlag. 201-220.

Schulte, K., Bögenholz, S. \& Watermann, R. (2008). Selbstwirksamkeitserwartungen und Pädagogisches Professionswissen im Verlauf des Lehramtsstudiums. In: Zeitschrift für Erziehungswissenschaft. 11 (2). 268-287.

Schüßler, I. $\left(2012^{3}\right)$. Nachhaltigkeit in der Weiterbildung. Baltmannsweiler: Schneider Hohengehren.

Schüssler, R., Schönring, A., Schwier, V., Schicht, S., Gold, J., \& Weyland, U. (Hrsg.) (2017). Forschendes Lernen im Praxissemester. Zugänge, Erfahrungen, Konzepte. Bad Heilbrunn: Klinkhardt Verlag.

Schützenmeister, J. (2008). Professionalisierung und Polyvalenz in der Lehrerausbildung. Marburg: Tectum.

Schuy, R. (2019). https://clevermemo.com/blog/grow-modell/(2019-05-26).

Schwänke, U. (1988). Der Beruf des Lehrers. Professionalisierung und Autonomie im historischen Prozess. Weinheim: Juventa Verlag.

Schwarzer, R. \& Jerusalem, M. (2002). Das Konzept der Selbstwirksamkeit. In: Jerusalem, M. \& Hopf, D. (Hrsg.) (2002). Selbstwirksamkeit und Motivationsprozesse in Bildungsinstitutionen. Weinheim: Beltz Verlag. 28-53.

Schweer, M. (2004). Vertrauen und soziale Unterstützung in der pädagogischen Beziehung. Bildung und Erziehung. 57 (3). 279-288.

Seel, H. (2010). Einführung in die Schulgeschichte Österreichs. Innsbruck: Studienverlag.

Seidel, T. (2015). Klassenführung. In: Wild, E. \& Möller, J. (Hrsg.) (2015). Pädagogische Psychologie. Berlin: Springer Verlag. 107-119.

Seifert, A., Hilligus, A.H. \& Schaper, N. (2009). Entwicklung und psychometrische Überprüfung eines Messinstrumentes zur Erfassung pädagogischer Kompetenzen in der universitären Lehrerbildung. Lehrerbildung auf dem Prüfstand. 2 (1). 82-103.

Seifried, J. \& Ziegler, B. (2009). Domänenbezogene Professionalität. In: Zlatkin-Troitschanskaia, O., Beck, K., Sembill, D., Nickolaus, R. \& Mulder, R. (2009). Lehrpro-fessionalität: Bedingungen, Genese, Wirkungen und ihre Messung. Weinheim, Basel: Beltz Verlag. 83-92.

Shavelson, R.J., Hubner, J.J. \& Stanton, G.C. (1976). Self-concept: Validation of construct of interpretations. Review of Educational Research. 46. 407-411.

Shulman, L.S. (1986). Those Who Understand. Knowledge Growth in Teaching. In: Educational Researcher 15 (2). 4-14.

Shulman, L.S. (1987). Knowledge and teaching. Foundations of the new reform. In: Harvard Educational Review 57 (1). $1-22$.

Simpson, T., Hastings, W.W. \& Hill, B. (2007). I knew that she was watching me: the professional benefits of mentoring. Teachers and Teaching: Theory and Practice. 13 (5). 481-498.

Smith, P.J. (2003). Workplace learning and flexible delivery. In: Review of Educational Research. 73 (1). 53-58.

Stadelmann, M. (2006). Differenz oder Vermittlung in der Lehrerbildung? Das Verhältnis von Theorie und Praxis im Urteil von Praktikumslehrpersonen der Primar- und Sekundarstufe I. Bern: Haupt Verlag.

Standop, J. \& Jürgens, E. (2015). Unterricht planen, gestalten und evaluieren. Bad Heilbrunn: Klinkhardt Verlag.

Stangl, W. (2019). Lernen. Abgerufen unter: https://psychologie.stangl.eu/definition/Lernen. shtml (2019-08-13).

Statistik Austria (2018). Übertritte von der Primarstufe in die Sekundarstufe I im Schuljahr 2017/18 Abgerufen unter: file://C:/Users/ELISAB 1/AppData/Local/Temp/uebertritte_von_der_primarstufe_in_die_sekundarstufe_i_ im_schuljahr_201718.pdf(2018-12-04).

Statistik Austria (2018). Prozentueller Anteil der Schultypen in der 5. Schulstufe von 1980 bis 2018. Abgerufen unter: https://www.statistik.at/web_de/statistiken/menschen_und_gesellschaft/bildung_und_kultur/formales_bildungswesen/schulen_schulbesuch/index.html (2018-12-27).

Statistik Austria (2018). Verteilung der Schülerinnen und Schüler in der 9. Schulstufe 2017/18 - Vergleich mit den Jahren 1980/81, 1990/91,2000/01 und 2010/11 bis 2016/17. Abgerufen unter: https://www.statistik.at/web_de/ statistiken/menschen_und_gesellschaft/bildung_und_kultur/formales_bildungswesen/schulen_schulbesuch/index.html (2018-12-27).

Statistik Austria (2018). Abgerufen unter: https://www.studiversum.at/fileadmin/user_upload/Studierenden-Sozialerhebung/1_Studierenden_Sozialerhebung_2015_Band1_AnfaengerInnen.pdf(2018-12-04).

Steinke, I. (2013). Gütekriterien qualitativer Forschung. In: Flick, U., Kardorff, E.v. \& Steinke, I. (Hrsg.) (2013). Qualitative Forschung. Ein Handbuch. Reinbek: Rowohlt Verlag. 319-331.

Stöger, H., Ziegler, A. \& Schminke, D. (Eds.) (2009). Mentoring. Theoretische Hintergründe, empirische Befunde und praktische Anwendungen. Lengerich: Pabst. 
Strauss, A. (1991). Grundlagen qualitativer Sozialforschung. Datenanalyse und Theoriebildung in der empirischen soziologischen Forschung. München: Wilhelm Fink Verlag.

Strauss, A. \& Corbin, B. (1996). Grounded Theory: Grundlagen Qualitativer Sozialforschung. Weinheim: Beltz Verlag. Psychologie Verlags Union.

Strübing, J. $\left(2014^{3}\right)$. Grounded Theory: Zur sozialtheoretischen und epistemologischen Fundierung eines pragmatistischen Forschungsstils. Wiesbaden: Springer Verlag.

Tashakkori, A. \& Teddlie, Ch. (Hrsg.) (2003a). Handbook of Mixed Methods in Social \& Behavioral Research. Thousand Oaks. CA.: Sage.

Teichholz, J.G. (2007). Eine unerwartete Annäherung - Postmoderne Therapie, Säuglingsforschung und das psychoanalytische Unbewusste. Selbstpsychologie. 8 (29/30). 263-285.

Teml, H. (2002). Von der « Schulpraktischen Ausbildung“ zu den „Schulpraktischen Studien“. In: Klement, K., Lobendanz, A. \& Teml, H. (Hrsg.) (2002). Schulpraktische Studien. Beiträge zur Qualitätsentwicklung in der Lehrerbildung unter Berücksichtigung europäischer Perspektiven. Innsbruck: Studienverlag. 9-32.

Teml, H. \& Teml, H. (2011). Praxisberatung. Coaching und Mentoring in pädagogischen Ausbildungsfeldern. Innsbruck: Studienverlag.

Terhart, E. (1991). Pädagogisches Wissen. Überlegungen zu seiner Vielfalt, Funktion und sprachlichen Form am Beispiel des Lehrerwissens. In: Oelkers, J., Tenorth, H.-E. (Hrsg.) (1991). Pädagogisches Wissen. In: Zeitschrift für Pädagogik. 27. Heft. Weinheim, Basel: Beltz Verlag. 129-141.

Terhart, E. (1993). Lehrerwissen: Aufbau, Genese, Funktion. In: Forum Lehrerfortbildung. Heft 24-25. 94-101.

Terhart, E. (1993). Pädagogisches Wissen. Überlegungen zu einer Vielfalt, Funktion und sprachlichen Form am Beispiel des Lehrerwissens. In: Oelkers, J. \& Tenorth, H.-E. (Hrsg.) (1993). Pädagogisches Wissen. Weinheim: Beltz Verlag. 129-141.

Terhart, E. (2001). Lehrerberuf und Lehrerbildung. Forschungsbefunde, Problemanalysen, Reformkonzepte. Weinheim: Beltz Verlag.

Terhart, E. (2007). Strukturprobleme der Lehrerausbildung in Deutschland. In: Ohidy, A., Terhart, E. \& Zsonai, J. (Hrsg.) (2007). Lehrerbild und Lehrerbildung. Praxis und Perspektiven der Lehrerausbildung in Deutschland und Ungarn. Wiesbaden: Verlag für Sozialwissenschaften. 45-65.

Terhart, E. (2009). Didaktik. Eine Einführung. Stuttgart: Reclam jun.

Terhart, E. (2010). Personalauswahl, Personaleinsatz und Personalentwicklung an Schulen. In: Altrichter, H. \& Maag Merki, K. (Hrsg.) (2010). Neue Steuerung im Schulsystem - Ein Handbuch. Wiesbaden: Verlag der Sozialwissenschaften. 255-276.

Terhart, E., Bennewitz, H. \& Rothland, M. (2011). Handbuch der Forschung zum Lehrerberuf. Münster: Waxmann Verlag.

Terhart, E. (2012). Wie wirkt Lehrerbildung? In: Zeitschrift für Bildungsforschung 2. Salzburg: ÖFEB. Springer Verlag. 2-23.

Terhart, E., Schulze, F., Kunina-Habenicht, O., Dicke, T., Förster, D., Lohse-Bossenz, H. \& Leutner, D. (2012). Bildungswissenschaftliches Wissen und der Erwerb professioneller Kompetenz in der Lehramtsausbildung - Eine Kurzdarstellung des BilWiss-Projekts. Lehrerbildung auf dem Prüfstand. 5 (1). 96-106.

Terhart, E. (2013). Erziehungswissenschaft und Lehrerbildung. Münster: Waxmann Verlag.

Timperley, H. (2001). Mentoring conversations designed to promote student teacher learning. Asia-Pacific Journal of Teacher Education. 29 (2). 111-123.

Topsch, W. (2004b). Schulpraxis in der Lehrerbildung. In: Blömeke, S., Reinhold, P., Tulodziecki, G. \& Wildt, J. (Hrsg.) (2004). Handbuch Lehrerbildung. Bad Heilbrunn: Klinkhardt Verlag. 476-486.

Trapp, E.C. (1780). Versuch einer Pädagogik. Berlin: Nicolai Verlag.

Trapp, E.C. (1977). Versuch einer Pädagogik. In: Herrmann, U. (Hrsg.) (1977). Schöninghs Sammlung Pädagogischer Schriften. Quellen zur Geschichte der Pädagogik. Neuauflage. Paderborn: Schöningh Verlag.

Trautmann, M. (Hrsg.) (2004). Entwicklungsaufgaben im Bildungsgang. Wiesbaden: Verlag für Sozialwissenschaften.

Trojer, P. (2018). Wer wird Lehrer/Lehrerin? Konzepte der Berufswahl und Befunde zur Entwicklung des Berufswunsches Lehrer/in und ihre Bedeutung für das Studium. Bad Heilbrunn: Klinkhardt Verlag.

Turban, D.B. \& Lee, F.K. (2007). The Role of Personality in Mentoring Relationships: Formation, Dynamics, and Outcomes. In: Ragins, B.R. \& Kram, K.E. (Hrsg.) (2007). The Handbook of Mentoring at Work. Theory, Research, and Practice. London: Sage Publications. 21-50.

Ullrich, C.G. (1999). Deutungsmusteranalyse und diskursives Interview. In: Zeitschrift für Soziologie, 28. Bielefeld: Lucius \& Lucius Verlagsgesellschaft. 429-447.

United Nations Educational, Scientific and Cultural Organization (UNESCO) (1968). Recommendation concerning the status of teachers. Adopted by the special intergovernmental conference on the status of teachers. Paris. 5. October 1966. London: Department of Education and Science. 
Van Es, E.A. \& Sherin, M.G. (2008). Mathematics teachers'„learning to notice” in the context of a video club. Teaching and Teacher Education. 17 (7), 783-805.

Van Ophuysen, S., Behrmann, L., Bloh, B., Homt, M. \& Schmidt, J. (2017). Die universitäre Vorbereitung angehender Lehrkräfte auf Forschendes Lernen im schulischen Berufsalltag.In: Journal for educational research online. 9 (2). 276-305.

Verden, N. (1997). Rollentheorie. Ein Abriss ihrer Hauptvertreter Ralf Dahrendorf, Talcott Parsons und Erving Goffman. Norderstedt: Grin Verlag.

Voss, T. \& Kunter, M. (2011). Pädagogisch-psychologisches Wissen von Lehrkräften. In: Kunter, M., Baumert, J., Blum, W., Klusmann, U., Krauss, S. \& Neubrand, M. (Hrsg.) (2011). Professionelle Kompetenz von Lehrkräften - Ergebnisse des Forschungsprogramms COACTIV. Münster: Waxmann Verlag. 193-214. Schwerpunkt

Voss, T., Kunina-Habenicht, O., Hoehne, V. \& Kunter, M. (2015). Pädagogisches Wissen von Lehrkräften: Empirische Zugänge und Befunde. In: Zeitschrift für Erziehungswissenschaft. Wiesbaden: Springer Fachmedien.

Wang, J. \& Odell, S.J. (2002). Mentored learning to teach according to standards-based reform: a critical review. Review of Educational Research. 72 (3). 481-546.

Wang, J., Strong, M. \& Odell, S.J. (2004). Mentor-novice conversation about teaching: A comparison of two U.S. and two Chinese cases. Teacher College Record. 106 (4).775-813.

Warner, L.M. \& Schwarzer, R. (2009). Selbstwirksamkeit bei Lehrkräften. In: Zlatkin-Troitschanskaia, O., Beck, K., Sembill, D., Nickolaus, R. \& Mulder, R. (2009). Lehrprofessionalität: Bedingungen, Genese, Wirkungen und ihre Messung. Weinheim, Basel: Beltz Verlag. 629-640.

Weinert, F.E. (2001). Concepts of competence. A conceptual clarification. In: Fychen, D. \& Salganik, I. (Hrsg.) (2001). Defining and selecting key competences. Göttingen: Hogrefe \& Huber Verlag. 45-65.

Wellhöfer, P.R. (2018). Gruppendynamik und soziales Lernen. Theorie und Praxis der Arbeit mit Gruppen. München: UVK Verlag.

Wenddorf, M. (2018). Führungstechniken, Führungsstile, Führungsmethoden für junge Führungskräfte. Führungskompetenz verstehen, lernen und entwickeln. Wroclaw: Amazon Fulfillment.

Werler, T. (2014). Aktuelle Entwicklungen der Lehrerbildung in den skandinavischen Staaten. In: Döbert, H., Von Kopp, B. \& Weishaupt, H. (Hrsg.) (2014). Innovative Ansätze der Lehrer-bildung im Ausland. Münster, Berlin: Waxmann Verlag. 109-133.

Werther, S. (2016). Shared leadership. In: Au von, C. (Hrsg.) (2016). Leadership und angewandte Psychologie. Wiesbaden: Springer Verlag.

Westphal, A., Lohse-Bossenz, H., Vock, M. \& Lenske, G. (2018). Was wissen Studierende über Klassenführung? Lerngelegenheiten und Lerngewinne in Studium und Praxissemester. In: Rothland, M. \& Schaper, N. (2018). Lehrerbildung auf dem Prüfstand. 11 (1). Landau: Verlag Empirische Pädagogik. 132-151.

Wewer, K. (2009). Personalentwicklung durch Mentoring. Hamburg: Diplomica Verlag.

Weyland, U. (2010). Zur Intentionalität schulpraktischer Studien im Kontext universitärer Lehrerbildung. Paderborn: Eusl Verlag.

Weyland, B. \& Kraler, Ch. (2011). Mentorinnen und Mentoren im Spannungsfeld eigener und fremder professioneller Entwicklung. Mentorinnen- und Mentorentätigkeit - Chance zur Persönlichkeitsbildung und Professionalisierung zukünftiger Lehrerinnen und Lehrer. Universität Trier und Universität Innsbruck: Mentorentag der Universität Frankfurt am Main. (2011-10-04).

Wieser, C. (2013). Abgerufen unter: http://othes.univie.ac.at/29675/1/2013-08-10_0147204.pdf. (2019-02-03).

Wildt, J. (2005). Auf dem Weg zu einer Didaktik der Lehrerbildung? In: Beiträge der Lehrerbildung 23 (2). $183-190$.

Williams, E.A., Butt, G.W., Gray, C., Leach, S., Marr, A. \& Soares, A. (1998). Mentors' use of dialogue within a secondary initial teacher education partnership. Educational Review. 3 (50). 225-239.

Wilson, S.M., Floden, R.E. \& Ferrini-Mundi, J. (2001). Teacher Preparation Research. Current Knowledge, Gaps and Recommendations. Washington.

Wilson, S.M. \& Youngs, P. (2005). Research on Accountability Processes in Teacher Education. In: Cochran-Smith, M. \& Zeichner, K.M. (2005). Studying teacher education. The report of the AERA Panel on Research and Teacher Education. Executive summary. Mahwah: Erlbaum. 591-643.

Wisbert, R. (2006). Das Schulpraktikum und die Theorie-Praxis-Frage in der Lehrerbildung. In: Beyer, K., Wisbert, R., Plöger, W., Wasmuth, K-U. \& Anhalt, E. (2006). Schulpraktikum - Ein-führung in die theoriegeleitete Planung, Durchführung und Reflexion. Stuttgart, Baltmannsweiler: Schneider Verlag Hohengehren.

Wisniewski, B. (2016). Psychologie für die Lehrerbildung. Bad Heilbrunn, Stuttgart: Klinkhardt Verlag.

Wissenschaftsrat (2001). Empfehlungen zur künftigen Struktur der Lehrerausbildung. Berlin.

Wolff, S. $\left(2012^{9}\right.$ ). Wege ins Feld und ihre Varianten. In: Flick, U., Kardorff, E.v. \& Steinke, I. (Hrsg.) (2012 ${ }^{9}$ ): Qualitative Forschung. Ein Handbuch. Reinbek: Rowohlt. 337-353. 
Woolfolk Hoy, A. \& Burke Spero, R. (2005). Changes in teacher efficacy during the early years of teaching. A comparison of four measures. Teaching and Teacher Education. 21 (4). 343-356.

Yankov, A. (2009). Auswertung nach den Regeln der Grounded Theory. Norderstedt: Grin Verlag.

Zee, M. \& Koomen, H.M.Y. (2016). Teacher self-efficacy and its effects on classroom processes. student academic adjustment, and teacher well-being. Review of Educational Research. 86 (4). 981-1015.

Zeichner, K. \& Liston, D. (1987). Teaching student teachers to reflect. Harvard Educational Review. 57 (1). 23-49.

Zeichner, K.M. (2010). Rethinking the connections between campus courses and field experiences in college- and university-based teacher education. Journal of Teacher Education. 61. 89-99.

Zeit online: Abgerufen unter: https://blog.zeit.de/schueler/2015/10/22/sokrates-philosophie-der-antike/(2019-0415).

Ziegler, A. (2009). Mentoring: Konzeptuelle Grundlagen und Wirksamkeitsanalyse. In: Stöger, H., Ziegler, A. \& Schminke, D. (Eds.) (2009). Mentoring. Theoretische Hintergründe, empirische Befunde und praktische Anwendungen. Lengerich: Pabst. 7-29.

Ziegler, E. (2008). Die Rollentheorie aus sozialpsychologischer Sicht. Norderstedt: Grin Verlag.

Zlatkin-Troitschanskaia, O., Beck, K., Sembill, D., Nickolaus, R. \& Mulder, R. (2009). Lehrprofessionalität: Bedingungen, Genese, Wirkungen und ihre Messung. Weinheim, Basel: Beltz Verlag.

Zymek, B. (2008). Geschichte des Schulwesens und des Lehrerberufs. In: Helsper, W. \& Böhme, J. (Hrsg.) (2008). Handbuch der Schulforschung. Wiesbaden: Verlag der Sozialwissenschaften. 


\section{Danksagung}

Die vorliegende Arbeit beschäftigt sich mit Mentoring als einer kooperativen und erfahrungsbasierten Lernform im Rahmen der schulpraktischen Anteile in der Lehrer/innenausbildung. Mentor/innen stehen Studierenden als Begleiter/innen, Berater/innen und Unterstützer/ innen zwischen den theoriebezogenen hochschulischen und schulischen Ausbildungselementen im Berufsfeld Schule zur Verfügung und nehmen in der Wahrnehmung von Mentee und Ausbildungsinstitutionen einen hohen Stellenwert ein (Arnold et al. 2011; Cramer 2012; Bach 2013; Ostermann 2015). Über die Wirksamkeit und insbesondere über die Begleitungs- und Betreuungsqualität werden unterschiedliche Diskussionen geführt (Schubarth et al. 2012; Bach 2013; Reintjes et al. 2018), zumal auch Zielsetzungen, Anforderungsprofile und Modelle der Schulpraktika in der Ausbildungslandschaft divergieren. Die Studie rekonstruiert und identifiziert mittels Grounded Theory Gelingensbedingungen schulpraktischer Mentoringprozesse aus Lehrenden- und Studierendenperspektive und liefert konzeptionelle und praxisrelevante Anhaltspunkte und Impulse für Mentor/innen, Mentee und Ausbildungsinstitutionen.

In der Zeit der Anfertigung der Arbeit unterstützten wertvolle Wegbegleiter/innen - meine persönlichen Mentor/innen - die Umsetzungspläne. Mein besonderer Dank gilt Christian Kraler, der von Anbeginn meine Gedankengänge aufgrund meiner beruflichen Sozialisation in der Lehrer/innenausbildung auf dieses Thema lenkte und mich im weiteren Verlauf stets konstruktiv und fachlich zeitintensiv betreute. Den Befragten danke ich für Offenheit, Zeit und das Vertrauen. Für Gespräche, kritische Anregungen, die Möglichkeit der flexiblen Zeiteinteilung, für Literaturhinweise und Korrekturlesen danke ich Peter Trojer, Regina Brandl, Elisabeth Ostermann, Thilo Grund, Günther Bader, Klaus Greier, Klaus Sonnweber, Hubert Brenn, Stefan Rädiker und Isolde Woolley. Auch meine Familie unterstützte mich in den letzten Jahren und stand dem wissenschaftlichen Forschungsvorhaben wohlwollend gegenüber. Ich bedanke mich bei meinem Mann Wolfgang und unserem Sohn Florian dafür herzlich. 
Die vorliegende Arbeit beschäftigt sich mit Mentoring in der Lehrer:innenausbildung als einer kooperativen und erfahrungsbasierten Lernform im Rahmen der Schulpraxis. Mentor:innen stehen Studierenden begleitend, unterstützend und beratend zwischen den grundlagenorientierten hochschulischen und schulpraktischen Ausbildungselementen im Berufsfeld zur Verfügung. Im Band werden grundlegende Aspekte des Mentorings im Rahmen der Lehramtsausbildung zur Diskussion gestellt und systematisch aufgearbeitet. Darauf aufbauend werden Ergebnisse einer Rekonstruktionsstudie zu Gelingensbedingungen schulpraktischer Mentoringprozesse aus Lehrenden- und Studierendenperspektive dargestellt. Theoretische Überlegungen und empirische Befunde werden in ein praxisbezogenes Modell zu Gelingensbedingungen integriert. Die Arbeit möchte damit einen Beitrag zur aktuellen Diskussion um Mentoring in der Lehrer:innenbildung sowie deren Weiterentwicklung leisten.

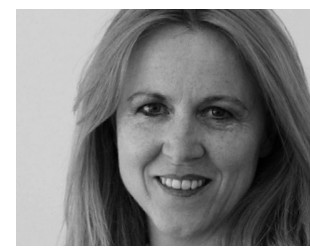

Die Autorin

Elisabeth Haas, BEd Mag. nat. PhD, Jahrgang 1969, Lehramtsstudien Volksschule und Sonderschule, Studium Psychologie, Institutsleiterin für Pädagogisch Praktische Studien und Schulforschung an der KPH Edith Stein (Österreich); Konzeption und Durchführung Hochschullehrgang Mentoring; Forschungsschwerpunkte: Lehrer:innenbildungsforschung; Bildungspsychologie; Pädagogisch Praktische Studien. 